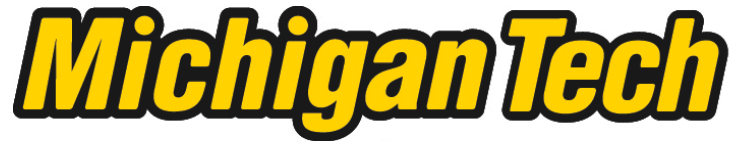 \\ Michigan Technological University Create the Future Digital Commons @ Michigan Tech
}

2014

\section{Nano-engineering of composite material via reactive mechanical alloying/milling (RMA/M)}

Edward Andrew Laitila

Michigan Technological University

Follow this and additional works at: https://digitalcommons.mtu.edu/etds

Part of the Materials Science and Engineering Commons

Copyright 2014 Edward Andrew Laitila

\section{Recommended Citation}

Laitila, Edward Andrew, "Nano-engineering of composite material via reactive mechanical alloying/milling (RMA/M)", Dissertation, Michigan Technological University, 2014.

https://doi.org/10.37099/mtu.dc.etds/949

Follow this and additional works at: https://digitalcommons.mtu.edu/etds

Part of the Materials Science and Engineering Commons 


\title{
NANO-ENGINEERING OF COMPOSITE MATERIAL VIA REACTIVE MECHANICAL ALLOYING/MILLING (RMA/M)
}

\author{
By \\ Edward Andrew Laitila
}

\section{A DISSERTATION}

Submitted in partial fulfillment of the requirements for the degree of DOCTOR OF PHILOSOPHY

In Materials Science and Engineering MICHIGAN TECHNOLOGICAL UNIVERSITY

2014

(C) 2014 Edward A. Laitila 
This dissertation has been approved in partial fulfillment of the requirements for the Degree of DOCTOR OF PHILOSOPHY in Materials Science and Engineering.

Dissertation Advisor:

Committee Member:

Committee Member:

Committee Member:

Department Chair:
Dr. Donald E. Mikkola

Dr. Stephen L. Kampe

Dr. Walter W. Milligan

Dr. Michael E. Mullins

Dr. Stephen L. Kampe 


\section{Dedication}

"To my father, who passed away days after my defense, whom while not formally educated was a very intelligent man, who taught me independence, showing me how hard work pays off, and how to work with my hands........thank you, for building the foundation that lead me to a passion I enjoy, your quick wit, and critical thinking skills rival anyone."

"To a person in my life who I developed such a special relationship with, that most never get to experience in their lifetime...this work is also dedicated to my supervisor, my teacher, my colleague, my advisor, my mentor, and more importantly my friend Dr. Donald E. Mikkola, who also taught me three very important words: think about it!"

"Lastly and most importantly, I finally dedicate this work to my lovely wife, whose compassion and understanding during this long ordeal, allowed me to complete this work as a side part or our life.....without your love, support, and encouragement, this would have never happened." 


\section{Table of Contents}

Approval Page ............................................................................................................................... 2

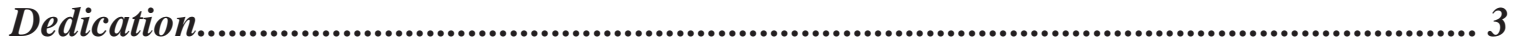

List of Figures ...................................................................................................................................... 4

List of Tables ............................................................................................... 11

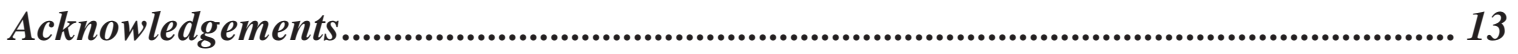

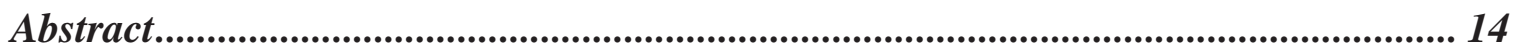

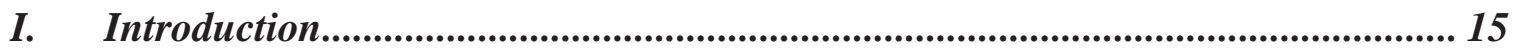

A. Chromium-Modified Titanium Trialuminide............................................................................16

B. Strengthening Mechanisms Investigated ............................................................................. 19

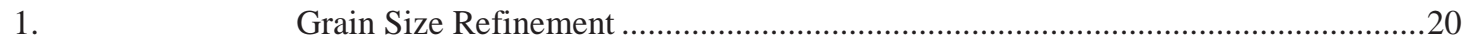

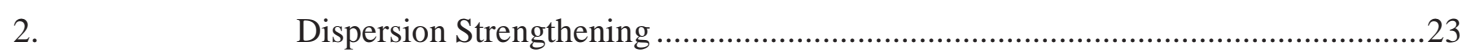

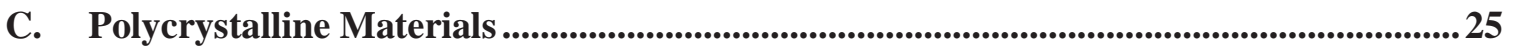

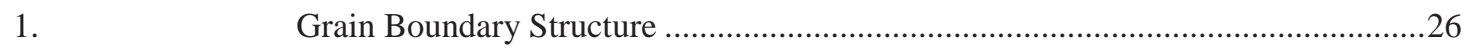

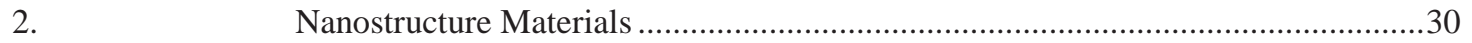

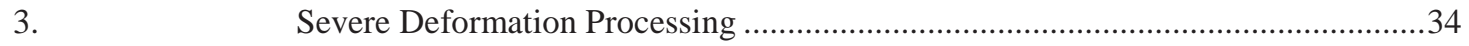

D. Mechanical Alloying.....................................................................................................39

1. Reactive Mechanical Alloying/Milling (RMA/M) ................................................43

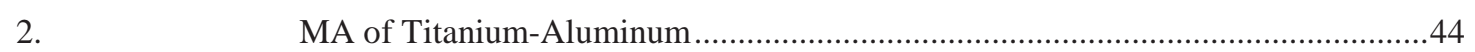

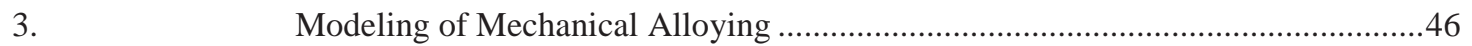

E. Chromium-Modified Titanium Trialuminide Matrix Composites..................................52

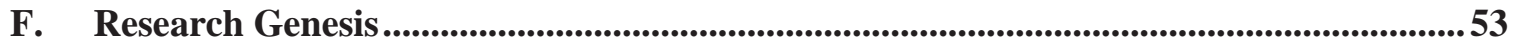

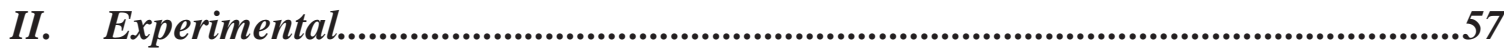

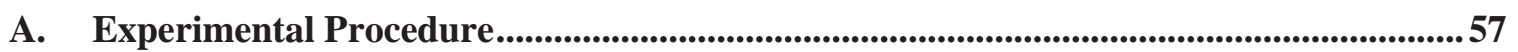

1. Production of Cubic Trialuminide Powder - Reading Alloy ..................................57

2. Production of Cubic Trialuminide Powder - Master Alloy …………......................58

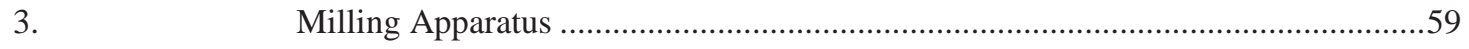

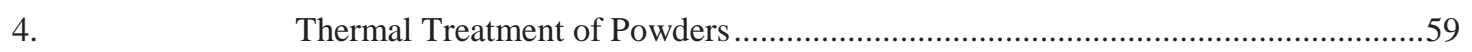

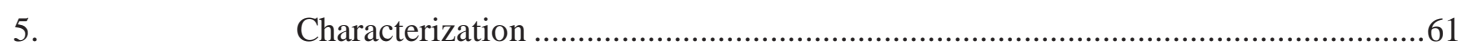

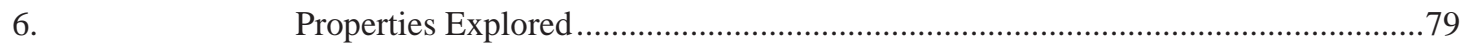

B. Reactive Mechanical Alloying/Milling (RMA/M) Processing Experiments.....................82

1. Reactive Mechanical Milling (RMM) of Pre-Alloyed Powders with Fixed Excess Titanium Addition in an Argon Atmosphere 
2. Reactive Mechanical Alloying (RMA) of Elemental Powders with Excess Titanium in an Argon Atmosphere.....

3. Reactive Mechanical Milling (RMM) of Pre-Alloyed Powders with Systematic Increases in Excess Titanium and RMM Time in an Argon Atmosphere

C. Consolidation of RMM and MM Powders by Cold Isostatic Press (CIP) and Hot Isostatic Press (HIP) Processing ..................................................................................................... 88

\section{Experimental Results of the Powder Processing by Reactive Mechanical} Alloying/Milling.

A. Reactive Mechanical Milling (RMM) of Pre-Alloyed Powders with Fixed Excess Titanium RMMXh9TiAr Series ..............................................................................................93

1.

As-Milled Powders.

B. Reactive Mechanical Alloying (RMA) of RMA20h8.2TiAr Elemental Powders of $\mathrm{Al}_{66} \mathrm{Cr}_{9} \mathrm{Ti}_{25}$ with Excess Titanium in an Argon Atmosphere...................................................133

1. X-ray Power Diffraction Phase Analysis of As-Milled and Annealed Powder.....133

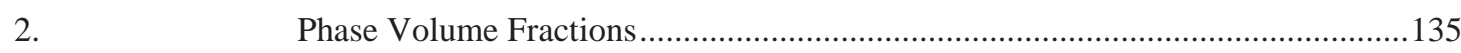

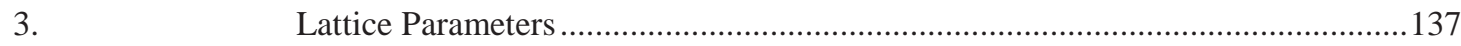

4. Peak Broadening Analysis of Profile Fit X-ray Diffraction Peaks by the WarrenAverbach Fourier Method

C. Results from Reactive Mechanical Milling (RMM) of Pre-Alloyed Powders with Systematic Increases in Excess Titanium in an Argon Atmosphere

D. Results from Consolidated Materials ...........................................................................141

IV. Discussion..............................................................................................188

A. Nano-Scale Polycrystalline Materials Produced by Severe Deformation ..................... 190 1. Structural Characterization of the Nanometer-Scale Powders Produced by the RMA/M Process 191

2. Nano-Scale Grain Boundary Structure ……........................................................217

B. The Reactive Mechanical Alloying/Milling Process .........................................................2232

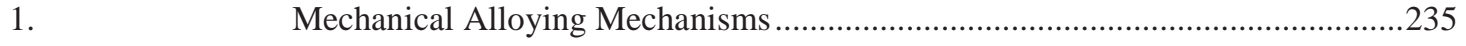

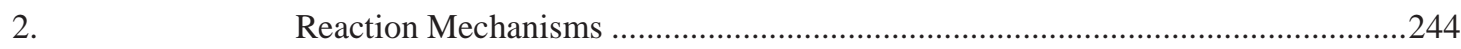

C. Nano-Engineering of Composite Materials or Nano-Engineering of Bulk Consolidated

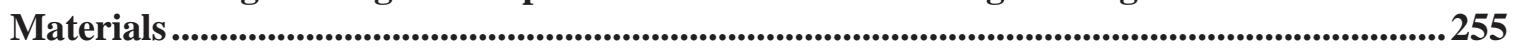

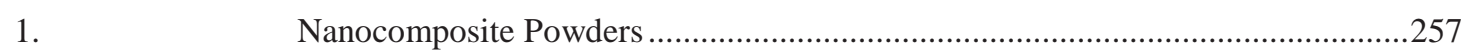

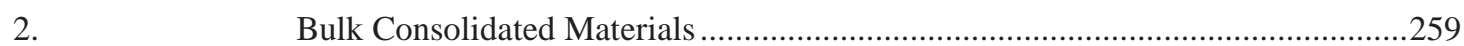




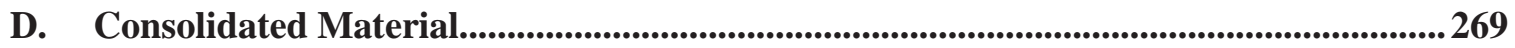

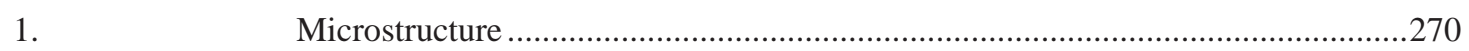

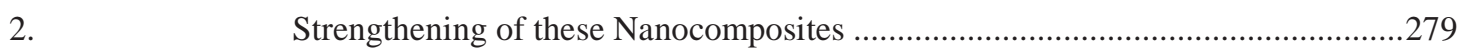

V. Conclusions.................................................................................................284

VI. Potential of this Processing Method............................................................................ 287

VII. Future Work.............................................................................................2290

VIII. Appendix

A. Standard Files................................................................................................................................. 291

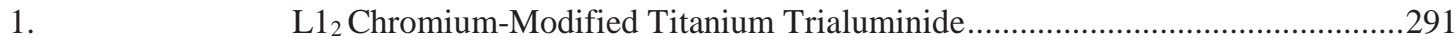

2. L1 ${ }_{2}$ Chromium-modified Titanium Trialuminide ................................................22

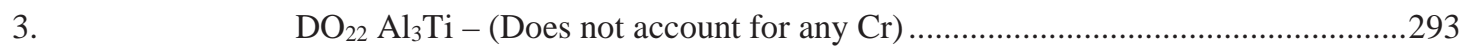

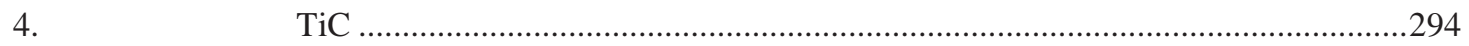

5. TiN

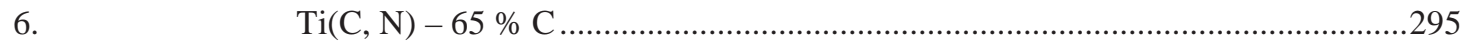

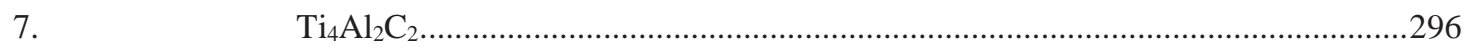

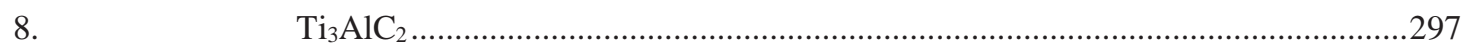

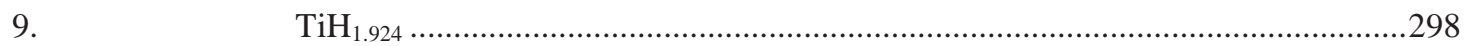

10.

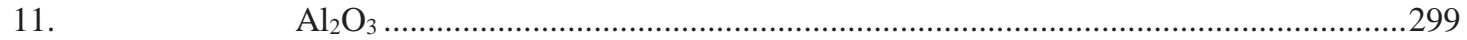

B. Mechanical Milling to Produce Fine-Grain Single-Phase Chromium-modified Titanium Trialuminide ..............................................................................................................301

1. Single-phase Powder Metallurgy $\mathrm{Al}_{66} \mathrm{Cr}_{9} \mathrm{Ti}_{25}$ Alloy ………….............................301

C. RMMXh9TiAr Annealed Powder XRD Analysis.................................................................. 304

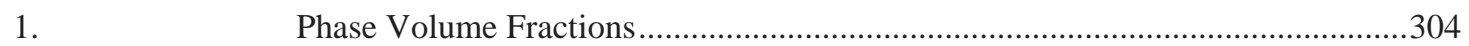

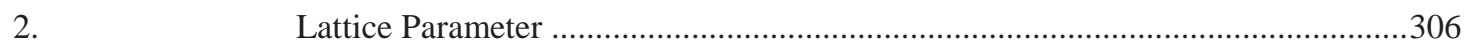

D. Phase Analysis of Consolidated Material.....................................................................307

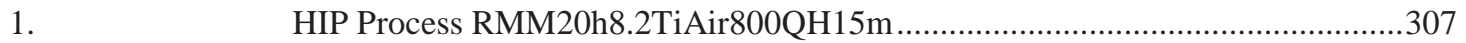

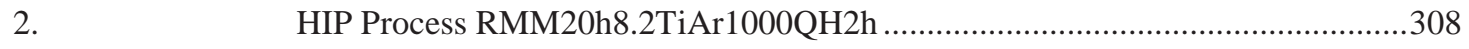

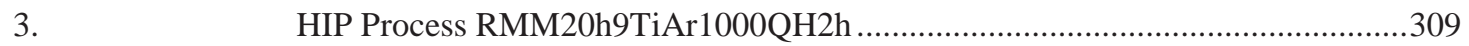

4. Consolidated Materials from Systematic Increases in Excess Titanium and RMM

Time in an Argon Atmosphere High Carbide Content (Long RMM Time Processing in Argon)..........311

E. NGB Calculation for RMA Processing...................................................................317

1. RMA20h8.2TiAr Elemental Starting Powder ......................................................

F. Role of Grain Boundary Structure in Superplastic Deformation....................................320

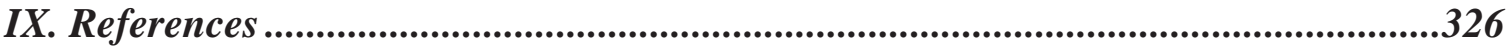




\section{List of Figures}

Figure I-I a) $\mathrm{LI}_{2}$ Crystal Structure, b) $\mathrm{LI}_{2}$ to $\mathrm{DO}_{22}$ Crystallographic Relationship, and c)

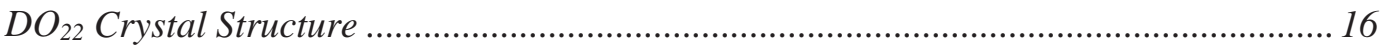

Figure I-2 a) SPEX ${ }^{T M}$ Mill b) Attritor Mill c) Ball Mill ...................................................... 41

Figure I-3 Fundamental Welding/Fracture Ball to Ball Collision Model [99]................. 47

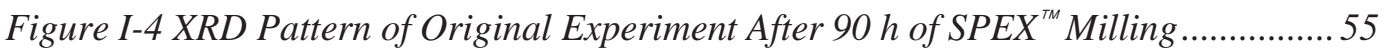

Figure I-5 XRD Patterns of the Original Experiment for 36 h to 90 h MA Times............ 55

Figure I-6 Integrated Background Intensity Increase with MA Time ............................... 56

Figure II-1 Side-Drifted Powder Diffraction Sample Holder.......................................... 62

Figure II-2 Complex Pattern for 40 h RMM Time Sample RMM40h9TiAr..................... 64

Figure II-3 Example of Profile Fitting of the RMM40h9TiAr Sample RMM Time 40 h... 66

Figure II-4 Comparison of Profile Fit and Raw Data for the Crystallite Size Fourier

Coefficients for Copper <100> ..................................................................................... 75

Figure III-1 XRD Pattern for Initial, $0.5 \mathrm{~h}$, and $1 \mathrm{~h}$ RMM Processing Times

(RMMOh9TiAr, RMM0.5h9TiAr, RMM1h9TiAr)_......................................................... 94

Figure III-2 XRD Pattern for 5 h, 10 h, 15 h, 20 h, 25 h, 30 h, and 40 h RMM Times..... 95

Figure III-3 RMMXh9TiAr Series all RMM Samples ...................................................... 96

Figure III-4 XRD Phase ID RMMOh9TiAr Initial Mixture .............................................. 98

Figure III-5 XRD Phase ID RMM0.5h9TiAr RMM Time 0.5 h ..................................... 98

Figure III-6 XRD Phase ID RMMIh9TiAr RMM Time 1 h .......................................... 99

Figure III-7 XRD Phase ID RMM5h9TiAr RMM Time 5 h ............................................ 99

Figure III-8 XRD Phase ID RMM10h9TiAr RMM Time 10 h ....................................... 100

Figure III-9 XRD Phase ID RMM15h9TiAr RMM Time 15 h ...................................... 100

Figure III-10 XRD Phase ID RMM20h9TiAr RMM Time 20 h.................................... 101

Figure III-11 XRD Phase ID RMM25h9TiAr RMM Time 25 h.................................... 101

Figure III-12 XRD Phase ID RMM30h9TiAr RMM Time 30 h................................... 102

Figure III-13 XRD Phase ID RMM40h9TiAr RMM Time 40 h ..................................... 102

Figure III-14 Profile Fit of RMM20h9TiAr RMM Time 20 h........................................ 103

Figure III-15 Volume Fraction for RMMXh9TiAr Series of RMM Times ...................... 105

Figure III-16 L1 $1_{2}$ Intermetallic Lattice Parameter ...................................................... 107

Figure III-17 RMMXh9TiAr Series Warren-Averbach Crystallite Size Analysis ........... 109

Figure III-18 RMMXh9TiAr Series Warren-Averbach Microstrain Analysis ................ 111

Figure III-19 X-ray Powder Diffraction Pattern Background Increase ......................... 115 
Figure III-20 RMMXh9TiAr Series Background Intensity Area for As-Milled and Annealed Samples for all RMM Times......................................................................... 116

Figure III-21 Total Crystalline Diffracted Intensity for the RMMXh9TIAr Series Asmilled and Annealed Samples ...................................................................................... 118

Figure III-22 Particle Size Distribution of RMM0.5h9TiAr As-Milled Powder............. 121

Figure III-23 Particle Size Distribution of RMM25h9TiAr As-Milled Powder.............. 122

Figure III-24 Particle Size Distribution of RMM40h9TiAr As-Milled Powder.............. 122

Figure III-25 Infant Aggolomerate Particle RMM10h9TiAr As-Milled 700X................ 124

Figure III-26 As-Milled Powder Particle RMM0.5h9TiAr 2kX................................... 124

Figure III-27 As-Milled Individual Powder Particles for 10, 25, 40, and 60 h RMM Times (the 10 and 25 h same excess titanium, $40 \mathrm{~h} 2 \mathrm{X}, 60 \mathrm{~h} 3 \mathrm{X}$ amount)............................... 125

Figure III-28 Particle Cavity in RMM1h9TiAr As-milled Powder Particle 13kX.......... 126

Figure III-29 Continued Cavity Closure in RMM25h9TiAr As-millled Powder Particle $8 k X$

Figure III-30 High Magnification Micrograph of Surface Features of As-milled RMM10h9TiAr Powder Particle 15kX. 128

Figure III-31 Surface Smeared Particles Showing Flow Lines in As-milled RMM10h9TiAr Powder Particle 19kX. 128

Figure III-32 Areas of Small Particle Cold Welding and Brittle Fracture Surfaces on Asmilled Particle RMM1h9TiAr 1.8kX. 130 Figure III-33 Crack and Fracture Surface on As-milled RMM0.5h9TiAr Powder Particle $6 k X$ 131

Figure III-34 Cracks and Fractures As-milled Powder Particle of 40 h RMM Time with 16.8 wt. pct. Titanium $4.5 \mathrm{kX}$ 132

Figure III-35 Diffraction Pattern of RMA20h8.2TiAr As-Milled with Identified Phases134 Figure III-36 Diffraction Pattern of RMA20h8.2TiAr Vacuum Annealed with Identified

Phases 135

Figure III-37 Ternary Titanium Aluminum Chromium Phase Diagram Determined from HIPed Materials at $1200{ }^{\circ} \mathrm{C} 172 \mathrm{MPa} 2 \mathrm{~h}$ [16] 137

Figure III-38 As-Milled Systematic Increase in Excess Titanium and RMM Time ........ 140 Figure III-39 Carbon Content Dependence on RMM Time .......................................... 143 Figure III-40 Iron Content Dependence on RMM Time .............................................. 145 Figure III-41 Volume Fraction Dependence of $\mathrm{L1}_{2}$ Intermetallic with RMM Time ....... 150 
Figure III-42 Carbon Content Calculated from XRD Volume Fraction Analysis and LECO Elemental Analysis.

Figure III-43 Crystallite Size Results from the Scherrer and Warren-Averbach Methods as a Function of RMM Time 157

Figure III-44 Microstrain Data in the <111> Direction as a Function of RMM Time.. 158

Figure III-45 TEM Micrograph of Sample 20/1000/MA 50 kX Magnification 159

Figure III-46 TEM Micrograph of Sample 40/1000/RA 60 kX Magnification 160

Figure III-47 TEM Micrograph of Sample 40/1000/RA 80 kX Magnification 161

Figure III-48 TEM Image of Carbide Edge for Sample 40/1000/RA 300 kX.... 163

Figure III-49 FESEM Micrograph of Sample 20/1000/MA.......................................... 165

Figure III-50 FESEM Micrograph of Sample 20/1000/RA .......................................... 165

Figure III-51 FESEM Micrograph of Sample 40/1000/RA ........................................... 166

Figure III-52 FESEM Micrograph of Sample 60/1000/RA ............................................ 166

Figure III-53 Density of Alloys HIPed at $1000^{\circ} \mathrm{C}$ with Increasing Complex Carbide Volume Fraction 172

Figure III-54 Vickers Hardness as a Function of Carbon Content for Samples Consolidated at $1000{ }^{\circ} \mathrm{C}$ for $2 \mathrm{~h}$................................................................................. 175

Figure III-55 Compression Curves for Cr9 and MM3h Samples .................................. 178

Figure III-56 Compression Curves for RMM Samples Consolidated at $1000^{\circ} \mathrm{C}$............ 179 Figure III-57 Changes in Strain Hardening Rate as a Function of the Square Root of the Dislocation Density 181

Figure III-58 Engineering Stress-Strain Curve Sample 40/1000/RA at $900{ }^{\circ} \mathrm{C}$ 182 Figure III-59 Macro Photomicrograph of $900{ }^{\circ}$ C Compression Sample 20/1000/RA (right) and a Untested Sample (left) with Loading Direction Vertical. 183

Figure III-60 Young's and Shear Moduli Change with Carbon Content. 185

Figure III-61 Poisson's Ratio Change with Carbon Content. 186

Figure III-62 CTE as a Function of Volume Fraction of Carbide and Therefore Carbon Content. 187

Figure IV-1Comparison of As-Milled Powder to Annealed As-Milled Powder 192 Figure IV-2 Interference Functions W(r) for interatomic distances. (a) Long-range ordered crystalline structure; (b) short-range ordered amorphous structure; (c) random neither long-range nor short-range ordered structure [160]. 194 Figure IV-3 Corrected Volume Fraction Data for RMMXh9TiAr As-Milled Powders .. 205 
Figure IV-4 Two-Dimensional View of the Spherical Model for an Average Unit Volume of the Matrix - A Fundemental Unit Volume Building Block of the Microstructure....... 213

Figure IV-5 Microstructural Model of As-milled Powder with TiC in Black [] ............. 225

Figure IV-6 Surface Smearing As-Milled RMM Time 1 h ............................................. 238

Figure IV-7 Extrapolated Carbon Uptake with RMM Time …....................................... 259

Figure IV-8 Density Changes with Total Carbide Content ............................................ 262

Figure IV-9 Young's Modulus Change with Total Carbide Content................................ 264

Figure IV-10 Shear Modulus Change with Total Carbide Content ................................ 265

Figure IV-11 Poisson's Ratio Change with Total Carbide Content ................................ 266

Figure IV-12 CTE with Total Carbide Content …......................................................... 267

Figure IV-13 20 h RMM Time HIPed at $800{ }^{\circ} \mathrm{C}$ Sample 20/800/MA ............................. 273

Figure IV-14 Yield Strength as a Function of Grain Size/Crystallite Size for Chromium

Modified Titaniun Trialuminide Samples ...................................................................... 282

Figure VI-1 Iron Carbon RMM 160 h CIP- HIP Consolidated at $950{ }^{\circ} \mathrm{C}$....................... 289

Figure VIII-1 XRD Pattern for the MM3h Sample with Phase Identification ................. 303

Figure VIII-2 L1 Intermetallic Lattice Parameter of Annealed Powders for all RMM

Times. 307

Figure VIII-3 XRD Pattern for HIP Sample 20/800/MA-A with Phase Identification ... 308

Figure VIII-4 XRD Pattern for HIP Sample 20/1000/M with Phase Identification ........ 309

Figure VIII-5 XRD Pattern for HIP Sample 20/1000/RA with Phase Identification...... 311

Figure VIII-6 XRD Pattern for HIP Sample 40/1000/RA with Phase Identification....... 312

Figure VIII-7 XRD Pattern for HIP Sample 60/1000/RA with Phase Identification....... 314

Figure VIII-8 XRD Pattern for HIP Sample 100/1000/RA with Phase Identification .... 315

Figure VIII-9 XRD Pattern for HIP Sample 120/1000/RA with Phase Identification.... 317 


\section{List of Tables}

Table I-1 Yield Strength, Grain Size, and Dispersoid Size of IMC Materials ................. 53

Table II-1 Comparison of Warren-Averbach Results for Copper Profile Fit Data vs Raw

Data 75

Table II-2 RMMXh9TiAr Series Component Weights..... 84

Table II-3 RMMXh9TiAr Series X-ray Diffraction Patameters. 85

Table II-4 RMA20h8.2TiAr Powder Composition and Media Specifications. 86

Table II-5 RMM Processing Parameters. .88

Table II-6 Consolidated Sample Identification and Quick HIP Parameters.... 90

Table II-7 Consolidated Sample Identification and Low Pressure HIP Parameters........ 91

Table II-8 HIP Process Number Sample Codes................................................................ 92

Table II-9 XRD Scan Parameters for Consolidated Material ........................................... 92

Table III-1 Phase Analysis of XRD Patterns for RMMXh9TiAr Series ........................... 97

Table III-2 Volume Fractions of Phases for RMMXh9TiAr Series of RMM Times........ 104

Table III-3 Dislocation Density Estimates for RMMXh9TiAr Series.............................. 112

Table III-4 Crystallite Size Estimates of the TiC Particles.............................................. 113

Table III-5 Particle Size Analysis of RMMxh9TiAr Series of As-Milled Powders ......... 121

Table III-6 Phase Volume Fraction Estimates in RMA20h8.2TiAr As-Milled Powder.. 136

Table III-7 Phase Volume Fraction in RMA20h8.2TiAr Annealed Powder ................... 136

Table III-8 Lattice Parameter Measurements RMA20h8.2TiAr Samples...................... 138

Table III-9 Warren-Averbach Fourier Analysis RMA20h8.2TiAr As-Milled Powder.... 139

Table III-10 Carbon, Oxygen, and Nitrogen Content .................................................. 142

Table III-11 Iron Content in Consolidated Samples........................................................ 144

Table III-12 Phase Analysis of Consolidated Samples RMM in Argon .......................... 147

Table III-13 L1 $\mathrm{L}_{2}$ Intermetallic Lattice Parameter for Consolidated Materials ............... 148

Table III-14 Volume Fractions of Consolidated Samples.............................................. 150

Table III-15 Carbon Content Calculated from XRD Volume Fraction Data and Elemental Carbon Analysis 151

Table III-16 Warren-Averbach Peak Broadening Determination of $\mathrm{LI}_{2}$ Intermetallic. 156 Table III-17 Dislocation Densities for Select Consolidated Samples.............................. 158

Table III-18 Complex Carbide Particle Measurements Consolidated Powders ............ 167

Table III-19 Crystallite Size Estimates of Carbide Phases............................................. 169

Table III-20 Archimedes Density Measurements........................................................ 171 


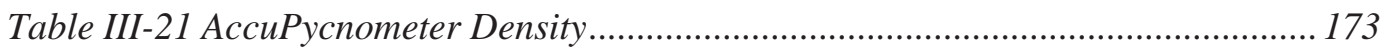

Table III-22 Vickers Hardness Measurements ........................................................... 174

Table III-23 Mechanical Properties at Room Temperature Determined from Compression

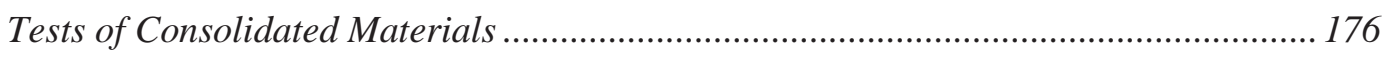

Table III-24 Strain Hardening Rate of Samples HIPed at $1000^{\circ} \mathrm{C}$................................. 180

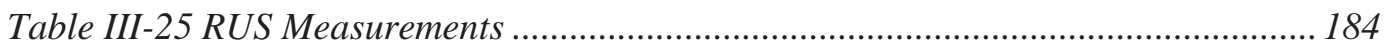

Table III-26 CTE Results Select Consolidated Samples .............................................. 187

Table IV-1 Integrated Background Area Correction ..................................................... 200

Table IV-2 Nano-grain Boundary Phase Volume Fraction within the L1 $1_{2}$ Intermetallic201

Table IV-3 Values for Correction Factor X ............................................................... 203

Table IV-4 Corrected or Actual Volume Fractions in the RMMXh9TiAr Series of As-

Milled Powders 203

Table IV-5 Volume Fractions of Original and Corrected Values for RMA20h8.2TiAr

Sample...... 206

Table IV-6 Spherical Model Parameters for Determination of the L1 2 Unit Volume..... 216

Table IV-7 Boundary Conditions of Properties Based on the Rule of Mixtures.............. 260

Table IV-8 Measured and Calculated Density Comparison ............................................ 261

Table IV-9 Comparison of Measured and Theoretical CTE .......................................... 268

Table IV-10 Results of Dislocation Density Calculations using the Arsenault Equation275

Table IV-11 Complex Carbide Aspect Ratio. 277

Table IV-12 Crystallite Size (Grain Size), Complex Carbide Content, Yield Strength and

Ultimate Compressive Strength of Select Alloys from this Study.................................... 281

Table IV-13 Delta Stress Change Determined from Dislcoation Densities.................... 283

Table VIII-1 Mechanical Milling Process Parameters................................................... 301

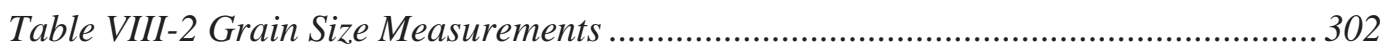

Table VIII-3 Volume Fraction Results for Sample MM3h ............................................ 304

Table VIII-4 Volume Fractions of RMMXh9TiAr Series As-Milled Powders, Degassed, then Annealed at $1000^{\circ} \mathrm{C}$ for Various RMM Times........................................................ 305

Table VIII-5 Data for Calculation of NGB Volume Fraction within Ductile Phases..... 319 


\section{Acknowledgments}

"To my loving and supportive wife who sacrificed so much, my sons, and daughter for sharing all their precious time which allowed me to complete this long journey, I will be forever grateful for their love and support making this all possible. To my advisor, Dr. Donald E. Mikkola, whose scientific help and grammatical abilities, were beyond reproach, and to his wife Sharon whom together their support, guidance, and friendship, have touched all facets of my life. A special thanks to Stephen Forsell, Matt King, Wes Kangas, and Kip Paxton, whom spent enormous amounts of their time providing unbridled assistance and input in the powder processing, that made this work so successful. An expression of thanks also to Dr. James Brusso, Dennis Moore, Owen Mills, Jim Payne, and Dr. Stephen Hackney, for their tremendous help with the characterization of these materials. A significant thanks to my committee composed of Prof. Walter Milligan, Prof. Stephen Kampe, and Prof. Michael Mullins, who all endured this lengthy document, providing their valuable time and exceptional input. Finally, I would be remiss to not thank the wonderful and helpful faculty, staff, and fellow graduate students from the Department of Metallurgy, to the Department of Materials Science and Engineering, all too numerous to mention. Finally, an especial thanks to the many young researchers, that have provided exploratory supporting data to this project, with support provided by the Research \& Engineering Apprentice Program (REAP) funded by the U.S. Army as part of the Army Educational Outreach Program (AEOP), and administered by the Academy of Applied Science. REAP provides a summer internship experience for high school students from underrepresented populations. Financial support was provided by Donald and Sharon Mikkola, the Department of Materials Science and Engineering, and the Research Excellence Fund administered by Michigan Technological University" 


\begin{abstract}
Attempts to strengthen a chromium-modified titanium trialuminide by a combination of grain size refinement and dispersoid strengthening led to a new means to synthesize such materials. This Reactive Mechanical Alloying/Milling process uses in situ reactions between the metallic powders and elements from a process control agent and/or a gaseous environment to assemble a dispersed small hard particle phase within the matrix by a bottom-up approach. In the current research milled powders of the trialuminide alloy along with titanium carbide were produced. The amount of the carbide can be varied widely with simple processing changes and in this case the milling process created trialuminide grain sizes and carbide particles that are the smallest known from such a process. Characterization of these materials required the development of x-ray diffraction means to determine particle sizes by deconvoluting and synthesizing components of the complex multiphase diffraction patterns and to carry out whole pattern analysis to analyze the diffuse scattering that developed from larger than usual highly defective grain boundary regions. These identified regions provide an important mass transport capability in the processing and not only facilitate the alloy development, but add to the understanding of the mechanical alloying process. Consolidation of the milled powder that consisted of small crystallites of the alloy and dispersed carbide particles two nanometers in size formed a unique, somewhat coarsened, microstructure producing an ultra-high strength solid material composed of the chromium-modified titanium trialuminide alloy matrix with small platelets of the complex carbides $\mathrm{Ti}_{2} \mathrm{AlC}$ and $\mathrm{Ti}_{3} \mathrm{AlC}_{2}$. This synthesis process provides the unique ability to nano-engineer a wide variety of composite materials, or special alloys, and has shown the ability to be extended to a wide variety of metallic materials.
\end{abstract}




\section{Introduction}

While exploring a research idea, it is fascinating when the initial results reveal new science or different "paths" leading to new discoveries. An example is the genesis of this research with an initial goal of strengthening a chromium-modified titanium trialuminide intermetallic by the mechanical alloying (MA) process. The solution to the failure of the initial MA attempts, adding a process control agent (PCA), provided a means for processing and enhancing new trialuminide materials. Investigation of long MA times revealed a significant amount of reaction product formed from elements in the metallic powders and the PCA along with significant grain size refinement. Simple processing changes allowed the systematic control of these in situ reactions between elements in the metallic powders, PCA, and gas environment, creating finely dispersed hard ceramic phases. Consequently, a new "path" of investigation was established to determine if this reaction can be controlled by simple process control changes in the MA process, including selecting and creating dispersed phases to alter the properties of the material. Manipulating the nature and content of these dispersed hard particles provides the potential to "engineer" the properties of these composite materials.

Characterization of these materials with nano-scale features required the development of improved x-ray diffraction pattern analysis techniques. The resulting observations provided a new way to view the grain boundary structure in these nanoscale grain size materials. More importantly, an understanding of a unique microstructure produced in these as-milled powders coupled with investigation of the macrostructure of powders has led to a means for understanding the large amounts of mass transport observed in the mechanical alloying/milling process. Important to the original goal, fully dense consolidated materials produced from these unprecedented fine-grain size powders maintained a nanometer scale structure resulting in an ultra-high strength material. The journey to document this exciting processing method and the understanding developed of these deformation nano-scale materials has been long; however, true science has no bounds. 


\section{A. Chromium-Modified Titanium Trialuminide}

Recent developments have improved the ductility of binary $\mathrm{Al}_{3} \mathrm{Ti}$ by ternary alloying with small additions of $4^{\text {th }}$ period transition elements, zinc, copper, nickel [1], iron $[2,3]$, palladium [4], manganese and chromium [5,6]. This occurs because the ternary alloying transforms the crystal structure from ordered tetragonal $\mathrm{DO}_{22}$ to the ordered cubic $\mathrm{L} 1_{2}$ crystal structure [7]. It is very important to note that the $\mathrm{L} 1_{2}$ crystal structure, as shown in Figure I-2 a), is an FCC derivative structure, and that the $\mathrm{DO}_{22}$ crystal structure can be developed by stacking two $\mathrm{L}_{2}$ unit cells and introducing $a$ $1 / 2<110>$-type antiphase boundary (APB) on the (001) plane between the two cubic cells (Figure I-1 b)). Interest in these alloys stems from the large amount of aluminum which results in lower density than common titanium aluminides with expected excellent density-compensated strengths along with excellent oxidation resistance.

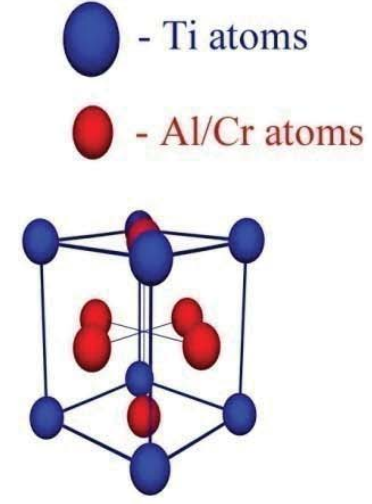

a)

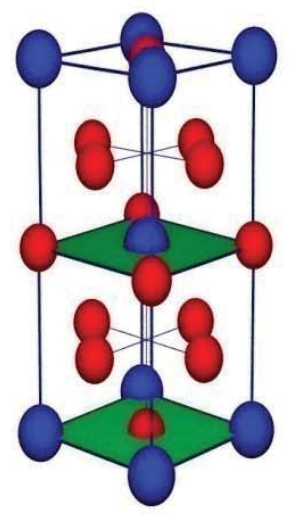

b)

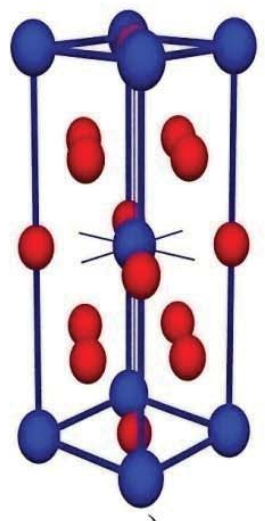

c)

Figure I-1 a) $\mathrm{LI}_{2}$ Crystal Structure, b) $\mathrm{L1}_{2}$ to $\mathrm{DO}_{22}$ Crystallographic Relationship, and c) $\mathrm{DO}_{22}$ Crystal Structure

Of particular interest are the chromium and manganese modified alloys, which have promising amounts of bend ductility compared to the $\mathrm{L}_{2}$ alloys formed by adding other $4^{\text {th }}$ period transition elements. These alloys have shown lower hardness, greatly increased resistance to cracking, and significant ductility in compression compared to the 
other $\mathrm{L}_{2}$ alloys, and the properties are especially attractive compared with the $\mathrm{DO}_{22}$ binary alloy [8]. More importantly, since the FCC structure is close packed it's expected that this atom arrangement contributes to strength to very high fractions of the melting temperature [9]. It should be noted that both the chromium and manganese modified $\mathrm{Al}_{3} \mathrm{Ti}$ alloys were developed at Michigan Technological University by Zhang, Nic, and Mikkola [5].

Of various $\mathrm{L}_{2}$ cubic modified titanium trialuminides $(\mathrm{Al}, \mathrm{X})_{3} \mathrm{Ti}, \mathrm{Nic}$ [7] determined that the $\mathrm{Al}_{66} \mathrm{Cr}_{9} \mathrm{Ti}_{25}$ alloy, referred to as as-cast, exhibited the most desirable and promising mechanical properties, exceeding those of the manganese-modified alloy developed earlier by Zhang [10]. One important comparison to make is that at room temperature the binary $\mathrm{Al}_{3} \mathrm{Ti}$ fails in compression in a brittle manner with a $0.2 \%$ yield strength of $169 \mathrm{MPa}$ contrasted with the compression response of the as-cast alloy of $\sim 19 \%$ ductility and a $0.2 \%$ yield strength of $263 \mathrm{MPa}$ [7]. Further, alloys modified with chromium have shown a small amount of tensile ductility as measured by bend testing, ranging from $0.5 \%$ to $0.9 \%[7,11]$ to as much as $1.25 \%$ with quaternary alloying [11]. Tensile testing of forged $\mathrm{Al}_{67} \mathrm{Cr}_{8} \mathrm{Ti}_{25}$ and $\mathrm{Al}_{66} \mathrm{Mn}_{9} \mathrm{Ti}_{25} \mathrm{Ll}_{2}$ cubic alloys both at room temperature and high temperatures, found zero ductility at room temperature, and $19 \%$ ductility at $800{ }^{\circ} \mathrm{C}[12,13]$. However, these authors reported the existence of $\mathrm{Ti}_{2} \mathrm{AlN}$ and $\mathrm{Ti}_{2} \mathrm{AlC}$ precipitates and dependent on the size (not reported) these phases can be stress concentrators. Additionally, Schneibel et al. [14] reported that the $\mathrm{Al}_{67} \mathrm{Cr}_{8} \mathrm{Ti}_{25}$ alloy was very notch sensitive, while Kumar found $\mathrm{Al}_{67} \mathrm{Mn}_{8} \mathrm{Ti}_{25}$ to be more notch sensitive then $\mathrm{Al}_{67} \mathrm{Cr}_{8} \mathrm{Ti}_{25}$.

Deformation behavior of single crystal $\mathrm{Al}_{66.8} \mathrm{Fe}_{5.8} \mathrm{Ti}_{27.4}$, a two-phase mixture of primarily $\mathrm{L}_{2}$ plus $\mathrm{Al}_{2} \mathrm{Ti}$, was compared to polycrystalline behavior of the same material by $\mathrm{Wu}$ and Pope [15]. The polycrystalline material was more ductile than the single crystal material. This was attributed to a brittle cleavage fracture mode, with the grain boundaries acting to blunt cracks. This suggests that a finer grain size is expected to make the material tougher. Klansky et al. [16] reported the single-phase region for the $\mathrm{L}_{2}$ alloy to be in the composition range of 7-13 wt. pct. chromium, substituted for aluminum, keeping the titanium level at 25 wt. pct. This argued against use of $\mathrm{Al}_{67} \mathrm{Cr}_{8} \mathrm{Ti}_{25}\left(\mathrm{Cr}_{8}\right)$ 
alloys based on the fact that the $\mathrm{Cr}_{8}$ composition is quite close to the single-phase field boundary, with the $\mathrm{Cr}_{9}$ - and $\mathrm{Cr}_{10}$-modified alloys now receiving more attention.

In summary, the chromium-modified $\mathrm{L1}_{2}$ cubic titanium trialuminides have shown good potential for high temperature applications. The $\mathrm{Cr}_{8}$-modified alloy has a moderate room temperature $0.2 \%$ offset yield strength of $259 \mathrm{MPa}$, but still retains $62 \%$ of the room temperature yield strength at $875^{\circ} \mathrm{C}$ [7]. The density of the $\mathrm{Cr}$-9-modified $\mathrm{L}_{2}$ cubic alloy is $3.68 \mathrm{~g} / \mathrm{cm}^{3}$, only $9.5 \%$ more than $\mathrm{Al}_{3} \mathrm{Ti}$, giving these alloys excellent density-compensated strengths [7]. Oxidation resistance of the chromium-modified alloys was evaluated by Parfitt et al. [17] using cyclic oxidation testing at $1200{ }^{\circ} \mathrm{C}$ in air. The $\mathrm{Al}_{67} \mathrm{Cr}_{8} \mathrm{Ti}_{25}$ alloy exhibited much better oxidation resistance than a common $\mathrm{NiAl}$ alloy, and considerably better oxidation resistance than the binary $\mathrm{Al}_{3} \mathrm{Ti}$ alloy. The behavior was comparable to that for a zirconium-doped NiAl alloy. Additionally, Hirukawa et al. [18] showed the superior oxidation resistance of the $\mathrm{Al}_{67} \mathrm{Cr}_{8} \mathrm{Ti}_{25}$ alloy over that of the binary $\mathrm{TiAl}$ at $1000{ }^{\circ} \mathrm{C}$. Further high temperature application support is given by Schneibel et al. [14] who found that the creep properties of $\mathrm{Al}_{67} \mathrm{Cr}_{8} \mathrm{Ti}_{25}$ at $927^{\circ} \mathrm{C}$ are comparable to binary TiAl creep properties at $827^{\circ} \mathrm{C}$.

Because of the excellent oxidation properties, one application of the chromiummodified cubic titanium trialuminide alloy that has been pursued is as a protective barrier coating for oxidation-prone structural materials. Waubik, Inc. has developed, on a commercial basis, thermal spray powders of the chromium-modified cubic titanium trialuminide alloy [19]. Preliminary studies by Dewald et al. [20], have successfully demonstrated the excellent oxidation protection provided to several commercial titaniumbased alloys in extreme temperature cyclic oxidation tests. Several other commercial alloy substrates have been successfully coated using the chromium-modified cubic titanium trialuminide spray powder alloys with excellent adhesion and oxidation protection [19]. Given these desirable properties of the chromium-modified cubic titanium trialuminide alloys, further improvements in the strength, "bulk" properties of the alloy, and wear resistance would be of considerable benefit to expand the potential application possibilities. 


\section{B. Strengthening Mechanisms Investigated}

Structural engineering applications of the chromium-modified titanium trialuminide, referred to as the $\mathrm{L}_{2}$ intermetallic alloy, are limited mainly by the moderate yield strength combined with low tensile ductility. One approach to make these alloys more attractive for a wider variety of engineering applications, despite the low ductility, is to dramatically increase the yield strength. Two traditional methods of achieving an increase in the yield strength and bulk properties of a material are by grain size refinement, giving a Hall-Petch type strengthening, or by impeding dislocation motion with a fine dispersion of hard second phase particles in the matrix commonly referred to as Orowan strengthening [21].

Darken [22] pointed out that the "theoretical strength" of a material, or the stress necessary for pure cleavage (pulling apart adjacent atomic planes) in tension (for a single crystal), is approximately one-tenth of Young's modulus. However, in pure shear, sliding one plane as a whole over another, as generally expected in materials, the "strength" can be estimated as one-thirtieth to one-tenth of the shear modulus. Furthermore, Darken mentioned that the highest tensile strengths of iron whiskers (i.e. material with no dislocations) were observed to be approximately one-fifteenth of shear modulus of the material, in agreement with the theoretical shear strength. The shear modulus for the chromium-modified titanium trialuminide [7] is $75 \mathrm{GPa}$ which corresponds to an approximate theoretical shear strength (at one-fifteenth) of $5 \mathrm{GPa}$, over a magnitude greater than the current yield strength of the polycrystalline material. Darken extrapolated the grain size versus strength behavior for mild steel and determined that for grain sizes of 3 - 4 atom diameters the flow stress agrees with the theoretical shear strength of iron. Importantly, Darken's observations reinforced the notion that the various means for impeding dislocation motion, e.g., grain size and dispersed particles have untapped potential to achieve greater fractions of theoretical strengths.

Once plastic deformation is initiated in large-grained polycrystalline materials, dislocations begin movement along specific crystallographic planes and directions determined by the crystal structure and grain size. This movement continues until 
impeded by some type of crystallographic disruption, which can be in the form of grain boundaries or incoherent hard particles. Impeding the motion results in an increase in the mechanical strength. The basic model of this strengthening is that the pile-up of mobile dislocations at these crystallographic barriers increases the necessary force for continued deformation arising from back stresses developed from the dislocation pile-ups. These forms of strengthening are pursued in this study by decreasing the distance dislocations move with smaller grain sizes and with dispersed incoherent hard particles.

\section{Grain Size Refinement}

The Hall-Petch expression (I.1) [23, 24], describes the fact that a reduction in the grain size of a material decreases the free motion distance of dislocations by a reduction in obstacle spacing. Because the strength of a material is increased by the inverse square root of the grain size, phenomenal strength increases can be observed. In addition to the experimental determination of the relationship, Hall [25] provided an interpretation of the relationship based on the dislocation pile-up mechanism. Cottrell et al. [26] determined that the force on an obstacle from an array of $n$ dislocations is proportional to $n$ times the external stress. Based on this model, and the model by Eshelby et al. [27] of free dislocations piled up against a fixed dislocation, Hall was able to mathematically justify his observations. In support of this observation, grain boundaries are incredibly effective as obstacles to dislocation motion, because they impede dislocation motion along the whole slip plane. As the grain size decreases these very effective barriers to dislocation motion become more numerous increasing the resistance to dislocation motion thereby strengthening the material. Typically as the grain size reduces to the micrometer range dramatic strengthening of the materials occurs [23]. However, when the grain size falls below about a micrometer the slope of the Hall-Petch plot begins to change due to a breakdown in the pile-up model as the dislocation spacings become a significant portion of the grain size.

Petch [28], applied an early form of the well-known Hall-Petch expression to interpret an observation that coarse-grained brittle steels were more prone to brittle fracture than fine-grained brittle steels. Petch was able to model the cleavage strength, or 
plastic strain at fracture, of steels as a function of grain size and found a linear dependence on the inverse square of the grain size. Petch suggested that "Griffith cracks" were identified with those glide planes in which dislocation movement was blocked by grain boundaries. Petch then related the yield point to the grain size, in agreement with the original findings by Hall [28] for the grain size dependence. Petch explained the behavior on the basis that the yield point is associated with a stress concentration produced by a blocked glide plane, as is the case during cleavage of fine-grained materials with the exception that cleavage involves normal stresses and the yield point involves shear stresses. Effectively, yielding during deformation occurs when dislocations in an adjacent grain not favorably oriented are activated by donation, or creation and subsequent emission of dislocations from the grain boundaries, or a dislocation pile-up producing a stress concentration. Darken [22] noted that there are several dislocation strengthening theories that can be used to explain the various observations.

$$
\begin{gathered}
\sigma_{Y . S .}=\sigma_{o}+k_{y} D^{-1 / 2} \quad \text { (I.1) } \\
\sigma_{Y . S .}=\text { the yield strength } \\
\sigma_{o}=\text { friction stress required to move individual } \\
\text { dislocations (also referred to the single crystal stress) } \\
k_{Y}=\text { stress needed to propagate yielding across } \\
\text { polycrystalline grains, friction opposing dislocation } \\
\text { propagation across grains or stress intensity factor } \\
D=\text { grain diameter }
\end{gathered}
$$

Suryanarayana [29] summarized the findings of many researchers related to possible deviations from the Hall-Petch relation, including an inverse response, which may occur for grain sizes below a critical value, usually below about $20 \mathrm{~nm}$. Schulson et al. [30] reported an exponent of -0.8 for the grain size dependence for the yield strength of $\mathrm{Ni}_{3} \mathrm{Al}$ alloys, contrary to most studies on a substantial variety of large-grained polycrystalline materials which agree with the Hall-Petch value of -0.5. Two versions of Hall-Petch models have been proposed, stress concentrations at the tip of dislocation pileups and dislocation network models that assume the slip can be transferred without restriction by grain boundaries. Based on the dislocation pile-up model, Pande et al. [31] 
suggested the grain size exponent can be between 0 and -1 for nanocrystalline materials as opposed to -0.5 for large-grained polycrystals. Further, while suggesting an inverse Hall-Petch behavior is possible, their model was based on assuming the barrier stress in the pile-up model is independent of grain size, citing needed investigation into this assumption.

Choksihi et al. [32] investigated the validity of the Hall-Petch relation in nanocrystalline materials and found materials with grain sizes down to a micrometer grain size adhered to the Hall-Petch relation. However, they observed discrepancies below these grain sizes, and a Hall-Petch plot including grain sizes of 7 and $16 \mathrm{~nm}$ for both copper and palladium gave a negative slope. Thus they cited the first inverse relationship to traditional Hall-Petch data behavior, i.e., using the -0.5 exponent the plots show negative slope as opposed to the normally positive slope. What is important here, controversy exists as to whether the exponent should be changed and/or the $k$ in the relationship modified by incorporating variables [33]. Based on the dislocation network model, Scattergood and Koch [34] modified the Hall-Petch relationship in order to account for deviations from the grain size exponent of -0.5 in nanometer grain size materials in the inverse Hall-Petch relationship; i.e. softening of the material. It should be noted that the modification by Scattergood and Koch only applies to grain sizes below a critical grain size at which this behavior occurs, with this critical grain size between 8 and $65 \mathrm{~nm}$ for the materials studied (copper and palladium both at $21 \mathrm{~nm}$ ). Chang et al. [35] argue that nanometer grain size samples of TiAl with a $96 \%$ density follow an inverse Hall-Petch relationship (negative slope) below a critical grain size of approximately 30 $\mathrm{nm}$ in agreement with Scattergood and Koch estimates.

Current key questions about the applicability of the typical Hall-Petch relationship concern changes to the grain boundary structure and hence grain boundary properties, as well as the existence of grain sizes below which a grain can no longer support dislocations. If a small grain size cannot support dislocations (or a very small number of dislocations) one possible explanation is that diffusional creep and grain boundary sliding [35] play a key role in the plastic deformation. Neglecting relaxation processes at the grain boundary, Nieh and Wadsworth [36] showed a breakdown of the Hall-Petch 
relationship when the grain size is of the order of the spacing of two dislocations, accounting for the critical grain size of $19 \mathrm{~nm}$ in copper and in agreement with work by Scattergood and Koch. Yip and Argon [37] determined "the strongest size" (grain size) for copper at $\sim 12 \mathrm{~nm}$ grain size, by developing a model based on the competition between dislocation mechanisms and grain boundary sliding during plastic deformation. This remains a controversial area for several reasons including the difficulty in creating "bulk" (fully dense) single-phase materials with nanometer grain sizes and the means for detailed characterizations of such fine structures.

\section{Dispersion Strengthening}

The conventional definition of a dispersed second phase or "dispersoid" is an inert, stable, insoluble phase in the matrix. Dispersion-strengthened metals most likely originated with the development of ductile tungsten lamp filaments (see Fink and Coolidge in 1910 [38]). Sintered Aluminum Product (SAP), which utilized reactive aluminum powder in an atmosphere to promote the oxidation reaction, was the next major development in dispersion strengthening in 1946. The process consisted of grinding in an atmosphere to produce an oxide film on $\mathrm{Al}$ powder. The internal oxide of aluminum is processed further by a dry grinding technique to break up the oxide particles and provide a homogeneous distribution of particles. The material is then sintered and hot worked producing a fine dispersion of oxide particles. Another important breakthrough was a chemical codeposition process for making $\mathrm{Ni}-\mathrm{ThO}_{2}$ at $\mathrm{Du}$ Pont which produced fine dispersions ranging from about 5 to $100 \mathrm{~nm}$ in size. Finally, in the 1970's Benjamin developed commercially available oxide dispersion strengthened gamma prime intermetallic nickel base superalloys by a mechanical alloying (MA) process. Murty and Ranganathan [39] reviewed MA over the previous 25 years, a processing method that has grown tremendously, partly because of the wide variety of materials that can be produced by this means. The technique has been developed considerably to refine structures owing to the interest in oxide dispersion strengthened (ODS) materials. Recently, the unique ability to extended solubility and create "amorphous" materials, nanocrystalline materials, intermetallics, and silicides has spurred further interest in MA. Reactive 
milling has received much smaller attention, but has grown from reduction techniques to a fair amount of work on oxide and nitride production by milling elemental powders.

Orowan [40] attributed the strengthening of a material by dispersions of "hard" particles to the bowing of dislocations around the particles, during which a critical value is reached at which point the dislocation breaks free leaving the particles encircled by dislocations in the wake of the motion. The stress field surrounding the particles developed by these encircling dislocations increases the effective dislocation-stopping diameter of the particles during successive dislocation motions. The fundamental equation developed by Orowan (Equation (I.2)) shows that the stress required to produce a critical semicircular shape in the dislocation encountering the particle depends on the inverse of the particle spacing. However, if the dispersoid becomes large, above about a micrometer, this can actually be a weakening mechanism by creating critical-sized flaws in the material. The important point to emphasize is that the resistance to dislocation motion is substantially increased as the spacing of the dispersed phase particles is substantially decreased, thereby increasing the strength of the material.

$$
\begin{aligned}
\tau_{\max } & \cong \frac{G b}{L} \quad(\mathrm{I} .2) \\
G & =\text { the shear modulus of the matrix } \\
b & =\text { Burger vector } \\
L & =\text { distance between particles }
\end{aligned}
$$

The key to creating a substantial increase in yield strength is to utilize a processing method that can produce fine-grain sizes, as well as small dispersoid diameter, and dispersoid spacings, thereby maximizing resistance to dislocation motion. Additionally, the ability to vary and increase the dispersoid volume fraction (while maintaining a small size) will not only increase resistance to dislocation motion, but in the case of large volume fractions the mechanical properties of the dispersed phase will begin to affect the "bulk" properties of the material. The MA process has shown to be very effective incorporating a fine-scale dispersion of second phase particles [39]. However, the latter process can also refine the grain size, which may alter traditional dispersion strengthening when the grain size reaches the order of the dispersoid size. 


\section{Polycrystalline Materials}

Common materials of interest often have polycrystalline microstructures with crystallographic defects such as the grain boundary (GB) between individual single crystal grains. These boundary regions have a higher energy than the crystalline grains and thus materials naturally tend toward large-grains to minimize their internal energy. In fact it is very difficult to create bulk materials with extremely fine-grain sizes particularly by typical thermal processes since diffusion facilitates grain growth. It is especially difficult to create grain sizes on the nano-scale (single digit nanometer size) and the efforts to evaluate the materials are complicated by the difficulties in characterizing these materials.

The model polycrystalline material consists of perfect single crystal grains, with a random orientation. More importantly, the grains must fit together geometrically so no spaces or voids exist (which would add to the internal energy) and are ideally modeled to have a tetrakaidecahedral shape (Lord Kelvin 1887); with the polyhedral facets meeting in intimate contact, fitting together to create a near theoretically $100 \%$ dense material (it should be acknowledged that while this GB volume is extremely small the material density must be just slightly lower than that of a perfect single crystal). The GBs of largegrain polycrystalline materials, those with grain sizes greater than a micrometer, consist

of two basic structures; high angle grain boundaries with special boundaries characterized by coincident site lattice points and low angle grain boundaries bridged by periodic dislocations. The grain boundary at each interface has some atoms that bond, but usually only a small fraction of the atomic density of the grain surface atoms.

A polycrystalline material, with no texture or preferred orientation, can thus be modeled as collection of single crystals with a tetrakaidecahedral shape, with random crystallographic orientations of each polyhedral facet at the grain boundary between two tetrakaidecahedron grains. The grains are held together by GB energy, with the boundary considered only a couple atomic layers thick [41], which varies in cohesiveness from material to material (and also with the type of boundary). An important point is that provided the grains are in a random crystallographic orientations the properties are 
generally isotropic in nature. The grain size is a very important microstructural feature because of the changes to the properties that can occur, such as the well-known HallPetch relationship described earlier.

Traditionally, materials with grain sizes above a micrometer are classified as large-grained materials. The second classification is for grain sizes in the sub-micrometer range often called ultra-fine grained materials (UFG). Finally, the last traditional category is the nanometer grain size materials, those materials with grain sizes or features of $100 \mathrm{~nm}$ or less (often the lower limit is considered to be about $2 \mathrm{~nm}$ ). In this work materials with grain sizes below $10 \mathrm{~nm}$ will be designated as nano-scale to separate this range from the more general classification for nanometer grain size materials. It should be noted that nanometer grain size materials have also been referred to as nanostructured or nanocrystalline materials, and other terms, such as nanoscale, nanophase, and ultrafine grained have also been used [42]. Of importance to the current work is the structural nature of the GBs and the changes to this structural as the grain size decreases, particularly to the nano-scale range.

\section{Grain Boundary Structure}

In general, materials can have different GB strengths and a GB energy that varies widely based on the GB structure [43]. This natural adhesive force (GB strength) holds the polyhedra together in a fully dense fashion. Grain boundary structures have been well documented for large-grained polycrystalline materials. Of particular interest to the current work is that as the grain size decreases into the sub-micrometer range properties that are typically structurally-insensitive begin to change, and as will be discussed these changes can be attributed to significant changes in the GBs. Further, it will be argued that as the grain size decreases toward the nano-scale range, these structural changes of the GBs continue, aspects of which will be defined by the current research.

In a typical large-grained polycrystalline material, the GBs have crystallographic order and structures consistent with the grains, with the only constraints being those needed to interface different crystallographic faces of grain facets with a boundary plane $[43,44]$. Consider two perfect crystal surfaces in contact that have different orientations, 
the atoms on each surface will be much too close or too far from their neighbors on the other side of the boundary compared to their equilibrium positions. Thus the atoms move to new positions (based on the energy atoms can even be removed) causing elastic strains a few lattice spacings away from the boundary [45]. Thus the GB is a transition between the two orientations which can be described as a radical readjustment of the bonds across the boundary and a thin layer of elastic distortions on either side of the boundary. More importantly, an infinite number of different grain boundaries can be formed depending on the orientation relationship between the two adjacent grains.

As mentioned, these boundaries have been well characterized in large-grained materials and classified into two fundamental types' low-angle grain boundaries (LAGBs) and high-angle grain boundaries (HAGBs). The structure of LAGBs is based on closely spaced dislocations with a spacing that depends on the Burgers vector and inversely on the orientation angle between the planes. There are two basic types of LAGBs based on the dislocations comprising the boundary: pure tilt composed of parallel edge dislocations and pure twist a cross grid of two types of screw dislocations. However, in general LAGBs can be a mixture of the two types. By definition the boundary planes of each grain facet start out having the same exact crystallography, but dislocation accumulation in the boundary (e.g. as a result of plastic deformation) can alter the orientation relationship between each grain facet. This angular orientation increases until the dislocation spacing reaches a limiting minimum distance between dislocation cores. This limitation typically occurs at an orientation difference of about $10^{\circ}-15^{\circ}$ [46], beyond which the energetics induce a transformation to the HAGB structure.

HAGBs consist of two types, general (or random) and special (or ordered). The general HAGB is thought to be composed of a random array of GB dislocations, having much smaller Burgers vectors than lattice dislocations, with this irregular pattern connecting the boundary. The irregular nature of the dislocations in general HAGBs creates a large free volume in the boundary, thus they typically have large GB energies. The structure of these general HAGBs often observed in very large-grained polycrystalline materials have not been studied as extensively as the more ordered grain boundaries [47]. 
Generally it has been shown that as the degree of order at the crystallographic interface increases, the free volume decreases, which can lower the GB energy as much as two orders of magnitude. The more ordered HAGBs are based on the concept of the coincident site lattice (CSL), a superlattice that forms based on coincidence of atoms from the corresponding crystallographic arrangements on both sides of each boundary. It is generally accepted that the concept of the CSL is important to understanding the structure of these special HAGBs, but it is merely based on geometry. For example, a CSL can result when two identical crystal lattices are rotated about a common crystallographic axis by some angle; so that several lattice points are coincident from both lattices and form a three-dimensional superlattice called the CSL. The CSL concept evolved to a more general O-lattice theory developed by Bollmann [44], with an important concept developed from the O-lattice theory the displacement shift complete (DSC) lattice that accounts for small deviations from a perfect lattice coincidence orientation. The importance here is that these descriptions of GBs are geometrically based and maintain some crystallographic relationship between the grains, and therefore periodically tie the two lattices together. These various models of the GB structure of large-grained polycrystalline materials have been proposed to describe the interface by geometric means, but more importantly, this can describe the changing free volume of the boundary. Additionally, intrinsic dislocation densities vary with the periodicity of the CSL boundaries. Essentially all of these considerations have pointed to an ordered crystalline superstructure common to each plane at the interface, resulting in various cohesive strengths, with all GBs existing at all temperatures at which the solid state is stable.

Simplistically, the CSL boundary is identified by the expression $\Sigma X$ where $X$ is an integer; the integer value is the number of coincidence sites in an elementary cell divided by the total number of all lattice sites in an elementary cell. A common upper limit used in GB identification is a CSL value is 541 . The CSL model details the geometry of the GB structure, not the energy of the boundary, which is much more elusive in regards to the physical reasoning for the different GB energies. One parameter important to GB energy is the free volume which scales well with the boundary energy. The geometrical 
basis for classifying the CSL has led to predicting the free volume, often providing good estimates of the GB energy.

As mentioned, lower values for the $\Sigma X$ in CSL boundaries will have a large fraction of coincident site atoms compared to the total atoms on the surface of each grain facet. Of interest is the increase in the percentage of CSL type boundaries with grain size reduction. For example, measurements by Watanabe [48] have shown a saturation of nearly $100 \%$ of the GBs of the CSL type when the grain size approaches a micrometer, with an apparent minimum of $13.3 \%$ of the GBs as CSLs for larger-grained materials. Watanabe attributed this change from large fractions of general HAGBs at larger grain sizes to more CSL type GBs at smaller grain sizes to the greater reduction in the GB energy as the surface area of the grains increases with the smaller grain sizes.

Work by Valiev's group [49, 50, 51] has suggested another type of GB structure with peculiar properties; that has been termed a "nonequilibrium grain boundary" (NEGB). This new type of GB structure was first observed in bulk samples created by severe plastic deformation once the grain size became sub-micrometer in size. This NEGB structure is identified by the presence of more dislocations than deemed crystallographically necessary to maintain compatibility across the GB; i.e., presence of extrinsic GB dislocations. Further, materials with NEGB show elastically distorted regions resulting from high dislocation densities near the boundaries. Importantly, materials with the NEGB structure have shown significant changes to properties that are typically considered insensitive to changes in the microstructure, such as Young's modulus, Curie temperature, saturation magnetization, and Debye temperature, all which decrease, while the diffusivity and solubility both show increases compared to largegrained materials $[52,53]$. These property changes as the grain size is reduced are indicative of changes to the atomic-scale crystal structure in the boundary regions as well as the effects of associated strain in the exterior of the grains. The structural changes over a larger volume of material will have more influence on the overall properties, expected as the grain size decreases further. Additionally, dramatic reductions in the temperature for initiating superplastic behavior have also been observed in these materials. 
Many materials begin to show the NEGB structure with grain sizes that approach the traditional "nanocrystalline size" range of materials, approximately $100 \mathrm{~nm}$ or less. Valiev's group adds that the GB energy is expected to increase based on the observed extrinsic GB dislocations present in this new GB structure of sub-micrometer to nanometer grained materials. Additionally, many studies of materials with NEGBs show regions of the GBs that appear disordered in nature with no visible periodic structure; of interest to the current work is the source of these disordered regions. Also important to the current work is the observation that the GB structure changes as the grain size decreases down to the upper nanometer range.

\section{2. $\quad$ Nanostructure Materials}

Gleiter [42] introduced the basic concept that nanocrystalline (nanometer size) materials (also called nanostructured materials) represent a new class of materials as a result of the high density of defect cores in the grain boundaries, with up to $50 \%$ of the atoms situated in the cores of these defects when grain sizes are in nano-scale range. Gleiter theorized that unique properties should result because a large fraction of atoms are within the GBs of nanocrystalline materials. Recall that deviations from the traditional Hall-Petch relationship or inverse Hall-Petch relationship are often reported for nanocrystalline materials, below some critical grain size [29]. As expected, the arrangement of this large fraction of atoms within the boundary regions is controversial with one camp suggesting the GB structure is similar to that of large-grained polycrystals while the other argues that the structure is different and more disordered than in largegrained polycrystals. These structure questions have been further investigated in the current work by developing x-ray powder diffraction methods to probe the structures produced in nano-scale materials.

Clearly, much interest has been generated in bringing materials into nonequilibrium states for processing since the normal thermodynamics and kinetics favor larger grain sizes to reduce surface energy. These nonequilibrium processing techniques provide additional means for manipulating the structure of materials, and the new properties developed with these nanocrystalline materials are thought to be due in part to 
the large fraction of atoms associated with the grain boundaries. The grain size range of sub-micrometer to upper nanometer materials have clearly exhibited changes in various structurally insensitive properties; therefore, materials in the true nano-scale grain size range would be expected to further enhance these property differences.

In general, nanometer grain size materials are considered as having at least one dimension in the $1-100 \mathrm{~nm}$ range [29]. However, structural characterization of materials with grain sizes in the nano-scale range is often lacking, in part because of the difficulties in observing these sizes, as well as in creating fully dense materials for study. More specifically, creating these nano-scale materials commonly requires bringing the material into nonequilibrium states during the processing. This can be approached in two fundamental ways; the top-down approach such as refining large grain sizes, or the bottom-up approach such as nucleation of critical size clusters of the phase of choice. The basic refinement technique utilized in the current research is mechanical alloying which has been used to create nano-scale grain sizes, by a top-down approach. Contrarily, but simultaneously, a reaction can occur during the mechanical alloying process that results in the formation of a ceramic phase (controlled by milling parameters), thus a bottom-up creation of nanoparticles of carbides. As will be shown the grain sizes of powders created by this reactive mechanical alloying procedure are in the $2-3 \mathrm{~nm}$ range, a range of grain sizes not as well characterized in the literature.

The top-down approach with mechanically alloying typically results in a limited crystallite size limit achieved after long milling times. Often, pure elemental materials mechanically alloyed have much larger limited crystallite size limits, around $10-20 \mathrm{~nm}$, whereas alloys and compounds have much smaller sizes, even extending into the nanoscale range. In the current work, it will be shown that the small crystallite sizes achieved in the $\mathrm{L}_{2}$ intermetallic are similar to the expected critical size nucleus of metals during homogeneous solidification, which appears to be a merging of the top-down and bottomup approaches, using a simple mechanical alloying process. Often the limits of the topdown approach, such as with pure elements, are larger than those achieved by the bottomup method, such as in solidification processes or reaction products. As an example, platinum catalysts are often formed by the bottom-up approach with the platinum-oxide 
colloidal method yielding crystal sizes of the order of $2 \mathrm{~nm}$ [54], whereas mechanically alloyed platinum alloys produced a minimum crystallite size of $15 \mathrm{~nm}$. However, it must be noted that these considerations also depends on mill energy [55].

One very interesting aspect of nanocrystalline materials is an increase in background intensity reported from x-ray diffraction experiments. Gleiter et al. [56], found an increase in the background intensity in nanocrystalline iron compared to a largegrained material. Their findings described a background intensity function that was smoothly decreasing with diffraction angle, but with an increased intensity throughout the $2 \theta$ range. They attributed this behavior to a "gas-like" structure of the atoms in the grain boundary regions of this material. This "gas-like" structure of the atoms, implying a random displacement of the atoms from ideal lattice positions, although used to describe the atomic arrangement, was not implying the atoms have the mobility (or that the arrangement has the density) of a true gas. The volume fraction of this "gas-like" structure of the atoms in the grain boundaries was estimated to be $50 \%$, based on volumes calculated using a $6 \mathrm{~nm}$ grain size assuming a $1 \mathrm{~nm}$ boundary thickness. In 2002 Suryanarayana [57] discussed the GB structure changes in nanocrystalline materials pointing out two schools of thought, one suggesting there is a change in the structure and the other that the structure is the same as with large-grain polycrystals, and stating that the latter has gained widespread acceptance. The analysis of mechanically alloyed powders in the current study, which creates nano-scale grain size polycrystalline particles on the order of ten micrometers in physical size, will address this and other issues providing new insight into the change in structure of GBs in materials with grain sizes approaching the nano-scale range.

\section{a) Diffusion in Nanostructured Materials}

Substitutional solid state diffusion in polycrystalline materials can be described by three basic mechanisms; lattice diffusion, GB diffusion, and surface diffusion. The diffusivity, $D$, of a material is dependent on these diffusion mechanisms and for a given temperature the magnitudes of the diffusivity along a free surface $\left(D_{s}\right)$, the GB $\left(D_{g}\right)$, and the lattice $\left(D_{l}\right)$ can be related as follows: 


$$
D_{s}>D_{g}>D_{l}
$$

Important in this description is the fact that the rate of surface diffusion is orders of magnitude greater than that of GB diffusion which is orders of magnitude greater than lattice diffusion. Conversely, the slope of the diffusivity with temperature is greatest for lattice diffusion, lower for grain boundary diffusion, and least for surface diffusion. In fact at low temperatures surface diffusion dominates, at mid-temperatures grain boundary diffusion dominates, and at high temperatures (typically $\mathrm{T} \geq 0.5 \mathrm{~T}_{\mathrm{m}}$ ) lattice diffusion dominates. Important to this study is that in large-grained polycrystalline materials an extremely small volume of the material is associated with the atoms that reside on the GBs rendering this diffusion mechanism extremely small on a volume basis compared to lattice or bulk diffusion. However, as the grain size decreases the volume of GB atoms becomes more significant in relation to the volume of lattice atoms affecting the diffusional process.

The Gleiter group [58] investigated diffusion in a nanocrystalline material, reporting that the diffusivity of nanocrystalline copper ( $8 \mathrm{~nm}$ grain size) was found at $353^{\circ} \mathrm{K}$ and $393{ }^{\circ} \mathrm{K}$ to be fourteen and sixteen orders of magnitude, respectively, larger than in bulk self-diffusion and about three orders of magnitude larger than with GB selfdiffusion in large-grained polycrystalline copper. The diffusivity of silver in copper (8 $\mathrm{nm}$ grain size from 303 to $373^{\circ} \mathrm{K}$ ) was also found to be two to four orders of magnitude faster than that for diffusion of silver in GBs of copper bicrystals, taken to be representative of large-grained polycrystals [59]. It is important to recognize that lattice diffusion in large-grained copper typically occurs above $\sim 678{ }^{\circ} \mathrm{K}\left(0.5 \mathrm{~T}_{\mathrm{m}}\right)$ with nanocrystalline materials also showing a significant reduction in diffusion temperature. These much larger than expected diffusivities, compared to that of typical GB diffusion in large-grained polycrystalline materials, adds support to the structural changes in the GBs of nanocrystalline materials compared to grain boundaries in large-grained polycrystalline material. 


\section{Severe Deformation Processing}

In severe deformation of materials the grain size can be reduced by deformation down to the to the nanometer regime. The grain refinement and mechanism of nanostructure formation has been described by Hellstern et al. [60] in mechanical milling of the AlRu intermetallic in a SPEX ${ }^{\mathrm{TM}}$ mill, based on TEM observations. It was noted in the early stages shear bands were observed in the internal microstructure of these powder particles due to the high deformation rates. These inhomogeneous shear bands had a large dislocation density with typical widths of $0.5-1.0 \mu \mathrm{m}$ creating grains of 8-14 nm in size within these regions. With continued milling the increased dislocation density leads to increased atomic level strains, and at a certain density within these heavily stained regions the crystal degenerates into subgrains that are separated by LAGBs, decreasing the lattice strain. After long milling times the material consists of only small grain sizes of 5 - $7 \mathrm{~nm}$ separated by HAGBs. Of particular importance is the completely random orientation of neighboring crystals indicating significant grain rotation as the GBs change from LAGB to HAGB as the deformation process proceeds. The current research will present another possible mechanism of grain refinement.

This grain refinement journey by deformation is expected to take the material through all the various deformation mechanisms and the reasons for these changes appear to be the relationship of the grain size with the dimensional effects of the deformation. The deformation in large-grained polycrystalline materials has been well established based on the motion of perfect dislocations that are typically nucleated in the grain interiors with minimal interaction with the GBs. This behavior continues down to about a micrometer in which case the deformation mechanism begins to changes.

Similarly, the Hall-Petch behavior only appears to be valid down to a micrometer grain size. Recall, the deformation mechanism in the Hall-Petch behavior is based on pile-ups at GBs which breaks down below about a micrometer indicating a mechanism change. The concept of dislocation pile-ups at GBs is taken to be applicable to largegrained polycrystalline materials with dislocations produced by Frank-Read sources operating in the grain interiors. Additionally, for these pile-ups to occur large grain sizes

are required as exemplified by the change in the slope of Hall-Petch relationship below a 
micrometer in grain size. Calculations of dislocation spacings for an iron-silicon bicrystal based on this model determined that the first and second dislocation spacing was between 2 and $14 \mathrm{~nm}$ while the distance for the $6^{\text {th }}$ dislocation in a pile-up was already at over $200 \mathrm{~nm}$ from the boundary, while for screw dislocations the $13^{\text {th }}$ dislocation in the pile-up is over $300 \mathrm{~nm}$ from the first [61]. This emphasizes that these sub-micrometer, and or smaller, sizes begin to have an effect on the nature of typical dislocation pile-up mechanism fundamental to the Hall-Petch relationship for large-grained materials.

As the deformation proceeds in polycrystalline metals the stress required to induce Frank-Read source operation increases, and the dislocation pile-ups distances become a significant fraction of the grain size. This causes changes in the deformation behavior as has been observed by the formation of a dislocation cell structure, particularly in FCC materials, within the grains having a cell size around the micrometer range to tenths of a micrometer. In cold-rolled aluminum the dislocation cell structure sizes developed are 1-2 $\mu \mathrm{m}$ [62]. Copper and copper alloys cold rolled to a $30 \%$ reduction have an average dislocation cell structure size of about $0.8 \mu \mathrm{m}$ [63]. Whereas severe deformation by shock loading and rolling of large-grained Mo-33\% Re alloy both showing similar development of a dislocation cell structure that reaches a minimum of about $0.4 \mu \mathrm{m}$ as determined by TEM [64, 65].

It is important to note that during deformation by cold-rolling grain rotation is often observed induced by the materials response to the severe deformation. Bulk materials can also be produced with sub-micrometer grains by severe plastic deformation (SPD) methods such as equal channel angular pressing (ECA), high pressure torsion (HPT), with very localized grain refinement from ball drop test, particle impact deformation, drilling, sliding wear, and shot peening [66]. The degree of deformation is loosely related to the amount of grain refinement observed from the deformation process. As such it has been noted that the ECA and HPT methods can also produce bulk materials in the lower sub-micrometer to upper nanometer grain size ranges. Methods such as wear processes, mechanical alloying, and surface deformation treatments can develop crystallite sizes in the lower nanometer range. In a study of stacking fault formation and twinning during SPD of FCC materials, analysis of experimental data, also 
confirmed by molecular-dynamic (MD) studies of nanocrystalline materials, determined that partial dislocations (stacking faults, twinning) dominated deformation mechanisms in these materials near the upper nanometer grain size [67].

Aluminum magnesium alloys deformed by HPT developed grain sizes from $120 \mathrm{~nm}$ to $55 \mathrm{~nm}$ for the highest magnesium content alloy [68]. Identified by HRTEM were low-angle, high-angle, equilibrium and non-equilibrium grain/subgrain boundaries, twin boundaries, stacking faults, full dislocations, and dipoles. Additionally, microtwins of about $1 \mathrm{~nm}$, or about 4 atomic layers, thick were also observed in the smallest grains. Three regions of deformation mechanisms were identified: 100-1000 nm grain sizes where traditional dislocation mechanisms dominate plastic deformation, intermediate nanometer regime of 10-100 $\mathrm{nm}$ where dislocation source operation and pile-ups are not expected to occur with normal slip replaced by partial dislocation activities and with deformation speculated to be initiated by GBs; and finally below $10 \mathrm{~nm}$ deformation is assumed to be controlled by GB processes such as grain rotations and grain boundary sliding. Finally, this study cited a high density of extrinsic $60^{\circ}$ dislocations frequently encountered in the form of dipoles at NEGB and grain/subgrain boundaries. Valiev et al. [49] have determined that the NEGB structure is indicative of a large number of extrinsic dislocations that are not geometrically necessary to maintain crystallographic compatibility across the GB. A relationship between extrinsic dislocations and the GB structure will be discussed later.

One method that appears to produce significant and severe deformation, similar to that obtained in the mechanical alloying process for long times, is the simple filing of metals, a singular event, creating a defect structure with crystallite sizes for some FCC materials as low as $3 \mathrm{~nm}$ with microstrains as large as $0.70 \%$, and approaching dislocation densities near those that will be reported in the current work [69]. This deformation process is very simple yet puts an incredible amount of defects into the material in a single deformation event unlike that observed in the MA process which can take many hours to achieve similar sizes on a homogeneous basis. The amount of deformation in a single filing event is fascinatingly large (based on the small crystallite size) and is also similar to the large amounts of deformation observed in wear debris and 
transfer layer produced from sliding wear processes [70] which can have similar crystallite sizes. Recall that a crystallite size of about $3 \mathrm{~nm}$ in both processes approaches the lower limit of the grain size observed in polycrystalline materials.

Youngdahl et al. [71] investigating deformation in a nanocrystalline copper during in situ deformation in the TEM found that dislocations were only seen passing through grain sizes around $30 \mathrm{~nm}$ or less with no signs of interior lattice dislocations. They also observed that there were a large number of partial dislocations producing stacking faults along with frequent observation of twins. In studies at MTU of in situ straining in the TEM of $10 \mathrm{~nm}$ and $110 \mathrm{~nm}$ grain size thin films of silver and gold, respectively, dislocations were observed both during and after in situ straining activity in the $110 \mathrm{~nm}$ grain sizes, whereas with the $10 \mathrm{~nm}$ grain size film no dislocations were observed either in situ or after straining [72]. Also, inhomogeneous grain rotation occurred in the $10 \mathrm{~nm}$ grain size film and fracture was intergranular, while fracture in the $110 \mathrm{~nm}$ grain size film was transgranular.

Swygenhoven et al. [73] in molecular-dynamic studies of deformation of nickel and copper nanostructure materials with grain sizes in the range of $3-12 \mathrm{~nm}$ observed a change in deformation mechanism with all deformation accommodated in the GBs for the smallest grain sizes. This fundamental change in deformation mechanism could reflect the inverse Hall-Petch behavior for grain sizes in the nano-scale range. Moleculardynamic analysis of the mechanical deformation of nanometer grain size aluminum shows this inverse Hall-Petch effect below $10 \mathrm{~nm}$ grain size with GB sliding dominating the plastic deformation [74]. In computer simulations investigating the softening during deformation of nanocrystalline metals at very small grain sizes significant GB sliding occurred with occasional partial dislocations nucleated at the GB traveling through the grain [75].

In the deformation of nanocrystalline nickel grown as thin films, with grain sizes ranging from several nanometers to $23 \mathrm{~nm}$, a change from partial dislocation motion to a GB-mediated plastic deformation occurred as the grain size decreased [76]. Specifically, this study determined that for the $10 \mathrm{~nm}$ grain size nickel films GB-mediated processes became the dominant deformation mode. This change in deformation mode with grain 
size illustrates the competition between deformation controlled by nucleation and motion of dislocations and deformation controlled by diffusion-assisted GB plasticity. These comments agree with the conclusions by Argon and Yip [37] discussed earlier who created a fundamental deformation model that determined "the strongest size" in copper to be $\sim 12 \mathrm{~nm}$ as the point at which both deformation mechanisms, GB-mediated and dislocation plasticity, have an equal contribution, with GB-mediated deformation dominant at the smaller sizes. The inverse Hall-Petch behavior has been attributed to the domination of GB-mediated deformation; however, it is important to state that there is still controversy related to the existence of this inverse behavior.

Milligan summarized deformation behavior in bulk nanocrystalline and ultrafinegrained metals and classified the mechanical behavior of FCC metals into four grain size regimes [77]. In the finest grain size class, "Nano-1", only GB-mediated processes are active with no lattice dislocations being observed in TEM. Below a grain size of about 10 $\mathrm{nm}$ the strength remains constant or decreases, in agreement with the "strongest size" by Argon and Yip [37]. The "Nano-2" grain sizes of about $20-30 \mathrm{~nm}$ deformation is dominated by partial dislocations that only pass through the grains. Grains are sheared by Shockley partials which are absorbed by the GB leaving a stacking fault behind. The third regime is the "Ultra-fine" extending from grain sizes of about $30 \mathrm{~nm}$ to $1 \mu \mathrm{m}$ in which plastic deformation proceeds mainly by dislocation nucleation from GB sources with a tension-compression asymmetry observed in strength behavior. This grain size regime shows increased strengths with smaller grain size; however, reasonable ductility is typically only found in grain sizes in excess of $100 \mathrm{~nm}$. Finally, the "Traditional" regime for grain sizes above $1 \mu \mathrm{m}$ where dislocations are nucleated from intragranular and GB sources with typical normal metallic deformation behavior.

Later Meyers et al. [78] in an overview of deformation of nanocrystalline metals based on experimentation and computations also summarized deformation based on grain size regions. The core-and-mantle model describes the sub-micrometer to $100 \mathrm{~nm}$ size range well. Typically for grain sizes greater than $1 \mu \mathrm{m}$ the increase in yield stress can be inversely related to the grain size $d^{-n}$ (Hall-Petch) where in this region $n=1 / 2$, and with grain size region between $1 \mu \mathrm{m}$ and $100 \mathrm{~nm}$ the exponent becomes smaller than $1 / 2$. For 
grain sizes of $100 \mathrm{~nm}$ to $20 \mathrm{~nm}$ the Hall-Petch slope continues to decrease with decreasing grain size. The GB ledge spacing becomes large with respect to the grain size and can no longer supply dislocations in this region. The dislocations can travel through the grain relatively unimpeded and annihilate at the opposing boundary, carried out without significant work hardening, leading to shear localization. With grain sizes between $20 \mathrm{~nm}$ and $1 \mathrm{~nm}$ GB deformation dominates the process with GB sliding becoming important.

In summary the grain size appears to have large effects on the deformation mechanisms. Materials below a micrometer begin to show changes in deformation mechanisms with plastic deformation proceeding mainly by dislocation emitted and absorbed by GBs with partial dislocation activity becoming more important as grain size decreases. Partial dislocations dominate the plasticity below about $100 \mathrm{~nm}$ and dislocation are no longer observed in the grain interiors below about $30-50 \mathrm{~nm}$. Below about $10 \mathrm{~nm}$ it appears that GB-mediated plasticity begins to dominate the deformation. Despite limited information it appears that GB structures coincidently also begin to change at the micrometer level where a saturation of the low energy CSL type ordered HAGBs is observed. Below this size the GB structure begins to further change toward the NEGB structure as the grain size approaches $100 \mathrm{~nm}$. These and other changes in GB structure will be further explored and a model developed of the GB structure that may help understand the GB-mediated plasticity that is thought to require GB diffusion to accommodate the GB deformation.

\section{Mechanical Alloying}

Benjamin [79] created a new process termed "mechanical alloying" (MA) in 1970 that evolved as an extension to mechanical working techniques developed earlier by Anders to create an oxide dispersion strengthened alloys. In the original work Benjamin [80] attempted to use MA to create a nickel coated refractory oxide that could be injected into a molten superalloy, but this failed due to rejection of unwetted oxide particles by the melt. However, several key concepts developed from these efforts; the welding and fracturing of metallic powders permitted fine powders of any composition to be 
manufactured from a mixture of elemental powders, alloys, or compounds. These concepts led to MA of powders in which the internal structure is refined while maintaining a larger overall coarse particle size, thus, for example, preventing pyrophoricity in these materials. Up to the time Benjamin developed the MA technique there were four major techniques to combine oxide dispersion strengthened materials in systems with relatively nonreactive elements; simple mechanical mixing, ignition surface coating, internal oxidation, and selective reduction. All of these techniques had particular problems; most important being the difficulty in producing dispersoids less than $5 \mu \mathrm{m}$ in size because of excessive growth of the dispersoid particles during processing.

The potential of MA as a new processing technique has now been widely investigated, and it is commonly used to accomplish a significant crystallite size reduction, in some cases down to the nano-scale, of pure and alloyed materials as well as to carry out solid state alloying of elemental powders [81]. Most importantly, this technique has developed a large variety of materials with previously unattainable microstructures. For example, iron and copper are immiscible in the solid state due in part to the positive heat of mixing [82]; however, extended solid solutions in these systems can be easily created by the MA process. Some other examples of uses of this unique processing method are synthesis of quasi-crystalline and nonequilibrium alloys, and amorphous alloys. Additionally, and important to the current study, a variety of chemical reactions have been observed in this near room temperature processing.

Mechanical alloying is a broad term that encompasses several individual techniques, and is commonly used to describe all of these techniques. Some detailed technique descriptors important to this study are mechanical milling, mechanical alloying, reactive mechanical alloying and reactive mechanical milling [81]. For completeness other technique descriptions include; mechanochemistry, mechanosynthesis, cryomilling, mechanical grinding, mechanical disordering, rod milling, mechanically activated annealing, double mechanical alloying, and mechanically activated self-propagating high-temperature synthesis. It is important to note that often in the literature the term "mechanical alloying" is applied generally and can include any of these specific techniques. However, as pointed out by Kaupp [83] MA is technically a 
subset of mechanochemistry which is more descriptive nomenclature for this family of processes.

The three main types of mills commonly used in MA are shown in Figure I-2. The powder capacity or "charge size" scales with the mills, the smallest capacity with the $\mathrm{SPEX}^{\mathrm{TM}}$ mill, followed by the attritor mill, and finally the largest capacity is typically the ball mill. The collision frequency and energy imparted to the powder particles is inversely related to the capacity with the largest amount of energy imparted in the SPEX ${ }^{\mathrm{TM}}$ mill, followed by the attritor mill, and finally the ball mill [81, 84]. Another popular high-energy mill is the planetary mill; with higher velocity of balls than in a SPEX ${ }^{\mathrm{TM}}$ mill, but the higher frequency of impacts in the $\mathrm{SPEX}^{\mathrm{TM}}$ mill provides greater transferable energies [81]. Milling times are varied to attain a given microstructure between the various mill types; additionally some microstructures cannot be attained with all mills. For example, during milling of an iron-chromium-manganese alloy in both an attritor mill and SPEX ${ }^{\mathrm{TM}}$ mill, the amount of observed ferrite and austenite was monitored. With $\mathrm{SPEX}^{\mathrm{TM}}$ milling the material was $100 \%$ austenite by 120 hours of milling but attritor milling could not reach $100 \%$ austenite [85].

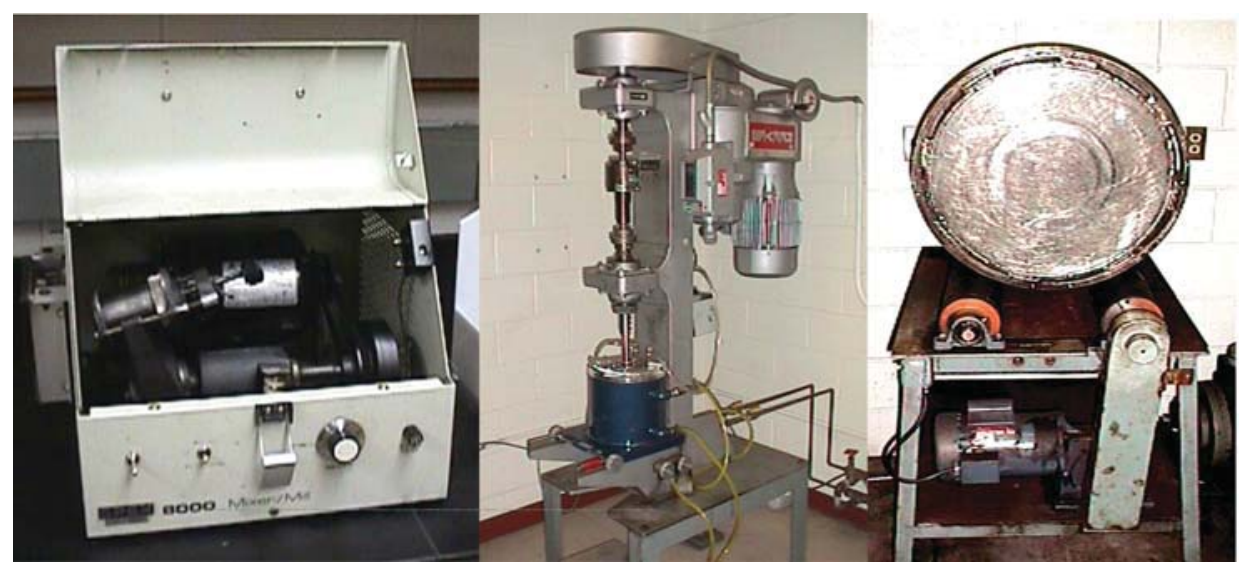

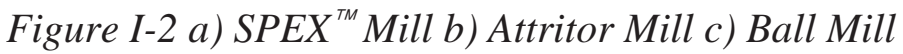

The first detailed report of the structural details of an "amorphous" type of material produced by MA of elemental powders was $\mathrm{Ni}_{60} \mathrm{Nb}_{40}$ by Koch et al. [86]. Although others had reported the formation of an "amorphous" type structure during MA, Koch et al. reported detailed x-ray diffraction studies and crystallization behavior. In 
particular, they compared the $\mathrm{x}$-ray diffraction (XRD) pattern of mechanically alloyed $\mathrm{Ni}_{60} \mathrm{Nb}_{40}$ with that of a liquid-quenched $\mathrm{Ni}_{60} \mathrm{Nb}_{40}$ alloy, and further observed exothermic heat effects derived from differential scanning calorimetry (DSC) of the mechanically alloyed samples. Of interest in these XRD results is the fact that although there are some similarities between the MA sample and the liquid-quenched sample several detailed differences stand out. In both samples the diffraction pattern appears similar to a typical amorphous pattern with one large peak at low angles and smaller modulations at the higher angles. The main XRD peak from the liquid quenched sample is symmetrical, whereas the main XRD peak for the MA sample is asymmetrical and is shifted slightly in angular position [86]. The DSC results from the work also show an exothermic peak for crystallization of the MA samples that occurs at lower temperatures than for the liquidquench samples and has a different shape. Important to note is that prior to the merging of the nickel $\{111\}$ and niobium $\{110\}$ diffraction peaks the crystallite size of the nickel was $1.2 \mathrm{~nm}$ and that of the niobium was $3.4 \mathrm{~nm}$. It was concluded that strong similarities to a liquid quenched amorphous material suggested amorphous formation, although the need for further work was mentioned.

Many researchers have studied and cited "amorphous-type" materials formed by MA based on XRD patterns, or DSC results, or a ring diffraction pattern produced in transmission electron microscopy (TEM) as indicators of "amorphous" structures. It should be pointed out that nano-scale crystallite sizes, more pronounced from multiple phases, can have an amorphous-like appearance in XRD. Furthermore, it's difficult to find a published direct TEM observation of an amorphous structure on a sample created by MA. This indicates that using a single type of analysis may not be adequate in identifying amorphous materials.

Many of the large array of materials processed by MA require the use of a process control agent (PCA), typically an organic liquid used to inhibit welding and to facilitate the alloying. For example, in ductile-ductile alloying the use of a PCA can create a much finer macroscopic particles size often by an order of magnitude or more. Without a PCA, some systems will amass large welded particles essentially isolating the inner material from the action of the MA process. The first detailed investigation into milling metallic 
powders in organic (and inorganic) liquids was by Arias [87] who concluded that metal powders cannot be comminuted in organic (or inorganic) liquids without becoming contaminated with the elements from the liquid. However, his research also established that it should be possible to select liquids that minimize the contamination or produce contaminants that can be removed by reduction procedures. While concerns about contamination by the PCA in MA is usually paramount [81], the current study will demonstrate that this phenomenon can be utilized to an advantage in the synthesis of new materials.

\section{Reactive Mechanical Alloying/Milling (RMA/M)}

Reactive mechanical alloying/milling has received less attention than other MA methods. Less than $1 \%$ of all MA papers from 1970 - 1994 specifically cite reaction milling, and almost all of the materials involved are dispersion-strengthened aluminum [88]. This interesting process involves a chemical reaction typically leading to the formation of compounds during the milling process. While reduction of metallic oxides and displacement reactions of metal oxides during the MA process are technically reactions, this process is often referred to in the literature as mechanochemical synthesis (or mechanosynthesis) $[89,90]$. The differentiation for RMA/M is that this process involves a chemical reaction typically leading to the formation of compounds between metallic powders and nonmetallic elements during the milling process. Several ceramics have been produced by RMM of elemental powders with nonmetal powders, or with elements from the milling atmosphere; however, often this requires additional processing to initiate these reactions after milling but the milling lowers the required temperatures. Murty and Ranganathan [39] report a variety of nitride ceramic particles formed by milling in liquid nitrogen or with nitrogen gas. Dispersions of AlN in an aluminum matrix have also been produced by mechanical milling in liquid nitrogen [91].

Whittenberger et al. [92] milled pre-alloyed powders of NiAl in liquid nitrogen, termed "cryomilling", which formed a dispersion of AlN in the NiAl matrix from the reaction between aluminum and nitrogen and allowed for systematic control of the volume fraction. Materials with volume fractions of 10 and $30 \%$ of AlN were produced 
by cryomilling followed by hot pressing and then hot isostatic pressing to full density. However, in order to preserve the matrix stoichiometry it was necessary to create a hyperstoichiometric aluminum NiAl pre-alloyed powder to achieve 30 vol. pct. AlN. Whittenberger et al. [93] analyzed the as-milled powder with XRD and suggested that an observed peak shift was caused by creation of a supersaturated nonequilibrium interstitial nitrogen solid solution during the milling process, and that AlN particles are formed with further thermomechanical processing. It is interesting to note that the original attempt to produce an ODS NiAl-based alloy by cryomilling pre-alloyed $\mathrm{NiAl}$ with yttria failed to produce a fine dispersion of yttria. However, after cryomilling a fine dispersion of AlN was observed in the microstructure, with a substantial increase in mechanical properties [94].

Jangg [95] first reported the use of reaction milling in the formation of dispersionstrengthened aluminum-carbon alloys. In this case the aluminum was milled with $2 \mathrm{wt}$. pct. graphite and also 2 wt. pct. carbon "lampblack" with no observed in situ reaction and completion of the reaction with aluminum and carbon required additional thermal processing. Jangg does suggest that the fine-scale composite of aluminum and carbon particles is an important part of the reaction process. The Jangg work shows that when both reactants are powders they are less likely to react in situ. However, it will be shown in the current work the proper choice of the reactants can achieve full reaction in situ during MA.

\section{MA of Titanium-Aluminum}

A majority of the MA processing of titanium-aluminum materials has focused on the TiAl alloy; however, there has been some work on alloys based on $\mathrm{Al}_{3} \mathrm{Ti}$. Important here is the MA study of a 75/25 wt. pct. mixture of aluminum titanium elemental powders in a $\mathrm{SPEX}^{\mathrm{TM}}$ mill by Srinivasan et al. [96]. Of particular interest is the use of a PCA, in this case hexanes $\left(\mathrm{C}_{6} \mathrm{H}_{14}\right)$, to prevent agglomeration or "welding" of ductile aluminum powder particles. Srinivasan et al. determined that in a 5-g powder charge approximately 0.1 to $0.2 \mathrm{~g}$ of hexanes is retained as elemental hydrogen and carbon. They concluded that hydrogen can be completely removed by vacuum annealing, with 
approximately 2 wt. pct. carbon retained in solution. Of further interest is the fact that the structure of the $\mathrm{Al}_{3} \mathrm{Ti}$ powders produced by this technique is the metastable binary cubic $\mathrm{L1}_{2}$ phase as opposed to the equilibrium $\mathrm{DO}_{22}$ phase. As will be shown, an important key to the current research is that the PCA can be used not only to enhance the milling of the material, but also to provide a mechanism for incorporating a fine-scale dispersion of carbides in the matrix.

Very little milling has been carried out on chromium-modified titanium trialuminides. Lee and Ahn [97] mechanically alloyed several elemental compositions of $\mathrm{Al}_{75-\mathrm{x}} \mathrm{Cr}_{\mathrm{X}} \mathrm{Ti}_{25}(\mathrm{X}=6,8,10,12$, and 14 wt. pct.) in an argon environment with a horizontal ball mill without a PCA. The structural evolution during the milling indicated the formation of a metastable FCC phase because the aluminum diffraction peaks shifted to slightly higher angles (smaller lattice parameter). TEM study confirmed the FCC phase with a grain size of tens of nanometers, along with fine embedded chromium particles. The matrix began to transform to the $\mathrm{L}_{2}$ structure after heating the powder at $400{ }^{\circ} \mathrm{C}$ for an hour as evidenced in TEM by the appearance of the 100 and 110 superlattice diffraction nodes. Additionally, the size of the chromium particles as viewed by TEM, decreased, indicating diffusion of chromium into the FCC phase, this agreed with the appearance of superlattice diffractions characteristic of the L1 2 structure. The as-milled powders were also hot pressed at $1200^{\circ} \mathrm{C}$ for 12 hours yielding a grain size of $1-3 \mu \mathrm{m}$. There were indications of oxide formation that may have been a factor in preserving the fine grain size.

Research by Heilmaier et al. [98] focused on enhancing the creep resistance of the $\mathrm{L}_{2}$ chromium-modified titanium trialuminides by adding an insoluble dispersed $\mathrm{Y}_{2} \mathrm{O}_{3}$ phase by means of MA prior to consolidation by vacuum hot pressing to 96 - $98 \%$ of theoretical density. Mechanical alloying experiments were performed on both elemental and ingot cast crushed pre-alloyed powders with the stoichiometry of $\mathrm{Al}_{67} \mathrm{Cr}_{8} \mathrm{Ti}_{25}$ and $\mathrm{Al}_{75} \mathrm{Ti}_{25}$ both with and without 3 vol. pct. of $\mathrm{Y}_{2} \mathrm{O}_{3}$. Further, all milling was accomplished in an argon environment using both a planetary ball mill and an attritor mill. Structural evolution was monitored by XRD, which after $150 \mathrm{~h}$ of milling elemental powders showed a nanostructured FCC-phase, the disappearance of the titanium phase, and a 
chromium phase with peaks sharper than the FCC-phase. The FCC phase peak positions were found to coincide with those from the $\mathrm{L}_{2}$ structure and there was evidence of superlattice peaks throughout most of the milling. Of interest here is the formation of the $\mathrm{L} 1_{2}$ structure in the binary material, even the pre-alloyed powder with the ordered tetragonal $\mathrm{DO}_{22}$ structure began to change into the cubic $\mathrm{L}_{2}$ within $10 \mathrm{~h}$ with complete phase change by $100 \mathrm{~h}$.

In both cases the $\mathrm{Y}_{2} \mathrm{O}_{3}$ dispersoids became much smaller in size with added milling time. The size, volume fraction, and distribution of the dispersoids are decisive factors in the creep resistance of ODS alloys. Typically the creep resistance (and strength) increases with a decrease in the dispersoid size. Samples consolidated, by hot pressing at $800{ }^{\circ} \mathrm{C}$ for $15 \mathrm{~min}$, having a matrix grain size of approximately $3 \mu \mathrm{m}$ and $\mathrm{Y}_{2} \mathrm{O}_{3}$ dispersoid sizes measured by XRD of $5 \mathrm{~nm}$, developed excellent properties. The room temperature Vickers hardness of the $\mathrm{Al}_{67} \mathrm{Cr}_{8} \mathrm{Ti}_{25}$ alloy with 3 vol. pct. $\mathrm{Y}_{2} \mathrm{O}_{3}$ was about triple that of single-phase $\mathrm{Al}_{67} \mathrm{Cr}_{8} \mathrm{Ti}_{25}$. Since the powder tended to "stick' to the vial walls reducing powder output, use of a PCA was thought necessary, but that had a twofold negative effect: delay of phase transitions and introduction of contaminants, particularly carbon which led to stoichiometry effects because of its bonding with the titanium. At first this observed contamination was a concern during planning of the current research, but as will be discussed later, carbides formed from intentional interactions with titanium, that allows simple changes to the MA parameters to control the final microstructure produced.

\section{Modeling of Mechanical Alloying}

The fundamental model of successful MA first brought forth by Benjamin [80] is a competition between cold welding and fracturing of particles caught between two given milling balls that collide. Gilman and Benjamin [99] gave a schematic representation of this competition, shown in Figure I-3, which depicts the physical event for various particles trapped between ball collisions during the milling process. Mechanical alloying experiments by Benjamin [80] established that the initial 4 - $7 \mu \mathrm{m}$ particle size of a prealloyed sample was altered by the processing, reaching a steady size of $100-130 \mu \mathrm{m}$ 
after prolonged milling. The powder particles become an agglomeration by a cold welding process of both smaller particles and fractured pieces thereby creating larger particles. Above a critical particle size the internal flaws were deemed large enough to induce fracture of the particles from the severe deformation imposed during the ball to ball collision. The suggestion is that the small particles are statistically less likely to contain a critical flaw size to cause fracture, thus promoting cold welding of the particles, whereas large particles have a statistically larger probability of containing a critical flaw size. This model has been used since the inception of MA with slight modifications that expand the details of these basic process of cold welding and fracture. As will be detailed in the current work a modified model has been developed that especially focuses on the cold welding aspect of MA.

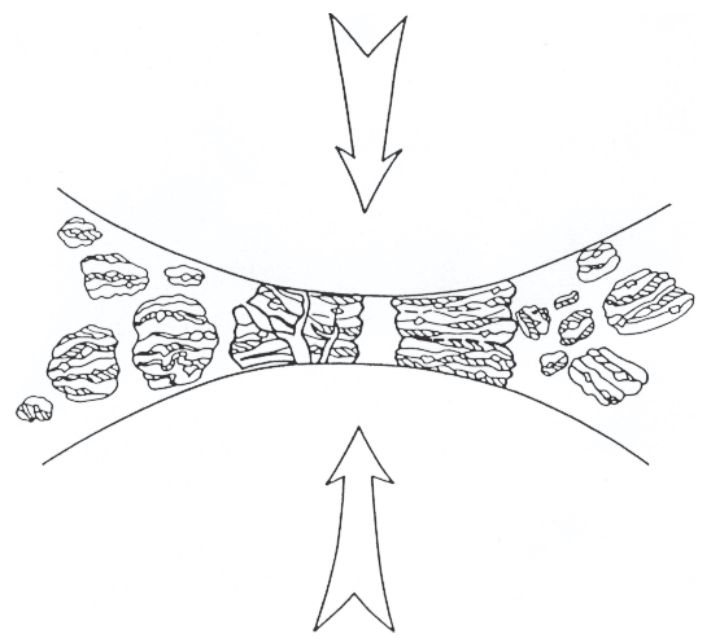

Figure I-3 Fundamental Welding/Fracture Ball to Ball Collision Model [99]

Most important in the early modeling of the process, for the case of ductile powders, was the development of a lamellar structure, with the spacing between the lamellae in the agglomerated materials being exponentially reduced to values typically below detection by optical microscopy. Physically this interpretation is reasonable based on the high frequency of impacts experienced by the ductile mixture of trapped particles during ball to ball, or vial to ball, collisions that causes a "kneading" of the mixture dependent on milling time. Since this processing develops microstructural scales below the resolution of optical microscopy, XRD studies have become important to characterize mechanically alloyed powders, which typically have crystallite sizes well below optical 
resolution values and can reach values as small as 2 - $20 \mathrm{~nm}$, orders of magnitude smaller than the physical powder particle sizes. Of particular interest in the current study is that the MA process reaches a lower limit of the crystallite sizes of about $2 \mathrm{~nm}$, a value that may be of fundamental interest.

Since Benjamin's early work, more sophisticated attempts have been developed to model the physics of mechanical alloying, and these fall into two basic groups. First, the modeling/simulation looks at two fundamental aspects, local modeling and global modeling. Local modeling considers the energy involved in collisions involving the powder particles, and global modeling applies the local models to predict events in a specific mill. Courtney [100] gives an overview of process modeling of MA with "welding" (or agglomeration) of the particles modeled by cold welding, similar to Benjamin, and fracture using forging models. Secondly, energy maps/milling maps have been developed to determine the conditions under which certain phase transformations occur. The current models study certain alloy systems to predict the conditions leading to specific transformations. Although studies have been system-specific, one conclusion [39] was that a dynamic equilibrium is reached which depends only on the energy involved during the milling, and is independent of the initial mixture.

Benjamin also concluded that the welding event must take place as one event to prevent the reocclusion of the fresh metal. Based on estimated strain energies, Benjamin suggested, local temperatures up to $800{ }^{\circ} \mathrm{C}$, with global temperatures up to $200{ }^{\circ} \mathrm{C}$, during the process. Since that time several models have estimated the macroscopic (overall) temperature and microscopic (instantaneous local) temperatures during the process. Macroscopic temperatures have been measured for a $\mathrm{SPEX}^{\mathrm{TM}}$ mill, with the maximum temperatures of the order of $50{ }^{\circ} \mathrm{C}$, in agreement with values determined in the current research. Microscopic temperature increases for a variety of materials, based off indirect calculated values, as compiled by Murty and Ranganathan [39], these values can range from $10^{\circ} \mathrm{C}$ to $400{ }^{\circ} \mathrm{C}$. Temperature measurements have been extremely difficult to perform and have importance in attempts to explain diffusion during MA but the general consensus is that the temperature is not a factor. For example, in modeling physics of the MA process Courtney [101] determine the localized temperature increase is modest with 
the particle cooling back to mill temperature well before experiencing another impact. However, because of the possibility of enhanced diffusion at the GB, as pointed out by the current work, this is not a simple problem!

\section{a) Diffusion in Mechanical Alloying}

The MA process has shown the unique ability for large amounts of mass transport as evidenced by the creation of non-equilibrium alloys and materials, extended solid solutions, amorphous structures, intermetallics, compounds, quasi-crystals, etc. [81] often accomplished from elemental constituents. The important aspect is that atomic homogenization, requiring mass transport, occurs in order to create these observed phases by means of a near room temperature process. In the early work the local temperatures were thought to be the major driving force in activating diffusion, but experiments have shown that the temperatures developed are far less than those required for the typical diffusion to occur. Subsequently, other aspects of diffusion activation and mass transport in general have been explored. To this day the most often cited model for diffusion in the MA process is that originally proposed by Benjamin, where diffusion was thought to have occurred when the observed lamellar spacing could not be resolved optically. Gilman and Benjamin [99] suggested that the severe cold work enhances diffusion by providing numerous sites of low activation energy pipe diffusion. When the lamellae are unresolved by optical methods diffusion is considered to have been operational because of the short diffusion distance; however, no supporting experimental evidence is available.

Martin and Gaffet [102] suggested ballistic diffusion being independent of temperature may account for the enhanced diffusion rates observed. In this model atomic mixing in MA can occur by repeated shearing of the particles which lowers the free energy configurations such as by alloying. Important to the current study was the conclusion stating the importance of the need to develop atomistic mechanisms that enables atomic mixing during MA in this near room temperature process. The model for mass transport developed in the current work provides such a mechanism. Shtremel [103] in a similar model defined "shear displacement" as a source of diffusion in MA without 
the need for traditional diffusion. Shtremel's interpretation suggested shear bands create the redistribution of atoms and accomplish homogenization based on dislocation motion without the need for diffusion. This anomalous diffusion involves plastic deformation creating jogs, which emit vacancies, thus increase the nonequilibrium vacancies which can increase diffusivity.

Another area of focus has been those mechanisms that decrease the activation energy for diffusion. One means for lowering the activation energy is the creation of high defect densities by the impacts in MA causing increased vacancy formation [104]. For example, considering diffusion in the Al-Cu system (large-grained polycrystal) with an activation energy of $126 \mathrm{~kJ} /$ mole the diffusivity $(D)$ at $800^{\circ} \mathrm{K}$ is $8.8968 \times 10^{-14}$; however, with defect diffusion considered, the activation energy is lowered to $82 \mathrm{~kJ} / \mathrm{mole}$ and the diffusivity at $521{ }^{\circ} \mathrm{K}$ is similar at $8.693 \times 10^{-14}$ this provides for a similar diffusion rate at a much lower temperature [104]. Lu et al. [105] concluded that MA significantly lowers the activation energy for diffusion based on short-circuit diffusion from the increased grain boundaries and surfaces. This will be discussed in more detail in connection with the model presented in the current work.

Gleiter [106] has shown that large potential gradients associated with the stress fields of dislocations can enhance diffusion in vicinity of dislocation cores even at low temperatures, and it should be noted that the MA process creates extremely large dislocation densities. There is certainly agreement that the MA process may depend on short-circuit diffusional processes like grain boundary diffusion, pipe diffusion, and surface diffusion, but experimental details of these processes are lacking. A simple model of pipe diffusion based on the fraction $f$ of atoms in the dislocation cores was proposed by Hart [107] as given in Equation (I.4). This model considered static dislocations, one of the first acknowledgements that diffusion in dislocations is a short-circuit diffusion which may occur at temperatures below the basic rule of thumb for lattice diffusion of $\mathrm{T}>0.5$ $\mathrm{T}_{\mathrm{m}} \mathrm{K}$ in a given material.

$$
D_{T}=D_{l}+f D_{P}
$$

In a similar manner, a model of diffusion focused on the role of plastic deformation in MA was developed more recently by Bhattacharya and Arzt [108]. They 
considered two fundamental contributions to diffusion, lattice and pipe diffusion (or dislocation core diffusion). Their total diffusivity expression is given as Equation (I.5), where $\beta$ is the core diffusivity factor, $\rho$ the dislocation density, and $b$ is the Burgers vector. Similar to the Hart model this separates the diffusion contributions from both the lattice and that for pipe diffusion.

$$
D=D_{l} \exp \left(-\frac{Q_{l}}{R T}\right)+\beta b^{2} \rho D_{c} \exp \left(-\frac{Q_{c}}{R T}\right)
$$

A more elaborate model based on pipe diffusion was proposed by Schwarz [109] in which a mechanism of low temperature diffusion in MA designated as "dislocation solute-pumping" (DSP), based on short-circuit static solute diffusion along dislocations that terminate at cold welded surfaces and leaving solute chains after the dislocation dynamically moves. It should be noted that in the MA process a period of time exists where powder particles do not participate in the media collisions and it is thought to be much longer than the time powder particles actually participate in impacts. This explained diffusion in the MA process once the grains are extremely small, as the model suggests that solutes only travel about $10 \mathrm{~nm}$. This model was limited to straight dislocations and was further advanced for curved dislocations by Estrin and Rabkin [110] who suggested the mechanism by Schwarz may operate for powders with particle sizes up to $100 \mathrm{~nm}$.

Short-circuit diffusion by pipe diffusion is an important mechanism of diffusion enhancement and with the large amount of dislocations and defects generated in the MA process it would be expected to be important. In the diffusion model by Schwarz [109] dislocation solute-pumping is an important mechanism to this model; however, "clogging" of dislocations can occur and impede the diffusion process [111]. This is particularly important if the dislocations are static since the solute has to visit the dislocation to participate in the enhanced diffusion. Moreover, Schwarz alluded to the possible participation of GBs and triple junctions which have much higher diffusivities than pipe diffusion as possible factors for future research (none found), this highlights the potential importance of the GBs to mass transport. 
In summary, the diffusion models to date are all based on grain sizes being extremely small for the models to explain the diffusion. These models while attractive mechanisms for localized diffusion cannot explain the large amount of mass transport that takes place in short durations of MA time. Much of the work to date is based on the fundamental view that the historic model of cold welding and fracture prevails. While this work was not intended to be a study of the diffusion process in MA, the results of the analysis will suggest a new diffusion mechanism that can also help explain the short-time mass transport effects observed. Additionally, this mechanism can also provide insight into the grain refinement occurring during this process.

\section{E. Chromium-Modified Titanium Trialuminide Matrix Composites}

There have been some studies of dispersion strengthening of the $\mathrm{L} 1_{2}$ intermetallics by other means than MA (Table I-1). The approaches differed from the current research in that many used a top-down approach of admixing dispersoid phase followed by standard powder metallurgy techniques. The $\mathrm{Al}_{67} \mathrm{Cr}_{8} \mathrm{Ti}_{25}$ and $20 \% \mathrm{TiB}_{2}$ sample involved an exothermic reaction step in the process to create the $\mathrm{TiB}_{2}$ from elemental powders. The MA work by Lee and Ahn [97] is included for comparison. Table I-1 lists reported yield strengths as well as grain size, dispersoid size, and basic processing method. These results demonstrate that while the mechanical properties can be dramatically enhanced by means of these basic strengthening mechanisms, further enhancement of the mechanical properties could be achieved by finer grain sizes and dispersoid sizes. 
Table I-1 Yield Strength, Grain Size, and Dispersoid Size of IMC Materials

\begin{tabular}{|c|c|c|c|c|c|}
\hline $\begin{array}{c}\text { Material } \\
\text { (Volume pct.) }\end{array}$ & $\begin{array}{c}\text { 0.2 \% Y.S. } \\
\text { Rm. Temp. } \\
\text { (MPa) }\end{array}$ & $\begin{array}{l}\text { Grain } \\
\text { Size } \\
(\mu \mathrm{m})\end{array}$ & $\begin{array}{c}\text { Dispersoid } \\
\text { Size } \\
(\mu \mathrm{m})\end{array}$ & $\begin{array}{l}\text { Processing } \\
\text { Method }\end{array}$ & Ref. \\
\hline $\mathrm{Al}_{66} \mathrm{Cr}_{9} \mathrm{Ti}_{25}$ & 245 & 270 & ** & Arc-melt & [123] \\
\hline $\mathrm{Al}_{67} \mathrm{Cr}_{8} \mathrm{Ti}_{25} *$ & 750 & 2 & ** & MA-P/M & [97] \\
\hline $\mathrm{Al}_{67} \mathrm{Cr}_{8} \mathrm{Ti}_{25}$ * & 320 & 30 & $* *$ & $\mathrm{P} / \mathrm{M}$ & [112] \\
\hline $\mathrm{Al}_{67} \mathrm{Cr}_{8} \mathrm{Ti}_{25} *$ & 490 & 50 & $* *$ & $\mathrm{P} / \mathrm{M}$ & [113] \\
\hline $\begin{array}{c}\mathrm{Al}_{67} \mathrm{Cr}_{8} \mathrm{Ti}_{25}{ }^{*}+ \\
10 \% \mathrm{Al}_{2} \mathrm{O}_{3}\end{array}$ & 470 & 50 & 35 & $\mathrm{P} / \mathrm{M}$ & [113] \\
\hline $\begin{array}{c}\mathrm{Al}_{67} \mathrm{Cr}_{8} \mathrm{Ti}_{25} *+ \\
30 \% \mathrm{Al}_{2} \mathrm{O}_{3}\end{array}$ & 970 & 50 & 35 & $\mathrm{P} / \mathrm{M}$ & [113] \\
\hline $\mathrm{Al}_{67} \mathrm{Cr}_{8} \mathrm{Ti}_{25} *$ & 900 & 4 & $* *$ & $\mathrm{P} / \mathrm{M}$ & [112] \\
\hline 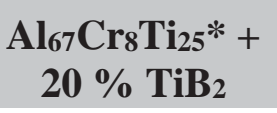 & 1500 & 4 & 0.5 & $\mathbf{P} / \mathbf{M}$ & [112] \\
\hline
\end{tabular}

\section{F. Research Genesis}

The initial exploratory experiment in the current work, which proved futile, was an attempt to mechanically alloy elemental powders or mechanically mill pre-alloyed powders of the $\mathrm{L}_{2}$ chromium-modified titanium trialuminide material developed in MTU laboratories $[6,7,8]$ ad-mixed with 10 vol. $\% \mathrm{TiB}_{2}$ ceramic powder in a dry milling environment. This failure was because of welding into large particles in the case of MA of elemental powders and "caking" into a hard mass of packed powder developed in the mechanical milling (MM) of pre-alloyed powder. Therefore, in both cases a majority of the powder was isolated from participating in the milling process. Attempts using the same approach with various combinations of elemental powders and pre-alloyed powders were also of no avail. Despite the potential of the oft-cited problems from contamination, it was decided to explore the use of a process control agent (PCA) to enhance the milling 
process. The selection of the PCA hexanes $\left(\mathrm{C}_{6} \mathrm{H}_{14}\right)$ was based on the work by Srinivasan et al [96] on MA of elemental aluminum and titanium in a SPEX ${ }^{\mathrm{TM}}$ mill (having the stoichiometry of $\mathrm{Al}_{3} \mathrm{Ti}$ ). This original experiment was based on MA of elemental powders (stoichiometry of $\mathrm{Al}_{67} \mathrm{Cr}_{8} \mathrm{Ti}_{25}$ ) and ad-mixing 10 vol. pct. $\mathrm{TiB}_{2}$ powder using 6 $\mathrm{ml}$ of hexanes as a PCA. The initial $9 \mathrm{~h}$ MA time proved successful as the powder particles remained small and the particles appeared free to participate in the MA process. In order to track microstructural changes the milling was followed by XRD analysis of the as-milled powder. To better evaluate this new MA process using hexanes as a PCA, the experiment was extended to long milling times based on $9 \mathrm{~h}$ test increments each followed by XRD analysis, repeated a total of 10 times with each charge comprised of the remaining powder with a fresh amount of the PCA hexanes for a total $90 \mathrm{~h}$.

This experiment resulted in a startling observation of the synthesis of a significant amount of $\operatorname{Ti}(\mathrm{C}, \mathrm{N})$ phase; a result of an in situ reaction between the titanium in the metallic powder with carbon from the liquid PCA and nitrogen from the air environment. Figure I-4 shows the XRD pattern from the $90 \mathrm{~h}$ MA as-milled powder which is dominated by the isomorphous $\operatorname{Ti}(\mathrm{C}, \mathrm{N})$ phase delineated by the superimposed JCPDS power diffraction database file "stick" patterns for TiC, and TiN. This uptake of elements was confirmed by a $42 \%$ increase in the weight of the dry as-milled powder. More importantly, based on Scherrer equation determination of the crystallite size the $\mathrm{Ti}(\mathrm{C}, \mathrm{N})$ phase particles were more than an order of magnitude smaller than the $\mathrm{TiB}_{2}$ phase particles at a $90 \mathrm{~h}$ milling time. At that time the use of the ad-mixed $\mathrm{TiB}_{2}$ phase was abandoned.

Of particular interest in this important experiment was the systematic increase in the intensity of the diffraction peaks from the $\operatorname{Ti}(\mathrm{C}, \mathrm{N})$ phase, caused by increases in the amount of this phase with milling time as shown in Figure I-5. This behavior suggests potential mechanisms of simple processing changes to vary the volume fraction of this hard dispersed phase. This is an incredibly important discovery in composite alloy development, whereby one has the ability to control and vary the amount of the reactant product based on simple changes to the MA parameters. In early milling times the movement of the aluminum diffraction peaks to higher angles indicates alloying with 
titanium with this new phase having a similar crystallite size to the $\operatorname{Ti}(\mathrm{C}, \mathrm{N})$. There are several other important features of the diffraction patterns.

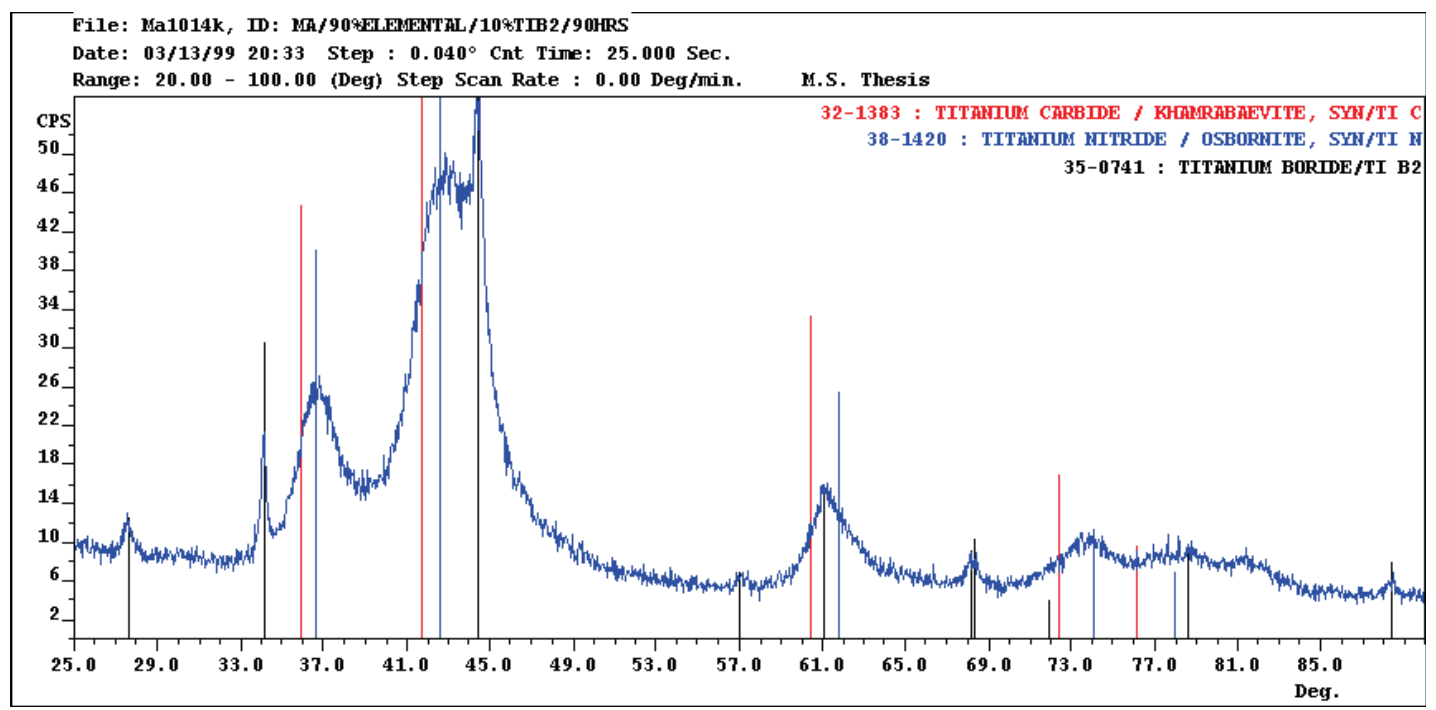

Figure I-4 XRD Pattern of Original Experiment After $90 \mathrm{~h}$ of SPEX ${ }^{T M}$ Milling

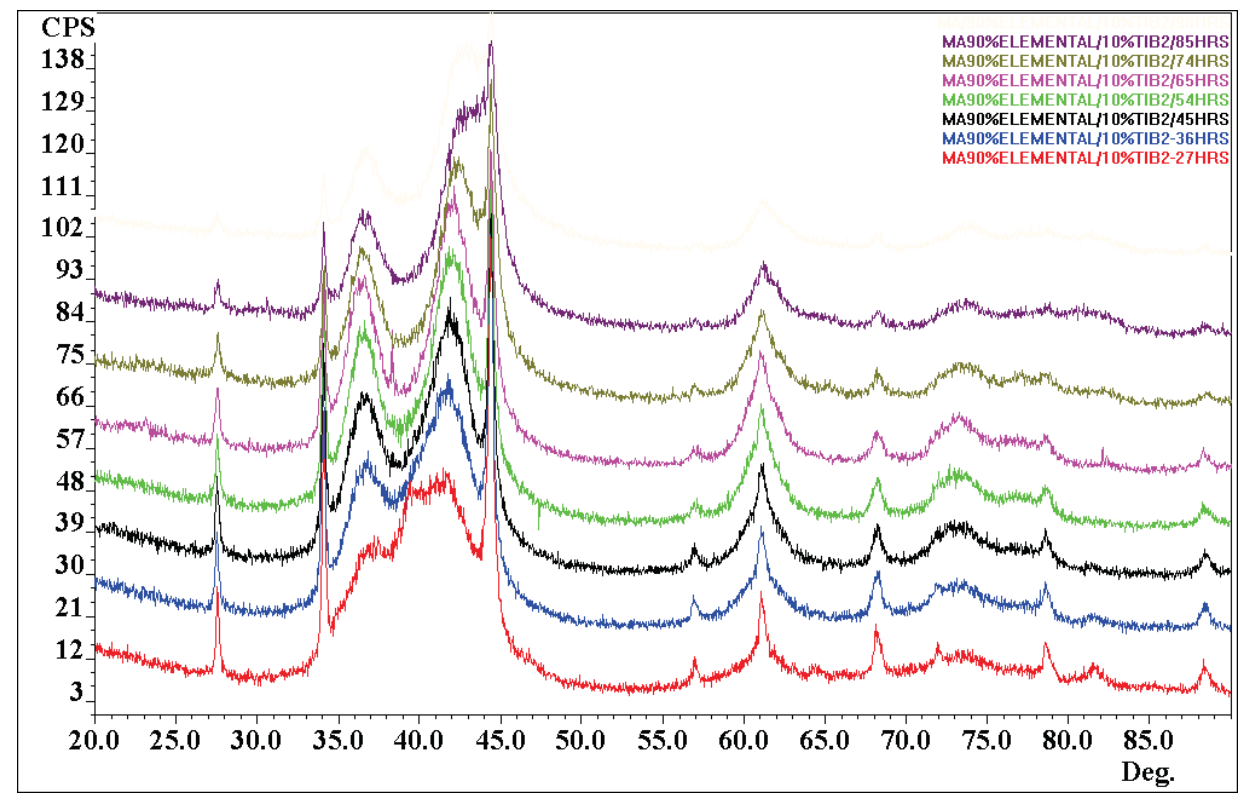

Figure I-5 XRD Patterns of the Original Experiment for $36 \mathrm{~h}$ to $90 \mathrm{~h} \mathrm{MA}$ Times

Comparing the XRD patterns from this series of experiments, a subtle but unusual scattering behavior was observed; a systematic increase of the XRD background 
throughout the whole $2 \theta$ range as the MA time increased. The background was modeled using an exponential decay function fitting from actual background data points with the integrated intensity under these background curves represented in Figure I-6. The intensity of the background increases rapidly up to about the 40 MA time then appears to saturate. Clearly, this scattering increase in the background of XRD patterns must originate from within a volume of atoms structurally arranged in a manner capable of accounting for this unexpected scattering. The source of this unusual scattering became a major focus of this work while aiding to provide a better understanding of the MA process.

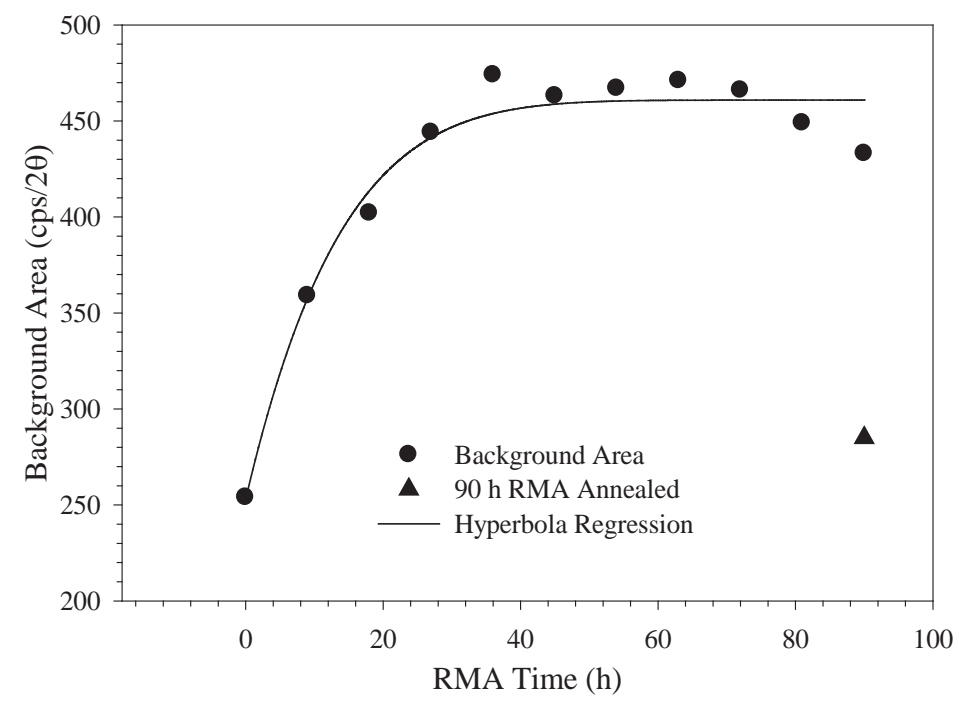

Figure I-6 Integrated Background Intensity Increase with MA Time

This original experiment provided the impetus for the current research to be presented which focused on the in situ reaction of elements in the charge powder with those from the liquid and gas environment to form new hard particle dispersed phases, a process that can be controlled by simple MA processing parameter changes. It may be important at this point to comment on the choice of the phrase "reactive mechanical alloying/milling" that will be used to describe this processing. Due to the in situ reactions during both the MA and MM processing in this new approach the choice of the word "reactive" seems appropriate thus leading to the terminology of reactive mechanical 
alloying/milling (RMA/M). The science of this research will concentrate on the structural evolution (atomic, micro, and macrostructure) of these powders during milling, its relation to the RMA/M process, and the properties of bulk consolidated samples synthesized from these new composite powders. This processing scheme provides a method of "nano-engineering" the powders by simple process control changes. While the focus is on the $\mathrm{L}_{2}$ intermetallic, this new processing approach has been successfully attempted on several other materials systems, opening the door to materials previously unavailable.

\section{Experimental}

The experimental approach is presented in three major sections, experiment procedures employed to characterize the materials created and evaluate important properties, experiments to systematically investigate the details of the newly developed RMA/M processing, and the means for consolidation of these powders developed with the new RMA/M processing method into bulk materials. Regarding terminology, the chromium-modified titanium trialuminide, or cubic $\mathrm{Al}_{3} \mathrm{Ti}$, will be referred to as the $\mathrm{L}_{2}$ intermetallic, unless otherwise specified. The powders resulting from a particular RMA/M process after drying under ventilation to remove the PCA hexanes are referred to as "as-milled powder".

\section{A. Experimental Procedure}

\section{Production of Cubic Trialuminide Powder - Reading Alloy}

As mentioned, several of the milling experiments utilized pre-alloyed $\mathrm{L}_{2}$ intermetallic material in powder form, referred to as the Reading Alloy. Reading Alloys Inc. (Reading, PA) manufactured large quantities of the $\mathrm{Al}_{66} \mathrm{Cr}_{9} \mathrm{Ti}_{25}$ alloy by exothermic reaction sintering of a mixture of the elemental components in the proper stoichiometry. Alloying was accomplished by physically mixing titanium sponge, aluminum flake, chromium pieces, and heating the mixture in an inert environment until an exothermic reaction initiated, which then sintered the material primarily as a single-phase $\mathrm{L}_{2}$ 
intermetallic. At this stage the alloy was in the form of sponge that was subsequently screened to -1 inch. Chemical analysis by Reading Alloys Inc. of the resultant product revealed near target amounts of aluminum and chromium and $24 \mathrm{wt}$. pct. titanium [114]. The material was prepared for use by crushing in a SPEX ${ }^{T M} 8000$ mixer/mill with two $12.7 \mathrm{~mm}$ diameter steel balls and screened to -200 mesh. Additional detailed chemical analysis for minor elements by Waubik Inc. (Hancock, MI) found the following values in wt. pct. approximately 0.25 carbon, 0.29 oxygen, 0.02 nitrogen, 0.14 iron, 0.14 silicon, 0.07 vanadium, and 0.02 manganese [114].

\section{Production of Cubic Trialuminide Powder - Master Alloy}

Grinding large pieces from a fractured arc-melted ingot produced the powder known as "Master Alloy". The first step in producing the initial arc-melted ingots was pressing the appropriate amount of each elemental powder in a closed-die Dake (Grand Haven, MI) Elec-Draulic II 150 ton Press at a pressure of 207 MPa. Pressing is necessary to minimize material ejection during initial application of the arcing electrode. The elemental starting materials to form the stoichiometry of $\mathrm{Al}_{66} \mathrm{Cr}_{9} \mathrm{Ti}_{25}$ had a minimum of $99.9 \%$ purity and were in the form of granules, flake, and sponge. Since aluminum has a low vapor pressure and because the arc is at very high temperatures, it was necessary to add 1.25 wt. pct. aluminum to offset the loss due to vaporization during arc-melt casting [7]. Use of large size elemental pieces in the compact further minimized material expulsion during initial arc application.

These pressed green compacts of the stoichiometric composition mixture and added aluminum were arc-melted on a copper chill plate using a nonconsumable tungsten electrode. Prior to melting, the chamber was purged five times by evacuating to $13 \mathrm{~Pa}$ followed by filling to $61 \mathrm{kPa}$ with filtered high purity argon. Pure titanium was then melted in the arc melt chamber to remove residual oxygen prior to melting the alloy charge. The alloy mixture was melted a minimum of 5 times and rotated between melt cycles to enhance homogeneity.

Powder of the alloy was produced by crushing slices of the arc-melted ingots in a hydraulic press followed by refinement of the particle size in a $\mathrm{SPEX}^{\mathrm{TM}} 8000 \mathrm{mixer} / \mathrm{mill}$ 
employing two $12.7 \mathrm{~mm}$ steel balls in a round ended vial. The crushed pieces were milled until the resultant powder size was -200 mesh, typically requiring several 5-10 minute intervals of milling.

\section{Milling Apparatus}

All samples in this study were reactively mechanically alloyed/milled in a SPEX ${ }^{\mathrm{TM}}$ (Edison, NJ) 8000 mixer/mill shown in Figure I-2 a). The mill was modified slightly by the addition of an electrical switch that allows the electrical timer to be bypassed in order to run the mill for extended periods of time. The interior shape of the milling vial is a cylinder $38 \mathrm{~mm}$ in diameter and $58 \mathrm{~mm}$ in depth with flat surfaces on the bottom and top. The nominal outside dimensions of the S-5 hardened tool steel vial are $51 \mathrm{~mm}$ in diameter and $70.3 \mathrm{~mm}$ in height. The spherical milling media was Grade 25, 52100 chromium steel, with dimensions of $6.35 \mathrm{~mm}$. The source of the milling media was Salem Specialty Ball Company of West Simsbury Connecticut. Several parameters were varied in the milling experiments. These are noted for specific milling experiments and include the number, size, and weight of the milling media, total powder charge weight, time in hours of milling, type of PCA, as well as the milling environment. The external temperature of the vial was monitored periodically throughout this research and found to average about $50^{\circ} \mathrm{C}$, which made the vial warm to the touch after milling.

\section{Thermal Treatment of Powders}

\section{a) Hydrogen Removal: “Degassing”}

Hydrogen removal, or "degassing", was accomplished by heating the powder to $500{ }^{\circ} \mathrm{C}$ in a dynamic vacuum [96]. A system to do this was constructed by modifying an existing quartz encapsulator system and building a furnace capable of a $1000{ }^{\circ} \mathrm{C}$ temperature. The furnace is attached to a Eurotherm PID controller with a silicon controlled rectifier power module. The modified quartz encapsulator consists of a manifold that accepts $9 \mathrm{~mm}, 12 \mathrm{~mm}, 15 \mathrm{~mm}, 19 \mathrm{~mm}$, and $25 \mathrm{~mm}$ diameter quartz tubes. The manifold is attached to mechanical and diffusion vacuum pumps capable of 
producing $\leq 10^{-4} \mathrm{~Pa}$ vacuum. This system, with a furnace designed to accept the largest diameter quartz tube, allows heating of the powder in a dynamic vacuum.

Regarding use, the powders are placed in a $15 \mathrm{~mm}$ diameter tube, approximately $50 \mathrm{~mm}$ long, with $\mathrm{Al}_{2} \mathrm{O}_{3}$ batting inserted in the open end of the tube. The $15 \mathrm{~mm}$ tube is placed in the larger $25 \mathrm{~mm}$ tube that is then necked down to a very small diameter at the tested length of the eventual sample ampoule and attached to the $25 \mathrm{~mm}$ position of the encapsulator manifold. The furnace is placed in a position so the powder is in the hot zone of the furnace where a vacuum of at least $10^{-4} \mathrm{~Pa}$ is established. The furnace is ramped up to $500{ }^{\circ} \mathrm{C}$ and the vacuum increases and becomes erratic after $250{ }^{\circ} \mathrm{C}$, expected from organic expulsion, then invigorates near $500{ }^{\circ} \mathrm{C}$ expected from hydrogen outgassing. Removal of heat is determined when the vacuum decreases close to the original value and the behavior is stable, which is typically less than $1 \mathrm{~h}$. The large tube is then sealed off by applying heat with a hydrogen-oxygen torch to soften the quartz tube until it collapses at the necked down region sealing the tube. This process leaves the powder in a vacuum inside a quartz ampoule.

\section{b) Heat Treatment: “Annealing”}

Thermally treated powders are used as a standard in an important empirical instrumental correction procedure used in a new XRD analysis technique to be discussed. These heat treatments, referred to as "annealing", were performed on the vacuum-sealed quartz ampoules resulting from the hydrogen degassing procedure described in the previous section. The quartz ampoules were placed in a box furnace at $1000{ }^{\circ} \mathrm{C}$ for $2 \mathrm{~h}$ (unless otherwise specified) and furnace cooled to room temperature. This procedure when necessary was applied to approximately half of the powder produced from each RMA/M process. Following this annealing treatment, for simplicity the powder is referred to as "annealed powder". 


\section{Characterization}

\section{a) X-ray Powder Diffraction (XRD)}

XRD was used extensively to monitor the structural changes during the RMA/M

process and to characterize the structure of the consolidated bulk materials. The powders were screened to -200 mesh to maintain a small particle size and provide the desirable random particle orientations. Annealed powders often required simple mechanical crushing prior to screening as a small amount of sintering occurred. Additionally, it's important to note that screening provides similarities in powder particle size for all powders analyzed, thus providing similar packing characteristics.

The solid specimens used for XRD were produced by cutting along the center of the consolidated HIP cans and cutting to a nominal length of $20 \mathrm{~mm}$ with a water-cooled precision wafering saw. This results in a sample that has a shape of a half of a cylinder $20 \mathrm{~mm}$ in length with the outer cylindrical portion clad with the copper can and tantalum foil. The alloy exposed surface of the specimens was ground on a 120 grit grinding wheel. In addition, the copper can and tantalum foil exposed on the alloy surface were removed so that only the consolidated alloy was exposed to x-ray illumination to prevent diffraction from the copper can or tantalum foil. Next, the surfaces of the specimens were mechanically ground with successively finer SiC papers through 1200 grit. Finally specimens were successively polished with $6 \mu \mathrm{m}$ diamond paste, and a $0.05 \mu \mathrm{m} \mathrm{Al}_{2} \mathrm{O}_{3}$ solution. These polished specimens were etched with Keller's micro etch (1 vol. pct. HF, 1.5 vol. pct. $\mathrm{HCL}, 2.5$ vol. pct. $\mathrm{HNO}_{3}$, balance distilled $\mathrm{H}_{2} \mathrm{O}$ ) for $5 \mathrm{~s}$. This final polish $\left(0.05 \mu \mathrm{m} \mathrm{Al}{ }_{2} \mathrm{O}_{3}\right.$ solution) and etch procedure was repeated 3 times, ending with an etch, this removes the deformed surface layer produced by the polishing process. The specimen was mounted with the length direction in the plane of the tube and detector, to ensure a constant diffraction volume down to $20^{\circ} 2 \theta$.

\section{(1) Data Collection}

X-ray diffraction data were collected with a Scintag Inc. (Division of Thermo ARL, Dearborn, MI) XDS-2000 $\theta / \theta$ powder diffractometer. The diffraction optics 
parameters included 1 - $2 \mathrm{~mm}$ beam slits, 0.5 - $0.3 \mathrm{~mm}$ receiving slits, and a graphite monochromator set at an angle of $26.6^{\circ} 2 \theta$. The x-ray tube with a 2000 watt $\mathrm{Cu}$ target was operated at a power setting of $45 \mathrm{kV}$ and $35 \mathrm{~mA}$. The powder samples were packed mechanically in a side-drifted holder developed and made at MTU. This side-drifted holder has outside dimensions of $42 \mathrm{~mm}$ in length by $30 \mathrm{~mm}$ in width, with the powder channel $40 \mathrm{~mm}$ by $19 \mathrm{~mm}$ wide, and $1.25 \mathrm{~mm}$ deep, as shown in Figure II-1. For loading, the holder is covered with a $3 \mathrm{~mm}$ thick glass plate and powder is inserted into the channel in the aluminum holder, periodically tapping the holder while filling the channel. The glass plate is released from the holder, with pressure still applied downward, by sliding the glass toward the closed end of the channel until it is completely removed from the holder. Proper packing will leave the powder surface exactly in the same plane as the surface of the holder insuring the proper sample surface placement.

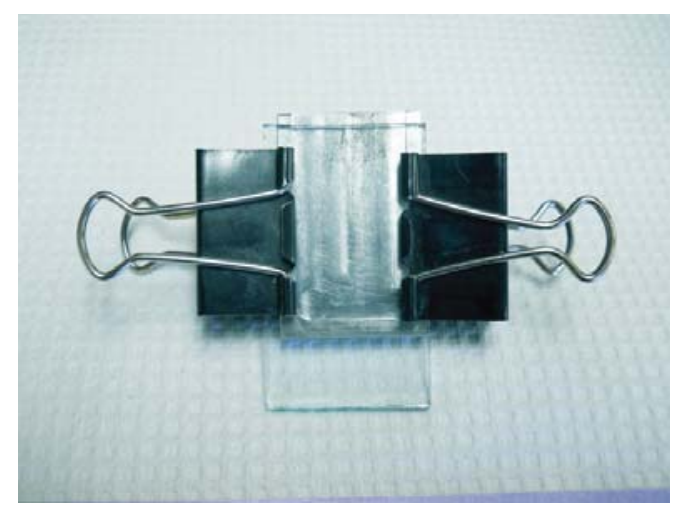

Figure II-1 Side-Drifted Powder Diffraction Sample Holder

Mounting of the consolidated solid specimens for XRD was accomplished using a channel specimen holder $30 \mathrm{~mm}$ wide, $40 \mathrm{~mm}$ long, and $20 \mathrm{~mm}$ deep. The top of the channel is mounted flush with the focal plane of the instrument. The specimen is held in this plane by pressing down on the top of the specimen (above the channel) with a $3 \mathrm{~mm}$ thick glass plate with the specimen supported by clay. The glass plate is held against the top of the channel for approximately $5 \mathrm{~m}$ to minimize any elastic deflection from the clay. It is important that the clay support be smaller than the dimensions of the specimen to avoid any exposure to the beam since clay provides a crystalline diffraction pattern.

Of fundamental importance in comparing diffracted intensities, for example in the calculation of phase volume fractions from diffraction data, is that the diffracting volume 
remains constant. In order to achieve a constant diffracting volume, it is necessary that the length of the sample be greater than the length of the beam at the lowest incident angle of interest. This beam length is defined along a direction in the surface of the sample that lies in the plane defined by the incident and diffracted beams, and changes with angle. The assumption of constant volume derives its validity from the increase in the depth of penetration of the beam with increasing diffraction angle and the corresponding decrease in the beam length at the specimen surface. This is imperative for the quantitative peak area measurements for the diffraction peaks from the various phases present, if the beam is larger than the sample significant intensity losses will occur. For the Scintag, Inc. XDS-2000 diffractometer, based on given optics, the length of the powder sample holder and the consolidated samples is longer than the beam down to at least $12^{\circ} 2 \theta$ for the powder holder and $20^{\circ} 2 \theta$ for the consolidated samples in these experiments.

Absolute intensity comparison of as-milled and annealed powder is required for a new XRD analysis method developed in this work. The powders have similar densities and therefore differences in weight of a powder, in the constant volume holder (infinite in terms of the beam volume), leads to varying numbers of atoms within the constant diffracted volume. Since x-ray scattering is electron dependent, the scattering power from a sample will be based largely on the total number of atoms and atom type, which is proportional to the weight of the powders in the constant volume holder. Powders under the force of gravity typically pack at $60 \%$ of the full density. All powders were packed using a constant tapping force method in the same holder, all passing -200 mesh screening. However, to get an estimate of this variation, as-milled powder samples was repacked five times each in the same holder and weighed after each packing, this resulted in a $95 \%$ confidence interval of $\pm 1 \%$ of the weight.

\section{(2) “Intelligent" Profile Fitting}

Peak profile fitting or "modeling" of XRD peaks is invaluable in the process of quantitative measurement of complex diffraction patterns. Often diffraction peaks from multiple phases can overlap, with the collected pattern representing a composite sum of 
all diffraction peaks, with peak profile fitting providing a mechanism of deconvoluting the individual diffraction peaks of the various phases. Typically diffraction peaks are sharp and despite the overlap a majority of the features from each individual diffraction peak are retained which allows for simple computer based development of the actual peak profiles. In the fit process these profile parameters are changed in the direction that minimizes errors between the profile sum and the actual XRD pattern, with highly crystalline diffractions the convergence is rapid and very decisive providing a good fit. However, the XRD patterns produced by the as-milled material in this work are extremely complex because of the extreme broadening of diffraction peaks from all phases coupled with overlap of patterns from as many as five phases creating a XRD pattern that has an "amorphous-like" appearance as demonstrated in Figure II-2.

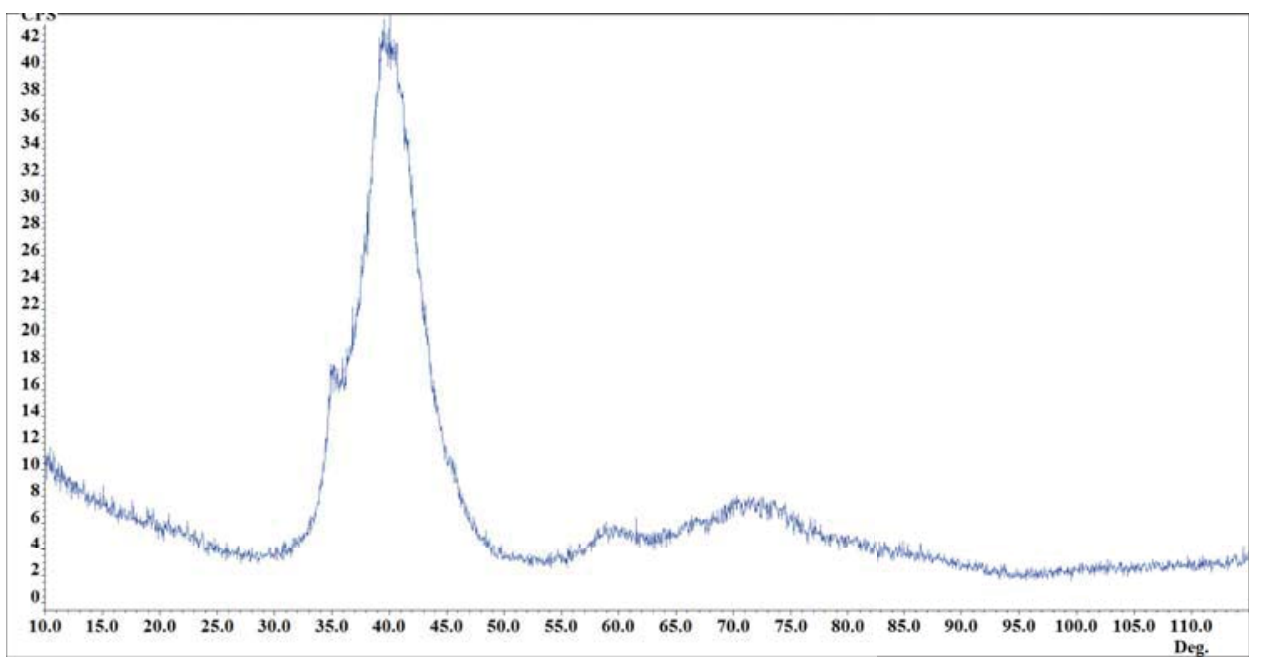

Figure II-2 Complex Pattern for 40 h RMM Time Sample RMM40h9TiAr

Two basic stand-alone methods of profile fitting diffraction peaks are incorporated into most software, one based on peak identification by computer algorithms, the other based on manual insertion of the peak shape and position, in both cases distinct features from each peak are required. Once the peak position is identified (by computer or manual insertion) a peak profile is inserted being a best guess of the expected peak shape and position. Often the position is accurate but other features are much further from the actual profiles. The fit process proceeds based on error minimization between the sum of the profiles with the diffraction pattern. In typical profile fitting the distinct features for each individual diffraction peak allow for easy 
modeling of the peak profiles; however, in the as-milled patterns the features are highly convoluted which could produce extremely large errors and many important diffraction peaks will not be identified. More importantly, this profile fitting is the first data reduction procedure accomplished prior to any XRD analysis and producing good fits are paramount to providing accurate calculations. Thus, the ability to extract individual peak information requires a different approach based on human interaction and knowledge of the phase structure; this procedure will be termed "intelligent" profile fitting.

The first step in the intelligent profile fitting process is phase identification to gain an understanding of the phases present as well as their peak positions; for example, the original experiment for long milling times provided important information about phases produced by reactions. Once an understanding of the phase structure is known the next step is to minimize the range of data and hence number of profiles to fit by reducing the diffraction pattern into ranges. Of upmost importance here is to ensure the end points of each range are at true background. This is followed by careful insertion of a peak profile as closely as possible to what is expected, paying particular attention to the position and expected breadth. Once satisfactory profiles are inserted error minimization provides proper fitting of the profiles; however, user interaction is necessary to insure the peaks are in the proper positions since the original choice of a profile can have profound impact on the result in this process because of the limited peak features in theses XRD patterns. Often this process has to be repeated with more careful insertion of the profiles in order to obtain a good fit; for example, lattice parameter determination helps to understand if the determined peak positions are all proper for a given phase. After copacetic peaks are obtained, this process is redone up to 5 separate times, along with a new background fit, with all quantitative peak data for each important XRD analysis derived from an average of multiple independent profile fits and analysis. The annealed and consolidated samples have well-defined diffraction peaks and follow standard practice of a single profile fitting unless otherwise noted.

In order to illustrate the complexity and success of this analysis an example of a satisfactory fit is provided in Figure II-3 showing the individual profiles developed for the identified phases for the same pattern provided in Figure II-2. It is quite easy to see 
especially in the case of the highest intensity in the $40^{\circ} 2 \theta$ range that a computer algorithm would have picked one large peak that is actually composed of more than one peak. This is more pronounced at the higher angles. Due to the lack of sufficient features the manual insertion of peaks has significant consequences since not only the position, but the intensity and shape all play a role in the final convergence of the iterative error minimizations, and for a good fit it would be expected that for each phase all peaks should have similar traits.

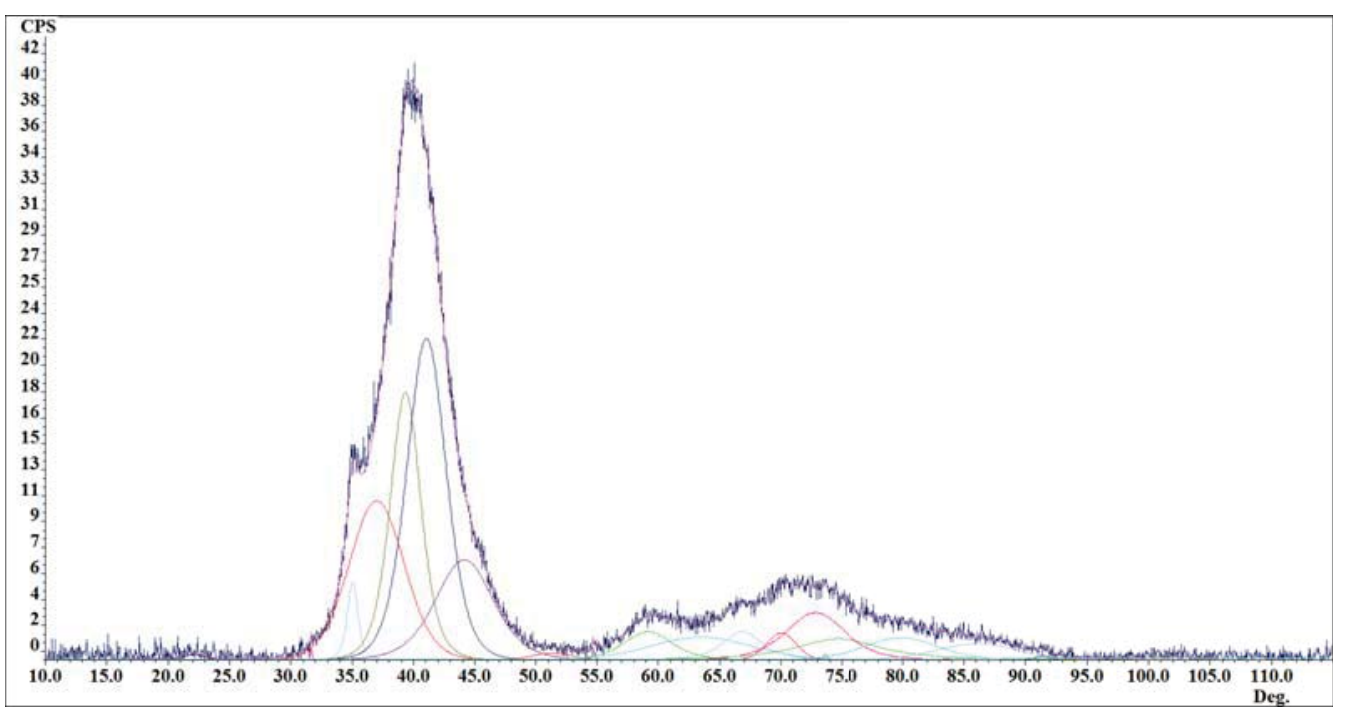

Figure II-3 Example of Profile Fitting of the RMM40h9TiAr Sample RMM Time $40 \mathrm{~h}$

Several different mathematical models can be utilized in profile fitting; however, throughout this research the symmetrical Pearson VII profile, a commonly preferred model for diffraction peaks is used. The unique aspect of the Pearson VII profile model is that no assumptions about peak shape, beyond symmetry, are necessary, in contrast with most other models that assume a profile shape (e.g. Cauchy, Gaussian, etc.). The mathematical expression has several parameters that are varied until an error minimized match is obtained with the raw data these include: a shape parameter allowing the general shape to change, a FWHM, intensity, and $2 \theta$ position of the peak maximum.

The attractiveness of the Pearson VII function is the shape independence, but the one drawback is that if diffraction peaks have any asymmetry that information is lost. While asymmetric functions are available; e.g., Split Pearson VII, the added peak parameters in the model made convergence even more difficult for these complex 
patterns. It is expected that the $\mathrm{L}_{2}$ intermetallic is the only phase present that could possibly have asymmetries and future work might attempt to provide for modeling of asymmetries.

\section{(3) Phase Identification}

Identification of phases is commonly accomplished by comparing the x-ray powder diffraction peaks established in each recorded pattern with the database for crystalline materials available in the JCPDS-ICDD powder diffraction file [115]. A match is determined if a specific number of diffraction peaks in the sample XRD pattern are accounted for by a particular phase from the JCPDS-ICDD powder diffraction file. Given the powder nature and small grain size in consolidated samples the intensity matches for stoichiometric alloys should also follow those of the database adding credence to the match. Of course, in this match process the intense peaks are important, especially when identifying small amounts of a phase. Since the phases in the JCDPSICDD powder diffraction file typically do not contain solid solution information, phases may be considered a match if the correct pattern is present but the peaks are consistently shifted to low or high angles on the sample pattern.

The computer database for the JCPDS-ICDD powder diffraction file used in this work ended with Set 40. However, hardcopy of JCPDS-ICDD data contained in sets above 40 were manually entered to form a user-generated computer database. Thus, this user database includes phases with all combinations of elements used in these experiments. In addition, for phases of interest not contained in the current JCPDS-ICDD database, or for cases where quality of the data for a phase is suspect, several other sources of diffraction information can be used. These sources include profile fitting XRD scans of known material, calculating patterns based on published structural or reference information, or taking information directly from published diffraction data. An example of this last source is the work of Nic [116] who carefully prepared a single-phase $\mathrm{L}_{2}$ chromium-modified cubic trialuminide from -400 mesh pressed powders to avoid extinction effects. His data are much more reliable then JCDPS-ICCD cards currently available for the $\mathrm{Al}_{67} \mathrm{Cr}_{8} \mathrm{Ti}_{25}$ phase (43-1099 and 43-1309). 


\section{(4) Direct Comparison Volume Fraction Determination}

Phase volume fractions were calculated by means of the x-ray powder diffraction direct comparison method. This allows quantification of the amount of any phase, regardless of the quantity present, or the true crystallite size, as long as it produces a measurable diffraction peak. With this technique, scattering from any phase is averaged over a constant diffracting volume giving a very good "bulk" representation of the phases present, provided a constant diffracting volume is maintained. However, this method for total phase volume fraction determination requires identification of all phases present, including those of a non-crystalline nature, and knowledge of the position coordinates of all atoms in the unit cell of each phase. It should be pointed out that volume fractions determined by other means can also be used in conjunction with the direct comparison results to formulate a complete phase volume fraction analysis. Thorough treatment of this technique can be found in several classic diffraction texts; e.g., [117, 118].

Volume fractions were determined with software called Volfract [119] developed at MTU, which has an algorithm based on the x-ray diffraction direct comparison method. The integrated area for each diffraction peak is measured to obtain the needed intensities as opposed to using the maximum peak intensity which depends on the peak shape and therefore can introduce large errors. The calculation of the $R$ factor, Equation (II.1), for a given $\{\mathrm{hkl}\}$ peak from each phase requires the determination of the structure factor. This requires knowledge of atomic scattering factor(s) and position coordinates of all atoms in the unit cell of each phase. In this treatment the atomic scattering factors were determined with the Hartree-Fock wave function and curve-fitted with the coefficients tabulated by Cromer [120]. All of the atom positions for known phases studied in this research were calculated from the point set determined for each phase, based on the particular point symmetry from Pearson's Handbook of Crystallographic Data for Intermetallic Phases [121], in conjunction with the crystallographic translation data in the International Tables for X-ray Crystallography [122].

$$
R_{\alpha}=\left(\frac{1}{v^{2}}\right)\left[\left|F^{2}\right| p\right]\left(\frac{1+\cos ^{2} 2 \alpha \cos ^{2} 2 \theta}{\sin ^{2} \theta \cos \theta}\right)\left(e^{-2 M}\right)
$$


Equation (II.2) shows the structure factor determined for a binary (two atom) ordered intermetallic alloy with a primitive cubic $\mathrm{L}_{2}$ structure assuming an $\mathrm{Al}_{3} \mathrm{Ti}$ stoichiometric alloy with atom positions determined from the four FCC translation vectors defining the positions of the titanium and 3 aluminum atoms, respectively $(0,0,0)$, $(1 / 2,0,1 / 2)(1 / 2,1 / 2,0)$, and $(0,1 / 2,1 / 2)$. Equation (II.3) represents the structure factor squared for the fundamental peaks with h,k,l unmixed. The structure factor squared, shown in Equation (II.4) for superlattice reflections (h,k,l mixed), is dependent on the difference in scattering factor. Of course this represents complete order in the alloy and the intensity for the h,k,l mixed diffractions will diminish as the alloy disorders, finally disappearing when the atom types occupy sites randomly.

$$
\begin{gathered}
F=f_{T i} e^{(0)}+f_{A l}\left(e^{-\pi i(h+k)}+e^{-\pi i(h+l)}+e^{-\pi i(k+l)}\right) \\
|F|^{2}=\left(f_{T i}+3 f_{A l}\right)^{2} \quad \text { (II.3) } \\
|F|^{2}=\left(f_{T i}-f_{A l}\right)^{2} \quad \text { (II.4) }
\end{gathered}
$$

The $R$ factor also contains other information based on peak position such as the Lorentz-Polarization factor which corrects for geometrical effects of intensity flux and wave polarization, also a correction for the scattering from the graphite crystal monochromator. The temperature correction, $e^{-2 M}$, included in the Volfract program computations, is a function of the particular diffraction peak and the average atomic vibrations, which can be represented by the Debye or Debye-Waller factor. This factor is strictly only valid for pure elements having the cubic crystal structure. Additionally, Debye temperature coefficients are typically tabulated for elemental materials and it is not valid to apply a rule of mixtures to the elemental component values in multiple element phases. However, Nic [123] has empirically determined a Debye temperature equivalent for the $\mathrm{L}_{2}$ material in site occupancy studies for use in the Volfract program. In that work, Nic empirically determined site occupancies for all the atomic sites for a variety of these trialuminide alloys.

The Volfract program requires a standard file providing important information for each phase such as the crystal structure, lattice parameter, atom type, site occupancy 
fraction, atomic weight, Debye temperature, atomic scattering factor, absorption edge, and number and location of atom positions. All standard files used in this work are given in Appendix VIII.A Standard Files. These values for the cubic $\mathrm{Al}_{66} \mathrm{Cr}_{9} \mathrm{Ti}_{25}$ alloy standard file were used in all calculations involving this phase except where noted. Since the phase has high intensity peaks with the largest temperature effect, correcting for temperature will minimize the total analysis error. All other multi-element phases will not use a Debye temperature correction. It should be noted this temperature correction is usually small, especially for high melting temperature materials. For example, there is a $10 \%$ decrease in the intensity of $90^{\circ} 2 \theta$ peaks from iron at $20^{\circ} \mathrm{C}$, and the correction approaches zero at low angles. Similar melting temperature alloys would have similar corrections, so it can be concluded that room temperature corrections are small, and the use of low angle peaks further minimizes the error from the corrections.

\section{(5) Lattice Parameter Calculations}

The method used for determining lattice parameters is a refinement process based on the reciprocal tensor algorithm of Schwarzenbach [124]. This method requires knowledge of the crystal system and indexed $\{$ hkl $\}$ diffraction planes for each phase. The calculations were performed with Scintag Inc. DMS-NT crystallography software option, version 1.36b. Lattice parameter calculations are accomplished on each individual profile fit to help in determination of good fit of the intelligent profile fitting model, with the results reported as an average of the various independent pattern profile fits.

\section{(6) Scherrer Crystallite Size Measurement}

The Scherrer Equation (II.5) provides a simple method to estimate the crystallite size of a material based on diffraction peak broadening. This calculation is commonly referred to as yielding the "particle size", which can be a misleading term since this technique actually measures the mean coherently diffracting domain distance (CDDD), or commonly called the crystallite size, in the diffracting volume. In some cases this can be related to the external physical particle size, usually in the case of single crystal powder particles. More often it represents a mean interdefect distance within the grains of 
polycrystalline materials as a result of defects such as dislocations and subgrain boundaries defining the CDDDs that are smaller than the grain size. Of course, these defects can be grain boundaries and thus can represent a grain size. This measurement in its simplest form requires several assumptions, the most important being that the broadening caused by microstrain or "non-uniform strain" is negligible. Additionally, the basic expression assumes a pseudo-spherical shape for the particles.

$$
\begin{gathered}
D=\frac{0.9 \lambda}{\beta \cos \theta} \quad \text { (II.5) } \\
\lambda=0.1540562 \mathrm{~nm} \\
\beta=\text { FWHM in radians } \\
\theta=\text { angle of peak } \\
D=\text { crystallite size nm }
\end{gathered}
$$

Calculation of the crystallite size using the Scherrer method is based simply on the full width at half maximum (FWHM) of each diffraction peak transformed into radians. Also broadening from the instrument must be taken into account to provide a corrected FWHM that represents the broadening due to crystallite size alone. Two common methods are used for the correction based on Gaussian and Lorentzian (Cauchy) peak shapes. However, these shapes typically represent the extremes of actual diffraction peak shapes encountered, which are often a mixture of the two shapes. Therefore, the instrumental broadening correction utilized in the current work is a geometric mean of the two forms of correction [125] (Equation (II.6)). It is important to note that instrumental broadening is best determined from well-annealed large crystallite size samples of the phases in question. Although many researchers have used different materials than the phase for which crystallite size is being determined, the broadening from different well-annealed materials can vary and is influenced by intrinsic broadening characteristics of that particular phase, therefore in this work measurements for reference purposes were made on the phase being studied in a well-annealed state wherever possible. The final crystallite size value reported for each phase represents an average of the values found from several diffraction peaks, as will be indicated. Experience in applying the Scherrer method has shown that the average value of the crystallite size for all diffraction peaks in the range collected is typically in close agreement with crystallite 
size results obtained from the Warren-Averbach Fourier analysis (which separates the effects of crystallite size and strain broadening). The Scherrer method assumes a zero microstrain; however, microstrains effects increase with peak angle.

$$
\begin{aligned}
& \beta=\sqrt{\left[\left(\sqrt{\beta_{M}^{2}-\beta_{I}^{2}}\right) *\left(\beta_{M}-\beta_{I}\right)\right]} \quad \text { (II.6) } \\
& \beta_{M}=\text { FWHM measured } \\
& \beta_{I}=\text { FWHM due to instrument broadening }
\end{aligned}
$$

\section{(7) Warren-Averbach Fourier Analysis}

The more detailed and complete Warren-Averbach (W-A) [126, 127, 128, 129] approach was utilized to separate and determine peak broadening caused by the CDDS, and the nonuniform strain or microstrain, developed in the crystalline material. This analysis was performed with software developed at MTU based on early work by Wagner [130]. The program calculates the complete set of Fourier coefficients for both sine and cosine functions that define the diffraction peak profiles for the sample of interest (e.g., a cold worked sample), as well as for an annealed sample, then applies a Stokes correction $[126,129]$ to remove instrumental broadening effects, yielding the true broadening of the diffraction peak. The diffraction pattern from an annealed sample provides a measure of the instrumental broadening due to the optics as well as any intrinsic defects in the material. As noted, the general requirement for instrumental broadening correction is a material that is essentially defect-free, with diffraction peak positions corresponding to the $2 \theta$ positions of the cold worked diffraction peaks.

In general, the W-A procedure involves determining the Fourier transform, by numerical integration of the broadened diffraction peaks, and the corresponding annealed peak for each $\{\mathrm{hkl}\}$ peak. The true broadening is then determined by dividing the broadened Fourier transform by the annealed Fourier transform for each $\{\mathrm{hkl}\}$ peak. The resulting transform represents the true broadening (due to the cold work and other effects) and this determination is referred to as the Stokes corrected transform. Because microstrain effects vary with order of diffraction, employing two diffraction peaks of the same $\{\mathrm{hkl}\}$ form permits mathematical separation of the effects of microstrains from CDDS broadening, a root-mean-square (rms) value. Specifically, the zeroth order 
intercepts for each pair of $\{\mathrm{hkl}\}$ peaks of Fourier coefficients for a given column length provide the crystallite size coefficients and the slopes of the same series of lines provide the microstrain coefficients. From these coefficients the crystallite size and microstrain are easily determined.

\section{(a) Warren-Averbach Fourier Analysis of Diffraction Peak Profile Fit Models}

The W-A approach requires isolated diffraction peaks in the raw data, but slight overlap on the tails on only one side of the diffraction peak can be accommodated by the software utilized. However, the complex nature of the as-milled diffraction patterns is not conducive to conventional W-A Fourier analysis using the raw data. In order to obtain information from the severe overlap of the diffraction peaks in patterns from as-milled powders in this research; individual and isolated peak profiles are created from the separated peaks accomplished by the intelligent profile fitting, Chapter II.A.5.a)(2).

The profile model chosen was the Pearson VII which, as mentioned earlier, does not assume a shape of the diffraction peaks (with the exception of symmetry). The mathematical model parameters for the Pearson VII function in Equation (II.7) can be obtained from the result of the intelligent profile fit analysis for each diffraction peak of interest. The variable $x$ is the chosen step size (user defined) with the $k$ parameter a function of the shape exponent $n$ and the FWHM $\beta$ with the value $2 \theta_{0}$ the maximum position of the diffraction peak. It should be pointed out that the intensity in this analysis is not important. Obtaining these parameters from the individual diffraction peaks determined from the intelligent profile fitting, the peaks can then be synthesized mathematically using an in-house FORTRAN program. These synthesized diffraction peak profiles can be input into the W-A program allowing the determination of the crystallite size and microstrain values. It should be noted that the synthesized profile is constructed only from the $\mathrm{K}_{\alpha 1}$ contribution to the collected diffraction peak. 


$$
\begin{array}{r}
\mathrm{I}_{2 \theta}=\frac{\mathrm{I}_{2 \theta_{o}}}{\left(1+k^{2} x^{2}\right)} \\
k=\frac{\left(2^{\left(\frac{1}{n}\right)}-1\right)^{\frac{1}{2}}}{\frac{\beta}{2}}
\end{array}
$$

It is important to note that this peak synthesis technique using the Pearson VII profiles has not been previously used in analysis of complex patterns and provides a unique method of allowing for characterization of the observed complex patterns. The sole reference related to this work is by Enzo et al. [131], they determined the W-A Fourier crystallite size and microstrain values for a variety of single-phase materials using a pseudo-Voigt function. The pseudo-Voigt function is written as a sum of Lorentz and Gaussian functions. In contrast, the Pearson VII function assumes no shape to the peak, and can provide a better description of the diffraction peaks profiles. Enzo et al. also found that such a profile fit model reduced the well-known "hook" effect common in the W-A Fourier analysis and also minimized the oscillations often found at longer column lengths.

The use of these synthesized Pearson VII profiles was validated by applying the same approach to pure copper powder that had undergone one hour of mechanical milling with the PCA hexanes in an argon environment. Since all peaks do not overlap in this case, W-A analysis was applied to both the Pearson VII profile fit data and the raw data in order to compare the results, shown in Table II-1. For the $<111>$ direction the two methods give the same crystallite size and a minimal difference in the microstrain at a value of $5 \mathrm{~nm}$. On the other hand, the $<100>$ direction values show a small difference for both the crystallite size and the microstrain at a value of $5 \mathrm{~nm}$. This has been attributed to the very low intensity of the $\{400\}$ diffraction peak, which despite a count time of 60 seconds had a statistical maximum variation of counts of $\sim 5 \mathrm{cps}$ vs. the peak height of only 8 cps. In such a case, slight changes in background choice can have significant effects on the shape of the peak profile. Of particular interest is the elimination of the oscillations in the Fourier coefficients for the $<100\rangle$ direction with synthesized peaks from profile fitting shown in Figure II-4. 
Table II-1 Comparison of Warren-Averbach Results for Copper Profile Fit Data vs Raw Data

\begin{tabular}{|c|c|c|c|c|}
\hline $\begin{array}{c}\text { Crystallographic } \\
\text { Direction }\end{array}$ & $\begin{array}{c}\text { CDDS } \\
(\mathrm{nm}) \\
\text { Profile Fit }\end{array}$ & $\begin{array}{c}\left\langle\varepsilon^{2} 5 \mathrm{~nm}\right\rangle^{1 / 2} \\
(\%) \\
\text { Profile Fit }\end{array}$ & $\begin{array}{c}\text { CDDS } \\
(\mathbf{n m}) \\
\text { Raw Data }\end{array}$ & $\begin{array}{c}\left\langle\varepsilon^{2} 5 \mathrm{~nm}\right\rangle^{1 / 2} \\
(\%)\end{array}$ \\
\hline$\{111\}-\{222\}$ & 22.2 & 0.204 & 22.2 & 0.208 \\
\hline$\{200\}-\{400\}$ & 10.8 & 0.220 & 12.0 & 0.290 \\
\hline
\end{tabular}

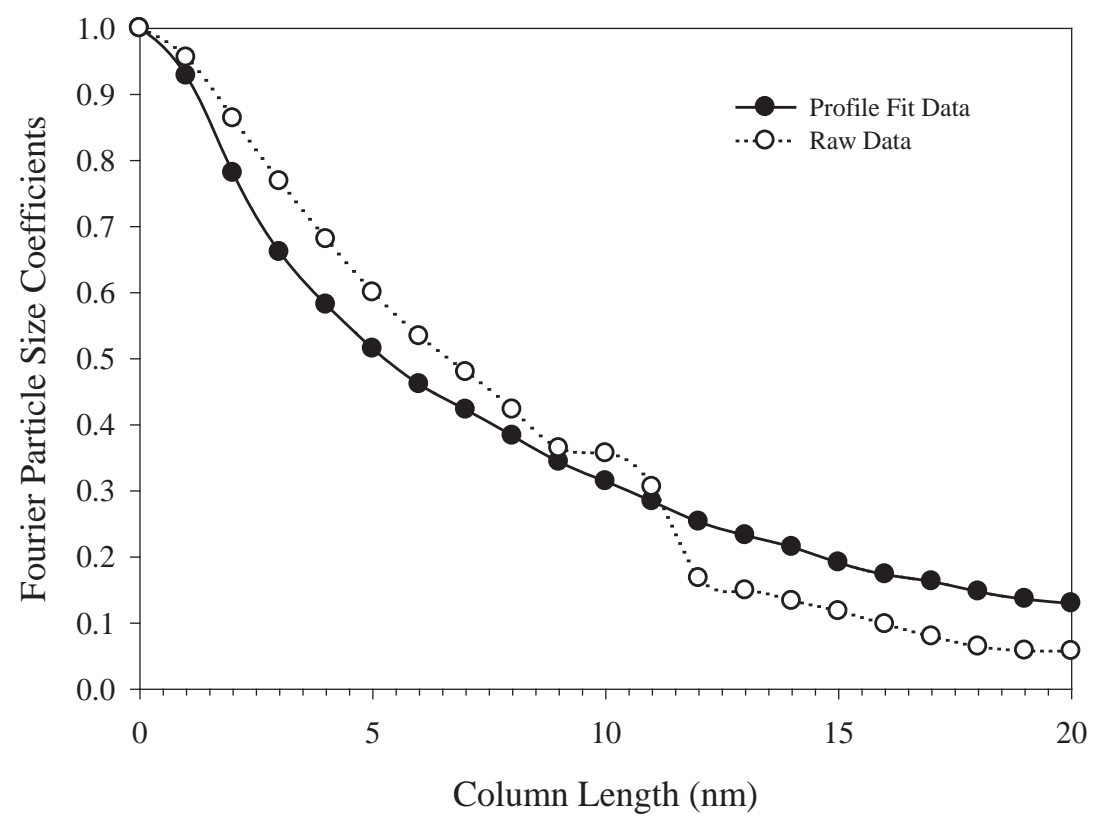

Figure II-4 Comparison of Profile Fit and Raw Data for the Crystallite Size Fourier Coefficients for Copper <100>

\section{(b) Dislocation Density Estimates}

Dislocation densities can be estimated from the crystallite size and microstrain data determined by W-A Fourier analysis, with the expression relating these quantities given in Equation (II.8). Where $b$ is the Burgers vector, $D$ is the crystallite size (using units of $\mathrm{mm}$ ), and the rms microstrain. Recall that the $\mathrm{L}_{2}$ intermetallic has an ordered or 
superlattice structure. Typical dislocation motion in these types of FCC-based structures would destroy the ordered relationship of the structure, hence dislocations move in pairs with an antiphase boundary (APB) between the two partials; the second dislocation restoring the ordered relationship destroyed by movement of the first. In the case of the $\mathrm{L}_{2} \mathrm{Al}_{66} \mathrm{Cr}_{9} \mathrm{Ti}_{25}$, full dislocations defined by $a<110>$ breakdown into partial dislocations of the $a / 3<112>$ type which have most commonly been observed as dominant room temperature mobile dislocations [132].

$$
\rho=\frac{2 \sqrt{3}\left\langle\varepsilon_{5 n m}^{2}\right\rangle^{1 / 2}}{b D}
$$

\section{b) Field Emission Scanning Electron Microscopy (FESEM)}

The macrostructure of some powder mixtures and microstructure of consolidated samples produced were studied using a Hitachi (Pleasanton, CA) S-4700 field emission scanning electron microscope to better understand the physical and structural aspects of the materials in question. The initial sample preparation of powders for observations, referred to as Method I, created samples by dropping a random sample of powder from a spatula on carbon tape mounted on aluminum holders, then gently spraying with filtered compressed air to remove excess powder. Subsequent sample preparations, Method II, used a higher flow rate by enclosing one gram of powder in a plastic container, with an air access hose, alongside an aluminum stub covered with a carbon-based adhesive. With the cover on the container filtered compressed air was blown into the access hose to create a thin film of powder on the surface of the stub. While both methods produce representative samples, Method II provides better particle separation. Micrographs of the powders were recorded with various magnifications, with the instrument in ultra-high resolution mode using a $1.5 \mathrm{~mm}$ working distance, $1 \mathrm{kV}$ acceleration voltage, and a $10 \mu \mathrm{A}$ emission current.

Consolidated samples for analysis were created by cutting disks from HIPed specimens with a precision wafering saw. These disks were mounted in Bakelite and mechanically ground with successively finer $\mathrm{SiC}$ paper through 1200 grit. The samples were then polished with $6 \mu \mathrm{m}$ diamond paste followed by $0.05 \mu \mathrm{m} \mathrm{Al}_{2} \mathrm{O}_{3}$ solution. After 
removal from the Bakelite mount, the specimen microstructure was revealed by etching with a modified Tuckers macroetchant (10 vol. pct. HCL, 10 vol. pct. $\mathrm{HNO}_{3}$, and 5 vol. pct. $\mathrm{HF}$ with the balance distilled $\mathrm{H}_{2} \mathrm{O}$ ) for approximately a second. It is interesting to point out the rapid nature of the etching process in the RMM consolidated samples compared to samples $\mathrm{Cr} 9$ and HIP3h both of which required a 45 s etch time. FESEM samples were mounted on standard Hitachi mounting plates and fastened by Super Glue ${ }^{\mathrm{TM}}$ with silver paint used to ensure conductivity between the sample and the mounting plate. Micrographs of the specimen surface were recorded at various magnifications, with the instrument in Ultra High Resolution mode using a $1.5 \mathrm{~mm}$ working distance, $1 \mathrm{kV}$ acceleration voltage, and a $10 \mu \mathrm{A}$ emission current.

\section{c) Transmission Electron Microscopy (TEM)}

The detailed microstructural features of the consolidated specimens were observed directly with TEM, utilizing the JEOL (Peabody, MA) 4000FX operating at $200 \mathrm{kV}$. Bright field (BF) imaging was employed to observe the microstructure, and $\mathrm{x}$ ray energy dispersive spectrometry (XEDS) provided localized chemical information. The microstructures of several specimens were also examined in BF imaging with a JEOL (Peabody, MA) 100CX TEM operating at $120 \mathrm{kV}$.

The procedure for creating thin foils from consolidated samples for TEM examination was based on the work of Zhang [10]. A rod with a diameter of $3 \mathrm{~mm}$ was cut from the consolidated material with an electro-discharge machine using a brass tube $3 \mathrm{~mm}$ in diameter. Disks $1 \mathrm{~mm}$ thick were cut from the rod with a precision wafering saw. These disks were ground with 400 and 600 grit $\mathrm{SiC}$ to a thickness less than $200 \mu \mathrm{m}$ by attaching them with Super Glue ${ }^{\mathrm{TM}}$ to a specially constructed jig. Finally, specimens were produced by electropolishing to perforation with a Struers Inc. (Cleveland, $\mathrm{OH}$ ) Tenupo-3 twin jet electropolisher. The electrolyte solution (5 vol. pct. perchloric acid, 35 vol. pct. butoxyethanol, and 60 vol. pct. methanol) was cooled to a temperature of -35 ${ }^{\circ} \mathrm{C}$. Typical settings for electropolishing were 35 - $40 \mathrm{~V}$ with a current density of 0.08 $0.15 \mathrm{~A} / \mathrm{cm}^{2}$. Perforation was typically achieved in 10 minutes. The perforated thin foils were immediately soaked in absolute ethanol after electropolishing to remove the anodic 
layer. Success rate for TEM specimen creation was $100 \%$; this unusual success rate was attributed to the extremely fine grain size.

\section{d) Chemical Analysis}

Several elements that can enter into the alloy as a result of the milling process were monitored. Most important to the process is the amount of carbon introduced as a result of the reactive milling process; however, nitrogen and oxygen are also of interest. The other possible chemical invasion involves iron from the milling media and vial. Selected samples were analyzed by two basic methods, inert gas fusion-gas chromatography (LECO) to monitor the light elements, and x-ray fluorescence spectrometry (XRF) to monitor iron and other elements with atomic numbers 9 (fluorine) and above.

\section{(1) Carbon, Nitrogen, and Oxygen Chemical Analysis}

Elements carbon, nitrogen, and oxygen were determined using a LECO (St. Joseph, MI) model C/S 224 for carbon/sulfur and a LECO model 136 for nitrogen/oxygen. Each analysis was performed on small pieces or powders (approximately $1 \mathrm{~g}$ ) with 3 independent measurements performed for each sample.

\section{(2) X-ray Fluorescence Spectrometry (XRF) Chemical Analysis}

Bulk chemical analysis of select consolidated samples, mainly to monitor iron contamination, was performed with a Thermo Electron Corporation (Dearborn, MI) ARL 9400 wavelength dispersive x-ray fluorescence spectrometer using UniQuant ${ }^{\circledR}(\mathrm{NH}$ Veldhoven, Netherlands) standardless quantitative analysis software. This analysis software is capable of analyzing for fluorine $(z=9)$ and higher atomic numbers (a total of 78 elements) without the need for standards. An important capability is that this analysis can be performed on small (less than $29 \mathrm{~mm}$ diameter), as well as irregular shaped samples. Analysis was performed on samples used in the XRD analysis with the surface prepared by polishing with $6 \mu \mathrm{m}$ diamond paste. Since the samples were smaller than the normal cassette opening this required developing means to hold the sample in place. Two methods were employed, one was to set the samples on a polymer film over the cassette 
opening, and the second was to Super Glue ${ }^{\mathrm{TM}}$ the samples to a plastic grid with Plexiglas backing to prevent interference from the cassette material. Instrument parameters were the default parameters used by the software.

\section{6. $\quad$ Properties Explored}

Properties investigated were density, hardness, compression stress/strain behavior, mechanical properties determined by ultrasonic measurements, and coefficient of thermal expansion (CTE). Because the consolidated samples weighed $15 \mathrm{~g}$ or less, in some cases, the size usually permitted testing only one sample instead of using multiple samples in destructive tests to provide statistics on experimental data. However, where possible multiple samples or measurements were utilized to provide statistics for that particular experimental data point.

\section{a) Density}

Density measurements on the consolidated samples were performed using the Archimedes method with sample sizes ranging from $\sim 1 \mathrm{~g}$ to $5 \mathrm{~g}$. In order to compare the density of the RMM powders to the consolidated material, gas displacement pycnometer density determination was accomplished on both powder and consolidated samples with weights ranging from $\sim 4 \mathrm{~g}$ to $9 \mathrm{~g}$.

\section{(1) Archimedes Method}

Dry samples were weighed to the nearest $0.0001 \mathrm{~g}$ on a Mettler (Columbus $\mathrm{OH}$ ) type H6 digCap160g analytical balance. A beaker of distilled water remained at room temperature for several days prior to testing and the temperature of the water was monitored to accurately determine the specific gravity. The samples were immersed in the beaker of distilled water in a platinum wire basket and shaken to remove any air bubbles. The immersed contents were weighed on a Mettler analytical balance to the nearest $0.0001 \mathrm{~g}$. Based on water temperature the specific gravity is determined and the density for each test can be calculated as shown in Equation (II.9). Ten measurements were taken to provide an average, with the sample thoroughly dried between measurements. 


$$
\rho=\frac{w t_{d r y}}{w t_{d r y}-w t_{w e t}} \times \text { SpecificGravity }
$$

\section{(2) Gas Displacement Pycnometer}

In order to measure the density of the powder material to compare with the consolidated sample, determinations were made with a Micromeritics (Norcross, GA) AccuPyc 1330 gas displacement pycnometer. The mass of each sample was the average of 5 measurements taken to the nearest $0.0001 \mathrm{~g}$ on a Mettler type H6 digCap160g analytical balance. The volumes measured by the AccuPyc 1330 are the average of 5 measurements calibrated with a stainless steel volume standard before each series of measurements.

\section{b) Vickers Hardness}

Vickers hardness was determined with an automated LECO Model V-100-C2 equipped with a $136^{\circ}$ diamond pyramid (DPH) indenter with load capabilities from 1 to $50 \mathrm{~kg}$. Instrument accuracy was verified before each series of measurements by confirming the hardness of the supplied test block 07-320. Reported values for the sample of interest represent an average of a minimum of ten measurements at loads below the cracking threshold. Independently, loads were varied from $1 \mathrm{~kg}$ up to the maximum load of $50 \mathrm{~kg}$ and the indentations examined for signs of cracking, and noting those loads at which cracking took place. The cracking load provides qualitative information about the fracture toughness of the material.

\section{c) Compression Stress/Strain Relationship}

The mechanical stress/strain properties were evaluated by compression testing to examine the effects of composition, structure, and processing, on strength and deformation behavior. Typically, composite samples weighed $15 \mathrm{~g}$ or less making compression testing attractive because of the small sample size. Yield strength, ultimate strength, work hardening rate, and strain to failure were parameters derived from these tests. Compression test specimens were sectioned from HIP-consolidated ingots using a precision wafering saw with a $\mathrm{SiC}$ blade. Specimens were produced in a rectangular 
parallelepiped geometry and nominal dimensions of 3 X 3 X $7 \mathrm{~mm}$. Sides and ends are made parallel, using a specially designed jig, by grinding with SiC through 1200 grit. Final surface preparation was accomplished by polishing with $6 \mu \mathrm{m}$ diamond paste, and then, with $0.05 \mu \mathrm{m}$ colloidal silica. All surface scratches were parallel to the applied load since all polishing was performed in this direction.

Compression tests were conducted using a computer-controlled Instron (Canton, MA) 4507 screw-type frame fitted with custom constructed cylindrical alumina rams with a diameter of $19.8 \mathrm{~mm}$ and $20 \mathrm{~cm}$ in length. Thin alumina buffering pads of the same diameter were in contact with the test specimens to prevent any possible damage to the main alumina rams. Careful alignment of the rams and alumina pads was accomplished to ensure that the pad surfaces were parallel. A clam shell resistance furnace was used to heat the specimen for the elevated temperature test. A Type-Kshielded thermocouple, in contact with the surface of the specimen, monitored the temperature. All samples were heated at approximately $0.25 \mathrm{~K} / \mathrm{s}$ and soaked for 30 minutes at the test temperature prior to testing. All compression testing was done with a nominal strain rate of $1 \times 10^{-4} \mathrm{~s}^{-1}$. Each sample height was carefully measured and multiplied by the strain rate to determine the appropriate crosshead velocity. Load vs. deflection data was digitally recorded using a calibrated $90 \mathrm{kN}$ load cell and the specimen-specific crosshead velocity. Compliance curves determining machine elastic deflection were developed before testing each specimen test by determining the load vs. deflection with the rams and pads together without a specimen. Finally, engineering stress-strain curves for the tests were calculated by mathematically correcting for the machine elastic deflection.

\section{d) Resonant Ultrasonic Spectroscopy (RUS)}

Isotropic elastic constants, Young's and shear moduli, as well as Poisson's ratio, were determined by RUS measurement for three samples of different carbide content. The samples had a nominal 4 X 4 X 8 mm rectangular parallelepiped geometry. Samples were cut from consolidated material with a precision wafering saw, and then polished on 240 grit $\mathrm{SiC}$ through $0.05 \mu \mathrm{m}$ colloidal silica using a specially designed jig to maintain 
parallel sides. RUS measurements were performed with an apparatus built by Le Claire [142]. Le Claire determined that RUS measurement error, based on two standard deviations, is an order of magnitude less than the more common Pulse Echo method for determining elastic constants.

\section{e) Coefficient of Thermal Expansion (CTE)}

Coefficient of thermal expansion of a material is the change in dimension divided by the original dimension times the temperature, that is strain per unit temperature often in units of $\mathrm{ppm} /{ }^{\circ} \mathrm{C}$. Equation (II.10) defines the relationship with the units provided by the instrument calculation method. Additionally, the CTE is dependent on the temperature of the material and hence results to compare materials are given either by a specific temperature or averaged over a temperature range. Of interest is the expected variation of CTE of these materials with changes in the carbide content. This determination was accomplished at the Institute of Materials Processing at Michigan Technological University. Measurements were made with a DuPont Instruments (Midland, MI) 943 Thermomechanical Analyzer attached to a Thermal Analyst 2100 with results determined for a specific temperature of $75^{\circ} \mathrm{C}$.

$$
\alpha=\frac{d \ell}{\ell \times d T}\left(u m / m^{o} C\right)
$$

\section{B. Reactive Mechanical Alloying/Milling (RMA/M) Processing Experiments}

In order to further understand the RMA/M process, including the reactions and structural changes that occur, processing has been observed in both air and argon environments and for both elemental and pre-alloyed powders. Recall this process was developed based on similar MA processing of binary titanium trialuminides with the PCA hexanes where it was determined that about $2 \mathrm{wt}$. pct. carbon is retained in a $20 \mathrm{~h} \mathrm{MA}$ time $[96,133]$. This information was used as a basis to determine the amount of excess titanium needed for a $20 \mathrm{~h}$ milling time and incorporated into all the experiments. In order to gain a basic understanding of the RMM processing, all parameters were fixed 
with the only variable an increase in the RMM time using excess titanium as determined necessary to react with the 2 wt. pct. carbon to form TiC. Economies of scale of the processing were investigated using elemental powders that were RMA for $20 \mathrm{~h}$, again using a similar amount of excess titanium. To study whether these fine-scale structures produced can be preserved in consolidation of bulk samples and to understand if large amounts of carbon that can be retained in this system, an experiment was developed that systematically varied both the amount of excess titanium and milling time; these samples were consolidated into fine-scale bulk materials and select properties were determined. Understanding of these properties produced showcases the simple nano-engineering capabilities of this unique processing method.

The sample codes used in this work use the first three letters to represent the processing method (RMA or RMM) which dictates whether elemental powders or prealloyed powders are the starting materials, followed by the milling time, amount of excess titanium in wt. pct., and finally argon or air atmosphere. The powders and PCA (hexanes in all cases) noted steel media with the specified atmosphere (either air or argon), are all milled in a $\mathrm{SPEX}^{\mathrm{TM}} 8000 \mathrm{mixer} / \mathrm{mill}$ for the appropriate time. For example in the experiment below (Chapter II.B.1), the sample code for a $20 \mathrm{~h}$ milling time is RMM20h9TiAr. Details of the milling procedure were given in Chapter II.A.3.

In all cases after each of these RMA/M runs the vial was opened in a fume hood. The contents of the vial were emptied on to a 35-mesh screen held over a stainless steel pan and shaken. The sample remained in the fume hood until all of the liquid hexanes evaporated. The loose as-milled powder, vial, ball media, and vial cover were all weighed on a Mettler Digital Scale with a precision of $0.01 \mathrm{~g}$.

\section{Reactive Mechanical Milling (RMM) of Pre-Alloyed Powders with Fixed Excess Titanium Addition in an Argon Atmosphere}

This experiment, designated as the RMMXh9TiAr Series, concentrated on RMM processing as a function of RMM time (hence the variable $\mathrm{X}$ ) thus focusing on providing a better understanding of the reaction with titanium and the carbon in the PCA 
established in the original experimentation. To eliminate the nitrogen reaction this experiment used an argon atmosphere to prevent the gas-powder reaction. Formation of the TiC during RMM with hexanes required additional titanium to prevent the expected depletion of titanium from the $\mathrm{L}_{2}$ intermetallic.

\section{a) Reactive Mechanical Milling (RMM) Parameters}

All of these milling experiments involved a mixture of pre-alloyed Reading Alloy Inc. powder with $9 \mathrm{wt}$. pct. additional elemental titanium. While theoretically only $8.2 \mathrm{wt}$. pct. titanium is required to react with $2 \mathrm{wt}$. pct. carbon to form the stoichiometric TiC (20 h milling time), the pre-alloyed Reading Alloy powder contained an initial carbon content of 0.25 wt. pct. warranting a slight titanium increase. Milling was accomplished using a SPEX ${ }^{\text {TM }} 8000$ mixer/mill using milling times $(X)$ of $0.5,1,5,10,15,20,25,30$, and $40 \mathrm{~h}$. Each charge composition is the same (Table II-2), with $18 \mathrm{ml}$ of the PCA hexanes, in an argon atmosphere.

Table II-2 RMMXh9TiAr Series Component Weights

\begin{tabular}{c|cc} 
Component & $\begin{array}{c}\text { Weight } \\
\text { (g) }\end{array}$ & Comments \\
\cline { 2 - 3 } Reading Alloy & 13.65 & -200 mesh \\
Titanium & 1.35 & Alfa 00384 -100 mesh $99.4 \%$ \\
\cline { 2 - 3 } Total Charge & 15.00 & \\
\cline { 2 - 3 } Media & $\mathbf{9 0 . 0 0}$ & $\mathbf{5 2 1 0 0}$ Steel 6.35 mm diameter \\
\end{tabular}

\section{b) X-ray Powder Diffraction (XRD)}

The structural evolution of the as-milled powders was carefully examined with XRD after each RMM time. The experimental XRD scan parameters are as listed in Table II-3. Note the progressively larger step size used for longer RMM times which allows for increased count times statistically aiding definition of the lower crystalline 
diffracted peak intensities. The sample preparation and mounting are described in Chapter II.A.5.a)(1).

Table II-3 RMMXh9TiAr Series X-ray Diffraction Patameters

\begin{tabular}{|c|c|c|c|}
\hline $\begin{array}{l}\text { RMM Time } \\
\text { (h) }\end{array}$ & $\begin{array}{c}\text { Scan angle } \\
\left(2 \theta^{\circ}\right)\end{array}$ & $\begin{array}{l}\text { Step Size } \\
\left(2 \theta^{\circ}\right)\end{array}$ & $\begin{array}{c}\text { Scan Count Time } \\
\text { (s) }\end{array}$ \\
\hline 0.0 & $10-110$ & 0.03 & 20 \\
\hline 0.5 & $10-120$ & 0.03 & 20 \\
\hline 1.0 & $10-115$ & 0.03 & 24 \\
\hline 5.0 & $10-115$ & 0.04 & 30 \\
\hline 10.0 & $10-115$ & 0.04 & 30 \\
\hline 15.0 & $10-115$ & 0.04 & 30 \\
\hline 20.0 & $10-115$ & 0.04 & 30 \\
\hline 25.0 & $10-115$ & 0.04 & 45 \\
\hline 30.0 & $10-115$ & 0.04 & 55 \\
\hline 40.0 & $10-115$ & 0.05 & 40 \\
\hline
\end{tabular}

Important to this work was an understanding of the x-ray background scattering of the annealed powder produced from as-milled powder from each RMM time. The XRD scan parameters were the same for all annealed powders, with data collected from $10-$ $120^{\circ} 2 \theta$, using a $0.03^{\circ} 2 \theta$ step size, and a 20 s count time.

\section{Reactive Mechanical Alloying (RMA) of Elemental Powders with Excess Titanium in an Argon Atmosphere}

Success in creating a dispersoid in the $\mathrm{L}_{2}$ intermetallic using elemental powders in the original experiment led to the exploratory experiment to investigate the RMA process. This process explored the means to effect some economies by reducing the cost by the amount needed to create pre-alloyed intermetallic powder. The original experiment 
verified that the cubic form of the $\mathrm{Al}_{3} \mathrm{Ti}$ phase could be produced by this process from elemental binary components.

\section{a) Reactive Mechanical Alloying Parameters}

For this elemental powder experiment, the parameters of powder weight, media weight, amount of hexanes, and carbon weight retained were based on work by Srinivasan et al. [96]. However, the total powder weight, mill media weight, and amount of hexanes were tripled in order to increase the amount of sample produced; although the charge size was the same as in RMM experiment, over a $50 \mathrm{wt}$. pct. increase in milling media was used with expectations of more impact volumes; however, the velocities may have been slightly decreased due to increase in volume of occupied space in the mill. Excess titanium in this case was $8.2 \mathrm{wt}$. pct. expected to consume the carbon resulting in $\sim 10$ wt. pct. TiC. This experiment was identified as RMA20h8.2TiAr with the initial charge composition given in Table II-4. These powder amounts produce the composition $\mathrm{Al}_{66} \mathrm{Cr}_{9} \mathrm{Ti}_{25}$ for the $\mathrm{L}_{2}$ intermetallic and the stated excess titanium.

\begin{tabular}{|c|c|c|c|}
\hline \multicolumn{4}{|c|}{ Table II-4 RMA20h8.2TiAr Powder Composition and Media Specifications } \\
\hline Component & $\begin{array}{c}\text { Weight } \\
\text { (g) }\end{array}$ & $\begin{array}{c}\text { Volume } \\
\text { (pct.) }\end{array}$ & Comments \\
\hline $\mathrm{Al}$ & 7.11 & 62.20 & Aldrich $202584-200$ mesh $99.95 \%$ \\
\hline $\mathrm{Cr}$ & 1.87 & 6.20 & Alfa $00077-48$ mesh $99.95 \%$ \\
\hline $\mathrm{Ti}$ & 6.02 & 31.60 & Alfa $00384-100$ mesh $99.4 \%$ \\
\hline Total Charge & 15.00 & & \\
\hline Media & 150.00 & & 52100 Steel $6.35 \mathrm{~mm}$ diameter \\
\hline
\end{tabular}




\section{b) X-ray Powder Diffraction}

$\mathrm{X}$-ray powder diffraction was utilized to observe the structure in the as-milled condition and the annealed condition. The as-milled powder diffraction data was collected from $10^{\circ}-90^{\circ} 2 \theta$, with a step size of $0.04^{\circ} 2 \theta$, and a count time of $28 \mathrm{~s}$. The annealed $\left(800^{\circ} \mathrm{C}\right.$ in this case) powder data was collected from $20^{\circ}-70^{\circ} 2 \theta$, a step size of $0.03^{\circ} 2 \theta$, and a count time of $25 \mathrm{~s}$.

\section{Reactive Mechanical Milling (RMM) of Pre-Alloyed Powders with Systematic Increases in Excess Titanium and RMM Time in an Argon Atmosphere}

The established RMM process results in the formation of titanium-based carbides (and nitrides when milled in air) during the milling process dispersed within the cubic $\mathrm{Al}_{66} \mathrm{Cr}_{9} \mathrm{Ti}_{25}$ ( $\mathrm{L}_{2}$ intermetallic). The results indicate that the amount of the carbides formed during the RMM process increases with milling time. Since titanium carbide is created during this processing the rate limiting factor is the availability of titanium, as shown by the results of the RMMxh9TiAr Series indicating saturation of the titanium carbide for longer RMM times and suggesting increased titanium amounts are needed to react with the expected absorbed carbon to avoid loss of titanium in the alloy and to keep the $\mathrm{L}_{2}$ intermetallic in the single-phase field. In this experiment increases in the amount of excess titanium will be accompanied by increases in RMM time. These alloys produced with variation in excess titanium and RMM time will be consolidated by a combination of cold and hot isostatic pressing thus comprising the bulk samples.

These RMM experiments were carried out in both air and argon environments. Details of the particular powder mixtures and the corresponding RMM process parameters are given in Table II-5, for both the Master Alloy and Reading Alloy powders used as pre-alloyed powders. For all cases milling media total weight was $90 \mathrm{~g}$. In order to investigate structure/property relationships in materials with higher carbide contents, the basis was an 8.2 wt. pct. Master Alloy (9 wt. pct. Reading alloy) with the $20 \mathrm{~h}$ RMM time both increased by factors of $2,3,5$, and 6 . 
$\mathrm{X}$-ray powder diffraction was utilized to characterize the structure in the as-milled condition. The as-milled powder diffraction data for all samples was collected from $10^{\circ}$ $120^{\circ} 2 \theta$, with a step size of $0.04^{\circ} 2 \theta$, and a count time of $30 \mathrm{~s}$.

\begin{tabular}{|c|c|c|c|c|c|}
\hline \multicolumn{6}{|c|}{ Table II-5 RMM Processing Parameters } \\
\hline RMM Process \# & $\begin{array}{l}\text { Initial Alloy } \\
\text { Composition } \\
\text { (wt. pct.) }\end{array}$ & $\begin{array}{c}\text { RMM } \\
\text { Atmosphere }\end{array}$ & $\begin{array}{c}\text { Powder } \\
\text { Charge } \\
\text { (g) }\end{array}$ & $\begin{array}{c}\text { Charge } \\
\text { Ratio }\end{array}$ & $\begin{array}{c}\text { RMM } \\
\text { Time } \\
\text { (h) }\end{array}$ \\
\hline RMM20h8.2TiAir & $\begin{array}{l}91.8 \% \text { Master } \\
\text { Alloy } 8.2 \% \mathrm{Ti}\end{array}$ & Air & 6.86 & 7 to 1 & 20 \\
\hline RMM20h8.2TiAr & $\begin{array}{c}91.8 \% \text { Master } \\
\text { Alloy } 8.2 \% \mathrm{Ti} *\end{array}$ & Argon & 15 & 6.7 to 1 & 20 \\
\hline RMM20h9TiAr & $\begin{array}{l}91.8 \% \text { Reading } \\
\text { Alloy } 8.2 \% \mathrm{Ti} *\end{array}$ & Argon & 15 & 6.7 to 1 & 20 \\
\hline RMM40h18TiAr & $\begin{array}{c}83.6 \% \text { Reading } \\
\text { Alloy } 16.4 \% \mathrm{Ti} \\
*\end{array}$ & Argon & 15 & 6.7 to 1 & 40 \\
\hline RMM60h27TiAr & $\begin{array}{l}73 \% \text { Reading } \\
\text { Alloy } 27 \% \mathrm{Ti}\end{array}$ & Argon & 15 & 6.5 to 1 & 60 \\
\hline RMM100h45TiAr & $\begin{array}{l}55 \% \text { Reading } \\
\text { Alloy } 45 \% \mathrm{Ti}\end{array}$ & Argon & 15 & 6.5 to 1 & 100 \\
\hline RMM120h54TiAr & $\begin{array}{l}46 \% \text { Reading } \\
\text { Alloy } 54 \% \mathrm{Ti}\end{array}$ & Argon & 15 & 6.5 to 1 & 120 \\
\hline
\end{tabular}

*Note samples were early in the experimental cycle before understanding chemical analysis of the Reading Alloys

\section{Consolidation of RMM and MM Powders by Cold Isostatic Press (CIP) and Hot Isostatic Press (HIP) Processing}

The original interest in this processing technique was for creating a consolidated dispersion strengthened alloy with a fine-grain size. In the experiment the powders derived from RMA/M processing were consolidated by powder metallurgy techniques of CIPing followed by HIPing, proving to be a process that holds promise for preserving some of fine-structure produced in the milling process.

As will be detailed, the RMM process developed resulted in reactions incorporating carbon, nitrogen (when milling in air), and hydrogen. The undesirable 
hydrogen was removed from the powder prior to consolidation by degassing in a vacuum as described in Chapter II.A.4.a).

The important details of the CIP procedure are as follows: post-processed or degassed reactive mechanically milled powders were placed in a 5/8" diameter CIP bag (a long rubber tube with one end closed) and a custom rubber stopper inserted to the point of contact with the powder, and secured with Nylon cable ties. The custom rubber stopper was fitted with filter paper on the insertion end with a glass tube through its center attached to Tygon tubing, which in turn was attached to a mechanical pump that applied a vacuum for approximately one hour. The vacuum was preserved by folding the Tygon tube and fastening it with Nylon cable ties. The CIP bag assembly was then CIPed in a National Forge Co. (Irvine, PA) model 100ksi CIP to a nominal pressure of $620 \mathrm{MPa}$ for approximately $0.5 \mathrm{~h}$.

The HIP process uses gas to apply pressure, while heating, to consolidate the powder and therefore requires encapsulation of the green powder compacts with a solid material. This solid gas-impermeable material is referred to as a "can". Two factors have been determined to aid in retaining the shape of the green compact; placing cylindrical plugs at each end of the can and selecting a can material that has a very low mechanical strength (or high ductility) at the HIP temperature. Thus, the can material is selected based on its melting temperature relative to the maximum HIP temperature. In this work HIP temperatures were $800{ }^{\circ} \mathrm{C}$ and $1000{ }^{\circ} \mathrm{C}$, therefore copper, with a melting temperature of $1085^{\circ} \mathrm{C}$, was selected based on excellent ductility at these temperatures.

The copper can encapsulation (or canning) procedure was completed in an argon glove box that maintains oxygen levels less than $1 \mathrm{ppm}$. The CIP samples remain sealed in the CIP bag and were opened in the glove box prior to insertion into the can. To prevent reaction of the copper can with the aluminum present in the samples a diffusion barrier was created by wrapping the CIP compacts in tantalum foil (the lack of a diffusion barrier caused catastrophic failure of the can at the $1000^{\circ} \mathrm{C}$ HIP temperature). The copper can was fabricated from $5 / 8$ " inside diameter refrigeration tubing. One end was crimped, in a special die, with a force of $67 \mathrm{kN}$ in a 4-post press and sealed with a TIG tungsten electrode welder. A copper plug of appropriate diameter was then inserted followed by 
the tantalum wrapped sample and another copper plug. The can was heated to $250{ }^{\circ} \mathrm{C}$ while a vacuum was developed by mechanical pumping for approximately one hour. Finally, the end attached to the vacuum pump was crimped and simultaneously cut and sealed using a TIG welder.

The canned powders, that had undergone RMM processing under various conditions, were HIPed for chosen temperatures, times, and pressures. The HIP consolidation was accomplished primarily with the unique capability at the Institute of Materials Processing at MTU, a high pressure hot isostatic press that can achieve pressures of $517 \mathrm{MPa}$ with fast temperature and pressure cycles referred to as the Quick HIP process. Table II-6 provides details of the consolidation of samples with the Quick HIP process. The RMM process designation is the prefix of the HIP process number with the first number after the atmosphere noting the HIPing temperature followed by the HIPing method and the time at those conditions. All of these Quick HIP samples had undergone RMM processing for $20 \mathrm{~h}$ with the exception of the RMM40h18TiAr sample processing for $40 \mathrm{~h}$. While the $800{ }^{\circ} \mathrm{C}$ sample data are provided, that material behaved in a brittle manner in compression and will not be further detailed, but it does have important implications as will be discussed.

\begin{tabular}{|c|c|c|c|c|}
\hline HIP Process Number & $\begin{array}{c}\text { RMM Process } \\
\text { Number }\end{array}$ & $\begin{array}{c}\text { HIP } \\
\text { Temp. } \\
\left({ }^{\circ} \mathrm{C}\right)\end{array}$ & $\begin{array}{c}\text { HIP } \\
\text { Pressure } \\
\text { (MPa) }\end{array}$ & $\begin{array}{l}\text { HIP } \\
\text { Time } \\
\text { (min) }\end{array}$ \\
\hline RMM20h8.2TiAir800QH15m & RMM20h8.2TiAir & 800 & 414 & 15 \\
\hline RMM20h8.2TiAr1000QH2h & RMM20h8.2TiAr & 1000 & 414 & 120 \\
\hline RMM20h8.2TiAr1000QH2h & RMM20h9TiAr & 1000 & 414 & 120 \\
\hline RMM40h16.4TiAr1000QH2h & RMM40h18TiAr & 1000 & 414 & 120 \\
\hline
\end{tabular}

Several additional RMM experiments intended to further investigate effects of compositional and parameter changes during the RMM processing involved consolidation with an ASEA Pressure Systems Inc. (London, OH) model SL-1 miniHIPper. Maximum compaction pressure obtainable with this HIP system is $172 \mathrm{MPa}$. 
Several samples were found to melt during HIPing at $1000{ }^{\circ} \mathrm{C}$ despite confirmation of the target temperature of $1000{ }^{\circ} \mathrm{C}$ by two thermocouples. Because the sample chamber in this HIP system is much smaller than that in the Quick HIP system, it is speculated that the melting resulted from thermal gradients associated with the sample chamber since the dimensions of the samples are near the size of the chamber length with the sample initially resting on chamber walls. This problem was ultimately avoided by careful placement of the sample in the center of the HIP chamber. The HIP parameters are detailed in Table II-7, with a similar naming convention as the Quick HIP process. However the low pressure HIP is distinguished by the use of the $\mathrm{H}$ designation in the HIP process number for the low pressure HIPing. The MM3hAr sample will not be further discussed; however, this treatment produced a fine-grained single-phase $\mathrm{L}_{2}$ intermetallic sample for mechanical property assessment.

\begin{tabular}{|ccccc|}
\hline Table II-7 Consolidated Sample Identification and Low Pressure HIP Parameters \\
\hline HIP Process Number & $\begin{array}{c}\text { RMM Process } \\
\text { Number }\end{array}$ & $\begin{array}{c}\text { HIP } \\
\text { Temp. } \\
\left({ }^{\circ} \mathbf{C}\right)\end{array}$ & $\begin{array}{c}\text { HIP } \\
\text { Pressure } \\
(\mathbf{M P a})\end{array}$ & $\begin{array}{c}\text { HIP } \\
\text { Time } \\
(\mathbf{m})\end{array}$ \\
\cline { 2 - 5 } RMM60h27TiAr1000H2h & RMM60h27TiAr & 1000 & 172 & 120 \\
\cline { 2 - 5 } RMM100h45TiAr1000H2h & RMM100h45TiAr & 1000 & 172 & 120 \\
RMM120h54TiAr1000H2h & RMM120h54TiAr & 1000 & 172 & 120 \\
\cline { 2 - 5 } MM3hArHIP & MM3hAr & $\mathbf{1 0 0 0}$ & $\mathbf{1 7 2}$ & $\mathbf{1 2 0}$ \\
\hline
\end{tabular}

The consolidated mass of material resulting from the process was cylindrical, as expected, with the shape of the copper end plugs clearly visible. As desired, the presence of the copper plugs combined with the soft nature of the can material resulted in preservation of the cylindrical shape originally produced during the CIP process. Due to the length of the HIP process number, when appropriate a sample code provided in Table II-8 was utilized for brevity. The abbreviated sample code is based on the RMM time, HIP temperature, and alloy type with MA corresponding to the Master Alloy and RA the Reading Alloy. Note the sample with the suffix -A has been milled in air. The RMM time provides an idea of the amount of excess titanium along with the alloy type. 
Table II-8 HIP Process Number Sample Codes

\begin{tabular}{cc} 
HIP Process Number & Sample Code \\
RMM20h8.2TiAir800QH15m & 20/800/MA-A \\
\cline { 2 - 2 } RMM20h8.2TiAr1000QH2h & 20/1000/MA \\
RMM20h9TiAr1000QH2h & $20 / 1000 / R A$ \\
RMM40h18TiAr1000QH2h & $40 / 1000 / R A$ \\
RMM60h27TiAr1000H2h & $60 / 1000 / R A$ \\
RMM100h45TiAr1000H2h & $100 / 1000 / R A$ \\
RMM120h54TiAr1000H2h & $120 / 1000 / R A$ \\
\cline { 2 - 2 } MM3hArHIP & MM3h
\end{tabular}

Experimental details of XRD data collection and analysis were discussed in Chapter II.A.5.a). Specific specimen XRD scan parameters are given in Table II-9 for each sample code.

Table II-9 XRD Scan Parameters for Consolidated Material

\begin{tabular}{|c|c|c|c|}
\hline Sample Code & $\begin{array}{c}\text { Scan angle } \\
\left(2 \theta^{\circ}\right)\end{array}$ & $\begin{array}{c}\text { Step Size } \\
\left(2 \theta^{\circ}\right)\end{array}$ & $\begin{array}{c}\text { Scan Count } \\
\text { Time (s) }\end{array}$ \\
\hline 20/800/MA-A & $10-100$ & 0.04 & 23 \\
\hline 20/1000/MA & $10-110$ & 0.03 & 30 \\
\hline 20/1000/RA & $10-110$ & 0.03 & 30 \\
\hline 40/1000/RA & $10-110$ & 0.03 & 30 \\
\hline 60/1000/RA & $10-110$ & 0.03 & 23 \\
\hline 100/1000/RA & $10-110$ & 0.03 & 22 \\
\hline 120/1000/RA & $10-85$ & 0.03 & 10 \\
\hline MM3h* & 18-105 & 0.02 & 25 \\
\hline
\end{tabular}

* Note this sample was mechanically milled for $3 \mathrm{~h}$ 


\section{Experimental Results of the Powder Processing by Reactive Mechanical Alloying/Milling}

For all RMA/M experiments the loose powder typically constituted over $80 \%$ by weight of the total powder with most of the remaining powder that adhered to the vial walls easily removed and included as as-milled powder. The total powder weight was determined by the mass difference of a clean vial assembly (including specified media) with one after the RMA/M processing which contains the remainder of the adhered powder on the vial assembly in addition to the actual weight of the loose powder. Loose powder recovery was slightly higher for the shortest milling time and gradually decreased with increased milling time.

\section{A. Reactive Mechanical Milling (RMM) of Pre- Alloyed Powders with Fixed Excess Titanium RMMXh9TiAr Series}

The purpose of these experiments was to explore the creation of TiC during RMM of a pre-alloyed $\mathrm{Al}_{66} \mathrm{Cr}_{9} \mathrm{Ti}_{25}$ powder with added titanium in the presence of the hexanes PCA in an inert atmosphere. The pre-alloyed (Reading Alloy Inc.) $\mathrm{Al}_{66} \mathrm{Cr}_{9} \mathrm{Ti}_{25}$ powder along with 9 wt. pct. excess titanium was milled for various RMM times of up to $40 \mathrm{~h}$ in an argon environment.

\section{As-Milled Powders}

\section{a) General Features of the X-ray Powder Diffraction Patterns}

Of particular interest is the structural evolution of this as-milled powder, designated as the RMMXh9TiAr Series, as a function of RMM processing time. The features of the diffraction patterns make it possible to divide them into to two types; for short RMM times the diffraction patterns have distinct crystalline features, whereas at longer times the diffraction peaks are heavily broadened and convoluted, with added peaks from the formation of new phases $\mathrm{TiC}$ and $\mathrm{TiH}_{1.92}$. In the first type the initial $\mathrm{L}_{2}$ 
intermetallic diffraction peaks broaden and a homogenizing effect appears to take place with the rapid disappearance of secondary phases (present in the original Reading Alloy) with the diffraction pattern moving toward that of a pure $\mathrm{L}_{2}$ cubic $\mathrm{Al}_{66} \mathrm{Cr}_{9} \mathrm{Ti}_{25}$ alloy. The XRD powder pattern for the initial mixture along with those for the $0.5 \mathrm{~h}$ and $1 \mathrm{~h} \mathrm{RMM}$ times is shown in Figure III-1. Significant broadening of the diffraction peaks can be seen in these initial stages of RMM processing, even at the shortest time of $0.5 \mathrm{~h}$. After a processing time of $1 \mathrm{~h}$, while broadening increases, it can be noted that the $\mathrm{D}_{22} \mathrm{Al}_{3} \mathrm{Ti}$ (200) diffraction peak at $\sim 47.5^{\circ} 2 \theta$, a $95 \%$ intensity peak for that phase, is no longer visible. However, the (002) and (101) diffraction peaks at $20.7^{\circ}$ and $25.3^{\circ} 2 \theta$ respectively, which do not overlap with $\mathrm{L}_{2}$ intermetallic diffraction peaks, while diminished, are still visible.

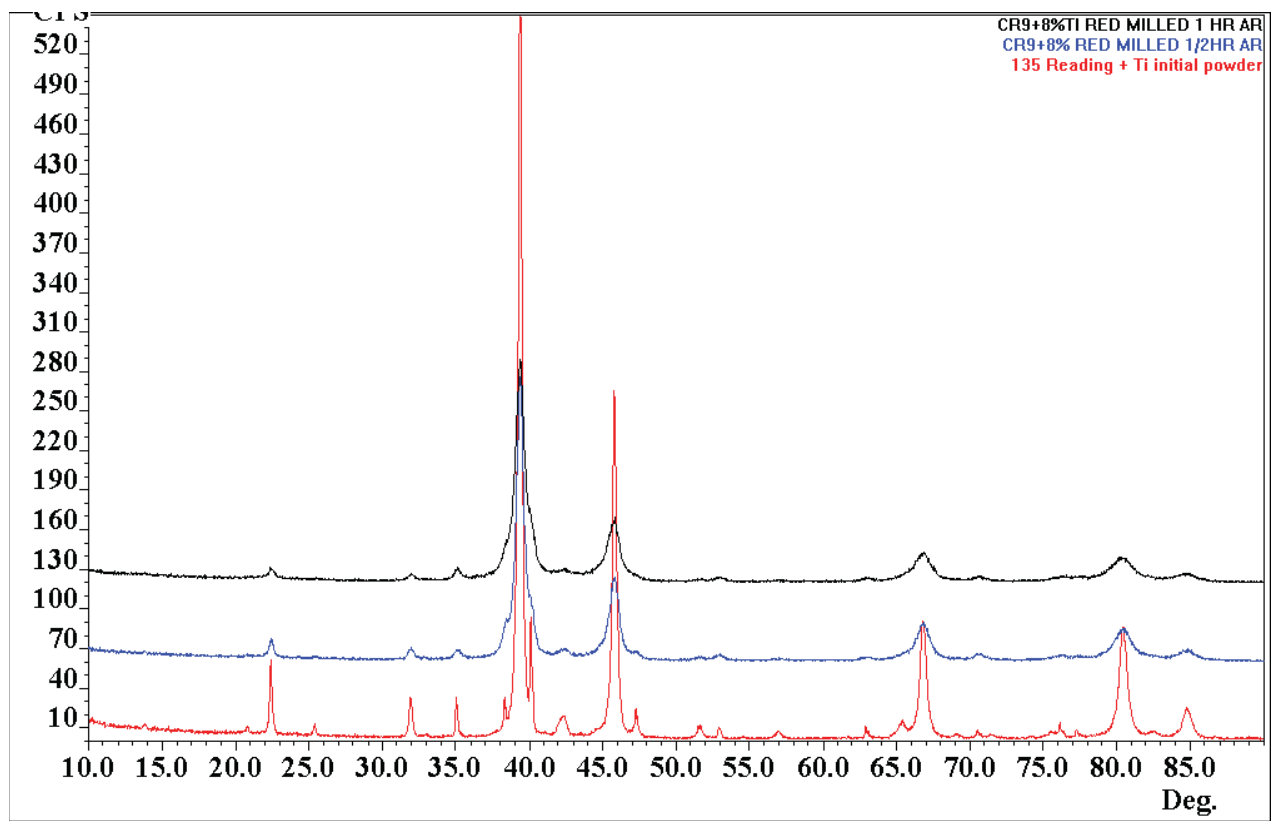

Figure III-1 XRD Pattern for Initial, $0.5 \mathrm{~h}$, and $1 \mathrm{~h}$ RMM Processing Times (RMMOh9TiAr, RMM0.5h9TiAr, RMM1h9TiAr)

The second type of diffraction pattern shows severe broadening of the $\mathrm{L}_{2}$ intermetallic diffraction peaks, as well as significant in situ formation of $\mathrm{TiC}$ and $\mathrm{TiH}_{1.92}$. This combination of phases results in two $2 \theta$ ranges, one from $\sim 30-50^{\circ} 2 \theta$ and the other from $\sim 55-90^{\circ} 2 \theta$, within which there is severe overlap of peaks from all three major 
phases as shown in Figure III-2 for increasing RMM times of 5, 10, 15, 20, 25, 30, and $40 \mathrm{~h}$. There is extensive broadening of the diffraction peaks for all RMM processing times above $5 \mathrm{~h}$, which combined with the fact that three of the phases are cubic with similar lattice parameters results in the noted complex convolution of the diffraction peaks. In all cases detailed diffraction pattern peak information was determined by the intelligent profile fitting as previously described in Chapter II.A.5.a)(2).

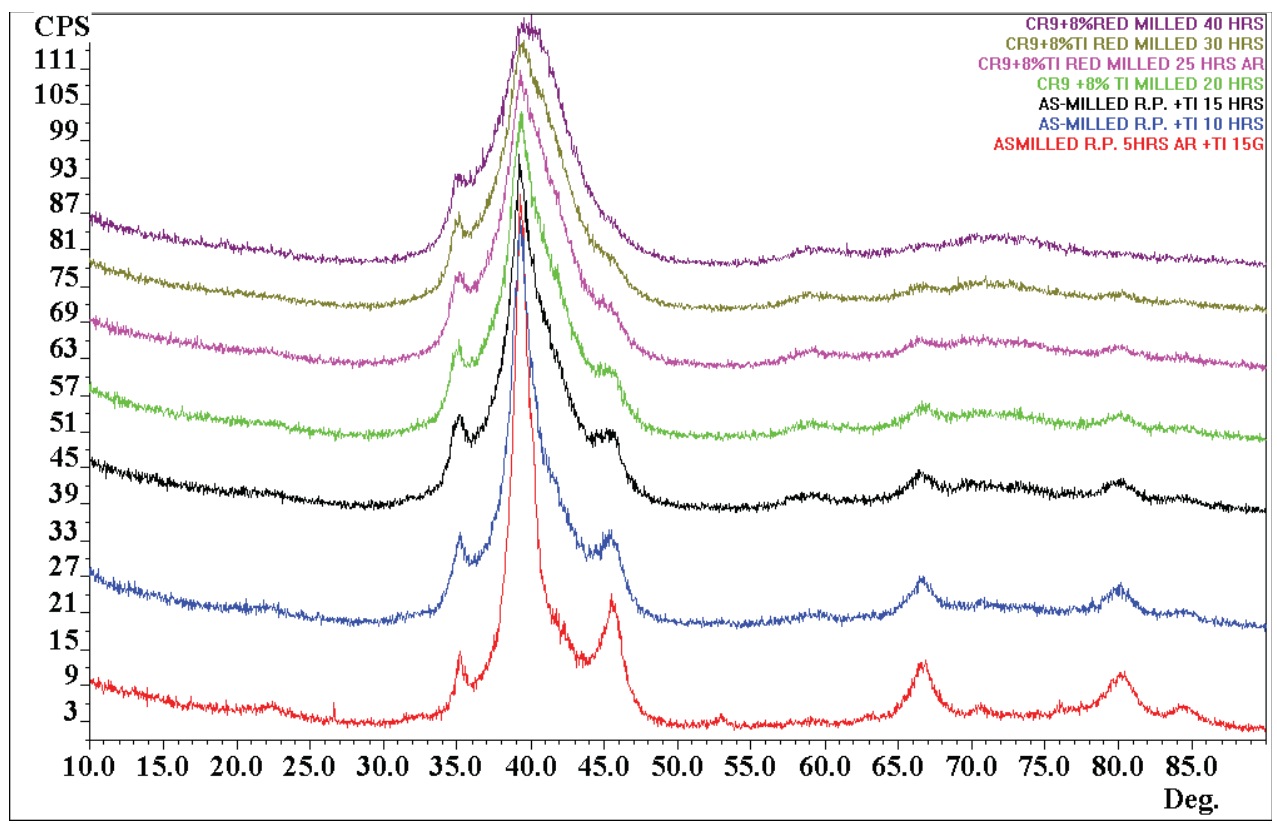

Figure III-2 XRD Pattern for $5 \mathrm{~h}, 10 \mathrm{~h}, 15 \mathrm{~h}, 20 \mathrm{~h}, 25 \mathrm{~h}, 30 \mathrm{~h}$, and $40 \mathrm{~h}$ RMM Times

Despite the extreme broadening of the diffraction peaks with RMM processing time, the ordered crystal structure is preserved in the $\mathrm{L}_{2}$ intermetallic as evidenced by the existence of the $\{100\}$ diffraction peak at $\sim 22.5^{\circ}$ shown in Figure III-3. For display purposes, the diffraction patterns shown in Figure III-3 are offset incrementally with increasing RMM processing times of $0,0.5,1,5,10,15,20,25$, and $30 \mathrm{~h}$. The $\{100\} \mathrm{L} 1_{2}$ intermetallic diffraction peak arising from atomic ordering of the crystal structure is still identifiable out to an RMM processing time of $30 \mathrm{~h}$, with very weak evidence of the $\{100\}$ diffraction peak at the RMM time of $40 \mathrm{~h}$, because of the severe broadening. Attempts to profile fit this very weak peak indicated a peak half breadth just over $4^{\circ} 2 \theta$, 
corresponding to a very small crystallite size (similar to the first two fundamental peak values).

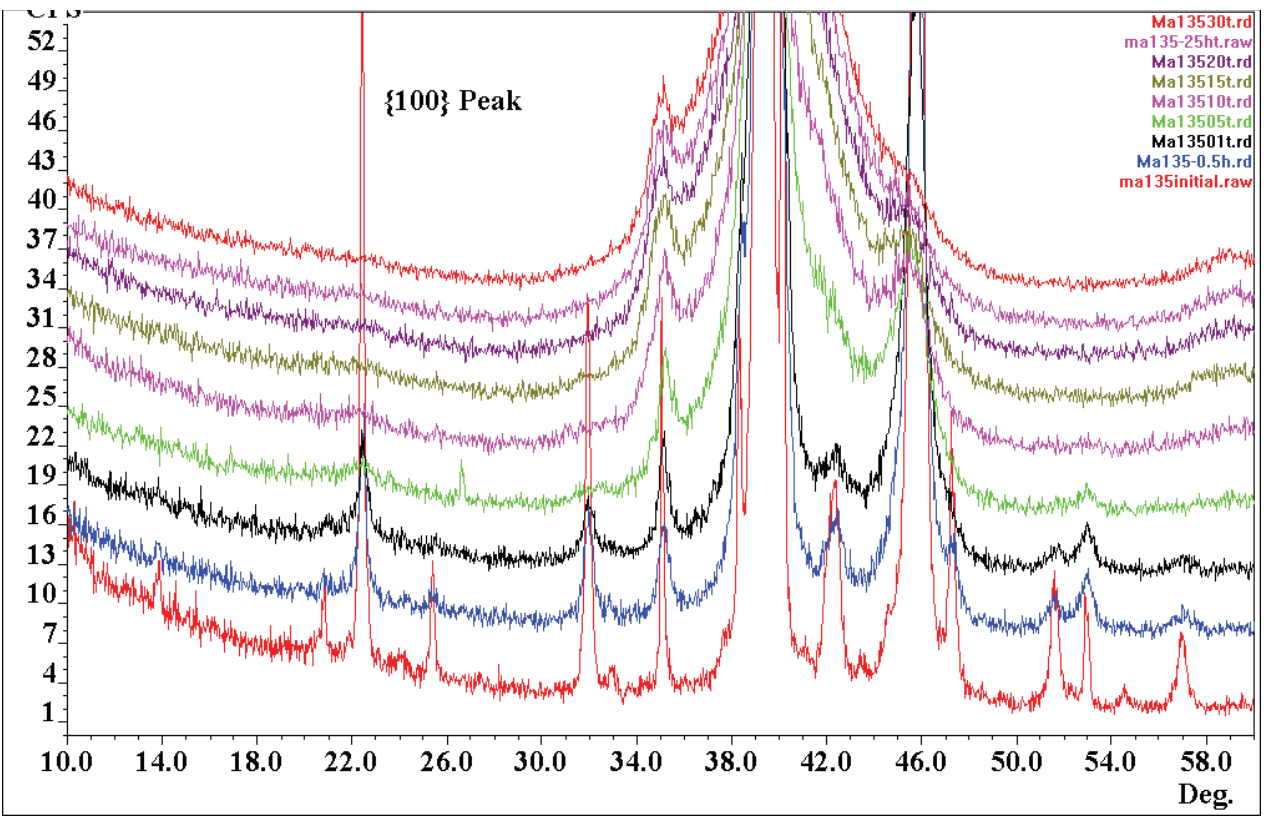

Figure III-3 RMMXh9TiAr Series all RMM Samples

\section{b) RMM Powder Phase Identification}

Table III-1 lists phases identified in the RMMXh9TiAr Series of samples as a function of RMM processing time. Phases were considered present when some diffraction peaks could be clearly established; recognizing that because of the large amount of broadening and peak overlap not all peaks could be positively identified (note that in the following comments the JCPDS-ICDD card numbers are shown in parenthesis). Given the nature of the patterns, it should be noted that small amounts of other phases could potentially be masked. The initial mixture of pre-alloyed Reading powders with 9 wt. pct. titanium added (44-1294) did contain a majority of $\mathrm{L}_{2}$ intermetallic [116], with very much smaller amounts of $\mathrm{Al}_{3} \mathrm{Ti}$ (37-1449), $\mathrm{Al}_{8} \mathrm{Cr}_{5}$ (29-15), $\mathrm{Al}_{2} \mathrm{O}_{3}(10-173)$, and $\mathrm{Ti}_{3} \mathrm{Al}(14-451)$.

In addition to these phases identified in Table III-1, the samples with RMM processing times of 0.5 and $1 \mathrm{~h}$ showed indications of the $\{220\}$ peaks for $\mathrm{TiH}_{1.92}(25$ 982) and TiC (32-1383); however, the peak intensities of these peaks are less than $1 \mathrm{cps}$ 
making identification and profile fitting questionable. As can be seen the added titanium has been consumed by carbide formation after $10 \mathrm{~h}$ of milling. The sample processed for $10 \mathrm{~h}$ gave indications of titanium; however, the (102) titanium peak intensity was less than 1 cps. This (102) titanium peak (13\% intensity on JCPDS-ICCD card) is the only titanium peak that does not overlap with other phases. It should be noted that with a peak intensity of $1 \mathrm{cps}$ the corresponding volume fraction is expected to be extremely small. Samples processed for $15,20,25,30$, and $40 \mathrm{~h}$ contained the $\mathrm{L} 1_{2}$ intermetallic, TiC (321383), and $\mathrm{TiH}_{1.92}$ (25-982). The formation of $\mathrm{TiC}(32-1383)$ and $\mathrm{TiH}_{1.92}$ (25-982) in the as-milled powders is of particular interest as is the apparent increase in the amount of TiC with RMM processing time.

\begin{tabular}{|c|c|c|c|c|c|c|}
\hline $\begin{array}{c}\text { RMM Time } \\
\text { X (h) }\end{array}$ & $\mathrm{L1}_{2}$ & Ti & $\begin{array}{l}\mathrm{DO}_{22} \\
\mathrm{Al}_{3} \mathrm{Ti}\end{array}$ & AlsCr5 & $\mathrm{TiC}$ & TiH1.92 \\
\hline $0 *$ & $X$ & $X$ & $X$ & $\mathrm{X}$ & & \\
\hline 0.5 & $X$ & $X$ & $X$ & & & \\
\hline 1 & $X$ & $X$ & $X$ & & & \\
\hline 5 & $X$ & X & & & X & X \\
\hline 10 & X & $X$ & & & $X$ & $X$ \\
\hline 15 & $X$ & & & & $X$ & X \\
\hline 20 & $X$ & & & & $X$ & $X$ \\
\hline 25 & $X$ & & & & $X$ & $X$ \\
\hline 30 & $X$ & & & & $X$ & $X$ \\
\hline 40 & $\mathbf{X}$ & & & & $\mathbf{X}$ & $\mathbf{X}$ \\
\hline
\end{tabular}

*Small amounts of $\mathrm{Al}_{2} \mathrm{O}_{3}$ and $\mathrm{Ti}_{3} \mathrm{Al}$ were also identified

The individual x-ray powder diffraction patterns are shown Figure III-4 through Figure III-13 for all RMM times. Figure III-4 shows the initial mixture that represents the starting composition and phase structure. Superimposed on each pattern are data from the pertinent JCPDS-ICDD powder diffraction cards (and in the case of the $\mathrm{L}_{2}$ phase an experimentally created card). With all of these XRD patterns the background has been 
removed by subtracting a cubic spline fit to the background with the resultant XRD pattern referred to as a net intensity file.

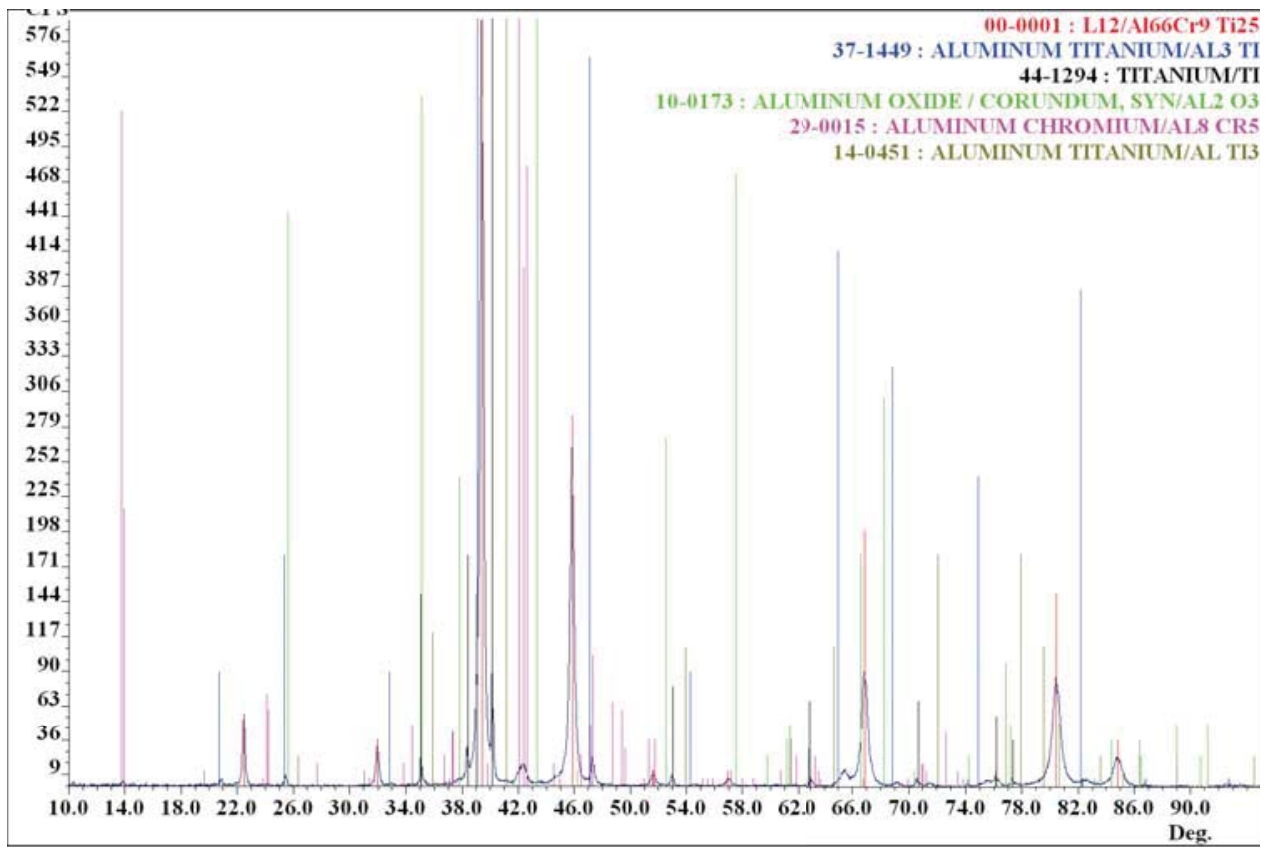

Figure III-4 XRD Phase ID RMMOh9TiAr Initial Mixture

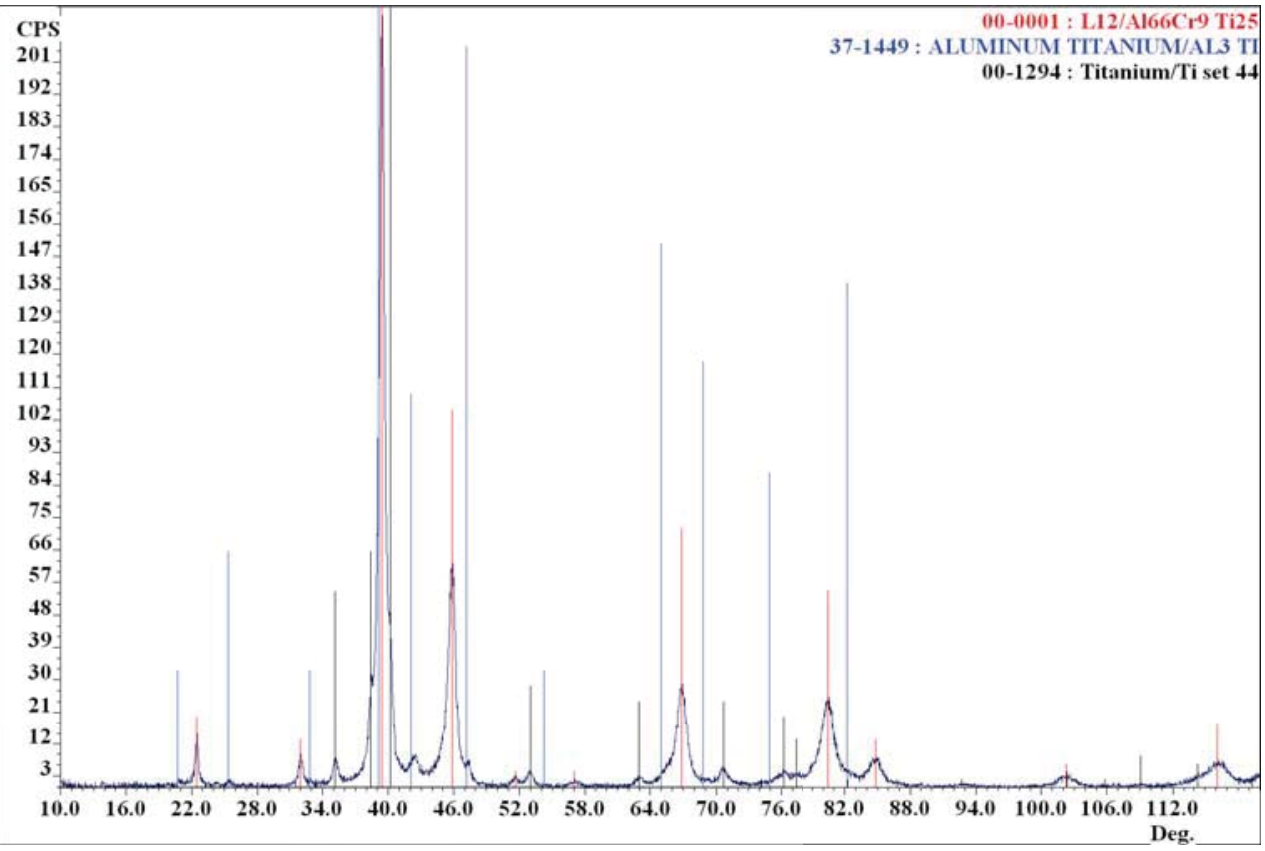

Figure III-5 XRD Phase ID RMM0.5h9TiAr RMM Time $0.5 \mathrm{~h}$ 


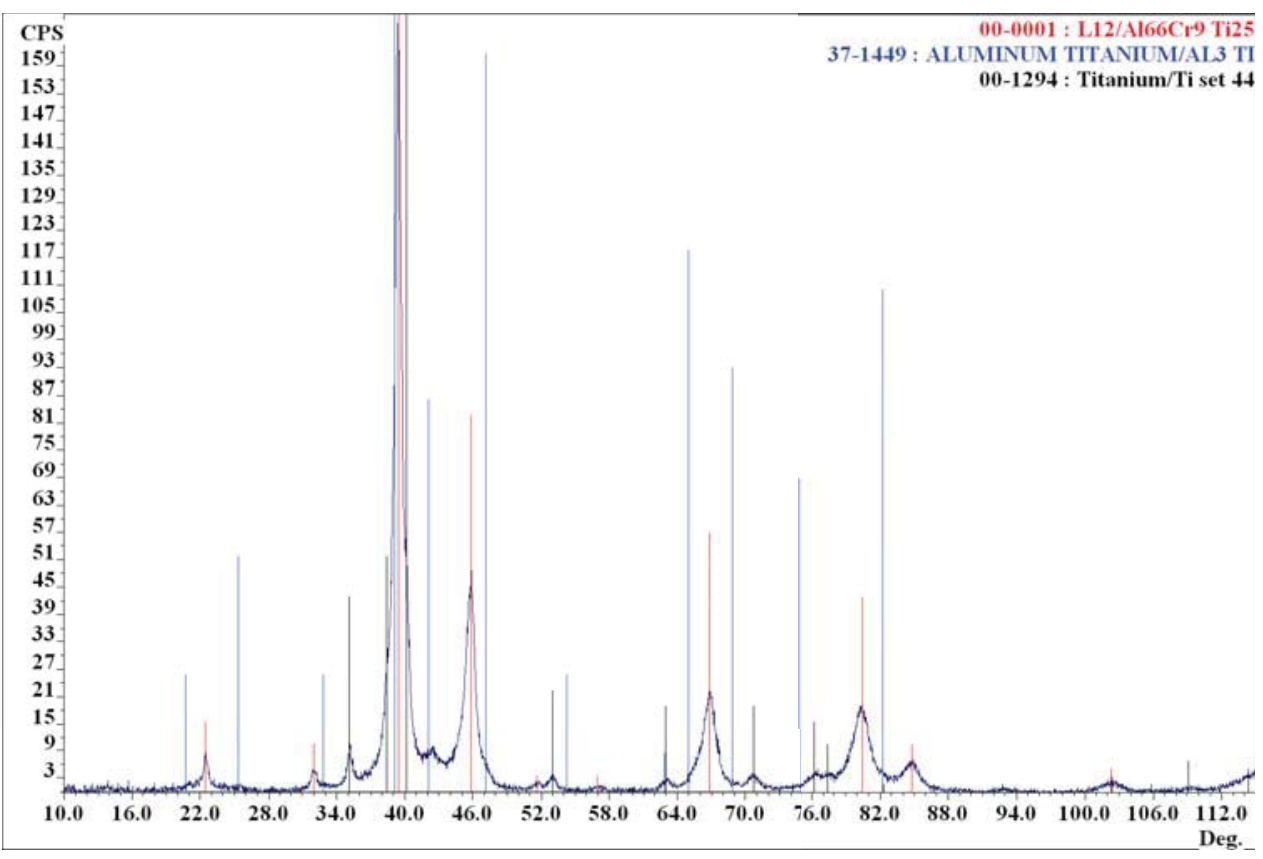

Figure III-6 XRD Phase ID RMM1h9TiAr RMM Time $1 \mathrm{~h}$

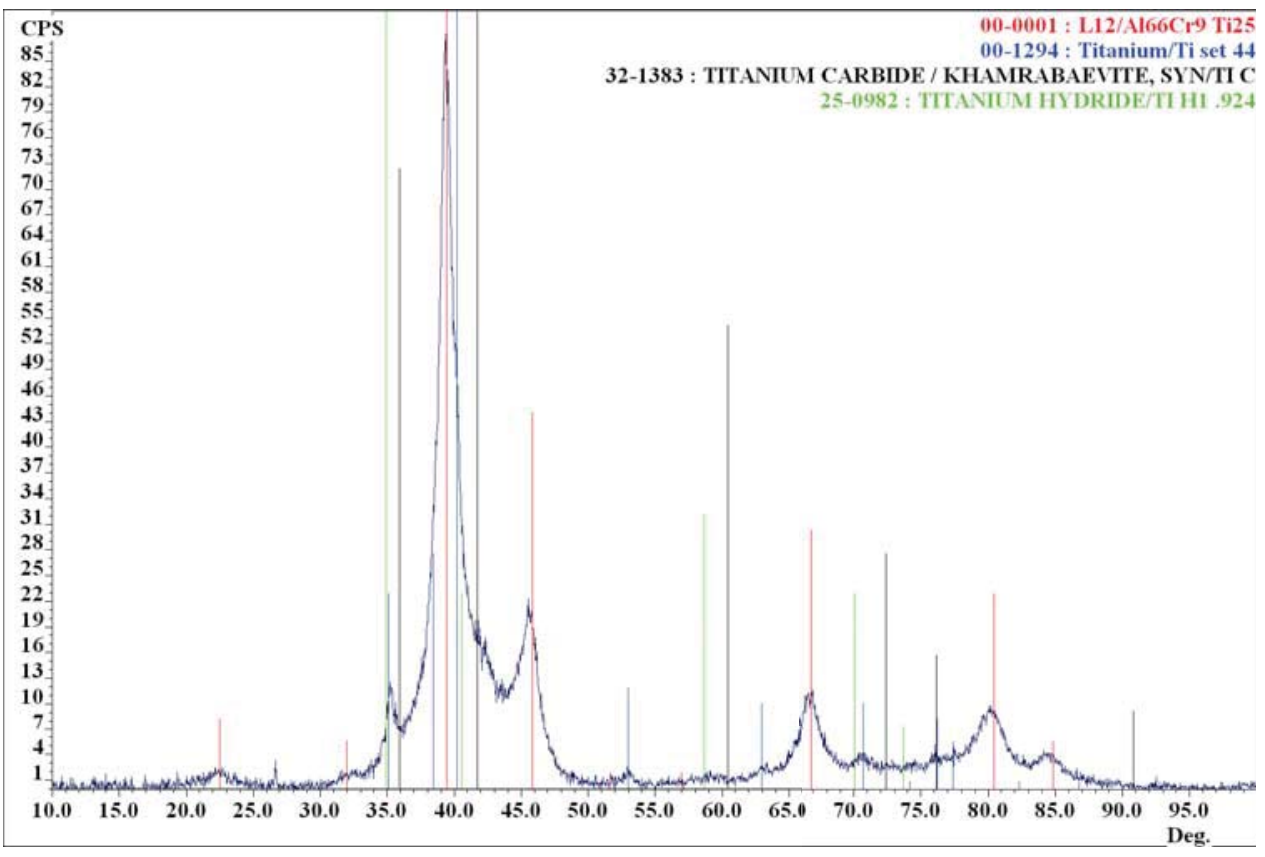

Figure III-7 XRD Phase ID RMM5h9TiAr RMM Time $5 \mathrm{~h}$ 


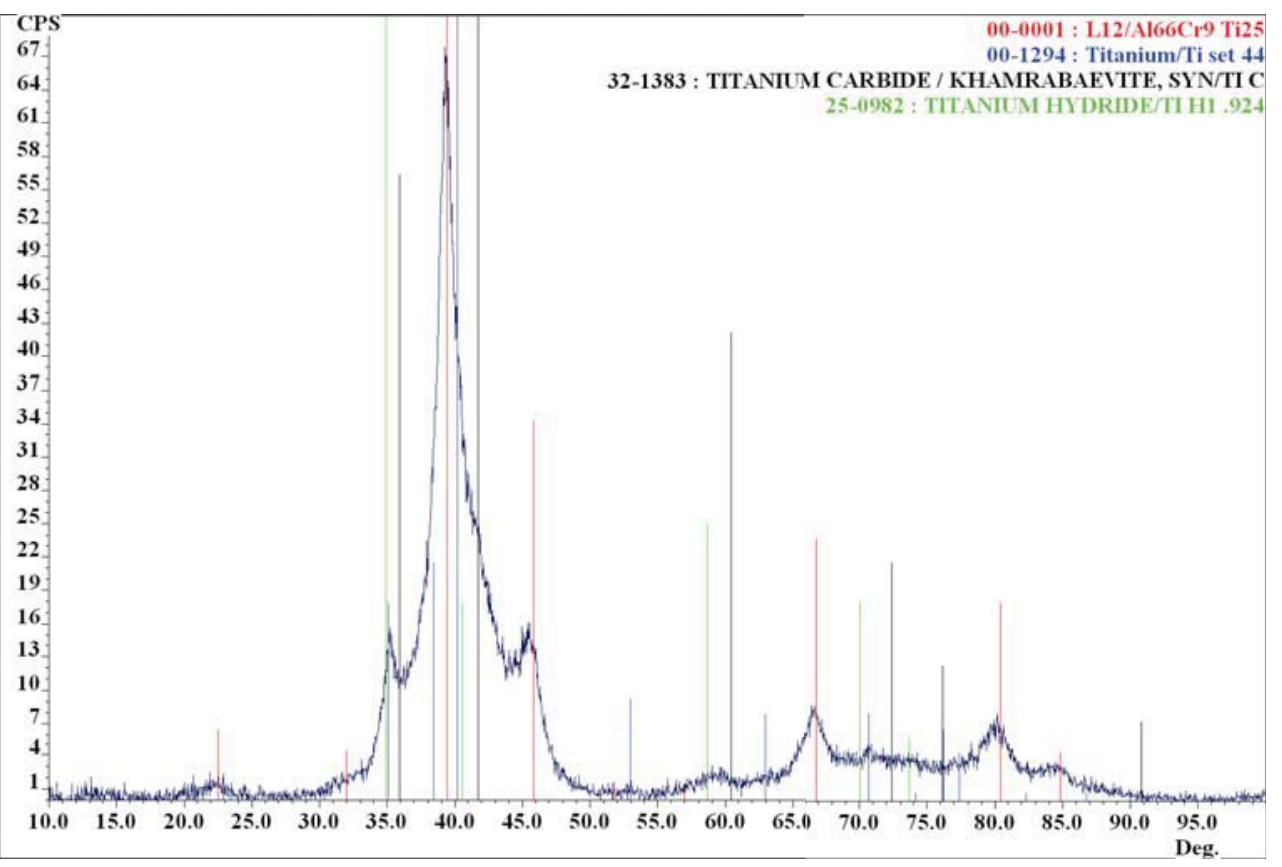

Figure III-8 XRD Phase ID RMM10h9TiAr RMM Time $10 \mathrm{~h}$

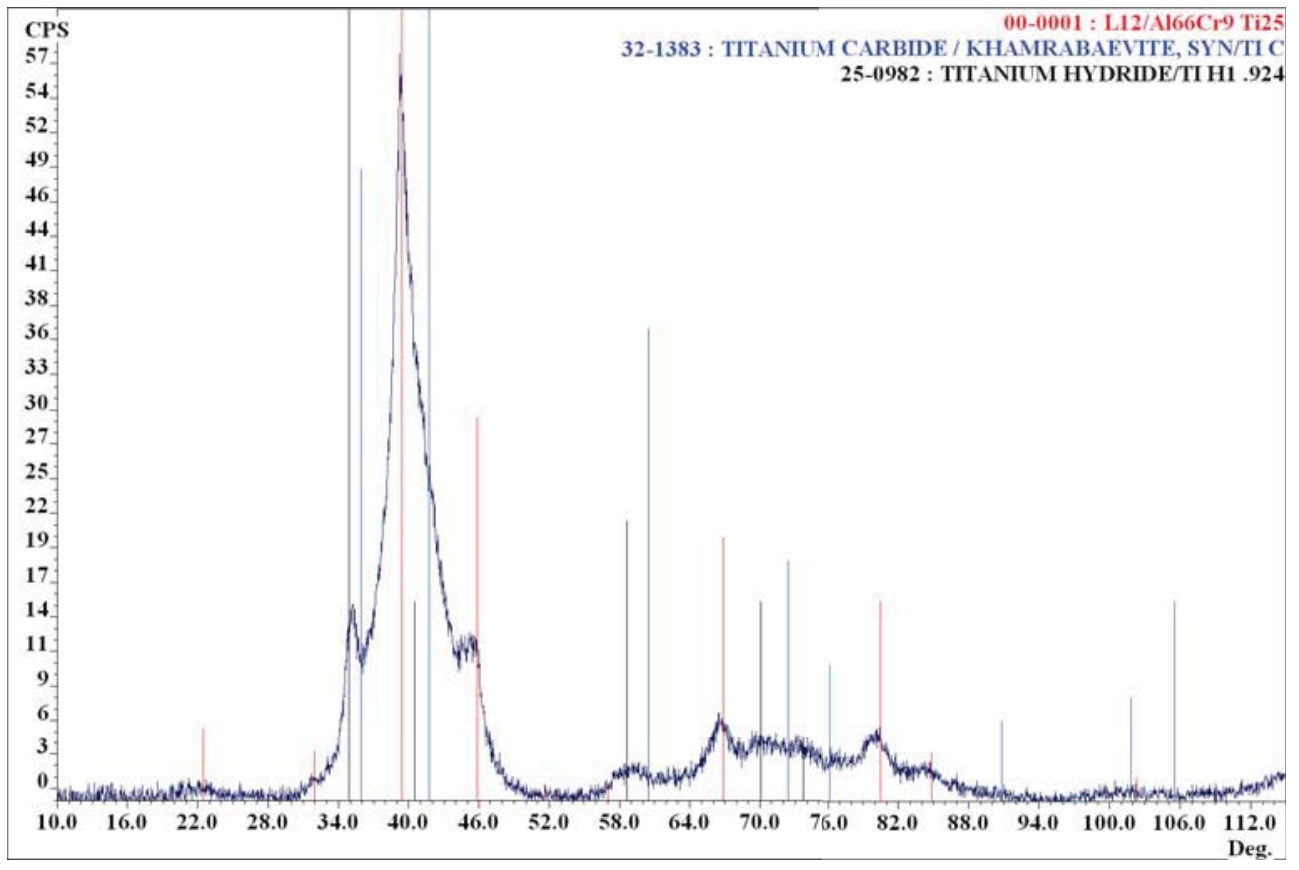

Figure III-9 XRD Phase ID RMM15h9TiAr RMM Time $15 \mathrm{~h}$ 


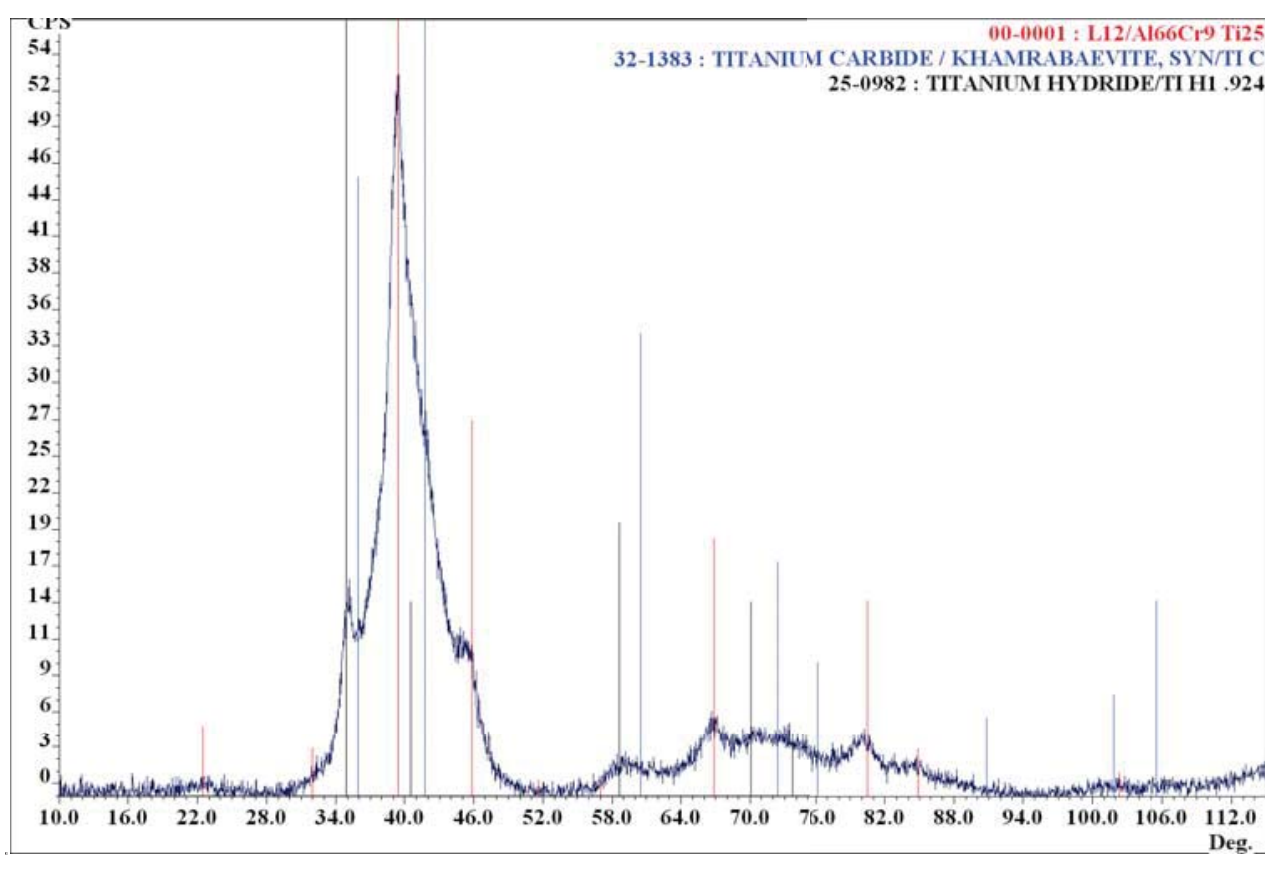

Figure III-10 XRD Phase ID RMM20h9TiAr RMM Time $20 \mathrm{~h}$

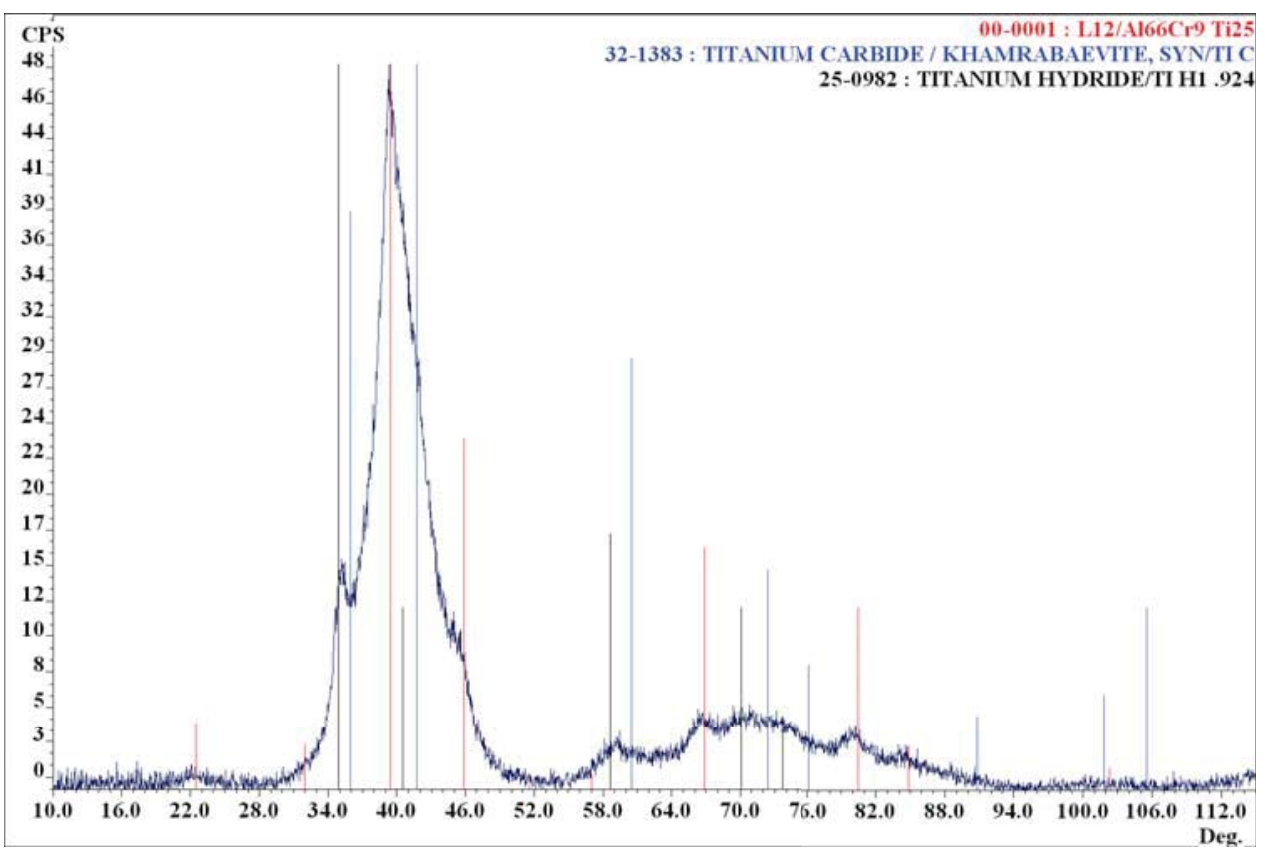

Figure III-11 XRD Phase ID RMM25h9TiAr RMM Time $25 \mathrm{~h}$ 


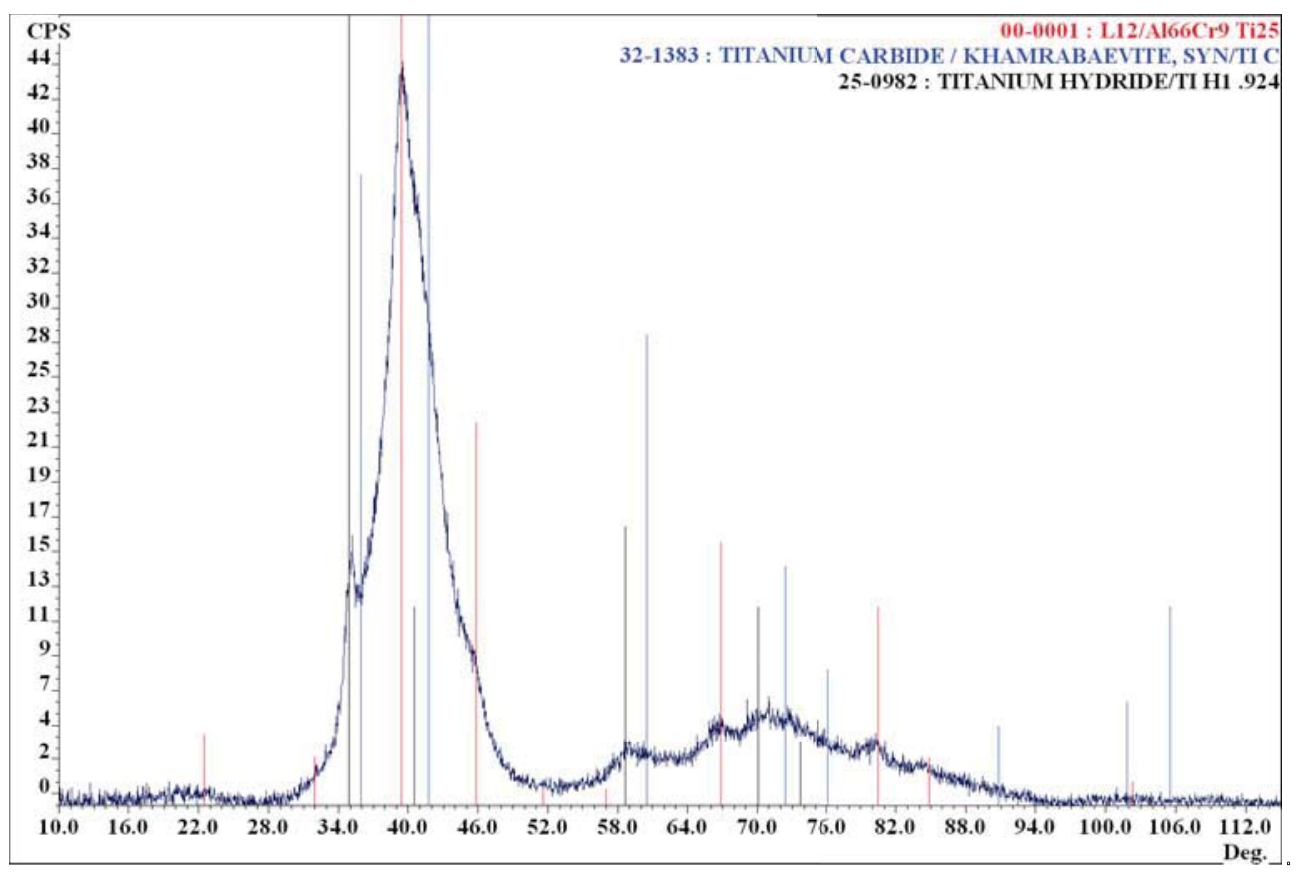

Figure III-12 XRD Phase ID RMM30h9TiAr RMM Time $30 \mathrm{~h}$

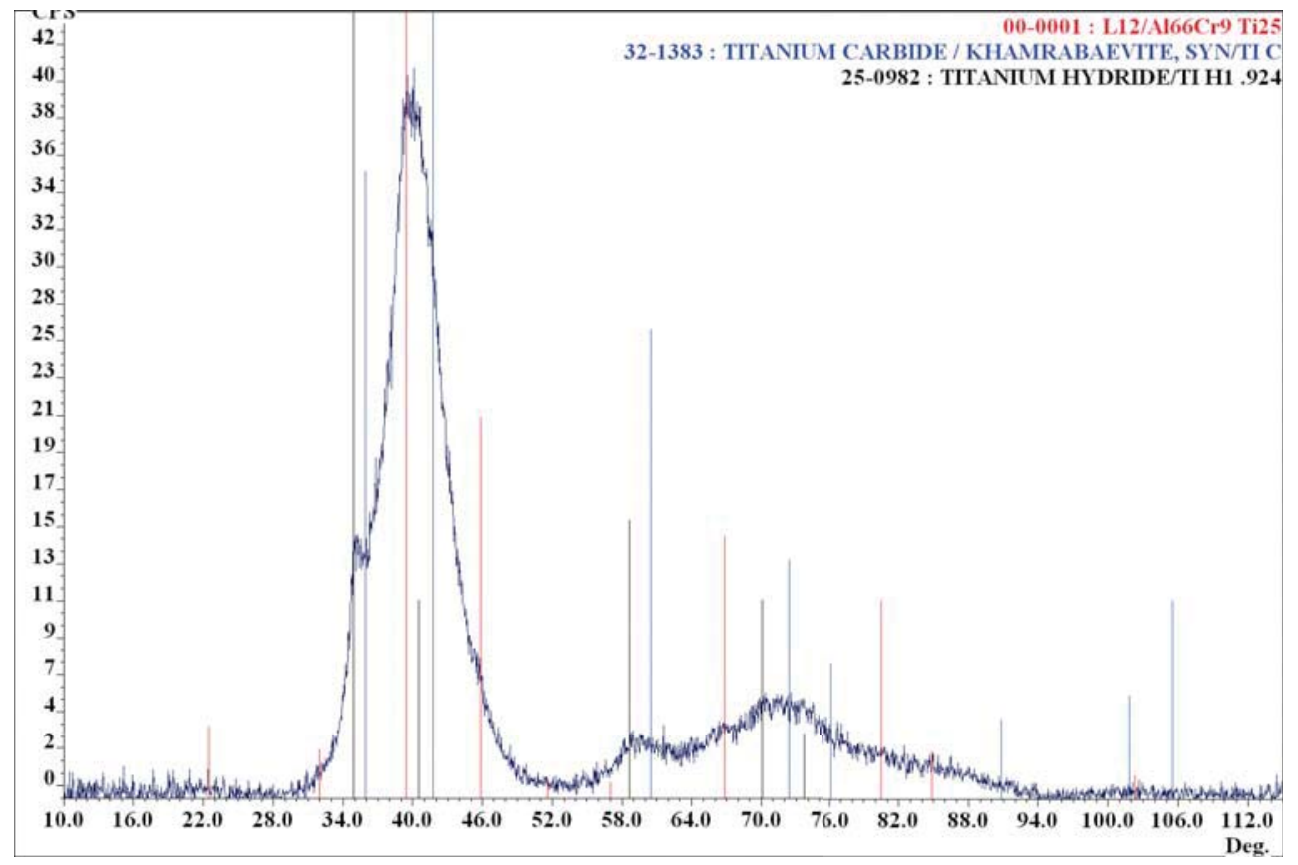

Figure III-13 XRD Phase ID RMM40h9TiAr RMM Time $40 \mathrm{~h}$ 


\section{c) Volume Fraction Estimates}

Volume fractions were determined as a function of RMM time for all identified phases with the direct comparison XRD method using area values for the respective XRD peaks derived from intelligent profile fits. The longer RMM times with severe broadening and convolution of the diffraction peaks do pose challenges to the intelligent profile fitting; however, even in those instances reasonable estimates are possible. For example, Figure III-14 shows the profile fit for the RMM time of $20 \mathrm{~h}$; the green and red curves in the range of $\sim 30^{\circ}-50^{\circ} 2 \theta$ are the $\{111\}$ and $\{200\}$ diffraction peaks of $\mathrm{TiC}$, while the peak at $\sim 60^{\circ} 2 \theta$ is the $\{220\}$ diffraction peak of TiC. The $\{111\}$ and $\{200\}$ diffraction peaks are not clearly visible in the original diffraction pattern posing a successfully met challenge for the software to determine those peak areas. While the exact position of the $\{220\}$ peak at $\sim 60^{\circ} 2 \theta$ is also difficult to determine, the peak has a quite well-defined intensity, with interference mainly from the $\{220\}$ of $\mathrm{TiH}_{1.92}$ located at a slightly smaller $2 \theta$ angle. Again, this difficulty was overcome.

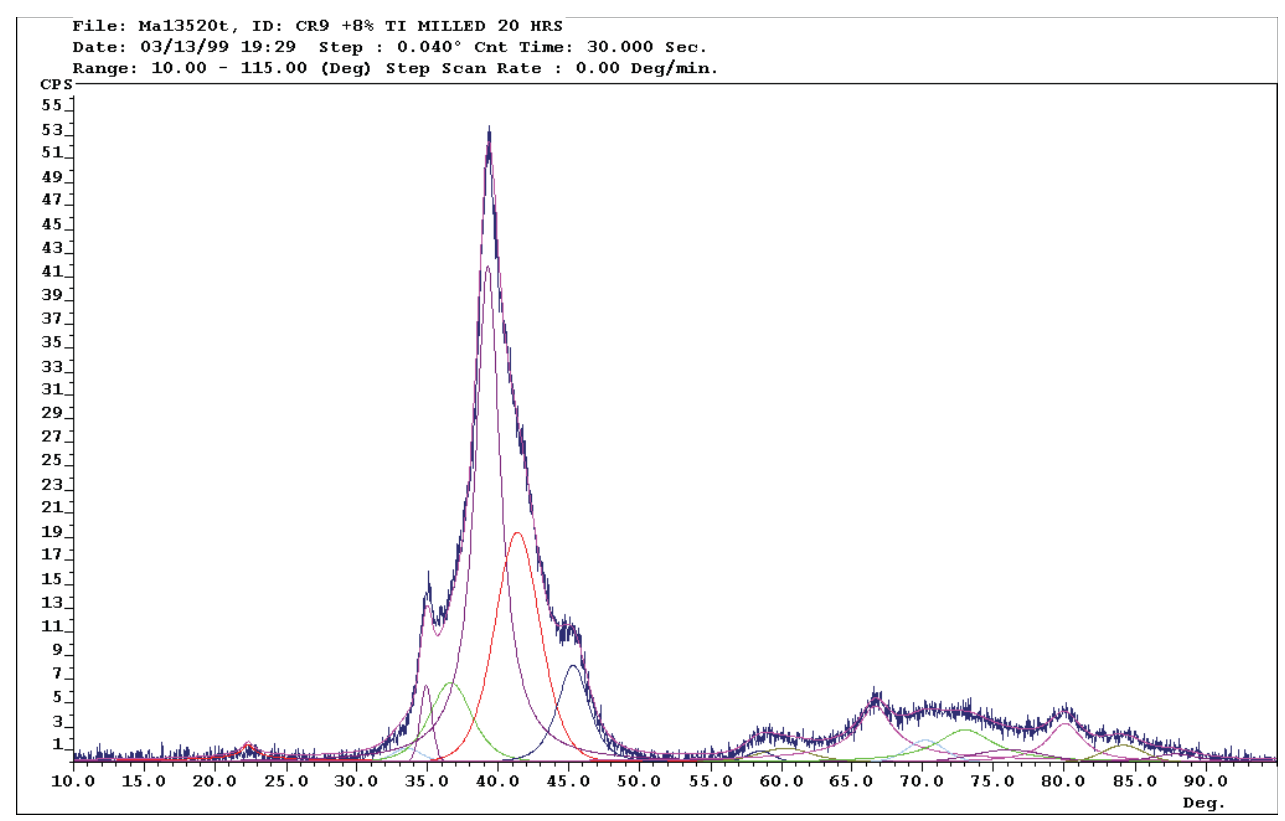

Figure III-14 Profile Fit of RMM20h9TiAr RMM Time $20 \mathrm{~h}$

In order to improve the estimate of the volume fractions, several intelligent profile fits for each RMM time were performed. Thus, the reported volume fraction results for each RMM time represent an average of the volume fractions results from several 
individual profile fits assuming stoichiometric phases. The reported volume fraction results include a $95 \%$ confidence intervals calculated based on the results for each individual profile fit for each RMM time.

Volume fraction results are tabulated in Table III- 2 for the as-milled RMMXh9TiAr Series of samples for RMM times from 0.5 to $40 \mathrm{~h}$. The initial mixture is designated as $0 \mathrm{~h}$, and contains an added volume fraction of 0.075 excess titanium, along with 0.11 of the binary phase $\mathrm{Al}_{3} \mathrm{Ti}$ (and noted elsewhere small amounts of other phases are present, i.e. $\mathrm{Al}_{8} \mathrm{Cr}_{5}, \mathrm{Al}_{2} \mathrm{O}_{3}$, and $\mathrm{Ti}_{3} \mathrm{Al}$ ). The $\mathrm{Al}_{3} \mathrm{Ti}$ phase present in the initial powder is no longer detectable in the $5 \mathrm{~h} \mathrm{RMM} \mathrm{sample,} \mathrm{while} \mathrm{titanium} \mathrm{is} \mathrm{no} \mathrm{longer} \mathrm{detectable} \mathrm{in}$ the $15 \mathrm{~h}$ sample. Regarding the lower limit of delectability of titanium, taken to be less than 0.01 , the (102) titanium peak at $\sim 53^{\circ} 2 \theta$ does not overlap with any other diffraction peak; however, since it is a low intensity peak for the material it is heavily influenced by the background selection which can be difficult to model in this region.

Table III-2 Volume Fractions of Phases for RMMXh9TiAr Series of RMM Times

\begin{tabular}{|c|c|c|c|c|c|}
\hline$d$ & & & me Frac & & \\
\hline $\begin{array}{c}\text { RMM Time } \mathbf{X} \\
\text { (h) }\end{array}$ & $\mathrm{L1}_{2}$ & $\mathrm{TiC}$ & TiH1.92 & $\mathbf{T i}$ & AlsTi \\
\hline 0 & 0.807 & & & 0.075 & 0.110 \\
\hline 0.5 & 0.798 & & & 0.087 & 0.115 \\
\hline 1 & 0.815 & & & 0.081 & 0.104 \\
\hline 5 & 0.854 & 0.071 & 0.011 & 0.064 & \\
\hline 10 & 0.807 & 0.125 & 0.038 & 0.030 & \\
\hline 15 & 0.754 & 0.195 & 0.052 & & \\
\hline 20 & 0.736 & 0.214 & 0.049 & & \\
\hline 25 & 0.695 & 0.231 & 0.074 & & \\
\hline 30 & 0.630 & 0.284 & 0.087 & & \\
\hline 40 & 0.623 & 0.287 & 0.090 & & \\
\hline
\end{tabular}

Changes in volume fractions for the RMMXh9TiAr Series of RMM times are shown graphically in Figure III-15. Of particular interest is the systematic increase in 
volume fraction of $\mathrm{TiC}$ with $\mathrm{RMM}$ time, which appears to approach a saturation level for the long RMM times. Extrapolation of the TiC volume fractions for 5, 10, and $15 \mathrm{~h} \mathrm{RMM}$ times to zero yields a time intercept very near to $0 \mathrm{~h}$. This suggests that $\mathrm{TiC}$ has likely formed during the RMM time of $0.5 \mathrm{~h}$ despite that fact a specific amount could not be detected in the XRD pattern with confidence for that time, and further that the TiC phase begins to form before the $\mathrm{TiH}_{1.92}$ phase. There is a slight increase in the volume fraction of the $\mathrm{L} 1_{2}$ intermetallic for the RMM time of $5 \mathrm{~h}$ compared to the initial amount present, which coincides with the disappearance of the tetragonal $\mathrm{DO}_{22} \mathrm{Al}_{3} \mathrm{Ti}$ phase possibly indicating absorption of these elements from $\mathrm{DO}_{22} \mathrm{Al}_{3} \mathrm{Ti}$ phase by the $\mathrm{L}_{2}$ intermetallic. However, this apparent increase can also be explained by other factors that will be detailed in the Discussion. Titanium was not detectable at the $15 \mathrm{~h} \mathrm{RMM} \mathrm{time,} \mathrm{and}$ extrapolation of the titanium volume fraction values to zero indicates that the last of the titanium disappears prior to the $15 \mathrm{~h}$ RMM time.

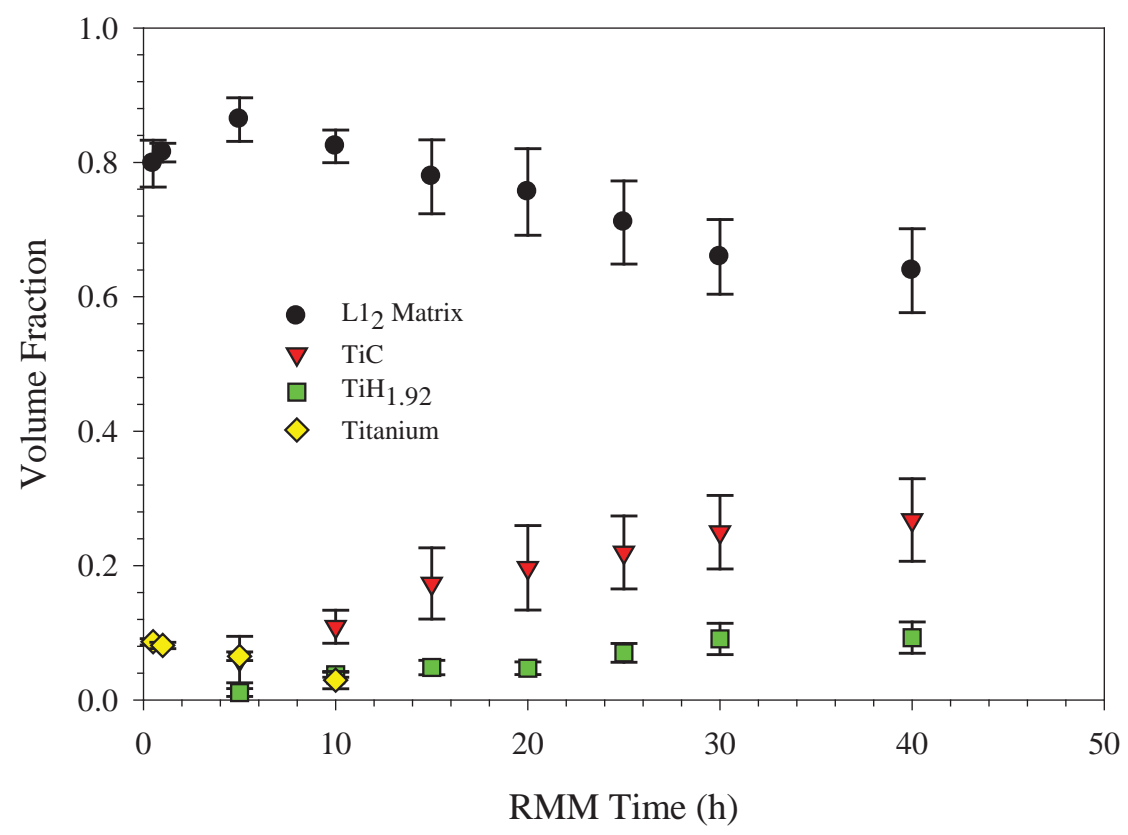

Figure III-15 Volume Fraction for RMMXh9TiAr Series of RMM Times 


\section{d) $\quad \mathrm{L1}_{2}$ Intermetallic Lattice Parameter}

Lattice parameters for the $\mathrm{L}_{2}$ intermetallic phase were determined using the Scintag Inc. DMS-NT crystallography (refinement) software option. Error reporting was determined from multiple lattice parameter determinations from the intelligent peak profile fitting for each sample providing a measure of the standard deviation. Because of the multiple phases and the peak broadening caused by the severe deformation not all $\{h k l\}$ diffraction peaks for the $\mathrm{L}_{2}$ intermetallic are observed for all RMM times. The superlattice peaks are generally weak, especially with decreases in long range order parameter, as are the $\{400\}$ peaks, making determination of these diffraction peaks difficult for some RMM times. However, the first five fundamental $\mathrm{L}_{2}$ diffraction peaks, $\{111\},\{200\},\{220\},\{311\}$, and $\{222\}$, are available for all RMM times. Positional error of the peak position based on differences between the calculated values and the actual peak positions grew with RMM time about an order of magnitude from $0.00002 \mathrm{~nm}$ in the initial powder analysis to a value of $0.0003 \mathrm{~nm}$ at $20 \mathrm{~h} \mathrm{RMM} \mathrm{time,}$ thus confirming very reasonable accuracy using the intelligent profile fitting approach.

As a point of reference, comparison of the methodology used here to that by Nic $[7,116]$ for the $\mathrm{Al}_{66} \mathrm{Cr}_{9} \mathrm{Ti}_{25}$ alloy was possible because the specimen he used was available (referred to as the $\mathrm{L}_{2}$ Standard Alloy). With the function refinement program used here and in his work the lattice parameter determined from the $\{100\},\{110\},\{200\}$, $\{220\}$ was $0.39565 \mathrm{~nm}$ compared to his value of $0.3957 \mathrm{~nm}$. A similar value for his specimen resulted from a Nelson-Riley extrapolation made with all the peaks out to the $\{400\}$ which was $0.39566 \mathrm{~nm}$.

The $\mathrm{L}_{2}$ intermetallic phase lattice parameter results from the milling experiment with standard deviations are shown in Figure III-16 for each RMM time. The initial mixture lattice parameter of $0.39588 \mathrm{~nm}$ is slightly larger than the $\mathrm{L}_{2}$ Standard Alloy of $0.39565 \mathrm{~nm}$ just reported. Some difference is anticipated because of the presence of $\mathrm{Al}_{3} \mathrm{Ti}$, the formation of which could change the composition of the ternary $\mathrm{L}_{2}$ intermetallic. As expected, the standard deviations increase with RMM time because of the increase in uncertainty of the peak positions of the $\mathrm{L}_{2}$ intermetallic as the peaks broaden and the diffraction pattern becomes more complex. There is a rapid initial 
increase in the $\mathrm{L} 1_{2}$ intermetallic lattice parameter upon milling, reaching a peak around a RMM time of $20 \mathrm{~h}$. According to Nic [7, 123] an increase (the equilibrium single-phase limit is 27 wt. pct.) in titanium content will result in an increase of the lattice parameter. Here the rapid increase in the $\mathrm{L}_{2}$ intermetallic lattice parameter occurs while there is a simultaneous rapid decrease in the excess elemental titanium resulting from the formation of the $\mathrm{TiC}$ and $\mathrm{TiH}_{1.92}$ phases with a possible increase in titanium in the $\mathrm{L}_{2}$ intermetallic by alloying as well.

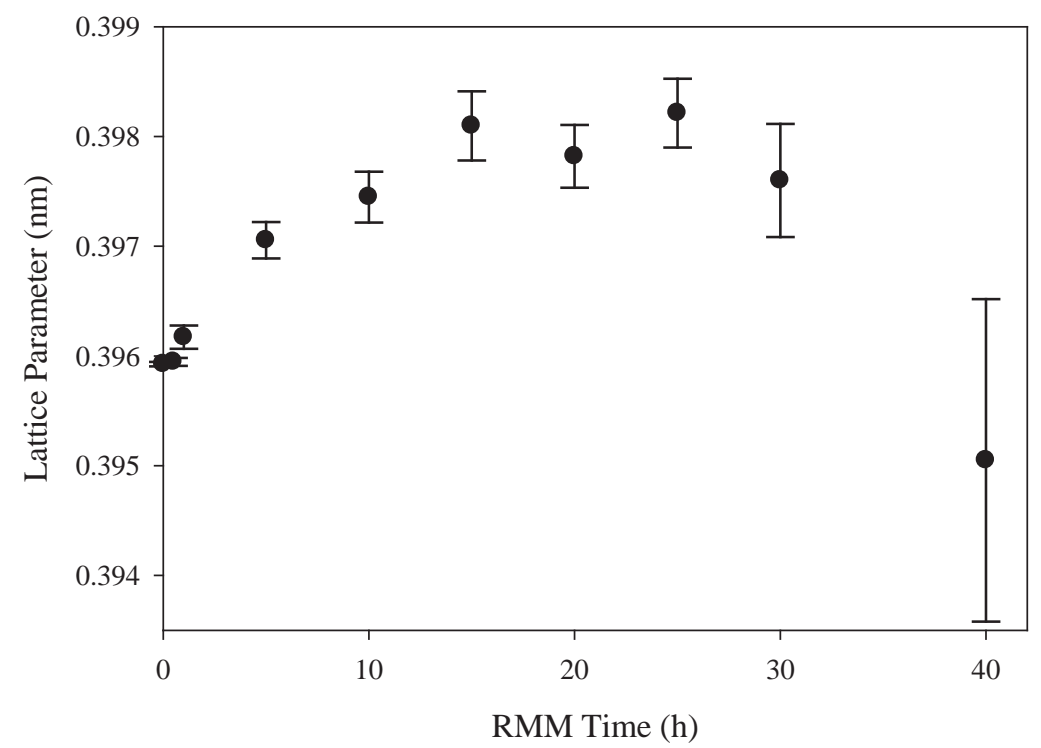

Figure III-16 L12 Intermetallic Lattice Parameter

There is a decrease in the lattice parameter of the $\mathrm{L}_{2}$ intermetallic after about $25 \mathrm{~h}$ of milling. According to Nic [7] the lattice parameter of the $\mathrm{L}_{2}$ intermetallic decreases with increasing chromium content. Based on the fixed composition of aluminum and chromium, elements that do not appear in other observed phases, their concentrations would increase within the given $\mathrm{L}_{2}$ intermetallic volume as titanium is extracted from the $\mathrm{L}_{2}$ intermetallic to contribute to carbide and hydride formation. Thus, the dynamic changes in titanium in the $\mathrm{L}_{2}$ intermetallic result from the significant role it plays in the reactions to form the titanium-based phases. Further, the MA processing can often create nonequilibrium alloys; therefore it can be expected to increase the extent of the $\mathrm{L}_{2}$ single-phase field based. This is supported by the observations in the RMA 
process experiment where the $\mathrm{L}_{2}$ cubic structure was formed from binary elements of aluminum and titanium in the proper stoichiometric ratio; this binary cubic alloying was also confirmed by Swartz et al. [133] using a similar mechanical alloying process.

\section{e) Peak Broadening Analysis of Profile Fit X-ray Diffraction Peaks by Warren-Averbach Fourier Method}

Estimates of the coherently diffracting domain size (CDDS), commonly referred to as crystallite size, and the microstrain were determined for the $\mathrm{L}_{2}$ intermetallic. This was accomplished by Warren-Averbach (W-A) analysis of individual diffraction peak models synthesized from Pearson VII functions determined from intelligent profile fitting, as described in ChapeterII.A.5.a)(7)(a). The W-A analysis was applied to the $\mathrm{CuK}_{\alpha 1}$ component of the $1^{\text {st }}$ and $2^{\text {nd }}$ order in the $<111>$ direction using the $\{111\}$ and $\{222\}$ diffraction peaks, and with the $1^{\text {st }}$ and $2^{\text {nd }}$ order in the $\langle 100\rangle$ direction using the $\{200\}$ and $\{400\}$ diffraction peaks of the $\mathrm{L}_{2}$ intermetallic. Instrumental broadening was removed by the Stokes method, using profile-fit synthesized peaks of well-annealed L1 2 chromium-modified cubic trialuminide, the $\mathrm{L}_{2}$ Standard. The W-A Fourier analysis method was applied to each of the profiles determined from the XRD data for each RMM processing time from 0.5 to $40 \mathrm{~h}$.

The crystallite size coefficients and microstrain coefficients are determined as described in Chapter II.A.5.a)(7) with each plotted as a function of column length. The crystallite size is determined by fitting a line to the linear portion of the crystallite size coefficients as a function of column length with extrapolation to the column length axis (at zero value of the crystallite size axis) for each order to establish the crystallite size data for each direction. The slope of the Fourier coefficients at each column length determines the root mean square (rms) microstrain for that column length. These rms microstrain data are displayed as a function of column length using a smooth curve fit through the values to obtain the value at $5 \mathrm{~nm}$ reported as the final rms microstrain (\%). The final crystallite size and microstrain data reported are averages of data from 2 to 5 W-A analyses for each RMM time from the independent intelligent profile fits on each sample to establish statistics. 
The results of the average crystallite size analysis are shown in Figure III-17 for all RMM processing times for the $<111>$ direction and up to an RMM processing time of $20 \mathrm{~h}$ for the $<100>$ direction because of the weak $\{400\}$ peak. In addition, $95 \%$ confidence interval error bars are determined for each data point, except for the 25 and $30 \mathrm{~h}$ RMM times had only two separate profile analyses for both times which gave the same results. For a processing time of $0.5 \mathrm{~h}$ the crystallite size was quite small, $10.5 \mathrm{~nm}$ for the $<100>$ direction and $11 \mathrm{~nm}$ for the $<111>$ direction. However, the size still decreased rapidly with increasing RMM processing time. Beyond $\sim 20 \mathrm{~h}$ the sizes approached a steady state average value of $\sim 2-3 \mathrm{~nm}$.

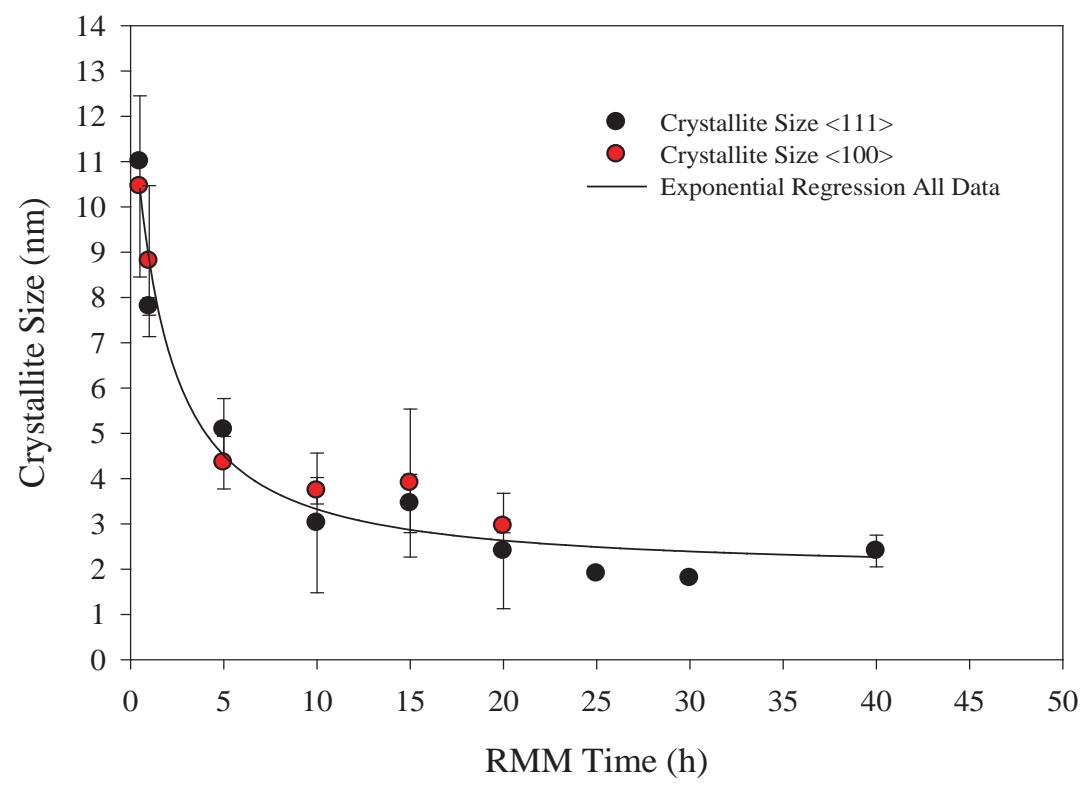

Figure III-17 RMMXh9TiAr Series Warren-Averbach Crystallite Size Analysis

The values for both crystallographic directions are essentially the same suggesting an isotropic crystallite size behavior in the nanometer-grain sized material. The macroscopic mechanical properties would be expected to be isotropic (random statistical nature to grain orientations in nanometer grain size) but the individual grains would be expected to retain the typical anisotropic behavior of the single crystal metallic grains. The change in crystallite size with RMM time is illustrated by an exponential decay regression curve shown in Figure III-17 determined with all of the data points for both directions. The crystallite size for the $40 \mathrm{~h}$ processing time shows a small increase with 
respect to the 25 to $30 \mathrm{~h}$ values; however, the behavior is uncertain. Original experiments for RMA times up to $90 \mathrm{~h}$ presented earlier did show similar crystallite size of $2 \mathrm{~nm}$ for all times.

Corresponding values of the average microstrain (\%), when they could be determined, are shown in Figure III-18. Again, $95 \%$ confidence intervals were determined, where appropriate, based on the statistics of multiple W-A analyses. In general, the microstrain increases in an exponential manner up to RMM processing times of $\sim 20 \mathrm{~h}$. The behavior for both crystallographic directions was similar with RMM time, indicating isotropic behavior. This is illustrated by the exponential regression curve determined for all data points and shown in Figure III-18. While it appears that the microstrain approaches a maximum, the value and error bars for the $40 \mathrm{~h}$ RMM time are below this maximum. Although this behavior is unexpected, it appears statistically significant and coupled with particle size behavior could be a topic that warrants further study.

Of significance from these estimates are the large microstrains in the material, even after only $0.5 \mathrm{~h}$ of RMM processing time, with the largest values reaching a $1 \%$ microstrain. These microstrain values are physically reasonable since compression tests of the consolidated samples (with much larger grain sizes) have shown elastic strains just over $1 \%$. Additionally, Ultrasonic Young's modulus measurements consistently gave values of $\sim 200 \mathrm{GPa}$, which when coupled with yield strengths of the order of $2 \mathrm{GPa}$ suggests attainable elastic strains of $\sim 1 \%$ in consolidated samples. In these powders grain sizes are much smaller (approximately 20 - 50 times smaller) than consolidated samples and based on a Hall-Petch type analysis suggests that attainable elastic strains could be much higher. Varin et al. [134] determined lattice strains approaching $1 \%$ for mechanically milled $\mathrm{L}_{2}$ manganese-modified titanium trialuminide showing similar microstrains despite a different type of mill. It can be noted that the Varin et al. value was calculated by the more approximate integral breadth Williamson-Hall method. 


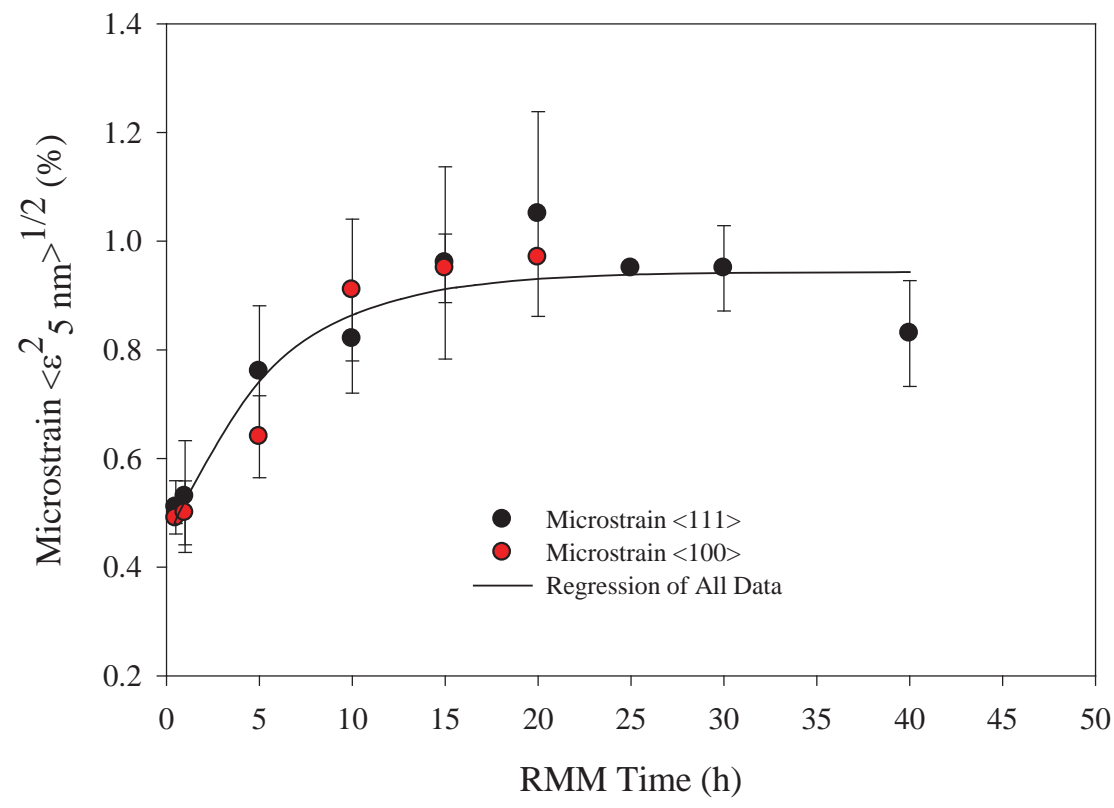

Figure III-18 RMMXh9TiAr Series Warren-Averbach Microstrain Analysis

Estimates of the dislocation densities using the Burgers vector of the total dislocation $a<110>$ and the smaller $a / 3<112>$ dislocation (commonly observed) using W-A data from the $\langle 111\rangle$ direction are summarized for all RMM times for the RMMXh0TiAr Series in Table III-3. As discussed earlier, small crystallite sizes can affect deformation mechanisms favoring a smaller Burgers. These dislocation densities are very large; in comparison, many heavily cold rolled metals have dislocation densities that are commonly an order of magnitude smaller [141]. In a study by Brusso [135] on shock loading of $\mathrm{Ni}_{3} \mathrm{Al}$ alloyed with boron (20 GPa pressure, $2.9 \mathrm{~km} / \mathrm{s}$ velocity, 34 and $130 \mathrm{~ns}$ shock durations) dislocation densities in these materials were from $5 \times 10^{8} \mathrm{~mm}^{-2}$ to $1.2 \times 10^{9} \mathrm{~mm}^{-2}$ developing a substructure arrangement with crystallite sizes between 49 to $80 \mathrm{~nm}$ in the $\langle 111\rangle$ direction and 20 to $50 \mathrm{~nm}$ in the $\langle 100\rangle$. These dislocation densities are an order of magnitude lower than those developed in the RMMXh9TiAr samples. The dislocation densities are very large at the shortest RMM time of $0.5 \mathrm{~h}$, similar to heavily cold rolled material, indicating a large amount of deformation has taken place during this very short time interval. The near constant dislocation density for RMM times over $15 \mathrm{~h}$ suggests a change in the deformation mechanism. An alternate view of this substructure and the role of dislocations in interpreting these results will be discussed later. 
Table III-3 Dislocation Density Estimates for RMMXh9TiAr Series

\begin{tabular}{|c|c|c|}
\hline $\begin{array}{c}\text { RMM Time } \\
\text { (h) }\end{array}$ & $\begin{array}{c}\text { Dislocation Density } \\
\qquad \begin{array}{c}(\mathrm{mm})^{-2} \\
a / 3<112>\end{array}\end{array}$ & $\begin{array}{l}\text { Dislocation Density } \\
\qquad \begin{array}{c}(\mathrm{mm})^{-2} \\
a<110>\end{array}\end{array}$ \\
\hline 0.5 & $4.6 \times 10^{9}$ & $2.6 \times 10^{9}$ \\
\hline 1 & $8.9 \times 10^{9}$ & $5.1 \times 10^{9}$ \\
\hline 5 & $1.6 \times 10^{10}$ & $9.4 \times 10^{9}$ \\
\hline 10 & $2.0 \times 10^{10}$ & $1.2 \times 10^{10}$ \\
\hline 15 & $2.6 \times 10^{10}$ & $1.5 \times 10^{10}$ \\
\hline 20 & $5.3 \times 10^{10}$ & $3.0 \times 10^{10}$ \\
\hline 25 & $5.4 \times 10^{10}$ & $3.1 \times 10^{10}$ \\
\hline 30 & $5.4 \times 10^{10}$ & $3.1 \times 10^{10}$ \\
\hline 40 & $3.8 \times 10^{10}$ & $2.2 \times 10^{10}$ \\
\hline
\end{tabular}

\section{f) Scherrer Crystallite Size Estimates of the TiC Phase}

Scherrer crystallite size measurements were accomplished on the TiC phase present in the RMMXh9TiAr Series for those RMM times having measurable amounts of TiC shown in the volume fraction data (Table III-2). The analysis was carried out in a manner described in Chapter II.A.5.a)(6). The crystallite sizes in Table III-4 represent an average of determinations from the first three diffraction peaks; $\{111\},\{200\}$, and $\{220\}$ using an average FHWM from multiple profile fits for each time. The crystallite size of $\sim 2 \mathrm{~nm}$ for the TiC particles is very similar to the values estimated in the original experiment as expected. Included in the results are the $95 \%$ confidence intervals based on averages of these first three diffraction peaks of the TiC. These crystallite size values are all similar except that for the $5 \mathrm{~h} \mathrm{RMM}$ time. Despite a crystallite size that is twice as large as other measurements the large $95 \%$ confidence interval of $3.5 \mathrm{~nm}$ makes any trend statistically difficult to establish. The expectation is that the crystallite size of these particles, created by a bottom-up approach of nucleation, would be similar. A concern is the difficulty of obtaining peak profiles in these complex diffraction patterns when the 
volume fraction of TiC is very small as is the case with the $5 \mathrm{~h}$ RMM time (Table III-2) or less.

Table III-4 Crystallite Size Estimates of the TiC Particles

\begin{tabular}{c|cc}
\hline $\begin{array}{c}\text { RMM Time } \\
(\mathbf{h})\end{array}$ & $\begin{array}{c}\text { Crystallite Size } \\
(\mathbf{n m})\end{array}$ & $\begin{array}{c}\mathbf{9 5} \% \text { Confidence } \\
(\mathbf{n m})\end{array}$ \\
\hline 5 & 4.1 & 3.5 \\
10 & 1.9 & 0.5 \\
15 & 1.9 & 0.6 \\
20 & 1.9 & 0.4 \\
\hline 25 & 1.7 & 0.5 \\
30 & 1.5 & 0.5 \\
$\mathbf{4 0}$ & $\mathbf{1 . 6}$ & $\mathbf{0 . 6}$ \\
\hline
\end{tabular}

The similar crystallite sizes in the original experiment and the RMMXh9TIAr Series demonstrate that the $\mathrm{TiC}$ (recall $\mathrm{Ti}(\mathrm{C}, \mathrm{N})$ in the original experiment) particle size remains the same with increased milling time and it appears that only the number of particles increases causing the increase in volume fraction. Given that the ideal lattice parameter is $0.43274 \mathrm{~nm}$ (JCPDS-ICDD 32-1383) only four to five unit cell sizes are required to reach the average crystallite size diameter of TiC particles. Based on volume, about 50 unit cells exist within the average $\mathrm{TiC}$ particle. It is interesting that the size of these average particles is very similar to the expected critical sized nuclei in homogeneous solidification (typically 1-2 nm) as will be discussed later. This suggests nucleation and formation of particles, but little or no growth.

\section{X-ray Powder Diffraction Analysis of Annealed Powder}

X-ray powder diffraction patterns were collected for the annealed version of the powders for all RMM times; however, diffraction data for the $15 \mathrm{~h}$ RMM time sample was not analyzed due to contamination of the powder during thermal treatment. Samples typically sintered lightly on annealing, but were easily crushed to smaller particles by milling for one minute and screened to -200 mesh similar to the as-milled powders. One 
important observation for all these XRD patterns was that there was no evidence of hydrogen-based phases indicating the degassing procedure (Chapter II.A.4.a) removed all hydrogen. The main use of the XRD data collected from the annealed powder is for a background scattering area correction for a new XRD analysis technique. However, volume fraction and lattice parameter data were compiled for these annealed powders and are reported in Appendix VIII.C as this data provides important information regarding the chemistry effects on the final phase structure produced for each RMM time, which will be addressed in the Discussion.

\section{X-ray Powder Diffraction Intensity Analysis}

In the original experiment, Figure I-6, Bragg intensities were measured along with increases in the background scattering. Apparent losses of Bragg scattering and increases of background scattering in the as-milled powder were investigated in more detail to better understand this effect and its relationship to the RMM process. Two assumptions underlie this analysis, one is that the density of the packed powders is constant, and the other is that the intensity produced by the instrument is stable. It is concluded that these assumptions are valid for two reasons: Chapter II.A.5.a)(1) showed only a $1 \%$ weight variation due to packing, and the data for all samples were collected on consecutive days minimizing variations in the intensity produced by the instrument, which typically decays extremely slowly with time. Although likely not necessary, the initial sample will be not be used in this analysis since that data was collected at a date much later than the data collected for the as-milled and annealed samples.

\section{a) Background Intensity Models}

Quantitative analysis of a diffraction pattern requires subtracting the background intensity. In general, the background intensity for a particular material should remain constant unless there are contributions of scattering from atoms no longer in crystalline positions. This will influence both the background and the crystalline peak intensity. In order to quantify the background intensity behavior for the RMMXh9TiAr Series, and the associated annealed samples, a regression fit was made to the background using a 
Polynomial Inverse Third Order function given in Equation (III.1). This function was determined to provide the best fit (R-value near 1) to the background from among several functions attempted that have a decaying shape similar to the background behavior over the $2 \theta$ range of interest. In the usual manner, ranges of data for intensity and $2 \theta$ that represent regions of the true background from the corresponding $\mathrm{x}$-ray powder diffraction scans were utilized in the regression analysis.

$$
f=I_{o}+\left(\frac{a}{2 \theta}\right)+\left(\frac{b}{(2 \theta)^{2}}\right)+\left(\frac{c}{(2 \theta)^{3}}\right)
$$

There is a visually evident gradual increase in the background of the diffraction pattern as the RMM time is increased, similar to the behavior observed in the original experiment. The background intensity model fits are shown in Figure III-19 for all RMM times. Of particular interest is the convergence of all the backgrounds to similar levels as the $2 \theta$ angle approaches $10^{\circ} 2 \theta$. This indicates that the background (i.e. diffuse intensity) increases occur into the higher $2 \theta$ ranges suggesting atoms rearranged into noncrystalline positions in a random manner as opposed modulated diffuse scattering characteristic of the effects of short-range order or clustering. As noted, the $\mathrm{L}_{2}$ intermetallic retains an ordered structure and doesn't contribute to the diffuse scattering. It is important to point out that the background changes in cps are very small on the order of $\leq 2 \mathrm{cps}$.

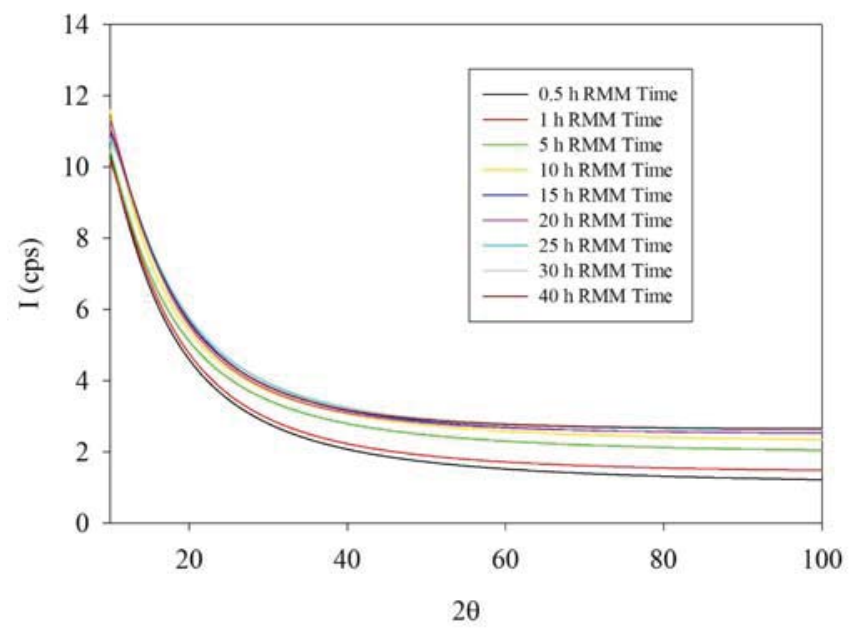

Figure III-19 X-ray Powder Diffraction Pattern Background Increase 
A quantitative measure of the background scattering can be determined by numerical integration of the individual background models for all as-milled and annealed RMMXh9TIAr samples as shown in Figure III-20. Also shown are estimated standard deviations along with a hyperbolic regression of the RMM data. As noted there is a sharp increase in the background area up to an RMM time of $15 \mathrm{~h}$ with similar areas for RMM times beyond $20 \mathrm{~h}$. In addition, the background area of the annealed samples for each RMM time are also shown for comparison, with the values remaining fairly constant for all times. It is interesting to note that the as-milled sample at a RMM time of only $0.5 \mathrm{~h}$ shows a significant increase in the background area over the annealed version. The observed background increase in the as-milled samples appears to be more prominent at the higher $2 \theta$ ranges, with increases throughout the $2 \theta$ range indicating a large spread of diffuse intensity. This suggests that the severe mechanical deformation results in large fractions of the atoms having a wide variations in position best described as "random" displacement of atoms from the expected crystalline positions.

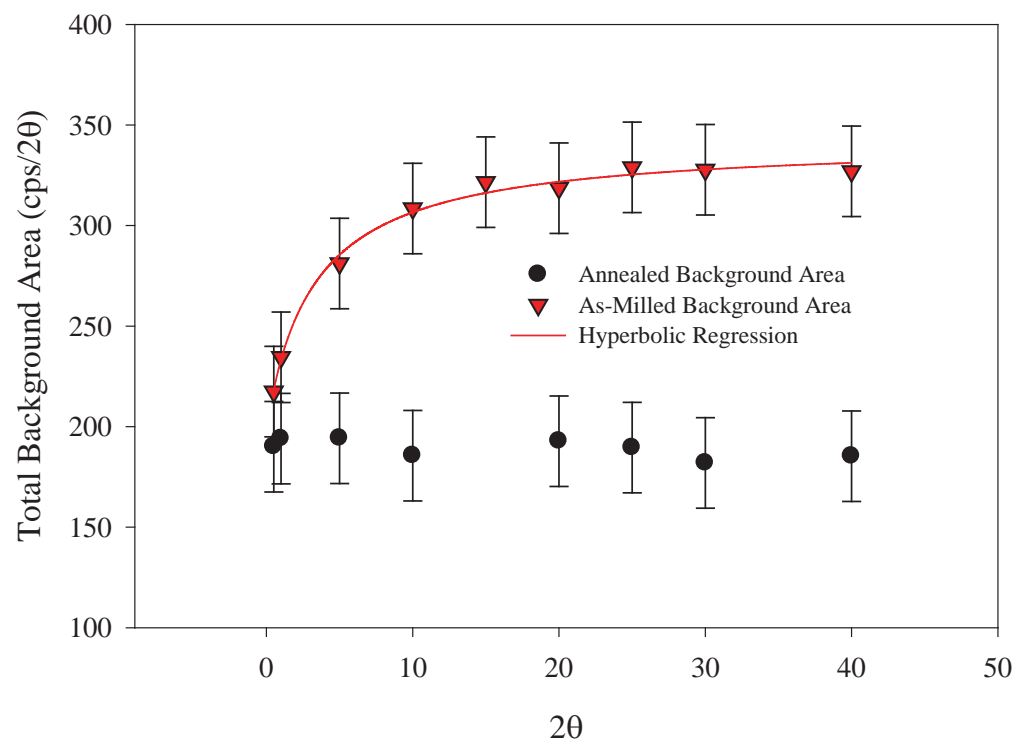

Figure III-20 RMMXh9TiAr Series Background Intensity Area for As-Milled and Annealed Samples for all RMM Times 


\section{b) Crystalline or “Bragg” Peak Area}

Theoretically, the total diffracted intensity, expressed as the area of the crystalline peaks, should remain constant provided the diffracting volume and the number of atoms in crystalline positions remains constant. This also assumes that the amount of each phase remains constant. In order to understand the changes in the total Bragg scattering intensity with RMM time, the total diffracted crystalline peak area was determined as the sum of all crystalline diffraction peaks using the individual peak areas determined from profile fitting analysis.

As RMM time increases the background level increases in conjunction with a rapid decrease in the total area of crystalline peaks, or Bragg scattering. The total crystalline diffracted intensity for both the RMM samples and the annealed samples are shown with $95 \%$ confidence intervals in Figure III-21. The crystalline total area for the starting mixtures of powders is also shown for comparison; however, these data were collected at a date much later than the other samples (therefore subject to small intensity changes due to tube intensity, alignment, etc.). Although direct comparison must be done with caution it still shows the magnitude of the decrease in crystalline area beyond $0.5 \mathrm{~h}$ of RMM time. The sample with a $0.5 \mathrm{~h}$ RMM time, for both as-milled and annealed powder conditions, shows approximately the same total crystalline diffracted area. In the case of the annealed and as-milled powders with a $1 \mathrm{~h}$ RMM time the total crystalline diffracted area decreases approximately $10 \%$ in the as-milled powder compared to the annealed powder. Beyond an RMM time of $5 \mathrm{~h}$ the decrease of total crystalline diffracted area of the annealed powder compared to the as-milled powder is in general approximately $20 \%$.

It is important to consider that phase changes have occurred in the annealed powder compared to the as-milled powder. This undoubtedly leads to a change in the total Bragg scattering; however, since the chemical composition is approximately the same, one would expect this change to be small. The only element not present in the annealed sample is hydrogen, which has a low atomic scattering factor, and is involved with a small volume fraction phase. The majority of the material for both the annealed and the as-milled powders is the $\mathrm{L} 1_{2}$ intermetallic. In fact, as will be shown, the phases 
present in the as-milled material are mostly cubic, whereas in the annealed material only the $\mathrm{L}_{2}$ intermetallic is cubic. Typically, for a similar chemical composition and a given amount, a phase with a cubic structure will have a much larger scattering factor than a non-cubic structure because of symmetry effects and therefore scatter more intensity. Again this supports the findings in which the total crystalline intensity is less in the asmilled powder than in the annealed powder.

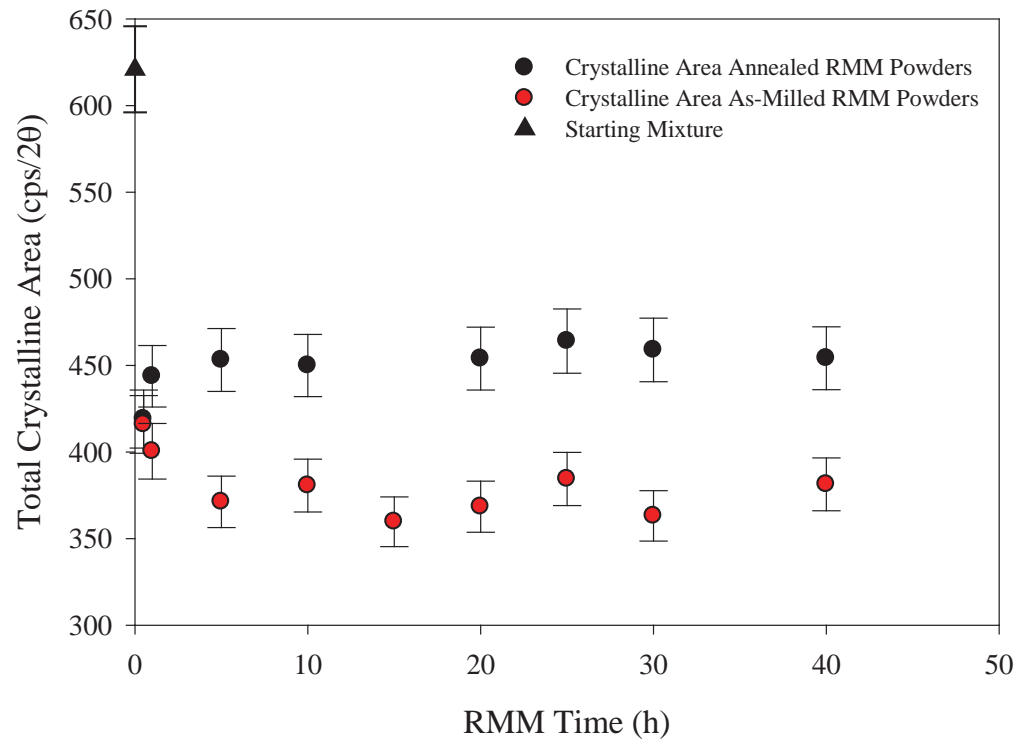

Figure III-21 Total Crystalline Diffracted Intensity for the RMMXh9TIAr Series As-milled and Annealed Samples

It is concluded that there is a significant decrease in Bragg scattering along with an associated increase in background scattering as a function of RMM time. As mentioned, one interpretation of this observation, especially for the background increase, is that large numbers of the atoms normally in crystalline positions are moved, on a per volume basis, to non-crystalline positions during the RMM process. Specifically, the general intensity increase of the background occurs because a significant number of atoms are randomly displaced in magnitude and direction to non-crystalline positions. This suggests that this process of RMA/M, which causes a significant volume fraction of such disorder, impacts the $\mathrm{x}$-ray scattering process and develops interesting 
microstructures that can be expected to result in unusual properties as will be discussed later.

\section{Macrostructure of Powders}

Few studies were found in the literature that provide information on the macrostructure of the as-milled powder particles from the MA process with most focusing only on physical size and shape. Furthermore, studies that examine the surface features of the as-milled powders in this process were vague. For example, one study indirectly mentioned smaller particles welded to the surface of larger particles in the MA of iron aluminides [136]. Details of the macrostructure, in particular particle morphology and the surface features, will be presented here mainly for the as-milled powders providing important new information about the MA process.

\section{a) Physical Powder Particle Size}

Powder particle size, that is, the average physical powder particle size, was determined from FESEM digital micrographs of the powders using an average from numerous fields of view of each sample. Measurements were accomplished using the public domain Image $\mathbf{J}$ software (developed at the U.S. National Institutes of Health and available on the Internet at http://rsb.info.nih.gov/nih-image/). Sample preparation techniques helped assume that a representative distribution was created along with a good dispersion of the particles. Often more than one specimen was created for each processing condition and the analyses were determined from at least a dozen images collected for each condition.

The Image J software was spatially calibrated using the micrometer markers provided in each image. Digital measurement of powder particles was accomplished by thresholding of the image contrast of interest followed by the creation of a binary image based on the thresholding. Often combinations of erosion and dilation, the Watershed command in Image $\mathrm{J}$, as well as the various manual image tools in Image $\mathrm{J}$, were used to help separate physically touching particles. The Image $\mathrm{J}$ software provides several types of measurement on these thresheld features, but direct methods for particle size 
dimensions are based on exterior measurements such as; perimeter, fit ellipse, and Feret's diameter. It was determined that the fit ellipse parameter provided the best description of the particle size of these powder particles, especially those with protrusions [137]. All results for the particle size used an average of the major and minor ellipse axes as the final particle diameter. These values from each particle were averaged for all particles measured for that sample to yield the average particle size along with a particle size distribution. Results are organized based on the as-milled RMM processing time.

These powder particles did not always appear to be dense and often significant cavities and pores were present within individual powder particles. Analysis of image measurement data supports the simple approximation of a spherical shape, with about a $25 \%$ difference between the major and minor axis of the elliptical fit for select measured particles. However, the perimeter of the particles appeared more irregular with small protrusions emanating from the particles often observed. What is of interest is the particles maintained a general spherical nature despite an expected asymmetrical shape due to the uniaxial impacts between powder, media, and/or vial walls during milling, which is commonly observed in MA of ductile powders. However, in this case, as will be discussed, the surface appears constructed of smaller particles welded to the surface which themselves have the more expected high aspect ratio shape.

Average particles sizes for RMMxh9TiAr samples with RMM times of 0.5, 1, 10, 25, 30 and $40 \mathrm{~h}$ are provided in Table III-5 along with the standard deviation and number of data points included in each analysis. What is interesting is the initial and rapid reduction of original powder particle size from -200 mesh, or $74 \mu \mathrm{m}$ or less, to about an order of magnitude reduction after the $0.5 \mathrm{~h} \mathrm{RMM}$ time, with no consistent change in the particle size with longer RMM time, suggesting a somewhat steady state size is reached of about $5 \mu \mathrm{m}$. It is important to note that numerous fines were observed with sizes on the order of the lower limit of resolution for the magnification chosen.

Of particular importance to the current work are the differences in the particle size distributions developed with increased milling time. It can be noted that fewer large particles and more numerous average size particles are observed in the particle size distribution histograms shown for the $0.5 \mathrm{~h}$ and $25 \mathrm{~h}$ RMM times in Figure III-22 and 
Figure III-23. A further reduction in the number of larger particles is observed in the particle size distribution for the $40 \mathrm{~h}$ RMM time in Figure III-24 with a narrowing of the distribution and a larger number of particles sizes nearer to the average. Additionally, as expected the distributions have an asymmetric nature that tends to decrease with milling time. It is interesting to note that this trend in the particle size distribution showing fewer large particles with RMM time coincides with the observation that more fines are produced as RMM time increases.

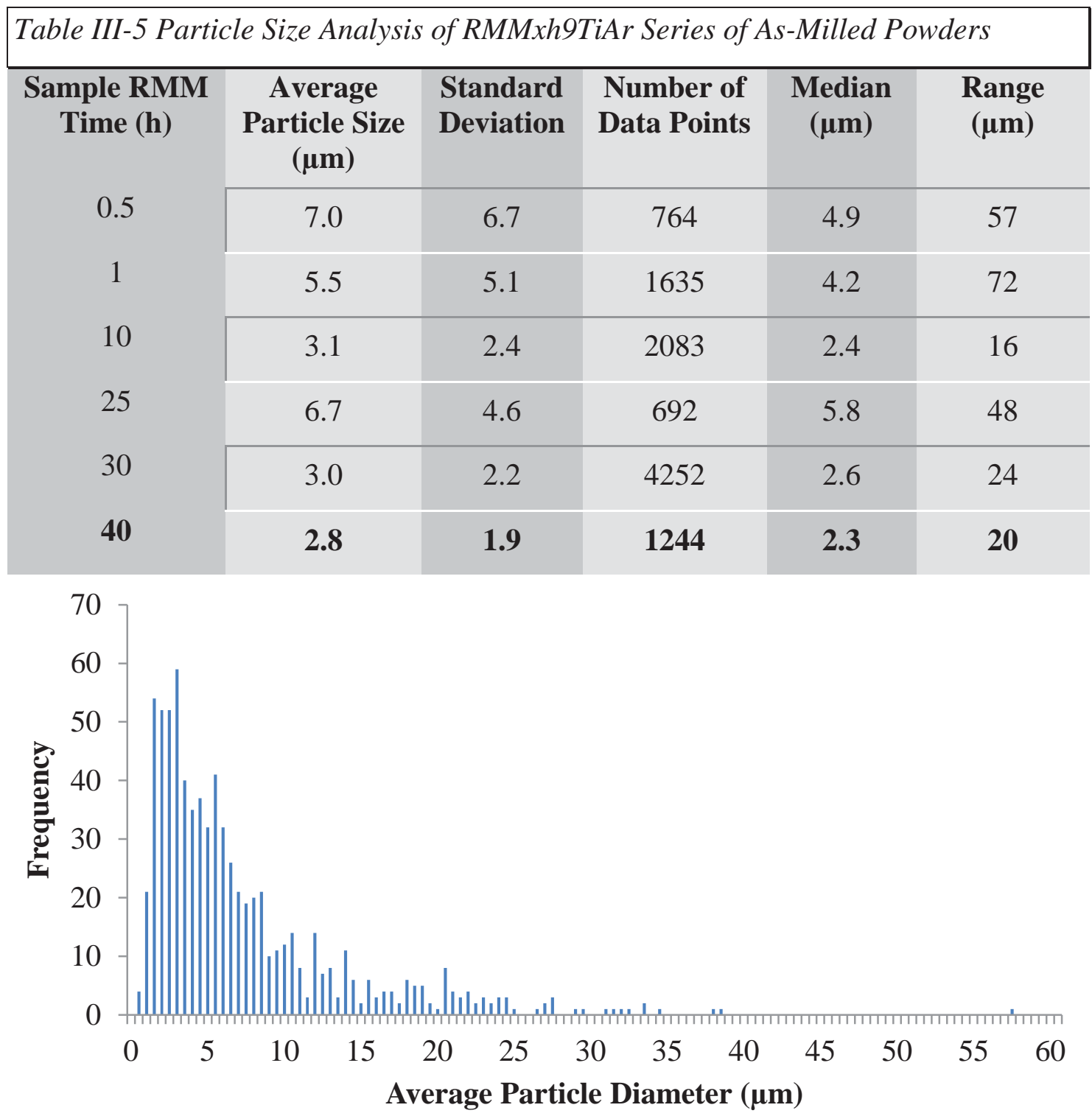

Figure III-22 Particle Size Distribution of RMM0.5h9TiAr As-Milled Powder 


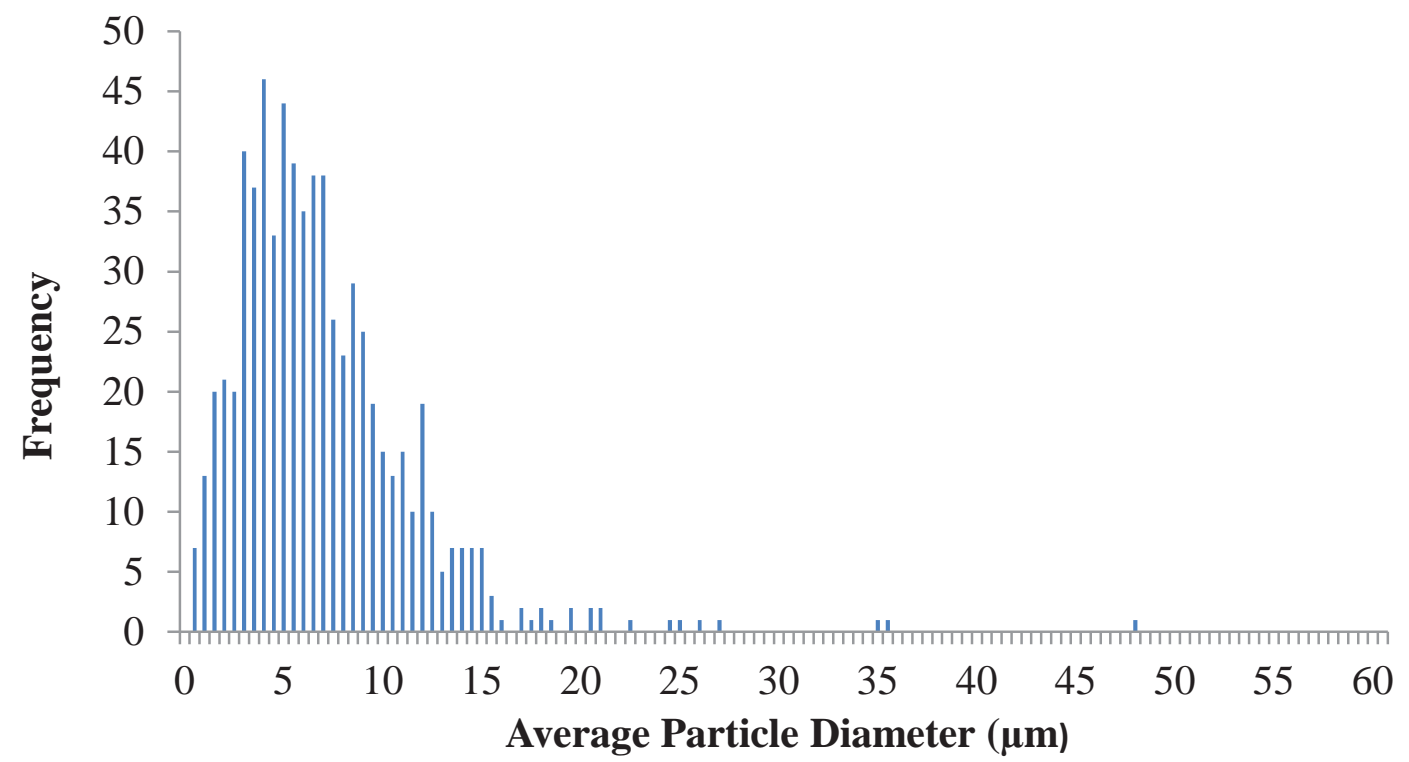

Figure III-23 Particle Size Distribution of RMM25h9TiAr As-Milled Powder

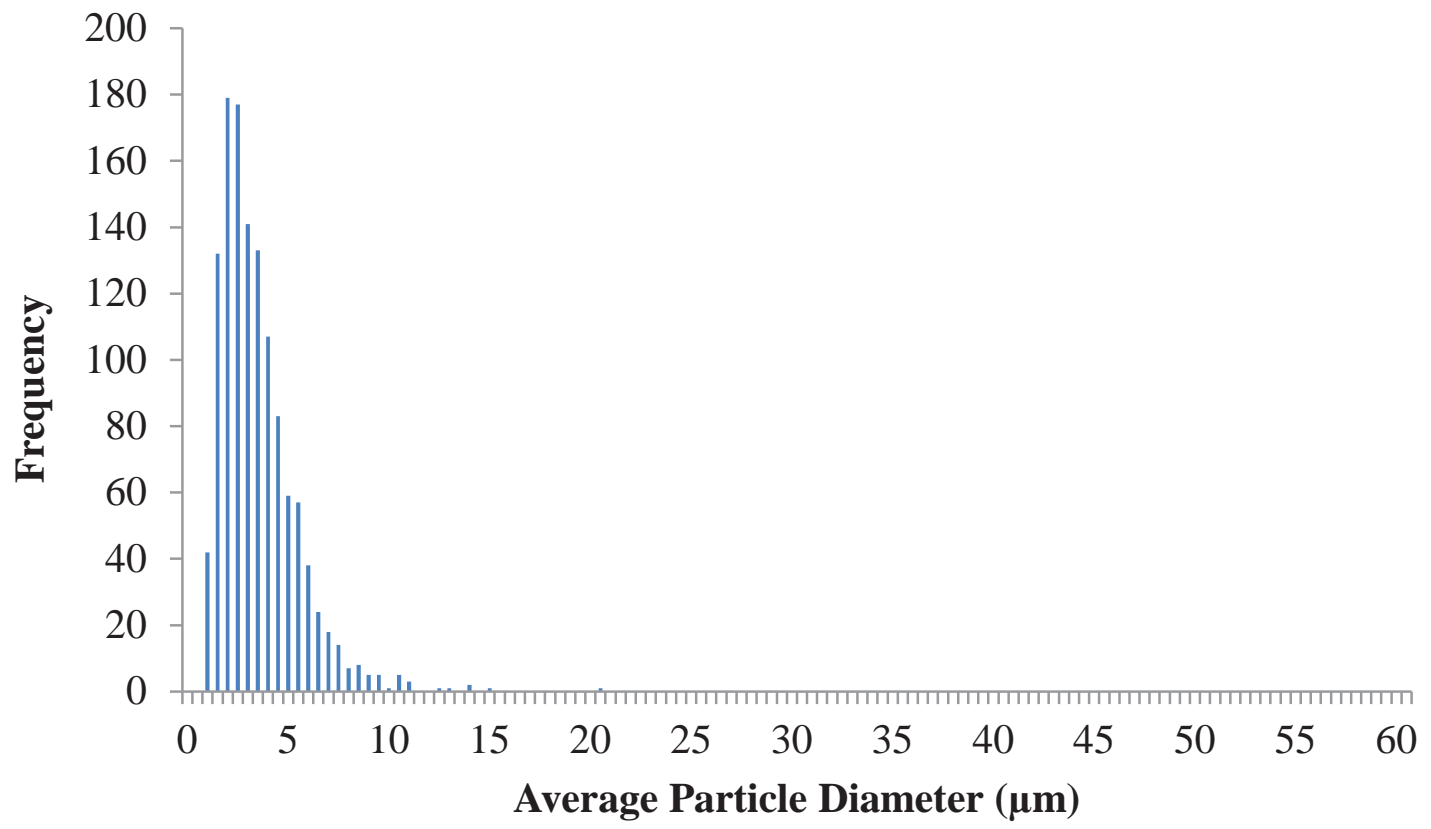

Figure III-24 Particle Size Distribution of RMM40h9TiAr As-Milled Powder 


\section{b) Particle Morphology}

Individual particle morphology was investigated to better understand the macrostructure of the powder particles as well as details of surface features. It is clear that the largest particles tended to be an obvious agglomeration of smaller particles. The general particle morphology does provide insight into the process involved in the formation of these composite powder materials during the RMA/M process. Details of the surface features may reflect the active mechanisms during the violent collisions between the powder particles and media. This aspect will be discussed later and may contribute to the understanding of the MA process.

\section{(a) Individual Particle Morphology}

The as-milled powders varied from low density particles that are formed from an agglomeration of a large number of smaller particles as shown in Figure III-25, termed "infant agglomerates", to much more dense particles as shown in Figure III-26. While not investigated here, it may be of interest to examine the relationship between infant agglomerates and the largest size particles as these particles are on the large end of the size distribution, but clearly composed of the small average size particles. This agglomeration is similar to that commonly observed in particulate processing as the particle size decreases the generally weak van der Waals forces take over and smaller particles tend to agglomerate, and as will be discussed later the nature of the surface may enhance this process. It is important to note that these various phases of particle agglomeration were evident in powders for all RMM times explored in this research; however, distribution analysis suggests fewer extremely large particles as milling time increases. 


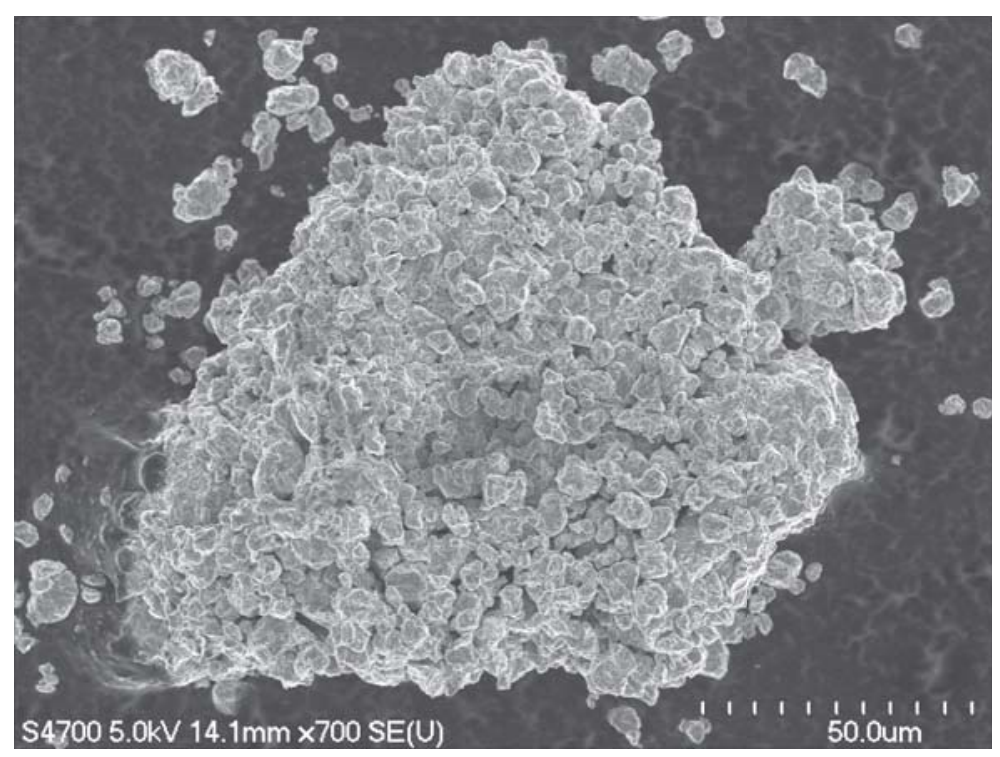

Figure III-25 Infant Aggolomerate Particle RMM10h9TiAr As-Milled 700X

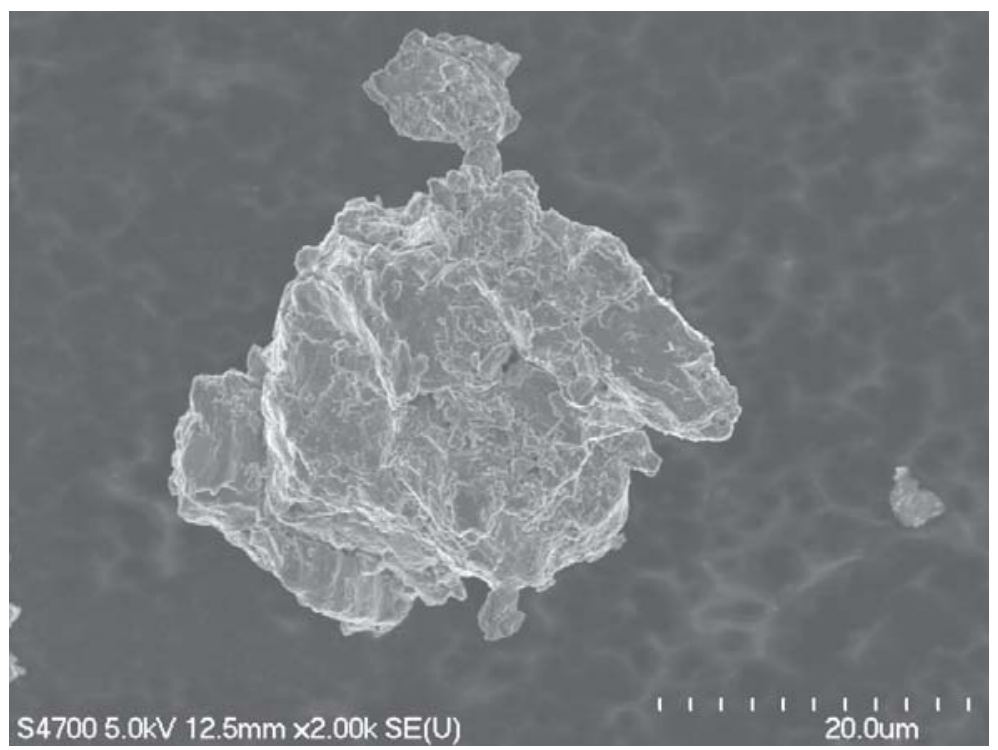

Figure III-26 As-Milled Powder Particle RMM0.5h9TiAr $2 k X$

Select samples of RMMXh9TiAr Series, 40, and $60 \mathrm{~h}$ RMM powder particles were imaged, the latter two RMM times contained two and three times the amount of elemental titanium as the RMMXh9TiAr Series at the start of milling with similar mechanical processing parameters except for the milling time. Based on a few images the $40 \mathrm{~h}$ and $60 \mathrm{~h}$ RMM times produced the same large particles consisting of an 
agglomeration of smaller particles. Because of the enormous amount of energy imparted to the powders during milling it might be expected that this type of structure would no longer exist at longer milling times. However, this type of agglomerated particle is present at all RMM times as shown in Figure III-27 for the 10, 25, 40, and $60 \mathrm{~h}$ RMM times. This observation suggests some type of cyclic process in which agglomeration is followed by densification, and fracture.

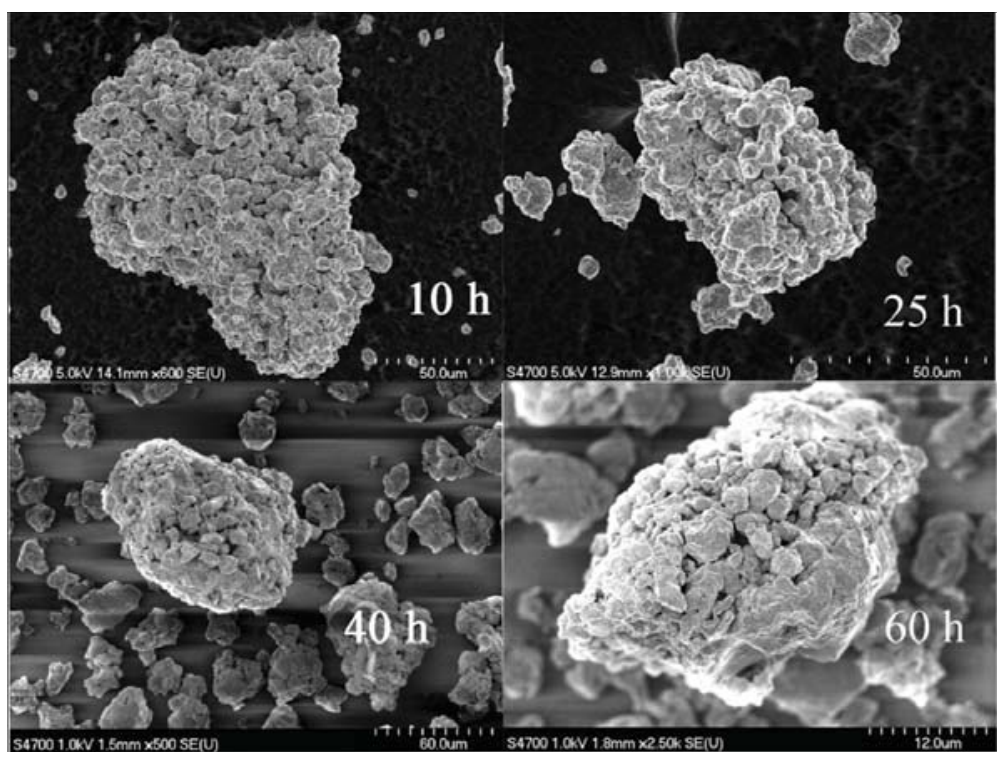

Figure III-27 As-Milled Individual Powder Particles for 10, 25, 40, and 60 h RMM Times (the 10 and 25 h same excess titanium, 40 h $2 X$, 60 h $3 X$ amount)

Infant agglomerates have a macrostructure consisting of a collection of smaller sized particles that are apparently mechanically pushed into contact. The "cavities" associated with the infant agglomerates are expected to have an interconnected structure and typically with a size of the order of micrometers. As the particles densify the cavities begin to show a more pore-like structure. This implies that diffusion occurs between agglomerated individual particles with the cavities beginning to shrink and close-off as shown in Figure III-28. Some particles as in Figure III-29 show more of a pore-like structure evolving from a cavity, seen here in the center of image, with a size of approximately $1 \mu \mathrm{m}$. With further densification these become more spherical or pore-like with sizes in the submicrometer to nanometer range. 


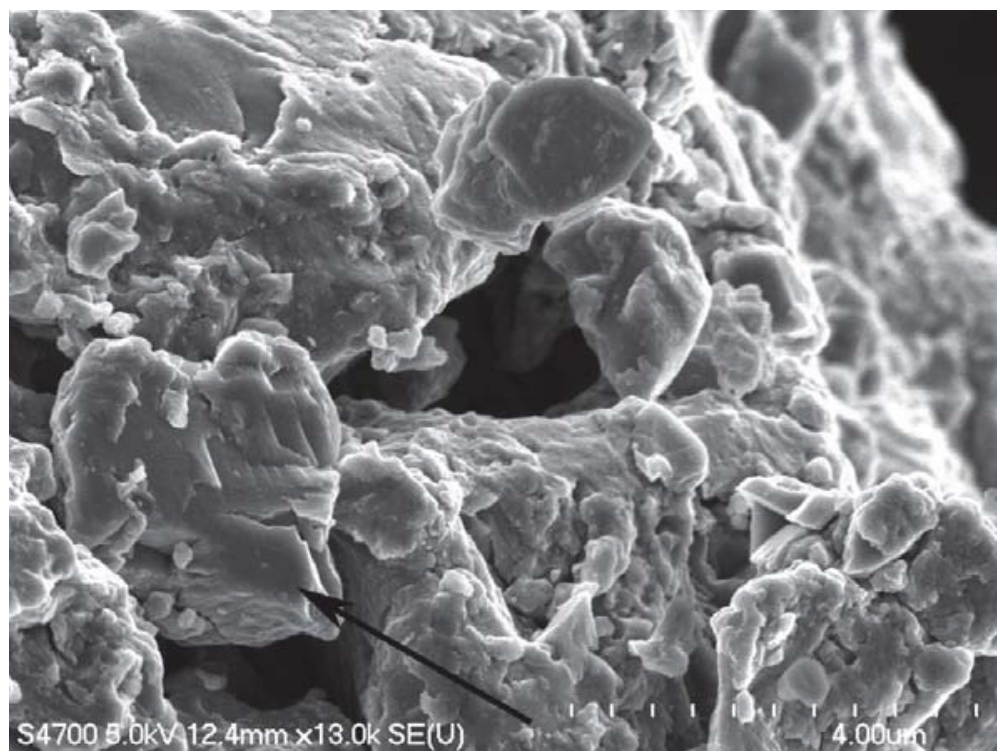

Figure III-28 Particle Cavity in RMM1h9TiAr As-milled Powder Particle 13kX

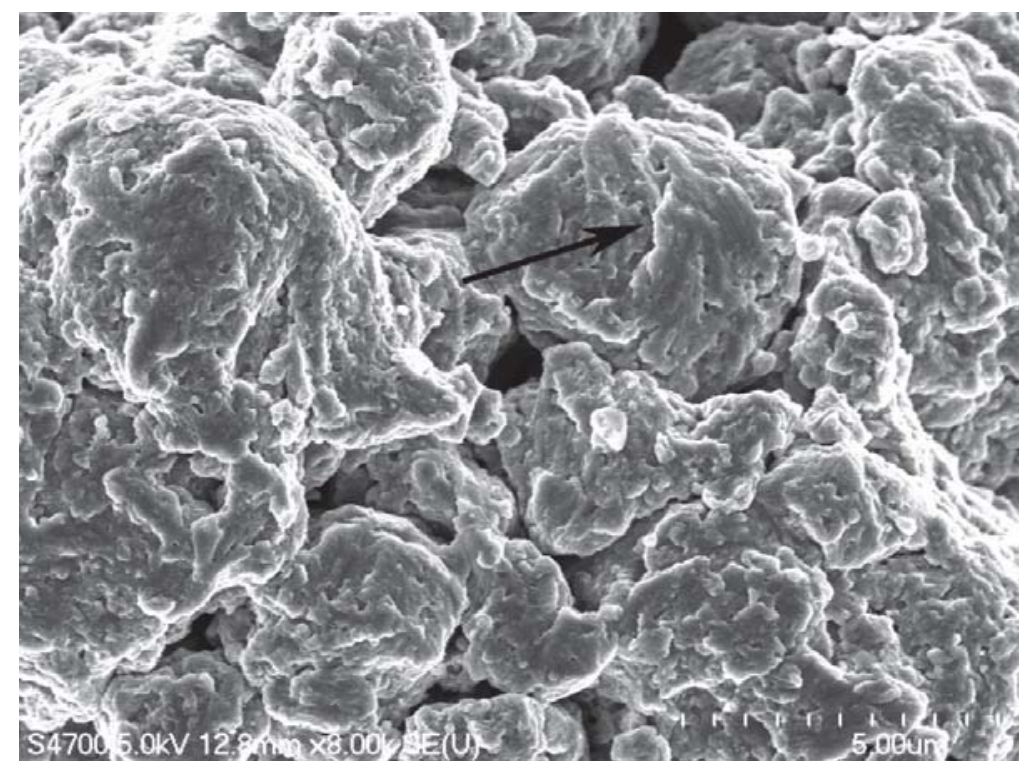

Figure III-29 Continued Cavity Closure in RMM25h9TiAr As-millled Powder Particle $8 k X$

\section{(b) Surface Morphology}

The surface morphology of the particles shows features not previously reported with similar high magnification details in the powders for all RMM times. A typical 
powder particle surface features islands of material that appear to be composed of many smaller highly compressed particles adhered to the surface. The focus here is on these compressed particles adhered to the surface that are expected to comprise one component of cold welding along with the large particle agglomeration presented in the previous section.

These islands of much smaller particles compressed onto the surface of the host particle occasionally show well-defined flow lines in the particle surface features, a behavior reminiscent of near liquid type viscous flow, similar to the feature highlighted by the black arrow in Figure III-29. It appears shear forces in this instance have caused the solid particle to flow as these are compressed onto the host surface with possible diffusion type bonding occurring at the interface. An average of a major and minor ellipse of the surface particle highlighted in Figure III-29 is about $2 \mu \mathrm{m}$ with a thickness of about $100 \mathrm{~nm}$ resulting in a large aspect ratio. Based on these measurements the volume of the particle can be estimated assuming a cylindrical shape. Equating this volume to that of a sphere allows for estimation of the diameter of the original particle size prior to compressing the particle onto the surface, resulting in a value just under a micrometer. This allows estimation of the particle deformation of about $75 \%$. These small pieces of material are impacted with sufficiently high energy to exceed the flow strength of the material causing the very large amount of deformation.

While not as well-defined, several of these flattened particles on the surface in Figure III-31 show a similar flow behavior suggesting that these surface agglomerated particles can undergo a shear event essentially "smearing" the particles onto the surface. Many of these surface features in Figure III-30 appear as flattened particles. Suggesting both shear and normal stresses can compress ("smear") smaller particles into the surface of host particles. Although not part of the original research plan, it is clear this behavior plays a significant role in MA and reaction mechanisms. 


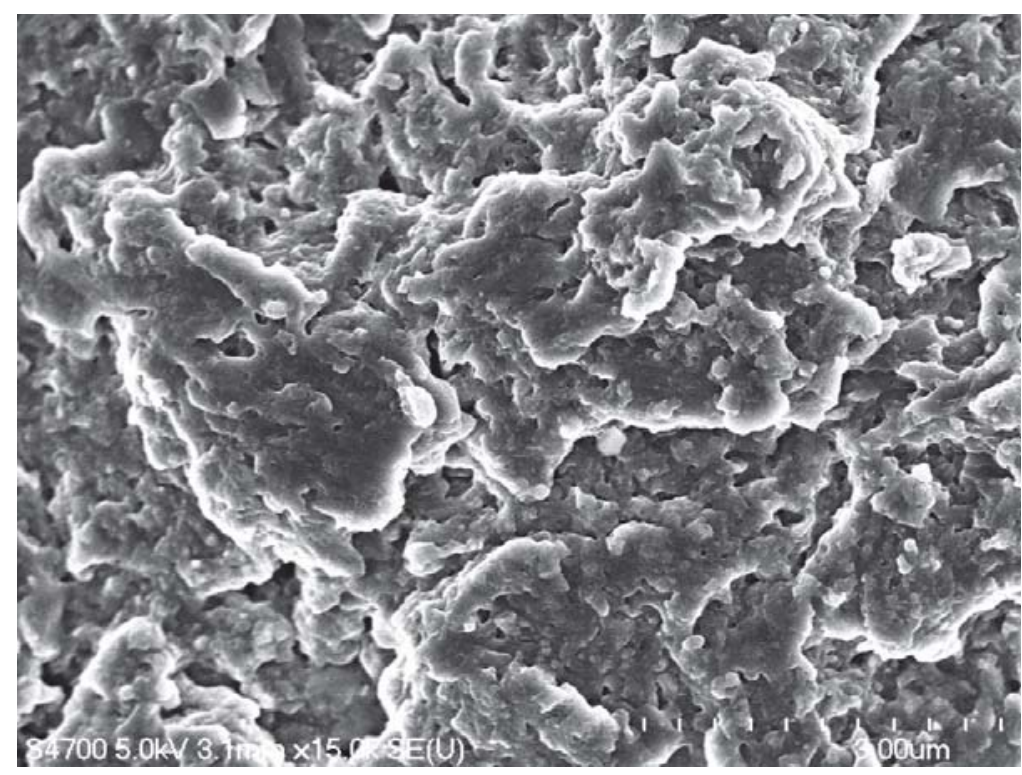

Figure III-30 High Magnification Micrograph of Surface Features of As-milled RMM10h9TiAr Powder Particle 15kX

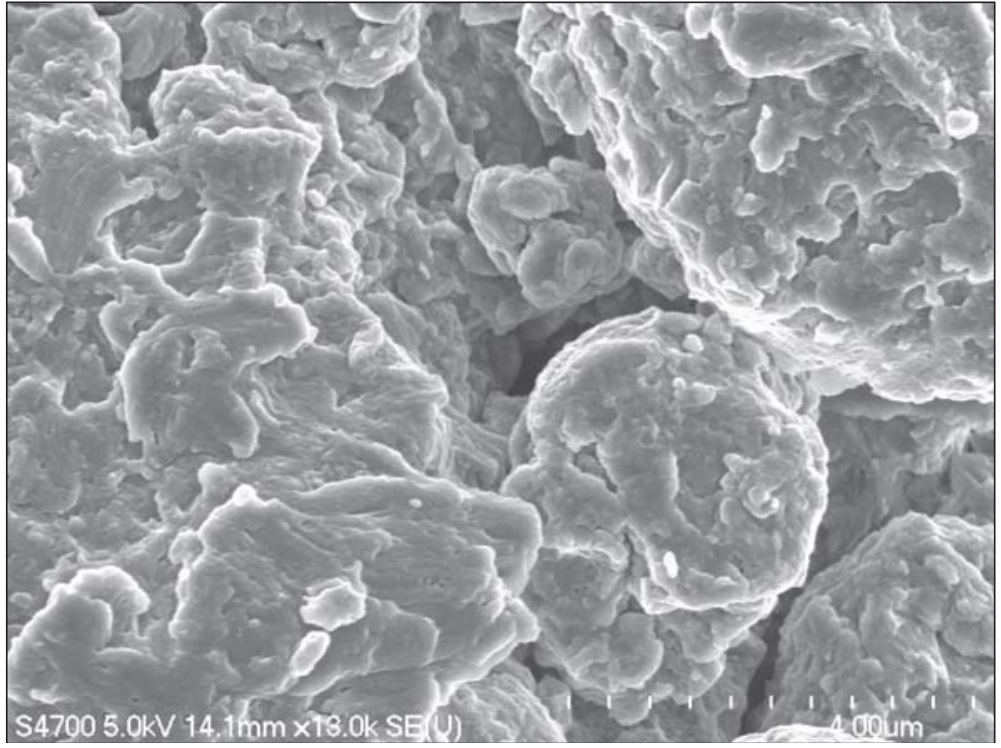

Figure III-31 Surface Smeared Particles Showing Flow Lines in As-milled RMM10h9TiAr Powder Particle 19kX

These surface smeared particles vary in size well into the nanometer range and appear cold welded to the surface playing an important role in the agglomeration to form larger particles with these surface features appearing at the point of particle to particle contact. An example is shown by the small particle adhered to the larger particle in the 
lower right hand quadrant of Figure III-31. In summary, particles from nanometer to micrometer size are impact welded to the surface of larger host particles essentially creating a small scale agglomeration process in addition to large particle agglomeration previously introduced; however, the frequency of this surface smearing behavior is expected to produce an extremely significant increase in interaction volume of the agglomeration process over that for the large particle behavior. These surface smearing processes provide a dynamic and cyclic behavior to the creation and nature of the physical particles making up the as-milled powder from the nano-scale level to micrometer level.

The particle surfaces also show areas of smeared particles in a "pile-up" type structure jutting out of the larger host particle surface, as denoted by the black arrows in Figure III-32, showing particle upon particle being stacked and flattened with these smaller particles appearing to be mechanically compressed onto the prior surface in a similar manner to the surface smearing. These larger agglomerates appeared to cyclically densify and fracture creating the various particles observed. It is interesting to note that during the longer milling times larger amounts of fines were noted upon vial opening (although a not quantified) suspected to be involved in the surface smearing and in the large particle cold welding. These fines are defined as particles that float upon vial opening in the fume hood, thus expected to be in the nanometer range.

Based on the model developed by Benjamin two mechanisms are fundamental to the MA process; cold welding and fracture. This consists of large particle agglomeration of ductile metals in which particles compress together, ultimately creating an internal lamellar microstructure. However, the cold welding in the current work has been described based on two mechanisms consisting of the large particles cold welding as suggested by Benjamin, but with a new surface agglomeration consisting of smaller particles cold welding to the surface of host particles. On the other hand, fracture behavior is more elusive as these surfaces become obscured leaving few footprints of the event. Considering the cyclical nature of the powder agglomerations and the basic understanding of MA being a fracture and welding dynamic, and along with the 
population of much smaller particle sizes in this processed material than the original powder particle sizes indicates fracture must take place.

Occasionally smaller flat planar areas have been observed on the surface of powder particles indicating a brittle type fracture highlighted by the arrow in Figure III-28. In this case fracture is occurring on what appears to be surface smeared particles, a possible source of the fines produced. Larger planar surfaces are observed on more densified particles, indicated by the white arrows in Figure III-32, similar to fracture surfaces. Smaller planar surfaces are observed in Figure III-33, white arrows, that shows larger fractures from the surface and the black arrow shows a crack developed within a small fracture surface.

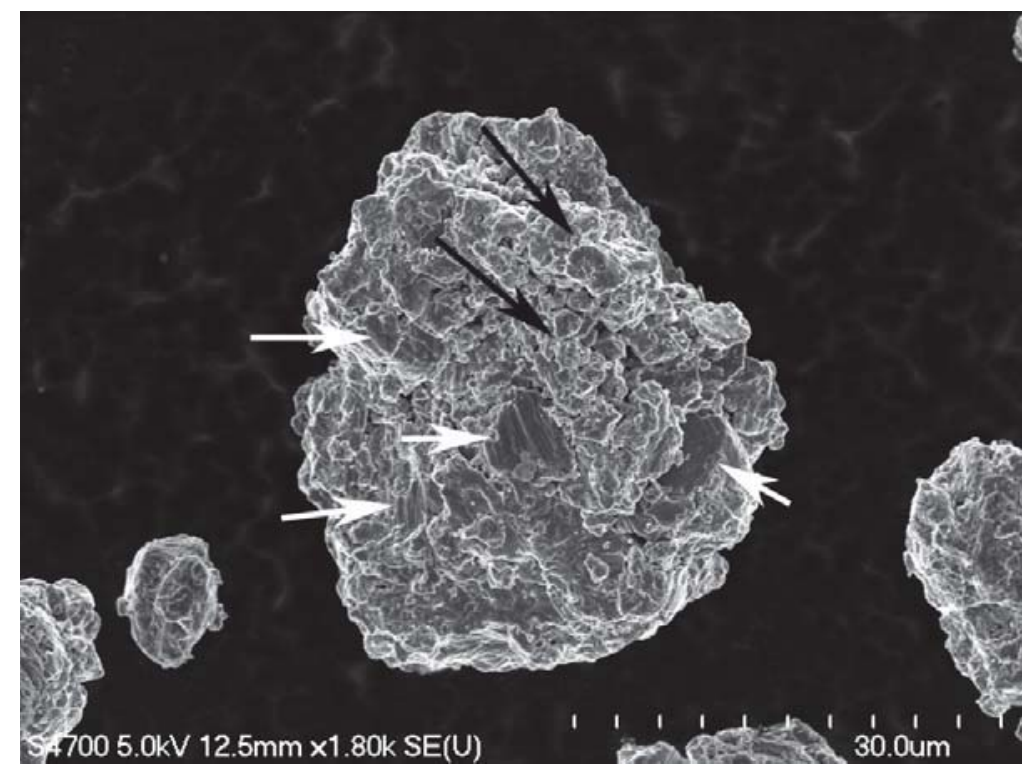

Figure III-32 Areas of Small Particle Cold Welding and Brittle Fracture Surfaces on As-milled Particle RMM1h9TiAr 1.8kX 


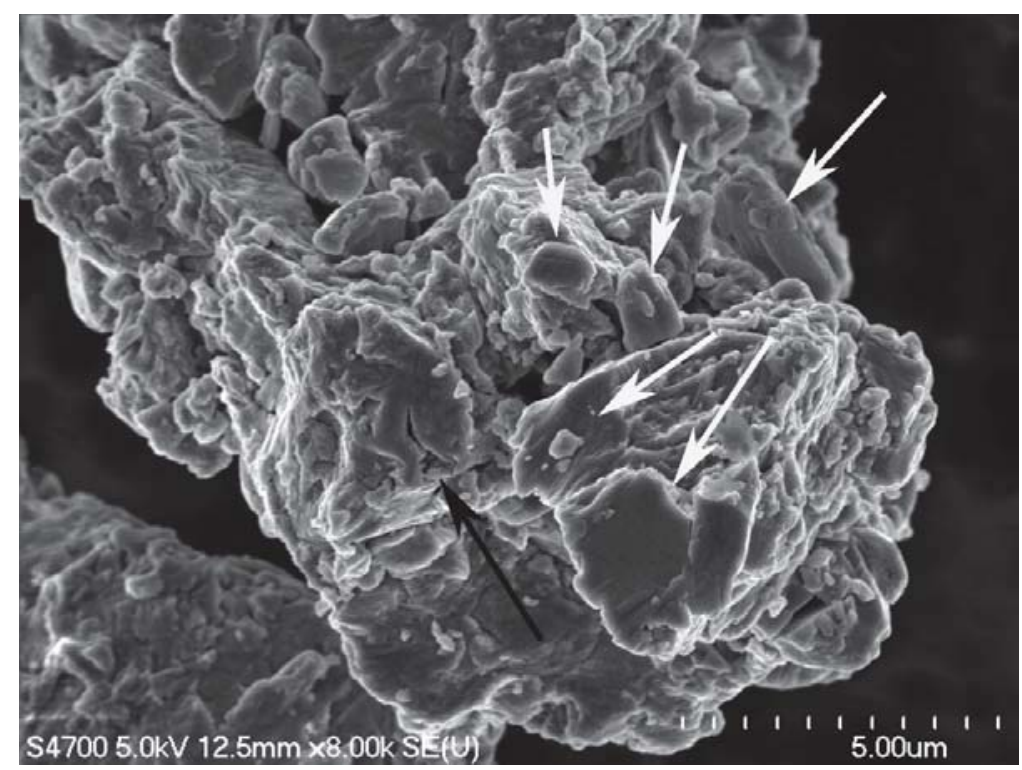

Figure III-33 Crack and Fracture Surface on As-milled RMM0.5h9TiAr Powder Particle $6 \mathrm{kX}$

Large cracks on a larger densified powder particle, such as indicated by the black arrows in Figure III-34, have also been observed. Here the left arrow shows a crack on what appears as a planar brittle fracture surface, as well as interparticle and intraparticle cracks shown by the black arrow on the right of the micrograph. Areas highlighted by the white arrows in Figure III-34 appear to be the result of pieces of material that broke away from the particle indicated by sharp corners and fracture surfaces in the interior cavities. It is expected that once the cracks form they propagate with continued impacts causing fracture of pieces of smaller physical particles away from the larger particles. With the extremely small crystallite size of the as-milled powders the mechanical properties are expected to be isotropic thus cracks would expect to be more directionally dependent on the macroforces involved as opposed to the crystallography. These larger macroscopic cracks were only visible on densified particles. 


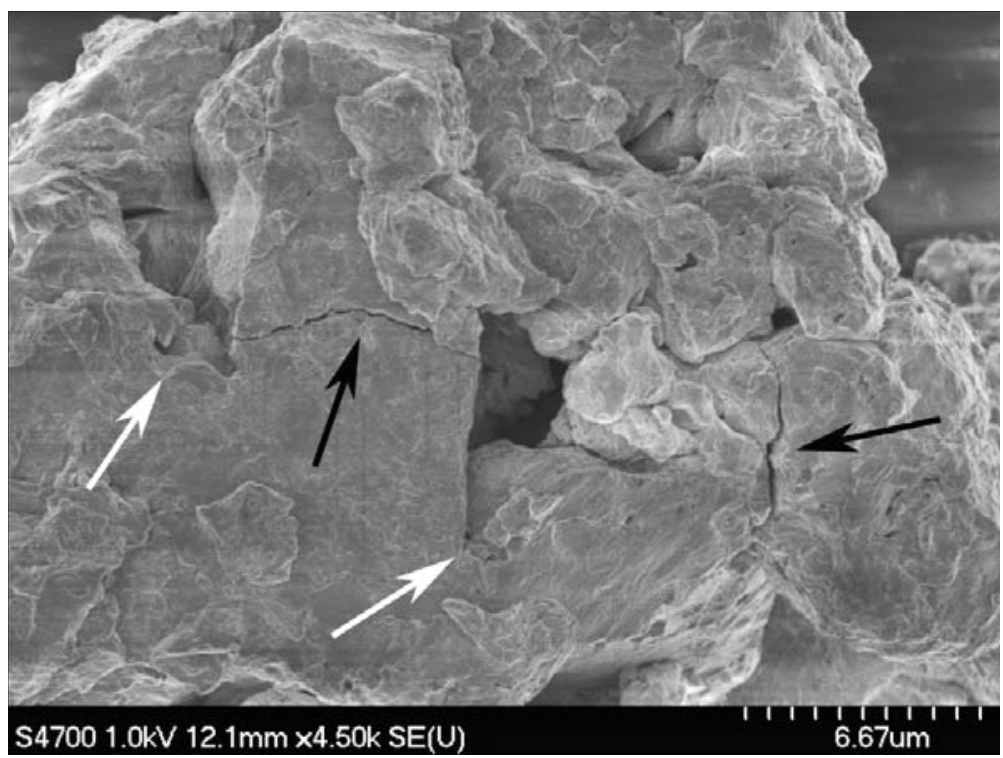

Figure III-34 Cracks and Fractures As-milled Powder Particle of 40 h RMM Time with 16.8 wt. pct. Titanium $4.5 \mathrm{kX}$

In summary, a variety of individual powder particle morphologies are developed in these mechanically alloyed as-milled powders from very low density highly agglomerated particles creating larger particles to very dense particles often smaller. Two fundamental cold welding processes occur; larger particles agglomerating and surface smearing of nanometer to micrometer fines creating a surface agglomeration. These smaller suspended fines are trapped by collisions and impact welded to the surface of host particles, often observed with extreme deformation by the flow lines in the solid material indicating large amounts of ductility. Fracturing of smaller pieces of larger particles are evidenced by the fracture surfaces and brittle cracks observed in the macrostructure of the largest particles down to smaller surface fractures into the nanometer range. These observations suggest a variety of mechanisms contribute to the basic cold welding and fracture processes in the MA process. These include macroscopic to microscopic level events causing dynamic changes in the size and physical nature of the particles that have highly reactive surfaces. 


\section{B. Reactive Mechanical Alloying (RMA) of RMA20h8.2TiAr Elemental Powders of $\mathrm{Al}_{66} \mathrm{Cr}_{9} \mathrm{Ti}_{25}$ with Excess Titanium in an Argon Atmosphere}

Given the success in producing titanium carbide particles in the $\mathrm{L}_{2}$ intermetallic this experiment examined the potential for RMA of the elemental feed powders needed to produce an $\mathrm{Al}_{66} \mathrm{Cr}_{9} \mathrm{Ti}_{25}$ alloy with a similar excess titanium to produce carbide. The elemental components to form the $\mathrm{Al}_{66} \mathrm{Cr}_{9} \mathrm{Ti}_{25}$ alloy, together with 8.2 wt. pct. excess titanium, were milled for $20 \mathrm{~h}$, with the sample being identified as RMA20h8.2TiAr.

\section{X-ray Power Diffraction Phase Analysis of As-Milled and Annealed Powder}

Phase analysis of RMA20h8.2TiAr sample based on the as-milled RMA (20 h) powder diffraction pattern, Figure III-35, established the existence of chromium, $\mathrm{TiH}_{1.92}$, $\mathrm{TiC}$, and an indication of titanium. The main diffraction peaks occurred in positions between those for pure aluminum and the $\mathrm{L}_{2}$ chromium-modified titanium trialuminide. The presence of the $\{100\}$ and $\{110\}$ superlattice peaks confirmed the existence of the $\mathrm{L1}_{2}$ structure. The strong presence of elemental chromium and the large shift in peak position from the normal $\mathrm{L}_{2}$ chromium-modified titanium trialuminide indicates that the $\mathrm{L1}_{2}$ intermetallic phase contained little or no chromium. As mentioned, Srinivasan et al. [96] observed formation of the $\mathrm{L}_{2}$ structure in mechanically alloyed $\mathrm{Al}_{75} \mathrm{Ti}_{25}$ elemental components in air. The $\{100\}$ superlattice peak presence was more pronounced and easier to distinguish compared to the same peak observed in the original RMA experiment with no excess titanium. This may indicate that formation of this structure is enhanced by the greater availability of titanium aided by the argon environment. The strong diffraction peaks from chromium indicate that this element does not participate in the alloying during the milling. Also, elemental titanium was still present after $20 \mathrm{~h}$ of RMA time, compared to the RMMXh9TiAr Series where the starting $\mathrm{L}_{2}$ intermetallic was pre-alloyed powder and titanium was exhausted by the $15 \mathrm{~h}$ RMM time. 


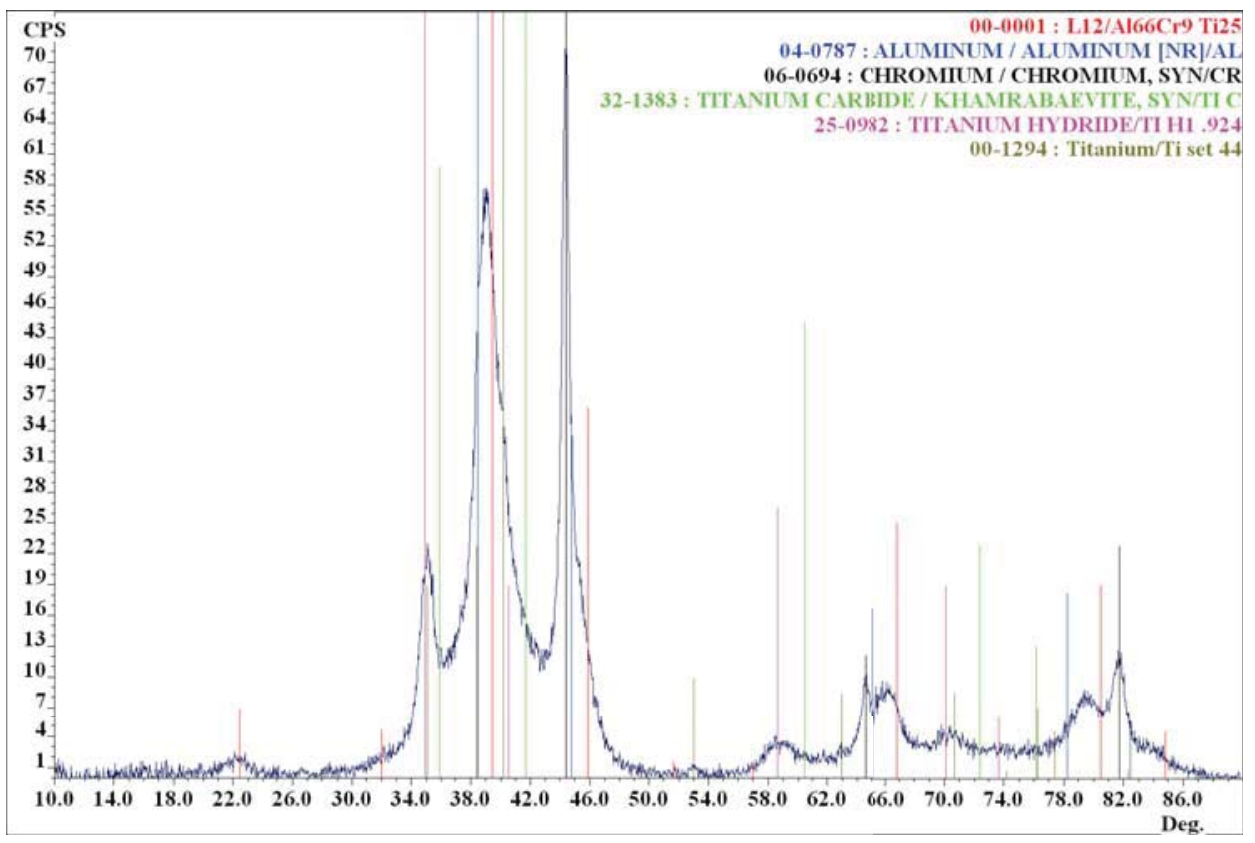

Figure III-35 Diffraction Pattern of RMA20h8.2TiAr As-Milled with Identified Phases

This RMA powder was thermally treated at $800{ }^{\circ} \mathrm{C}$ for $1 \mathrm{~h}$, which will be referred to as the "annealed" condition. Phase analysis of the annealed RMA powder diffraction pattern, Figure III-36, found the existence of the $\mathrm{L}_{2}$ chromium-modified cubic trialuminide, along with $\mathrm{Ti}_{3} \mathrm{AlC}_{2}, \mathrm{AlCr}_{2}$, and $\mathrm{Al}_{3} \mathrm{Ti}$. A small diffraction peak located at $26.6^{\circ} 2 \theta$ was attributed to contamination from the quartz used as a mill cleaning agent. There was also an unidentified peak at $44.4^{\circ} 2 \theta$; however, its intensity was less than 2 cps. In retrospect, this diffraction pattern should have been collected starting at $9^{\circ} 2 \theta$ in order to observe a very intense diffraction peak at $\sim 9.5^{\circ} 2 \theta$ from the $\mathrm{Ti}_{3} \mathrm{AlC}_{2}$ phase. This carbide phase is not included in the JCPDS-ICDD powder diffraction database, and crystallographic information was taken from Tzenov and Barsoum [138]. 


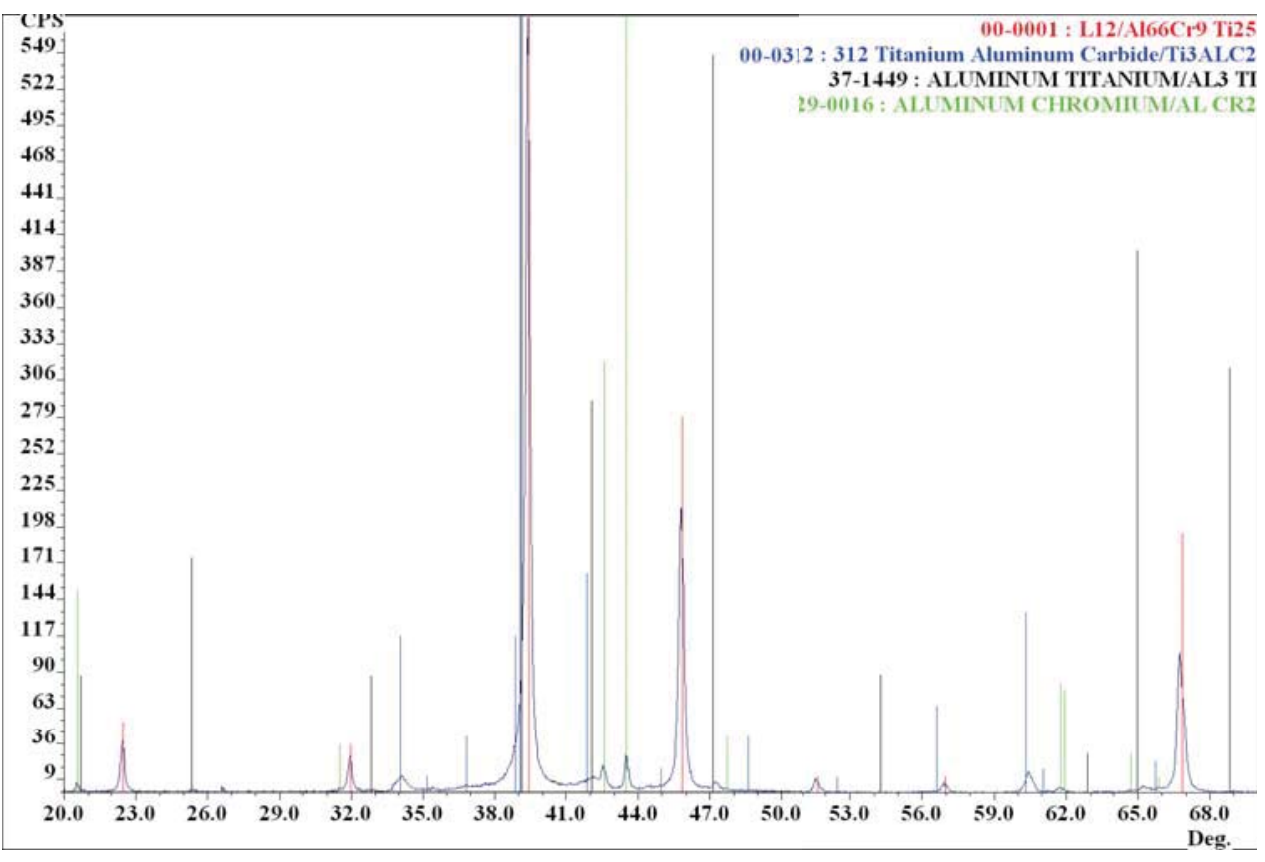

Figure III-36 Diffraction Pattern of RMA20h8.2TiAr Vacuum Annealed with Identified Phases

\section{2. $\quad$ Phase Volume Fractions}

Volume fraction estimates, determined for the as-milled powder by the direct comparison method using the Volfract program, are tabulated in Table III-6. The titanium (102) is an isolated peak and was the only titanium peak used. The $\{220\}$ peak for both the $\mathrm{TiC}$ and $\mathrm{TiH}_{1.92}$ phases were used and provided the most repeatable areas based on profile fitting although they do overlap with each other. Compared with the RMM20h9TiAr sample (similar milling time) there is a reduction in $\mathrm{TiC}$ and an increase in $\mathrm{TiH}_{1.92}$. The $\{200\}$ and $\{211\}$ peaks were used in the chromium volume fraction determination, and it can be noted that the volume fraction of 0.084 is larger than the 0.062 for the original mixture. An increase in the chromium is not physically reasonable, but as will be discussed this observation is an "apparent" increase due to the analysis and not a real increase. The standard file for the $\mathrm{L}_{2}$ intermetallic phase, utilized in the Volfract calculations, was based on a binary $\mathrm{L}_{2}$ cubic structure with a stoichiometric ratio of $\mathrm{Al}_{3} \mathrm{Ti}$. This was necessary since the main phase was cubic with peak positions shifted from those of the chromium-modified cubic trialuminide, superlattice peaks were 
present, and the fact that chromium did not appear to alloy with the $\mathrm{L} 1_{2}$ intermetallic. Diffraction peaks utilized from this phase in the volume fraction calculation were the $\{111\},\{311\}$, and the $\{222\}$.

\begin{tabular}{|c|cccc|}
\hline \multicolumn{4}{|c|}{ Table III-6 Phase Volume Fraction Estimates in RMA20h8.2TiAr As-Milled Powder } \\
\hline Al3Ti L12 & TiC & Cr & TiH1.92 & Ti \\
\hline $\mathbf{0 . 6 8 8}$ & $\mathbf{0 . 1 0 4}$ & $\mathbf{0 . 0 8 4}$ & $\mathbf{0 . 0 8 0}$ & $\mathbf{0 . 0 4 4}$ \\
\hline
\end{tabular}

Phase volume fractions for the annealed powder $\left(800^{\circ} \mathrm{C}\right.$ for $\left.1 \mathrm{~h}\right)$ were determined from the XRD powder pattern by the direct comparison method, again calculated using the Volfract program based on measured diffraction peak area. The calculation used three peaks each for the $\mathrm{L}_{2}$ intermetallic and carbide phases, while two peaks were used for the $\mathrm{AlCr}_{2}$ and $\mathrm{DO}_{22} \mathrm{Al}_{3} \mathrm{Ti}$ phase calculations. Values tabulated in Table III-7, show a volume fraction of 0.799 for the $\mathrm{L}_{2}$ intermetallic and 0.143 for the $\mathrm{Ti}_{3} \mathrm{AlC}_{2}$. Also, there was a volume fraction of 0.021 for the $\mathrm{AlCr}_{2}$ and 0.037 for the $\mathrm{Al}_{3} \mathrm{Ti}\left(\mathrm{DO}_{22}\right.$ structure), both of which are undesirable phases.

Table III-7 Phase Volume Fraction in RMA20h8.2TiAr Annealed Powder

\begin{tabular}{|c|c|c|c|}
\hline $\mathrm{Al}_{66} \mathrm{Cr} 9 \mathrm{Ti}_{25} \mathrm{L1}_{2}$ & $\mathrm{Ti}_{3} \mathrm{AlC}_{2}$ & $\mathrm{AlCr}_{2}$ & $\mathrm{Al}_{3} \mathrm{Ti}$ \\
\hline 0.799 & 0.143 & 0.021 & 0.037 \\
\hline
\end{tabular}

The amount of titanium required to form the determined volume fraction of the $\mathrm{Ti}_{3} \mathrm{AlC}_{2}$ phase, assuming stoichiometry, is $11.6 \mathrm{wt}$. pct. This value far exceeds the initial amount of 8.2 wt. pct. of excess elemental titanium. Therefore, the extra titanium needed to form the reported volume fraction of 0.143 of the carbide must come from the $\mathrm{L}_{2}$ intermetallic. This indicates that a portion of the $\mathrm{L} 1_{2}$ intermetallic alloy is forced out of the single-phase field (black area shown in Figure III-37) due to depletion of titanium and hence, accounts for the presence of $\mathrm{Al}_{3} \mathrm{Ti}$. The $\mathrm{Al}_{17} \mathrm{Cr}_{9}$ phase called for by the ternary phase diagram for a titanium-depleted $\mathrm{L}_{2}$ intermetallic is not observed suggesting the $\mathrm{AlCr}_{2}$ phase is the more stable form in this material. Additionally, the $\mathrm{Ti}_{3} \mathrm{AlC}_{2}$ phase also 
depletes the $\mathrm{L}_{2}$ intermetallic of aluminum which may favor aluminum chromium alloys with lower aluminum content.

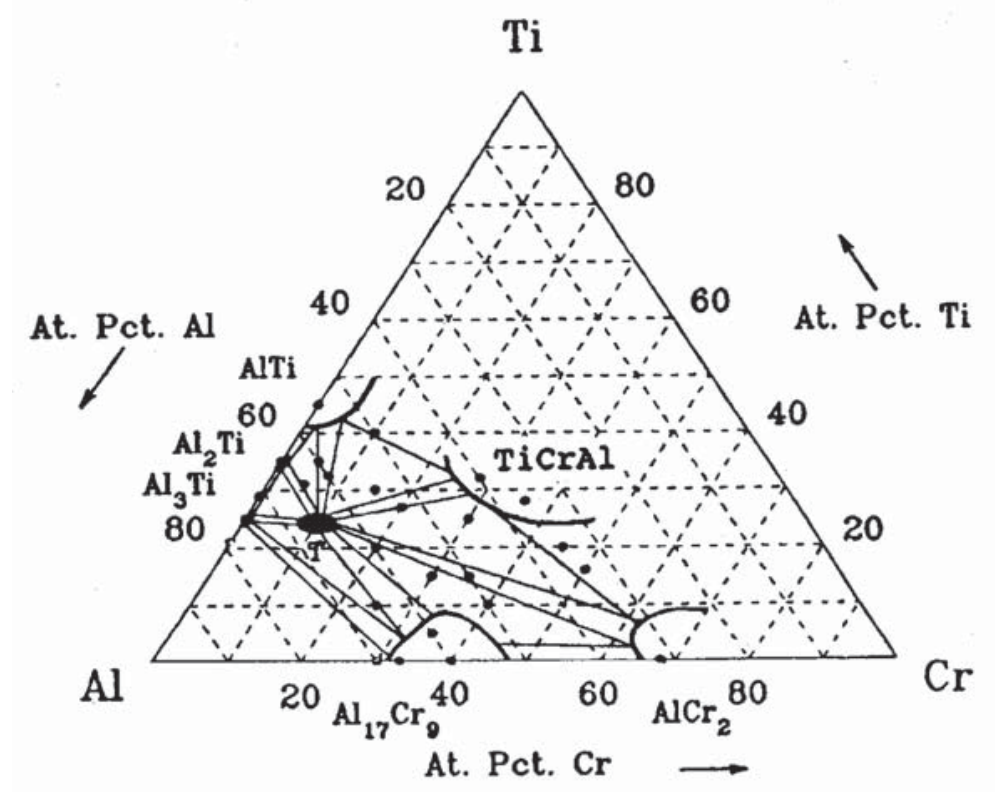

Figure III-37 Ternary Titanium Aluminum Chromium Phase Diagram Determined from HIPed Materials at $1200{ }^{\circ} \mathrm{C} 172 \mathrm{MPa} 2 \mathrm{~h}$ [16]

\section{Lattice Parameters}

Lattice parameters were determined for the $\mathrm{L}_{2}$ intermetallic phase in both the asmilled powder and the annealed powder. The diffraction peaks used for the as-milled powder lattice refinement process were the $\{100\},\{111\},\{200\},\{220\},\{311\}$, and $\{222\}$; whereas peaks used in the annealed powder lattice refinement process were the $\{100\},\{110\},\{111\},\{200\}$, and $\{220\}$.

The lattice parameter results are tabulated in Table III-8. Also shown for comparison are experimentally determined values for the $\mathrm{L1}_{2}$ instrumental broadening standard (calculated using the above refinement process and extrapolation method with a Nelson-Riley function with the same hkl diffraction peaks), and pure aluminum value published in the JCPDS-ICDD. There is a significant increase in the $\mathrm{L}_{2}$ intermetallic lattice parameter in the as-milled sample compared to the annealed sample. The as-milled $\mathrm{L}_{2}$ intermetallic lattice parameter lies between the value for pure aluminum and the $\mathrm{L}_{2}$ 
Standard. According to Nic [7] the lattice parameter of the $\mathrm{L} 1_{2}$ chromium-modified titanium trialuminide increases with decreasing chromium content. However, the largest value determined by Nic is still much less than the value found for the as-milled material. As noted, the diffraction pattern of the as-milled powder clearly shows the existence of a cubic intermetallic structure with superlattice peaks and no indication of an appreciable amount of alloying with chromium in the starting mixture, all of which support the existence of a binary $\mathrm{L}_{2}$ cubic titanium trialuminide. The observation of a binary cubic titanium trialuminide agrees with work by Srinivasan et al. [96], who reported a lattice parameter of $0.3967 \mathrm{~nm}$, much smaller than that for the as-milled powder and slightly larger than the value for the annealed powder.

Table III-8 Lattice Parameter Measurements RMA20h8.2TiAr Samples

\begin{tabular}{l|c}
\multicolumn{1}{c}{ Sample } & $\begin{array}{c}\text { Lattice Parameter } \\
\text { Refinement }(\mathbf{n m})\end{array}$ \\
\cline { 2 - 2 } RMA20h8.2TiAr As-Milled & 0.39973 \\
RMA20h8.2TiAr Annealed & 0.39618 \\
L1 $_{2}\left(\mathrm{Al}_{66} \mathrm{Cr}_{9} \mathrm{Ti}_{25}\right)$ Standard & 0.39565 \\
\cline { 2 - 2 } Aluminum JCPDS-ICDD* & $\mathbf{0 . 4 0 4 9 4}$ \\
*Published value &
\end{tabular}

The lattice parameter of the annealed sample is larger than the value determined for the $\mathrm{L}_{2}$ Standard. This suggests the composition of the $\mathrm{L} 1_{2}$ intermetallic changes from the target composition, which can be attributed to the loss of aluminum to the formation of the $\mathrm{Ti}_{3} \mathrm{AlC}_{2}$ carbide. This value for the annealed sample corresponds to the value given by Nic [116] for an $\mathrm{Al}_{63} \mathrm{Cr}_{10} \mathrm{Ti}_{27} \mathrm{~L}_{2}$ chromium-modified titanium trialuminide with a lattice parameter of $0.39603 \mathrm{~nm}$. It is likely that as aluminum decreases in the $\mathrm{L}_{2}$ intermetallic would effectively cause an increase in relative amounts of chromium and titanium. However, this simple analysis is complicated by the apparent loss of titanium in the $\mathrm{L}_{2}$ intermetallic since the total carbide formed exceeds the amount accounted for by the excess titanium originally added. The final analysis is that losses in titanium and aluminum in the alloy cause an increase in chromium content an effect similar to an 
$\mathrm{Al}_{62} \mathrm{Cr}_{13} \mathrm{Ti}_{25}$ alloy created by Nic [7] with a lattice parameter $0.3951 \mathrm{~nm}$. Some changes to the amount of each of the three elements in the $\mathrm{L}_{2}$ intermetallic is concluded.

\section{Peak Broadening Analysis of Profile Fit X-ray Diffraction Peaks by the Warren-Averbach Fourier Method}

Warren-Averbach Fourier analysis was applied to the as-milled RMA powder using synthesized diffraction peaks based on intelligent profile fitting as discussed in Chapter II.A.5.a)(7)(a). The analysis was performed in the <111> crystallographic direction with results tabulated in Table III-9, determined in a similar manner to that in Chapter III.A.1.e). The crystallite size (CDDS) in the as-milled powder is $4 \mathrm{~nm}$ with a large microstrain of $0.55 \%$. For comparison, the as-milled RMM20h9TiAr sample (starting with pre-alloyed $\mathrm{L}_{2}$ intermetallic powder) had a crystallite size of $2.4 \mathrm{~nm}$ and a microstrain of $1.05 \%$ by the same analysis technique.

Table III-9 Warren-Averbach Fourier Analysis RMA20h8.2TiAr As-Milled Powder

\begin{tabular}{|c|c|c|}
$\begin{array}{c}\text { Crystallographic } \\
\text { Direction }\end{array}$ & $\begin{array}{c}\text { CDDS }(\mathrm{nm}) \\
\text { Profile Fit }\end{array}$ & $\begin{array}{c}\left\langle\varepsilon^{2} 5 \mathrm{~nm}\right\rangle^{1 / 2}(\%) \\
\text { Profile Fit }\end{array}$ \\
\hline$<111>$ & $\mathbf{4 . 0}$ & 0.55 \\
\hline
\end{tabular}

The larger crystallite size and smaller microstrain value suggest that a significant amount of energy input during the milling process in the RMA20h8.2TiAr sample is consumed by the alloying process between aluminum and titanium, as opposed to deformation processes. All processing conditions were the same, except the media to charge ratio was 6 to 1 for the RMM20h9TiAr sample and 10 to 1 for the RMA20h8.2TiAr sample, thus slightly more energy was input into the latter. While the change in energy imparted has not been quantified, comparison of diffraction patterns from pre-alloyed powders with the two charge ratios in question show very similar features, supporting the hypothesis that the differences observed are due to the extra energy consumed by the alloying process in the RMA20h8.2TiAr sample. 


\section{Results from Reactive Mechanical Milling (RMM) of Pre-Alloyed Powders with Systematic Increases in Excess Titanium in an Argon Atmosphere}

The goal here was to examine whether the amount of carbides can be continually increased if titanium continues to be available. While exhaustive analysis of the as-milled powders was not accomplished in this experimental series, as these powders produced were intended for consolidation, XRD patterns are provided in Figure III-38 for all samples milled. Recall that for each $20 \mathrm{~h}$ milling time a multiple of 8.2 or 9 wt. pct. excess titanium is added, i.e. $60 \mathrm{~h}$ RMM has $27 \mathrm{wt}$. pct. excess titanium. Of importance as both the time and excess amount of titanium are increased is the large growth in the intensity of the $\mathrm{TiC}$ phase diffraction peaks while maintaining a similar shape and hence expected similar crystallite size for all RMM times. The XRD pattern is dominated by the TiC phase for the longest RMM time of $120 \mathrm{~h}$, for which 54 wt. pct. excess titanium was provided. Clearly, the results show that as long as titanium is available the reaction will continue until all titanium is exhausted.

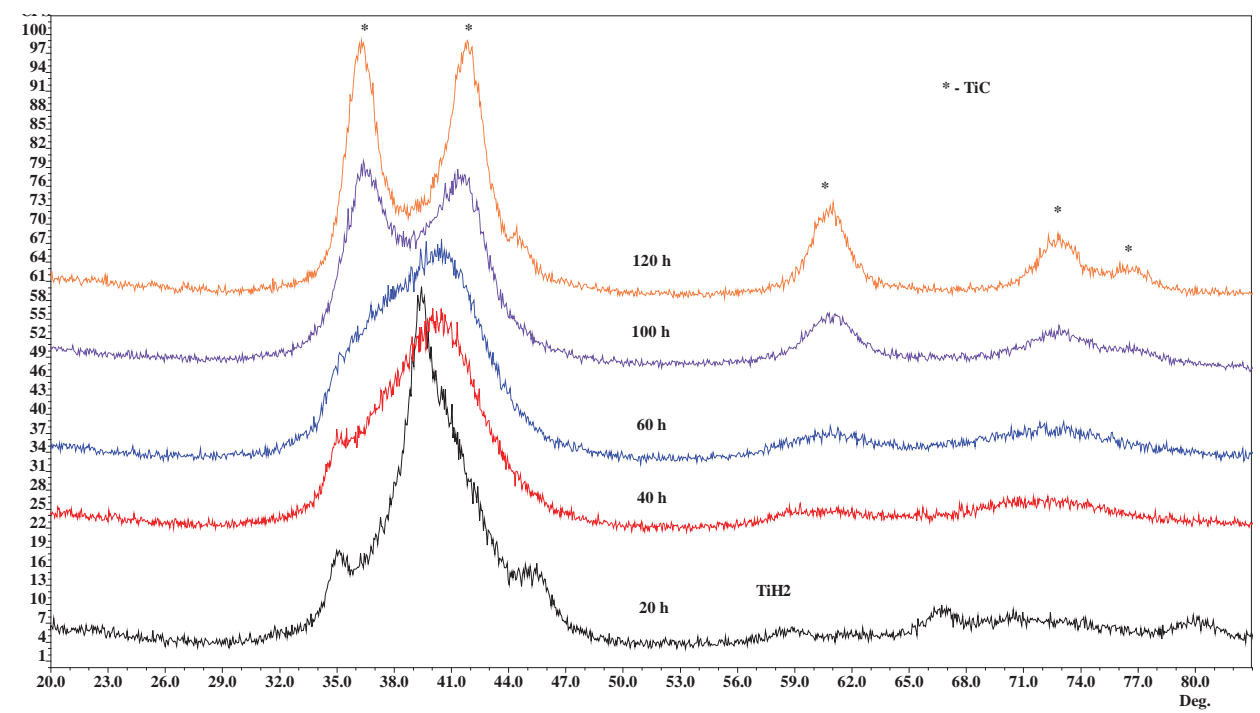

Figure III-38 As-Milled Systematic Increase in Excess Titanium and RMM Time 


\section{Results from Consolidated Materials}

The main objective of this part of the study has been to develop an understanding of the relationship between the microstructure, particularly the grain structure and carbide morphology, and the unique properties of these materials. The nanometer features in these materials require a combination of XRD, TEM, and FESEM observations to develop a useful understanding of the microstructures. The important result from the consolidated composite materials produced by this unique processing method are the very high hardness values with compressive strength values $\sim 10$ times the as-cast values for the single-phase $\mathrm{L}_{2}$ intermetallic alloy. Additionally, as will be shown, the ability to vary the carbide content permits control of engineering properties. Details of the processing are provided in Table II- 8 with the sample codes used to identify samples by the RMM time/HIP temperature/type of starting alloy (MA - Master Alloy and RA Reading Alloy).

\section{Characterization}

\section{a) Chemical Analysis}

Initial interest with this experiment was to establish and understand the uptake of the carbon to produce the carbide. The results of LECO chemical analysis for carbon, oxygen, and nitrogen in various materials are presented in Table III-10. Most values represent the mean of four analyses, although a few were derived from three, or two, analyses. As noted earlier, the 20/800/MA-A sample was RMM in an air environment, while all other samples were RMM in an argon environment. The RMM time is the first numerical value in the sample name code for the appropriate samples. Carbon contents were determined from vacuum annealed as-milled powders for samples 60/1000/RA, 100/1000/RA, and 120/1000/RA, while all other samples were analyzed using consolidated samples. The carbon content reported for the 20/1000/MA sample is an average of 4 analyses with 3 analyses from samples using a sample from a different HIP condition. Samples 20/1000/RA, 40/1000/RA, 60/1000/RA, 100/1000/RA, and 120/1000/RA all refer to the Reading Alloy starting powder, while all other samples were 
from the Master Alloy starting powder. It should be noted that the Reading Alloy powder originally contained approximately 0.25 wt. pct. carbon. As expected, the carbon content for the 20/1000/RA sample is larger than that for 20/1000/MA sample by about that amount.

Table III-10 Carbon, Oxygen, and Nitrogen Content

\begin{tabular}{|cccc} 
Sample & $\begin{array}{c}\text { Carbon } \\
\text { (wt. pct.) }\end{array}$ & $\begin{array}{c}\text { Oxygen } \\
\text { (wt. pct.) }\end{array}$ & $\begin{array}{c}\text { Nitrogen } \\
\text { (wt. pct.) }\end{array}$ \\
\hline 20/800/MA-A & 1.93 & 0.731 & 1.058 \\
\cline { 2 - 4 } 20/1000/MA & $1.59 *$ & 0.670 & 0.047 \\
\hline 20/1000/RA & 1.88 & - & - \\
40/1000/RA & 3.13 & 1.020 & 0.031 \\
60/1000/RA & 3.76 & - & - \\
\hline 100/1000/RA & 6.23 & - & - \\
\hline 120/1000/RA & $\mathbf{8 . 0 4}$ & - & - \\
\hline Average of several similar samples RMM under the same conditions
\end{tabular}

A significant result of these RMM experiments is that the amount of carbon increases regularly with the RMM time, with the carbon supplied by the PCA (hexanes), provided titanium is available. Figure III-39 shows the results of carbon content analysis of the samples that utilized the Reading Alloy starting powders and added for comparison is the result for the 20/1000/MA sample based on the Master Alloy starting powder (shown in red). Each data point also shows $95 \%$ confidence error bars based on multiple carbon analyses. The carbon content has a nearly linear behavior as demonstrated by the linear regression applied to the Reading Alloy data. The intercept of the linear regression (for these Reading Alloy starting powder samples) is 0.56 wt. pct., which is close to the initial carbon content in the Reading Alloy powder. Of primary interest to this study is that the carbon content scales closely with the RMM time, with an approximate doubling of the carbon content with a doubling of the RMM time. 


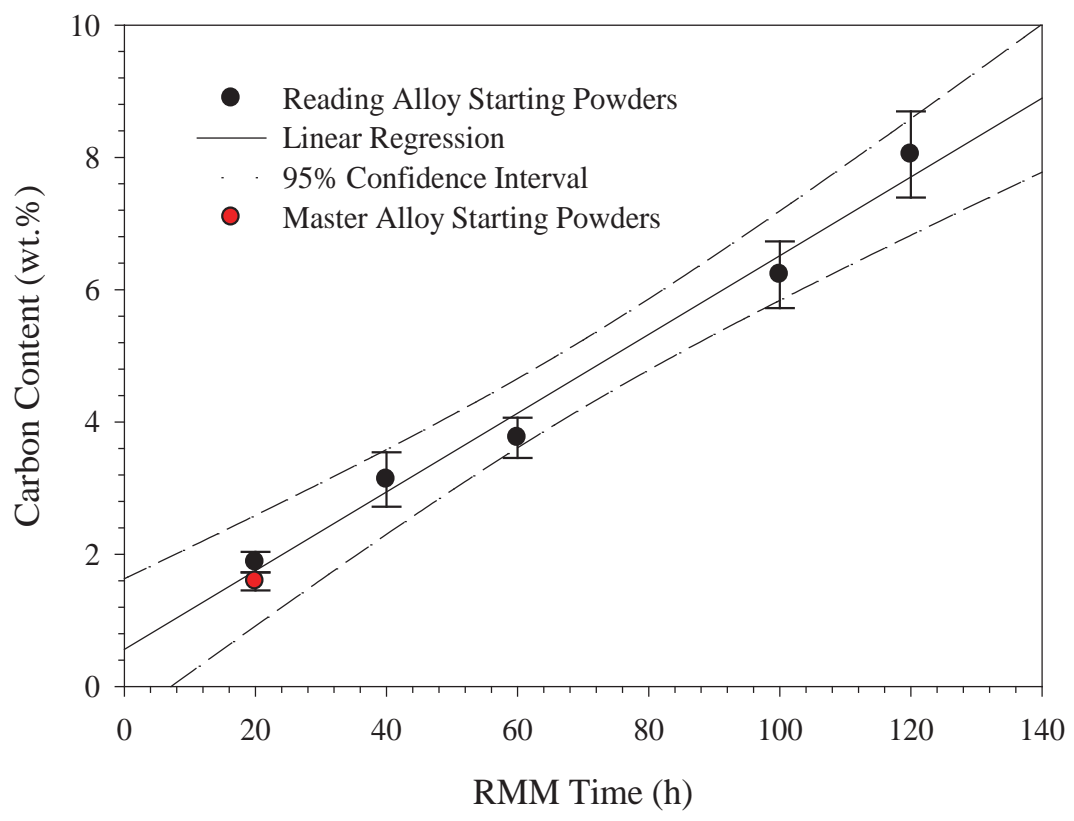

Figure III-39 Carbon Content Dependence on RMM Time

Sample 20/800/MA-A was milled in an air environment, which resulted in a nitrogen content that is approximately 10 times greater than that for the other samples. Of importance with that sample is the significant uptake of nitrogen from the air environment within the vial into phases present in the consolidated samples. The samples milled in an argon environment all show small but varying amounts of nitrogen, which is speculated to have been introduced during the mill vial sealing operation done in an argon atmosphere in a glove box. The glove box does remove oxygen by a sieve method, but nitrogen is not specifically removed, therefore varying amounts of nitrogen could be present dependent on the purge cycle of new argon introduced into the glove box.

The initial Reading Alloy, used in sample 40/1000/RA, has an oxygen content of approximately 0.29 wt. pct. while LECO analysis of large pieces of the Master Alloy gave an oxygen content of 0.074 wt. pct. Clearly, both starting materials had oxygen contents much less than reported for the milled alloys. The Master Alloy samples HIPed at two different temperatures, but processed the same, except the 20/800/MA-A was milled in air, had similar oxygen contents. Additionally, as the RMM time increases beyond $20 \mathrm{~h}$ the oxygen content increases to a value of $1.02 \mathrm{wt}$. pct. with the Reading 
Alloy; however, subtracting the preexisting oxygen content results in 0.73 wt. pct. oxygen uptake a similar value to that in the $20 \mathrm{~h}$ RMM time samples despite a doubling of the milling time. Oxygen contamination is suspected to be introduced during the various processing steps and most likely not related to the milling time.

Bulk chemical analysis by XRF using UniQuant ${ }^{\circledR}$ standardless analysis was accomplished for other elements on selected samples. Materials based on Reading Alloy starting powders all showed less than $\sim 0.09$ wt. pct. vanadium, and $\sim 0.02$ wt. pct. manganese. Sample 20/800/MA (sample not used in this study), the only one analyzed that used the Master Alloy starting powder, did not show any of these elements.

Of particular importance in this analysis is the iron content that is likely introduced by the wear of the vial and media used in the milling. Iron analyses are given in Table III-11, for various RMM times. As would be expected the iron content increases with RMM time. However, the levels are very low as demonstrated by the 20/800/MA sample with only 0.09 wt. pct. iron. For the same RMM time it can be seen that the 20/1000/RA sample is somewhat higher (0.17 wt. pct.) than the 20/800/MA sample RMM for the same milling time. The difference is attributed to that fact that the 20/1000/RA sample was based on Reading Alloy powders as starting materials and recall that this material had approximately $0.14 \mathrm{wt}$. pct. iron. This agrees quite well with the values presented here.

Table III-11 Iron Content in Consolidated Samples

\begin{tabular}{l|c}
\multirow{2}{*}{ Sample } & \multicolumn{1}{c}{$\begin{array}{c}\text { Iron } \\
\text { (wt. pct.) }\end{array}$} \\
\cline { 2 - 2 } 20/800/MA & 0.09 \\
20/1000/RA & 0.17 \\
60/1000/RA & 0.34 \\
\hline 100/1000/RA & 1.40 \\
\hline $\mathbf{1 2 0 / 1 0 0 0 / R A}$ & $\mathbf{6 . 3 0}$ \\
\hline
\end{tabular}


Figure III-40, shows the exponential increase in the iron content with RMM time for the samples (multiples of 9 wt. pct. Ti) that used the Reading Alloy as a starting powder. Despite the apparent dramatic increase at the RMM time of $120 \mathrm{~h}$ shown in Figure III-40, this level of iron is almost an order of magnitude lower than that for the original experiment at an RMA of $90 \mathrm{~h}$ in a material which had the hard ceramic $\mathrm{TiB}_{2}$ admixed. Importantly, at the shorter RMM times, iron contamination is very low and is easily solutionized by the $\mathrm{L}_{2}$ intermetallic material. Other aspects of the iron-based phases will be discussed later.

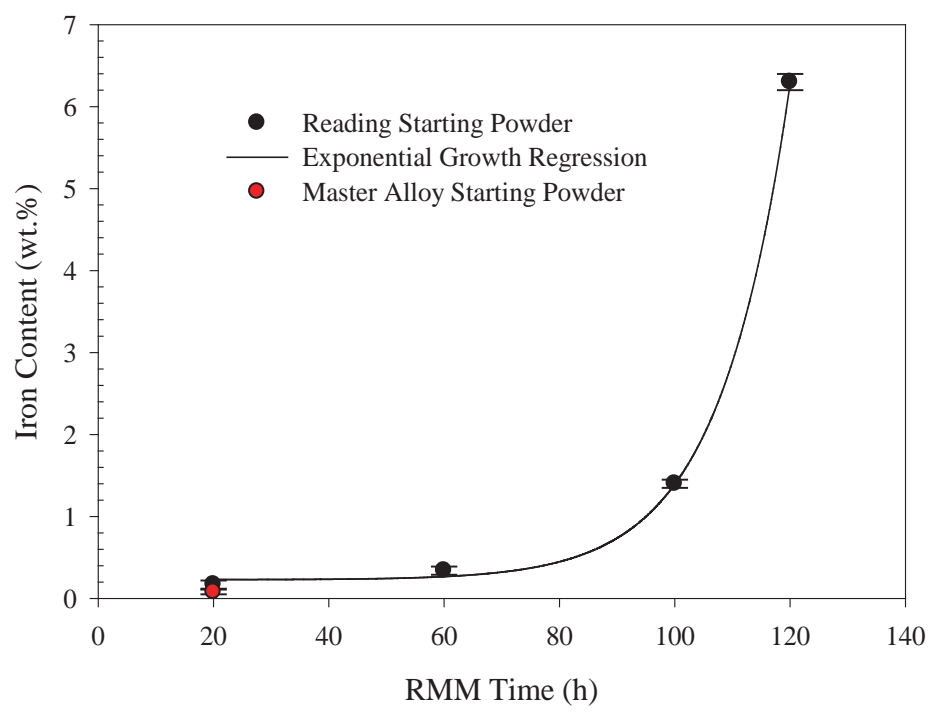

Figure III-40 Iron Content Dependence on RMM Time

\section{b) X-ray Powder Diffraction (XRD)}

X-ray powder diffraction was used to characterize the phase structure, phase lattice parameters, phase volume fractions, and crystallite sizes of both the $\mathrm{L}_{2}$ intermetallic (including presence of microstrains) and the carbide phase present in consolidated RMM materials.

\section{(1) Phase Analysis}

A detailed phase analysis of all consolidated samples is given in Appendix VIII.D. Important to note is that some of the identified phases were solid solutions and/or 
had stoichiometry effects that resulted in a systematic shift in the diffraction peak positions. The $\mathrm{Ti}_{3} \mathrm{AlC}_{2}$ (designated as 312) or n-phase and $\mathrm{Ti}_{2} \mathrm{AlC}$ (referred to as 211) or h-phase, are referred to collectively as "complex carbides", all had shifts in the diffraction peak positions compared to known values. In addition, when the $\mathrm{DO}_{22} \mathrm{Al}_{3} \mathrm{Ti}$ phase was observed it also had significant shifts in the diffraction peaks compared to known values. The 120/1000/RA sample had shifts in the diffraction peaks for the $\mathrm{L}_{2}$ chromium-modified trialuminide, and peak shifts were also observed for the TiC phase and the complex carbides. The 20/800/MA-A sample milled in an air environment was predominantly $\mathrm{L}_{2}$ chromium-modified trialuminide with a solid solution of $\mathrm{DO}_{22} \mathrm{Al}_{3} \mathrm{Ti}$ also present. In addition, the air processing produced $\mathrm{Ti}(\mathrm{C}, \mathrm{N})$ and $\mathrm{Ti}_{2} \mathrm{Al}(\mathrm{C}, \mathrm{N})$ carbonitride phases.

A summary of phases present for all consolidated samples (HIP $1000{ }^{\circ} \mathrm{C}$ and processed in argon) is given in Table III-12, with the $\mathrm{X}$ designating a positive identification of that phase. The 20/800/MA-A and MM3h are not included. It can be noted that the one carbide phase that is present in all samples listed is the $\mathrm{Ti}_{3} \mathrm{AlC}_{2}$ phase (heading 312). In the case of the 20/800/MA-A carbonitrides were formed in all cases as all carbide and nitride phases in this system were isomorphous. For example the TiC phase was present in 20/800/MA-A sample milled in air, but as a solid solution of $\mathrm{Ti}(\mathrm{C}, \mathrm{N})$. TiC does not appear in samples HIPed at $1000^{\circ} \mathrm{C}$ until an RMM time of $100 \mathrm{~h}$ where it appears in a very small amounts. TiC dominates the 120/1000/RA sample which also has a significant amount of an iron-bearing AlFe phase with the iron suspected to occur in significant amounts from the wear process on the milling chamber and media for long RMM process times (see Figure III-40). AlFe appeared in very small amounts in the samples corresponding to RMM times of 20 through $100 \mathrm{~h}$ that used the Reading Alloy powders attributed in that case to the initial iron in the Reading Alloy powder. The $\mathrm{Al}_{8} \mathrm{Cr}_{5}$ phase appeared only in the 120/1000/RA sample. 
Table III-12 Phase Analysis of Consolidated Samples RMM in Argon

\begin{tabular}{|c|c|c|c|c|c|c|c|c|c|}
\hline RMM/HIP/Alloy & $\mathbf{L 1 2}$ & 312 & 211 & $\mathrm{TiC}$ & Als Ti & AlCr2 & $\mathrm{Al}_{2} \mathrm{O}_{3}$ & AlFe & $\mathrm{Al}_{8} \mathrm{Cr} 5$ \\
\hline 20/1000/MA & $\mathrm{X}$ & $\mathrm{X}$ & $X$ & & & $\mathrm{X}$ & $\mathrm{X}$ & & \\
\hline $20 / 1000 / \mathrm{RA}^{1}$ & $\mathrm{X}$ & $\mathrm{X}$ & & & $\mathrm{X}$ & & $\mathrm{X}$ & $\mathrm{X}$ & \\
\hline $40 / 1000 / \mathrm{RA}^{1}$ & $\mathrm{X}$ & $\mathrm{X}$ & & & & $\mathrm{X}$ & $\mathrm{X}$ & $X$ & \\
\hline 60/1000/RA & $\mathrm{X}$ & $\mathrm{X}$ & $X$ & & $\mathrm{X}$ & $\mathrm{X}$ & $\mathrm{X}$ & $\mathrm{X}$ & \\
\hline 100/1000/RA & $\mathrm{X}$ & $\mathrm{X}$ & $\mathrm{X}$ & $\mathrm{X}$ & & $\mathrm{X}$ & $\mathrm{X}$ & $\mathrm{X}$ & \\
\hline $120 / 1000 / R^{2}$ & $\mathbf{X}$ & $\mathbf{X}$ & & $\mathbf{X}$ & & & & $\mathbf{X}$ & $\mathbf{X}$ \\
\hline
\end{tabular}

${ }^{1}$ Also contained indications of $\mathrm{Ti}_{3} \mathrm{Al}(\mathrm{C}, \mathrm{N})$

${ }^{2}$ Also contained $\mathrm{Ti}(\mathrm{Fe}, \mathrm{Cr})$

\section{(2) $\quad \mathbf{L 1}_{2}$ Intermetallic Lattice Parameters}

The lattice parameters for the $\mathrm{L}_{2}{ }_{2}$ intermetallic phase were determined for all samples consolidated at $1000^{\circ} \mathrm{C}$ by means of the lattice constant refinement process, discussed in Chapter II.A.5.a)(5), using the $\{100\},\{110\},\{111\},\{200\},\{220\}$, and \{311\} diffraction peaks, based on the limited scan range used for the 120/1000/RA sample, and the preference that the same diffraction peaks be used for all consolidated samples.

The results for the samples consolidated at $1000^{\circ} \mathrm{C}$ are presented in Table III- 13 . Initially, the lattice parameter remains quite constant, followed by a decrease at a RMM time of $100 \mathrm{~h}$ to a value comparable with that for an $\mathrm{Al}_{62} \mathrm{Cr}_{13} \mathrm{Ti}_{25}$ alloy created by Nic [7] (lattice parameter $0.3951 \mathrm{~nm}$ ) which lies at the single-phase solubility limit for chromium. An apparent increase in chromium is plausible since the formation of the complex carbides requires aluminum with the only source being the $\mathrm{L}_{2}$ intermetallic. This depletion of aluminum in the $\mathrm{L}_{2}$ intermetallic would effectively cause an increase in chromium content provided other chromium-based phases remain constant.

There is a further significant decrease in the lattice parameter for the RMM time of $120 \mathrm{~h}$. This value is much smaller than any lattice parameter observed by Nic [7, 116] in any $\mathrm{L}_{2}$ alloy synthesized; even alloys explored using elements other than chromium 
(e.g. iron). The dramatic change in the lattice parameter coincides with a significant increase in the carbide content. Therefore, the change appears to be associated with changes in the stoichiometry of the $\mathrm{L}_{2}$ intermetallic phase since the only other element entering the system is iron and this typically only has a small affect on the lattice parameter (e.g. Al-24.5Ti-9Fe lattice parameter is $0.3942 \mathrm{~nm}$ ). Most of the iron is tied up with the identified iron-bearing phase; however, the $120 \mathrm{~h}$ sample does have the largest amount.

\begin{tabular}{|c|c|}
\hline Table III-13 LI $_{2}$ Intermetallic Lattice Parameter for Consolidated Materials \\
\hline HIP Process & $\begin{array}{c}\text { Lattice Parameter } \\
(\mathbf{n m})\end{array}$ \\
\hline 20/1000/MA & 0.39576 \\
\hline 20/1000/RA & 0.39561 \\
\hline 40/1000/RA & 0.39577 \\
60/1000/RA & 0.39575 \\
\hline 100/1000/RA & 0.39538 \\
\hline $\mathbf{1 2 0 / 1 0 0 0 / R A}$ & $\mathbf{0 . 3 9 0 0 8}$ \\
\hline
\end{tabular}

As mentioned, the $\mathrm{L} 1_{2}$ intermetallic is expected to be aluminum deficient as a result of the complex carbide formation. As will be shown in phase volume fraction results, the amount of complex carbides comprises over one-half of the 100 RMM sample while the $\mathrm{L} 1_{2}$ intermetallic reduces to about one-third. The only source for aluminum is from the $\mathrm{L} 1_{2}$ intermetallic, and as expected this depletion of aluminum would increase with RMM time along with depletion of titanium since the complex carbides can require up to twice as much titanium as was experimentally accounted for based on the creation of TiC. Although volume fractions of the phases for the $120 \mathrm{~h}$ RMM sample were not determined because of the large number of phases present and the fundamental breakdown of some of the expected phases, it appears from the pattern that the amount of complex carbides is much less than present in the $100 \mathrm{~h} \mathrm{RMM} \mathrm{sample,} \mathrm{but} \mathrm{there} \mathrm{is} \mathrm{a} \mathrm{large}$ increase in the amount of TiC. Regarding the large decrease in the lattice parameter it might be speculated that the change from complex carbides to $\mathrm{TiC}$ indicates severe 
depletion of aluminum, causing a titanium substitution on aluminum sites. The major change in the $\mathrm{L}_{2}$ intermetallic lattice parameter combined with the change from a majority of complex carbides to a majority of $\mathrm{TiC}$, indicates a fundamental departure in the phase structure from the typical structure of $\mathrm{L}_{2}$ intermetallic and complex carbides observed for the alloys and therefore the $120 \mathrm{~h}$ RMM sample was not included in property studies.

\section{(3) Phase Volume Fractions}

Volume fractions of phases making up the consolidated samples were determined by the direct comparison XRD method for all samples except 20/800/MA-A and 120/1000/RA. Typically, three to five of the lowest angle diffraction peaks are utilized in the calculations for each phase.

Volume fraction results for all identified phases are summarized in Table III-14. As noted, because of the large number of phases encountered in the analysis, several abbreviations have been used in the Table headings. L1 2 denotes the chromium-modified titanium trialuminide intermetallic, 312 corresponds to the $\mathrm{Ti}_{3} \mathrm{AlC}_{2}$ carbide, 211 the $\mathrm{Ti}_{2} \mathrm{AlC}$ carbide, 311 the $\mathrm{Ti}_{3} \mathrm{Al}(\mathrm{C}, \mathrm{N})$ with the others being self-explanatory. As expected, for all samples shown, the primary microconstituents consist of the $\mathrm{L}_{2}$ intermetallic and the titanium-aluminum based complex carbides. More importantly, the total carbide content clearly increased with RMM time. The $\mathrm{L1}_{2}$ intermetallic is the major phase prior to the RMM time of $100 \mathrm{~h}$, at which the complex carbide becomes the majority phase, on a volume fraction basis this indicates a potential change from a intermetallic matrix composite (IMC) to a ceramic matrix composite (CMC); however, this is more clearly defined by the microstructure.

Since several carbide phases can be present in a given sample, it appeared useful to plot the volume fraction of the $\mathrm{L}_{2}$ intermetallic phase as a function of RMM time to display not only the reduction in volume fraction of this phase, but to show the increase in amount of carbide content. The result is shown in Figure III-41 along with a linear regression determined from all points except the 20/1000/RA sample shown in red on the graph. This point was omitted due to the appreciable amount of $\mathrm{Al}_{3} \mathrm{Ti}$ in that material. As 
can be seen the zero time intercept of the regression is unity as expected. The importance of this near-linear reduction in the $\mathrm{L}_{2}$ intermetallic phase with RMM time demonstrates that there is still further potential for increasing the amount of carbide. Of course, this assumes that other phases will not be formed in place of the carbides with further milling. Also, such behavior may require extra aluminum to be added for the complex carbide formation. It might be considered whether $100 \%$ carbide could be created provided the correct initial chemistry is established.

\begin{tabular}{|c|c|c|c|c|c|c|c|c|c|}
\hline \multicolumn{10}{|c|}{ Table III-14 Volume Fractions of Consolidated Samples } \\
\hline Sample & $\mathbf{L 1 2}$ & 312 & 211 & $\mathrm{TiC}$ & $\mathrm{Al}_{3} \mathbf{T i}$ & $\mathrm{AlCr}_{2}$ & $\mathrm{Al}_{2} \mathrm{O}_{3}$ & AlFe & 311 \\
\hline 20/1000/MA & 0.852 & 0.086 & 0.050 & & & 0.004 & 0.008 & & \\
\hline 20/1000/RA & 0.807 & 0.137 & & & 0.042 & & 0.006 & 0.002 & 0.006 \\
\hline 40/1000/RA & 0.733 & 0.243 & & & & 0.001 & 0.018 & 0.002 & 0.004 \\
\hline $60 / 1000 / \mathrm{RA}$ & 0.584 & 0.043 & 0.335 & & 0.014 & 0.006 & 0.018 & $<0.001$ & \\
\hline 100/1000/RA & 0.294 & 0.130 & 0.512 & 0.013 & & 0.025 & 0.023 & 0.002 & \\
\hline
\end{tabular}

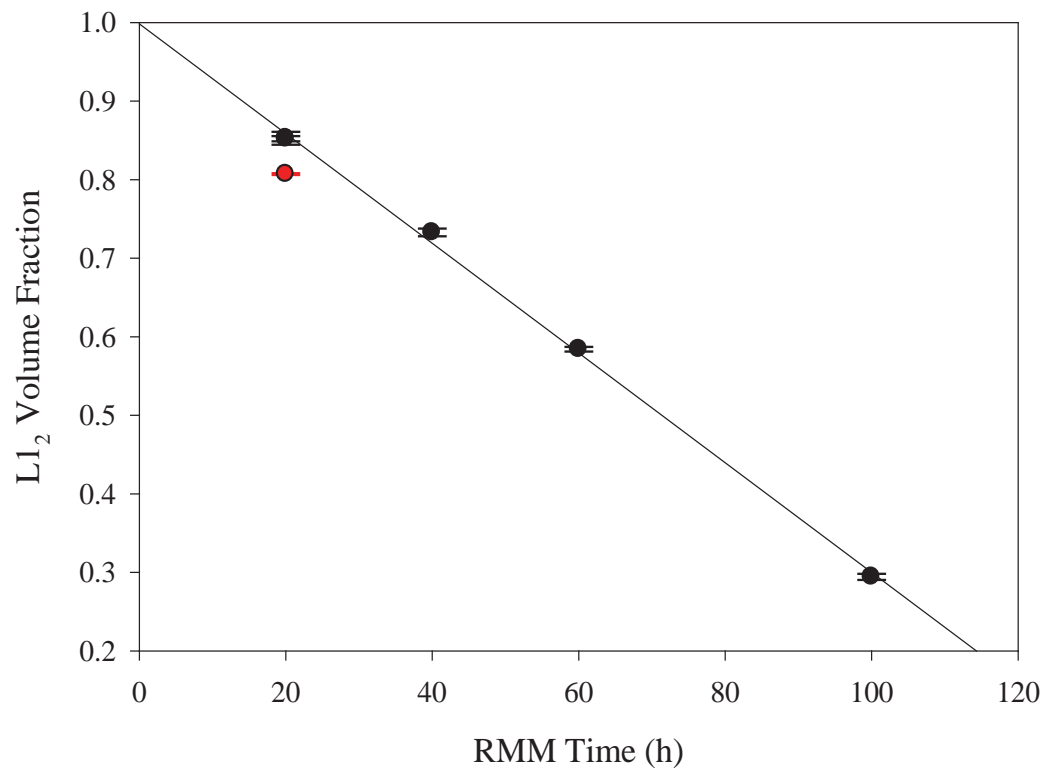

Figure III-41 Volume Fraction Dependence of L1 $1_{2}$ Intermetallic with RMM Time

The $\mathrm{Ti}_{3} \mathrm{AlC}_{2}$ phase occurs in all the samples, with nearly equal amounts of $\mathrm{Ti}_{3} \mathrm{AlC}_{2}$ and $\mathrm{Ti}_{2} \mathrm{AlC}$ in the 20/1000/MA sample. Samples 20/1000/RA and 40/1000/RA 
both contain only the $\mathrm{Ti}_{3} \mathrm{AlC}_{2}$ phase with the amount increasing with $\mathrm{RMM}$ time as expected (0.137 and 0.243, respectively). On the other hand, samples 60/1000/RA and $100 / 1000 / \mathrm{RA}$ both contain a majority of the $\mathrm{Ti}_{2} \mathrm{AlC}$ phase with smaller amounts of the $\mathrm{Ti}_{3} \mathrm{AlC}_{2}$ phase. Of interest is that the $\mathrm{Ti}_{3} \mathrm{AlC}_{2}$ phase forms at $1300{ }^{\circ} \mathrm{C}$ and is not present in the isothermal section at $1250{ }^{\circ} \mathrm{C}$ indicating stability only in a narrow temperature range [139]. Also, the 100/1000/RA sample contains 0.013 volume fraction of TiC, which as noted is at the point where the microstructure changes dramatically. If the depletion of aluminum in the $\mathrm{L}_{2}$ intermetallic is greater than the need for aluminum in the complex carbide a phase breakdown would be expected. It appears the dominant carbide becomes $\mathrm{TiC}$ and the 100/1000/RA sample is beginning to show effects of this aluminum depletion with the small amount of $\mathrm{TiC}$ that has formed is an indicator of this behavior.

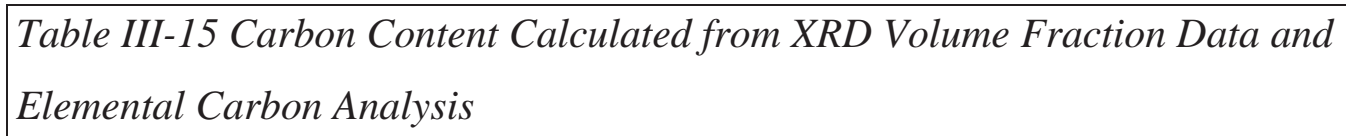

\begin{tabular}{|c|c|c|}
\hline RMM/HIP/Alloy & $\begin{array}{c}\text { Calculated Carbon } \\
\text { (wt. pct.) }\end{array}$ & $\begin{array}{l}\text { LECO Carbon } \\
\text { (wt. pet.) }\end{array}$ \\
\hline 20/800/MA & 1.44 & $1.59^{*}$ \\
\hline 20/1000/MA & 1.64 & $1.59^{*}$ \\
\hline 20/1000/RA & 1.85 & 1.88 \\
\hline 40/1000/RA & 3.29 & 3.13 \\
\hline 60/1000/RA & 3.68 & 3.76 \\
\hline 100/1000/RA & 6.73 & 6.23 \\
\hline
\end{tabular}

* Average of samples processed and consolidated in a similar manner.

In most cases, there is more than one carbide phase present in each sample. This makes it difficult to define the process of the carbide formation. One approach to track carbon content with RMM time is to calculate the total carbon content determined from the XRD volume fraction data assuming all carbon phases are stoichiometric. Results of this analysis are given in Table III-15, which compares the carbon content calculated 
from XRD volume fraction measurements with the results of the LECO carbon analysis. The calculated values deviated slightly from the values determined by LECO carbon analysis; however, Figure III-42 shows that the calculated data in most cases fell within the error limits of the LECO data. One means for accounting for the small difference between calculated values and those from the LECO determination could be the assumption that the carbide phases are stoichiometric. Certainly the carbide phases may not be stoichiometric, which would affect the calculated carbon contents. In fact, slight shifts in the diffraction peak positions from values expected suggest stoichiometric changes [138]. The linear behavior Figure III-42 provides agreement between the chemical analysis and volume fraction analysis reinforcing the simple linear uptake behavior of carbon with RMM time.

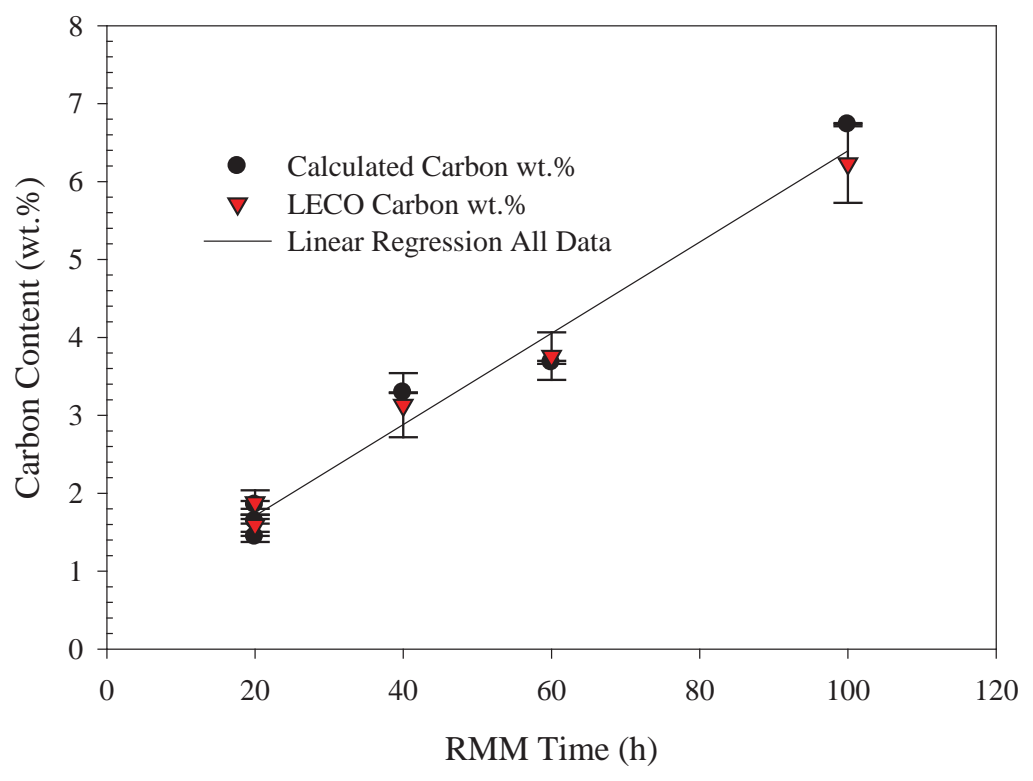

Figure III-42 Carbon Content Calculated from XRD Volume Fraction Analysis and LECO Elemental Analysis

The appearance of $\mathrm{Al}_{3} \mathrm{Ti}\left(\mathrm{DO}_{22}\right)$ can be used as an indicator of when the ideal milling time is exceeded significantly. Nic [116] has shown that a small decrease of 2 at. pct. titanium in the $\mathrm{L} 1_{2}$ chromium-modified titanium trialuminide phase, see Figure III-37, can lead to formation of $\mathrm{Al}_{3} \mathrm{Ti}$. It stands to reason that when a sample is over milled (recall that carbon uptake increases with RMM time) for a given amount of excess 
titanium this causes a reduction of titanium in the $\mathrm{L} 1_{2}$ intermetallic phase and hence formation of $\mathrm{Al}_{3} \mathrm{Ti}$. Both the 20/1000/RA and 60/1000/RA samples contain a small amount of $\mathrm{Al}_{3} \mathrm{Ti}$ indicating an RMM time too long for that composition.

It was found in the RMMXh9TiAr Series annealed powders, Appendix VIII.C, (which were created after the consolidated samples based on an RMM time of $20 \mathrm{~h}$ and 9 wt. pct. titanium), that an RMM time of $10 \mathrm{~h}$ contained only the $\mathrm{L}_{2}$ intermetallic and $\mathrm{Ti}_{2} \mathrm{AlC}$ with a very small amount of $\mathrm{Ti}_{3} \mathrm{AlC}_{2}$, while a small amount of $\mathrm{Al}_{2} \mathrm{O}_{3}$ (undesirable but a result of oxygen entering the system as discussed) was always present. While the desired microstructure is a two phase mixture consisting of $\mathrm{L}_{2}$ intermetallic and a complex carbide, the carbide can exists in two forms. In this series, the $10 \mathrm{~h}$ sample provided the closest to the target microstructure indicating that future experimentation of consolidated materials should be based around a $10 \mathrm{~h}$ RMM time with the stated excess amount of titanium with multiples thereafter. Another indication of exceeding the ideal milling time, for a given added titanium content, may be the presence of any aluminumchromium phases. In a study that was an extension of this work, Le Claire [140] also observed the presence of $\mathrm{AlCr}_{2}$ and associated this with a $\mathrm{RMM}$ time that was too long. All samples shown in Table III-14 have one or more of these indicator phases present, which suggests that for each of these compositions the RMM time may have been too long. However, it is important to note that these phases were typically present in very small amounts, which could lessen their impact on the properties of these materials.

Also present in several of the samples was the intermetallic phase AlFe which did not appear in the samples that were RMM using the Master Alloy as a starting powder. Those samples using the Reading Alloy starting powder, which contained a small amount of iron, all had a very small amount of this phase. However, it should be recalled that it was determined that iron content increases with RMM time, and iron enters the system in an exponentially increasing manner with RMM time, while the amount of the AlFe phase content remains fairly constant. It should be noted that the amounts of the phase were near to detection limits, and as such should be viewed as approximate values. Since no other iron-containing phases were found it has been concluded that the iron, especially for longer RMM times (with the exception of $120 \mathrm{~h} \mathrm{RMM} \mathrm{time),} \mathrm{becomes} \mathrm{a} \mathrm{solute} \mathrm{in} \mathrm{the}$ 
$\mathrm{L1}_{2}$ intermetallic. Iron when alloyed with $\mathrm{Al}_{3} \mathrm{Ti}$, much like chromium, creates the $\mathrm{L}_{2}$ titanium trialuminide [116].

The entire processing scheme attempted to avoid any oxygen contamination, which would likely lead to $\mathrm{Al}_{2} \mathrm{O}_{3}$ formation. Both the mill vial and the HIP "can" were sealed in an argon glove box prior to processing. The glove box had sieves for oxygen removal, and the level was monitored and maintained below 2 ppm. All samples had a small amount of $\mathrm{Al}_{2} \mathrm{O}_{3}$, with the amount increasing slightly with $\mathrm{RMM}$ time as seen by the increase in the $\mathrm{Al}_{2} \mathrm{O}_{3}$ phase for longer RMM times (40h, 60h, and 100h). Another source of oxygen involves contact of the powder with air when the vial is opened after the RMM process. Given the excellent oxidation resistance of the as-cast $\mathrm{L}_{2}$ chromiummodified titanium trialuminide [17] one would not expect this to be a problem, but the alloy does create a protective layer of $\mathrm{Al}_{2} \mathrm{O}_{3}$. One possibility is the reactive nature of the powder surface may enhance $\mathrm{Al}_{2} \mathrm{O}_{3}$ formation; however, this was not studied. As noted, the powder particle size decreases rapidly down to less than $10 \mu \mathrm{m}$ on milling and the appearance of fines (defined as powder that floats in the hood when the vial is opened) qualitatively increases with RMM time suggesting a possible increased reactive surface area when open to the air.

Another potential source of oxygen contamination occurs during the CIP procedure when the powder is evacuated with a mechanical pump and the potential exists for air to be trapped in the compacts. If this is the case, one would expect that more $\mathrm{Al}_{2} \mathrm{O}_{3}$ could be formed during longer HIP times as shown by comparing 20/800/MA and 20/1000/MA samples (similar RMM processing but different HIP time and temperature). One should expect this to be similar for all samples with the same HIP parameters and there does appear to be a small increase with HIPing time. Another possible source of oxygen occurs during the vacuum annealing or "degassing" for hydrogen removal. Since all of the samples were degassed in the same manner under a diffusion pump vacuum, little contamination was expected, but if some were introduced it seems it would be a near constant amount. Another possible source would be a leak in the milling vial during the RMM process, but this would tend to cause variations in the amount of oxygen uptake which were not observed. Additionally, nitrogen would be observed and a vacuum was 
always present when the vial lid was opened indicating a good seal in the vial. It is expected that the observed $\mathrm{Al}_{2} \mathrm{O}_{3}$ contamination results from some combination of contributions from all of these sources. However, although this phase is unwanted, it is present in very small amounts and further, is thought to have little effect on the properties of interest. A small amount of oxide is a common constituent of powder processed materials that include oxide formers.

A very small amount of a $\mathrm{Ti}_{3} \mathrm{Al}(\mathrm{C}, \mathrm{N})$ phase was detected in the 20/1000/RA and 40/1000/RA samples. It is speculated that this occurred because the glove box removes oxygen, but not nitrogen, and the concentration of nitrogen may increase with time as the argon gas in the glove box is reused over a period of time. Since both of these samples were created about the same time this suggests detectable levels of nitrogen were taken up during glove box use at that particular time. However, this identification is based on peak position movements from the $\mathrm{T}_{3} \mathrm{AlC}$ positions, and changes in carbon stoichiometry can also cause a similar shift.

\section{(4) Peak Broadening Analysis of Profile Fit X-ray Diffraction Peaks by Warren-Averbach Fourier Method}

Crystallite size and microstrains for the $\mathrm{L}_{2}$ intermetallic were determined for selected consolidated samples. One complication is that diffraction peaks from both complex carbide phases occur around the $\{111\}$ and $\{400\}$ diffraction peaks of the $\mathrm{L}_{2}$ intermetallic and become more prominent with increased RMM time as the amount of carbide phase increases. These interferences created difficulties with the microstrain data for the $\langle 100\rangle$ direction for several samples and therefore these were not reported.

Averages of several determinations of the crystallite size in the $\langle 111\rangle$ and $\langle 100\rangle$ directions for the $\mathrm{L}_{2}$ intermetallic, along with the microstrain in the $<111>$ direction, are tabulated in Table III-16. The W-A results show a decrease in crystallite size with RMM time for samples HIPed under the same conditions. At the same time, the microstrain increases with increasing RMM time. These microstrains are very large, especially considering that consolidation temperature is $1000^{\circ} \mathrm{C}$ for $2 \mathrm{~h}$. This suggests a high and somewhat stable defect concentration in the material. Although the maximum microstrain 
is $0.25 \%$; it will be shown that the elastic strain limit in these materials is approximately $1 \%$, confirming that such elastic strains can be supported by these materials.

\begin{tabular}{|c|c|c|c|}
\hline Sample & $\begin{array}{c}<111>\text { Crystallite } \\
\text { Size }(\mathrm{nm})\end{array}$ & $\begin{array}{c}<100>\text { Crystallite } \\
\text { Size }(\mathrm{nm})\end{array}$ & $\begin{array}{l}\left\langle\varepsilon^{2} 5 \mathrm{~nm}\right\rangle^{1 / 2}(\%) \\
<111\rangle \text { Direction }\end{array}$ \\
\hline 20/1000/MA & 118 & 101 & 0.11 \\
\hline 20/1000/RA & 111 & 90 & 0.12 \\
\hline 40/1000/RA & 86 & 60 & 0.15 \\
\hline 60/1000/RA & 68 & 52 & 0.21 \\
\hline 100/1000/RA & 41 & 49 & 0.25 \\
\hline
\end{tabular}

The crystallite size determinations made with both the Scherrer method and the W-A method are shown together in Figure III-43 as a function of RMM time. Also shown for each analysis is an exponential decay regression and $95 \%$ confidence intervals found from multiple deconvolutions of the diffraction peak profiles used in the W-A analysis. Of importance in this comparison is that the $\mathrm{W}$-A results are in good agreement with those found with the Scherrer method using an average of the first four fundamental diffraction peaks in each sample. As expected, the W-A results in the $\langle 111\rangle$ direction were slightly larger than those in the $\langle 100\rangle$ direction. However, the $\langle 111\rangle$ direction crystallite sizes appear to converge toward a size similar to that obtained for the <100> direction with milling time, and this sample had the largest microstrains. This convergence of crystallite size with RMM time suggests that the crystallites became more equiaxed as the RMM time increases. It is important to recall that Scherrer data is computed assuming spherical crystallites and the fact that the Scherrer results are very near to the average of the two crystallographic directions that converge to similar sizes supports the conclusion that the crystallites approach a more equiaxed shape. 


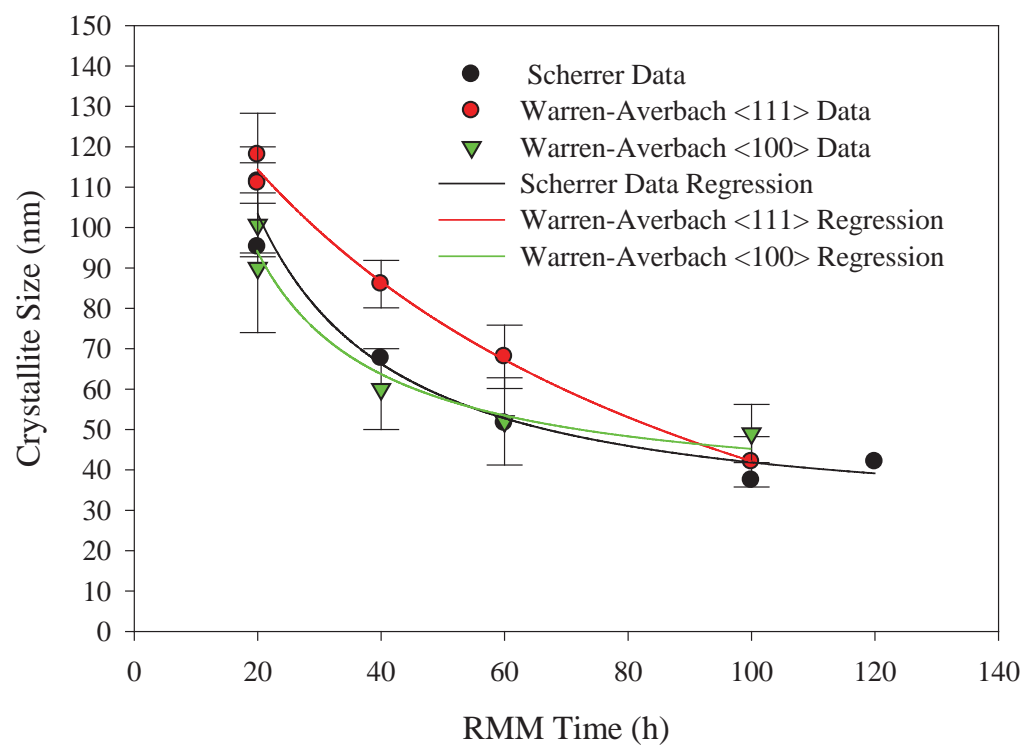

Figure III-43 Crystallite Size Results from the Scherrer and Warren-Averbach Methods as a Function of RMM Time

The <111> microstrains (Table III-16) show a parabolic type increase with time as shown in Figure III-44. The regression fit shown was performed on all data present, with $95 \%$ confidence intervals shown for each data point. The increase in uncertainty with RMM time shows the sensitivity of the microstrain determination to the variation in profile fits as a result of the broader and much less intense diffraction peaks involved, especially those of second order at high angles. Compounding these difficulties is the fact that the carbide content increases so that peak overlap problems involving the carbide and $\mathrm{L}_{2}$ intermetallic diffraction peaks become more pronounced. 


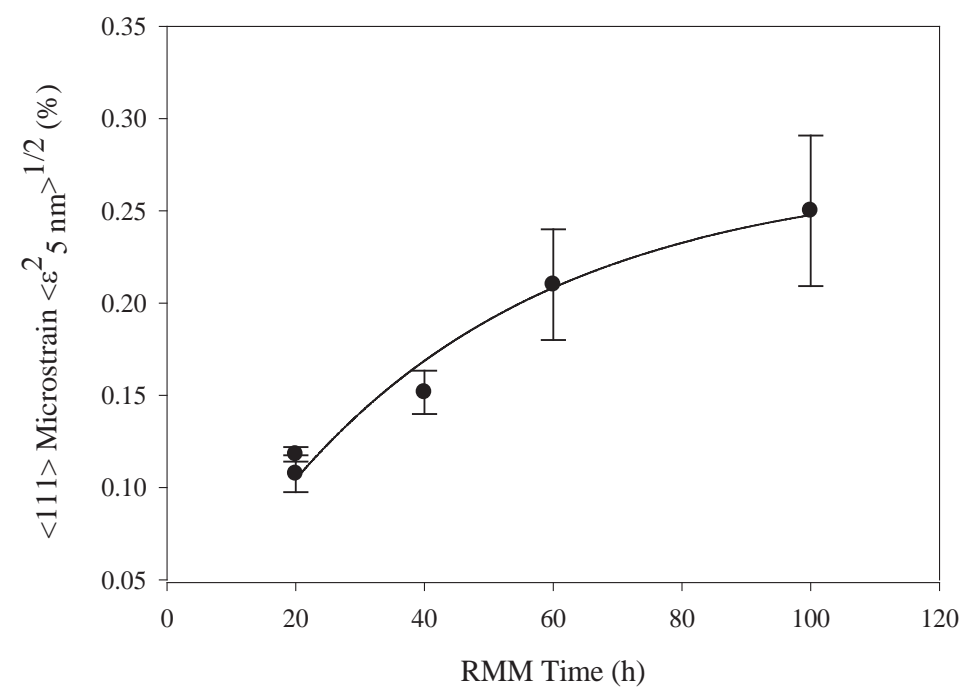

Figure III-44 Microstrain Data in the <111> Direction as a Function of RMM Time

Estimates of dislocation densities were made by means described earlier (see Chapter II.A.5.a)(7)(b)) with a Burgers vector $a<110>$ and the results of these calculations for the $\langle 111\rangle$ direction are presented in Table III-17. The dislocation densities for all samples are quite high especially considering the consolidation process is expected to produce an annealed material. Typically, the densities of dislocations in heavily cold rolled metals are in the range of $\sim 5 \times 10^{9} \mathrm{~mm}^{-2}$ [141], only about an order of magnitude larger than these values. More importantly, the dislocation density increases with RMM time and therefore with the carbide content.

Table III-17 Dislocation Densities for Select Consolidated Samples

\begin{tabular}{|c|c|} 
Sample & $\begin{array}{c}\text { Dislocation Density } \\
(\mathbf{m m})^{-2}\end{array}$ \\
\hline 20/1000/MA & $5.6 \times 10^{7}$ \\
\hline 20/1000/RA & $6.6 \times 10^{7}$ \\
\hline $40 / 1000 / R A$ & $1.1 \times 10^{8}$ \\
\cline { 2 - 2 } 60/1000/RA & $1.9 \times 10^{8}$ \\
\hline 100/1000/RA & $\mathbf{3 . 7} \times \mathbf{1 0}^{8}$ \\
\hline
\end{tabular}




\section{c) Transmission Electron Microscopy (TEM)}

Transmission electron microscopy analysis was performed on samples 20/1000/MA, and 40/1000/RA with example microstructures shown in Figure III-45 and Figure III-46, with the dimensions in the nanometer range. HIP sample 20/1000/MA consists of the $\mathrm{L} 1_{2}$ intermetallic grains, platelet-like features (appear to be rod-like, but the feature in the upper right hand corner of the micrograph is slightly tilted showing a platelet feature), and small spherical features. These platelets are mostly the $\mathrm{Ti}_{3} \mathrm{AlC}_{2}$ and $\mathrm{Ti}_{2} \mathrm{AlC}$ carbide phases, referred to as complex carbides. It can be noted that there were a number of smaller spherically-shaped particles, which based on XRD analysis would most likely be $\mathrm{AlCr}_{2}$ particles. Recall that $\mathrm{Al}_{2} \mathrm{O}_{3}$ phase while observed in XRD has been found in some samples to be very large particles that are difficult to observe because of the small volume fraction. Of course, these small particles in Figure III-45 could also be a very small amount of $\mathrm{TiC}$ that was not detectable by XRD.

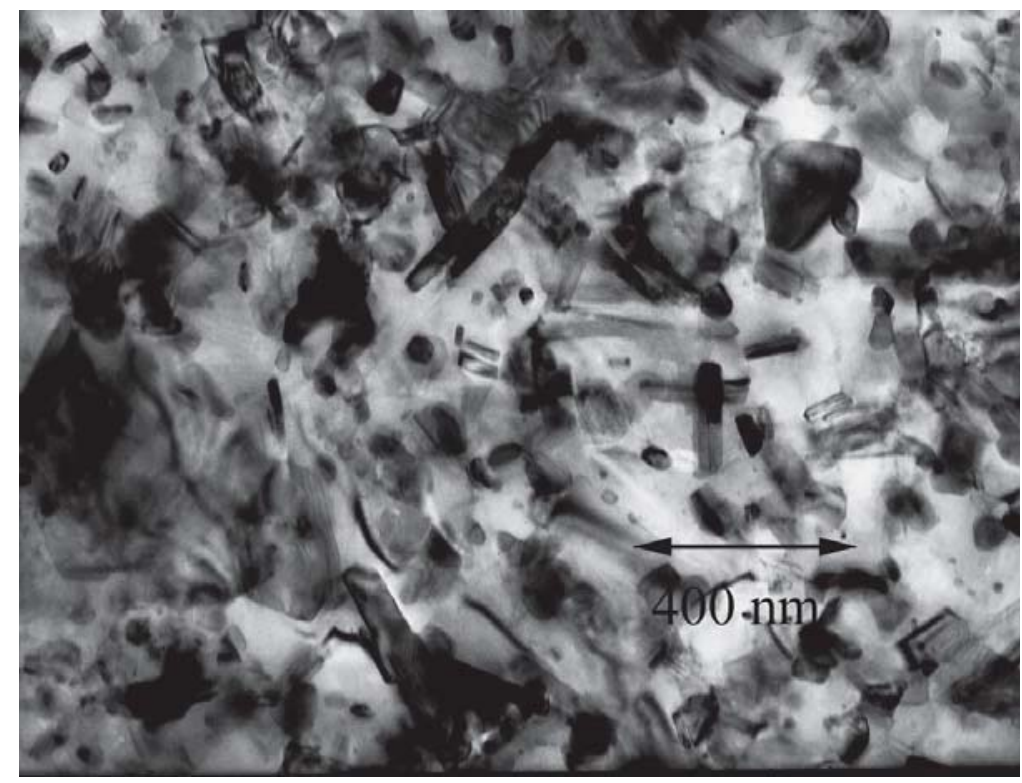

Figure III-45 TEM Micrograph of Sample 20/1000/MA 50 kX Magnification

The microstructure of the 40/1000/RA sample, shown in Figure III-46 and Figure III-47, is slightly different than that of the previous sample. In particular, the platelet $\mathrm{Ti}_{3} \mathrm{AlC}_{2}$ carbide phase (recall that this was the main carbide identified in XRD analysis) appears much larger in both diameter and thickness than in the previous samples. 
Additionally, qualitative energy dispersive analysis of these platelet particles resulted in titanium and aluminum emissions (carbon was not analyzed) with titanium approximately 3 times higher in intensity again indicating these as $\mathrm{Ti}_{3} \mathrm{AlC}_{2}$ particles. The features in the Figure III-46 micrograph look more distinct compared to the previous micrograph.

Within most $\mathrm{L}_{2}$ intermetallic grains there are contrast variations indicating some type of defect structure. In this sample, a majority of the grains were surrounded by carbide particles. However, areas of distinct grain boundaries that do not have carbide particles were observed. Also, occasionally, spherical particles appeared within the $\mathrm{L}_{2}$ intermetallic grains and on boundaries with carbides. Based on XRD phase analysis these particles are most likely $\mathrm{AlCr}_{2}, \mathrm{Ti}_{3} \mathrm{Al}(\mathrm{C}, \mathrm{N})$, and/or AlFe particles. A different field of view at a slightly higher magnification, Figure III-47, again shows a majority of grains bounded by carbides in addition to grain boundaries, with contrast variations within the majority of grains.

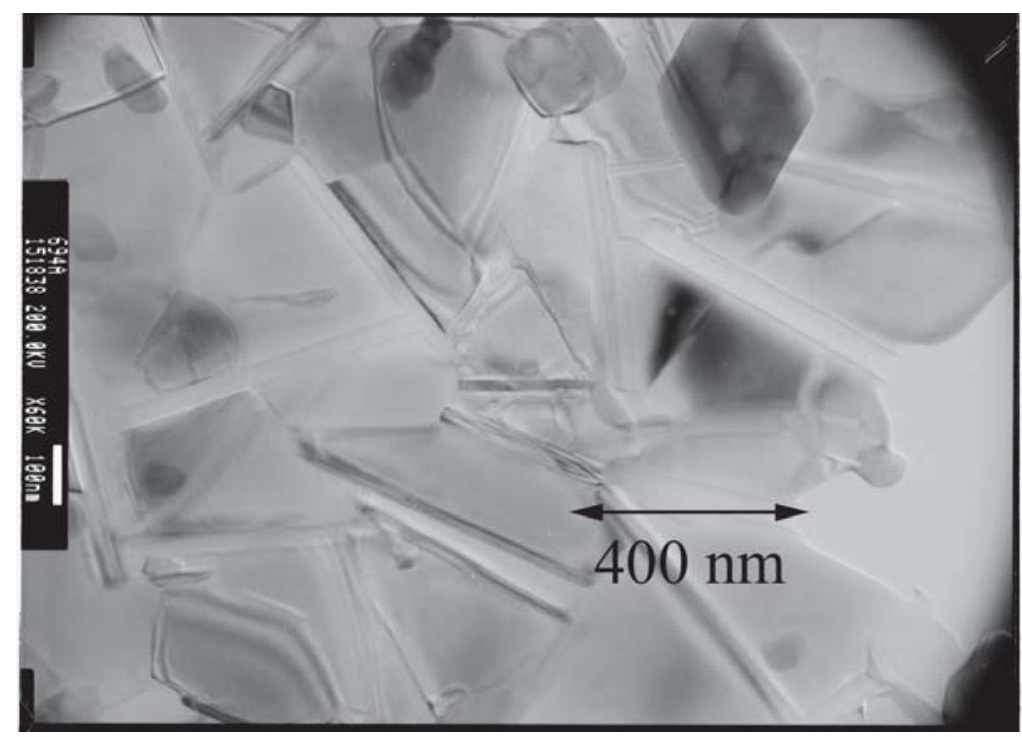

Figure III-46 TEM Micrograph of Sample 40/1000/RA 60 kX Magnification 


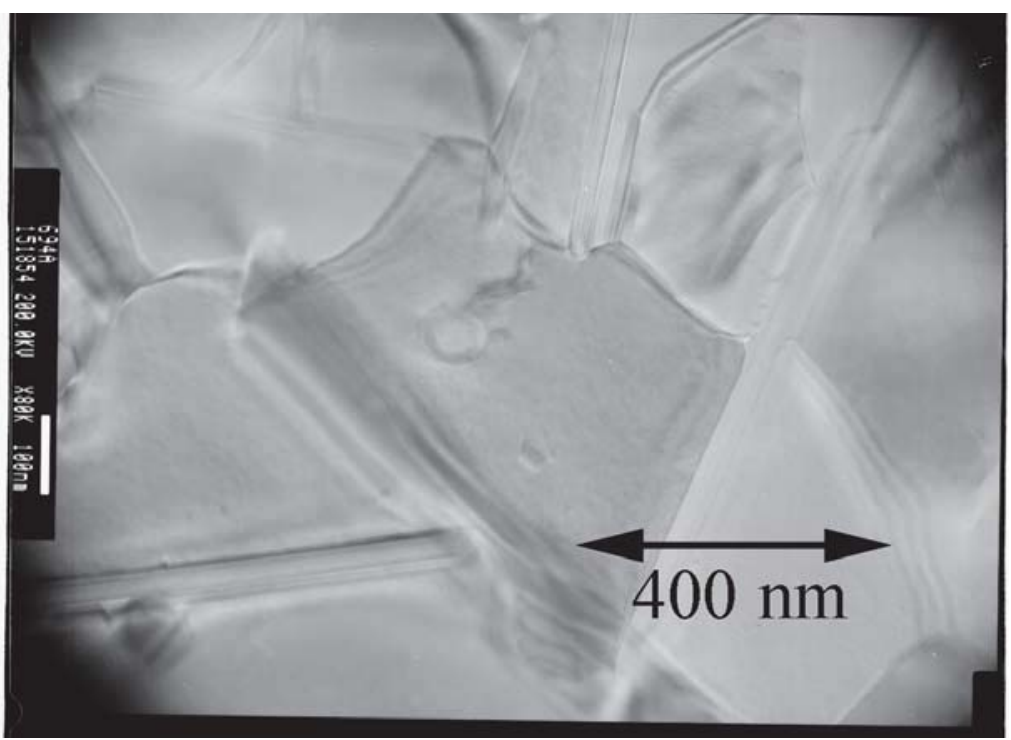

Figure III-47 TEM Micrograph of Sample 40/1000/RA 80 kX Magnification

Quantitative metallography was performed on several fields of view to estimate

the mean linear grain size. This is accomplished by using the linear intercept method illustrated in Equation (III.2), where $V$ is the volume fraction of the $\mathrm{L}_{2}$ intermetallic, $L_{0}$ is the line length, and $N$ is the number of grains intersected. The mean of 14 measurements of random line placement gave a value of $169 \mathrm{~nm}$. Therefore, assuming spherical grains yields a grain size of $249 \mathrm{~nm}$. Recall that the crystallite size for this sample was in the range of $60-86 \mathrm{~nm}$, much smaller than this grain size estimate, but the variations in contrast within the grains support the presence of some type of defects within the grains resulting in a smaller crystallite size than the grain size. It should be pointed out that some features in the micrographs overlap despite the fact that the fields of view are in a thin region adjacent to a hole in the TEM specimen.

$$
\bar{L}=V \times\left(\frac{L_{0}}{N}\right)
$$

One very large particle was also found in this sample which was of the order of a micrometer and qualitative energy dispersive analysis established only aluminum. Again, the light elements were not analyzed, but it was concluded that this very large particle was $\mathrm{Al}_{2} \mathrm{O}_{3}$. It is very interesting that these $\mathrm{Al}_{2} \mathrm{O}_{3}$ particles were so large compared with 
all other phases in the microstructure and more importantly as discussed that the amount of this phase increases slightly with RMM time as shown in Table III-14.

\section{d) Complex Carbide Morphology}

\section{(1) Transmission Electron Microscopy (TEM)}

Transmission electron microscopy image analysis established that both the $\mathrm{Ti}_{3} \mathrm{AlC}_{2}$ and $\mathrm{Ti}_{2} \mathrm{AlC}$ carbides have a platelet morphology. Figure III-48 is a TEM micrograph at $300 \mathrm{KX}$ magnification showing the edge of a platelet-shaped $\mathrm{Ti}_{3} \mathrm{AlC}_{2}$ carbide particle in 40/1000/RA sample. Additionally, the TEM image established that these complex carbides not only have a platelet shape, but are single crystals.

Measurement of the lattice spacing in this image of the edge (smallest dimension) of the carbide particle gave a value of $0.929 \mathrm{~nm}$ which corresponds to the (002) lattice spacing. This yields a "c" lattice parameter of $1.858 \mathrm{~nm}$ very close to the value for "c" obtained by Tzenov et al. [138] of $1.8487 \mathrm{~nm}$. Recall that the XRD pattern for this carbide had diffraction peaks that were slightly displaced from predictions based on the data given by Tzenov et al. This particular platelet thickness is about $40 \mathrm{~nm}$. Despite the fact that no lattice images of the $\mathrm{Ti}_{2} \mathrm{AlC}$ carbide were developed, it was concluded that the edge or thin direction of the plates involves the (001) planes similar to the $\mathrm{Ti}_{3} \mathrm{AlC}_{2}$ carbide.

Recall that the two carbides have similar hexagonal crystal structures.

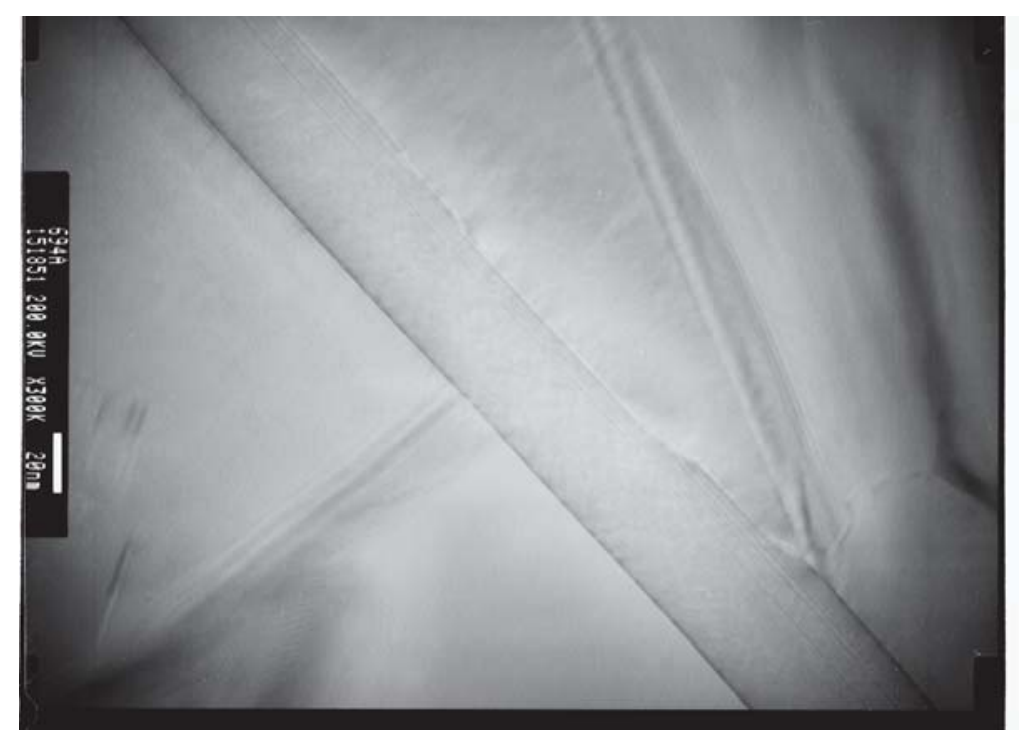


Figure III-48 TEM Image of Carbide Edge for Sample 40/1000/RA 300 kX

\section{(2) Field Emission Scanning Electron Microscopy (FESEM)}

The morphology of the complex carbides was also examined with an FESEM on etched consolidated samples from argon processing for RMM times of 20, 40, and $60 \mathrm{~h}$. The fine-scale structure resulted in extremely fast removal of the $\mathrm{L} 1_{2}$ intermetallic material with a Tuckers etchant, revealing the three-dimensional morphology of the complex carbides.

Example micrographs of the various carbide structures are shown in Figure III-49 through Figure III-52 at a magnification of 50kX. Recall, that the 20/1000/MA, and 20/1000/RA samples were RMM processed for the same time. The 20/1000/MA sample (Figure III-49) has a complex carbide morphology in which contact of the carbide platelets is minimal. The 20/1000/MA sample also shows a larger number of very small particles that generally appear to agglomerate along bands on individual carbide particles. This localized collection of these particles indicates this is a result of precipitation of small particles in the etchant as it evaporates or is displaced in the washing procedure. Most likely these are small L1 2 intermetallic particles, or particles from other phases, that are not completely dissolved by the etchant. These deposits, or particles, were also present in the 60/1000/RA sample (Figure III-52) and it appears there are a few on the 20/1000/RA sample shown in Figure III-50. The sporadic observation of these particles supports the argument that these are residual particles from the etching process in which the times and washing are somewhat varied being difficult to monitor.

For Reading Alloy samples of 20, 40, and 60 RMM times the complex carbide platelet structure is larger and the platelets appear to be more interconnected than observed in the Master Alloy RMM for $20 \mathrm{~h}$. Two of the samples, 20/1000/MA and 20/1000/RA, were RMM processed for the same time of $20 \mathrm{~h}$ and consolidated with the same parameters using the QHIP. However, as shown in Figure III-50 the 20/1000/RA sample appears to have a more interconnected carbide structure. Recall that this sample had a slightly greater carbon content than the 20/1000/MA sample, and thus, an increased volume fraction of carbide. In addition, only the $\mathrm{Ti}_{3} \mathrm{AlC}_{2}$ carbide phase was present in the 
20/1000/RA sample, whereas the 20/1000/MA sample had both the $\mathrm{Ti}_{3} \mathrm{AlC}_{2}$ and $\mathrm{Ti}_{2} \mathrm{AlC}$ carbide phases. The 40/1000/RA sample (Figure III-51) had a complex carbide structure similar to sample 20/1000/RA, but the amount of carbide was greater because of the increased carbon content. The 60/1000/RA sample (Figure III-52) shows a similar interconnected complex carbide structure with an increase in carbide volume fraction over the other samples.

An interesting observation was that the carbide particles may have some ductility since all the polished edges of the carbides have material that extends over the actual edge of the carbide particles indicating some flow of the material caused by the heavy localized deformation imposed during polishing. This is expected based on the metalliclike behavior observed in these materials, for example electrical conductivity (i.e. lack of charging in FESEM).

The carbide platelets appear to have an interconnected structure especially in the case of large volume fractions of carbides; where the platelets clearly impinge upon one another. Detailed examination of the micrographs shows a majority of the carbide platelets touch, but do not appear to be bonded, as observed by a clear boundary between some platelets. In the plane of polishing some of the carbides appear to be interconnected; however, carbide deformation obscures this boundary region by smearing. These carbides are considered to be random in orientation; however, many of the platelets that lie in the plane of the polish are most likely etched off the sample distorting the orientation of the carbide platelets as a majority observed in the micrograph lie on edge. 


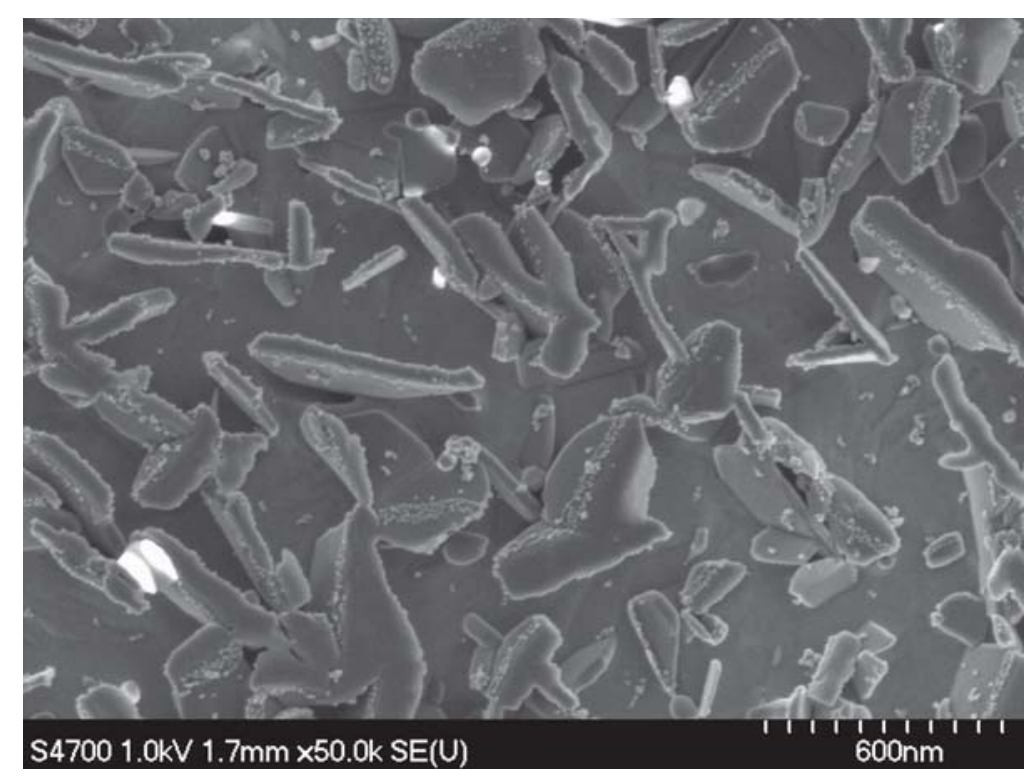

Figure III-49 FESEM Micrograph of Sample 20/1000/MA

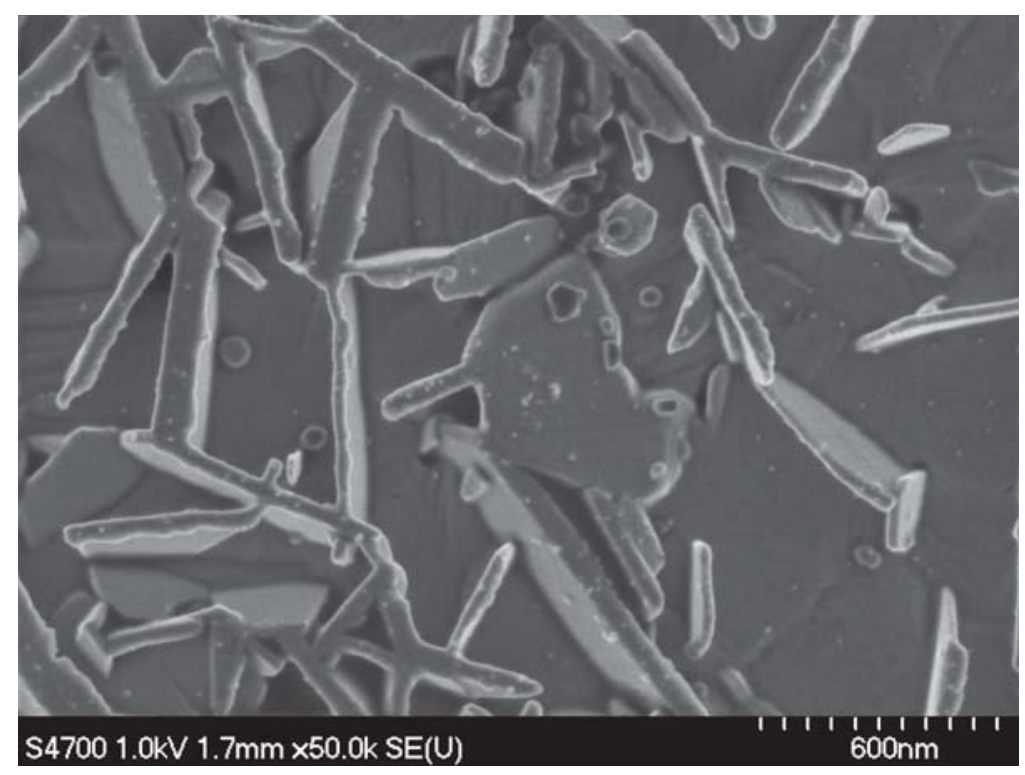

Figure III-50 FESEM Micrograph of Sample 20/1000/RA 


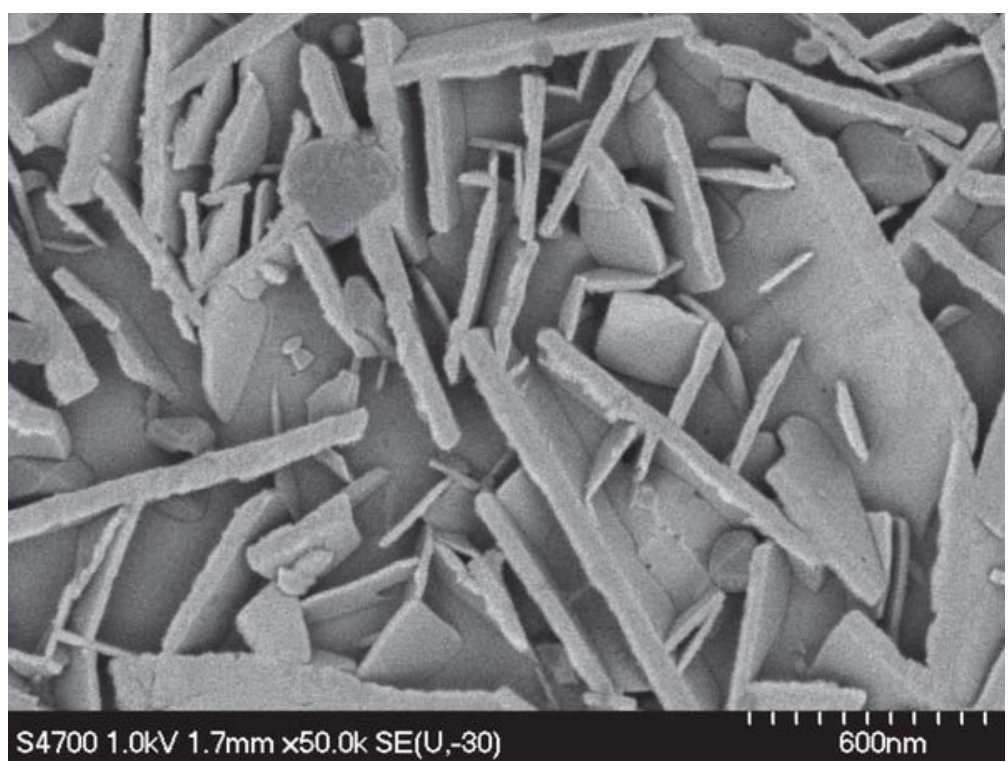

Figure III-51 FESEM Micrograph of Sample 40/1000/RA

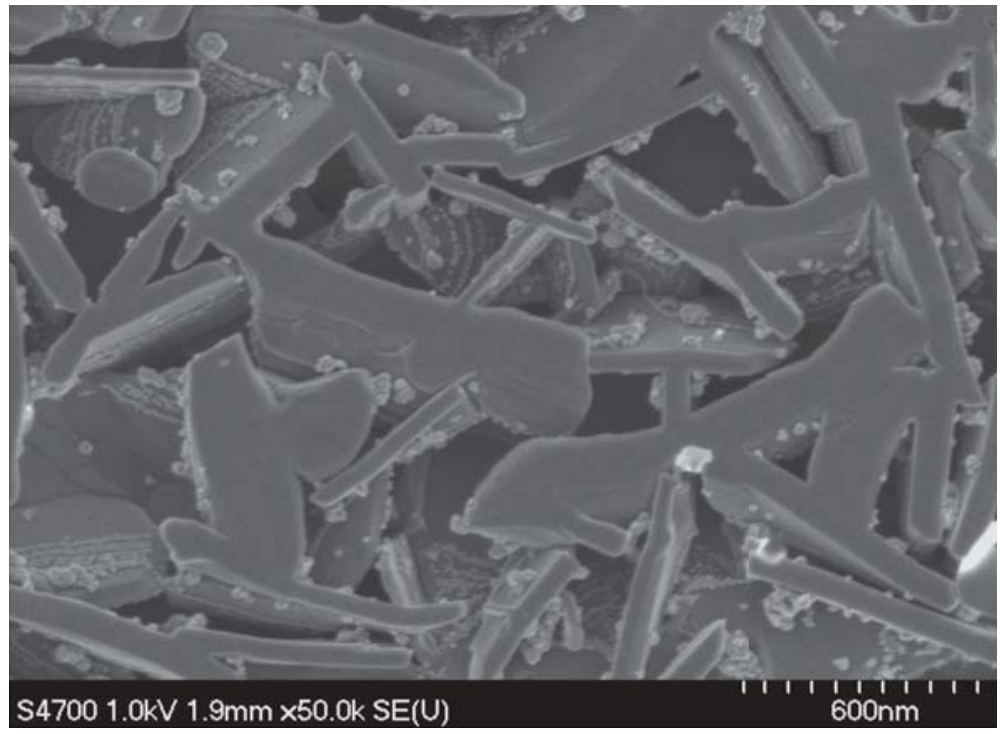

Figure III-52 FESEM Micrograph of Sample 60/1000/RA

Quantitative image analysis was performed on several micrographs of each

sample using Scion Image (Frederick, Maryland) version 4.0.2 image analysis software. The diameter and thickness of the complex carbide platelets were measured directly and represented an average of a minimum of 50 diameter measurements and 20 thickness measurements. In addition, mean linear interparticle spacing was measured. For this measurement, it is important that the intersections of particles with test lines be 
accomplished in the polishing plane because of the three dimensional nature of the images. This can be accomplished by taking measurements of carbides defining the plane of the polish. The spacing values, $\lambda$, represent an average of a minimum of 20 randomly placed lines on several micrographs using the relationship in Equation (III.3). Where $N_{L}($ particle $)=$ number of particles divided by test line length.

$$
\lambda=\frac{\left[1-V_{V}(\text { particle })\right]}{N_{L}(\text { particle })}
$$

Results of this quantitative image analysis are given in Table III-18. The 20/1000/RA sample, although consolidated under the same conditions as the 20/1000/MA sample, had 2.7 times the complex carbide platelet diameter with a near doubling of the thickness. However, there was a slight decrease in the interparticle spacing which agrees with a slight decrease in the L12 intermetallic crystallite size as shown in both Figure III-43 and Table III-16. The complex carbide dimensions increased slightly with RMM time as shown by the data in Table III-18 for the 40/1000/RA and 60/1000/RA samples. However, as expected the interparticle spacing decreased with RMM time. In general the interparticle spacing follows the behavior of the $\mathrm{L}_{2}$ intermetallic crystallite size which also decreases with RMM time as was shown in Figure III-43 and Table III-16.

\begin{tabular}{|c|ccc|}
\hline \multicolumn{2}{|c|}{ Table III-18 Complex } & Carbide Particle Measurements Consolidated Powders \\
\hline Sample & $\begin{array}{c}\text { Diameter } \\
(\mathbf{n m})\end{array}$ & $\begin{array}{c}\text { Thickness } \\
(\mathbf{n m})\end{array}$ & $\begin{array}{r}\text { Interparticle } \\
\text { Spacing (nm) }\end{array}$ \\
\hline 20/1000/MA & 160 & 16 & 428 \\
\cline { 2 - 4 } 20/1000/RA & 431 & 29 & 341 \\
40/1000/RA & 443 & 33 & 258 \\
60/1000/RA & $\mathbf{4 9 6}$ & $\mathbf{4 8}$ & $\mathbf{1 9 4}$ \\
\hline
\end{tabular}

However, as can be noted in making these comparisons, the crystallite size is smaller than the interparticle spacing, with this issue addressed in the discussion of the 
consolidated material. Observations with TEM, particularly of the 40/1000/RA sample, suggest that many of the individual grains are bounded by carbide particles, although contrast variations exist indicating defect-type contrast in the grains. Quantitative estimates of these grain sizes on TEM micrographs for sample 40/1000/RA, assuming spherical grains, gave $249 \mathrm{~nm}$, very similar to the interparticle spacing determined for this sample. This suggests that the interparticle spacing may be a measure of the average grain size in the 40/1000/RA sample. It is suspected that in all samples having the appearance of an interconnected complex carbide structure the interparticle spacing reflects the grain size; however, as will be shown these carbide platelets while appearing mechanically interconnected under high temperature can move suggesting they do not appear strongly bonded and therefore under proper conditions may be independent of each other. It is not clear whether this also applies to the 20/1000/MA sample.

\section{(3) Scherrer Crystallite Size Estimates}

Scherrer crystallite size measurements were made on the carbide phases formed in the consolidated samples. Most of these diffraction observations on the complex carbides were based on single peaks, because all of the diffraction peaks fall very close to other diffraction peaks. Instrumental broadening was corrected by comparison with an asreceived -325 mesh TiC powder, as discussed previously (e.g. Chapter II.A.5.a)(6)). It should be noted that the TiC powder was also used for an instrumental broadening correction for the complex carbide phases because single-phase complex carbide material was not available. Since, the (100) and (002) complex carbide diffraction peaks occurred at different $2 \theta$ values than diffraction peaks for the TiC standard, the FWHM data for the TiC was fit with a cubic polynomial regression to determine the FWHM values for the angles of interest.

The $\mathrm{TiC}$ values are reported for all samples having the $\mathrm{TiC}$ phase present. Because the TiC phase appeared as spherical particles in the TEM images, the Scherrer results represent averages of the first two TiC diffraction peaks, $\{111\}$ and $\{200\}$. The results of these crystallite size estimates are presented in Table III-19 for all consolidated samples. The TiC appeared in the sample milled for $100 \mathrm{~h}$ with a small crystallite size of 
$\sim 19 \mathrm{~nm}$, it grew in size and amount in the $120 \mathrm{~h}$ RMM time. The existence of the TiC phase in these samples has been attributed for the most part to the competition for the aluminum between the complex carbide and the $\mathrm{L}_{2}$ intermetallic phase.

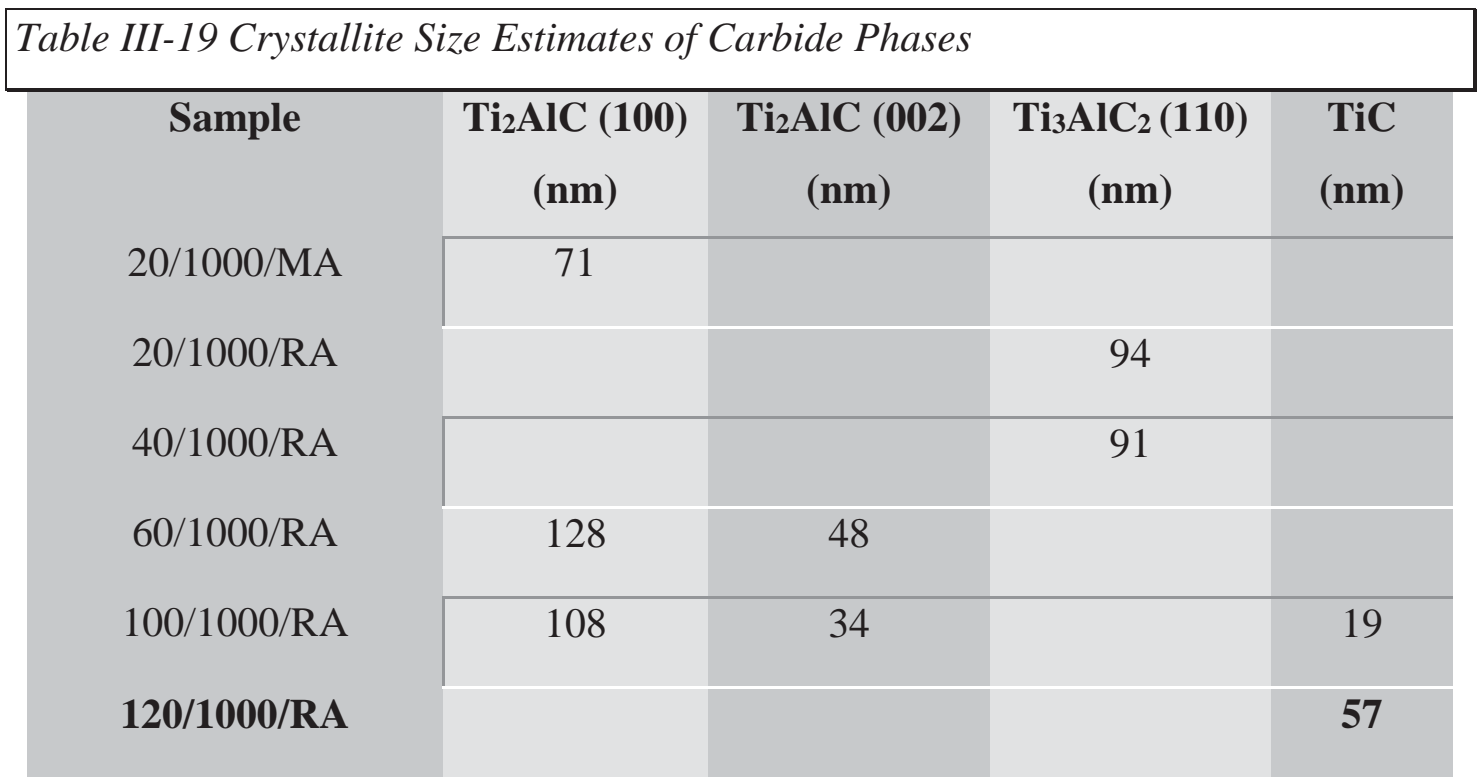

${ }^{*}$ Note this is the $\operatorname{Ti}(\mathrm{C}, \mathrm{N})$ phase

Crystallite sizes for the complex carbides depend on crystallographic orientation because of the platelet shape of these particles established with TEM observations that showed the direction of the platelet thickness corresponds to the [001] direction or normal to the (001) crystallographic plane. Additionally, a majority of the samples contained both of the complex carbide phases which have peaks that overlap. The best separation of these overlapping diffraction peaks occurs for the (002) diffraction peak for both complex carbides. However, these diffraction peaks were not always available or adequate. In those instances the (100) and (110) diffraction peaks were used, which if the platelet is considered as a single crystal, represent the diameter of the platelet since both plane normals are perpendicular to the [001] or "c" direction in the hexagonal crystal structure.

The crystallite size in the directions normal to the thickness in both complex carbide phases are about one fourth of the physical platelet diameter, Table III-18, with the exception of the 20/1000/MA which is only about one half of the value from TEM metallography. It is interesting that these are similar to the $\mathrm{L} 1_{2}$ intermetallic crystallite 
size to grain size ratio. What is important in this regard is that the diameter of the complex carbide platelets are beginning to mechanically impinge in the 20/1000/MA sample, Figure III-49, becoming more prominent with increased RMM time due to volume fraction increases with RMM time. As noted in Table III-18 the thickness of the platelets increases with increased complex carbide content. The crystallite size determined for the (002) plane of the $\mathrm{Ti}_{2} \mathrm{AlC}$ phase in the 60/1000/RA sample is in agreement with the physical thickness determined from TEM micrographs (Table III-18) with the same value of $48 \mathrm{~nm}$; however, the thickness shown by crystallite size in the 100/1000/RA sample shows an unexpected decrease. While micrographs of the 100/1000RA were not available, the volume fraction increases and thus thickness would also be expect to also increase (note the trend in physical thickness in Table III-18).

The crystallite size of the (101) pyramidal plane (not shown in the table) of the $\mathrm{Ti}_{3} \mathrm{AlC}_{2}$ carbide in the 120/1000/RA sample resulted in a value of $\sim 28 \mathrm{~nm}$ indicating a further decrease in the crystallite size in this phase compared to the $100 \mathrm{~h}$ RMM time sample which has a crystalline size twice as large for the same plane. It can be concluded that dislocations or some type of crystallographic defect is generated in the complex carbides related to the impingement of these platelets.

\section{2. $\quad$ Properties of Consolidated Materials}

\section{a) Density}

\section{(1) Archimedes}

Densities of the consolidated samples were determined by the Archimedes method and the results for each material, summarized in Table III-20, represent an average of ten measurements (one exception is the $\mathrm{Cr} 9$ sample for which three measurements were averaged). Also included in Table III-20 are the HIP parameters of temperature and time. Nic [116] determined a density of $3.682 \mathrm{~g} / \mathrm{cm}^{3}$ for as-cast $\mathrm{Al}_{66} \mathrm{Cr}_{9} \mathrm{Ti}_{25}$. The target composition for $\mathrm{Cr} 9$ and $\mathrm{MM} 3 \mathrm{~h}$ samples was $\mathrm{Al}_{66} \mathrm{Cr}_{9} \mathrm{Ti}_{25}$, but both have slightly greater density than the as-cast material. This should not be unexpected since Nic [116] noted a very small amount of porosity in the as-cast HIP-homogenized 
samples due in part to solutionization of unwanted phases. Also, a slightly higher density for the HIP3h sample is expected because that material has a small amount of the more dense $\mathrm{Ti}_{2} \mathrm{AlN}$ phase. A similar detailed phase analysis of the $\mathrm{Cr} 9$ material was not accomplished. It is interesting that a sample (not reported) similar to the 20/1000/MA sample but HIPed at $800{ }^{\circ} \mathrm{C}$ was only $0.6 \%$ less in density but also contained some TiC phase suggesting these alloys are nearly fully dense at this lower HIP temperature; however, these samples reported no ductility in compression.

Table III-20 Archimedes Density Measurements

\begin{tabular}{c|ccc} 
Sample & $\begin{array}{c}\text { Density } \\
\left(\mathbf{g} / \mathbf{c m}^{\mathbf{3}}\right)\end{array}$ & $\begin{array}{c}\text { Temp } \\
\left({ }^{\circ} \mathbf{C}\right)\end{array}$ & $\begin{array}{c}\text { Time } \\
(\mathbf{m i n})\end{array}$ \\
\cline { 2 - 4 } 20/1000/MA & 3.7815 & 1000 & 120 \\
\hline 20/1000/RA & 3.7647 & 1000 & 120 \\
\hline 40/1000/RA & 3.8230 & 1000 & 120 \\
\cline { 2 - 4 } 60/1000/RA & 3.8736 & 1000 & 120 \\
\hline \multirow{2}{*}{ 100/1000/RA } & 4.0497 & 1000 & 120 \\
\hline Cr 9 & 3.7076 & 1200 & 120 \\
\cline { 2 - 4 } MM3h & $\mathbf{3 . 6 9 0 7}$ & $\mathbf{1 0 0 0}$ & $\mathbf{1 2 0}$ \\
\hline
\end{tabular}

The carbide content can be increased by increasing the RMM time (as discussed in Chapter III.D.1.a); this has been accomplished with a systematic increase in the excess titanium in the starting mixture. The density of $\mathrm{Ti}_{3} \mathrm{AlC}_{2}$ is $4.2 \mathrm{~g} / \mathrm{cm}^{3}$ [138] and that of $\mathrm{Ti}_{2} \mathrm{AlC}$ is $4.113 \mathrm{~g} / \mathrm{cm}^{3}$ (JCPDS-ICDD database), and both of these densities are greater than that of the $\mathrm{L}_{2}$ intermetallic phase. In Figure III-53 the dependence of the densities for samples HIPed at $1000{ }^{\circ} \mathrm{C}$ on total complex carbide volume fraction is shown, along with a linear regression fit to the data. For each sample, the total carbide volume fraction was taken as the total of the $\mathrm{Ti}_{3} \mathrm{AlC}_{2}$ phase and the $\mathrm{Ti}_{2} \mathrm{AlC}$ phase. The $\mathrm{Cr} 9$ sample has been used to represent the density for a carbide volume fraction of zero. As the complex carbide content increases, the density increases linearly. Among the materials; the 
20/1000/RA and 40/1000/RA samples contained only the $\mathrm{Ti}_{3} \mathrm{AlC}_{2}$ phase, while the others contained mixtures of the two complex carbides. This density increase directly reflects the increase in carbide content with RMM time, which is expected since the density should follow a rule of mixtures approaching the density of the pure complex carbides. In fact, the extrapolation of the linear regression to a volume fraction value of unity yields a density of $4.2 \mathrm{~g} / \mathrm{cm}^{3}$, the density of the $\mathrm{Ti}_{3} \mathrm{AlC}_{2}$ phase.

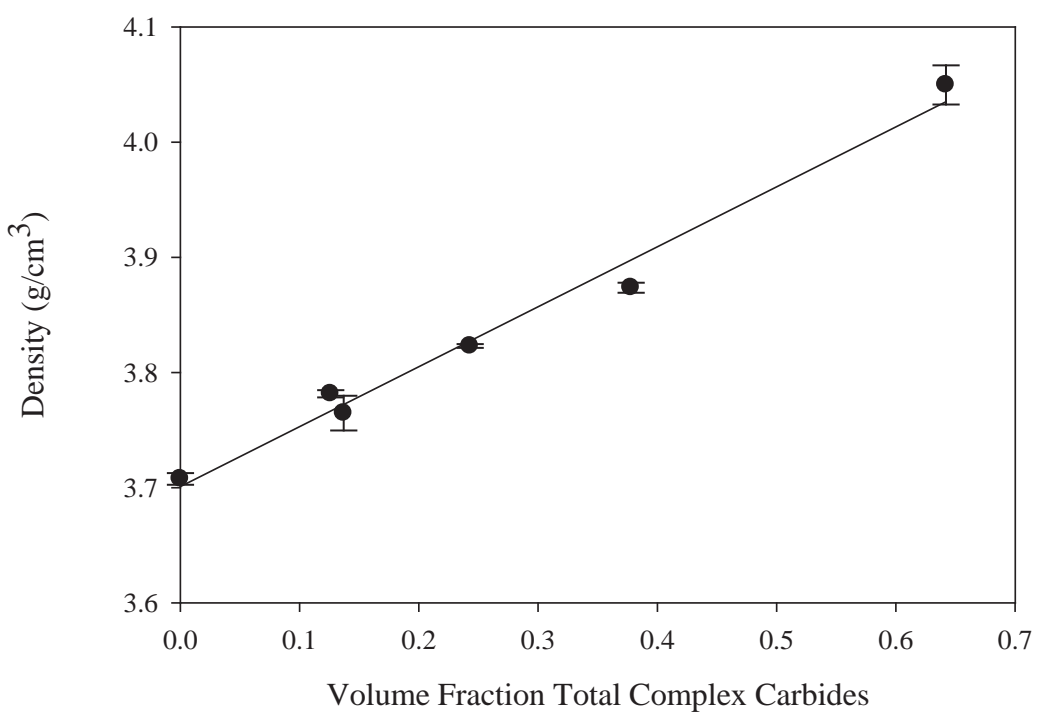

Figure III-53 Density of Alloys HIPed at $1000^{\circ} \mathrm{C}$ with Increasing Complex Carbide Volume Fraction

\section{(2) AccuPycnometer}

Density measurements using the gas displacement method provide a means for comparing the density of materials in the as-milled powder and consolidated forms. The powder samples chosen for comparison were for the two samples RMM processed material in air at $20 \mathrm{~h}$ time with the consolidated sample $\mathrm{HIPed}$ at $800^{\circ} \mathrm{C}$ for $15 \mathrm{~min}$, along with a powder RMM for $20 \mathrm{~h}$ in argon consolidated at $800^{\circ} \mathrm{C}$ but for $2 \mathrm{~h}$ (not reported). Table III-21 summarizes the average of ten density measurements for each sample. The powder density in both cases is slightly lower than the consolidated samples. Determination of the average density for both consolidated and as-milled samples shows only a $2.6 \%$ decrease in the density of the as-milled powder. Based on the gas 
displacement method this suggests a small amount of closed porosity in the interior of powder particles. Any open (connected or not connected) porosity in the powder would not contribute to a lower density measurement.

Table III-21 AccuPycnometer Density

\begin{tabular}{|c|cccc}
\hline \multirow{2}{*}{ Process Numbers } & $\begin{array}{c}\text { Density } \\
\left(\mathbf{g} / \mathbf{c m}^{\mathbf{3}}\right)\end{array}$ & $\begin{array}{c}\mathbf{\pm 9 5} \% \\
\text { Confidence }\end{array}$ & $\begin{array}{c}\text { Temp } \\
\left({ }^{\circ} \mathbf{C}\right)\end{array}$ & $\begin{array}{c}\text { Time } \\
(\mathbf{m i n})\end{array}$ \\
\cline { 2 - 6 } RMM20h8.2TiAir800QH15m & 3.7421 & 0.000558 & 800 & 15 \\
RMM20h8.2TiAir (powder) & 3.5969 & 0.00093 & NA & NA \\
RMM20h8.2TiAr800H2h* & 3.7503 & 0.000992 & 800 & 120 \\
\cline { 2 - 6 } RMM20h8.2TiAr (powder) & $\mathbf{3 . 6 9 1}$ & $\mathbf{0 . 0 0 2 5 4 1}$ & NA & NA \\
\hline
\end{tabular}

*This sample has not been discussed note $2 \mathrm{~h}$ HIP time

\section{b) Vickers Hardness}

Vickers hardness values determined by averaging ten measurements are tabulated in Table III-22 along with equivalent SI stress units. Sample Cr9 has been taken to represent a single-phase $\mathrm{Al}_{66} \mathrm{Cr}_{9} \mathrm{Ti}_{25}$ alloy consolidated by powder metallurgy CIP and HIP processing of a pre-alloyed Praxair powder [142]. Also shown in the Table are crack loads; i.e., the Vickers load at which crack(s) initially form, with loads varied from 1 to $50 \mathrm{~kg}$ for this test. The as-cast $\mathrm{Al}_{66} \mathrm{Cr}_{9} \mathrm{Ti}_{25}$ single-phase alloy after homogenization by HIP processing has a VHN of 166 [116]. The hardness of the $\mathrm{Cr} 9$ sample is somewhat greater, $217 \mathrm{VHN}$, and this is attributed to a finer grain size. Assuming spherical grains, the $\mathrm{Cr} 9$ grain diameter is $19 \mu \mathrm{m}$ compared to $\sim 200 \mu \mathrm{m}$ (equivalent spherical grain size determined from elongated grain data) for the as-cast sample [116]. The greater hardness for the MM3h sample compared to the $\mathrm{Cr} 9$ is again attributed to a finer grain size; recall that in this case the grain diameter was $9 \mu \mathrm{m}$, in addition, a small amount of secondary phases was found. In both of these samples there were no visible cracks at the highest load of $50 \mathrm{~kg}$; this behavior is similar to that found for the as-cast material [116]. 
Table III-22 Vickers Hardness Measurements

\begin{tabular}{c|cccc}
\hline \multirow{2}{*}{$\begin{array}{c}\text { Sample } \\
\text { Cr9 }\end{array}$} & VHN & GPa & $\begin{array}{c}\text { Crack Load } \\
(\mathbf{k g})\end{array}$ & $\begin{array}{c}\text { VHN } \mathbf{4} \text { 95 \% } \\
\text { Confidence }\end{array}$ \\
\cline { 2 - 5 } MM3h & 217 & 2.1 & No cracks & 3 \\
\hline 20/1000/MA & 347 & 3.4 & No cracks & 5 \\
\hline 20/1000/RA & 564 & 5.5 & $5 \mathrm{~kg}$ & 7 \\
\hline 40/1000/RA & 615 & 6.0 & $5 \mathrm{~kg}$ & 4 \\
\hline 60/1000/RA & 640 & 6.3 & $5 \mathrm{~kg}$ & 5 \\
\hline 100/1000/RA & $\mathbf{7 1 5}$ & $\mathbf{7 . 0}$ & $\mathbf{1 0 ~ k g}$ & 7 \\
\hline
\end{tabular}

The samples HIPed at $1000{ }^{\circ} \mathrm{C}$ for $2 \mathrm{~h}$ have increasing amounts of carbide phases with the exception of the 20/1000/MA and 20/1000/RA that are similar. Although the 20/1000/MA and 20/1000/RA samples had similar hardness values, it is interesting that the 20/1000/MA sample was slightly harder despite a slightly larger crystallite size and a lesser amount of secondary phases. The 20/1000/MA had both complex carbides, whereas 20/1000/RA had only the $\mathrm{Ti}_{3} \mathrm{AlC}_{2}$ carbide; however, the reason for this small increase in hardness is not clear.

There is a near-linear increase in the hardness with increasing carbon content, essentially carbide content, as shown in Figure III-54. The hardness of the 20/1000/RA sample (lower point) falls below this linear regression by a small amount; however, the difference with the 20/1000/MA sample (just above it) is less than $4 \%$. All of these samples had a cracking load of $5 \mathrm{~kg}$, except the 100/1000/RA sample with the largest amount of carbide phases, which had a higher cracking load at $10 \mathrm{~kg}$. Increase in the amount of the carbides at that level apparently leads to significant crack blunting and an increase in crack resistance. 


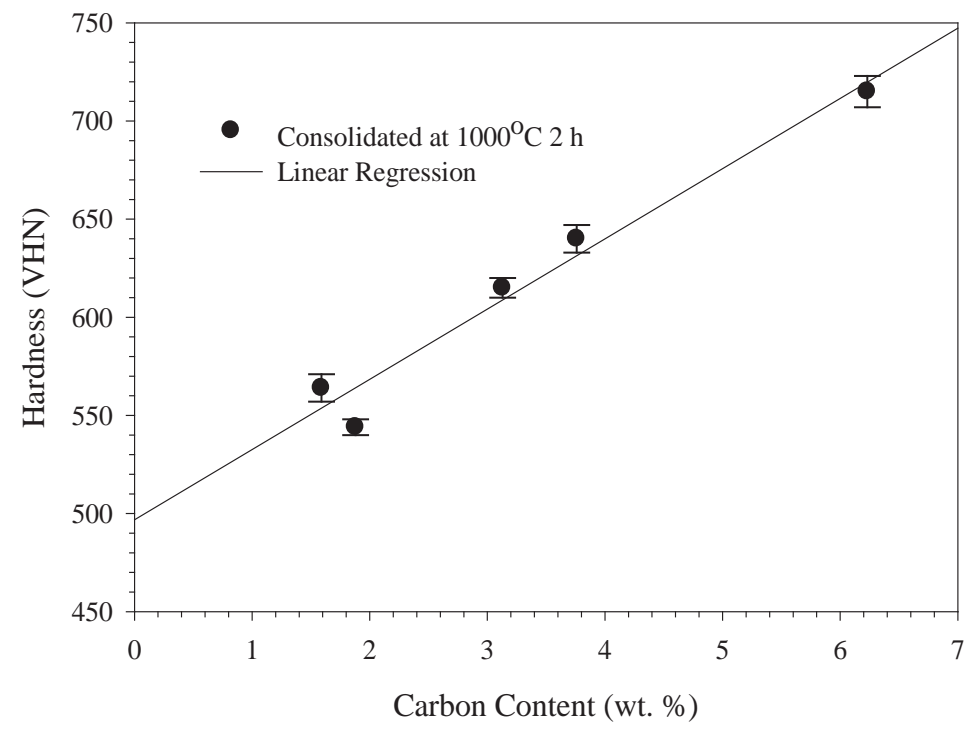

Figure III-54 Vickers Hardness as a Function of Carbon Content for Samples Consolidated at $1000{ }^{\circ} \mathrm{C}$ for $2 \mathrm{~h}$

\section{c) Compression Stress/Strain Relationships}

Because of the relatively small size of the consolidated samples, compression testing was employed to further define the mechanical properties of these materials. Often only one test was performed on each sample, with the results of the multiple tests averaged where applicable. Most engineering stress-strain curves presented were corrected for machine compliance. This was accomplished by curve fitting load versus displacement machine compliance curves and subtracting these experimental machine displacements from the recorded curves for each specimen based on an equivalent load. Despite this rigorous method, which was partly an attempt to generate modulus information from these engineering stress-strain curves, it was concluded that this approach does not provide reliable modulus values.

\section{(1) Room Temperature}

Room temperature $0.2 \%$ strain compressive yield strengths along with ultimate compressive strengths (UCS) and strains to fracture are summarized in Table III-23. Included for comparison are values for the as-cast $\mathrm{Al}_{66} \mathrm{Cr}_{9} \mathrm{Ti}_{25}$ as reported by Nic [7]. The 
strain rate was $10^{-4} \mathrm{~s}^{-1}$ for all samples tested. The first three samples represent singlephase materials with decreasing grain size; all other samples are RMM in argon.

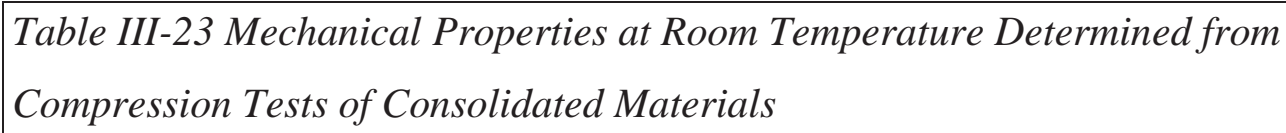

\begin{tabular}{|c|c|c|c|c|}
\hline Sample & $\begin{array}{c}\text { RMM Time } \\
\text { (h) }\end{array}$ & $\begin{array}{c}\text { YS } \\
(\mathbf{G P a})\end{array}$ & $\begin{array}{c}\text { UCS } \\
(\mathbf{G P a})\end{array}$ & $\begin{array}{c}\text { Strain to Fracture } \\
(\%)\end{array}$ \\
\hline As-Cast Cr9 [7, 116] & NA & 0.245 & 0.791 & 19.0 \\
\hline $\mathrm{Cr} 9$ & NA & 0.398 & 1.990 & 26.5 \\
\hline MM3h & $*$ & 0.990 & 1.930 & 19.3 \\
\hline 20/1000/MA & 20 & 2.060 & 2.210 & 7.4 \\
\hline 20/1000/RA & 20 & 1.910 & 2.150 & 8.4 \\
\hline 40/1000/RA & 40 & 2.170 & 2.590 & 4.4 \\
\hline 60/1000/RA & 60 & 2.400 & 2.570 & 2.2 \\
\hline 100/1000/RA ** & 100 & 2.145 & 2.390 & 2.3 \\
\hline
\end{tabular}

* 3 h dry milling

** All data an average of two compression tests

YS $-0.2 \%$ Yield Stress

UCS - Ultimate Compression Strength

Strain to Fracture - total strain at fracture

As one would expect, the yield strength of the $\mathrm{Cr} 9$ sample increased significantly over the as-cast material, with this increase attributed to the finer grain size. Also of interest is the larger UCS $(1.99 \mathrm{GPa}$ compared to $0.791 \mathrm{GPa})$ and greater total strain to fracture $(26.5 \%$ as opposed to $19 \%)$ for this material. The improvement in ductility might indicate that microstructural defects present in the as-cast material are minimized by HIP homogenization in the powder metallurgy $\mathrm{Cr} 9$ sample. The MM3h sample has a more dramatic increase in yield strength, nearly three times that of the $\mathrm{Cr} 9$ sample. A majority of this dramatic increase in strength has been attributed to a further refinement of the grain structure; however, a very small amount of hard, finely dispersed, second phase(s) was present. Despite the high strength the total strain to fracture of the MM3h sample is very similar to that for the as-cast alloy. In agreement with the compression 
data are the actual physically measured final strains in the $\mathrm{Cr} 9$ and MM3h samples of $26.1 \%$ and $18.7 \%$ respectively, showing expected elastic relaxation. It is very interesting that the UCS for the MM3h and Cr9 samples was of the order of the yield strength values attained in the RMM materials.

All of the RMM/1000 materials have nearly a ten-fold increase in yield strength over the as-cast material. With regard to increasing RMM time, there was approximately a $10 \%$ increase in yield strength for each $20 \mathrm{~h}$ increase in RMM time up to $60 \mathrm{~h}$. However, there is a slight drop in yield strength for the sample RMM for $100 \mathrm{~h}$ compared to the $60 \mathrm{~h}$ RMM time. Recall the hardness testing, the $100 \mathrm{~h}$ sample had the largest load to cracking for all RMM samples. Along with the increase in strength with RMM time there was a regular decrease in the total strain to fracture; but again the change did not continue to $100 \mathrm{~h}$ RMM time as $60 \mathrm{~h}$ and $100 \mathrm{~h}$ samples had similar strains to fracture. After testing the 20/1000/MA sample a corner of the original sample fractured when the test was ended, all other RMM samples failed catastrophically. The UCS reached a maximum of about $2.6 \mathrm{GPa}$ for the $40 \mathrm{~h}$ and $60 \mathrm{~h}$ RMM times, with a slightly smaller strength for the $100 \mathrm{~h}$ RMM time.

A polycrystalline $\mathrm{Ti}_{2} \mathrm{AlC}$ material [143] showed a hexagonal layer structure with similar crystallography to the observed platelets in the consolidated samples. These single-phase carbides had a compressive room temperature strength of $763 \mathrm{MPa}$ with shearing along the layered directions that are weakly bonded. The laminated grains in that study were 5-10 $\mu \mathrm{m}$ in thickness, of a hexagonal shape, but composed of a number of micro-laminates (size not given). While the crystallography is similar to the complex carbides in the consolidated materials here, the thicknesses are much larger. Both of the complex carbides have metallic characteristics such as conductivity, and have a mechanical behavior showing measurable ductility as opposed to the expected brittle nature of most ceramic materials $[144,264]$. Of importance is the fact that the maximum yield strength of a fine grained $\mathrm{L} 1_{2}$ powder processed intermetallic was approaching 1 $\mathrm{GPa}$; however yield strengths over twice that value have been reached in the consolidated carbide containing alloys. 
The compression engineering stress-strain curves for both the $\mathrm{Cr} 9$ and $\mathrm{MM} 3 \mathrm{~h}$ samples are shown in Figure III-55. There appeared to be a small yield drop for the $\mathrm{Cr} 9$ sample, and a similar, but less pronounced, behavior for the MM3h sample. Similar stress-strain curves for all RMM times HIPed at $1000{ }^{\circ} \mathrm{C}$ except the $120 \mathrm{~h}$ RMM time are shown in Figure III-56. Both the 20/1000/MA and 20/1000/RA samples are very similar (20/1000/RA slightly lower yield strength in agreement with hardness) with a defined yielding at the onset of plastic flow as seen with the $\mathrm{Cr} 9$ and MM3h samples, but followed by only a modest strain hardening up to fracture. As the carbide content was increased further the yield point was less defined with a progressively larger region of curvature as the compression curve transitioned from the elastic region to the plastic deformation region.

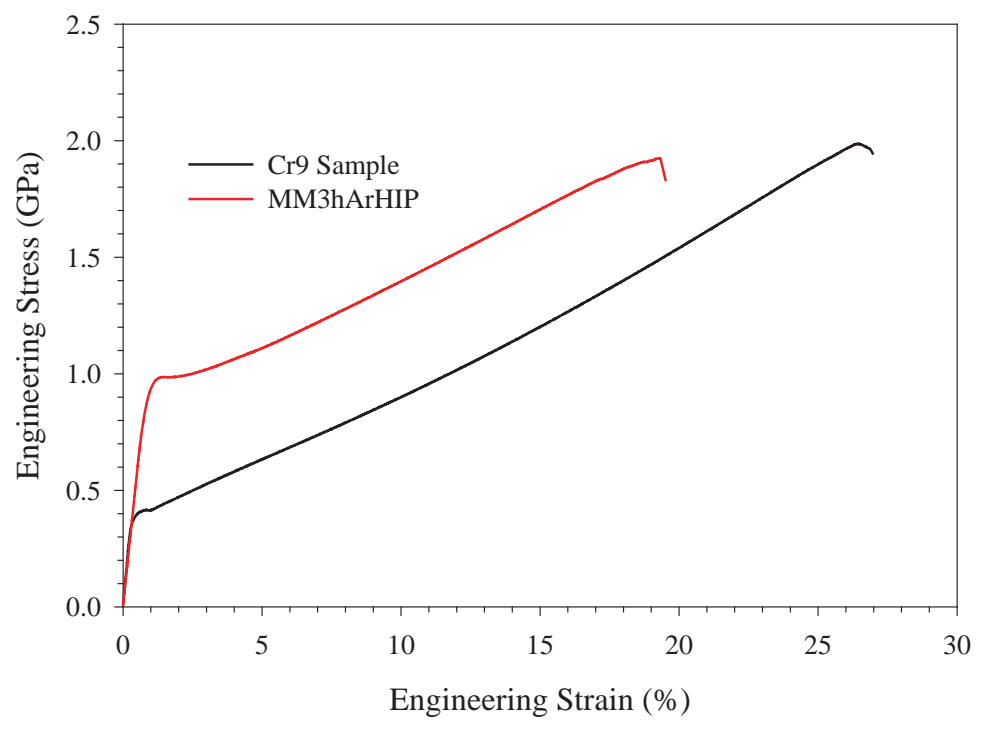

Figure III-55 Compression Curves for Cr9 and MM3h Samples 


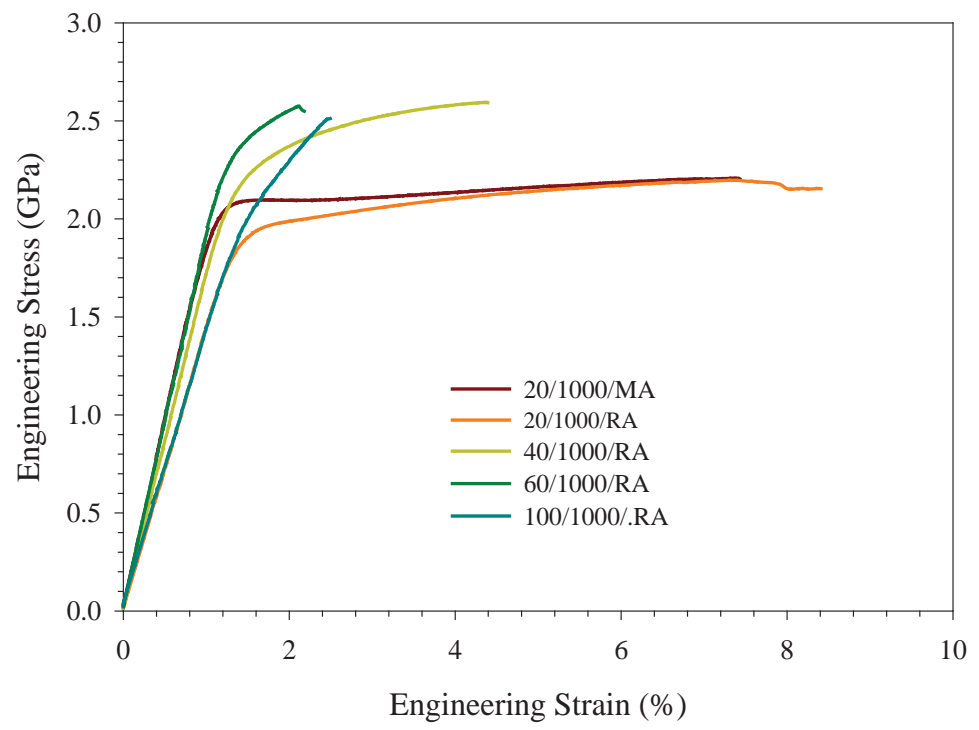

Figure III-56 Compression Curves for RMM Samples Consolidated at $1000^{\circ} \mathrm{C}$

The strain hardening rate (SHR) was determined from the slope of the stressstrain curve in the near-linear portion of the plastic flow region. Table III-24 lists the SHR values determined for all samples HIPed at $1000^{\circ} \mathrm{C}$. Also shown for comparison is a value from $\mathrm{Nic}$ [116] for the as-cast $\mathrm{Al}_{66} \mathrm{Cr}_{9} \mathrm{Ti}_{25}$ alloy, which was collected in a similar manner (i.e. compression, same instrument and apparatus, same strain rate calculation). Both the $\mathrm{Cr} 9$ and MM3h samples had a greater SHR than the as-cast alloy; this is expected because of the smaller grain size. The SHRs for the 20/1000/MA and 20/1000/RA samples were less than those for the Cr9 and MM3h samples, and were slightly less than that of the as-cast sample. Comparing microstructures with the $\mathrm{Cr} 9$ and MM3h samples, the 20/1000/MA and 20/1000/RA samples had a significantly smaller crystallite size and grain size, along with significant amounts of nanometer carbides. One would expect the smaller grain size in the 20/1000/MA and 20/1000/RA samples to increase the SHR; however, the decrease in SHR clearly indicates the activation of dislocation sources, which must be related to the major difference between these two groups of samples, that is, the carbide structure. Recall the earlier observations showing that the 20/1000/MA sample had a more discontinuous platelet-shaped carbide structure whereas the 20/1000/RA had a more interconnected-type platelet-shaped carbide structure. Since the two have a similar amount of carbides, but the carbides are slightly 
smaller in size and have less interconnection in the 20/1000/MA material it would have larger carbide surface area. If the carbide surfaces facilitate dislocation generation, it would account for the slightly lower SHR in the 20/1000/MA sample compared to the 20/1000/RA sample.

Table III-24 Strain Hardening Rate of Samples HIPed at $1000^{\circ} \mathrm{C}$

\begin{tabular}{c|c} 
Sample & $\begin{array}{c}\text { Strain Hardening Rate } \\
\text { (GPa) }\end{array}$ \\
Cr9 & 4.62 \\
As-Cast HIP Cr9 [116] & 6.20 \\
\hline MM3hArHIP & 6.00 \\
\cline { 2 - 2 } 20/1000/MA & 2.57 \\
\hline 20/1000/RA & 4.03 \\
\cline { 2 - 2 } 40/1000/RA & 8.62 \\
\hline 60/1000/RA & 24.76 \\
\cline { 2 - 2 } 100/1000/RA & $\mathbf{4 9 . 7 8}$ \\
\hline
\end{tabular}

Of particular interest is the fact that the SHR for all of the RMM materials increased with the total volume fraction of complex carbides. Recall that the crystallite size decreased with increased carbide content and the carbides had a strongly interconnected-type structure for the higher carbide content samples. Additionally, the dislocation density increased with carbide content (see Table III-17). Many studies of work hardening have shown a linear relationship between the flow stress $\tau$ and the square root of the dislocation density $(\sqrt{\rho})$ [145]. An increase in flow stress is reflected in an increase in SHR. The relationship of SHR with $\sqrt{\rho}$ in Figure III-57, clearly indicates a near-linear relationship. 


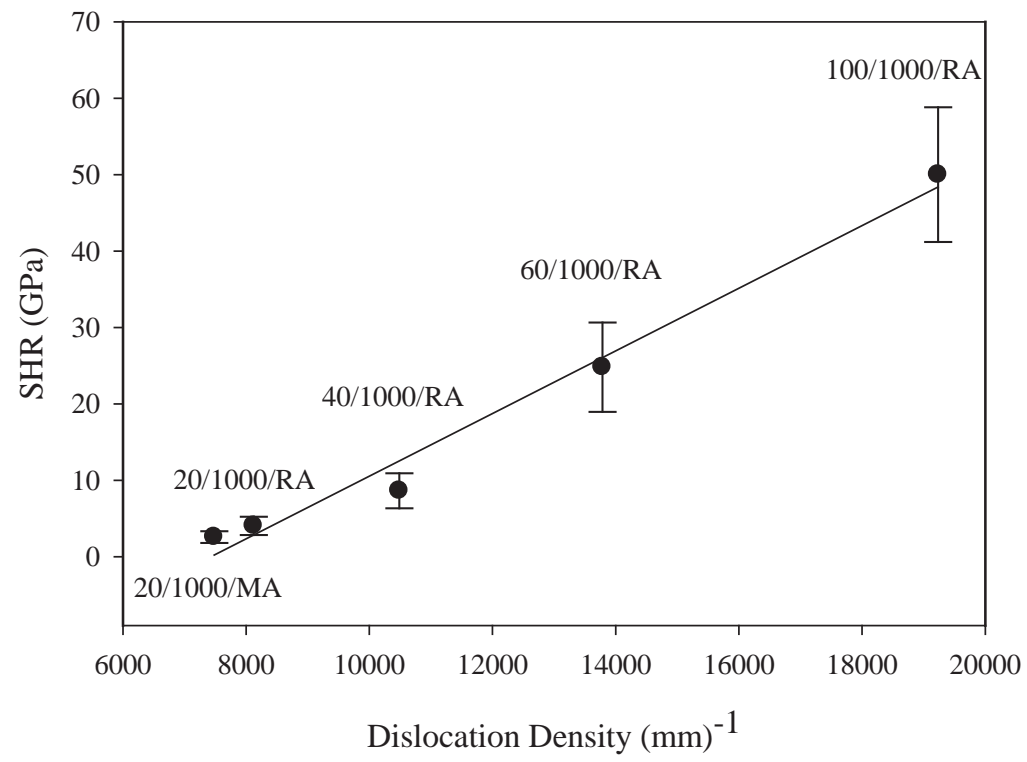

Figure III-57 Changes in Strain Hardening Rate as a Function of the Square Root of the Dislocation Density

Although the carbides (at least for lower volume fractions) appear to facilitate dislocation source operation, at the higher volume fractions of carbides the increase in dislocation density, along with the decrease in crystallite size, seem to lead to substantial increases in SHR. Additionally, the strongly interconnected-like morphology of the carbides may also play a role in the nature and operation of the dislocation sources. As the amount of complex carbide increases the surface area in contact with the $\mathrm{L}_{2}$ intermetallic decreases. Since the $\mathrm{L}_{2}$ intermetallic is typically a single crystal grain of about $350 \mathrm{~nm}$ or less, and dislocation generation for these grain sizes is typically from boundaries, as the carbide amount increases there is less surface to generate dislocations as shown by the increase in SHR. Superplastic behavior of these materials in high temperature compression tests, suggests that the interconnected-like carbide morphology can be modeled as independent platelets that can move independently with deformation. This ability may contribute to the increase in SHR. 


\section{(2) Superplastic Behavior}

The recorded high temperature engineering stress-strain curve for the 40/1000/RA sample, not corrected for compliance, is shown in Figure III-58. The material had an extremely low yield strength of $30 \mathrm{MPa}$, and the test was terminated at a strain of $\sim 45 \%$. After the onset of plastic flow there was a stress reduction followed by a gradually increase in stress level. At the same time the actual cross-sectional area was increasing. The apparent rise in stress near the end of the curve was actually caused by the extreme increase in the cross-sectional area and making the engineering stress appear larger. At the same time the actual measured strain from sample dimensional changes was $60 \%$, much larger than indicated by the compression test. Based on the difference between the strain at test termination and the measured strain, the yield strength must have been less than the $12 \mathrm{MPa}$ constant preload. Consequently, the material flowed plastically at the constant load of $12 \mathrm{MPa}$ during heating, concluding that the material is superplastic near the temperature $880{ }^{\circ} \mathrm{C}$. This response involves more than a 100 fold decrease in yield strength of the material from ambient temperature.

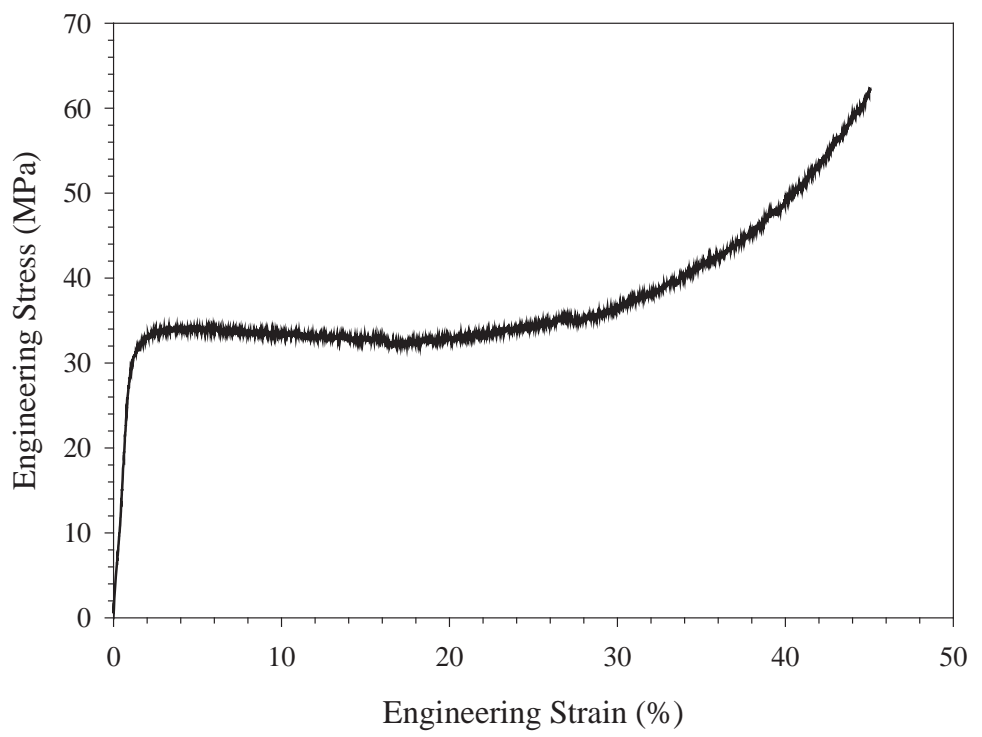

Figure III-58 Engineering Stress-Strain Curve Sample 40/1000/RA at $900{ }^{\circ} \mathrm{C}$

A macro picture of the $20 / 1000 / \mathrm{RA}$ sample after a $900{ }^{\circ} \mathrm{C}$ compression test is shown on the right in Figure III-59, also shown for comparison on the left is an untested 
sample of similar initial dimensions along with the experimental direction of the compressive stress. The physical strain measured for this sample was $61 \%$, and clearly as seen by the image of the compressed sample, this sample could have been strained further but the test was terminated. There were no visible signs of mechanical degradation in the surface of the compressed sample on the right, with the irregularity seen on the left edge of this sample simply created by material flowing around a thermocouple in contact with the surface. Since the test was terminated at $23 \%$ instrument strain, much less than the strain measured from the sample, the constant load of $12 \mathrm{MPa}$, maintained during heating, again clearly caused plastic flow. This large amount of deformation further supports the assertion that these carbide particles are not in a rigid interconnected structure, but are independent and free to rearrange at this temperature.

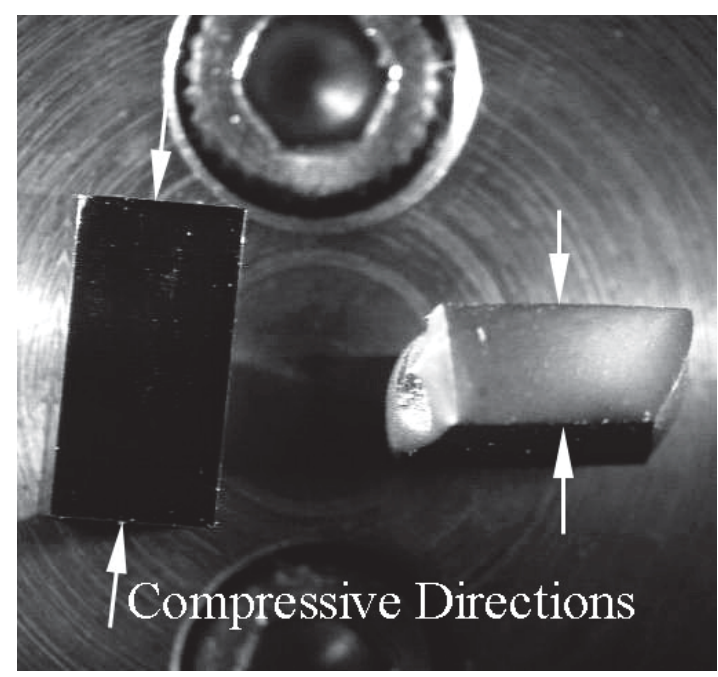

Figure III-59 Macro Photomicrograph of $900{ }^{\circ}$ C Compression Sample 20/1000/RA (right) and a Untested Sample (left) with Loading Direction Vertical

A compressive specimen was cut from the superplastically deformed 20/1000/RA sample. Due to the small amount of material the resultant sample dimensions were 1.7 $\mathrm{mm}$ X $2.4 \mathrm{~mm}$ X $4.2 \mathrm{~mm}$ high. The sample was tested in compression at room temperature with the same parameters as all other tests. The $0.2 \%$ yield strength was 1.76 GPa, with a UCS of $2.02 \mathrm{GPa}$, while the strain to failure was $\sim 6 \%$, along with a SHR of $7.7 \mathrm{GPa}$. Comparing these results to the compressive data at room temperature of the original 20/1000/RA sample, the yield strength only decreased by $\sim 8 \%$ with a smaller difference in the UCS. These results support the idea that these samples can 
undergo a superplastic forming operation, while still maintaining most of the high initial strengths. However, it is not clear how the microstructure changes as it might be expected that the position of the complex carbide platelets are influenced by the applied stress direction. Additionally, the strain to failure is a bit less $(6 \%$ versus $8 \%$ in original sample), while the SHR at 7.7 GPa is larger than the original $4 \mathrm{GPa}$ for the 20/1000/RA sample. One interpretation of these interesting results is an increase in dislocation density arising from the superplastic deformation, which is supported by the higher SHR and lower strain to fracture, with a possible combination of a change in platelet microstructure and a growth mechanism causing the lower yield strength.

\section{d) Resonant Ultrasonic Spectroscopy (RUS)}

Young's and shear moduli along with Poisson's ratio were measured by means of RUS on the Cr9, 20/1000/MA, and 40/1000/RA samples; the results are presented in Table III-25. The Cr9 sample was found to give values similar to those reported for the $\mathrm{Al}_{66} \mathrm{Cr}_{9} \mathrm{Ti}_{25}$ chromium-modified titanium trialuminide by Nic [116]. Because of a lack of adequate material from the original 20/1000/MA sample, measurements were made on a sample which was processed and consolidated in the exact same manner as the original 20/1000/MA.

Table III-25 RUS Measurements

\begin{tabular}{|c|c|c|c|}
\hline Sample & $\begin{array}{c}\text { Young's Modulus } \\
\text { (GPa) }\end{array}$ & $\begin{array}{c}\text { Shear Modulus } \\
\text { (GPa) }\end{array}$ & $\begin{array}{c}\text { Poisson's Ratio } \\
\text { (GPa) }\end{array}$ \\
\hline $\mathrm{Cr} 9$ & 184 & 75 & 0.224 \\
\hline 20/1000/MA & 197 & 81 & 0.214 \\
\hline 40/1000/RA & 208 & 86 & 0.199 \\
\hline
\end{tabular}

Both the Young's and shear moduli increase with increasing RMM time (and therefore, carbide content), while the Poisson's ratio decreases. The near-linear change in Young's and shear moduli with carbon content is shown graphically in Figure III-60. However, it is evident by the better fit with a quadratic regression over a linear regression 
that there is a slight curvature to the data. The Poisson's ratio also shows more curvature to the behavior with carbon content shown in Figure III-61, along with a quadratic regression. The 20/1000/MA sample has both complex carbides while the 40/1000/RA only has the 312 complex carbide. Also the microstructures are slightly different with a more interconnected carbide structure in the 40/1000/RA sample. The conclusion drawn from the results is that the elastic constants and Poisson's ratio of the material change with carbon content and therefore, the carbide content, which is determined largely by the RMM time. These increases in moduli with increasing carbide content reflect the increase in the "stiffness" of the material as a function of increasing carbide content.

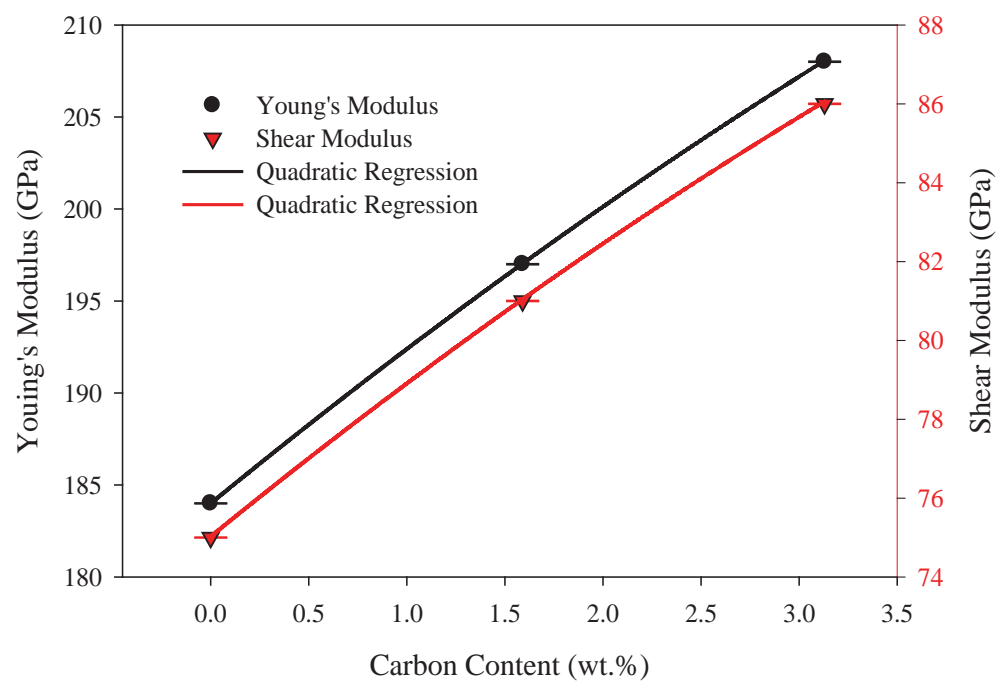

Figure III-60 Young's and Shear Moduli Change with Carbon Content

It can be noted that the 40/1000/RA sample has a modulus very similar to low carbon and low alloy steels (207 GPa); however, the Poisson's ratio is about two-thirds that for steels (0.30) [146]. The Young's modulus reported for the $\mathrm{Ti}_{3} \mathrm{AlC}_{2}$ material is $289 \mathrm{GPa}$ [147], and that for the $\mathrm{Ti}_{2} \mathrm{AlC}$ material is $277 \mathrm{GPa}$ (determined by RUS measurements) [148]. Additionally, the Poisson's ratio determined by RUS is 0.2 for $\mathrm{Ti}_{3} \mathrm{AlC}_{2}$ and 0.169 for $\mathrm{Ti}_{2} \mathrm{AlC}[148]$. 


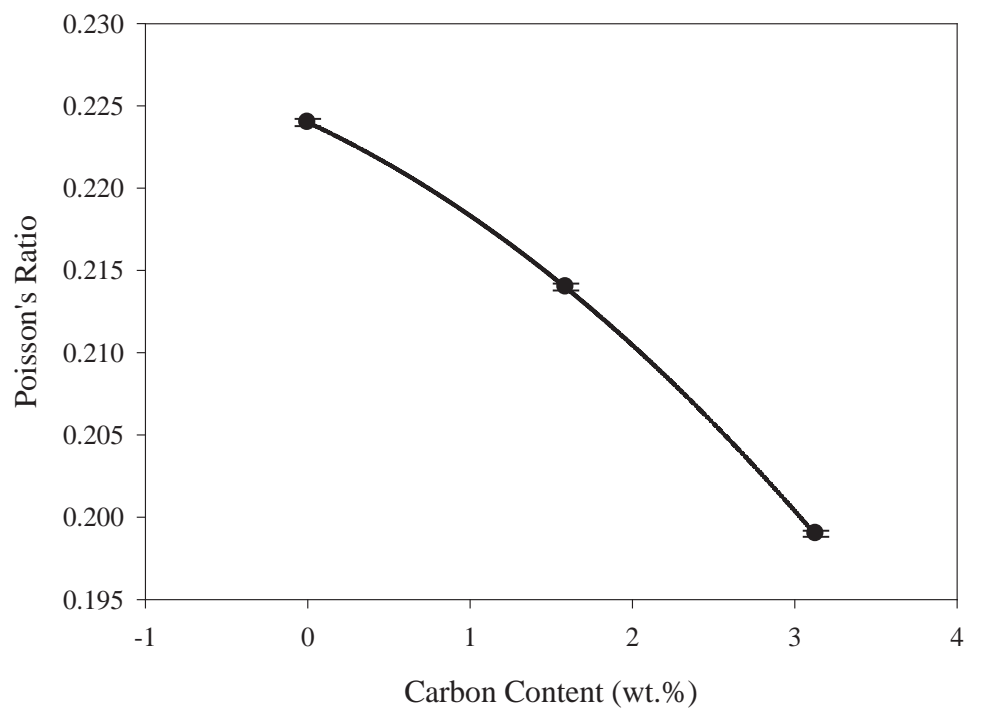

Figure III-61 Poisson's Ratio Change with Carbon Content

\section{e) Coefficient of Thermal Expansion (CTE)}

The CTE of a material can be a very important parameter, particularly when considering the material for coating purposes. Dewald et al. [19, 20, 149] have established that the $\mathrm{Al}_{66} \mathrm{Cr}_{9} \mathrm{Ti}_{25}$ chromium-modified titanium trialuminide alloy can serve as a very effective coating material on a variety of substrates. Additionally, preliminary work using a scaled-up version of the RMM process as a thermal spray coating has established improved wear and abrasion using a low carbide content material similar to 20/1000/MA sample [150].

The results presented in Table III-26 are the average of two CTE determinations at $75^{\circ} \mathrm{C}$ for each sample. The $\mathrm{CTE}$ for the $\mathrm{Cr} 9$ sample represents a value for the singlephase $\mathrm{L}_{2}$ intermetallic, while samples 20/1000/RA and 40/1000/RA (RMM times of $20 \mathrm{~h}$ and $40 \mathrm{~h}$, respectively) are materials with increasing carbide content. There is a systematic decrease in the CTE with increasing carbide content. This behavior is shown in Figure III-62 for the CTE as a function of the volume fraction of $\mathrm{Ti}_{3} \mathrm{AlC}_{2}(312)$ carbide, along with a quadratic regression of the data showing the nonlinear behavior. The CTE decreases by approximately $6 \%$ for the 20/1000/RA sample and by approximately $15 \%$ for the 40/1000/RA sample from the value of the $\mathrm{L}_{2}$ intermetallic 
given by the $\mathrm{Cr} 9$ sample. More importantly, the functional behavior suggests the decrease in the CTE for these samples would become progressively larger with increased carbide content, approaching the value for the carbide, which for $\mathrm{Ti}_{3} \mathrm{AlC}_{2}$ is $8.8\left(\mu \mathrm{m} / \mathrm{m}^{\circ} \mathrm{C}\right)$ [147] while that for $\mathrm{Ti}_{2} \mathrm{AlC}$ is $9.62\left(\mu \mathrm{m} / \mathrm{m}^{\circ} \mathrm{C}\right)$ [151]. It is interesting that many steels, CTE's from 12.3 to 10.8 [146], as well as titanium-base and nickel-base alloys, would be in the range of compatibility with CTE obtainable with these alloys.

Table III-26 CTE Results Select Consolidated Samples

\begin{tabular}{cc} 
Sample & $\begin{array}{c}\text { CTE } \\
\left(\mu \mathrm{m} / \mathbf{m}^{\circ} \mathbf{C}\right)\end{array}$ \\
\cline { 2 - 2 } Cr9 & 12.3 \\
\hline 20/1000/RA & 11.6 \\
\hline 40/1000/RA & $\mathbf{1 0 . 5}$
\end{tabular}

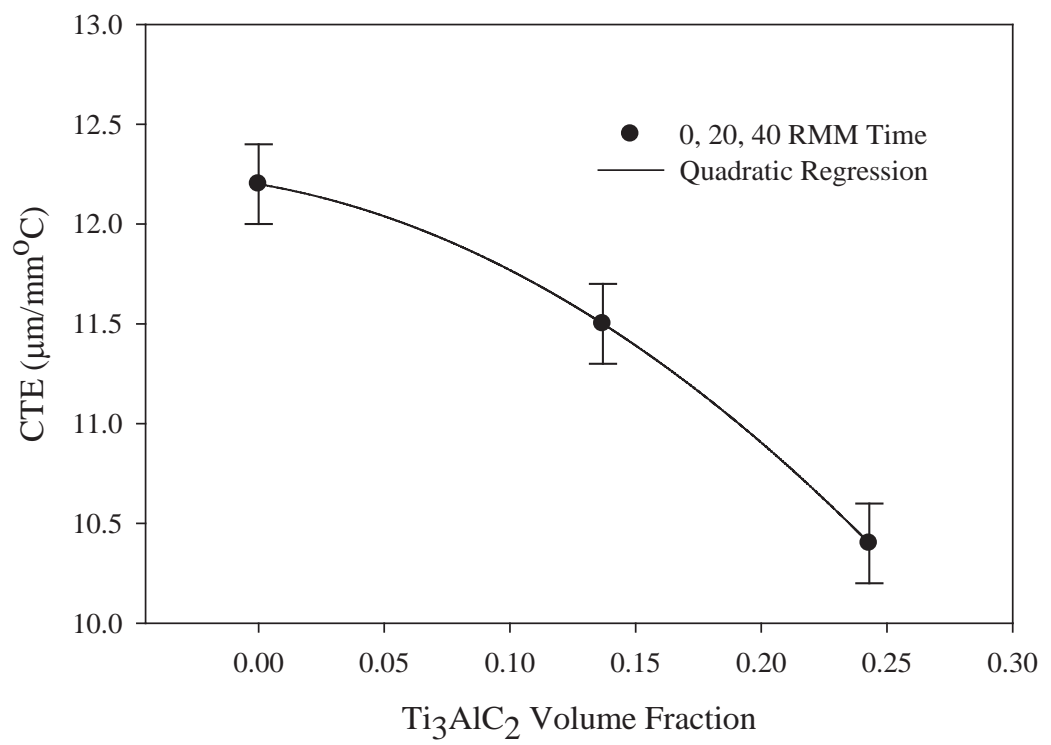

Figure III-62 CTE as a Function of Volume Fraction of Carbide and Therefore Carbon Content 


\section{Discussion}

Feynman in his lecture "There's Plenty of Room at the Bottom" [152] December $29^{\text {th }}, 1959$ pondered the idea of size reductions down to the Angstrom $(0.1 \mathrm{~nm})$ level and how this would dramatically change our way of life. For example, by reducing the Encyclopedia Britannica by 25,000 times would allow all pages to be printed on the head of pin allowing for easier data transfer. He also reminded us of the fact that DNA contains massive amounts of data on an extremely small scale. Feynman discussed creating scaled-down machines that would then create more scaled-down machines thus creating a parallel arrangement to manipulate on the atomic scale; this has been done on a rudimentary scale by IBM with a specially built scanning tunneling microscope [153]. Additionally, recognizing that as the size of particles approaches various limits changes in the behavior will ensue; for example, as particles become smaller they begin to agglomerate as van der Waals forces begin to dominate causing attraction. The current work has focused on the smallest size microstructural features of a polycrystalline material and contemplated how that size relates to our understanding of defects in relation to properties.

In the materials world Gleiter in 1989 [42] suggested that as the grain size of materials decreases to the smallest sizes known to exist a new structural arrangement must occur simply because of the large volume of atoms that now occupy the grain boundary (GB) region; and he further postulated that materials will have new properties based on these structural changes. Several researchers have observed such changes to the typical GB structure with reductions in the grain size. However, the structural details of the GBs are controversial, with one school of thought based on structural characterization of the GB structure by XRD suggesting a "gas-like" GB structure (Gleiter et al. [56]), while others (Eastman and Thompson [154]) counter, that the GB structure is similar to that in large-grained polycrystalline materials. In a discussion of concerns and issues in such nanocrystalline materials Suryanarayana [57] suggested that the latter idea favoring GB structures similar to large-grain size polycrystals has gained widespread acceptance with a more thorough treatment of solidification-developed type structures by Seigel and 
Thomas [155]. The current research provides evidence of a different GB structure and defect arrangement through development of a quantitative measurement technique to measure the volume fraction of these atomic arrangements in the GB regions in nanoscale grain size materials, those reaching the smallest expected grain size for a polycrystalline material. It is argued that these structural changes in GBs can be correlated with observed behavioral changes in materials with grain size reduction, particularly as a result of severe deformation processes.

It has been demonstrated that the reactive mechanical alloying/milling (RMA/M) process allows for simple processing changes to control the reaction between metallic powders and environmental elements in essentially atomic scale processes occurring at room temperature. This includes creation of hard particles at the smallest size that crystalline materials can exist. This reaction is based on dissociation of organic molecules, mass transport, and nucleation in a solid at essentially room temperature. Detailed macrostructural and microstructural analysis has led to potential mechanisms to explain this mass transport that gives rise at room temperature to the reactions occurring during the RMA/M process. Importantly these ideas can be applied to other materials phenomena near room temperature and requiring mass transport.

This new processing technique of RMA/M applied to $\mathrm{L}_{2}$ intermetallic system can yield hard particle reaction products, thus generating a bottom-up approach to dispersoid synthesis at room temperature. In addition, the study of these RMA/M processing effects on as-milled powders has provided a new understanding of the mechanisms involved in the mechanical alloying process. Finally, consolidated bulk specimens from the powders created with this new processing approach provide not only incredible strength levels, but demonstrate the simple ability to nano-engineer a variety of composite powders that can be utilized in consolidation processes with the ability to retain much of the fine-scale structure.

Early focus on polycrystalline materials detailed the understanding of behavioral mechanisms in large-grain size materials; however, as the grain size decreased these behavioral mechanisms became less well understood. For example, details of several materials phenomena that occur at the nano-scale level are lacking, such as the mass 
transport in mechanical alloying (MA), the wear process at the point of contact, the severe deformation introduced into metal filings in a single event, details of cold welding, deformation mechanism changes, and reduction of superplasticity temperature with decreased grain size. All of these have some relationship with extremely small grain sizes; i.e. those near the lower limits of grain size for polycrystalline materials. Perhaps it is time for some new ways of viewing the behavior of these extremely fine particle materials. Three primary results that have emerged from this research are the ability to create composite materials with any volume fraction by manipulating simple RMA/M processing parameters, secondly, an improved understanding of the substructures of severely deformed nano-scale materials, and finally, a new approach to understanding the large amount of mass transport that occurs during the mechanical alloying process.

\section{A. Nano-Scale Polycrystalline Materials Produced by Severe Deformation}

While the primary goal of engineering the microstructure of materials based on simple processing changes was realized, the detailed structural study of the as-milled product from the RMA/M processing led to several basic questions concerning the structural changes and behavior of materials as they approach their smallest size limits. Recall Milligan [77] divided polycrystalline materials into four grain size regimes; largegrain size materials those with grain sizes above about a micrometer, sub-micrometer grain size materials those down to $30 \mathrm{~nm}$, the "Nano-2" grain size between about 20 to 30 $\mathrm{nm}$, and lastly "Nano-1" below $10 \mathrm{~nm}$ whereby GB plasticity dominates. While much agreement exists for large-grain size materials about grain boundary (GB) structure and deformation mechanisms, as the grain size is reduced there is much less agreement with regard to the GB structure and associated deformation mechanisms especially as the grain size reaches the nano-scale (recall this involves single-digit nanometer sizes). Importantly in this grain size regime the controversial inverse Hall-Petch behavior was observed. The nano-scale range achieved in this work certainly coincides with controversial areas of grain boundary structure and deformation mechanisms that permit ductility at these small sizes, and mechanisms to achieve this GB-mediated plasticity will be suggested. 


\section{Structural Characterization of the Nanometer-Scale Powders Produced by the RMA/M Process}

As the structural features of deformed materials reach into the nano-scale range characterization becomes more difficult especially by direct observation techniques. Beyond imaging resolution limitations, the very small volume of material that can be analyzed and the fact that removal of three-dimensional constraints may alter structural features also create difficulties. X-ray powder diffraction (XRD) analysis is essentially a two-point probe method of summing the scattering from every pair of atoms in a significant sized diffracting volume, thus providing an average of the structural aspects of the material in a three-dimensional environment form. A major difficulty with XRD analysis is the diffraction peak broadening arising from both the small crystallite size and the large microstrains characteristic of these materials, which are of interest, can be obscured by the presence of multiple phases which result in highly convoluted diffraction patterns that are difficult to analyze. This problem was addressed here with a combination of intelligent profile fitting (Chapter II.A.5.a)(2)) whereby the peaks for all phases were modeled with Pearson VII functions in appropriate positions dictated by phase analysis, and synthesis of these diffraction peak models allowed the detailed XRD characterization from the structural analysis of the Bragg diffraction peaks.

The discovery of the significant systematic increase in XRD background scattering with RMA/M time coupled with the peak broadening information provided important details concerning the extent and the atomic-scale structure of grain boundaries (GBs) in nano-scale grain size materials. Specifically, modeling the change in background scattering led to developing a new quantitative analysis technique describing the source of this new scattering. This structural model of large GB regions in these nanoscale materials provides for effective mass transport that contributes to properties and process. Combined with the surface studies investigations of the as-milled powders the enhanced mass transport makes it possible to postulate new mechanisms important to the MA and RMA/M processes. 


\section{a) XRD Background Intensity Analysis}

Typically, diffraction effects observed from small crystallites with associated large microstrains show a marked decrease in the maximum diffracted peak intensity with greatly increased broadening. However, the integrated diffraction peak area representing the diffracted energy should remain constant as the broadening develops provided the diffraction occurs from a similar volume of atoms. Careful examination of the diffraction patterns for the original experiment and the RMMXh9TiAr Series of experiments show a clear trend of increasing background scattering with RMA/M time along with an associated decrease in Bragg scattering, Figure III-20, and Figure III-21. While phase changes would appear to complicate this issue, detailed analysis shows that the trend still remains. The origin of these changes in background scattering will be discussed along with its potential implications for the subsequent quantitative $\mathrm{x}$-ray diffraction calculations. XRD patterns (Figure IV-1) for a sample RMM for $25 \mathrm{~h}$ along with the same sample annealed at $1000^{\circ} \mathrm{C}$ for $2 \mathrm{~h}$ in a vacuum demonstrate the two fundamental aspects of XRD patterns for multiphase materials with grain sizes in the nano-scale regime; overlapping diffraction peaks and elevated background intensity.

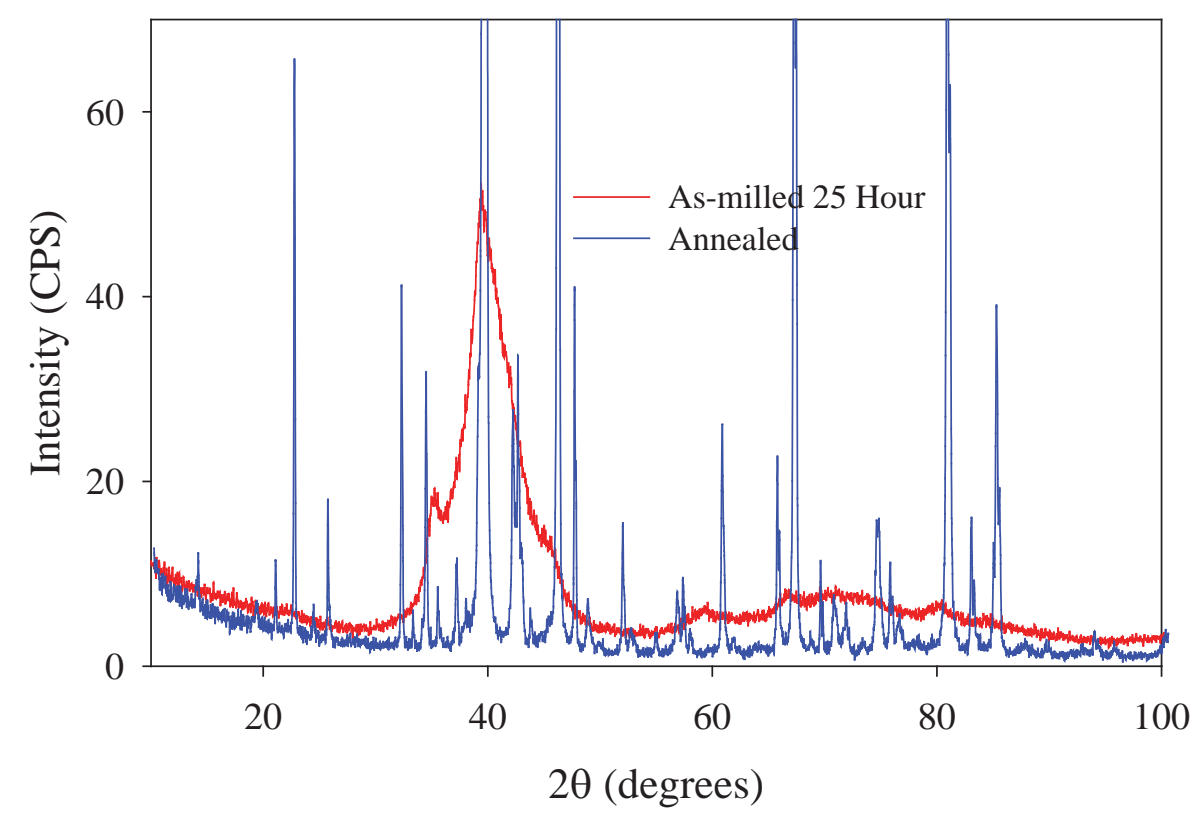

Figure IV-1Comparison of As-Milled Powder to Annealed As-Milled Powder 
Various reasons can be cited for changes in the background intensity of diffraction patterns that are usually associated with a compensating change in the Bragg (or peak) diffracted intensity. One possible contribution to background scattering is that arising from the introduction of an amorphous phase; i.e. a phase characterized by shortrange order of atoms only over a few interatomic distances, but with the atoms randomly arranged over longer distances. X-ray scattering from amorphous phases produce broad modulations in the background that are centered on specific $2 \theta$ values according to the interatomic distances. The x-ray observations for the samples examined did not show any discernible amorphous-type scattering. More importantly, pair distribution function (PDF) analysis, typically used to characterize amorphous structures, was applied to the original experiment with all atomic distances observed attributed to crystalline phases indicating no detectable amounts of an amorphous phase.

Other sources of diffuse background scattering that depend on the nature of the sample include the Laue Monotonic and Huang scattering. The Laue Monotonic is a direct result of the presence of two or more elements. Thus, this diffuse scattering for a random distribution of these compositional differences produces a smooth continuous decrease in intensity with $2 \theta$ angle. Huang scattering [156] arises from the strain fields around defects like vacancies and dislocations as well as around solute atoms [157] and produces diffuse scattering only very near the Bragg peaks.

Wooster [158] considered two groups of effects giving rise to diffuse scattering from crystalline materials; one from thermally excited elastic waves, and the other from static atomic arrangements. Thermally excited elastic waves cause diffuse scattering very near to Bragg diffraction peaks (typically on the tails of the peaks); whereas static atomic displacements can create diffuse scattering far away from the Bragg diffraction peaks. Lattice imperfections of various kinds also contribute to diffuse scattering [118] and would be considered as sources arising from static atomic displacements. Because the diffuse scattering observed in the current study generally results in background intensity increases throughout the $2 \theta$ range, the observed increase in diffuse scattering from the asmilled powders has been attributed to random static atomic displacements. 
In general structural terms, it has been common to consider that two basic types of scattering can occur from solid materials with two or more atom types; one arising from local order, and the other from long-range order. It should be pointed out that with multiple atom types short-range order can also be associated with clustering of like neighbors and local ordering of unlike neighbors; however, these would give rise to modulated scattering and will not be considered further. The third type of arrangement while typically only observed in a gas, results in random arrangement of the atoms. These three fundamental x-ray scattering effects are illustrated in Figure IV-2 by the interference functions for various interatomic distances in each of these types of atomic arrangements for a single element system. Assuming simple structures and single element configurations Figure IV-2 (a) shows the specific interatomic distances delivered by the interference function for a crystalline material with long-range order, while (b) shows the corresponding interference function for an amorphous version of the material having only short-range order. The interference function shows the characteristics of the diffraction patterns of the respective materials. Figure IV-2 (c) shows the same probability function for a structure devoid of long-range or short-range order (i.e., a perfect gas).

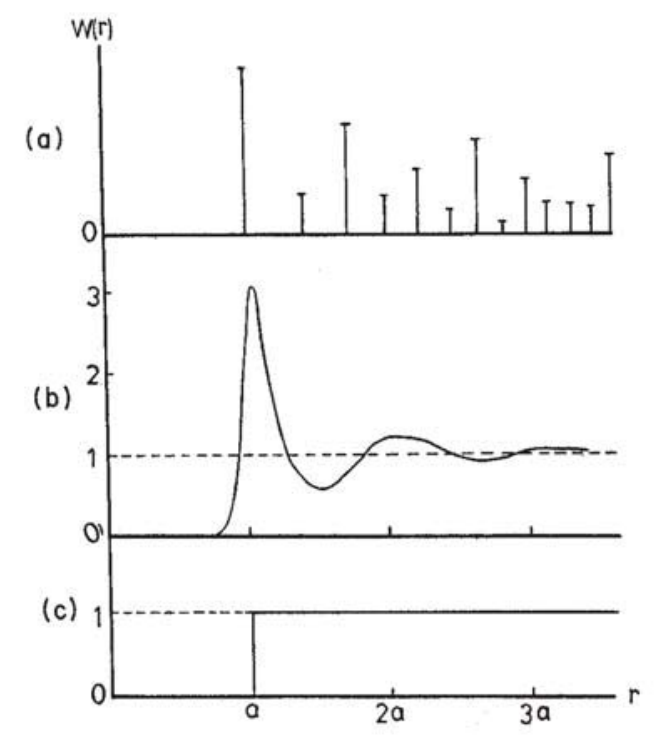

Figure IV-2 Interference Functions W(r) for interatomic distances. (a) Long-range ordered crystalline structure; (b) short-range ordered amorphous structure; (c) random neither long-range nor short-range ordered structure [159]. 
In general, the interference function for scattering by a collection of atoms is represented in Equation (IV.1) based on the Debye formula where $x_{m}$ are the interatomic distances summed over $m$ atoms, and where the $r$ vector is defined in Equation (IV.2) for x-ray powder diffraction. Given the actual solid-state structure, the idea of a completely random gas-like interatomic distances is not spatially possible based on the finite size of the GB region on the order of a nanometer. The observed background scattering changes in Figure III-19 represent a scattering similar to a gas-like arrangement of constant changes throughout the $2 \theta$ range. However, this difference becomes less at lower angles, hence greater atom distances, and slightly greater at higher angles, or smaller atom distances. Thus this increased scattering can best be described generically by random static displacements of the atoms.

$$
\begin{array}{r}
W_{r}(r)=1+\frac{2}{N} \sum_{m}\left(1+\frac{\sin 2 \pi r x_{m}}{2 \pi r x_{m}}\right) \\
r=\frac{2 \sin \theta}{\lambda} \quad \text { (IV.2) }
\end{array}
$$

Experimentally, several researchers have noted increases in the x-ray background intensity for nano-scale materials; however, they have provided only a variety of qualitative explanations. For example, Gleiter et al. [56] reported an increase in the background scattering for a compacted "vapor phase" iron material of $\sim 6 \mathrm{~nm}$ grain size and attributed this scattering to a "gas-like" arrangement of the atoms at the GBs. The volume fractions of these gas-like arrangements of the atoms was estimated to be about $50 \%$.

Later, the Gleiter research group [159] presented some x-ray diffraction work in which the interference function was calculated for $6 \mathrm{~nm}$ iron crystals and compared with data collected for a sample of iron with a similar grain size. The calculated interference function was based on cube-shaped BCC iron crystallites with an interfacial component of four layers of atoms using two different structures for the atoms within these layers. One case assumed short-range order of the interfacial layer of atoms with the other assumed randomly displaced atoms (no short-range or long-range order). The best 
correlation between the observed interference function and the calculated interference function resulted from assuming randomly displaced atoms in the boundary layer.

In further study by the Gleiter group [160], the GB arrangement of the atoms in both nanocrystalline iron and copper was explored using the extended $\mathrm{x}$-ray absorption fine structure (EXAFS) method. Looking at the first oscillation in the EXAFS spectrum allows estimates of GB atoms of between 10 - $30 \%$ for a crystallite sizes ranging from $10-24 \mathrm{~nm}$, with about $30 \%$ reduction for the $10 \mathrm{~nm}$ grain size and about $10 \%$ for $24 \mathrm{~nm}$ grain size, showcasing the increase in GB atoms with grain size reduction.

Eastman and Thompson [161] characterized nano-scaled materials by combining $\mathrm{X}$-ray diffraction and computer simulation, and challenged the results by the Gleiter group. In the Eastman study, patterns were recorded for nanostructured palladium with a crystallite size between 5 - $7 \mathrm{~nm}$ and these were compared with computer simulated diffraction patterns. The materials were created by an evaporation-solidification process that produced $5 \mathrm{~nm}$ grain size powders that were cold pressed at $1.4 \mathrm{GPa}$ to form a compact $9 \mathrm{~mm}$ in diameter with $70-80 \%$ density with a $\{111\}$ texture. It should be noted that in the as-milled powder in the current study the density difference with a fully consolidated materials is only 2 - $4 \%$; therefore a much smaller void volume.

Additionally, the sample in the Eastman work was mounted on a glass slide which produced artifacts in the diffuse scattering. The calculation was based on the kinematical diffracted amplitude using a one-dimensional structure for simplicity. They concluded that the random displacement of the atoms in the GB regions alone could not account for the observed decreases in peak to background ratio, but that voids in the grains, or a combination of voids in the grains and random displacements of the grain boundary atoms, could account for these changes. There was no observation of positional changes to the diffraction peaks.

In these studies by the Gleiter et al., and Eastman and Thompson, there was general agreement concerning a reduction of the peak to background ratio with decreasing grain size in these single-phase elemental nanocrystalline materials. Further, the agreement extended to the conclusion that the diffuse background intensity increase originated from GB atoms. However, the arrangement of the atoms in the GB was still 
uncertain. Of interest to the current work are the reported estimates of $10-50 \%$ by volume of atoms located in the GB regions. Also, the studies conclude that the GBs in these materials may be composed of randomly displaced atoms that have neither short-range nor long-range order. One very important observation from the as-milled powders processed by RMA/M in the current study is the quantitative establishment of the systematic increase in background intensity with decreasing grain size.

\section{(a) New X-ray Analysis Procedure: Quantitative Analysis of the Background Scattering in XRD Patterns of Nano-Scale Materials}

The analysis technique developed as part of the current study was presented at the Denver X-ray Conference 2007 and subsequently published in The Powder Diffraction Journal [162]. The basis of the analysis lies in the measurement of the background area of the as-milled powder compared to that of an annealed powder to provide empirical corrections in the background scattering caused by other common background scattering sources. It should be noted initially that care must be taken when using absolute intensities of powder patterns for analysis since this approach is fraught with potential errors. Even instrument stability can cause intensity variations. However, the natural decrease in the intensity occurs very slowly with time, hence running consecutive scans of as-milled and same annealed powders ensures they are in the stable region considering the $0.005 \%$ voltage regulation and $0.01 \%$ current regulation. Use of the same optics defines the diffracting volume as well as provides consistent intensity flux. It is important that the sample size be maintained larger than the diffracting volume to ensure a constant volume of atoms scattering. The number of atoms in the volume is controlled by the density, which in a loose powder is defined by the packing density of the powder. Practices needed to insure a nearly constant volume were discussed in Chapter II.A.5.a)(1). In general, great care must be taken to ensure proper sample mounting.

While background scattering is commonly removed empirically, two possible sources of concern to be considered are differences in chemistry and surface area. The chemistries of the as-milled and annealed powders are the same except for small amounts 
of hydrogen (less than a few tenths of a percent) which is the lowest scattering element and would not be to significantly affect the x-ray background scattering. Surface area differences can be another source of background scattering due to atoms on the surface having unsatisfied bonds and hence possible displacements from ideal positions. However, the powders are screened to a similar size mesh and therefore are expected to have a similar surface area.

The detailed characterizations presented earlier have established that the RMA/M powder is composed of a nano-scale grain size $\mathrm{L}_{2}$ intermetallic phase with increasing amounts of titanium carbides (much smaller amounts of titanium hydrides) and an increasing amount of diffuse scattering with grain size reduction attributed to the atomic structure of the GB region. Determining the nature of this arrangement of atoms is vital to providing a detailed understanding of the structure and properties of deformed nanocrystalline materials, particularly in the nano-scale region. Clearly the diffuse scattering observed in the current study arises from some type of random static atomic displacements within the GB region. As a consequence, these GB regions can be treated as a new phase for analysis purposes. During further discussion here these regions will be referred to as nano-grain boundary (or NGB) regions.

\section{(i) Volume Fraction of Nano-Grain Boundaries}

To date there has been no known quantification of the NGB region utilizing $x$-ray powder diffraction analysis. For example, the Gleiter group used changes to the first EXAFS peak to estimate the amount of atoms in these regions, while no quantification was done by XRD. In the current study the measurements of the total crystalline and background intensities, that is, a whole pattern analysis, reflects the actual number of atoms in the random static displacement configuration at the GB regions. The model for this calculation is based on work by Ruland [163] for calculating the weight fraction of the crystallinity in a material that contains an amorphous phase (of the same composition). This is represented by the ratio of the total integrated intensity of the crystalline material to the total integrated coherent intensity (which includes the diffuse background scattering). Equation (IV.3) shows the relationship developed by Ruland 
where $s$ is the reciprocal space vector and the factor $K$ is a correction factor for diffuse scattering contributions from both the thermal diffuse scattering and lattice imperfections. Additionally, a correction for the incoherent scattering must be applied.

$$
X_{\text {crystalline }}=\frac{\int_{0}^{\infty} s^{2} I_{\text {crystalline }} d s}{\int_{0}^{\infty} s^{2} I_{\text {total }} d s} \times K
$$

While the $K$ factor correction, which includes incoherent (or Compton) scattering, thermal diffuse scattering, and lattice imperfections should be applied, the calculation is highly complex and requires several assumptions. An effective empirical correction can be applied by subtracting the background integrated area of thermally annealed as-milled powders from the as-milled powders for each RMM time. Ruland's expression relates to the weight fraction of crystalline material assuming similar composition (and therefore similar density), with the intensities determined by integrating a unit volume over $s$ space. Here the result will be on a volume fraction basis, with the corresponding modified Ruland equation with the applicable integration interval equated to the volume of NGB region within the $\mathrm{L}_{2}$ intermetallic $\left(V_{N G B-L 12}\right)$ as follows:

$$
V_{N G B-L 12}=\frac{\int_{10}^{100} f_{N G B}(2 \theta) d 2 \theta}{\int_{10}^{100} I_{\text {Total }}(2 \theta) d 2 \theta}
$$

\section{(b) RMMXh9TiAr Series Reading Alloy Starting Powder with Fixed Amount of Titanium}

Determination of the NGB region volume fraction will be first illustrated using the RMMXh9TiAr Series (Chapter II.B.1) for several reasons. Most importantly, the less complicated phase structure, particularly for long RMM times, consisting of only the $\mathrm{L}_{2}$ intermetallic, $\mathrm{TiC}$, and $\mathrm{TiH}_{1.92}$. Since the $\mathrm{TiC}$ and $\mathrm{TiH}_{1.92}$ phases form as a result of nucleation and growth events and both are ceramic, the NGBs can be assumed to occur in the $\mathrm{L}_{2}$ intermetallic, with both phases having the similar chemistry. The first step in quantification of the NGB region within the $\mathrm{L}_{2}$ intermetallic is subtraction of the 
annealed x-ray background integrated area from the as-milled $\mathrm{x}$-ray background integrated area, Equation (IV.5), establishing the integrated area of the NGB region designated by $f_{N G B}(2 \theta)$, the numerator in Equation (IV.4). The background functions were previously defined in Equation (III.1). Additionally, corrections such as for LorentzPolarization factor can be applied to the intensity prior to integration. Results of this process are given in Table IV-1 for the RMMXh9TiAr Series of samples.

\section{Table IV-1 Integrated Background Area Correction}

\begin{tabular}{|c|c|c|c|}
\hline $\begin{array}{l}\text { RMM Time } \\
\text { (h) }\end{array}$ & 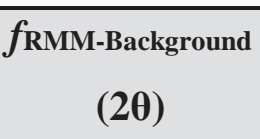 & $\begin{array}{c}f_{\text {Anneal-Background }} \\
\text { (20) }\end{array}$ & $\begin{array}{l}f_{\mathrm{NGB}} \\
(\mathbf{2 \theta})\end{array}$ \\
\hline 0.5 & 217 & 190 & 27 \\
\hline 1 & 235 & 194 & 41 \\
\hline 5 & 281 & 194 & 87 \\
\hline 10 & 308 & 186 & 123 \\
\hline 15 & 322 & $189^{*}$ & 132 \\
\hline 20 & 319 & 193 & 126 \\
\hline 25 & 329 & 190 & 139 \\
\hline 30 & 328 & 182 & 146 \\
\hline 40 & 327 & 185 & 142 \\
\hline
\end{tabular}

* This is an average of all annealed data

The sum of all $\mathrm{L}_{2}$ intermetallic Bragg peak areas comprising the crystalline integrated area with the NGB integrated area within the $\mathrm{L} 1_{2}$ intermetallic designated by the functions $I_{L 12}(2 \theta)$ and $f_{N G B}(2 \theta)$ respectively in Equation (IV.6) establish the value for $I_{\text {Total }}(2 \theta)$ the denominator in Equation (IV.4). The volume fraction of the NGB region within the $\mathrm{L}_{2}$ intermetallic can then be computed with Equation (IV.4) yielding the value of $V_{N G B-L 12}$ based on all Bragg diffraction peaks for the $2 \theta$ range. Results of this calculation are given in Table IV-2. It is important to note that this calculation only determines the volume fraction of the NGB region in the $\mathrm{L}_{2}$ intermetallic and does not determine the NGB region volume fraction in the whole sample [162]. 


$$
\begin{gathered}
\int_{10}^{100} f_{N G B}(2 \theta) d 2 \theta=\int_{10}^{100} f_{R M M-B a c k g r o u n d}(2 \theta) d 2 \theta-\int_{10}^{100} f_{\text {Anneal-Background }}(2 \theta) d 2 \theta \\
\int_{10}^{100} I_{\text {Total }}(2 \theta) d 2 \theta=\int_{10}^{100} I_{L 1_{2}}(2 \theta) d 2 \theta+\int_{10}^{100} f_{N G B}(2 \theta) d 2 \theta
\end{gathered}
$$

\begin{tabular}{|c|c|c|c|}
\hline $\begin{array}{c}\text { RMM Time } \\
\text { (h) }\end{array}$ & L12 Bragg Area & $V_{N G B-L 12}$ & $\begin{array}{c}V_{L 12-N G B} \text { Volume Fraction } \\
\left(1-V_{N G B-L 12}\right)\end{array}$ \\
\hline 0.5 & 331 & 0.08 & 0.92 \\
\hline 1 & 326 & 0.11 & 0.89 \\
\hline 5 & 244 & 0.26 & 0.74 \\
\hline 10 & 196 & 0.39 & 0.61 \\
\hline 15 & 195 & 0.40 & 0.60 \\
\hline 20 & 201 & 0.38 & 0.62 \\
\hline 25 & 203 & 0.41 & 0.59 \\
\hline 30 & 157 & 0.48 & 0.52 \\
\hline 40 & 154 & 0.48 & 0.52 \\
\hline
\end{tabular}

Of particular interest in these results $\left(V_{N G B-L 12}\right)$ is the fact that approximately half of the $\mathrm{L}_{2}$ material is ultimately composed of the NGB region. This agrees well with estimates by the Gleiter group [160] of 10 - $30 \%$ of the volume of atoms associated with the GBs in slightly larger grain size materials. The determination of the total volume fraction of all constituents requires recalculation of the original volume fraction data (given in Table III-2) taking into account the volume fraction of the NGB region. The direct comparison method utilized in the original volume fraction determinations (Chapter III.A.1.c)) are based only on the integrated area from the crystalline Bragg diffraction peaks for all phases. More importantly, the direct comparison method assumes that the volume fraction of the various crystalline phases in the diffraction volume have a sum equal to unity. Therefore, the determination of a significant amount of atoms that 
exist in the NGB region requires renormalization of the original volume fraction data for all the phases present. Essentially this calculation ensures that a "conservation of scattering volume" is not violated as would be the case if these atoms in non-crystalline positions are not taken into account in the x-ray scattering quantification process.

The recalculation of the total volume fraction of all phases, including the NGB region, requires that the sum of all phases, including the NGB region, be equal to one. This calculation will be shown for the RMMXh9TiAr Series of samples for RMM times greater than $10 \mathrm{~h}$ in which case the titanium phase is not present simplifying calculations. However, all RMM times will be recalculated, with the determination for the RMM times of $10 \mathrm{~h}$ and less having the same requirement that the volume fraction(s) for additional phase(s) be included in the sum equal to one and for additional phase(s) simply add a term(s) $V_{\text {phase }} \times X$, where $X$ is the correction factor applied to the original volume fraction data. Again, the formation of the NGB region is assumed to take place only within the $\mathrm{L}_{2}$ intermetallic phase.

Equation (IV.7) takes into account the actual volume fraction of NGB region, $V_{N G B}$, in the total volume fraction sum. The $X$ factor is the correction factor that must be applied to the original volume fraction data given in Table III-2. The actual volume fractions are then the result of the original volume fraction values multiplied by the correction factor $X$. The determination of the volume of the NGB region in the whole sample, $V_{N G B}$, is shown as Equation (IV.8) with the value of $V_{N G B-L 12}$, calculated in Table IV-2; i.e., the volume fraction of NGB region contained only within the $\mathrm{L}_{2}$ intermetallic. The $X$ factor is the same correction factor needed to recalculate the actual volume fractions shown in Equation (IV.7). The values of $X$ are determined by substituting for $V_{N G B}$ in Equation (IV.7) with the expression given in Equation (IV.8) and algebraically solving for $X$. For each RMM time, $X$ is determined, and tabulated in Table IV-3, which enables the true volume fraction of all phases to be determined with those results shown in Table IV-4 for the RMMXh9TiAr Series of as-milled powders.

$$
\begin{array}{r}
1=\left(V_{L 1_{2}} \times \mathrm{X}\right)+\left(V_{T i C} \times \mathrm{X}\right)+\left(V_{T i H_{1.92}} \times \mathrm{X}\right)+V_{N G B} \\
V_{N G B}=V_{L 1_{2}} \times \mathrm{X} \times V_{N G B-L 12}
\end{array}
$$


Table IV-3 Values for Correction Factor X

\begin{tabular}{c|c} 
RMM Time (h) & $\boldsymbol{X}$ \\
\hline 0.5 & 0.94 \\
\cline { 2 - 2 } 5 & 0.92 \\
\cline { 2 - 2 } 10 & 0.81 \\
15 & 0.76 \\
\hline 20 & 0.76 \\
\hline 25 & 0.77 \\
\hline 30 & 0.78 \\
40 & 0.76 \\
\hline
\end{tabular}

Table IV-4 Corrected or Actual Volume Fractions in the RMMXh9TiAr Series of AsMilled Powders

\begin{tabular}{|c|c|c|c|c|c|c|}
\hline & \multicolumn{6}{|c|}{ Corrected Volume Fractions } \\
\hline $\begin{array}{c}\text { RMM } \\
\text { Time (h) }\end{array}$ & $\mathbf{L 1 2}$ & TiC & $\mathrm{TiH}_{1.92}$ & $\mathbf{T i}$ & $\mathrm{Al}_{3} \mathrm{Ti}$ & NGB \\
\hline 0.5 & 0.752 & & & 0.082 & 0.109 & 0.058 \\
\hline 1 & 0.747 & & & 0.074 & 0.096 & 0.083 \\
\hline 5 & 0.704 & 0.049 & 0.009 & 0.053 & & 0.185 \\
\hline 10 & 0.625 & 0.083 & 0.029 & 0.023 & & 0.241 \\
\hline 15 & 0.592 & 0.132 & 0.037 & & & 0.239 \\
\hline 20 & 0.586 & 0.152 & 0.037 & & & 0.225 \\
\hline 25 & 0.551 & 0.170 & 0.054 & & & 0.224 \\
\hline 30 & 0.501 & 0.190 & 0.069 & & & 0.241 \\
\hline 40 & 0.489 & 0.205 & 0.071 & & & 0.234 \\
\hline
\end{tabular}


As expected, the corrected volume fractions show significant reductions from the original uncorrected volume fraction data for the RMMXh9TiAr Series of samples given in Table III-2. The most important volume fraction, that of the NGB region, increases dramatically with milling time reaching a final value of about 0.25 in the sample after 10 h. The corrected volume fractions are shown in Table IV-4. As can be noted, there was a significant amount of the NGB region for the $0.5 \mathrm{~h}$ RMM time.

The error bars associated with the values for the amount of NGB present were propagated errors based on estimates of the ability to resolve and model the background area. The behavior of the amount of NGB follows closely with the microstrain and inversely with the crystallite size; however, it does appear to reach a constant value in a slightly shorter processing time of 10 hours as opposed to $~ 20$ hours from broadening effects. However, the error bars for the crystallite size and microstrain determinations were quite large. A plausible interpretation appears to be that the true grain size remains constant after $10 \mathrm{~h}$ RMM time, but the number of defects around the crystallites increases as a result of the continuing deformation resulting from the milling. It is clear that about one quarter of the material by volume consists of the NGB region for longer RMM times which can have a significant impact on the behavior of the material.

The corrected volume fractions are shown in Figure IV-3 for all phases in the RMMXh9TiAr Series of as-milled powders with the exception of the $\mathrm{DO}_{22} \mathrm{Al}_{3} \mathrm{Ti}$ phase [162] which has disappeared within $5 \mathrm{~h}$. It is suggested that elements from the $\mathrm{DO}_{22}$ $\mathrm{Al}_{3} \mathrm{Ti}$ phase are solutionized and absorbed by the reactions that occur with RMM time. An important change to be noted in comparison with the original volume fraction data (Figure III-15) is the behavior of the $\mathrm{L}_{2}$ intermetallic. The corrected volume fraction of the $\mathrm{L}_{2}$ intermetallic phase decreases systematically with RMM time, such a decrease is expected because the amount of TiC increases exponentially. The original uncorrected behavior in Figure III-15 had an initial increase in the amount of $\mathrm{L}_{2}$ intermetallic at small RMM times followed by a regular decrease. Originally it was thought that this might be attributed to absorption of the elements from the $\mathrm{Al}_{3} \mathrm{Ti}$ phase by the $\mathrm{L}_{2}$ intermetallic. However, this was prior to considering the NGB region, which rapidly 
increases at low RMM times. Once this correction for NGB is made the $\mathrm{L}_{2}$ intermetallic has an expected smooth decrease with RMM time.

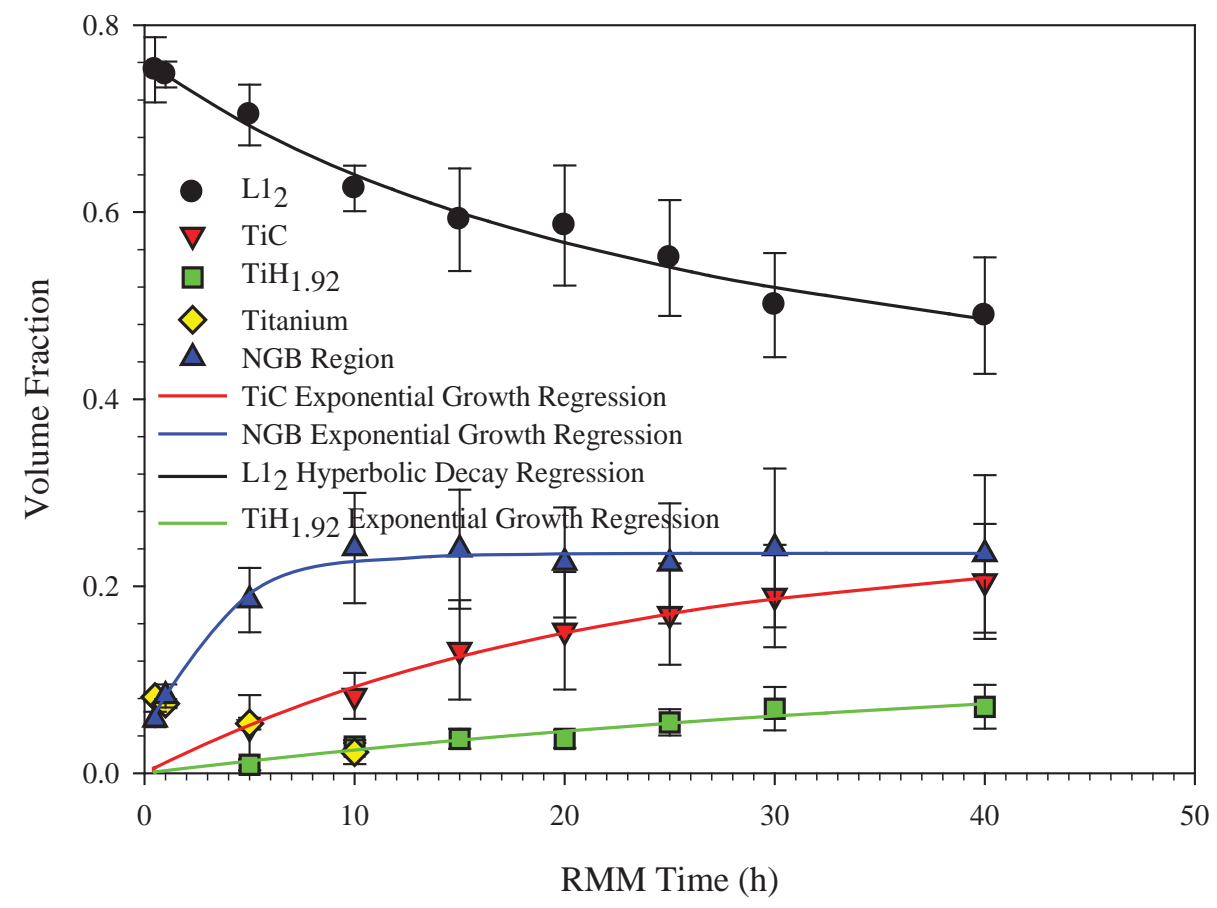

Figure IV-3 Corrected Volume Fraction Data for RMMXh9TiAr As-Milled Powders

Although the corrected volume fractions of titanium, $\mathrm{TiC}$, and $\mathrm{TiH}_{1.92}$ are slightly reduced the behavior is similar to the uncorrected data. The TiC increases with RMM time, but reaches a slightly lower value with longer RMM times. The nature of the change with RMM time is expected given that the amount of titanium available for reaction with the hexanes is fixed (both the added excess and amount in $\mathrm{L}_{2}$ intermetallic). The maximum $\mathrm{TiC}$ volume fraction, after correction, is 0.205 compared to 0.284 in the original calculations, which would change the calculated carbon content retained based on volume fraction data assuming stoichiometry. The loss of titanium was similar to that in the original data except that the titanium volume fraction reached zero at a $13.5 \mathrm{~h}$ RMM time as compared to $15 \mathrm{~h}$ determined with the original data. This agrees with the fact that no titanium was detected in the XRD pattern for the $15 \mathrm{~h} \mathrm{RMM} \mathrm{time.}$ 


\section{(c) RMA20h8.2TiAr Elemental Starting Powder}

The NGB content was also determined for the RMA20h8.2TiAr sample (Chapter II.B.2), which after a $20 \mathrm{~h}$ RMA time consisted of elemental titanium and chromium phases, in addition to the $\mathrm{L}_{2}$ intermetallic, $\mathrm{TiC}$, and $\mathrm{TiH}_{1.92}$. In order to calculate the NGB content it is necessary to determine within which phase(s) the NGB's form. In order to understand the possible range of values for the amount of the NGB region, two corrections will be determined; Case 1 where only the $\mathrm{L}_{2}$ intermetallic contains NGB regions and Case 2 where all ductile phases are assumed to contain the NGB regions. Details of these calculations are available in Appendix VIII.E.

The result of the NGB amount just in the L12 intermetallic, Table VIII-5, for Case 1 shows that 0.197 of the $\mathrm{L}_{2}$ intermetallic is attributed to the NGB region in the RMA20h8.2TiAr powder while Table IV-2 shows the RMM20h9TiAr (RMM $20 \mathrm{~h}$ ) powder has a NGB region volume fraction of 0.38 within only the $\mathrm{L}_{2}$ intermetallic (not the total volume fraction within the powder) which is a $48 \%$ decrease in NGB amount. One reason for this difference is the crystallite size of the RMA material is twice that of the RMM material for the same milling time, which leads to a larger volume fraction of the NGB in the smaller crystallite size. The corrected volume fractions for the total sample are shown in Table IV-5 along with the original volume fraction determinations. In all cases XRD volume fraction analysis of materials with nano-scale grain sizes must compensate for this arrangement of NGB atoms (i.e., new phase) and the associated scattering by this new phase makes it possible to quantify the amount of these NGB regions.

\begin{tabular}{|c|cccccc|}
\hline $\begin{array}{l}\text { Table IV-5 Volume Fractions of Original and Corrected Values for RMA20h8.2TiAr } \\
\text { Sample }\end{array}$ \\
\hline RMA20h8.2TiAr & $\mathbf{L 1 2}$ & TiC & Cr & TiH1.92 & Ti & NGB \\
\cline { 2 - 7 } Original & 0.688 & 0.104 & 0.084 & 0.080 & 0.044 & - \\
Corrected Case 1 & 0.606 & 0.092 & 0.074 & 0.070 & 0.039 & 0.119 \\
\cline { 2 - 7 } Corrected Case 2 & $\mathbf{0 . 6 0 8}$ & $\mathbf{0 . 0 9 2}$ & $\mathbf{0 . 0 7 4}$ & $\mathbf{0 . 0 7 1}$ & $\mathbf{0 . 0 3 9}$ & $\mathbf{0 . 1 1 7}$
\end{tabular}




\section{b) Microstructure}

The microstructure of the as-milled powders is extremely difficult to characterize by conventional means of imaging because of several factors, most notably the nanoscale grain size. While some HRTEM micrographs of nano-scale materials are available, a complicating factor is the nature of these high energy regions making up the GBs may cause alterations of the structure when the grains are no longer in a three-dimensional environment with relaxations expected due to the release of these constraints by specimen preparation. Direct observation was attempted in late stages of this work; however, it was not very useful because of the small grain size that leads to images of numerous overlapping crystallites. Despite these limitations, the microstructure of the RMMXh9TiAr Series of as-milled powders will be summarized based on the average structure information provided by detailed XRD analysis, which provides structural information in the natural three-dimensional environment of the material. While property measurements of the as-milled powders were not possible, the large amount of NGB region is expected to play a major role in determining the properties and behavior of these materials.

\section{(1) RMMXh9TiAr Series of As-Milled Powder}

In this series the initial milling conditions were the focus in milling the Reading Alloy $\mathrm{L}_{2}$ intermetallic with a fixed amount of extra titanium powder of 9 wt. pct. added. It was designated as a milling process instead of alloying process since the $\mathrm{L}_{2}$ intermetallic was pre-alloyed. However, as mentioned there are changes in the amount of titanium in the $\mathrm{L} 1_{2}$ intermetallic shown by lattice parameter changes when titanium is extracted to form TiC well after the elemental titanium is depleted at long RMM times. The Master Alloy and the Reading Alloy appear to have similar carbon uptakes with RMM time when the intrinsic difference in carbon content is taken into account. The Master Alloy starts out with a much larger grain size, shown by very little diffraction peak broadening in this alloy, while the Reading Alloy formed by a rapid solidification

process has a crystallite size of about $30 \mathrm{~nm}$. Both powders were initially screened using 200 mesh so the physical particle sizes are less than $74 \mu \mathrm{m}$. Despite the large difference 
in the initial grain structure of the powders the results from milling appear to be quite similar in terms of the crystallite size and amount of reactant absorbed.

Although a carbon analysis of the RMMXh9TiAr Series was not determined, the RMMXh9TiAr sample milled for $20 \mathrm{~h}$ is comparable on an RMM basis to the powder used in the consolidation of the 20/1000/RA sample. LECO chemical analysis of that material gave a carbon content of 1.88 wt. pct. Given the volume fraction data from the consolidated 20/1000/RA sample, assuming stoichiometry of the phases, yields a carbon content of 1.84 wt. pct., indicating very good agreement between chemical and structural analyses. It should be noted that a similar consolidated sample 20/1000/MA, that was milled for $20 \mathrm{~h}$ using the Master Alloy (hence no initial carbon) had a carbon content of 1.56 wt. pct. Adding the initial carbon content present in the Reading Alloy, 0.25 wt. pct., gives a value of $1.81 \mathrm{wt}$. pct. providing good agreement with that of the 20/1000/RA sample. This suggests that a total of about $1.5 \mathrm{wt}$. pct. carbon is absorbed on RMM processing for $20 \mathrm{~h}$ under the given milling conditions. The goal of the RMMXh9TiAr Series was to provide an improved understanding of the structural evolution during the RMM process with a fixed amount of excess titanium for milling times beyond the target amount expected from the base $20 \mathrm{~h}$ carbon reaction. Larger amounts of $\mathrm{TiC}$ can be formed at longer times, but lack of titanium will limit TiC production.

\section{(a) TiC Phase}

The amount of carbon can also be determined from volume fraction data for the RMM20h9TiAr (20 h RMM) sample assuming stoichiometry of the TiC phase. The amount calculated is expected to be near the measured value $1.88 \mathrm{wt}$. pct. carbon for the consolidated 20/1000/RA sample processed similarly. Therefore, given the estimated volume fractions, Table III-2, for the three phases that are present, $\mathrm{L}_{2}$ intermetallic, stoichiometric $\mathrm{TiC}$, and $\mathrm{TiH}_{1.92}$, a carbon content of 5.37 wt. pct. results. On the other hand, if the volume fractions are corrected to account for the NBG phase, as determined in the previous discussion shown in Table IV-4, a value of 3.88 wt. pct. carbon is obtained. Both of these values are much larger than that measured in the consolidated materials for a similar processing time. 
The reason for this discrepancy is that non-stoichiometric titanium carbide can exist within the limits; $\mathrm{TiC}_{X} 0.47 \leq X \leq 1$ [164]. Therefore, using the minimum carbon needed to maintain the TiC phase occurs for $\mathrm{X}=0.47$, which translates into 2.52 wt. pct. carbon based on estimated volume fractions in Table III-2, meaning that not enough carbon is available to maintain the $\mathrm{TiC}$ phase at those expected volume fractions. However, using the corrected volume fraction data allowing for the NBG phase (Table IV-4), a value of 1.82 wt. pct. carbon is determined, strongly suggesting that the stoichiometry is very near $\mathrm{TiC}_{0.47}$ in the $20 \mathrm{~h}$ as-milled sample. It appears that this lower carbon stoichiometry of the $\mathrm{TiC}$ is common with this process. This result further validates the NGB analysis in the previous section in that the volume fraction data corrected for the presence of the NGB phase provide a more reasonable carbon estimate. Additionally, the lattice parameter of the TiC phase determined for the RMM20h9TiAr sample was $0.430996 \mathrm{~nm}$ in agreement with the value for a $\mathrm{TiC}_{0.48}$ phase of $0.4309 \mathrm{~nm}$, created by planetary ball milling elemental titanium and carbon powders [165]. These results support a non-stoichiometric carbide that exists near the lower carbon limit of its phase stability range.

Microstructurally, the TiC particles appear to be isolated with an apparent spheroidal geometry considering the cubic crystal structure and a crystallite size that is very constant at about $2 \mathrm{~nm}$ for all RMM times, Table III-4. These are among the smallest sized crystalline particles that have been reported in the literature. For example, platinum catalysts are often formed by a bottom-up approach using the platinum-oxide colloidal method that yields extremely small crystal sizes on the order of $2 \mathrm{~nm}$ [54]. Calculation of critical size nuclei during homogeneous solidification of $\mathrm{TiC}$, using the average homogeneous solidification homologous temperature for metals [166], gives a diameter of $2.4 \mathrm{~nm}$ in agreement with the value obtained in the current study. This suggests that the TiC particles are analogous to the nuclei with little or no growth occurring.

The asymptotic approach of the amount of $\mathrm{TiC}$ to an end value in the as-milled RMMXh9TiAr Series is suggested to result from an equilibrium reached between the titanium in the dispersoid and the titanium in the $\mathrm{L}_{2}$ intermetallic. At an RMM time of 
$15 \mathrm{~h}$ the added free elemental titanium is completely consumed, with the titanium needed for $\mathrm{TiC}$ formation then extracted from the $\mathrm{L} 1_{2}$ intermetallic. In fact, the observed changes in the lattice parameter of the $\mathrm{L}_{2}$ intermetallic (Figure III-16) can be explained by these changes in the titanium content. Since these dispersoids are similar in size to the expected grain size of the $\mathrm{L}_{2}$ intermetallic phase, they cannot exist within the grains of the $\mathrm{L}_{2}$ intermetallic hence creating an interesting material that is a nano-scale composite; becoming a new type of dispersoid-based material since the dispersoid is similar to the grain size of the matrix. For completeness, it should be mentioned again that the RMA/M processed powders contain very small amounts of the titanium hydride; however, for simplicity, and noted removal of hydrogen, this phase was not discussed in the current study.

\section{(b) $\quad \mathrm{L}_{2}$ Intermetallic}

This phase goes through extensive grain refinement in response to the severe mechanical deformation of the mechanical alloying process, which reaches the crystallite size asymptotically toward a value near $2 \mathrm{~nm}$. These crystallite sizes (Figure III-17) are among the smallest sizes reported in the literature for crystalline materials. In addition to the grain size refinement, the dislocation density with associated microstrains, increases toward limiting values as shown in Figure III-18 and Table III-3, respectively. It is important to note that the dislocation densities are an order of magnitude larger than those commonly observed in heavily cold rolled metals or shock loaded metals [135]. This extremely fine-scale defect substructure produced by the cold working can be modeled assuming the equiaxed grains to be an ideal polyhedral (e.g., tetrakaidecahedral) grain shape, whereby a $2 \mathrm{~nm}$ grain size translates into the edge length of the GB area facets on the surface of the grain as being about two unit cells in length. It is important to note that with these few atoms on each grain boundary facets, establishment of typical largegrained CSL type GB structure appears geometrically problematic, again suggesting the GB structure must change with grain size reduction. This also translates into a grain volume of about 67 unit cells, or approximately 268 atoms comprising the average crystallite with $27 \%$ of atoms on the surface of each crystallite. 
In a review of mechanical alloying/milling by Murthy and Ranganathan [39] the minimum grain size for HCP metals appeared similar for all elements tested around 14 $\mathrm{nm}$ with a drop to about $8 \mathrm{~nm}$ for the BCC metals tested. In agreement, pure metals with the BCC and HCP structures prepared by $\mathrm{SPEX}^{\mathrm{TM}}$ ball milling produced a minimum grain size in BCC of $9 \mathrm{~nm}$ and in HCP $13 \mathrm{~nm}$ [167]. However, the more ductile FCC phases such as high melting point iridium has a grain sizes around $6 \mathrm{~nm}$ while low melting point aluminum about $22 \mathrm{~nm}$ [168]. Pure ruthenium and an AlRu alloy were SPEX ${ }^{\mathrm{TM}}$ milled for $64 \mathrm{~h}$ with a smaller crystallite size observed in the AlRu alloy relative to the pure ruthenium by about $25 \%$ for each milling time [169]. The crystallite size decreased rapidly up to $1 \mathrm{~h}$ milling time, then the decrease became linear, but at a lower rate with a final crystallite size of 5 to $7 \mathrm{~nm}$ for the AlRu alloy and 11 to $14 \mathrm{~nm}$ for pure ruthenium. Alloys of $\mathrm{Ni}_{3} \mathrm{Al} \mathrm{SPEX}^{\mathrm{TM}}$ milled showed crystallite sizes by XRD calculated from the first two diffraction peaks of about $4 \mathrm{~nm}$ while TEM analysis gave grain sizes of 2 - 4 nm [170]. Planetary ball milling of a mixture of elemental titanium and aluminum (one to one ratio) powders produced $1.5-5 \mathrm{~nm}$ crystallite sizes determined from the width of transmission electron diffractions spots [171]. However, milling of elemental powder blends with stoichiometries of $\mathrm{Al}_{75} \mathrm{X}_{8} \mathrm{Ti}_{25}$ (X various transition elements) in a planetary milling in toluene (PCA) is interesting in that for the longest milling time the alloy with chromium had a crystallite size of about $2 \mathrm{~nm}$ [172]. That is similar to that determined in the RMMXh9TiAr Series for longer times, thus the $2 \mathrm{~nm}$ crystallite size appears to be among the smallest crystallite and grain sizes reported in the literature for polycrystalline materials. Calculation of the critical size nuclei in homogeneous solidification for $\mathrm{Al}_{3} \mathrm{Ti}$, using the homologous temperature for metals [166], resulted in a similar value of a $2 \mathrm{~nm}$ diameter.

These crystallite sizes describe the mean volume of "perfect" single crystal pieces of the $\mathrm{L}_{2}$ intermetallic and the coherent defects present in the specimen. The possible presence of point defects within these volumes is ignored and has not been studied. Additionally, the $\mathrm{L} 1_{2}$ intermetallic retains a significant amount of long range order out to long milling times. There are an extremely large number of dislocations produced in these materials with likely plastic debris accumulation from the severe deformation. As 
has been discussed, dislocations and other linear defects were not observed in the interior of $8 \mathrm{~nm}$ grains during deformation of gold films [173] supporting the idea that these small crystallite volumes are essentially dislocation free. Consequently, the extremely large dislocation density observed in the RMMXh9TiAr samples must reflect the large density of defects that accumulate at the periphery of the perfect crystals. These regions must maintain some coherency with the lattices as these dislocation densities are derived from the diffraction peak broadening essentially separate from the NGB region which produces diffuse scattering. However, the high energy of the NGB region may project strains into the crystallite.

\section{(c) Modeling the Microstructure}

The average microstructure in these materials with nano-scale features can be modeled by creating a representative "unit volume", essentially a microstructural building block of the material analogous to a unit cell. Basing the model on spherical shapes, the analysis provides a simple means to illustrate the sizes of these various regions; the "perfect" crystals, coherent defects, and the NGB region. Considering a spherical unit volume of material, neglecting the presence of $\mathrm{TiC}$ (and the hydride), to represent the matrix microstructure to show the spatial relationships between these three regions (Figure IV-4). Here the crystallite is defined by the radius from the origin $r_{c}$ and the region of the coherent defect structure from $r_{c}$ to the radius of the defective region $r_{d}$. It is important to note that $r_{d}$ is the same as the crystallite size derived by XRD analysis. Surrounding this volume of each coherent matrix particle is the NGB region separating the grains of the polycrystalline material, with the radius $r_{N G B}$ defined as the radius from the center of a grain to the center of the grain boundary region, creating a repeatable unit volume of the $\mathrm{L}_{2}$ intermetallic microstructure. While it is understood that packing of spheres is not exact and that a model using a polyhedral shape is preferred, the result of the simple spherical analysis provides a reasonable estimate since the spherical shape approximates higher order polyhedra such as a tetrakaidecahedron. Currently, this simplistic model will be taken to serve for discussion purposes. 
Equation (IV.9) shows the volume relationships for a unit volume of material, Vol $_{\text {Total }}$, described by the radius from the center of a crystallite to the midpoint of the GB region. The volume of the $\mathrm{L1}_{2}$ intermetallic can be further defined, Equation (IV.10), as the volume of crystalline material (based experimental crystallite size data), with the volume of defects on the periphery of the perfect crystal interior, both contributing to the observed coherent diffraction, or crystalline scattering. These values described by the model can be derived provided the volume of defect structure surrounding each crystallite is known, requiring knowledge of defect densities assuming they occur outside of the perfect crystal interior.

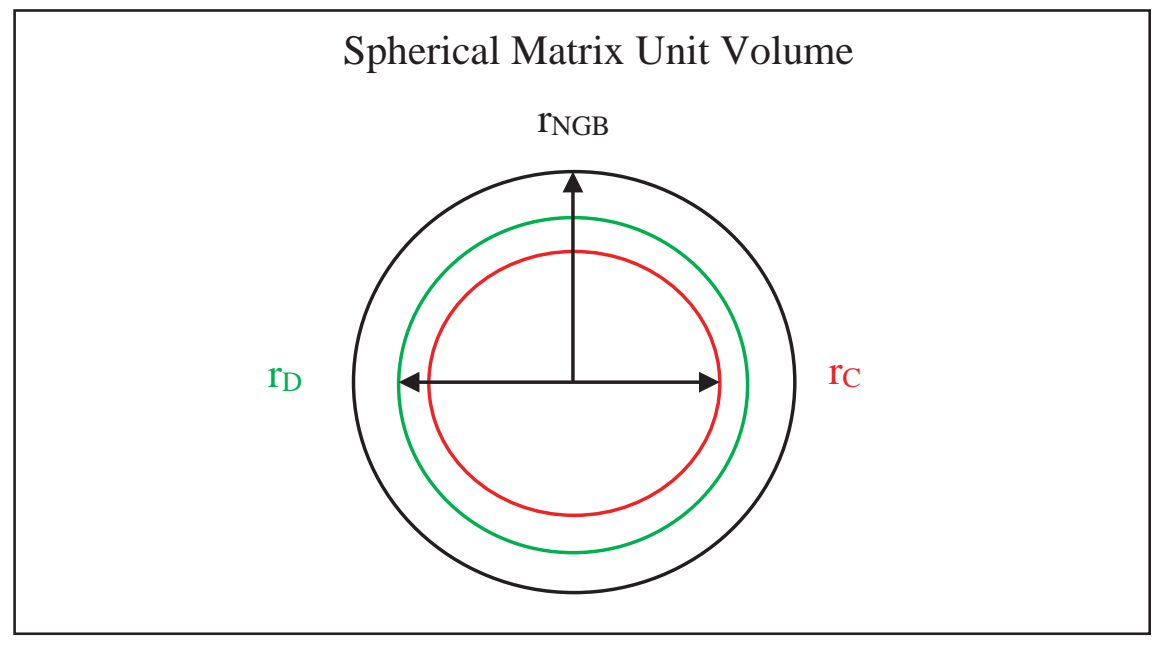

Figure IV-4 Two-Dimensional View of the Spherical Model for an Average Unit Volume of the Matrix - A Fundemental Unit Volume Building Block of the Microstructure

$$
\begin{gathered}
V o l_{\text {Total }}=V o l_{N G B}+V o l_{L 12} \\
V o l_{L 12}=V o l_{\text {Defects }}+V o l_{\text {Crystal }}
\end{gathered}
$$

A major difficulty is determining the volume of this defect region; however, it can be estimated if the core size of a dislocation is estimated, combined with the experimentally determined dislocation density. The dislocation density is a line length of defect per unit volume, as shown in Equation (IV.11), or dislocation intersection per unit area, so that knowing the cross-sectional area of the end of the dislocation allows for determination of the volume of the dislocation line, or area of intersections, from the 213 
experimentally obtained dislocation density $\rho$. However, while the dislocation intersections per unit area and line length per unit volume are equal for parallel dislocations, for a random arrangement the unit volume density is twice the unit area density [141]. Since these defects are expected to be random, the volume fraction of defects would be expected to be twice that of the intersections per unit area.

$$
\rho=\frac{l}{V_{D}}
$$

The experimental data determined is for parallel dislocations so that the final density is twice the value calculated from the dislocation density data. Taking the Burgers vector for an $a<110>$ dislocation as $0.2798 \mathrm{~nm}$, and assuming a dislocation core size of $4 b$, the area of the end of the dislocation can be readily determined as $3.94 \times 10^{-12} \mathrm{~mm}^{2}$. Multiplying this times the dislocation density, $5.4 \times 10^{10} \mathrm{~mm}^{-2}$, or the linear defect length, results in a volume fraction of 0.21 for defects in relation to defect-free crystal, based on a parallel dislocation determination. A more realistic value would be twice this for the expected random defect arrangement [141] or a value of 0.42 , which then by definition is the volume fraction of defects in the $\mathrm{L}_{2}$ intermetallic. Because the range of core sizes commonly suggested can vary from $b$ to $4 b$ and the Burger's vector used here is the largest observed, the value of the volume fraction might be expected to be overestimated.

In the following analysis $\mathrm{Vol}$ is the volume of a specific portion and $\mathrm{V}$ is the volume fraction of that portion, where $V_{o l} l_{L 12}$ is the volume of coherent $\mathrm{L}_{2}$ intermetallic making up the crystallite (the defect and perfect crystal regions). The volume of the

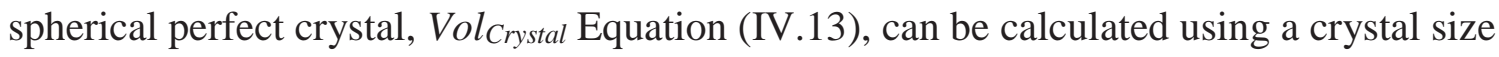
for $r_{C}$. With the determined volume fraction of defects along with undisturbed crystal (the crystallites) and understanding that the defects surround the periphery of the defect-free crystal, it can be surmised that Vol Crystal $_{1}$ is equal to the factors Vol $\operatorname{ll2}_{2} V_{\text {Crystal }}$ shown in Equation (IV.12) and hence the volume of the $\mathrm{L}_{2}$ intermetallic, allowing $\operatorname{Vol}_{L / 2}$ to be determined. Inserting a perfect crystal size of $2 \mathrm{~nm}(r=1 \mathrm{~nm})$, reasonable based on slightly larger crystallite size and minimum grain size expected, a volume of $4.19 \mathrm{~nm}^{3}$ is determined for $V_{\text {ol }}$ rystal with $V_{\text {Crystal }}$ being equal to $1-0.42$ or a value of 0.58 . This results in a $V_{L l} l_{L 12}$ of $7.28 \mathrm{~nm}^{3}$ or the volume of an average coherent portion of a grain of 
the $\mathrm{L}_{2}$ intermetallic. Using this value for the $\operatorname{Vol}_{L 12}$ in the proper equation in Equations (IV.13) allows for the determination of $r_{D}$ or the radius to the interface between defects and the NGB region. In a similar manner, once $V_{o l} l_{L 12}$ is known, Vol ${ }_{\text {Total }}$ can be determined using Equation (IV.9) and Equation (IV.14) knowing the corrected volume fraction data determined in Table IV-4, the value of Vol Total is determined to be $14 \mathrm{~nm}^{3}$.

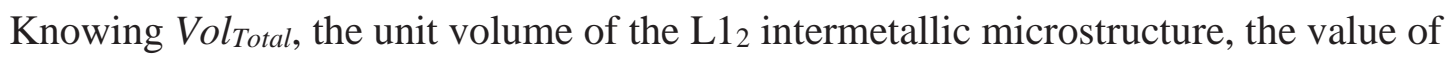
$r_{N G B}$ can be determined using the proper equation in Equation (IV.13).

$$
\begin{gathered}
V_{\text {ol }}=\operatorname{Vol}_{L 12} V_{\text {Defects }}+\operatorname{Vol}_{L 12} V_{\text {Crystal }} \\
\operatorname{Vol}_{\text {Total }}=\frac{4 \pi}{3}\left(r_{N G B}\right)^{3} \\
\operatorname{Vol}_{L 12}=\frac{4 \pi}{3}\left(r_{D}\right)^{3} \\
\operatorname{Vol}_{\text {Crystal }}=\frac{4 \pi}{3}\left(r_{C}\right)^{3} \\
V_{\text {ool }}=\operatorname{Vol}_{\text {Total }} V_{\text {NGB }}+\operatorname{Vol}_{\text {Total }} V_{L 12}
\end{gathered}
$$

Considering only the $\mathrm{L}_{2}$ intermetallic phase as the basis of the model, these radius values can be computed using the corrected volume fraction data determined previously, where the crystalline volume fraction is 0.52 while the NGB volume fraction is 0.48 . Results of this determination using the unit volume representation of the microstructure for a crystallite size and microstrain reaching saturation given in the example of $2 \mathrm{~nm}$ perfect crystal size is provided in Table IV-6 along with relevant factors. Additionally, the crystallite size measured varied between 2 and $3 \mathrm{~nm}$ for longer times with data for the $3 \mathrm{~nm}$ perfect crystal size also provided for comparison. For the $2 \mathrm{~nm}$ crystallite size with a defect radius $1.20 \mathrm{~nm}$ or a $20 \%$ linear increase for a $42 \%$ volume increase. The average atomic radius for a chromium-modified titanium trialuminide unit cell with the $\mathrm{L}_{2}$ structure is $0.140 \mathrm{~nm}$, similar to the atomic radii of aluminum $(0.143 \mathrm{~nm})$ and titanium $(0.144 \mathrm{~nm})$, suggesting the defect region is over one atomic layer thick, two layers in the $3 \mathrm{~nm}$ crystallite size. The NGB layer for the $2 \mathrm{~nm}$ crystallite size is about $0.6 \mathrm{~nm}$ thick whereas for the $3 \mathrm{~nm}$ crystallite size the NGB layer is $0.8 \mathrm{~nm}$ thick, close to estimates of a size of just under $1 \mathrm{~nm}$ in nanometer materials 
[29, 56, 174]. This also agrees with Mössbauer spectroscopy of FCC and BCC metals having grain sizes below $10 \mathrm{~nm}$ showing a GB width of $0.5 \mathrm{~nm}$ and $1 \mathrm{~nm}$ respectively [175].

Table IV-6 Spherical Model Parameters for Determination of the $\mathrm{L1}_{2}$ Unit Volume

\begin{tabular}{|c|cccc}
\hline & \multicolumn{5}{c}{ Model Parameters } \\
\hline Region & $\begin{array}{c}\text { Radius } \\
(\mathbf{n m})\end{array}$ & $\begin{array}{c}\text { Radius } \\
(\mathbf{n m})\end{array}$ & $\boldsymbol{V}_{\boldsymbol{C}} \& \boldsymbol{V}_{\boldsymbol{D}}$ & $\boldsymbol{V}_{\mathbf{L 1 2}} \& \boldsymbol{V}_{\text {NGB }}$ \\
\hline$r_{C}$ & 1.0 & 1.5 & 0.58 & \\
\hline$r_{D}$ & 1.2 & 1.8 & 0.42 & 0.52 \\
$r_{N G B}$ & $\mathbf{1 . 5}$ & $\mathbf{2 . 2}$ & & $\mathbf{0 . 4 8}$ \\
\hline
\end{tabular}

An interesting question to ask is what is the grain size of a material? In largegrained materials the GB is composed of planar facets of atoms in contact, or an atomically thin GB region. The grain size is readily determined by the size of the grain to the GB centers which is basically the facet interface between two grains; although ledges can form distorting the definition of the center of the grain, but in relation to the size of the grain these have little effect on the measurement. More important, the defects are in the grain interior. As the nanometer range is approached NEGB begin to dominate the structure showing a very wavy nature to the GBs along with added extrinsic dislocations providing more uncertainty in the grain size measurement. Additionally, as has been pointed out by Valiev and Alexandrov [176] stress fields that surround the GB region begin to distort measurement of the grain size. The NGBs are also expected to induce strain fields but this has not been defined. As the grain size goes into the nano-scale range the GB thickness and defect region surrounding the grains are now of the order of the grain size further distorting the measurement of the grain size.

The grain size in nano-scale materials is defined by the center to center distance of the NGB region producing an average and repeatable unit volume of the main microconstituent feature consisting of perfect crystals, coherent defect region, and NGB region, that is, incoherent region within the $\mathrm{L}_{2}$ intermetallic phase. This average volume 
assumes constant thickness of the NGB region. However it is quite expected that these NGB regions are not geometrically uniform in width. In HRTEM on nanometer palladium and titanium thin films, similar disordered atom regions of about $0.5 \mathrm{~nm}$ thickness were observed, but disordering was more concentrated at triple-point of the GBs suggesting these triple-points may be more significant [177]. Of importance from this study is the increase in triple-junctions as the grain size is reduced, with a maximum of triple junctions expected in these as-milled materials shown to have a more disordered structure than the planar GBs.

If one considers a close-packed arrangement of these unit volume elements, it is possible to construct the average physical powder particle minus the expected pores and point defects that likely exist. Although the absolute values determined have some limitations, the results still provide useful insight concerning the defects on the periphery of the crystallites. A possible means for accommodating the huge number of defects in these materials is by distributing them over the large surface area created by the extremely small crystallites size. In this manner the thickness of this disturbed region is minimized by the large surface area despite the significant volume of disturbed $\mathrm{L}_{2}$ intermetallic.

\section{Nano-Scale Grain Boundary Structure}

A clear conclusion is that the observed additional diffuse $\mathrm{x}$-ray scattering that occurs can be attributed to the GB regions with the current analysis of the source of this behavior best described by random static displacements of the atoms. These microstructures are produced by severe deformation suggesting that the changes noted in GB structure are signatures of deformation mechanism changes as the material approaches the lower limit of grain size. A hypothesis on how these NGBs develop is presented here based on the severe deformation processes. The proposed model of these regions leads to an important conclusion that the structure of this NGB region is not only primarily responsible for the mass transport observed in MA processing, but also for facilitating the GB-mediated plasticity noted to occur in materials with nano-scale grain 
sizes. It will also be suggested that this GB structure can account for the required mass transport during GB plasticity near room temperature.

Since the suggestion by Gleiter in 1984 that the GB structure of nano-scale materials may have a "gas-like" structure, additional studies of the GB structure of nanocrystalline materials have yielded controversial results. Siegel and Thomas in 1992 [155] looking at solidification structures of nano-scale grain sizes in HRTEM suggested the GBs are similar to those in large-grain size materials. Later Ranganathan et al. in 2001 [177] studied palladium and titanium thin films with HRTEM suggesting that the GB cannot be generalized as "gas-like" structural disorder, but rather that the GB structure is similar to that in large-grain size materials. However, in their work specific interfaces do exist that show large amounts of disorder which was suggested to arise from triple junctions contributing to the overall presence of the observed disorder. Finally, Suryanarayana in 2002 [57] cited the work of Siegel and Thomas [155] and that of Ranganathan et al. [177] and declared that there is wide-spread acceptance that the GB structure in nanocrystalline materials is the same as in large-grain size materials, despite the difficulties of creating a bulk nano-scale structure. It should be pointed out that the average as-milled powder particle size in the current work is close to $10 \mu \mathrm{m}$ and has a grain size of around 2 - $3 \mathrm{~nm}$ so each powder particle can be treated as a version of a bulk material. The powder particles sizes are about 3 orders of magnitude larger than the asmilled powder crystallite sizes which are close to the grain size as was discussed.

In a review of nanostructured materials Gleiter in 2000 [178] maintained that the atomic structure of the GBs differs in boundary density and nearest-neighbor coordination relative to the perfect crystal. Furthermore, the deformation in nanocrystalline materials was successfully modeled with molecular-dynamics where the GBs were assumed to have a high energy highly disordered atomic structure [179]. As the grain size decreased a "strongest size", or maximum yield strength was observed, and as the grain size was further decreased the strength also decreased showing a transition to GB plasticity below this critical grain size. Later in reviewing the deformation of nanocrystalline materials using molecular-dynamic modeling the Gleiter group [180] showed that the grain orientation in nanocrystalline materials (particularly those on the 
lower end of the nanometer range) is random and the GBs are generally of high energy as opposed to the special low energy GBs. Additionally, a radial distribution function calculated for the GB structure was similar to that for a pure amorphous material. They concluded that in the spirit of Rosehain's $[181,182]$ historic "amorphous-cement" model for large-grained materials developed in 1913, and considering the randomly ordered grain orientations in nanocrystalline materials, that the grains in FCC metals are connected by a glassy, glue-like phase.

One other happening that further supports the idea of changes in the GB structure as the grain size decreases has been the acceptance of the NEGB structure suggested by Valiev [183, 184]. The Valiev group in 2012 [185] pointed out the variety of convincing evidence establishing the non-equilibrium state of HAGBs in sub-micrometer to nanometer size range (referred to as ultra-fine grain materials). For example, the NEGB structure acquired more acceptance based on atomistic simulations by Van Swygenhoven et al. [186, 187] who concluded that an important factor is the capability of the nanometer GB to act as a source and sink for dislocations. In a molecular-dynamics simulation of $12 \mathrm{~nm}$ grain sized nickel, when introducing disorder into the GBs found that simulations with these boundary structures resulted in a larger plastic strain in deformation (as well as an increase in effective strength) [188] than obtained with no extrinsic defects in the GB. Tucker and McDowell [189] also observed changes in the deformation behavior in atomistic simulations while exploring the effects of NEGBs. They concluded that the increased free volume of these boundaries alters the structure as well as mechanical behavior including lowering the activation energy for GB sliding via atomic shuffling.

To better understand the evolution of the NGB region, a detailed analysis of the NEGB structure is in order with this being defined as essentially a larger-volume GB with a higher energy than those present in large-grained polycrystalline materials of the same composition [190]. Typically GBs in large-grained polycrystalline materials are very narrow, on the order of an atom in width. Detailed definitions are based on the compatibility of the crystallographic parameters between grains [190]. An NEGB has incompatible crystallographic parameters, whereas GBs possessing compatible 
crystallographic parameters are deemed equilibrium GBs. Of the GB defects leading to incompatibility, GB dislocations are the most studied and fall into two groups; intrinsic grain boundary dislocations (IGBDs) and extrinsic grain boundary dislocations (EGBDs). The role of IGBDs is fundamental in the establishment of crystal geometry for a given set of parameters for a boundary, while GBs composed of IGBDs are equilibrium GBs with regard to crystallographic compatibility parameters. However, EGBDs are not required GB features for compatibility of crystallographic parameters, and as such, are associated with nonequilibrium GBs.

Based on experimental evidence, these EGBDs are generally a consequence of the interaction of lattice dislocations with a GB. A trapped lattice dislocation (TLD) is a special case of an EGBD and these TLDs are considered key components to the creation of an NEGB. Consequently, NEGBs can be identified by an excess number of grain boundary dislocations over what is needed to maintain crystallographic compatibility across the boundary; i.e. the EGBDs. The presence of impurities and alloying elements increases the TLD stability. Therefore, the introduction of new elements and the presence of alloying elements in the current work would seem to favor conditions for stable TLDs.

In severe plastic deformation of metals that produces sub-micrometer grain size bulk materials, the information presented by the Valiev group [191] also showed that the background integrated intensity found with x-ray diffraction increased as the crystallite size decreased. Integrated background area for a $166 \mathrm{~nm}$ crystallite size sample increased $\sim 6 \%$ over that for the large-grained material and $\sim 8 \%$ for an $83 \mathrm{~nm}$ crystallite size. However, they did not detail the source or importance of this background scattering. What is important to note here is that materials with the NEGBs require the existence of EGBDs, suggesting that the accumulation of these defects may well be the source of the diffuse background scattering that is observed. Understanding that the crystallite sizes studied by the Valiev group were between one to two orders of magnitude larger than in the current research, accounts for why the amount of background scattering observed was small. For example, the change in integrated background area in the RMMXh9TiAr Series of samples in the current work approaches an increase of $74 \%$ over the annealed 
powder. Although the area change is large, it does arise from a very small intensity (cps) increase in the background that can be easily ignored if not carefully examined.

In the Introduction it was mentioned that Watanabe [48] described the GB structure change as the micrometer range is approached to be nearly $100 \%$ CSL or low energy GBs, thought to compensate for the added GB volume to minimize the internal energy. Additionally, work by Valiev has shown the GB structure again changes as the grain size is further refined into the sub-micrometer to upper nanometer (referred to as ultra-fine grained materials by Valiev) range detailed by the NEGB structure forming high energy GBs. As the grain size reduces further into the sub-micrometer regime the GB volume is increasing and it appears GB energy also increases, with this relationship not clear. However, the internal energy must increase enhancing the driving force to increase the grain size as is commonly observed in these ultra-fine grain sizes.

The Valiev group [192, 193], interpreted the structure resulting from severe plastic deformation (SPD), developing ultra-fine grained materials, as being composed of NEGBs with a heavily strained outer layer of the grains near the NEGBs. Importantly, dislocations must have existed within the grain interior in these materials with the grains actively participating in the deformation process. However, in the model of nano-scale grain sizes presented in the current work the grain interior is ultimately expected to have little participation in the deformation process indicating one difference between materials with NEGBs and those having NGBs. This distinction can be related to the operation of Frank-Read sources for dislocation generation, which for the theoretical shear strength of the $\mathrm{L}_{2}$ intermetallic computed to be $5 \mathrm{GPa}$, suggests there is essentially no dislocation generation below a grain size of about $4 \mathrm{~nm}$ in the $\mathrm{L} 1_{2}$ crystals. Because the observed crystallite size (or grain size) was established to be at or below this value for long RMM times dislocation motion through the grains may not occur and the crystallite could be viewed as a hard phase in the material.

In 1971 Balluffi et al. [194] concluded that EGBDs can be formed in large-grain size polycrystals by plastic deformation. Later, Wyrzykowski and Grabski [195] pointed out that the GBs becomes a haven for EGBDs under plastic strain, using plastic strain to intentionally introduce EGBDs. Intuitively as the deformation proceeds by defect 
generation and motion the grains are further refined into the nanometer range with most of the deformation activity occurring in the GB regions with the expectation that EGBDs would accumulate in the GBs giving increasingly stronger dislocation interactions. It is suggested here that as these GBs are infused with plastic deformation defects (i.e. EGBDs) until a "limiting" defect density is achieved. This point could well be reached when GB-mediated plasticity begins to dominate, thus not only facilitating the transition to GB plasticity processes, but also providing an environment of enhanced mass transport which combined with the high strain rate in the MA process can lead to the superplasticlike behavior observed on the as-milled powder particle surfaces. Thus, another difference between NGB and NEGB structures could be the defect density reaching a somewhat uniform maximum in the NGB structure and while being highly variable in the NEGB structure found for larger grain sizes. This issue of GB structure and superplasticity is more thoroughly treated in Appendix VIII.E. To summarize, experimental observations in the literature suggest that as the grain size is decreased into the sub-micrometer to nanometer ranges the temperature for initiating superplastic behavior decreases, with GB changes a key to this behavior. It is suggested here that the large volume fraction and structural nature of the NGB regions allows for the room temperature superplastic-like behavior that occurs on the surface of the powder particles under the high strain rates of milling.

The evidence for the accumulation of high densities of EGBDs in the GBs in a random manner is suggested to be shown from an XRD scattering perspective as a significant volume fraction of material with random static atomic displacements in the GB regions. Since a relatively large volume fraction of the NGB structure has developed in these nano-scale grain size materials it is expected that properties typically thought of as structure-insensitive would also change. Recall that as mentioned earlier, changes to these structure-insensitive properties have been observed with materials having the NEGB structure. The NGB structure suggested here brings forth interesting questions about details of these defects and defect arrangements that will require further investigation. At this point, it appears that this high density of defects in the GB regions may be made up of a wide variety of defects and combinations of defects. Included in the 
latter may be disclinations formed from arrays of ordinary dislocations. These would add additional components to the net strains. High resolution TEM by Koch et al. [170] has suggested the presence of disclinations at triple junctions in mechanically alloyed $\mathrm{Ni}_{3} \mathrm{Al}$.

\section{a) Proposed Model of Nano-Scale Materials Produced by the RMA/M Process}

Basic microstructural changes occur as the grain size is reduced into the nanoscale size range initiated by changes in the deformation mechanisms. These include significant changes in the GB structure. Based primarily on XRD analysis, yielding an average structural representation of the three-dimensional native structure, a model of the nano-scale as-milled powders can be described. The microstructure formed in the $\mathrm{L}_{2}$ intermetallic phase of the material having been divided up into regions consisting of the perfect crystal grain interior encircled by a coherent array of defects with both comprising a "crystallite", and all of which are surrounded by the NGB structure. Finally, increasing amounts of $\mathrm{TiC}$ are introduced into the microstructure resulting from reaction of the PCA with the added excess titanium. Here the model discussed will be restricted to the RMMXh9TiAr experimental series with the fixed initial excess amount of titanium providing a volume fraction of $\mathrm{TiC}$ asymptotically approaching 0.20 for the $40 \mathrm{~h} \mathrm{RMM}$ time. Essentially a steady state structure was produced that is less complicated than that at the short times. The model will ignore the $\mathrm{TiH}_{2}$ that is present in small amounts since the hydrogen is readily removed in post powder processing.

The $\mathrm{L}_{2}$ crystallites and the $\mathrm{TiC}$ particles are similar in size and both can be viewed mechanically has "hard" particles that generally do not ultimately participate in further deformation by means of dislocation motion; however, the $\mathrm{L} 1_{2}$ crystallites are expected to participate through grain rotation with rotation of the $\mathrm{TiC}$ particles also possible. The NGB region, having random atomic disorder, makes up a significant volume fraction considered microstructurally as a contiguous three-dimensional phase surrounding these hard "inert" particles and capable of a pseudoplastic mechanical behavior. In this case the NGB region becomes the matrix of the composite material. This pseudoplastic behavior can be of a type observed with fluids where they become less 
viscous as stress is applied. Although such behavior has not been reported in the literature for a solid at low temperatures, it best describes the large deformation behavior of the surface-smeared powder particles. The nature of the surface smearing clearly shows a large amount of plastic flow has taken place in these particles during RMM processing, a superplastic-like room temperature behavior in which GBs and GB diffusion play a large role. The dramatic mass transport enhancement provided by the GBs determined to exist in these nano-scale structures, coupled with the high strain rate of the milling process, effectively lowers the temperature needed to initiate superplastic behavior to room temperature.

This structure acts in a manner analogous to the ball bearing concept in rotational mechanical assemblies with the NGB region now the contiguous matrix material (or race) as having enhanced mass transport with pseudoplastic mechanical behavior, surrounding the hard particles (balls). Although the degree of coherency of the TiC particles is unknown, the $\mathrm{TiC}$ is expected to be in contact with NGB regions of the $\mathrm{L}_{2}$ intermetallic. As will be discussed in the next section, the $\mathrm{TiC}$ is thought to nucleate at triple junctions, or higher order grain nodes, thus surrounded by the NGB region. The result of this microstructure is similar in structure to the classic Rosehain's [181,182] historic "amorphous-cement" model, revisited by Gleiter, with a much larger volume experimentally determined to be associated with the GB region in these nano-scale materials. Rosehain's model from 1913 was first postulated for large-grain size materials and it is interesting how this first simplistic GB model resembles what is determined to develop now in these nano-scale materials.

A two-dimensional idealized schematic microstructural model can be created by cutting through the center of an array of grains with an average size having the idealized tetrakaidecahedral shape thereby creating a series of hexagonal shapes that geometrically fit together, but are separated by the NGB region. However, in the case of the nano-scale microstructure developed, the NGB region is nearly $1 \mathrm{~nm}$ in width adjacent to the coherent defect region at the surface of the crystallite. This is represented in the generated microstructure using a $2 \mathrm{~nm}$ average interior crystal size surrounded by a layer of defects comprising the crystallite as determined in Chapter IV.A.1.b)(1)(c), with the space 
between these regions filled by the large volume fraction of the NGB region. This simplistic average microstructure is shown in Figure IV-5, with the periodic atoms in crystalline grain interiors represented by close-packed planes. The micrograph model mainly represents the various microstructural volume changes; the crystal interior, coherent defects surrounding these interiors, and the NGB region of random static displacements of the atoms. While the atom configuration is not exact, it does dramatize the atomic volume changes expected from grain interior through the NGB region. Additionally, the atom representation within the perfect crystal grain interior is close to a true representation with no orientation differences.

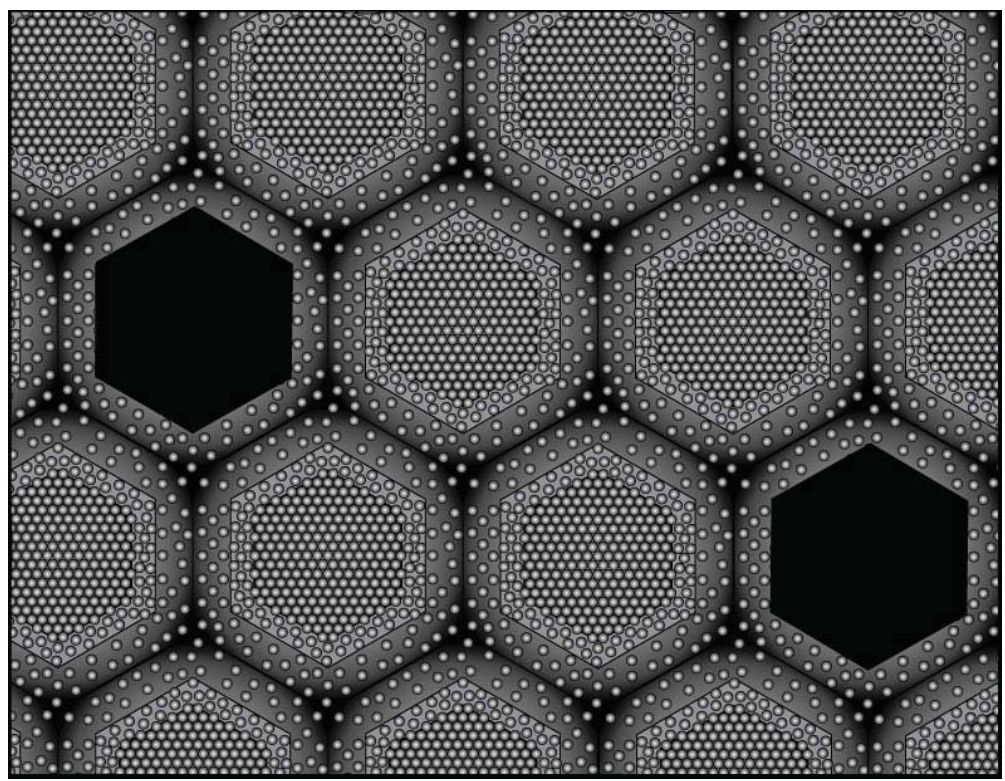

Figure IV-5 Microstructural Model of As-milled Powder with TiC in Black [196]

Based on the size of the TiC particles they can appropriately replace $\mathrm{L}_{2}$ crystallites and are represented by the black hexagons in Figure IV-5, having a similar hard particle behavior as the $\mathrm{L}_{2}$ intermetallic grain interior. Of course the volume fraction of $\mathrm{TiC}$ would determine the population, in this depiction the $\mathrm{TiC}$ volume fraction is similar to that produced from a $20 \mathrm{~h} \mathrm{RMM} \mathrm{time.} \mathrm{The} \mathrm{intent} \mathrm{of} \mathrm{this} \mathrm{structural} \mathrm{model} \mathrm{is}$ to showcase the contiguous nature of the NGB region which has a significant impact on the mass transport observed at these finest grain sizes. 


\section{b) Mass Transport in Nano-Scale materials}

In large-grained polycrystalline materials the GB diffusivity is a factor of about 10 greater than diffusivity in the bulk lattice [197]. However, because of the extremely small surface area of the GBs they play a very small role in the diffusion process which is dominated by bulk lattice diffusion. One of the first models to consider that the total diffusion is composed of the lattice diffusion and the fraction of the GB diffusion based on the GB width divided by the grain size $(d)$, is shown in Equation (IV.15), where the total diffusivity is expressed as a function of grain size [198]. This basic expression was used to compare the diffusivity in polycrystalline alloys in the nickel-aluminum system as a function of grain size [199]. This work, using the GB diffusivity $\left(D_{b}\right)$ that is similar to that in large-grain size materials, showed that the diffusivity rapidly increases as the grain size decreases. For example, if the diffusivity is determined for a $25 \mu \mathrm{m}$ grain size at 893 ${ }^{\circ} \mathrm{C}$, a similar diffusivity can be calculated for a $55 \mathrm{~nm}$ grain size at a much lower temperature of $127^{\circ} \mathrm{C}$. This suggests that similar diffusivities expected at higher temperatures (above $0.5 \mathrm{~T}_{\mathrm{M}}$ ) in large-grain size materials can be achieved at much lower temperatures in nanometer grain size materials. The importance of this work demonstrates the effects that increasing GB volume fraction has on the overall diffusivity.

$$
\begin{aligned}
& D_{\text {eff }}=(1-F) D_{l}+F D_{b} \\
& F=\frac{2 \delta}{d}
\end{aligned}
$$

Valiev et al. [200] have shown that a significant increase in dynamic mobility of atoms occurs in ultrafine-grained (UFG) materials (sub-micrometer). Grain growth occurs in UFG copper at a lower temperature of $0.32 \mathrm{~T}_{\mathrm{M}}$ as opposed to large-grained material. This was attributed to the GB dislocation structure (NEGBs) along with the significant elastic strains near the GBs. The diffusion of copper into nickel was compared for a $300 \mathrm{~nm}$ grain size developed by severe plastic deformation, with the NEGB structure, to a large-grain sample with the GB diffusivity determined to be about four orders of magnitude larger [201]. This increased diffusion in the GB region of materials 
with the NEGB structure has also been modeled more recently, describing the NEGB as a chaotic system of trapped lattice dislocations, which enhances the diffusivity [202].

As expected diffusion rates in nanocrystalline materials are very much greater than that in large-grained materials. In fact Horváth et al. [58] found that GB diffusion in an $8 \mathrm{~nm}$ grain size sample is 3 orders of magnitude faster than GB diffusion in a bicrystal, and more importantly 14 to 16 orders of magnitude faster than bulk diffusion in a largegrained material, attributed to the large amount of GB region. Certainly these observations apply to NGB structures, as well to NEGB structures. The mechanisms of these increased diffusivities are not well established although many references point to decreases in the activation energy as a common theme. In the work by Horváth et al. [58] the activation enthalpies for self-diffusivities in copper was shown to be similar to those for surface diffusion, $0.64 \mathrm{eV}$ and $0.69 \mathrm{eV}$, respectively. A continuous decrease in activation enthalpies for self-diffusivities with grain size is illustrated by $\{111\}$ diffusion in single crystal $(1.98 \mathrm{eV})$, polycrystal $(1.06 \mathrm{eV})$, and nanocrystal $(0.64 \mathrm{eV})$.

In studying the diffusion of silver in copper the Gleiter group [59] found that the interfacial component exhibits little expected short-range order or long-range order. The silver GB diffusivity in copper was determined to be 2 to 4 orders of magnitude faster than that of GB diffusion in a copper bicrystal. This suggests that GB diffusion in nanometer materials is much more rapid than in large-grained materials, which can be attributed to the boundary structure allowing these much more rapid diffusivities particularly at lower temperatures. An interesting aspect of their results is that the activation enthalpies in the temperature range of $303 \mathrm{~K}$ to $343 \mathrm{~K}$ are lower, $0.39 \mathrm{eV}$, than for higher temperatures of $353 \mathrm{~K}$ to $373 \mathrm{~K}, 0.63 \mathrm{eV}$, implying that the higher temperatures may affect the GB structure. This supports findings that temperature increases during MA increase the time to amorphization of intermetallics with the GBs expected to be driving force in that process [203].

The first reported diffusivity measurements in an MA powder were for copper in an Al-7.8 wt. pct. Ti-0.3 wt. pct. Fe alloy [204]. The MA powder was pressed in a diamond anvil at $3 \mathrm{GPa}$ resulting a crystallite size of $22 \mathrm{~nm}$. The activation energy for GB diffusion divided by that for volume diffusion in large-grained polycrystals was 
found to be between 0.6 to 0.8 for a twist GB, while in the nanocrystalline material this ratio is about 0.28 , which considering diffusion at $300 \mathrm{~K}$ translates into an increase of 9 orders of magnitude in diffusivity over that for volume diffusion of copper in single crystal aluminum. The authors concluded that this value is "sufficiently huge" to enhance reactions that occur in nanocrystalline materials. At this time there have only been a few cases of diffusivity measurements in nanometer and sub-micrometer grain size materials. The Gleiter group [59] concluded a large configurational entropy of the interfacial component could be responsible for the lower activation enthalpy. Similarly the NGB structure can be expected to also provide for a lower activation enthalpy perhaps providing diffusivities closer to that expected for surface diffusion.

A basic understanding of the diffusion equation and how these structural changes can affect the terms is needed. In the fundamental equation given in Equation (IV.16) for substitutional diffusion, the upper expression breaks down the activation energy into its parts consisting of the change in enthalpy of mobility $\left(H_{m}\right)$ and vacancy formation $\left(H_{v}\right)$ [46]. As just mentioned, a large decrease in activation energy in nano-scale materials has been reported, often reaching near that for surface diffusion, with a large impact on the material diffusivity, extending to much lower temperatures, in this case near room temperature. It can be argued that the more open random-like structure of the NGB region lowers both the mobility and vacancy formation energies thus decreasing the activation energy. Vacancies might be expected to be more prevalent in the NGB region, a high energy highly defective GB structure.

$$
\begin{aligned}
& D_{A}=D_{O} e-\left(\frac{\Delta H_{m}+\Delta H_{v}}{R T}\right) \\
& D_{A}=D_{O} e\left(\frac{-Q}{R T}\right)
\end{aligned}
$$

Besides expected increases in diffusivity from a much lower activation energy, the value of the $D_{O}$ can also increase in these nanometer materials despite the fact that these values are typically taken to be temperature and structure insensitive. A common definition of $D_{O}$ for substitutional diffusion is given in Equation (IV.17) where $v$ is the 
vibrational frequency and $z$ and $\alpha$ are constants of the material based on the number of nearest neighbors and the lattice parameter [46]. Reported values of $D_{O}$ can vary for a material, but it is usually treated as a constant and not an important diffusivity variable. This minor role in the diffusivity may reflect the means by which the constant is measured. As noted, differences in the $D_{O}$ in similar systems are commonly observed. Of interest here is whether the GB structure changes may also lead to changes to in $D_{O}$. It has been reported that $\mathrm{ZnS}$ nanometer particles of $3.4 \mathrm{~nm}$ size have an Einstein vibration frequency that is $62 \%$ higher than in the bulk $\mathrm{ZnS}$ [205]. Such changes should be reflected in the value of $D_{O}$ as well.

$$
D_{0}=\frac{1}{6} \alpha^{2} z v \exp \frac{\Delta S_{m}+\Delta S_{v}}{R}
$$

The terms in the exponent, the entropy of creating a vacancy $\left(S_{v}\right)$ and movement of the vacancy $\left(S_{m}\right)$ which are positive, can play a much larger role in increasing the value of $D_{O}$. The increased energy and disordered nature to the NGB region might be considered to affect both the entropy terms. The more disordered structure in the GBs of nanometer grain sized materials could lead to larger entropies, as also pointed out by the Gleiter group [59]. Similar to the pipe diffusion enhancement these grain boundary regions can undoubtedly enhance diffusion. The important point here is that the increases in diffusivity could easily be very large. In a recent review of the MA process it was stated that defects are the impetus of increased entropy $\Delta \mathrm{S}_{\mathrm{m}}[104]$.

The establishment of an NGB phase in nanomaterials has significant thermodynamic and kinetic implications for the behavior of such materials. This arises from the enhanced diffusion provided by the large volume of NGB material that creates "short-circuit" paths for diffusion along with contributions from the defect structure surrounding the crystal grain interior. Additionally, the very short diffusion distances associated with the small grain size of 2 - $3 \mathrm{~nm}$ (5 - 8 unit cells in length) contribute to the enhanced diffusion. In molecular-dynamic studies of the inverse Hall-Petch behavior in nanometer materials a general high-energy HAGB was used as the GB model [206]. While confirming previous critical grain sizes for inverse Hall-Petch behavior the Gleiter group determined that Coble creep is responsible for this behavior. As noted earlier, there 
has been general agreement that this behavior is attributable to GB-mediated plasticity; however, specific mechanisms are lacking. Based on conventional understanding of GB

plasticity in materials GB sliding must be accommodated by GB diffusion creep [207] and GB diffusional creep requires GB sliding [208]. Based on the diffusion enhancement by the NGB structure and the surrounding defect structure of the crystallites it is concluded here that this is the diffusional mechanism that supports GB sliding in these small grain sizes at temperatures near room temperature.

Fundamental quantification of how different GB structures affect the diffusivity will be needed to better understand these phenomena. One aspect of interest to note is that Nazarov et al. [209] considered the long-range stress fields produced by the high energy of NEGB structures and determined that these extend into the grains. The asmilled powders in the current study show extremely large elastic strains with a large fraction of atoms affected because of the much smaller grain size in these materials, which could also contribute to enhanced diffusivity. The NGB structure being regarded as saturated with EGBDs would act similarly in a more pronounced manner along with the extremely small grain size suggesting that enhancements to the bulk diffusion are likely to be increased allowing for diffusion at much lower temperatures.

\section{c) Diffusion Model}

A brief commentary concerning modeling of the diffusion in the $\mathrm{L}_{2}$ intermetallic, which is generally the majority phase for most RMA/M times is offered here. Other phases, such as the TiC produced, will be ignored in these comments. The focus of the observations concerned with diffusion enhancement at room temperature has been on activation energy decreases in the NGB regions of the as-milled material. The current work has established that the microstructure of the of the $\mathrm{L}_{2}$ intermetallic in the asmilled powder consists of $\sim 2 \mathrm{~nm}$ sized crystals surrounded by a layer of coherent defects with a saturation of EGBDs in GBs creating a highly defective "random" arrangement of the atoms in the boundary regions. Each of these three components of the microstructure is expected to have different diffusion characteristics so that the total diffusivity based on the volume fraction contributions might be represented as Equation (IV.18). 
$D_{L 12}=\left[V_{C r y s t a l} D_{C} \exp \left(-\frac{Q_{C}}{R T f_{C}(\dot{\varepsilon})}\right)+V_{\text {Defects }} D_{D} \exp \left(-\frac{Q_{D}}{R T f_{D}(\dot{\varepsilon})}\right)+V_{N G B} D_{N G B} \exp \left(-\frac{Q_{N G B}}{R T f_{N G B}(\dot{\varepsilon})}\right)\right]$

Because of the unusual structure of the GB region, the large density of defects on the periphery of the crystal interiors, and the small crystal size with elastic strains in the crystal interior, it certainly can be expected that the individual activation energies are substantially reduced in comparison to large-grained materials. Based on the microstructure model, the NGB region becomes the matrix "phase" creating a contiguous three-dimensional network of this phase that separates the crystallites. The much lower activation energy for diffusion expected based on the structural considerations, creates a large volume three-dimensional contiguous network of highly effective short-circuit diffusion paths throughout the material.

The surface smearing observed during milling suggests that superplastic deformation occurs, driven by the high strain rate imposed on the material and the enhanced diffusivity. In this regard, it should be mentioned that studies of the deformation of large-grain size metals showed the strain in the vicinity of the GBs differs dramatically from that in the grain interior with the strain continuous across the boundary, but with a sharp gradient at the boundary of the grain [210]. Importantly, triple junctions are noted to increase dramatically as the grain sizes approach the nano-scale region with these also being sources of stress concentrations [211]. Therefore, under high strain rates large stress gradients would occur in the GB regions giving rise to large increases in diffusivity. In a study of the inverse Hall-Petch relation in nanocrystalline materials a new model was proposed, incorporating a GB volume fraction component, that assumes that lattice distortions along the GBs can cause internal stresses and these high internal stresses along the grain boundaries can initiate GB yielding [212]. In atomistic simulations of GB sliding in pure and magnesium doped aluminum bicrystals, it was concluded that a higher energy GB slides at a lower stress with a lower viscosity and has a smaller activation energy [213]. Finally, in describing relaxations in NEGBs it was suggested that shear stresses within a GB caused by non-optimized rigid-body translations (from the NEGB structure) can be accommodated by diffusive flow of atoms 
along a GB [214]. These effects result in an effective lowering of the activation energy in the diffusion model expression, thus allowing for a more rapid mass transport at room temperature. While the exact form of this function is not understood, its effects have been observed.

In Fick's first law of diffusion the concentration gradient plays a large role in the diffusive flux. It can be surmised that the extremely small microstructural features and the expected rapid flow in the NGB region could set up extremely large concentration gradients affecting the diffusional flow. The incoherent defect region is expected to behave similar to pipe diffusion models such as dislocation solute-pumping [215], but an enhanced version of pipe diffusion. Because of the complex nature of these defect regions and the dynamic nature of the process, the possible role of strain rate is not clear. However, the strain gradients within the grains and the fact that the GBs support higher stresses can result in a strain rate sensitivity. Strain rate functions for each region have been included in the expression to note that possible contribution.

In a model based on the volumetric analysis of an average microstructural unit of the $\mathrm{L}_{2}$ intermetallic material the unit is made up of: perfect crystal $30 \%$, defect region $22 \%$, and NGB region $48 \%$. Thus, about half of the volume of the $\mathrm{L}_{2}$ intermetallic consists of the three-dimensional network of extremely rapid diffusivity along with nearby regions supporting rapid diffusion. Furthermore, as previously discussed, it can be expected that changes such as larger $D_{0}$ values may also result. The NGB region about one half of the volume, dominates the diffusion with the smallest activation energy and largest $D_{0}$ value. This GB structure may be the key to understanding deformation behavior especially the superplastic temperature decreases with grain size, a decrease that has also been documented in the current work in Appendix VIII.E.

\section{B. The Reactive Mechanical Alloying/Milling Process}

One of the most important aspects of this research, from a practical standpoint of synthesis of materials, is the establishment of a simple approach for in situ hard particle formation during the MA process. Contamination has historically been a concern in MA 
when adding a PCA; however, this new process intentionally takes advantage of the elemental uptake from the PCA (also in some cases from the gas environment) to create new compounds providing a bottom up approach to composite formation. In this new RMA/M process there are two mechanisms at work, those of the MA process and those of the reaction process. The fundamental model of the MA mechanism of cold welding and fracture, Figure I-3, has been used for well over 30 years to fundamentally explain the MA process. While several modifications have been presented they are basically variations of the same basic model. A new modification will be presented here that can account for the rapid mass transport observed in the MA processing that cannot be easily explained by the original cold welding and fracture model or the subsequent diffusion models developed that can only account for a diffusion contribution once the scale of the structure is below $100 \mathrm{~nm}$, or most often $10 \mathrm{~nm}$. Additionally, important to this synthesis process are some interesting reaction mechanisms gleaned from the experiments in this work building a foundation in the understanding of reactive milling.

Mechanical alloying can be envisioned as a room temperature microforging process occurring with each impact of powder between milling media (as well as vial surfaces) with the volume of the impacted region a small quantity based on statistical probability that is governed by several milling parameters; such as type of mill, charge ratio, and amount and type of media. For example, in these experiments commonly $90 \mathrm{~g}$ of milling media (referred to as "balls", that are about one gram each) with $15 \mathrm{~g}$ of the powder mixture in a closed container were moved rapidly in a figure-eight pattern. The Courtney group [216] modeling the physics of the SPEX ${ }^{\mathrm{TM}}$ mill, suggested that the collision time for each impacted volume of powder is on the order of $10^{-5} \mathrm{~s}$ with a maximum radius of contact felt by each impact event of ball to ball of several hundred micrometers. The average particle size in the current work is just under $10 \mu \mathrm{m}$ meaning about 50 particles could undergo each individual impact. The maximum number of impacts per ball, while complex to determine, was estimated for a SPEX ${ }^{\mathrm{TM}}$ to be about 250 impacts per second [217] for each ball (milling media). These continual events involve statistical fractions of the total powder volume undergoing impact in each individual event. The complex deformation imposed on the material by high-strain rates, 
typically suggested to be $10^{3}$ to $10^{4} \mathrm{~s}^{-1}[218,216]$, in random singular mechanical events during the MA process are well known to lead to large amount of mass transport with atomic-scale homogenization. As such, dictated by the properties of the material in question, it can be expected that mechanical working of a material would eventually lead to fracture.

In the generally accepted model by Gilman and Benjamin [81, 99], schematically represented in Figure I-3 for a ductile-ductile metallic powder system, showing the lamellae formation developed in the microstructure of the powder particles resulting from individual powder particles deforming from ball to ball impact modeling uniaxial events; however, based on the motion in SPEX ${ }^{\mathrm{TM}}$ mill shearing impacts are also expected to occur. These lamellae are assumed to form by the cold welding of flattened spherical powder particles with deformation creating these lamella estimated to be about $75 \%$ in these particles (see Chapter III.A.4.b)(b)), which is indeed a sufficient requirement for cold welding with fracture suggested to create "fresh" or clean metallic surfaces also necessary for cold welding [219]. In the Benjamin and Volin model [219], the mass transport is suggested to occur once the lamellae thickness decreases below optical resolution (sub-micrometer lamellae, milling time several hours) where diffusion is assumed to take place based on the short distance; however, a detailed mechanism was not identified. While several modifications in the details of the early models have been proposed to date there is still no explanation for the large amount of mass transport that occurs early in the milling process, as evidenced by the observed material changes.

Suryanarayana [81] described the various basic types of powder material systems encountered in MA: ductile-ductile, brittle-ductile, and brittle-brittle. The alloying is easier to describe, such as with the Gilman and Benjamin model, when extensive cold welding is expected from ductile-ductile metallic components, while in a brittle-brittle system cold welding typically is not expected to occur, although alloying has been observed. Speculations as to the mechanism of alloying in these brittle-brittle systems are based on plastic deformation, surface deformation, hydrostatic stress in the powders, and microdeformation in defect-free volumes [81]. The model presented here is developed for essentially a brittle-brittle system, in that regard the RMM of the $\mathrm{L} 1_{2}$ intermetallic will be 
considered; thus with this model it is expected to applicable to all of the basic types of metallic systems in MA: ductile-ductile, brittle-ductile, and brittle-brittle.

As has been discussed, the unique aspect of the MA process originates from the innate ability to easily create alloys and compounds, including those that are stable while being far from thermodynamic equilibrium, as well as those for which the components are typically immiscible. For example, in the current work MA of elemental aluminum and titanium powders in the proper ratios the $\mathrm{Ll}_{2}$ (cubic) ordered intermetallic $\mathrm{Al}_{3} \mathrm{Ti}$ can be produced from the binary components. As noted earlier, the equilibrium phase diagram shows only the $\mathrm{DO}_{22}$ (tetragonal) $\mathrm{Al}_{3} \mathrm{Ti}$ phase. Several other reactions have also been identified as a result of the MA process, producing compounds from metallic and non-metallic powders, or by milling in various environments such as liquid nitrogen or nitrogen gas. The import aspect of all of these phenomenological observations in MA is the requirement for a mass transport mechanism(s) in order to homogenize the various elemental species at an atomistic level. As has been stated previously, the local temperatures in MA are well below the temperature required to activate traditional diffusion, which is usually taken as $0.5 \mathrm{~T}_{\mathrm{M}}$. Given this background, the question arises as to how does the MA process accomplish the requisite mass transport; or more specifically, create an environment that exceeds normal expectations of atom movement?

\section{Mechanical Alloying Mechanisms}

The results of the particle morphology study in the current system established that the agglomeration process can occur in two ways; larger particle agglomeration on a macroscopic scale and surface events analogous to agglomeration on a microscopic scale. Large particle agglomeration of cold welding and hence flattening (in a ductile-ductile system) has been part of the fundamental model in MA used to explain the mass transport with the lamellar microstructure internal to the powder particles. In the system studied here, the large particle agglomeration is observed cyclically with milling time and a slower process of densification of the infant agglomerates is observed. In this case, these contact regions become successively larger between agglomerate particles as observed by the closing of cavities. However, unlike the ductile-ductile system these particle 
gradually undergo deformation with much less flattening of the large particles. While this contributes to the process, it is a much slower process of intermixing of the material since multiple impacts slowly densify the particles. With each single impact event there is a gradual increase in the contact area of the interface between two particles which involves a small fraction of the total volume of both particles. However, an interesting observation in this area is what appears to be a surface activated bonding exemplified by the small particle in the center of Figure III-31 with a surface that appears bonded to the neighboring larger particle. The current work has focused on the surface events of smaller particles agglomerating onto the surfaces which appears to be the most important MA mechanism, particularly for the more brittle system studied.

\section{a) Surface Events}

The study of the morphology of the powder particles during the RMA/M process clearly established that the surfaces of all particles are littered with highly deformed particles suggesting large amounts of mass transport have occurred. This mass transport can be explained by the smaller particles having the nano-scale structure, with the NGB regions, providing enhanced mass transport and deformation under a high strain rate as presented in the diffusion model in Equation (IV.18). In severe deformation processes such as filing [69], or in surface mechanical attrition treatment (SMAT), a technique of high frequency ultrasonic vibration of steel shot on the surface of a bulk material [220], a nano-scale deformation structure is developed in metals and are therefore expected to have the NGB structure. Another example, is in the fine grinding of hard metals, the chiplike debris produced from the process has a grain size on the order of $10 \mathrm{~nm}$ [221]. However, one area of materials science that has not been addressed is the mechanism that causes the nano-scale structure to be formed at the point of contact of these intense plastic deformation processes.

Important to this point is that the MA process has a slightly higher strain rate than measured for the SMAT process which can be a reflection of the amount of deformation imposed. For example, SMAT of copper found the near surface as having the highest strain rate of $\sim 10^{2-3} \mathrm{~s}^{-1}$ with lower strain rates as the depth from the surface of the 
material is increased [222]. As a result the grain size (TEM) at the surface is about $10 \mathrm{~nm}$ while at a depth of $25 \mu \mathrm{m}$ below the surface the grain size is about $100 \mathrm{~nm}$. In the case of MA the nano-scale microstructure is clearly produced; however, the nano-scale grain size only becomes homogeneous after long periods of time. Thus, it can be expected that the surface impacts will cause deformation into the surface of the powder particles. Additionally, the creation of these nano-scale microstructures are likely to be an important mechanism in grain refinement in the MA processing, as localized rapid grain refinement down to the lower nanometer range is readily observed.

In the RMM processing of the $\mathrm{L}_{2}$ intermetallic the high strain rates with large amounts of deformation push this material to the deformation limit, based on the fact the grain size is refined down to the minimum grain size for polycrystalline materials and these materials develop near theoretical dislocation densities. The theoretical density can be calculated using Equation (IV.19) [223], the theoretical shear stress $\left(t_{t h}\right)$ was determined on page $19(5 \mathrm{GPa})$ for the $\mathrm{L} 1_{2}$ intermetallic, with an alpha $(\alpha)$ of 0.5 common for FCC materials and Burgers vector $(b)$ for a $a / 3<112>$ dislocation results in a theoretical density of $2 \times 10^{11} \mathrm{~mm}^{-2}$. The densities developed in these materials of about $5 X 10^{10} \mathrm{~mm}^{-2}$ (see Table III-3) approach this value. As previously discussed, for this grain size regime dislocation motion is limited with the material expected to deform by GB plasticity. This essentially creates a "deformation kernel", i.e. a limit of the deformation structure. The observations of these surface features show that large amounts of surface deformation occur and often these surface smeared particles show a distinct flow. This suggests a superplastic-like behavior as no cracks are observed with the deformation far exceeding the normal plasticity abilities of the $\mathrm{L} 1_{2}$ intermetallic. These surface events have shown two basic types of morphology, those that appear like a liquid-type viscous flow expected from more normal surface forces and those with flow lines from a more shear type force.

$$
t_{t h} \approx \alpha G b \sqrt{\rho}
$$

These surface features of the as-milled powder particles are dominated by what appears to be much smaller particles physically smeared or pressed onto the surface of 
the larger particles as noted by the flow lines in many surface features suggesting severe shear deformation. The surface smeared particles appear with different flow line directions as would be expected from the high frequency of random impacts to the surface expected by the chaotic milling motion. Additionally, small cracks were visible suggesting fracture occurs on these surface small features (in addition to larger particle fracture). Figure IV-6 shows the different directions in flow lines and small visible cracks in select areas. While few studies in MA address these surface issues, some observations have been made in this regard. In the MA of iron-aluminum alloys, although the pictures are not clear, it is suggested that small particles are welded to the surface of larger particles probably by the high deformation [224]. In the planetary milling of iron and cobalt some flattened particles were noticed on a fracture surface of a large particle a result of shear forces from balls causing cold welding, fracture, and rewelding believing this layered structure plays an important role in interdiffusion and true alloy formation [225]. However, no further discussion of particle morphology or microstructure was provided in either study.

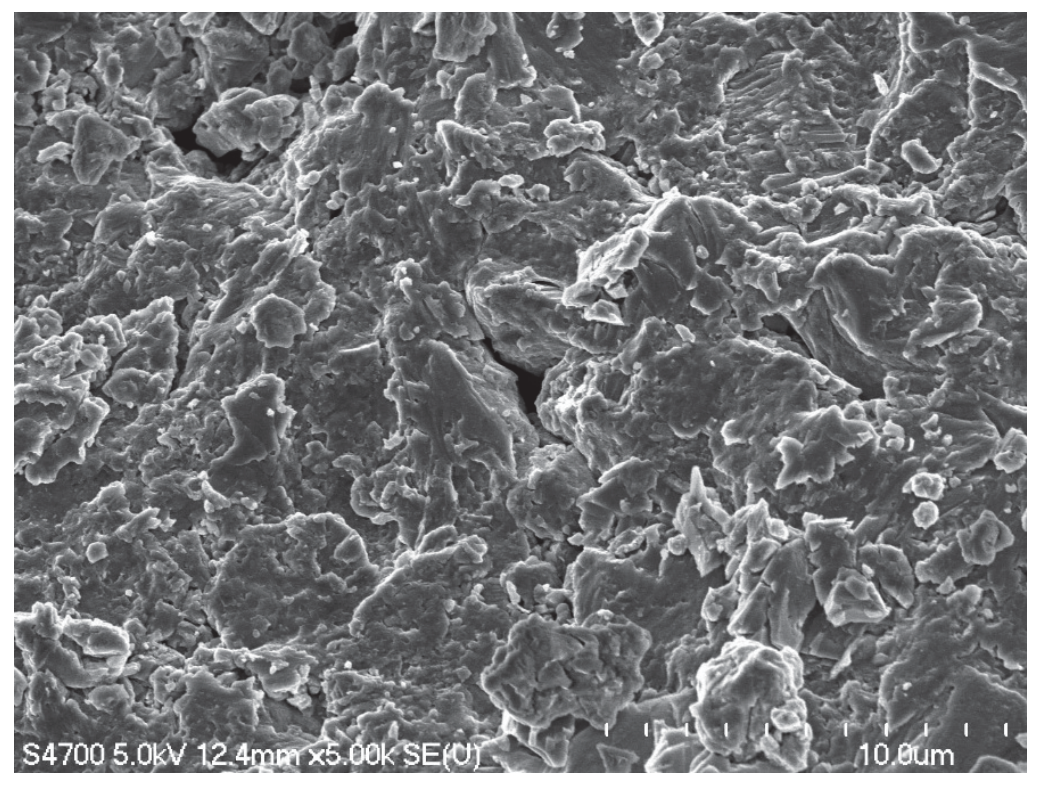

Figure IV-6 Surface Smearing As-Milled RMM Time $1 \mathrm{~h}$

Often in the literature on MA processes cold welding is cited to occur based on the availability of "fresh" metallic surfaces produced by fracturing. However, this has not been addressed in the brittle-brittle systems. Benjamin and Volin [219] did discuss this 
issue on the basis of large particle agglomeration in a ductile-ductile system. However, their basis was merely a $71 \%$ deformation in the large particles. In a typical cold welding process, high pressures are applied to cause deformation between two clean fresh materials surfaces in a vacuum at room temperature creating a solid state bond. Immense pressures are needed in air, but lighter pressures have been known to bond metals in an ultra-high vacuum (UHV) using flat ductile atomically clean materials. For example. nanocrystalline gold wires between $3-10 \mathrm{~nm}$ in diameter in UHV have been shown to produce exceptional cold welds with relatively low pressures (compared to large-grain samples) applied for mere seconds provided the wires are of the same orientation, with atomic diffusion and surface relaxation considered important factors in the process [226].

It should be pointed out that much of the research on cold welding is geared more toward phenomenological aspects of parameters that affect the bond strength and the integrity of the bond such as pressure, surface preparation, etc. A brief summary of mass transport in the cold welding process will be presented based on a review by Bay [227]. Bay summarized the bonding mechanisms of the cold welding of scratch brushed materials based on a two-step mechanism in which the cover layers are breached and the virgin metal is exposed to the cracks of the cover layer (contaminant film), then the virgin metal is extruded through the cracks and the cold weld joints are established from the contact surfaces of the virgin material from both metals. Bay also pointed out that at least a $40 \%$ expansion in the plane of the cold welding joint is necessary to create a good bond, indicating that a large amount of deformation is required to accomplish cold welding. Based on the current research this large deformation localized at the surface may well produce the NGB structure, thus creating a high mass transport material which can be expected to facilitate the cold welding process.

An example of cold welding in the brittle-brittle metallic system in this study, that can be explained by the surface wear model, is the smeared particle highlighted by the black arrow in Figure III-29, showing about $75 \%$ deformation which meets the usual criterion of $40 \%$ to produce a good bond during cold welding (with a good vacuum). It is interesting that this process also occurs while milling in air suggesting added help by the enhanced mass transport of the particles. The necessary pressure comes from the media 
impact on the particles with the expected NGB structure having the expected rapid room temperature mass transport, thus satisfying the necessary cold welding requirements for this brittle system. While the research here has shown that it is possible that the particle impacted could form the NGB structure, thus providing enhanced mass transport, based on the required large amount of deformation in a single impact it is not clear if the particle must have this structure prior to impact. More importantly, as previously pointed out, the contact time of one event has been suggested to be $10^{-5} \mathrm{~s}$ and it appears this process allows for a cold weld in these short durations, this appears to be the result of the enhanced mass transport of the nano-scale structures present in the surface impacted particles. While the surfaces can obtain this nano-scale structure from the impacts, surface agglomeration is highly expected from impacted particles possessing the nanoscale structure. However, a source for these particles having the nano-scale structure will be identified.

\section{b) Sliding Wear and Mechanical Alloying}

The work by Rigney et al.[228] on sliding wear is worth noting because of the similarities he noted between the transfer (or tribo) layer of sliding wear of two metals and the MA process developed by Benjamin. In order to test this hypothesis the Rigney group mechanically alloyed copper powder using steel balls and compared that microstructure with the transfer layer developed during sliding wear of M2 tool steel on copper. During both experiments he claimed a similar temperature of about $60{ }^{\circ} \mathrm{C}$ was reached globally with both developing a fine substructure. The grain size of the transfer layer of sliding wear surface is similar to the smallest grain sizes observed in the MA powder particles as determined by TEM analysis. The transfer layer has an average grain size less than $10 \mathrm{~nm}$, and while the MA particles had grain sizes as small as that, a majority of the grain sizes in the MA process were much larger. The Rigney group concluded both processes were similar, but that the MA process provides an inhomogeneous deformation microstructure as opposed to the transfer layer in sliding wear having a homogeneous deformation in the surface layer. Unfortunately, the analysis 
of the MA process was limited to only the internal particle microstructure and milling time was not given.

Heilmann et al. [229] determined that in sliding wear this surface or tribo layer and the wear debris have the similar nanocrystalline grain size, same composition, and same mixture of phases. They also concluded that the wear debris particles are derived from the transfer layer and irrespective of size or shape are composed of an intimate mixture of both the block and ring material [229]. The grain sizes of the wear particles and the transfer layer are generally between $3-50 \mathrm{~nm}$ in mixed material systems; however very little is known about the properties of the mechanically mixed material produced by sliding [70]. This layer extends to a depth of about $1 \mu \mathrm{m}$ from the surface, below which is a larger subgrain boundary type structure. One can speculate that in MA processing the material is heavily deformed to about a micrometer depth from the particle surface, but a corresponding view of the MA process has not been investigated.

One aspect of the similarities between sliding wear of metals and the MA process that has not been discussed is production of wear-type debris. While this was not initially studied, an indirect observation during the RMA/M process has shown more fines (including powder particles that float in the air) are observed with longer milling times when opening the vial in a fume hood. This is also supported by the increased frequency of smaller particles in the particle size distributions with increased milling time as shown in Figure III-22 through Figure III-24. Additionally, along with a distribution of the large particles, there are extremely small particles observed in the SEM powder mounts that are near in size to the resolution limit. These smaller particles are suspected to be wear-type particles. In the sliding wear of metals the wear debris can range in physical particle size from nanometers to centimeters [70]. In the beginning of the RMA/M process powder particles were on average $74 \mu \mathrm{m}$ in size and quickly reduced to below $10 \mu \mathrm{m}$ further supporting creation of small particles of wear-type debris. Particle shapes of wear debris in wear processes can include: cutting chips, spheres, cylinders, flakes, plates, ribbons, and irregular clusters and chunks. While wear debris has not been studied as much as other aspects of the wear process, what is of most interest is that wear debris with grain sizes down to $3 \mathrm{~nm}$ are frequently observed. This indicates that wear debris has the NGB 
structure and the associated dramatically enhanced diffusivity making these particles conducive to cold welding as observed. Therefore, it is postulated that wear-type debris particles produced at the point of contact of the deformation process is an instrumental part of the MA process and manifest themselves in the heavily deformed surface events. Thus, a source of these particles is readily available for accomplishing a significant part of the alloying and grain refinement process in MA.

In an attempt to understand these small grain sizes observed in the wear debris Rigney [228] looked at the bow-out shear strength from a Frank-Read source by setting it equal to the theoretical shear strength for copper to determine the smallest distance to generate a dislocation (distance $L$ Equation (I.2)) resulting in values of about $3 \mathrm{~nm}$ similar to the smallest grain sizes reported in the transfer layer and wear debris from sliding wear. However, the materials (transfer layer, wear debris, etc.) would be expected to be very hard (based on work hardening), but it was determined that the wear debris was not hard enough to mechanically press into the transfer layer creating an inconsistency that remains unexplained [228]. Localized shear may create these particles, but it would be expected that the transfer pieces would not be thicker than the sub-grain size developed, but are actually much larger. A factor that has been introduced to explain this is grain rotation, as variable angles across subboundaries of $20-30^{\circ}$ are observed, texture softening of the particles is suggested [230]. As will be presented this can also be explained by these particles having an NGB structure and hence would be much softer than predicted especially under a high strain rate.

The grain size of wear debris can vary according to the amount of deformation. No mechanism to date has been able to explain the rapid grain size reduction that is observed in extreme deformation conditions such as during surface deformation treatments, filing, wear, and the expected surface of MA powder particles. These very fine grain size structures developed are a phenomenological result of the natural response of the material to the extreme deformation overloading with better understanding of this mechanism important to all severe deformation processes. Of importance is a quote from the wear review by Rigney [70]: "In fact, plastic deformation and changes in structure during the early stages of sliding may be precursors to processes such as transfer and 
mechanical mixing in which the chemical composition of the near-surface material is modified, together with further changes in structure, and further deformation of the tribochemically modified material". What is interesting is that this supports the idea that material with the NGB structure produced by deformation can lead to the observations of alloying in the transfer layer. Again, this suggests that the surface impacts of these structurally-changed wear particles and transfer layer may well be the dominant mode of mass transport observed during the MA process. These wear-type particles would be expected to originate at the point of contact of the impacts with powder particles under the most stressful conditions thus also creating a transfer layer on the surfaces of the powder particles.

In general, the MA process has been shown to be very similar to the sliding wear process, but the MA process can have up to an order of magnitude increase in strain rate. The "fines" that appear to be produced as a function of the RMA/M time can be related to the products of wear debris occurring from the sliding wear-type shearing of neighboring powder particles. In the initial stages of the RMA/M process these particles are composed of elemental, or the pre-alloyed chemistry, with expected large grain sizes. As the milling proceeds, these particle surfaces are expected to become similar to the transfer or tribo layer observed in sliding wear along with production of wear-type debris. It should be pointed out that in the sliding wear process the wear debris can be ejected; however, in the MA process such particles (debris) are contained by the closed system thus remaining an integral part of the process. Thus the MA process is suggested to take advantage of these two mechanisms both dependent on surface events; the shearing action of individual large powder particles creating a transfer layers and interactions with smaller wear-type debris being impacted into the surface.

The traditional case often cited in the literature as the main mechanism of mass transport in the MA process has been considered as occurring at the cold welding interfaces of large particle agglomeration (ductile-ductile systems), which have a small interfacial area with respect to the volume of the particles. Intuitively, this suggests that the rapid grain refinement and mass transport observed during the MA process cannot be explained by this mechanism. A new mechanism has been identified based on surface 
events (or surface agglomeration) of wear-type debris on the particle surface along with tribo layer formation. The structure of the wear-type debris is such that diffusion enhancements are expected along with a very large surface area to volume ratio, also invigorating reactivity. Additionally, this mechanism can also explain the cold welding observed in MA of brittle-brittle systems, which to date has not been sufficiently explained. It is proposed that in addition to large particle agglomeration and localized diffusion, a majority of the mass transport and grain refinement during the MA process occurs due to these numerous surface wear-type events between large particles and weartype debris from the severe mechanical impacts sustained with each individual impact and the "tribo layer" produced by these wear-type processes.

\section{Reaction Mechanisms}

The RMM processing technique as carried out here is a controllable in situ reaction creating hard particles from the elements available in the powders, liquid PCA, and gas environment. There have been few studies of the systematic increase of reaction product in MA such as observed here in the RMA/M processing. The focus in the $\mathrm{L}_{2}$ intermetallic system studied here has been the systematic increase of the stable TiC phase provided appropriate amounts of titanium and the PCA hexanes are available. The principles developed are expected to translate to other reactions that occur such as the nitrogen uptake in air environments. With the in situ reactions observed during the $\mathrm{RMA} / \mathrm{M}$ process it is expected that the temperature is very near room temperature; and despite this, a significant amount of mass transport occurs rapidly within a large volume of powder forming the titanium carbide. Although reactions with PCA and gaseous molecules have been observed previously a comprehensive understanding of reaction mechanisms has been lacking. Some comments founded in basic surface science will be offered here.

In reducing $\mathrm{CuO}$ with calcium by means of mechanochemical processing Schaffer and McCormick [231] observed a reduction in reaction temperature as the crystallite size decreased. They assumed that the refinement of the crystallite size was the major factor in the observed reduction in reaction temperature. In a later study, static disorder was 
separated from thermal vibrations using atomic displacement parameters which are a measure of the mean square displacement of an atom from its ideal position.

Measurement of $\mathrm{CuO}$ reduction by $\mathrm{MA}$ processing determined that the lattice strain from the static displacements of atoms accounts for half of the activation energy, while other defects, dislocations, and GBs account for the remaining reduction [232]. However, these samples were only milled in a $\mathrm{SPEX}^{\mathrm{TM}}$ mill for 36 minutes using a low 3 to 1 charge ratio (far less powder impacted with each cycle) with no crystallite size data given. Based on the model of the microstructure for the RMM powders presented here in Chapter IV.A.1.b), this strain or static displacement would be expected to occur at the interface of the defect region surrounding the crystalline grains.

Other studies have shown similar behavior. The oxidation rate of aluminum is two orders of magnitude faster at $90^{\circ} \mathrm{C}$ in nanometer-scale aluminum that was mechanically alloyed compared to a large-grained aluminum sample [233]. Also in that work it was determined that the temperature for synthesis of aluminum carbide was dramatically reduced from $1200{ }^{\circ} \mathrm{C}$ down to $300{ }^{\circ} \mathrm{C}$ after MA processing to a $15 \mathrm{~nm}$ crystallite size. This structure can also be expected to have the NGB structure. Complete in situ reactions were observed in the mechanosynthesis of aluminum with chromia, reducing the chromia into chromium and creating alumina [234]. This reaction typically requires $1200{ }^{\circ} \mathrm{C}$ to occur; however, it occurs at room temperature when the crystallite size is about $10 \mathrm{~nm}$. Aluminum and titanium powders were reaction milled, for both $25 \mathrm{wt}$. pct. and $75 \mathrm{wt}$. pct. aluminum, in an ammonia $\left(\mathrm{NH}_{3}\right)$ [235]. Based on XRD analysis, TiN and AlN were observed in the as-milled product along with a $\mathrm{Ti}(\mathrm{Al}, \mathrm{N})$ solid solution (based on the current work these peaks appear close to the $\mathrm{L}_{1} 2$ intermetallic peak positions) when 75 wt. pct. aluminum is present. The grain surfaces were determined to absorb a large quantity of $\mathrm{NH}_{3}$ based on a noted decrease in pressure in the first stages of milling (86 h was considered the first stage). The paper concluded that for longer milling times the $\mathrm{NH}_{3}$ is adsorbed and dissociates on the particle surfaces promoting the nitride reactions. The formation of a TiN compound was also observed by Itsukaichi et al. [236] during ball milling of aluminum titanium elemental powders in air. 
A conclusion presented in the above work on chromia reduction is that repeated milling enhances reactivity by the creation of a large amount of interfaces and defects, a familiar theme used when explaining reaction mechanisms (and alloying) during milling. In all of these cases the advent of the NGB structure created by surface events along with the enhanced mass transport can be a mechanism that is helpful to understanding these reaction processes. This suggests that the increased NGB structure developed, with this enhanced mass transport, significantly reduces the reaction activation energy requirements. Based on this trend, these data suggest even smaller crystallite sizes could possibly change the reaction further toward ambient temperature. This supports the important hypothesis that the NGB structure plays a significant role in the behavior of the materials particularly as the grain sizes reach the lower nanometer range.

Mechanochemistry is defined as a branch of solid-state chemistry where intermolecular bonds are mechanically broken. Technically MA and MM can be considered a subset of mechanochemistry, but often in the literature this is associated with displacement reactions. Kaupp [237] described the reactivity of molecular solids under mechanochemical milling by a general three-step process: diffusion of reactants through a mobile phase (gas, eutectic, or amorphous solid) resulting in chemical reaction, nucleation and growth of the product phase, and product separation to expose fresh reactant surface. While comprehensive experiments were beyond the current work, suggestions as to possible mechanisms that can induce these observed, essentially room temperature reactions, will be addressed. What is of significance is that the requirement for these reactions to occur is that the organic liquid $\mathrm{C}_{6} \mathrm{H}_{14}$ molecules (and $\mathrm{N}_{2}$ gas molecules for milling in air) must first be dissociated and then mass transport is required to incorporate a large enough concentration of reactants to nucleate the reaction product(s). Thus, the reaction process can be broken down into three basic steps; dissociation of the liquid or gaseous molecules, mass transport, followed by nucleation of products from localized regions of reactants. 


\section{a) Dissociation}

The first step in the reaction process is adsorption of the PCA or gas onto the surface of the powder particles. The surface area and porosity, or pore volume, play complementary parts in the adsorption phenomenon [238]. Adsorption can be physisorption or chemisorption, with chemisorption expected to play a direct role in the dissociation. In order to understand the wetting of the powder by hexanes a simple drop test method [239] was used on both milled material and initial admixed powders showing immediate wetting of the powder. Absorption may also play a role as the pores and cavities in particles can have a capillary effect pulling the liquid PCA and gas molecules into these regions. Thus a two-step process of contact with the liquids/gases and powders can be envisioned; one based on adsorption on the surface and the second based on the capillary effect which would cause absorption (interface could be adsorbed) into pores and cavities of the less dense particles. Recall that in the surface wear model wear-type debris is superplastically deformed onto the existing surface by cold welding along with creation of a transfer layer both trapping adsorbed PCA at the cold welding interface. Subsequent particle impacts in both cases on larger particles can seal pores and cavities containing trapped liquid/gas molecules. The smearing of wear-type debris traps molecules adsorbed onto the surface along with the sealing of micropores could be expected to cause an increased pressure by the continued deformation thus compressing the trapped liquid/gas molecules and aiding the dissociation process.

The amount of carbide increases in a linear fashion with milling time provided that a source of titanium is readily available, but more importantly it appears to occur very rapidly. In the RMMXh9TiAr experimental series at the shortest times of 0.5 and $1 \mathrm{~h}$ it was likely below the detection limits determined by the difficulty and uncertainty in profile fitting these diffraction peaks. A small amount of $\mathrm{TiC}$ was indicated based on extrapolation of the volume fraction data. $\mathrm{TiH}_{2}$ was also created, but in smaller proportions based on the stoichiometry of the hexanes $\mathrm{C}_{6} \mathrm{H}_{14}$. In general, the reactions should occur following the stoichiometry of the PCA; however there is a clear choice for carbon over the hydrogen. This may well be due to the lower stability of the hydride as indicated by the dissociation of the titanium hydride and easy removal of hydrogen gas 
by a vacuum annealing at a temperature of $500{ }^{\circ} \mathrm{C}$. The results clearly show a preference for $\mathrm{TiC}$ over the $\mathrm{TiH}_{1.92}$ with excess dissociated hydrogen evolved as a gas. However, other alkane molecules in the liquid or gas could be present which would indicate if (molecular) cracking was occurring reducing the hexanes to other alkanes; analysis of the liquid and gas was not accomplished. In early experiments by Arias [87] PCA contamination was found during mechanical milling of either chromium or nickel in nheptane $\left(\mathrm{C}_{7} \mathrm{H}_{14}\right)$ with the gas composition mainly $\mathrm{H}_{2}$ with some $\mathrm{CH}_{4}$. A small amount of alkanes were also found in the liquid. While a mechanism was not discussed for the reactions, they were postulated to be caused by some type of cracking processing on the metal surfaces.

Cracking, particularly of hydrocarbons, is a common practice in industry where heavy molecules are broken down into lighter molecules. However, cracking typically employs both a high temperature and pressure, and while the presence of a catalyst can dramatically increase the reaction rate, more importantly it allows for lower processing temperatures and pressures. While the work by Arias suggested cracking as a mechanism, the in situ formation of $\mathrm{TiC}$ and $\mathrm{TiH}_{1.92}$ as proposed in the current work signifies a direct dissociation of the hexanes (or gas) occurring with the metallic titanium powder reacting with elements from the hexanes as shown by the unbalanced Equation (IV.20). The question mark in the equation was inserted since it is unknown in this system if other alkanes are produced or if other isomers of the hexanes are produced.

$$
\mathrm{C}_{6} \mathrm{H}_{14}+\mathrm{Ti}+\mathrm{Al}_{66} \mathrm{Cr}_{9} \mathrm{Ti}_{25}+\mathrm{Ar}=\mathrm{Al}_{66} \mathrm{Cr}_{9} \mathrm{Ti}_{(25)}+\mathrm{Ti}+\mathrm{TiC}+\mathrm{TiH}_{2}+\mathrm{Ar}+\mathrm{H}_{2}+?
$$

In the reaction of nitrogen gas with niobium and tantalum forming the respective nitrides, the reaction was explained by chemisorption of nitrogen onto clean metal surfaces created by ball milling as the fundamental process governing the reaction with defects generated during MA providing the diffusion mechanism of the adsorbed nitrogen [240]. However, details of how this is accomplished were not discussed. It is believed here based on the surface events that the powders have highly reactive surfaces with these surfaces representing the NGB structure important to these reactions. 


\section{(1) Catalysis}

One concept that has not been given much attention in the MA field is the idea that dissociation can be accelerated by a catalytic effect. Often in the literature describing reactions and alloying in MA processing it is suggested to be a result of "fresh metal" surfaces cyclically brought into contact, an argument similar to that of cold welding. A catalyst by definition increases the rate of reaction and plays a very important role in the reactions observed. Ideally the catalyst does not participate in the product of the reaction, but merely facilitates a new low energy path dissociating organic molecules to the needed forms by intermediate reactions. These lower energy pathways provide the desired product while increasing the kinetics of the reaction, but a catalyst that bonds with molecules or elements in the product is considered poisoned, not a desirable effect as the catalyst will eventually be consumed [241]. However, in this case the undesirable poisoned catalytic effect is important since combination of the high diffusivity in the NGB structure and cyclic nature would provide new "fresh" catalytic surfaces; in addition, it is suspected that the NGB structure could enhance the catalytic effect. The importance of the catalytic effect can be demonstrated by the ability to dissociate isolated molecules of $\mathrm{H}_{2} \mathrm{O}$, which normally requires a temperature on the order of thousands of degree $\mathrm{K}$, whereas in contact with a platinum surface this reaction spontaneously occurs at ambient temperature [241]. It should be pointed out that an exploratory experiment was carried out based on the RMM process of milling nickel in liquid $\mathrm{H}_{2} \mathrm{O}$ dissociating the $\mathrm{H}_{2} \mathrm{O}$ during the processing with the nickel solutionizing a significant amount of hydrogen at ambient temperature [242].

In the MA experiments the first step of the reaction process is the dissociation of the elemental components of the hexanes (or gases) in order to react with the metallic powder to form the TiC. How this dissociation occurs during MA has not been addressed. What is clear is that these reactions involve only titanium coming from the metallic powders with aluminum and chromium playing no role in the as-milled reaction product. Titanium, which is expected to be more reactive, since it forms the observed reaction products with elements from the PCA (or gas phase). While these observations do not delve into the kinetic parameters, often the goal of investigating catalytic mechanisms, 
what is important here is that the titanium is an attractive catalyst for hydrocarbons like hexanes, as are most transition elements to varying degrees [243].

Heterogeneous catalysts are often nanometer-sized metal surfaces that can reduce the bond strength of certain gas and liquid phases and encourage several orders of magnitude increase in reactivity in their presence. The catalytic effect requires adsorption of the liquid or gas onto the catalytic surface. While this can involve either physisorbed or chemisorbed molecules; it typically occurs from chemisorption as the catalyst often briefly participates in the reaction providing a low energy pathway for the reaction to proceed to its final product. It has been established that the hexanes in particular readily wet the surface of these powder particles. The adsorption is dependent on the partial pressure, process pressure, and process temperature, with data for adsorption isotherms showing increased adsorption with pressure. As discussed, both adsorbed molecules trapped by the wear-type debris impacts and the capillary effect filling pores that are trapped by closing particles would result in an increased pressure and with it an expected increase in adsorption. This may allow these closed pores to participate in the adsorption; however, this would only be expected to occur at the stagnant pore surface and thus the catalyst could be poisoned limiting the reaction. Although this occurs, it is considered secondary to the trapped PCA/gas from surface smearing of wear-type debris or from the transfer layer. The trapped surface adsorption by wear-type debris and transfer layer would be expected to be more important kinetically as the frequency and dissociation would be expected to be a cyclic within more numerous continual events exposing the reactive catalytic surface.

Dissociation was observed during the reaction milling of aluminum and titanium in an ammonia $\left(\mathrm{NH}_{3}\right)$ atmosphere but when milling only the ammonia no dissociation was observed [235]. This result adds support to the current assertion that the powders in this environment are catalytic in nature and therefore contribute to the dissociation. It is important to reiterate the conclusion that the microstructure of the powders contains the NGB regions which are highly reactive due in large part to the expected increase in diffusivity in these areas. The presence of surface defects, including steps and other forms of missing atoms, can cause the remaining atoms to have less overlap of the $\mathrm{d}$ band 
electron orbitals important to bonding and the catalytic effect, which shifts the $\mathrm{d}$ band upward in energy leading to a stronger catalytic behavior [243]. Certainly, the surface of the powder particles appears to be an important aspect of the reaction process with the NGB structure exposed to the surface further increasing the reactivity, due in part to an increase in the d-band energy.

Surface defects have been noted to increase the sticking coefficient in the case of gas contact, thus increasing the likelihood of adsorption of gaseous molecules. The rate of adsorption of a gas on a surface is determined by the rate of collision between the gas and the surface and by the sticking coefficient. Surface defects can cause dramatic increases in the reaction coefficient of the catalyst; for example, a study of the dissociation of $\mathrm{N}_{2}$ on ruthenium shows orders of magnitude increase in sticking coefficient and a one third decrease in activation energy compared to the same surface in which no defects are present [244]. The sticking coefficient can also change as the surface becomes occupied by reactants, but in the MA process it would be expected that the dynamic impact actions of the process, on the order of milliseconds apart, incorporating different surfaces would not allow the surface to become fully occupied by the adsorbing species. Of course, fresh surfaces of the metallic powders are naturally produced by the dynamic milling process.

Elastic strains at the surface can also affect the chemisorption of molecules on the surface. To investigate these effects a base metal was coated with various other metals having different lattice parameters, both larger and smaller than the base metal, resulting in strain effects of compression and tension in the surface determined by the difference in lattice parameter between the coating and the base metal [245]. Calculations of the dband energy, thought to play a critical role in reactions of catalysts, shows that in tension the average coordination diminishes and the d-band overlap decreases. This causes a narrowing of the d-band and since the degree of d-band filling remains constant the dband increases in energy to compensate. This increased energy during tension therefore causes increased reactivity, whereas compressive strains have the opposite effect. Intuitively one would expect the surface of MA powders to have compressive strains based on impact of the milling media on the surface similar to shot peening. However, in 
the case of the small particles smeared onto the surface which show a flow component from shear stresses deforming the material which cold welds to the surface. After release of the shear stress with the interface constrained by the cold welding the recovery of the elastic strains in the smeared particle could create tensile strains in these surface smeared particles. Consequently, this may enhance the reactivity of the surface increasing the catalytic activity of the material.

A two-step process of dissociation is suggested to occur; one by layer(s) of PCA or gas trapped at the interface of the surface wear events and the closing of microscopic pores on the surface contained by strong surface interactions with a possible rising pressure as the deformation continues. The cold welding of wear-type particles smeared onto the surface and the creation of the transfer layer trapping the PCA and gas would be expected to provide enough activation energy to dissociate the molecules during these surface wear events and increased pressure, along with contact of the reactive high mass transport NGB structure and catalytic nature of the metal. The constant large deformation favors the production of fresh metallic surfaces through fractures. In the case of the closed pores, while a pressure increase is expected to enhance dissociation, the reaction most likely only occurs at the interface and it can be expected that new or fresh metallic catalytic surfaces are more difficult to produce in this environment. Thus, this alternative provides a much smaller contribution to the overall dissociation, particularly on a volume basis. It is important to note that the type of metallic catalyst can also be important. For example, in the RMM of pure iron [246] the carbon uptake is less for a given RMM time (using similar milling conditions) than observed in the present case with titanium. Since titanium is known to be an effective catalyst this further supports the suggestion of catalytic enhancement of the reaction with the trialuminide alloy. This suggests an important requirement for reactions to occur in the RMA/M processing, that the metallic powders be composed of a transition element having a strong catalytic character.

\section{b) Mass Transport}

The dissociated atoms must concentrate by a mass transport mechanism that is active at the near ambient temperature of the milling process. The NGB structure is 
capable of large mass transport enhancement and can be considered to be important in the process. The maximum mass transport dominated by the NGB region occurs during the continual impact of surface events and begins immediately upon milling. The samples milled for $0.5 \mathrm{~h}$ had these homogeneous surface features.

One main consequence of this domination of the surface wear-type process is the intimate contact of dissociated atoms with the NGB regions. The catalytic effect of titanium atoms in the NGB structure and deformed regions which have a large surface area to volume ratio make these regions attractive for the reaction process. The necessary mass transport must occur either by dissociated carbon atoms or by diffusion of $\mathrm{TiC}$ molecules. The catalytic effect suggests that TiC molecules are formed during dissociation; however, a possible surface mechanism of transporting the carbon atoms is by a "chain gang" approach where carbon is moved dynamically by sharing bonds with stationary titanium atoms. A method like this would be preferred as the larger size of the TiC molecules would have very slow diffusivities even with the more rapid diffusivity within the NGB region and would be expected to play a minor role in mass transport. Once the carbon atoms are incorporated into the NGB structure interstitial diffusion of the carbon through the large contiguous three-dimensional NGB network in the nanoscale microstructure providing a superhighway of rapid diffusivity, at or near room temperature, enhanced by the strain variations of the dynamic MA process.

As noted previously, triple junctions (and larger order nodes) increase with grain size reduction and are often associated with stress concentrations, can act as nucleation sites, and have enhanced diffusivity [211]. Of interest here is that these triple junctions also become part of the NGB structure and being stress concentrators are speculated to create stress gradients within the NGB regions during MA impacts. These stress gradients drive the diffusion of the carbon atoms accumulating at the triple junction regions (and high order nodes) thus providing a vehicle to localize the required amount of carbon for forming the carbide nucleus. The NGB region could very well be the mobile phase, mentioned by Kaupp [237] that provides a vehicle for mass transport. 


\section{c) Nucleation of Reaction Products}

During homogeneous solidification from a supercooled liquid nucleation occurs once a cluster of the requisite number of atoms to create a critical size nuclei organize into lattice positions with the crystal spontaneously growing in response to the cooling rate. Similarly, in MA processing, it can be surmised that once sufficient atoms of both reactants are concentrated locally with the ability to organize into the lattice positions a spontaneous nucleation of TiC crystallites occurs. The decrease in entropy among these high concentrations of carbon and titanium atoms can decrease the free energy in this region providing one means for driving the TiC formation.

Triple junction volumes are larger than the typical GB regions, and given that the GBs are estimated to be nearly $1 \mathrm{~nm}$ in extent, it would not be surprising that sizes of these triple junctions and higher order nodes are close to the size of $\mathrm{TiC}$ nuclei. In addition to a source of carbon atoms, a sufficient amount of titanium is also required, and based on interstitial carbon diffusion dominance, the titanium may well come from regions surrounding the triple point junctions diffusing along the NGB regions. The average crystallite size of the carbide is similar to that of the $\mathrm{L}_{2}$ intermetallic, about 2 $\mathrm{nm}$. This requires about 50 unit cells per average crystallite and with four chemical formula units per unit cell amounting to about 200 titanium and 200 carbon atoms. However, the carbon stoichiometry in the $\mathrm{TiC}$ was determined to be near the lower limit for phase stability with only about $50 \%$ of carbon sites occupied, or about 100 carbon atoms, thus lessening the requirement for carbon diffusion.

The required source for 100 carbon atoms equates to dissociation of only about 16 molecules of hexanes $\left(\mathrm{C}_{6} \mathrm{H}_{14}\right)$ to yield enough carbon for an average TiC crystallite. Considering the average carbon bond length of a hexane molecular chain to be about $0.5 \mathrm{~nm}$ results in a chain length segment of about $8 \mathrm{~nm}$ for enough carbon to produce a TiC nucleus. This amount can easily obtained by the amount of PCA adsorbed onto the surface involved in a surface smearing or transfer layer event. Based on the contact area observed for the islands of material at the surfaces, the extent of these surface events is of the order of micrometers, or less, suggesting that each surface event could easily lead to numerous nucleation events. 
Reaction rates typically follow an Arrhenius-type expression so that as the reaction temperature decreases there must have been an associated decrease in the activation energy for the reaction to occur. Clearly MA processing has shown reaction temperature reductions that must come from decreases in activation energy. The catalytic effects along with the usual structural nature of the NGB regions, deformation substructures, and the extremely small grain sizes, all contribute to such a reduction in activation energy providing for the conditions to nucleate TiC. While this is simply an endeavor to evoke discussion of the fundamental phenomena involved, it may serve to guide further research to fully characterize the reaction mechanisms observed in the RMA/M process.

\section{Nano-Engineering of Composite Materials or Nano-Engineering of Bulk Consolidated Materials}

As stated recently by Greer et al. [247] "Materials sciences are entering an era in which specific properties of a materials are obtained not only from its processing but also by controlling of the architecture of its constituents, often with sub-micron dimensions." An important aspect of this research was the observation of in situ reactions that occurred during the RMA/M in which elements from the process control agent (PCA) and environment react with the metallic elements in the starting powders. This in situ reaction during the processing was discovered to create large controllable amounts of carbides based solely on simple processing changes. Of utmost importance is not only the creation of a nano-scale microstructure in the powders, but a nanometer microstructure in fully dense consolidated samples. While this investigation has concentrated on the larger volume fractions of these phases, the ability to create small amounts of product reactants is not precluded. In fact, this new RMA/M process provides the ability to "nanoengineer" the ratio of composite constituents in a material on a nanometer scale. These new "process controlled composite materials" allow for simple engineering of the properties by precise control of composite content and structure through simple control of the RMA/M parameters. 
In the system milled in argon, the primary reaction product of intent was the $\mathrm{TiC}$ compound formed from the carbon extracted from the PCA and the titanium in the initial powder mixture which yields $\mathrm{TiC}$ particles about $2 \mathrm{~nm}$ in diameter. Of importance, was the discovery that the amount of carbon taken up, and therefore carbide formed, scales directly with the RMA/M time as shown in Figure III-42 (and also Figure IV-7). Importantly, the dependence appears to be linear. The initial TiC nano-scale particles are taken to have a near spheroidal shape, which upon consolidation by HIPing with the chromium-modified titanium trialuminide forms platelets of complex carbides that impinge on a nanometer scale. As will be discussed, the architecture of the microconstituents may also be controllable to some degree, but with limitations; however, structural features in the nanometer regime can be easily realized with this new process.

Several properties of the consolidated materials show systematic changes with the volume fraction of carbide present in the composites formed by the $\mathrm{L}_{2}$ intermetallic with a distribution of complex carbide(s) platelets. With longer milling times the volume fractions for the carbide can exceed that of the $\mathrm{L}_{2}$ intermetallic. In forming the consolidated material two versions of carbides grow into larger platelets both having the hexagonal crystal structures designated as the $312\left(\mathrm{Ti}_{3} \mathrm{AlC}_{2}\right)$ and $211\left(\mathrm{Ti}_{2} \mathrm{AlC}\right)$ complex carbides. These complex carbides are ternary compounds requiring aluminum which is extracted from the $\mathrm{L} 1_{2}$ intermetallic, thus altering its chemistry and possibly its properties.

In the simplest case, two-phase composites can have properties based on the rule of mixtures. This establishes boundary conditions, or limits, on the expected property values for these materials created by this process for a given system; however, incorporation of other phases can alter these limits expanding the engineering possibilities available through this type of RMA/M processing of materials. Some of the properties measured for the current consolidated samples do closely follow a simple rule of mixtures between the $\mathrm{L} 1_{2}$ intermetallic and the amount of the carbide(s), while other properties have a non-linear behavior with carbide content. In any case, the properties have simple functional relationships with carbide content and hence the ability to predict 
properties for a given carbide amount. Other properties, particularly those dependent on the microstructure, such as hardness and yield strength, can exceed the properties of the constituents to a significant degree and may be less predictable.

\section{Nanocomposite Powders}

The powders created for the nanocomposite with long RMA/M times resulted in similar microstructural features of about $2 \mathrm{~nm}$ for the L12 intermetallic crystallite size and the carbide particle size with the amount of carbide variable, making for simple controllable nano-engineering based on simple processing changes. Applying this RMA/M processing to other systems has shown a similar behavior of increasing amount of elements taken up from the liquid and gas with RMA/M time, suggesting that this nano-engineering approach can be applied to a large array of systems, thus opening the door to nano-engineering materials in a very straightforward manner. Further, it was established that a surprisingly large fraction of matrix phase atoms reside on the grain boundaries which have a different structure and therefore different properties than the crystalline grains. Consequently, the grain boundaries can alter the properties of the material in a significant manner. This includes typically structure-insensitive properties such as elastic moduli, Debye temperature, etc. In summary, it should be further emphasized that it has been concluded that these nano-engineering principles can be useful to develop a variety of properties in a range of materials.

Understanding the control of the microstructural development during the RMA/M

process is key to understanding how to nano-engineer these powders to develop a specific property and to aid in the prediction of the microstructure in the consolidated state. Of fundamental importance is the amount of the various phases present including the grain boundary regions as a "phase". In the system studied here the physical particle size remains in the micrometer range during all milling times and does vary somewhat with milling times, but no specific or desirable pattern emerges. Recall the crystallite size of the intermetallic matrix decreases with RMA/M time but approaches a constant size, with these sizes near the apparent limit for minimum grain sizes in polycrystalline materials. At the same time, the microstrains increase exponentially with RMA/M time which 
establishes increasing dislocation densities and other defects in the grain boundary regions. Many of these and other features determine the ability to develop and control the engineered system.

The RMA/M powders studied are attractive for use as protective coatings; however, the small $15 \mathrm{~g}$ batches produced are only useful for research purposes. Although not discussed here, the RMM process was scaled up to create large batches of powder using a large capacity ball mill, in order to establish whether large amounts of powder can be produced with the intent for this powder to be used as a feed stock in a plasma spray coating process. Although longer milling times were necessary for a given carbon uptake this process was very successful. Beyond the excellent oxidation protection provided by the intermetallic phase, the composites formed with variable amount of carbides have been shown to dramatically increase hardness and thus it is expected that wear resistance would also increase. Moreover, the ability to alter the volume fraction of carbides with this process makes it possible to "engineer" the thermal coefficient of expansion (CTE); hence, a means to match the coating CTE with that of the substrate material, thus minimizing coating degradation. This carbide variability can be demonstrated by extrapolation of the carbon content as a function of RMM time, Figure IV-7, showing that potentially up to a $100 \%$ TiC could be produced; additionally, $100 \%$ of the 312 or 211 phases can be accomplished in a much shorter time. It is interesting that extrapolation of to zero of the $\mathrm{L}_{2}$ intermetallic volume fraction produced in the consolidated materials in Figure III-41 results in an RMM time of $145 \mathrm{~h}$ very similar to the time to reach a $100 \%$ of the 211 complex carbide phase. These composite powders produced from the scaled-up process were applied as a coating, with extremely fine microstructures and carbide sizes smaller than the coating thickness, showed outstanding properties [248]. Wear resistance of these coatings was superior to current commercial coatings, while holding the promise of excellent oxidation resistance. 


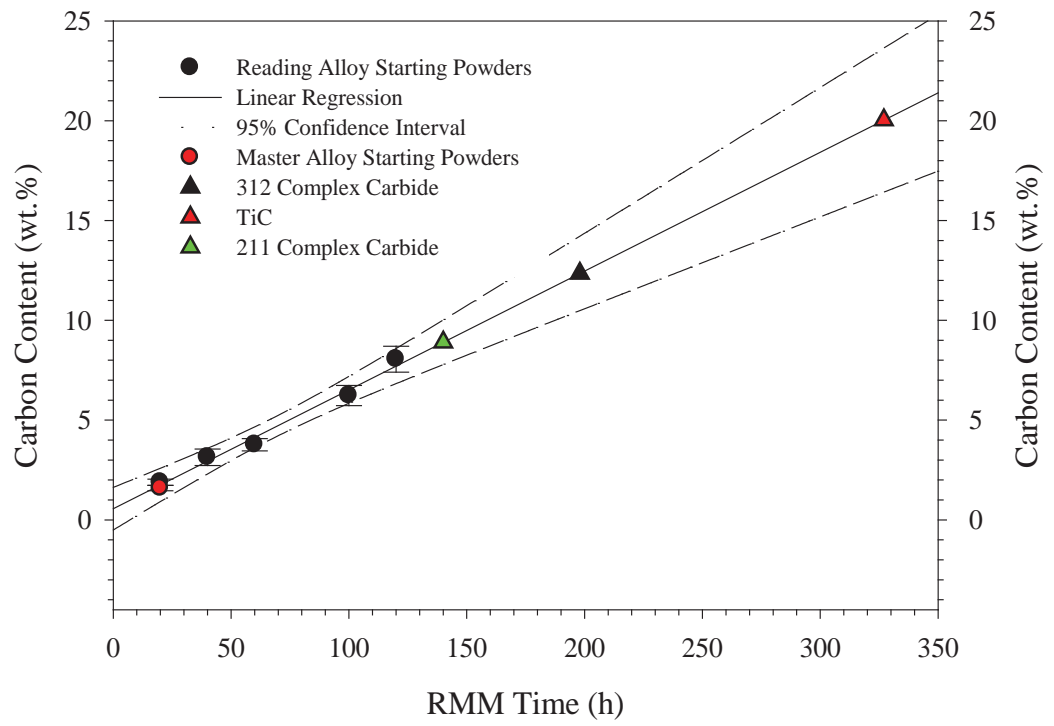

Figure IV-7 Extrapolated Carbon Uptake with RMM Time

\section{Bulk Consolidated Materials}

The more predictable properties, for example those expected to follow a rule of mixtures, depend simply on the volume fraction of the composite constituents. In the current system the pure $\mathrm{Al}_{66} \mathrm{Cr}_{9} \mathrm{Ti}_{25} \mathrm{~L}_{2}$ phase and pure complex carbide phase establish expected limits for the property changes. Some examples are shown in Table IV-7. The maximum percentage change for several properties that are achievable is shown for the complex carbide phase that has the largest change. While there are limits to the values, the range is large enough to consider when matching consolidated materials with certain needs.

Some brief comments about another property seem appropriate since the $\mathrm{L}_{2}$ intermetallic has been well documented to have outstanding oxidation properties and the complex carbides have also shown excellent oxidation properties [249, 250]. Le Claire [140] determined the oxidation resistance was close to that of the $\mathrm{Al}_{66} \mathrm{Cr}_{9} \mathrm{Ti}_{25} \mathrm{~L}_{2}$ phase for a sample with a volume fraction of 0.1 of the 211 complex carbide at $1007^{\circ} \mathrm{C}$. These complex or ternary carbides have a layered structure with a combination of metal- and ceramic-like properties; like metals, conductive thermally and electrically, thermally shock resistant, machinable, but damage tolerant; conversely like ceramics, low density, 
high elastic stiffness, a refractory, and oxidation resistant. They are part of a large family of layered structures with transition metals, group-A elements, and carbon and nitrogen [251]. The RMA/M processing in air produced a quaternary compound $\mathrm{Ti}_{2} \mathrm{Al}(\mathrm{C}, \mathrm{N})$, a solid solution forming the second phase in the composites of the consolidated materials. One important aspect of such phases with aluminum, a group-A element, is the formation of a protective $\mathrm{Al}_{2} \mathrm{O}_{3}$ layer and a higher diffusivity with aluminum in these compounds. The aluminum in the matrix is the only source for these complex carbides with the expected movement of this element increasing with RMA/M time. The combination of excellent properties in both composite microconstituents makes these chromiummodified titanium trialuminide complex carbide composites very attractive.

Table IV-7 Boundary Conditions of Properties Based on the Rule of Mixtures

\begin{tabular}{|c|c|c|c|c|}
\hline & \multicolumn{4}{|c|}{ Property Values for Each Phase } \\
\hline Property & $\mathbf{L 1}_{2}$ & $\mathrm{Ti}_{3} \mathrm{AlC}_{2}$ & $\mathrm{Ti}_{2} \mathrm{AlC}$ & $\operatorname{Max} \Delta \%$ \\
\hline $\begin{array}{l}\text { Density } \\
\left(\mathrm{g} / \mathrm{cm}^{3}\right)\end{array}$ & 3.708 & $4.21[252]$ & $4.11[253]$ & 12 \\
\hline $\begin{array}{l}\text { Young's Modulus } \\
\text { (GPa) }\end{array}$ & 184 & 289 [148] & 277 [148] & 36 \\
\hline $\begin{array}{l}\text { Shear Modulus } \\
\text { (GPA) }\end{array}$ & 75 & 124 [148] & 118.8 [148] & 40 \\
\hline $\begin{array}{c}\text { Poisson's Ratio } \\
\text { (v) }\end{array}$ & 0.224 & 0.200 [148] & $0.169[148]$ & -33 \\
\hline $\begin{array}{c}\text { CTE } \\
\left(\mu \mathbf{m} / \mathbf{m}^{\circ} \mathbf{C}\right)\end{array}$ & 12.3 & 8.8 [147] & $9.6[151]$ & -40 \\
\hline
\end{tabular}

After the very simple RMA/M processing, powders were consolidated using a combination of CIPing and HIPing with the extremely fine-scale microstructural sizes that exist in the degassed powders expected to dramatically enhance consolidation. Consolidation was attempted at $600{ }^{\circ} \mathrm{C}$ and did show significant necking between particles, but not enough mass transport for the short time duration chosen resulting in a porous-like samples with low densities. Most consolidated samples had densities slightly larger than those computed based on volume fraction data assuming stoichiometry for the 
phases present (Table IV-8). While electron microscopy did not focus on porosity in these alloys, there were no indications of porosity in the observations made. Of importance is that the measured density is slightly larger than that determined by calculations (with the exception of the 40/1000/RA sample having a opposite difference of $0.02 \%$ ). This suggests that these alloys are essentially fully dense. The small discrepancies between the measured and calculated values can be attributed to stoichiometry differences within the complex phases. For example, the $\mathrm{L}_{2}$ intermetallic is expected to lose aluminum to the complex carbide with titanium changes in these alloys produced, which would cause an effective enrichment of the heavier element chromium and therefore a slight increase in the density of the $\mathrm{L} 1_{2}$ intermetallic. Further work in these lower temperature regimes considering longer consolidation times may lead to ductility with a finer microstructure than has already been established.

Table IV-8 Measured and Calculated Density Comparison

\begin{tabular}{|c|c|c|}
\hline Sample & $\begin{array}{c}\text { Measured } \\
\text { Density } \\
\left(\mathrm{g} / \mathrm{cm}^{3}\right)\end{array}$ & $\begin{array}{c}\text { Calculated } \\
\text { Density } \\
\left(\mathrm{g} / \mathrm{cm}^{3}\right)\end{array}$ \\
\hline 20/1000/MA & 3.7815 & 3.7578 \\
\hline 20/1000/RA & 3.7647 & 3.7478 \\
\hline 40/1000/RA & 3.8230 & 3.8240 \\
\hline 60/1000/RA & 3.8736 & 3.8660 \\
\hline 100/1000/RA & 4.0497 & 4.0397 \\
\hline
\end{tabular}

In retrospect, this process leading to very high volume fractions of complex carbide based on control of the initial metallic powder stoichiometry and corresponding RMM time to reach the required carbon content is not unexpected. The properties of the 312 and 211 carbides do have small differences and the type of carbide in this system will determine the final limit reached. Furthermore, control of the chemical composition of the initial powders (to be discussed) may allow for selection of either of the complex carbides observed. The property-engineering diagrams developed include properties of 
both carbides to provide the expected limits for this particular system and also provide details of the relationship of the property value with observed carbide content for those properties measured in the consolidated materials.

\section{a) Physical Density Changes with Carbide Content}

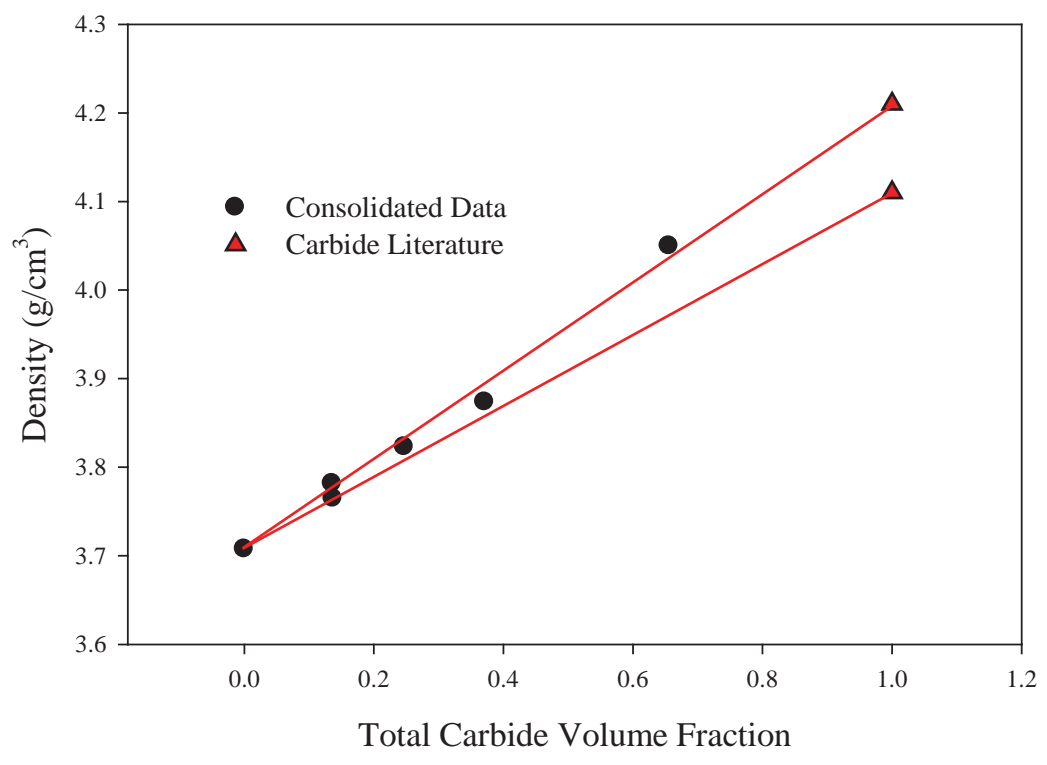

Figure IV-8 Density Changes with Total Carbide Content

The density of the consolidated material, assuming $100 \%$ densification during processing, has a near-linear trend following a rule of mixtures behavior as observed in Figure III-53. The measured densities, using the $\mathrm{Cr} 9$ sample as the pure $\mathrm{L}_{2}$ intermetallic are shown in Figure IV-8 along with the boundary conditions defined by the zero carbon content $\mathrm{Cr} 9 \mathrm{~L}_{2}$ intermetallic and the values in the literature for both complex carbides representing $100 \%$ of the respective carbides. The observations fall essentially within these limits. It should be recalled that there were some changes in proportions of the complex carbides in most samples. It is interesting that the 60/1000/RA sample which falls slightly outside these limits did have a couple of percent of other phases. 


\section{b) Mechanical Properties}

\section{(1) Elastic Moduli}

The most widely used model for predictions of the elastic moduli, $E$, of a composite material is based on the rule of mixtures approach shown in the Equation (IV.21) for a two-phase composite, with $V$ the volume fraction of each phase. However, there are several more sophisticated models that take into account other aspects such as the inclusion morphology and orientation, along with the elastic moduli and volume fractions of the constituents; the Hashin-Shrikman, Mori-Tanaka-type mean-field approaches, and the classical self-consistent schemes [254]. Also incorporated into these models can be porosity as well as other microstructural features, with some of these models directed toward specific situations. Several assumptions are often made with these various models such as; perfect bonding between constituents, surface variations of reinforcement do not deviate grossly, and constituents are isotropic. Of course inhomogeneous materials can lead to severe overestimates of the Young's moduli using the rule of mixtures approach, but given the near-linear behavior of the measurements as a first approximation this provides a simple method of prediction for this system.

$$
E_{e f f}=V_{A} E_{A}+V_{B} E_{B}
$$

The measured values of the Young's modulus show a near-linear dependence on the amount of carbide as shown in Figure IV-9. Recall, from Figure III-60 a slight curvature was noted in these results possibly due to the anisotropic nature of the moduli in the single crystal complex carbide platelets. The values do fall within the limits established by the value for the pure $\mathrm{L} 1_{2}$ intermetallic with the published values for each of the complex carbides also shown in red. 


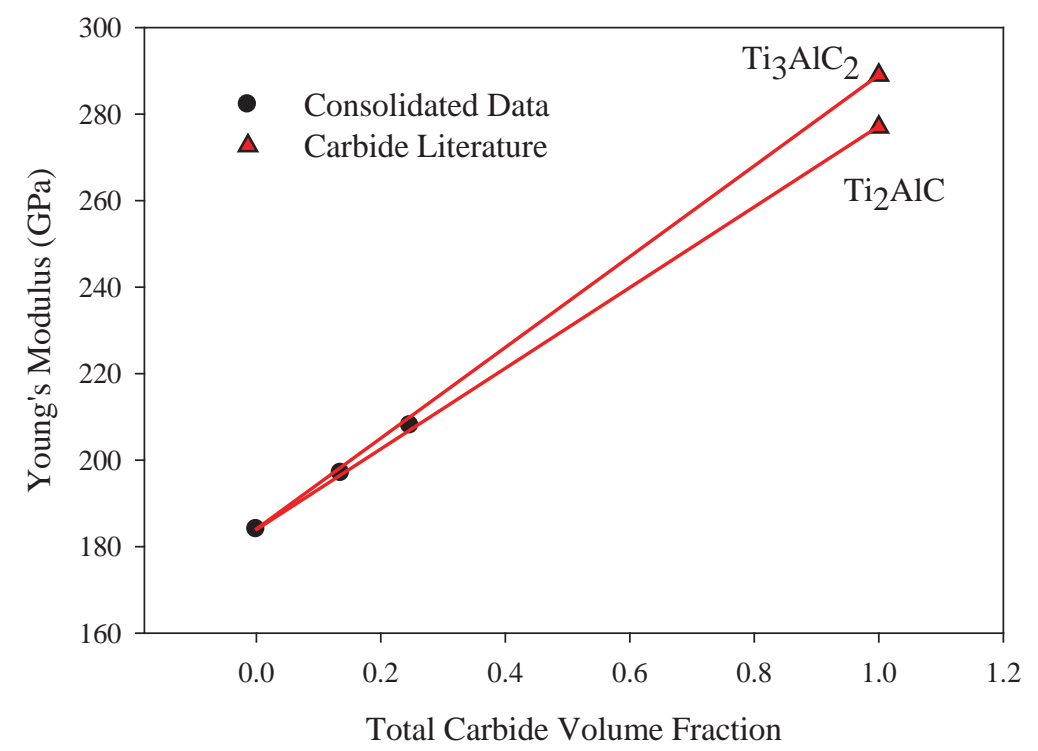

Figure IV-9 Young's Modulus Change with Total Carbide Content

The shear modulus (Figure IV-10) has a similar behavior to the Young's modulus with total carbide content, with the data falling within the limits (red lines) defined by the single phase $\mathrm{L}_{2}$ intermetallic (zero carbide) and the values in the literature for the complex carbides (shown in red), with these limits assumed to follow a simple linear relationship. As with the Young's modulus both consolidated composites show a shear modulus slightly less than predicted by the 312 limit (since these samples both contain this carbide phase), this may be due to the extremely fine-scale microstructural features and large aspect ratio of the single crystal complex carbide array. 


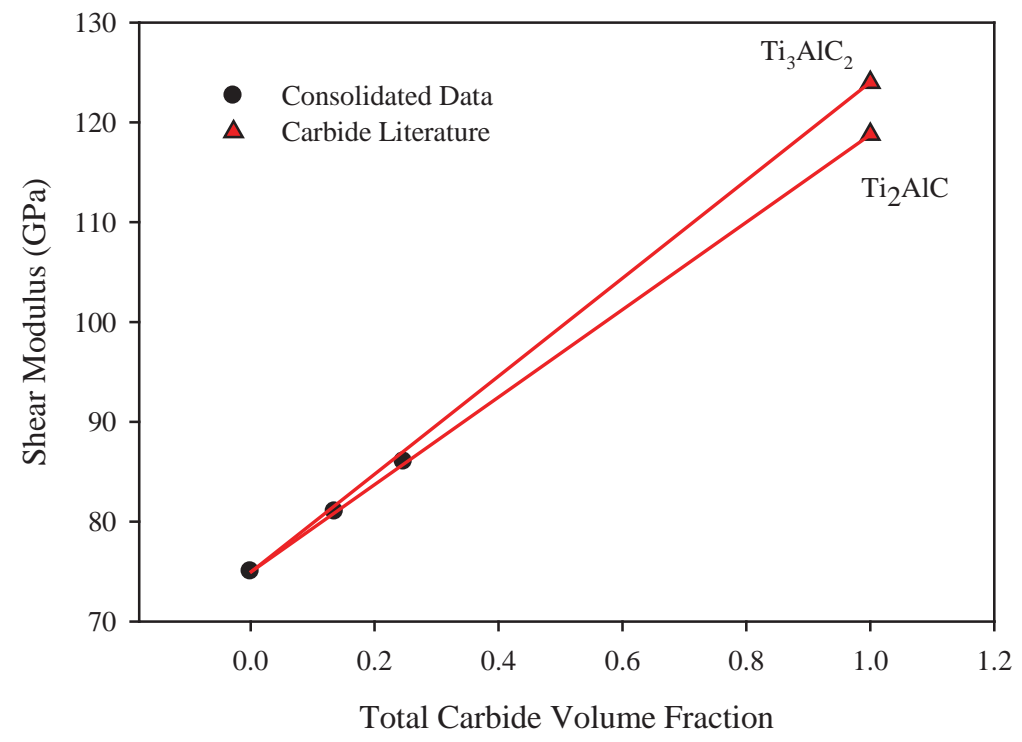

Figure IV-10 Shear Modulus Change with Total Carbide Content

\section{(2) Poisson's Ratio}

While the modulus changes follow a near-linear rule of mixtures the Poisson's ratio deviates significantly from this behavior, with the values measured for the 20/1000/RA and 40/1000/RA samples (20 and 40 RMM times respectively) showing a non-linear parabolic behavior, successively falling further below the linear lower limit established by the pure $\mathrm{L}_{2}$ intermetallic and the 211 complex carbide as shown in Figure IV-11. It should be noted that both of these samples contain the 312 complex carbide, represented by the upper limit line well above the measured values. Higher carbide contents would likely be helpful to fully understand this behavior. While the trend is systematic, phenomenological interpretation may be necessary to determine a predictable function. 


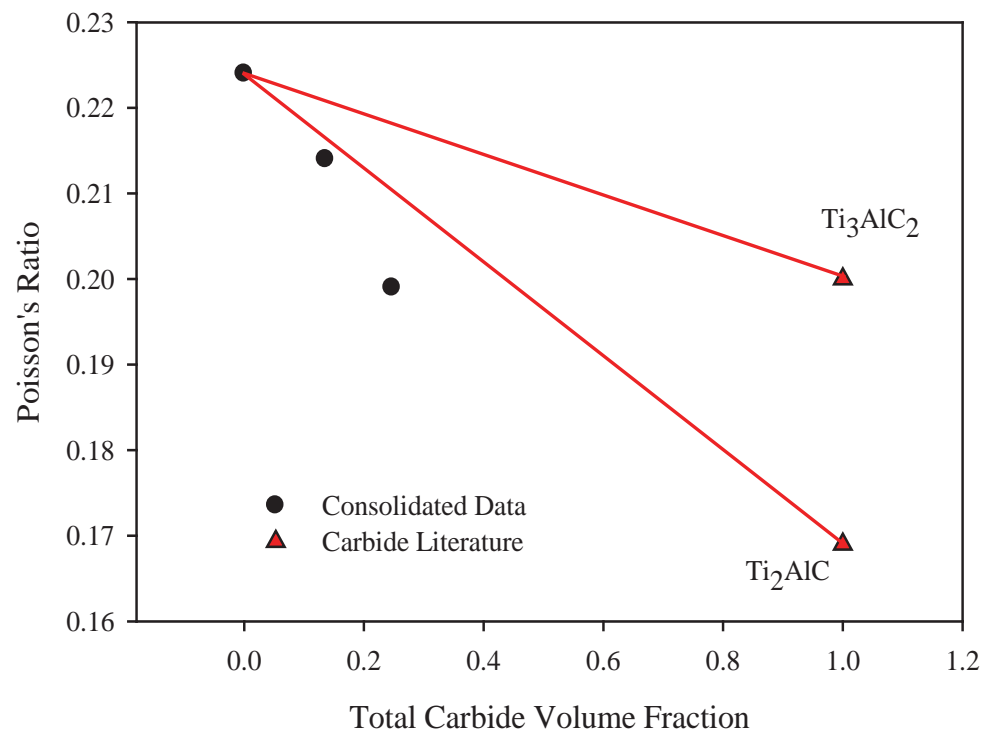

Figure IV-11 Poisson's Ratio Change with Total Carbide Content

The Poisson's ratio is defined mathematically as $v=-\epsilon_{t /} \epsilon_{l}$, a ratio between transverse elastic strain and longitudinal (loading direction) elastic strain [255]. Materials that have a network structure of anisotropic properties as well as foams and twodimensional structures have been shown to have a negative Poisson's ratio [256]. The three-dimensional arrays of these complex carbide single crystals disks may very well lower the Poisson's ratio by providing a negative contribution based on this complex carbide structure interacting with the positive value expected for the $\mathrm{L}_{2}$ intermetallic. It is expected that the non-linear behavior can be interpreted as resulting from these microstructural effects. In a related observation the temperature dependence of the elastic properties for a PbTe alloy is linear for the Young's and shear modulus, but the Poisson's ratio shows a parabolic decreasing behavior with temperature; however doping with $0.031 \%$ of $\mathrm{PbI}_{2}$ shows a parabolic (opposite curvature) increasing behavior with temperature [257]. This illustrates that the microstructure can play a significant role in the Poisson's ratio. 


\section{c) Coefficient of Thermal Expansion (CTE)}

The CTE measured for these materials also shows a non-linear parabolic behavior, similar to that of the Poisson's ratio, with the values outside the simplistic linear limits established by a rule of mixtures between the $\mathrm{L} 1_{2}$ intermetallic and the two complex carbides as shown in Figure IV-12. It is important to note that this systematic change in CTE with carbide content allows for the possibility of engineering the CTE of these composite materials. This ability to engineer the CTE has also been shown commercially, in an unrelated process, by CPS corporation (Dunkirk, N.Y.) which developed a process of CTE matching by modifying the particular ratio of the aluminum$\mathrm{SiC}$ composite for use in electronic devices and assemblies [258]. A more rigorous theoretical estimation of the mean CTEs of a composite can be achieved using a modified rule of mixtures that incorporates the bulk modulus described in both Equation (IV.22) and Equation (IV.23) [259]. Here $E$ is the Young's modulus, $v$ is Poisson's ratio, $V$ is the volume fraction of the appropriate phase; the matrix $(m)$ or the carbide $(c)$.

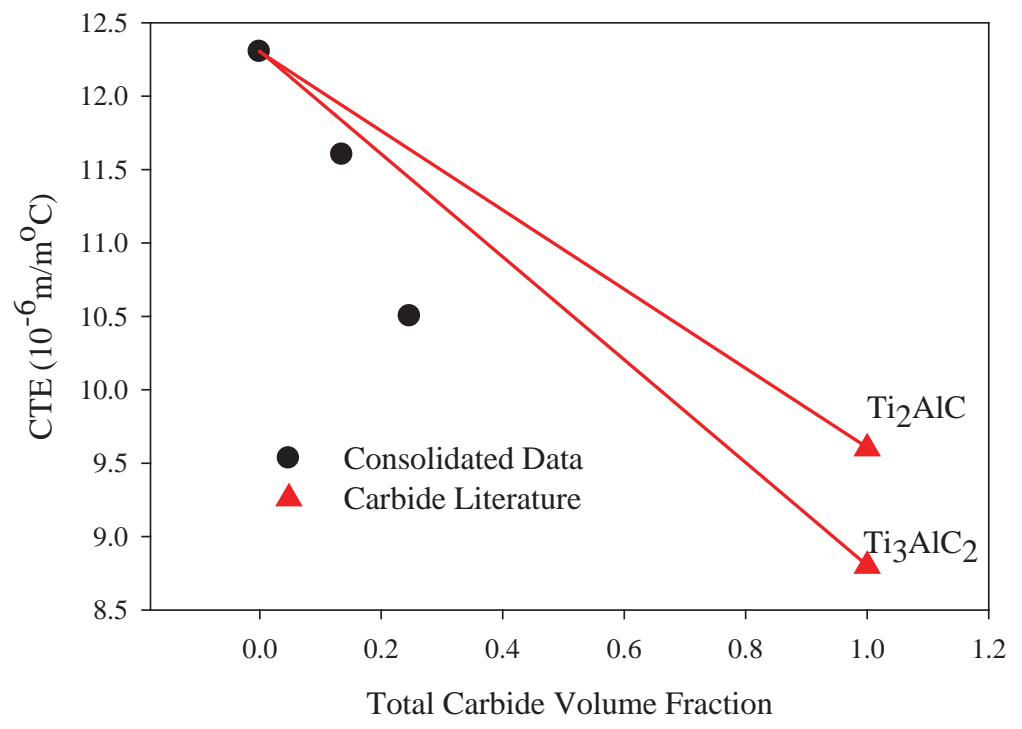

Figure IV-12 CTE with Total Carbide Content 


$$
\begin{gathered}
\alpha=\frac{\left(\alpha_{m} K_{m} V_{m}+\alpha_{c} K_{c} V_{c}\right)}{\left(K_{m} V_{m}+K_{c} V_{c}\right)} \\
K=\frac{E}{3(1-2 v)}
\end{gathered}
$$

Values for the theoretical CTE calculated in this manner based on the measured volume fraction and elastic constants, combined with elastic constants data from the literature for the 312 complex carbide in these alloys, are presented in Table IV-9. The theoretical value for the 20/1000/RA sample agrees with that measured. However, the value for the 40/1000/RA sample is predicted to be considerably larger than the measured value. Since the Poisson's ratio plays an important role in the theoretical calculation, the microstructural effects are expected to play a role in the CTE with an anticipated anisotropic behavior contribution from the complex carbides that have the hexagonal crystal structure. The structural array of complex carbides single crystal platelets lowers the expected Poisson's ratio of the carbide causing $K_{c}$ in Equation (IV.23) to increase. Since $K_{c}$ in the denominator of Equation (IV.22) dominates the value in the numerator, the calculated value of the CTE would be lowered in agreement with the results in Table IV-9.

Table IV-9 Comparison of Measured and Theoretical CTE

\begin{tabular}{ccc}
\hline $\begin{array}{c}\text { Sample - RMM } \\
\text { Time (h) }\end{array}$ & $\begin{array}{c}\text { CTE Measured } \\
\left(\mathbf{1 0}^{-\mathbf{6}} \mathbf{m} / \mathbf{m}^{\mathbf{0}} \mathbf{C}\right)\end{array}$ & $\begin{array}{c}\text { CTE Theoretical } \\
\left(\mathbf{1 0}^{-6} \mathbf{m} / \mathbf{m}^{\mathbf{0}} \mathbf{C}\right)\end{array}$ \\
\hline $692(20)$ & 11.5 & 11.5 \\
$\mathbf{6 9 4}(\mathbf{4 0})$ & $\mathbf{1 0 . 4}$ & $\mathbf{1 1 . 1}$ \\
\hline
\end{tabular}

The CTE values shown for the 211 and 312 complex carbides in Figure IV-12 is for a polycrystalline material. Given the hexagonal crystal structure, differences are expected based on the direction in the single crystal. The CTE for the 211 phase has been determined from high-temperature XRD data showing large differences between the "a" direction at $7.1 \mathrm{X} 10^{-6} /{ }^{\circ} \mathrm{C}$ and the "c" direction at $10 \mathrm{X} 10^{-6} /{ }^{\circ} \mathrm{C}[260]$, and the behavior of the 312 phase might be expected to be similar. This single crystal array of complex carbide platelets make contact at the edges and considering the large aspect ratio it might 
be expected that the CTE of the microstructure would be controlled by expansion in the "a" direction, a much lower value agreeing with the measured data showing a lower than expected CTE. It would be interesting to determine the values for higher carbide content samples and see if this behavior continues to be lower than expected.

While the measurements presented here may not all be optimal for precise prediction of engineering properties, the results support the concept of the ability to accurately predict the amount of carbides produced during this processing which can then be used to predict the resultant composite properties. While a simple rule of mixtures applies for some properties, others have shown deviations from the simple rule of mixtures as well as from current models based on a two-phase system. These deviations most likely result from aspects of the unique microstructures produced in these materials. A more complete understanding of the effects of parameters involved in the creation of the microstructure may permit developing guidelines for controlling these reactions and also to improve the ability to be able to predict the engineered property. An important ability of the process developed here based on the RMA/M process is that it allows access to unique fine-scale microstructures on the nanometer level, perhaps developing uncharted ranges of properties.

\section{Consolidated Material}

Often in polycrystalline materials the microstructure determines the strength of the material. What is remarkable about the consolidated materials produced in this study is the extremely large (tenfold) increase in yield strength of the material over that of an as-cast material of the pure $\mathrm{L}_{2}$ chromium-modified titanium trialuminide, which is a result of the unique microstructure developed. Considered in another way, the ultimate compressive strength in these materials (about 2.5 GPa) reaches about one-half of the theoretical shear strength estimated to be $5 \mathrm{GPa}$ for the $\mathrm{L} 1_{2}$ chromium-modified titanium trialuminide (see Chapter I.B). Since these materials reach a significant fraction of the theoretical shear strength they can be considered as ultra-high strength materials. The strengths in this case are similar to those observed for iron whiskers (defect free iron single crystals) from the pioneering work by Brenner [261]. However, the remarkably 
high strength is attained by the common powder metallurgy processes of CIPing and HIPing to consolidate the degassed powders produced by the RMM processing. These strengths approach, or exceed, those of ultra-high strength steels that require special heat treatment procedures [146]; however, the density-compensated strengths far exceed those for the ultra-high strength steels.

These initial attempts at creating these new materials reached strength levels that are among the highest strengths attained in polycrystalline materials using very simple processing methods and without any optimization of the microstructure and/or chemistry. The original goal to strengthen the $\mathrm{L}_{2}$ intermetallic has not only been achieved, but exceeded, with the ultra-high strength of this material derived from the common powder processing consolidation techniques of CIPing and HIPing; however, the key is the significant role the as-milled powder plays in the development of the microstructure. Several strengthening mechanisms will be discussed in relation to the microstructure developed in these materials.

\section{Microstructure}

The equilibrium microstructures expected for these consolidated materials consist of the $\mathrm{Li}_{2}$ intermetallic and the $\mathrm{Ti}_{2} \mathrm{AlC}$ carbide in varying amounts dependent on the RMM processing time. While this exact microstructure was not realized, it is clear that optimization of the initial chemistry is important in determining the microstructural changes as the carbon uptake increases as a function of RMM time. The microstructure of the consolidated composites produced in this project consists of two important features, a random array of complex carbide platelets (that make contact at higher carbon contents) with the $\mathrm{L}_{2}$ intermetallic filling the interplatelet spaces. Milling time allows for increased amounts of the complex ternary carbide group of compounds composed of two different stoichiometries, $\mathrm{Ti}_{2} \mathrm{AlC}$ and/or $\mathrm{Ti}_{3} \mathrm{AlC}_{2}$ referred to as the 211 and 312 complex carbides, respectively. As previously mentioned the 312 complex carbide is a metastable phase that has only been shown to form near temperatures of $1300{ }^{\circ} \mathrm{C}$ [139]. Here the phase has developed in alloys consolidated at lower temperatures. 
Some important volume fraction results, Appendix VIII.C.1, for the annealed powders from the RMMXh9TiAr Series experiment show that RMM times between 5 and $10 \mathrm{~h}$ provide the closest microstructure to that expected when adding $9 \mathrm{wt}$. pct. excess titanium with the intermetallic powders (i.e. a two-phase mixture of intermetallic and carbide material). This agrees with a similar RMM experiment by Le Claire [140] who determined an ideal milling time of $6 \mathrm{~h}$. Recall the original establishment of $9 \mathrm{wt}$. pct. excess titanium to be added to create the $\mathrm{TiC}$ phase was taken from the literature for a similar titanium trialuminide milling experiment which estimated 2 wt. pct. carbon was retained for a $20 \mathrm{~h}$ milling time [133]. Based on stoichiometry, this is about one-half of the required amount of titanium to produce the 211 complex carbide. This suggests that formation of the 312 carbide resulted from the lack of sufficient titanium since it is closer to the one to one ratio of titanium to carbon originally determined for $\mathrm{TiC}$ formation compared with the two to one ratio required for the 211 complex carbide. Thus, increased excess titanium for the $20 \mathrm{~h}$ RMM time frame (or lower RMM time for the $9 \mathrm{wt}$. pct. titanium) would be required to create the desired "ideal" microstructure. Such studies would supplement work by Le Claire [140] who examined the kinetics of the carbon uptake for the RMA/M process developed in this study and found that ball density as well as the type of PCA can both significantly enhance the carbon uptake. This is an area that requires more understanding. The importance of the shorter RMA/M times to reach a given carbon content is to prevent iron contamination, an issue that can develop at longer milling times.

The presence of secondary phases in the consolidated samples can be an indicator of whether the proper amounts of aluminum and titanium are available for a given set of milling conditions. On the other hand, oxygen and iron appear as contaminants. As has been discussed, $\mathrm{Al}_{2} \mathrm{O}_{3}$ results from air contact with the as-milled powders during processing. It is possible to eliminate or reduce this unwanted phase. However, such amounts of alumina are found in most powder processed materials that contain aluminum. The presence of iron-based phases results from iron that naturally wears from the milling media and becomes significant at the very long milling times. In the current work iron did not appear as a significant secondary phase until the $120 \mathrm{~h}$ RMM time, 
when a large amount of about 6 wt. pct. iron was observed. The small amount of iron produced during shorter RMM times can be easily solutionized in the $\mathrm{L}_{2}$ intermetallic phase as transition metals can substitute freely for chromium.

The presence of phases containing aluminum, titanium, and/or chromium are good indicators of improper chemistry and/or milling times for a given set of milling conditions. For example, Le Claire [140] noted that if carbon is too low $\mathrm{Al}_{2} \mathrm{Ti}$ forms as excess titanium reacts with $\mathrm{L}_{2}$ intermetallic, while if carbon is too high $\mathrm{AlCr}_{2}$ forms as the 211 carbide phase requires more titanium which is extracted from the $\mathrm{L}_{2}$ intermetallic. There are more complex issues that arise during milling. For example, in the phase analysis of the annealed powders from the RMMXh9TiAr Series (Appendix VIII.C.1) the $\mathrm{Al}_{3}$ Ti phase ( $\mathrm{DO}_{22}$ crystal structure) was observed and the amount increased in samples milled for the longer times indicating a milling time that it is too long for that chemistry. Adding complexity is that both aluminum and titanium can be extracted from the $\mathrm{L}_{2}$ intermetallic phase to be incorporated into the complex carbide phase. Adjustments of aluminum content may mitigate appearance of the aluminum-chromium alloy phases produced in several of these alloys. As noted earlier there is a significant breakdown in the microstructure for the RMM time of $120 \mathrm{~h}$. This sample contained 54 $\%$ elemental titanium in the initial mixture, but the lack of sufficient aluminum to maintain the stoichiometry of the complex carbide structure caused a significant amount of the $\mathrm{TiC}$ phase to form in the alloy. Thus, presence of the $\mathrm{TiC}$ phase can also indicate lack of adequate aluminum. However, it should be noted that the $\mathrm{L}_{2}$ structure of the intermetallic was visible throughout all the RMM times despite the extraction of some aluminum from the $\mathrm{L}_{2}$ intermetallic to form the complex carbides.

The microstructure discussion will now focus on the main phases present which are the $\mathrm{L}_{2}$ intermetallic and the complex carbides. During consolidation it is expected that the small TiC particles formed during milling act as nuclei to form the complex carbide. What is extremely important here is that these TiC particles, which have a $2 \mathrm{~nm}$ size of the order of the critical size nuclei in homogeneous solidification of $\mathrm{TiC}$, provide an extremely large density of nuclei for the complex carbide formation, a unique aspect of the RMA/M processed powders. The important positive aspect is that these TiC nuclei 
are much smaller and more numerous than in typical powder consolidation processes in which the far fewer nuclei lead to a coarser microstructure with a smaller number of larger particles. These numerous and extremely fine nuclei can be considered as a primary reason for the fine-scale microstructure produced despite the quite aggressive high temperature annealing treatment during consolidation.

Further, while the degassed powders were not presented in this study, XRD analysis of such powders revealed that the TiC remained at the same particle size of about $2 \mathrm{~nm}$ indicating no growth of the TiC during this $500^{\circ} \mathrm{C}$ processing. Then, in support of this general conclusion about microstructure is a comparison with the sample 20/800/MA, which was not included in the results. It was consolidated at the $800{ }^{\circ} \mathrm{C}$ for $15 \mathrm{~min}$ and processed in the exact same manner as the 20/1000/MA (also RMM time of $20 \mathrm{~h}$ ). As expected this sample has the smallest size complex carbides that are dispersed and isolated with platelet shapes. The orientation is expected to be random with the etching process lifting many of the more normal complex carbides off the surface distorting the orientation represented in the micrograph. Sample 20/1000/MA (Figure III-49), consolidated at $1000{ }^{\circ} \mathrm{C}$, has much larger complex carbides that begin to grow particularly normal to the (001) plane eventually causing these platelets to make contact, thus impeding the growth process.

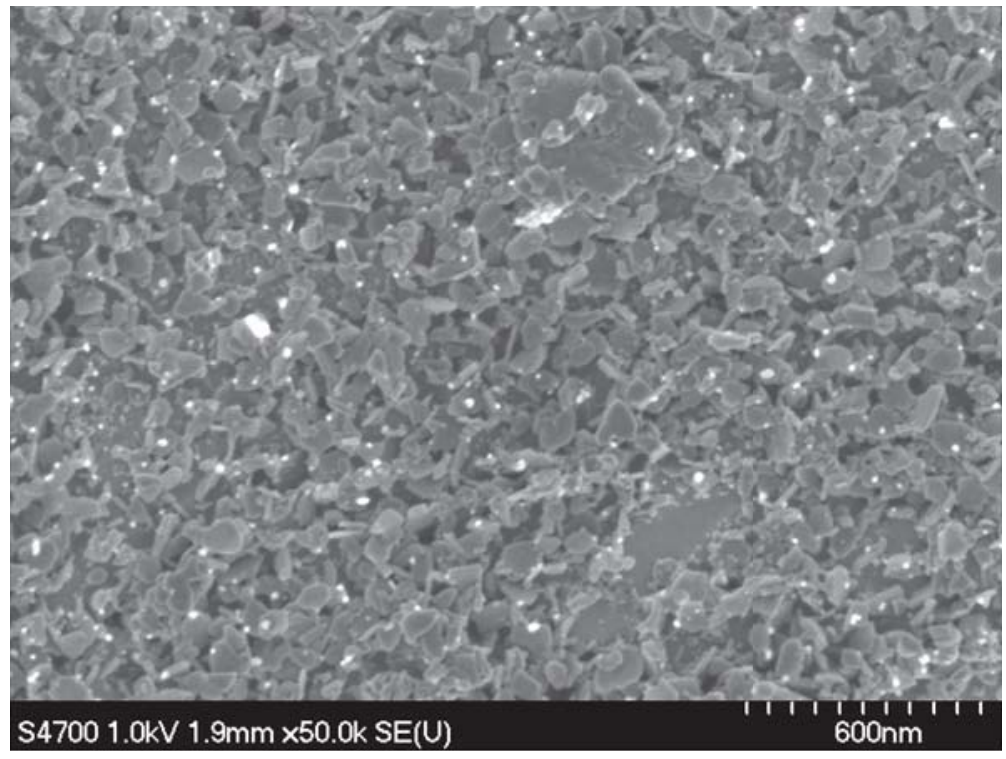

Figure IV-13 20 h RMM Time HIPed at $800{ }^{\circ} \mathrm{C}$ Sample 20/800/MA 
Intuitively, one would expect that these annealed structures would be essentially defect free. However, the $\mathrm{L}_{2}$ intermetallic has a very large dislocation density only about an order of magnitude less than that for heavily cold worked materials for the larger carbide content samples. Additionally, the crystallite sizes of the complex carbide particles are smaller than the physical sizes (Chapter III.D.1.d)(3)) indicating that dislocations are present. Because of the difference in CTE between the carbide particles and the $\mathrm{L}_{2}$ intermetallic, the most likely cause of dislocation generation is the cooling of the sample from the $1000{ }^{\circ} \mathrm{C}$ temperature. A dislocation generation model for this case termed the Prismatic Punch Model was developed by Arsenault [262] whereby the dislocation density generated is based on two important terms; the strain developed, which is determined by the CTE difference, and the surface area of the particles. This is shown in Equation (IV.24), along with an important variable $A_{n}$ the number or particles per unit matrix volume. Arsenault considered $\mathrm{SiC}$ in a platelet form dispersed within an aluminum matrix which represents a large six to one CTE ratio. Calculations with SiC platelets down to the micrometer range show that the dislocation density increases rapidly with decreases in the size of the SiC. A dislocation density of $7 \times 10^{6} \mathrm{~mm}^{-2}$ was determined for a 0.20 volume fraction and a $1 \mu \mathrm{m}$ particle size by Arsenault [262], about one to two orders of magnitude less than found in the consolidated samples in the current study.

$$
\begin{gathered}
\rho=\frac{2 \varepsilon A_{n}}{b} S \quad \text { (IV.24) } \\
A_{n}=\text { no. of particles } \\
S=\text { Particle area } \\
\varepsilon=\frac{\Delta C T E \times \Delta T}{2} \\
b=\text { Burgers vector }
\end{gathered}
$$

Considering the difference in the CTE for the consolidated microconstituents in the current materials, using an average value for both complex carbides compared to the value for the $\mathrm{L}_{2}$ intermetallic, gives a CTE ratio of about three to four. As noted, the CTE ratio in the Arsenault calculations is much larger at a six to one ratio. However, the 
much larger dislocation densities developed in the current work can be expected based on the much larger difference in the surface area to volume ratio $A_{n} S$ from the smaller sizes observed in the complex carbide microstructure. These are very large surface areas compared to those in Arsenault's calculation. Also, it should be noted that since the array of complex carbide platelets have a smaller coefficient of thermal expansion it would be expected that upon cooling the interspatial $\mathrm{L}_{2}$ intermetallic is under a tensile stress, while a compressive stress is imposed on the complex carbides.

Calculation of the dislocation density generated in the $\mathrm{L}_{2}$ intermetallic by the cooling process for select consolidated samples was accomplished based on data provided earlier. The size information for the complex carbides are from Table III-18 (also in Table IV-11), along with the volume fraction data from Table III-14, using the CTE for the appropriate phases (using the CTE for the major carbide present) provided on page 195, a delta temperature of $975^{\circ} \mathrm{C}$, and an $a / 3<112>$ Burgers vector. The results are summarized in Table IV-10 with the calculated results agreeing quite well with those measured from the peak profile analysis indicating the CTE mismatch is the major source of dislocation generation in these alloys.

Table IV-10 Results of Dislocation Density Calculations using the Arsenault Equation

\begin{tabular}{|c|c|c|}
\hline Sample & $\begin{array}{c}\text { Calculated } \\
\text { Dislocation Density } \\
\left(\mathrm{mm}^{-2}\right)\end{array}$ & $\begin{array}{c}\text { Measured Dislocation } \\
\text { Density (Table III-17) } \\
\qquad\left(\mathrm{mm}^{-2}\right)\end{array}$ \\
\hline 20/1000/MA & $1.90 \times 10^{8}$ & $5.6 \times 10^{7}$ \\
\hline 20/1000/RA & $1.16 \times 10^{8}$ & $6.6 \times 10^{7}$ \\
\hline 40/1000/RA & $2.06 \times 10^{8}$ & $1.1 \times 10^{8}$ \\
\hline 60/1000/RA & $1.75 \times 10^{8}$ & $1.9 \times 10^{8}$ \\
\hline
\end{tabular}

The $\mathrm{L}_{2}$ intermetallic phase in these composite materials appears to fill the voids between the carbide platelet arrays that have interparticle distances between 429 to $194 \mathrm{~nm}$ (Table III-18) with the largest spacing corresponded to the shortest RMM time. In some cases, more prominent at the lower carbide contents (20 h RMM times), GBs 
were observed but these lessened with increased amounts of complex carbide. The crystallite size of these consolidated materials decreases from a value of about $100 \mathrm{~nm}$ down to about $50 \mathrm{~nm}$ (Table III-16) as the RMM time and carbide content increases. It is interesting to note that the interparticle carbide spacing to crystallite size maintains about a 4 to 1 ratio for all samples measured. Analysis by TEM suggests these interparticle regions in the materials with larger carbide contents are generally single crystal grains with defect-originated contrast variations observed within these grains.

Results of Scherrer crystallite size measurements of the complex carbides found a smaller crystallite size in the diameter of the platelets than measured from SEM images. In the [001] direction, the thickness was similar until the RMM time of $100 \mathrm{~h}$ when a smaller crystallite size was developed in this direction than measured directly from the images, commensurate with large increases in the carbide volume fraction. Since these complex carbides are known to have some ductility and have metallic-like behavior $[144,264]$, dislocations can also be expected to be generated in this phase from the CTE mismatch upon cooling.

It has been clearly established that there is a linear increase in the amount of the complex carbides formed with RMM time in these consolidated materials. While the details of work at $800{ }^{\circ} \mathrm{C}$ have not been discussed, sample 20/800/MA (microstructure shown in Figure IV-13) was consolidated at $800^{\circ} \mathrm{C}$ for $15 \mathrm{~min}$ after being processed with an RMM time of $20 \mathrm{~h}$. As expected this sample has the smallest size complex carbides that are in a platelet form and dispersed in an isolated manner. However, larger amounts of carbides produced at longer RMM times and then consolidated at a higher temperature create a three-dimensional array of larger complex carbide platelets. It appears growth in theses single crystal platelets is more rapid in the "a" direction and with the growth at the higher HIP temperature, these platelets impinge forming an interface along the platelet edges. Often in the complex carbides a platelet edge will meet at random angles to the large surface of the platelets (normal to the edge). In some cases, the interfaces of these touching platelets in the FESEM micrographs (Figure III-49 through Figure III-52) show clear delineations, although areas with no delineations can be the result of plastic flow of the carbide platelets obscuring the interface. This is in agreement with compression 
testing reported on the pure 211 complex carbide that showed a $4.8 \%$ plastic strain at room temperature [143]. An important point, is that the superplastic properties of these consolidated materials at high temperatures (e.g., $900^{\circ} \mathrm{C}$ ) requires the accommodation of the movement of these complex carbide platelets to allow the large deformation observed. Strong bonding between the impinged platelets would create a more rigid structure tending to inhibit the amount of deformation observed during superplastic deformation. This suggests that while mechanical contact occurs between individual platelets the lack of significant bonding allows for these platelets to move in an independent manner under the proper conditions.

The aspect ratio of the carbide platelets, listed in Table IV-11, increases from the low temperature 20/800/MA sample to the higher temperature 20/1000/RA sample which has the complex carbides firmly in contact. The value then decreases slightly as the amount of the complex carbide increases and the growth of the diameter of platelets is impeded by the physical contact of the platelets. This obstruction causes more rapid growth in the [001] direction. The platelets then create a three-dimensional contiguous complex carbide structure with mechanical contact between platelet edges. It is likely that this three-dimensional array of carbide platelets adds to the strengthening observed in these alloys.

Table IV-11 Complex Carbide Aspect Ratio

\begin{tabular}{c|ccc} 
Sample & $\begin{array}{c}\text { Diameter } \\
(\mathbf{n m})\end{array}$ & $\begin{array}{c}\text { Thickness } \\
(\mathbf{n m})\end{array}$ & $\begin{array}{c}\text { Aspect Ratio } \\
\text { (Dia./Thick.) }\end{array}$ \\
\hline 20/800/MA & 48 & 6 & 8 \\
\cline { 2 - 4 } $20 / 1000 / M A$ & 160 & 16 & 10 \\
\hline 20/1000/RA & 431 & 29 & 15 \\
\cline { 2 - 4 } 40/1000/RA & 443 & 33 & 13 \\
\hline \multirow{6}{*0/1000/RA}{} & $\mathbf{4 9 6}$ & $\mathbf{4 8}$ & $\mathbf{1 0}$ \\
\hline
\end{tabular}


The microstructure commonly observed in polycrystalline $\mathrm{Ti}_{2} \mathrm{AlC}(211)$ is a hexagonal shaped micro-laminate [143], while no size information was given beyond a 5 - $10 \mu \mathrm{m}$ grain size with the grains having a hexagonal shape (the unit cell is hexagonal). A scanning electron microscope (SEM) micrograph of a transgranular fracture surface shows a layered structure. Further, the thickness of these layers was measured from an SEM micrograph in the publication as having an average value of about $375 \mathrm{~nm}$, a value near the lower limit of resolution for that micrograph. These layers are thicker than those observed in all of the consolidated material in the current work suggesting more growth of the single crystal platelets is possible.

The volume fraction of the carbide exceeds that of the $\mathrm{L}_{2}$ intermetallic by the $100 \mathrm{~h}$ RMM time suggesting that on a volume basis the carbide could become the matrix. The contiguous nature of the three-dimensional array of complex carbide platelets developing by the $20 \mathrm{~h}$ RMM time would technically define the complex carbide as the matrix of the material, which then becomes a ceramic matrix composite. This carbide formation process brings up the interesting potential that provided proper compensation for aluminum is established it could allow approaching $100 \%$ complex carbide. Extrapolation of the linear carbon uptake (Figure IV-7) to obtain the required stoichiometric amount of carbon needed to form only the 211 complex carbide results in an RMM time of $150 \mathrm{~h}$ for the conditions used in the current study. The ease and simplicity of this approach is quite appealing since it can be difficult to synthesize these compounds by other means $[263,264]$ and using this RMM/A technique and consolidation method could facilitate development of a new method of creating of these pure complex carbides at lower temperatures with the potential of creating a fine-scale microstructure. As mentioned earlier the milling can be easily scaled up with a large mill such as a ball mill.

In summary, the basic microstructural model for these consolidated materials (those HIPed at $1000^{\circ} \mathrm{C}$ ) is a contiguous three-dimensional array of single crystal complex carbide platelets with the $\mathrm{L}_{2}$ intermetallic occupying the space between these carbide platelets. The $\mathrm{L}_{2}$ intermetallic has a significant defect density especially considering the consolidation temperature and the long time at that temperature 
suggesting that these defects have been introduced in the cooling process. The important result is that the crystallite size and some carbide dimensions are in the nanometer range, thus a fine-scale structure has been preserved in the material, despite an aggressive consolidation temperature.

\section{Strengthening of these Nanocomposites}

Given the nature of the microstructure with the essentially contiguous complex carbides which have a much larger modulus compared to the interspatial $\mathrm{L}_{2}$ intermetallic phase, the load carrying capacity could initially be determined by the carbide array. However, these carbide platelets are single crystal in nature, supported by single-crystal, or polycrystal grains of the $\mathrm{L}_{2}$ intermetallic between them, creating a support system dependent on the materials interaction. Once the complex carbide structure begins to fail, destroying the contiguity, the $\mathrm{L}_{2}$ intermetallic will play a larger role in the plastic deformation behavior and the strengthening is more complex. There is an increase in the microstrains in the intermetallic prior to deformation that scales with the increase in carbide content as the RMM time of the powder increases. As noted, this increase in the carbide content can produce more dislocations on cooling from consolidation temperature because of the CTE mismatch which can affect the strengthening. In addition, any remnant residual stresses could also affect the strength.

These consolidated composite materials far exceeded the initial strengthening expectations with high yield strengths that exceed all values reported for other similar type materials in Table I-1. Although the strain to fracture was significant for the $20 \mathrm{~h}$ samples it decreased as the complex carbide content is increased, but it was surprising that any ductility was observed at these strength levels. Also, it is possible that additional slight improvements in the ductility may result from reducing the small amounts of oxide that formed during processing, as well as minimizing secondary phases from improper chemistry. The yield strength increases by about $20 \%$ as the complex carbide volume fraction increases up to 0.38 , but the strength drops for the sample with a complex carbide content of 0.64 (sample 100/1000/RA). This may be attributable to larger amounts of unwanted secondary phases produced at the longer RMM times. 
The two main strengthening mechanisms originally considered for the $\mathrm{L}_{2}$ intermetallic were grain size refinement and dispersion strengthening. While the crystallite size was refined to some unexpectedly small values, the dispersion strengthening was not realized in a classical dispersed-particle sense where the phase would occur within the grains of the $\mathrm{L}_{2}$ intermetallic in order to increase the stress required for dislocation generation and motion. However, the unique microstructure of the consolidated samples gives a different strengthening mechanism. Certainly, it could be that the complex carbides formed act as crack arrestors in the material during deformation which was an original question, but the model would likely differ from the common classical model given the microstructure.

The ultra-high strength is of primary interest and will be discussed in terms of a comparison of grain size effects with those of increasing the amount of complex carbides. In addition to grain refinement strengthening, the strength of composite materials in a simplistic sense should follow a rule of mixtures [265]. Recall, the compressive yield strength of the chromium-modified titanium trialuminide was $245 \mathrm{MPa}$ for a $200 \mu \mathrm{m}$ grain size, leaving about $1.8 \mathrm{GPa}$ to be attributed to grain size reduction or other aspects of the microstructure. The compressive strength of polycrystalline 211 carbide $\mathrm{Ti}_{2} \mathrm{AlC}$ has been reported to be about $540 \mathrm{~Pa}$, with Zhou and Wang [266] measuring a maximum stress at failure of $763 \mathrm{MPa}$. Similar results were found for a fabricated polycrystalline 312 carbide $\mathrm{Ti}_{3} \mathrm{AlC}_{2}$ with a maximum compressive strength upon failure of $764 \mathrm{MPa}$ [267]

Despite the variable nature of the information, a Hall-Petch relationship can be considered for the three single-phase samples in this study; a chill-cast, the $\mathrm{Cr} 9$ sample composed of HIPed as-received $\mathrm{L}_{2}$ intermetallic powder, and $\mathrm{HIPed} \mathrm{L}_{2}$ intermetallic powder milled for $3 \mathrm{~h}$ in an argon environment. Relevant data for these samples as well as the consolidated samples is provided in Table IV-12. These samples represent successively decreasing grain sizes permitting a Hall-Petch plot to be developed, represented by the black data points in Figure IV-14. It was also thought instructive to include the yield strength as a function of crystallite size of the intermetallic phase for the composite samples with different amounts of carbide as shown in red in Figure IV-14. 
These appear as two intersecting line segments defined by each group of materials. Clearly the traditional plot for the single-phase intermetallics show that the grain refinement could theoretically account for much of the observed strengthening. The extrapolated single-phase data intersects the consolidated data at a grain size of about one micrometer where the traditional Hall-Petch relationship has been suggested to show a decreasing slope, and in this case the increasing volume fraction of carbides lessens the effect of this strengthening mechanism. Additionally, in the consolidated composite materials the intermetallic phase grains are isolated and typically bounded by single crystal complex carbides and their substructure consists of significant dislocation densities produced from the CTE mismatch with the carbides. One other consideration is whether dislocations have been generated within the complex carbides and what their role might be.

\begin{tabular}{|c|c|c|c|c|}
\hline \multicolumn{5}{|c|}{$\begin{array}{l}\text { Table IV-12 Crystallite Size (Grain Size), Complex Carbide Content, Yield Strength } \\
\text { and Ultimate Compressive Strength of Select Alloys from this Study }\end{array}$} \\
\hline Sample & $\begin{array}{l}\text { Crystallite Size } \\
\text { Average (nm) }\end{array}$ & $\begin{array}{l}\text { Carbide } \\
\text { Volume } \\
\text { Fraction }\end{array}$ & $\begin{array}{c}\text { Yield } \\
\text { Strength } \\
\text { (GPa) }\end{array}$ & $\begin{array}{c}\text { Ultimate } \\
\text { Compressive } \\
\text { Strength (GPa) }\end{array}$ \\
\hline As-Cast HIP [7] & 200,000 & 0.0 & 0.245 & 0.791 \\
\hline $\mathrm{Cr} 9$ & 12,000 & 0.0 & 0.398 & 1.990 \\
\hline MM3hHIP & 6,000 & 0.0 & 0.990 & 1.930 \\
\hline 20/1000/MA & 110 & 0.136 & 2.060 & 0.510 \\
\hline 20/1000/RA & 95 & 0.137 & 1.910 & 2.210 \\
\hline 40/1000/RA & 73 & 0.243 & 2.170 & 2.150 \\
\hline 60/1000/RA & 60 & 0.378 & 2.400 & 2.590 \\
\hline 100/1000/RA & 45 & 0.642 & 2.145 & 2.570 \\
\hline
\end{tabular}




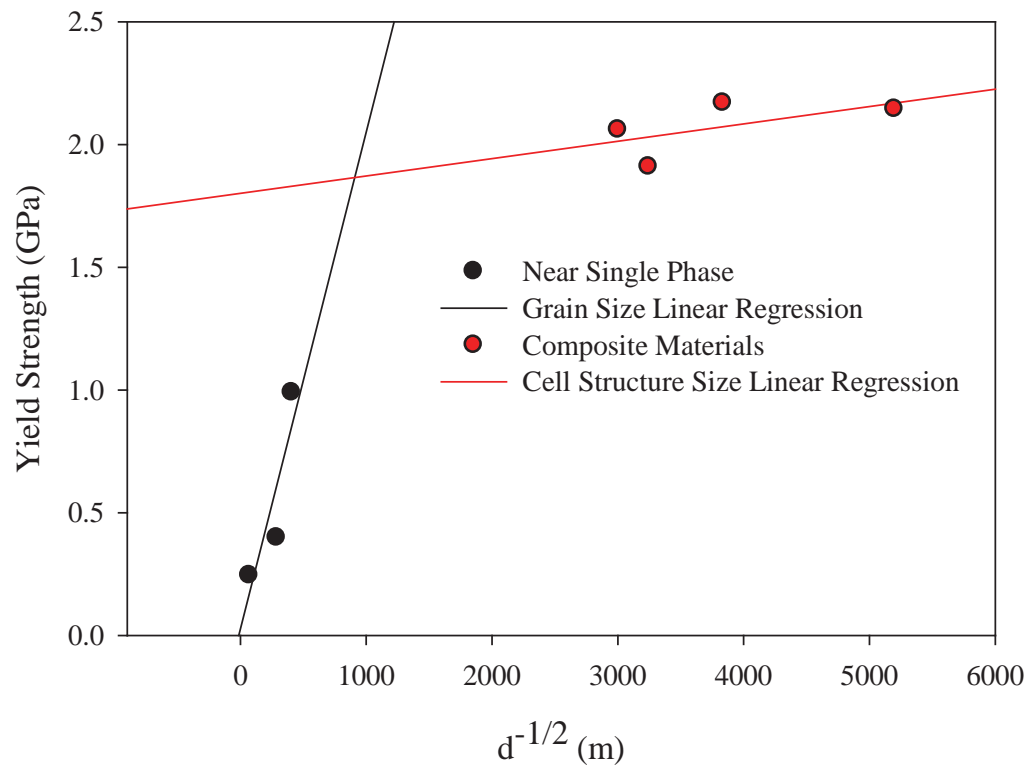

Figure IV-14 Yield Strength as a Function of Grain Size/Crystallite Size for Chromium Modified Titaniun Trialuminide Samples

The strengthening from these dislocation densities produced from the CTE mismatch in these composites can be related to the square root of the dislocation density as shown by Equation (IV.25) [262]. Using a $\beta$ of 1.25 for aluminum [268], and an $a / 3<112>$ Burgers vector, calculations we made for dislocations densities for both those calculated and measured available in Table IV-8. Results of these calculations are listed in Table IV-13 showing a maximum of about a $0.5 \mathrm{GPa}$ strengthening contribution from this simplistic model. Adding the larger compressive strength of the complex carbide material (764 MPa) results in a total strength of $1.2 \mathrm{GPa}$ suggesting that other mechanisms add to the strengthening. The small grain size (expected to be a maximum of about $500 \mathrm{~nm}$ ) can be expected to provide additional strengthening; however, the amount of this contribution is not clear. One aspect of the microstructure that may further add to the strengthening is the three-dimensional arrangement of these single crystal complex carbides with the softer $\mathrm{L}_{2}$ intermetallic as a buffer between the complex carbide platelets. Under stress elastic deformation may cause these complex carbide platelets to buckle with this deformation being accommodated by the $\mathrm{L}_{2}$ intermetallic in a hydrostatic manner. While the single-crystal nature of the platelets and a majority of the 
$\mathrm{L}_{2}$ comprised of single crystals with a substructure in these interplatelet regions, this unique microstructure may also add to the strengthening in these composite materials.

$$
\Delta \sigma=\beta \mu b \rho^{1 / 2}
$$

\begin{tabular}{|c|c|c|c|c|}
\hline Sample & $\begin{array}{c}\text { Calculated } \\
\text { Dislocation } \\
\text { Density } \\
\left(\mathbf{m m}^{-2}\right)\end{array}$ & $\begin{array}{c}\text { Measured } \\
\text { Dislocation } \\
\text { Density } \\
\left(\mathrm{mm}^{-2}\right)\end{array}$ & $\begin{array}{c}\Delta \sigma \text { Calculated } \\
\text { Dislocation } \\
\text { Density } \\
(\mathbf{G P a})\end{array}$ & $\begin{array}{c}\Delta \sigma \text { Measured } \\
\text { Dislocation } \\
\text { Density } \\
(\text { GPa })\end{array}$ \\
\hline 20/1000/MA & $1.90 \times 10^{8}$ & $5.6 \times 10^{7}$ & 0.4 & 0.2 \\
\hline 20/1000/RA & $1.16 \times 10^{8}$ & $6.6 \times 10^{7}$ & 0.3 & 0.2 \\
\hline 40/1000/RA & $2.06 \times 10^{8}$ & $1.1 \times 10^{8}$ & 0.4 & 0.3 \\
\hline 60/1000/RA & $1.75 \times 10^{8}$ & $1.9 \times 10^{8}$ & 0.3 & 0.4 \\
\hline $\begin{array}{c}\text { 100/1000/R } \\
\mathrm{A}\end{array}$ & N/A & $3.7 \times 10^{8}$ & N/A & 0.5 \\
\hline
\end{tabular}

Funkenbusch and Courtney [269] modeled the excess strength observed in twophase composites over that expected based on the strain hardening behavior of each phase. They concluded that both additional and geometrically necessary dislocations are generated during the deformation process which enhances the strength. This also may play a role in the $\mathrm{L}_{2}$ intermetallic composites as the SHR increases with the amount of complex carbide, indicating that more dislocations are produced during as well as before deformation based on the increased complex carbide content. In summary, the contributions of both the three-dimensional array of contiguous carbide single-crystal platelets and that of the high dislocation density deformed $\mathrm{L}_{2}$ intermetallic regions separating the carbides to the strengthening of the composite material are effective, complex and may be unique. 


\section{Conclusions}

1) The reactive mechanical alloying/milling (RMA/M) process developed here has the unique ability to control in situ chemical reactions between metallic powders and molecules of either a liquid or gaseous nature to produce composites or compounds of a very wide range of compositions.

2) Intelligent profile fitting provides effective means to quantify fundamental diffraction peak parameters for each phase from the diffraction pattern for a nanoscale multiphase material consisting of similar crystal structures. The ability to separate and define the x-ray diffraction pattern for each phase permits detailed peak profile analyses, thus the ability to establish structural information for fine scale and severely deformed microstructures.

3) The extremely small crystallite sizes (e.g., $2 \mathrm{~nm}$ ) and very large microstrains of about $1 \%$ appear to represent limits for the substructure of the severely deformed materials studied. These small crystallites might be referred to as "deformation kernels".

4) The nano-scale microstructures produced in as-milled powders of the $\mathrm{L}_{2}$ chromium-modified titanium trialuminide studied reach the limiting size with wide high energy grain boundary regions consisting of a variety of dislocations and other defects. These regions approaching half of the material volume give rise to diffuse background scattering from which it was possible through a new whole pattern analysis to be characterized as having a random static displacement of the atoms in the region, which is consistent with the high defect density giving rise to important additional diffuse scattering in these nano-scale materials.

5) The microstructures of these as-milled powders of the $\mathrm{L}_{2}$ intermetallic produced from this severe deformation process consist of crystallites that are defect free, but surrounded by a layer of coherent defects that are adjacent to the NGB matrix region.

6) The large volume fraction of high energy NBG regions provides mass transport in these materials that can account for several processes occurring during mechanical 
alloying (MA) and other treatments. For example, the NGB structure can account for GB-mediated deformation processes that enhance plasticity and decrease the temperatures for onset of superplasticity. Such behavior is also thought to be important in other processes, including cold welding and sliding wear. This enhanced mass transport and plasticity also provides a basis for the structural and chemical changes that occur in the MA.

7) Surface reactivity and catalytic behavior in milled powders appear to be important basic mechanisms in reactive milling. Molecules of an organic PCA or gaseous material must dissociate on the surface of the rapidly wetted particles. These individual surface events in the presence of effective mass transport and local pressure provide an environment that produces nuclei of the reactant products. A wide variety of phases, such as carbides, nitrides, oxides, etc. can be produced as fine particles in the milled powder.

8) The RMA/M processed powders provide for unique control of nucleation and growth of phases in subsequent processing, for example, by dominating nucleation events with numerous nuclei which by default reduces the growth while allowing for easy access to fine-scale structures developed from processes that typically create larger-scale microstructures.

9) The material powders created by the RMA/M process can be consolidated by common isostatic and hot isostatic pressing to produce fully dense materials. The nano-scale nature of the milled powder gives access to producing refined microstructures with unusual properties. As shown here, ultra-high strengths of the processed $\mathrm{L}_{2}$ intermetallic, approach about one half of the theoretical shear strength, which is a particularly impressive density-compensated strength.

10) In the consolidated $\mathrm{L}_{2}$ intermetallic containing titanium carbide the deformation induced by the CTE mismatch between the intermetallic and the carbide on cooling from the consolidation temperature introduced dislocations into the microstructure providing an additional deformation and strengthening mechanism.

11) In the consolidated microstructure formed at $1000{ }^{\circ} \mathrm{C}$ the titanium carbide platelets grow to form a contiguous array when the carbide volume fraction is about 0.15 , 
thus, rather than an intermetallic matrix composite, it can be considered a ceramic matrix composite with the intermetallic phase filling the intercarbide space.

12) The RMA/M process provides a simple means to nano-engineer powders by control of processing variables to provide special feedstock for additional processing, such as the ability to match the coefficient of thermal expansion of a base material for coating purposes. 


\section{Potential of this Processing Method}

This processing technique has opened the door to a vast array of possible materials that can be synthesized. The most important aspect of this method is the ability to control the reaction products that manifest the nano-engineering by a self-assembly method manipulated by simple alteration of RMA/M process variables. Tangential studies utilizing this unique RMA/M process have explored several different starting elemental powders, such as copper-titanium, titanium, aluminum with low levels of titanium, nickel, and iron. For example, strengthening of copper conductors was done by RMM of copper and titanium in hexanes forming small titanium carbides particles in the milled and also in the consolidation materials using a similar CIP and HIP method [270]. Other experiments have shown that iron carbides can be systematically formed with RMM processing of iron powders in hexanes; and actually effectively creating variable carbon content alloys from steels to cast irons based solely on the RMM time. Synthesizing these new plain carbon steels and cast irons by this process opens new possibilities for these typically conventional materials [246]. Hydrogen storage can also be accomplished by this RMM process using pure nickel powders and water as the PCA, creating solid hydrogen storage within powder particles quite easily [242]. Work by Courtney and Murphy [271] based on this discovery established that NbC can be formed in situ during milling with hexanes. These few diverse experiments demonstrate the potential for the wide variety of materials that can be synthesized by this means. Of utmost importance is the fact that this simple technique of RMA/M allows access to a wide range of material properties in a potentially unlimited number of new materials.

The analysis of the reaction mechanisms, previously discussed, are not thought to be thorough but more of a preliminary look at a neglected complex area of research in the MA field. This reaction process appears to start by rapid wetting of the powder surface with the majority of the reactions (and alloying) occurring from these identified surface events as previously described. These surface events are enhanced by wear-type debris having the enhanced mass transport because of the NGB structure. The current experiments suggest that at least some metallic powders can act as a catalyst in the 
process and that could be a key to understanding other potential and unique systems. It is thought that the surface is highly reactive as result of the surface events during milling and has unlimited wettability in many instances, but this issue needs investigation. It should be repeated that the sizes produced in the as-milled product are among of the smallest sizes known to exist for crystalline solids. This is expected to enhance the consolidation process by providing more nucleation sites for potential equilibrium reactions that may occur and thus help maintain a fine-scale structure upon consolidation.

These other powder mixture systems that were explored using a basic recipe similar to that developed in the current study of an RMA/M process that is; using a 6 to 1 media to charge ratio with similar amounts of PCA hexanes and metallic powder with the milling done in a $\mathrm{SPEX}^{\mathrm{TM}}$ mill. The importance is derived from the fact that they demonstrate the unique ability to create new materials by this a simple processing method involving severe deformation with intimate contact of metallic surfaces and various elements in both the gaseous and liquidus states. It should be noted that in earlier work at MTU Le Claire [140] has shown that changes to the milling parameters (PCA, media density, etc.) can influence reaction kinetics. Also, other PCAs such as water were shown to react suggesting that many other liquids can potentially be used, thus expanding the possible materials that can be synthesized. Just as important is the observation that enhanced reactivity developed by surface events is key to the potential for a wider array of systems.

Regarding scale-up of the process it should be noted that this RMM process for the $\mathrm{L}_{2}$ intermetallic in a hydrocarbon has been scaled up to produce batches of $2.2 \mathrm{~kg}$ of as-milled powder with a ball mill at MTU [19]; however, this mill accomplished a chosen carbon uptake with an RMM time of $96 \mathrm{~h}$, compared to producing a $15 \mathrm{~g}$ batch with the higher energy SPEX ${ }^{\mathrm{TM}}$ mill that only took $20 \mathrm{~h}$. Of course the large batch was equivalent in amount of powder produced to almost 150 of the small charges.

The type and amount of the powder-liquid-gas reactions that could occur is beyond imagination suggesting a very large number of possible composite/alloy materials can be developed using this technique. As noted, another far-reaching aspect of the RMA/M process is the extremely small sized particles that do not appear to grow and 
typically occur in the reaction products in the as-milled powders. These numerous small nuclei are very attractive for further phase transformations under more equilibrium conditions, for example, during powder consolidation processes or other thermally based processes. Managing or limiting the growth to preserve or create fine-scale unique microstructures should be easily accomplished. As an example, the microstructure of a consolidated RMM powder of pure iron milled in hexanes for a $160 \mathrm{~h}$, degassed, consolidated with basic CIP and HIP processing produced a cast iron with about 4 wt. pct. carbon and formed an interesting microstructure with about $60 \%$ by volume of cementite. What is remarkable about the microstructure produced, micrograph shown in Figure VI-1, is that cementite shows a floweret type structure in a contiguous ferrite phase, both with nanometer scale features, with $40 \mathrm{~nm}$ carbides sizes with ferrite sizes of about $17 \mathrm{~nm}$. The micrograph shown is of a polished/etched sample using the FESEM, note the $200 \mathrm{~nm}$ marker.

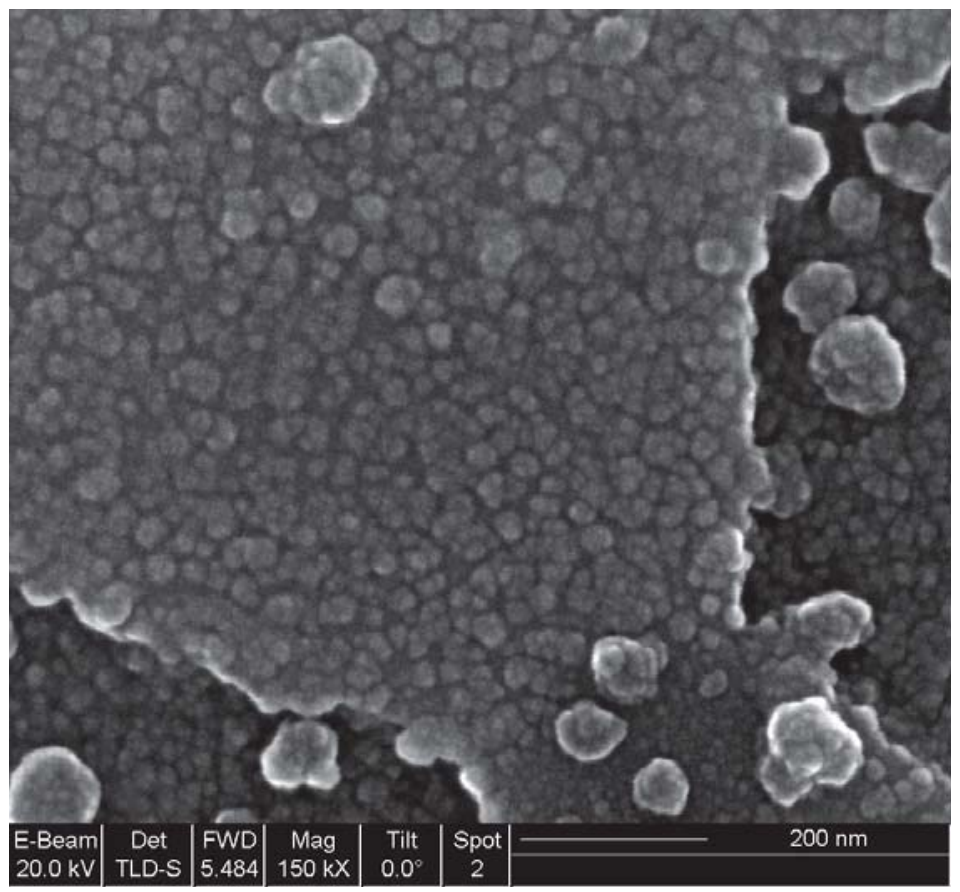

Figure VI-1 Iron Carbon RMM 160 h CIP- HIP Consolidated at $950^{\circ} \mathrm{C}$ 


\section{Future Work}

In the quest for scientific knowledge more questions are often raised than answered. This project has raised several questions, some of which have formed the basis for these comments. A more detailed characterization of the NGB regions is a lofty goal, perhaps by direct observation with electron imaging as well as further development of the new XRD approach. Additionally, apply these new XRD characterization techniques and direct observation to the degassed powders (used in consolidation) to develop a better understanding of the microstructure in these important powders. It may be worth considering calorimetry as a tool as well. Attempting to learn more about the NGB is a challenging area for several reasons, including the difficulty with sample preparation and imaging resolution with the electron imaging.

Design some experiments to explore kinetics of the reactions and optimize the process variables associated with the RMA/M processing method, including chemical control to prevent oxidation, choices of process control agents and atmosphere, as well as the mill and the media. Produce a series of the chromium-titanium trialuminide/carbide powders with careful control of chemical compositions to establish the range of twophase microstructures that can be produced and to evaluate the response of the various asmilled powders to different consolidation methods.

Creation of a series of chromium-titanium trialuminide/carbide composites based on the optimized procedures that encompasses the transition from isolated carbide particles to impinged carbide networks for determination of mechanical properties in relation to the characterized microstructure. Evaluate the mechanical properties in detail as a function of temperature and strain rate. Include fractography and consider acoustical emission information as a possible means to explore the behavior of the carbide platelets during deformation and fracture.

Finally, a survey of potential composite materials may suggest an ideal choice, not only based on the RMA/M process, but considering other aspects such as expected properties, along with ease of microstructural characterization and property evaluation. 


\section{Appendix}

\section{A. Standard Files}

The following text corresponds to the standard files required for all phases used in the determination of the volume fractions using the Volfract program.

\section{1. $\mathrm{L1}_{2}$ Chromium-Modified Titanium Trialuminide}

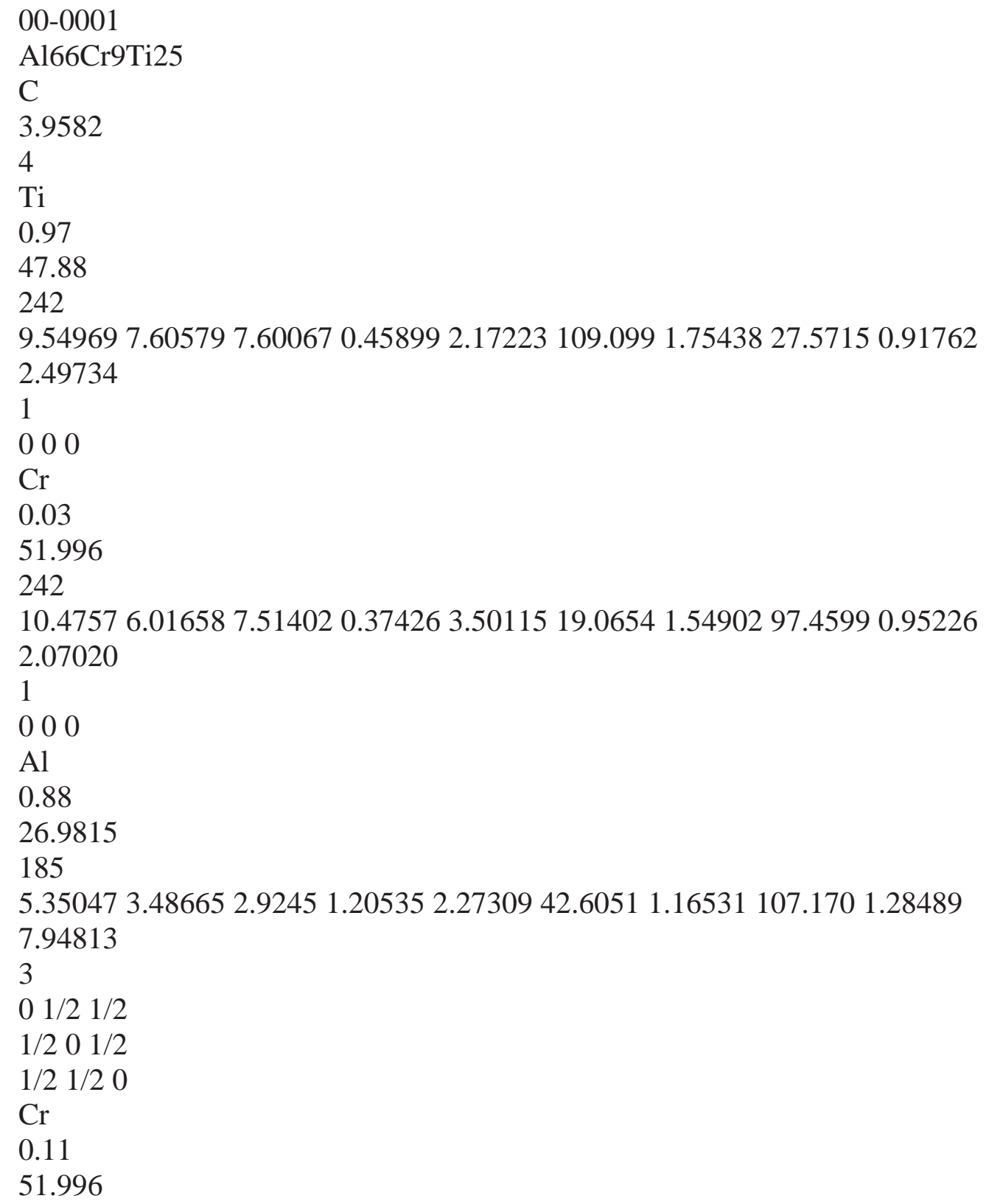


242

10.47576 .016587 .514020 .374263 .5011519 .06541 .5490297 .45990 .95226

2.07020

3

$01 / 21 / 2$

$1 / 201 / 2$

$1 / 21 / 20$

$\mathrm{Ti}$

0.01

47.88

242

9.549697 .605797 .600670 .458992 .17223109 .0991 .7543827 .57150 .91762

2.49734

3

$01 / 21 / 2$

$1 / 201 / 2$

$1 / 21 / 20$

\section{2. $\quad \mathrm{L}_{2}$ Chromium-modified Titanium Trialuminide}

Used for the calculation of R factors for the LRO parameter determination (S) considering perfect order in the $\mathrm{Al}_{67} \mathrm{Cr}_{9} \mathrm{Ti}_{25}$.

00-0001

Al66Cr9Ti25

C

3.9582

3

Ti

1.0

47.88

178

$9.549697 .605797 .600670 .458992 .17223109 .0991 .7543827 .5715 \quad 0.91762$

2.49734

1

000

$\mathrm{Al}$

0.87

26.9815

185

5.350473 .486652 .92451 .205352 .2730942 .60511 .16531107 .1701 .28489 7.94813

3

$01 / 21 / 2$

$1 / 201 / 2$ 
$1 / 21 / 20$

$\mathrm{Cr}$

0.13

51.996

242

$\begin{array}{llll}10.4757 & 6.016587 .514020 .37426 & 3.5011519 .06541 .5490297 .4599 & 0.95226\end{array}$ 2.07020

3

$01 / 21 / 2$

$1 / 201 / 2$

$1 / 21 / 20$

\section{3. $\mathrm{DO}_{22} \mathrm{Al}_{3} \mathrm{Ti}$ - (Does not account for any $\mathrm{Cr}$ )}

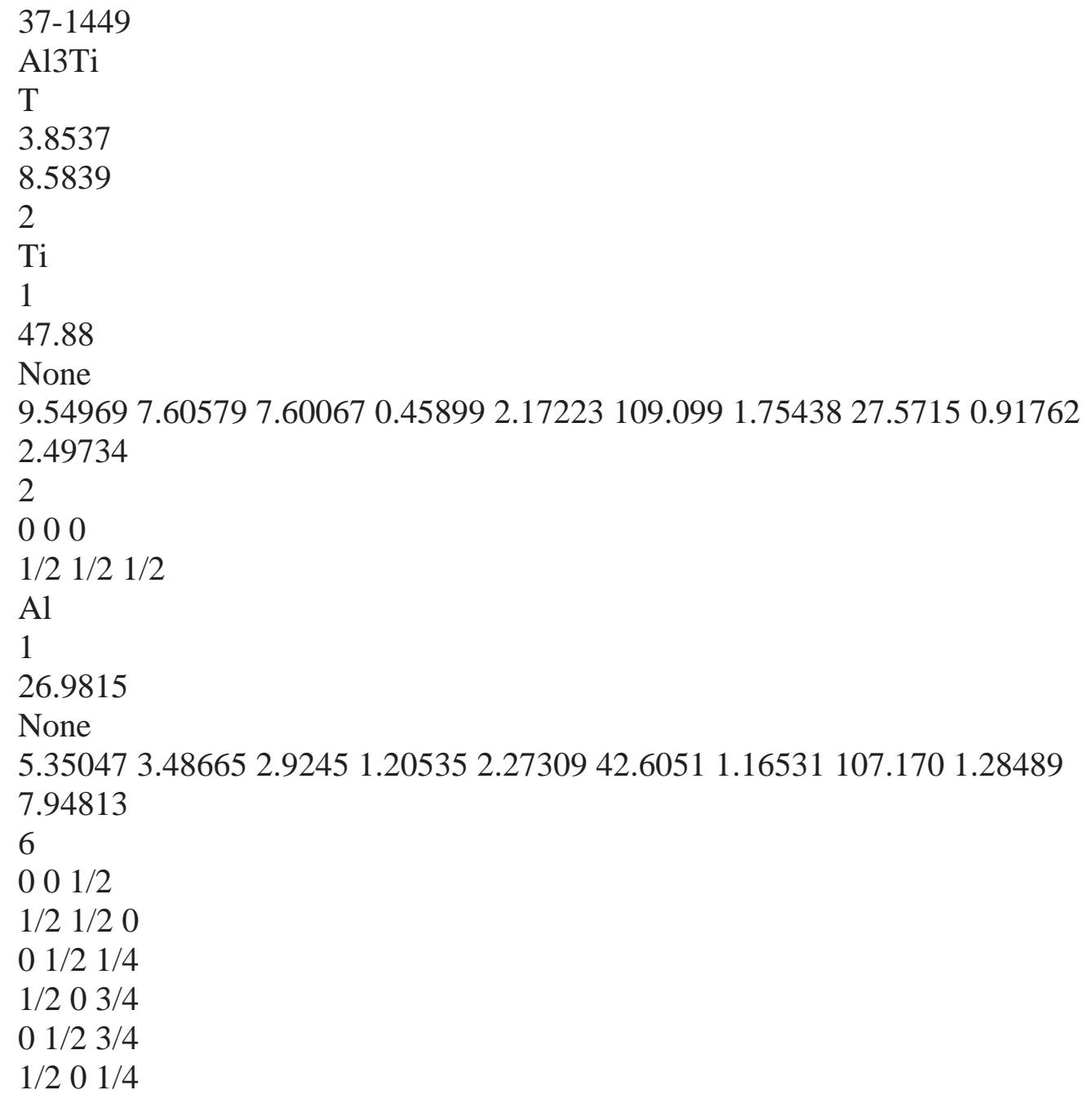




\section{4. $\quad$ TiC}

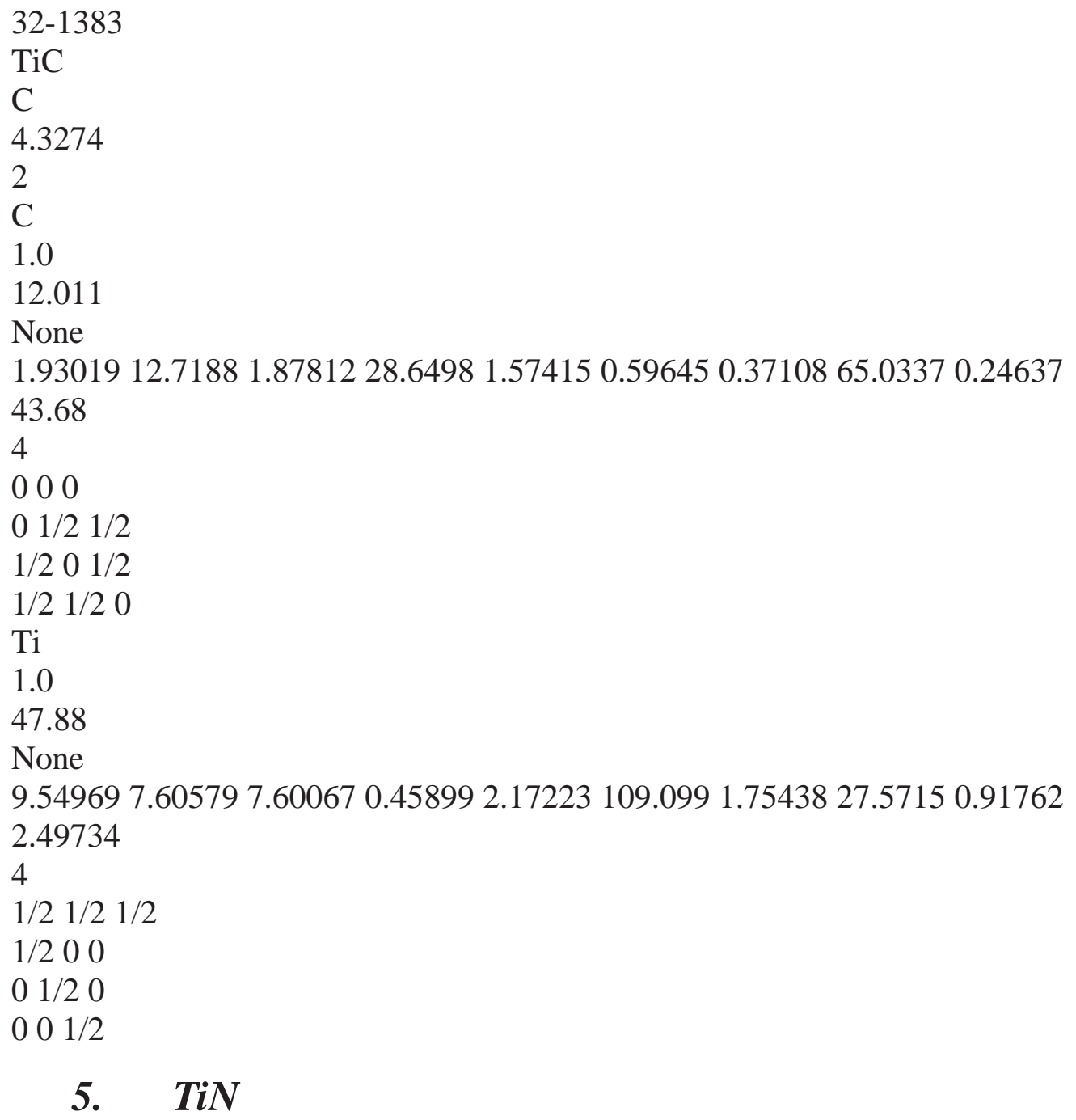




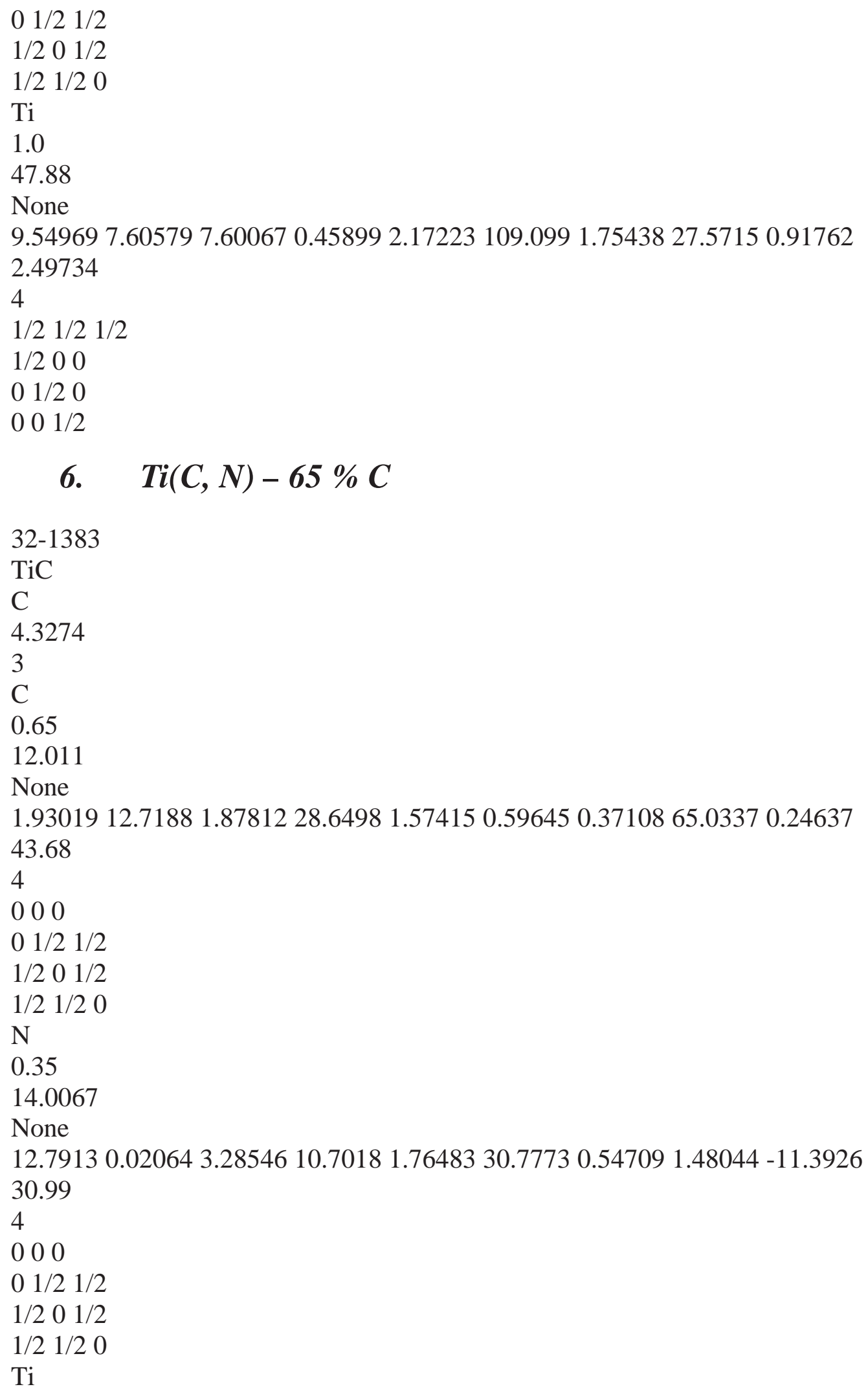




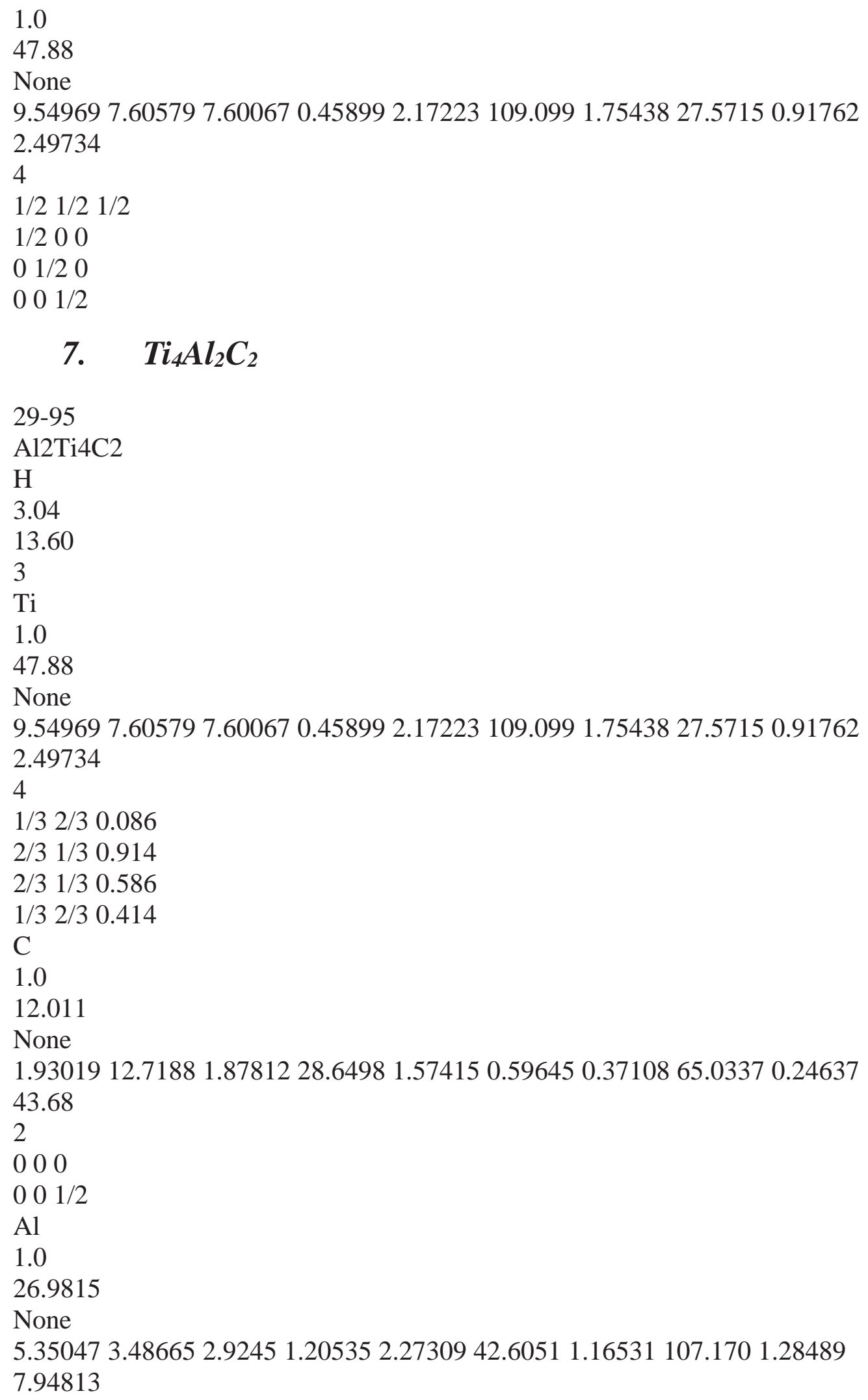




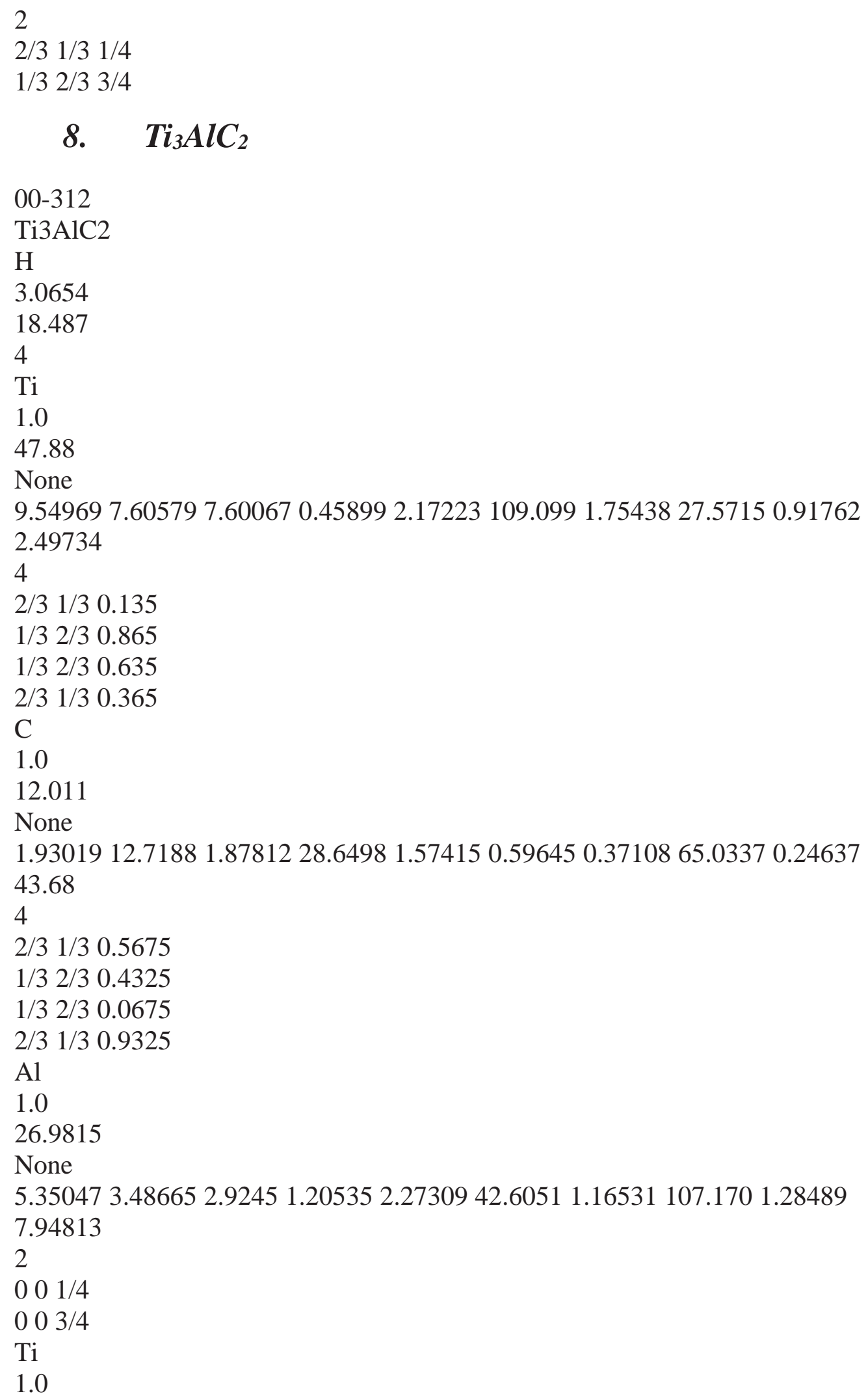




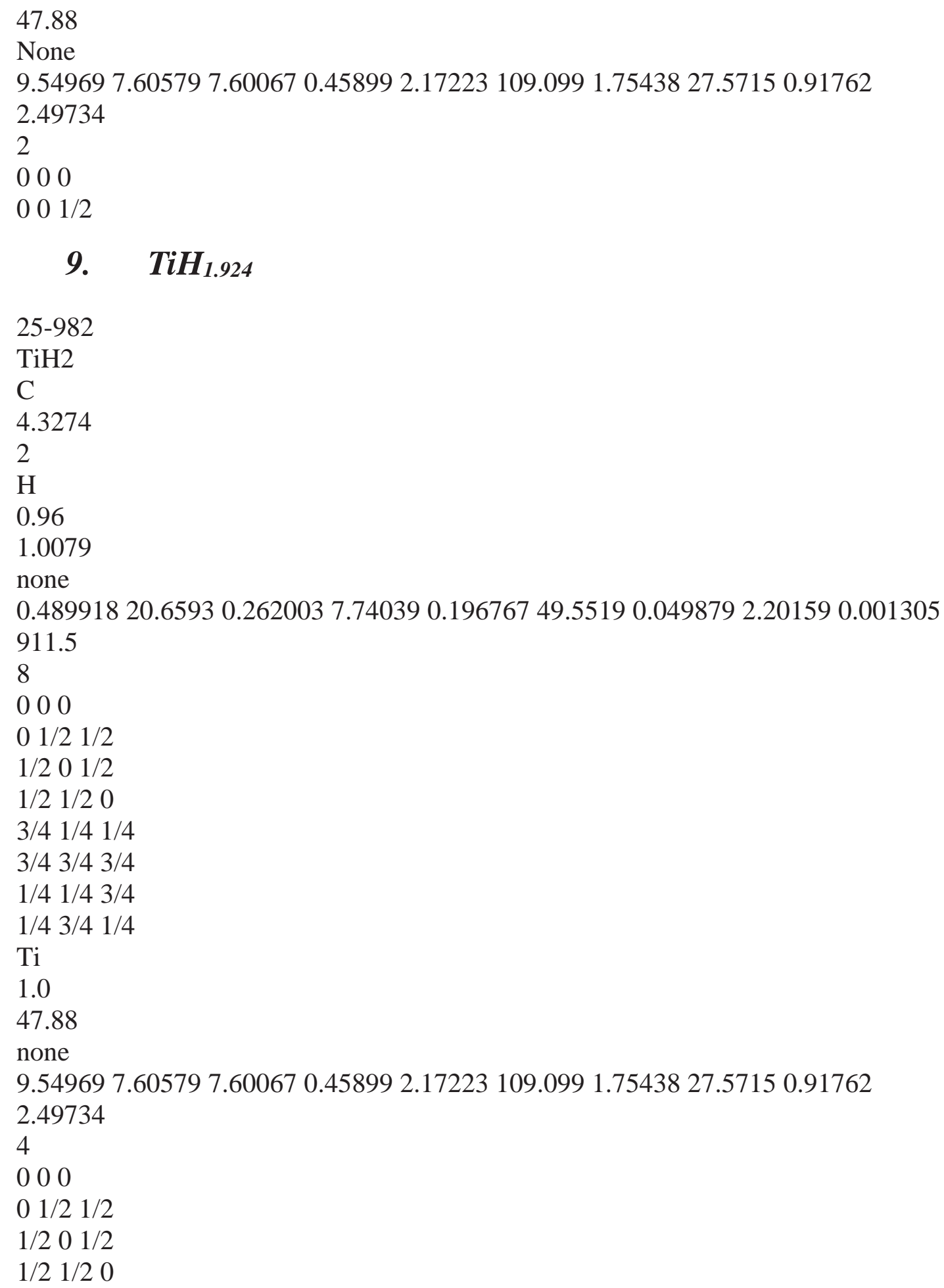

\section{0. $\mathrm{AlCr}_{2}$}

29-16 p 


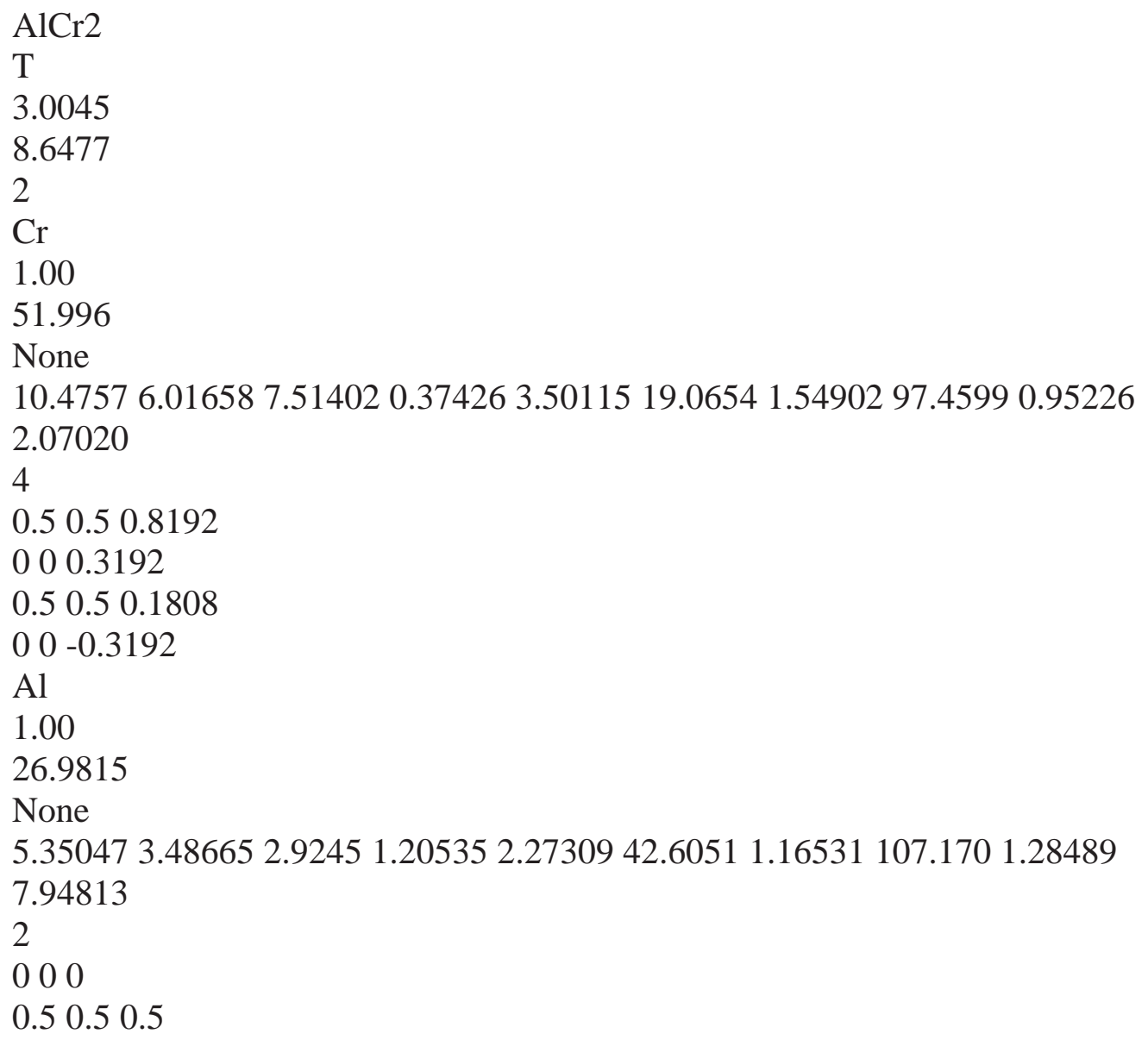


$\begin{array}{llll}1 / 3 & 2 / 3 & 0.019\end{array}$

$\begin{array}{lll}1 / 3 & 2 / 3 & 0.3144\end{array}$

$1 / 32 / 3 \quad 0.519$

$\begin{array}{llll}1 / 3 & 2 / 3 & 0.8144\end{array}$

$2 / 31 / 3 \quad 0.6856$

$2 / 31 / 3-0.019$

$2 / 31 / 3 \quad 0.1856$

$2 / 31 / 3 \quad 0.481$

$\mathrm{O}-2$

1.0

15.9994

None

3.2256318 .49913 .017176 .65681 .425530 .405890 .9052561 .1889

0.42362 23.32

18

$\begin{array}{lll}0.3064 & 0 & 1 / 4\end{array}$

$\begin{array}{lll}0 & 0.3064 & 1 / 4\end{array}$

$-0.3064-0.30641 / 4$

$\begin{array}{lll}-0.3064 & 0 & 3 / 4\end{array}$

$\begin{array}{ll}0 & -0.30643 / 4\end{array}$

$\begin{array}{lllll}0.3064 & 0.3064 & 3 / 4\end{array}$

$\begin{array}{lll}0.6397 & 2 / 3 & 0.9167\end{array}$

$\begin{array}{lll}1 / 3 & 0.9731 & 0.9167\end{array}$

$\begin{array}{lll}0.0269 & 0.3603 & 0.9167\end{array}$

$\begin{array}{llll}0.0269 & 2 / 3 & 0.4167\end{array}$

$1 / 3 \quad 0.36030 .4167$

$\begin{array}{lll}0.6397 & 0.9731 & 0.4167\end{array}$

$\begin{array}{llll}0.9731 & 1 / 3 & 0.5833\end{array}$

$2 / 3 \quad 0.63970 .5833$

0.36030 .02690 .5833

$\begin{array}{llll}0.3603 & 1 / 3 & 0.0833\end{array}$

$\begin{array}{lll}2 / 3 & 0.0269 & 0.0833\end{array}$

$\begin{array}{lll}0.9731 & 0.63970 .0833\end{array}$ 


\section{B. Mechanical Milling to Produce Fine-Grain Single-Phase Chromium-modified Titanium Trialuminide}

In order to evaluate the effects of grain size on the mechanical properties mechanical milling (MM) was performed on an $\mathrm{Al}_{66} \mathrm{Cr}_{9} \mathrm{Ti}_{25}$ alloy powder in a dry (no PCA) argon environment. The alloy powder was commercially produced by Praxair, Inc. Details of the milling conditions are given in Table VIII-1. The goal was to produce a fine-grained single-phase material of the $\mathrm{L}_{2}$ intermetallic to provide a base material in evaluating structure/property relationships. In order to minimize packing of the powder in dry MM the vial was periodically rotated and impacted mechanically.

Table VIII-1 Mechanical Milling Process Parameters

\begin{tabular}{|c|c|c|c|c|c|}
\hline $\begin{array}{c}\text { Process } \\
\text { Number }\end{array}$ & $\begin{array}{c}\text { Initial Alloy } \\
\text { Composition } \\
\text { (wt. pct.) }\end{array}$ & $\begin{array}{c}\text { RMM } \\
\text { Atmosphere }\end{array}$ & $\begin{array}{c}\text { Powder } \\
\text { Charge } \\
(\mathbf{g})\end{array}$ & $\begin{array}{c}\text { Ball- } \\
\text { Charge } \\
\text { Ratio }\end{array}$ & $\begin{array}{c}\text { RMM } \\
\text { Time } \\
\text { (h) }\end{array}$ \\
\hline MM3hAr & $\begin{array}{c}\mathbf{1 0 0 \%} \\
\text { Praxair }\end{array}$ & Argon & 15 & 6.7 to 1 & 3 \\
\hline
\end{tabular}

\section{Single-phase Powder Metallurgy $\mathrm{Al}_{66} \mathrm{Cr}_{9} \mathrm{Ti}_{25}$ Alloy}

In addition, for comparison a single-phase $\mathrm{Al}_{66} \mathrm{Cr}_{9} \mathrm{Ti}_{25}$ alloy was procured from the work of Le Claire [142]. This sample had been created by powder metallurgy processing of $\mathrm{Al}_{66} \mathrm{Cr}_{9} \mathrm{Ti}_{25}$ alloy powder from Praxair, Inc. with cold isostatic pressing (CIP) followed by hot isostatic pressing (HIP). The resulting sample was designated as Cr9 for this research. Again it should be emphasized that this sample was not created by any mechanical milling/alloying techniques, but rather by traditional powder metallurgy techniques from atomized powder.

\section{a) Grain Size Measurements}

Optical metallography was used to determine the grain size of the MM3h and $\mathrm{Cr}$ 9 samples. Mean linear grain intercepts were determined using a 10X eyepiece with a 
circular reticle of $10.5 \mathrm{~mm}$ line length in conjunction with a 50X objective lens. The number of grain intersections with the reticle was determined for a minimum of ten fields of view. The values presented in Table VIII-2 for the mean linear intercepts represent an average for all reticle intersections. The grains in both samples are equiaxed in shape. Actual grain sizes determined from the mean linear intercept assuming spherical grains [41] are presented in Table VIII-2.

\begin{tabular}{|c|cc|}
\hline \multicolumn{3}{|c|}{ Table VIII-2 Grain Size Measurements } \\
\hline Sample & $\begin{array}{c}\text { Mean Linear Intercept } \\
(\mu \mathbf{m})\end{array}$ & $\begin{array}{c}\text { Spherical Grain Size } \\
(\mu \mathbf{m})\end{array}$ \\
\cline { 2 - 3 } MM3h & 6 & 9 \\
Cr9 & $\mathbf{1 3}$ & 19 \\
\end{tabular}

\section{b) 3 Hour Mechanical Milling in Argon MM3h}

This MM processing was done in argon eliminating the air. As expected, the majority phase in the XRD pattern (Figure VIII-1) was the L1 2 chromium-modified trialuminide (00-0001). Several secondary phases appeared, but in very small quantities as indicated by the extremely low intensities. The most significant of these phases was $\mathrm{Ti}_{2} \mathrm{AlN}$ (18-70) and it should be pointed out that this phase is very similar to and isomorphous with $\mathrm{Ti}_{2} \mathrm{AlC}$. Observation of this phase was not unexpected since Nic [116] had found this phase during TEM analysis of as-cast $\mathrm{L}_{2}$ chromium-modified trialuminide. Regarding the origin of the phase, based on several argon milling experiments, it has been suggested that nitrogen levels may rise over time in the argon that is recycled in the glove box with only the oxygen reduced.

Also detectable were $\mathrm{Al}_{2} \mathrm{O}_{3}(10-173)$ and $\mathrm{TiN}$ (38-1420) as well as a small amount of tantalum that apparently came from incomplete removal of the tantalum foil used as a diffusion barrier in the HIP process. 


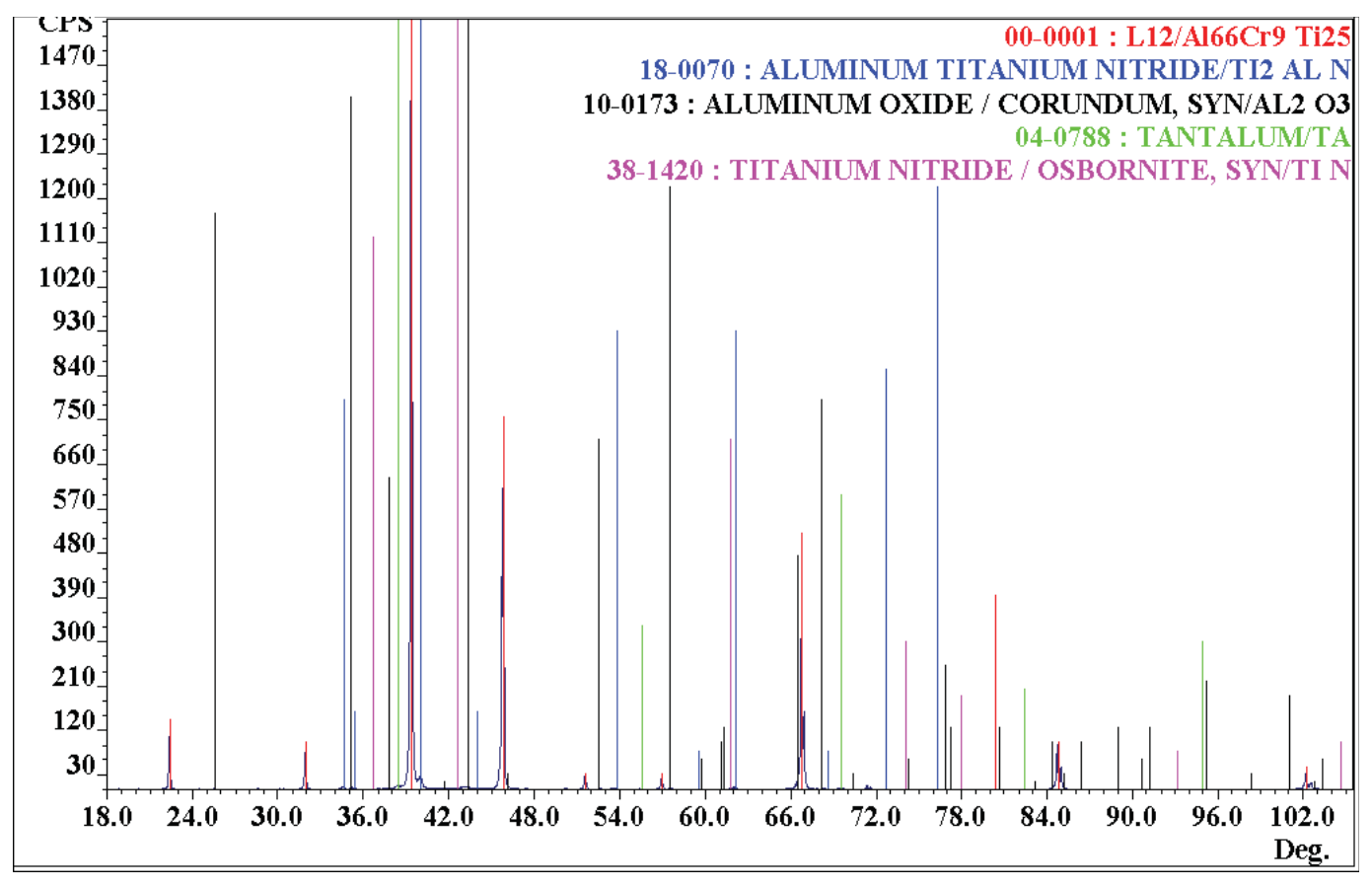

Figure VIII-1 XRD Pattern for the MM3h Sample with Phase Identification

\section{c) MM3h Sample}

The purpose for producing this sample was to create a single-phase material with a smaller grain size than available with both the as-cast material and the powder metallurgy sample Cr9, for use in a Hall-Petch type analysis. Unfortunately, the milling and processing caused the creation of some secondary phases. In order to quantify the amount of these identified phases, volume fractions were determined with the XRD direct comparison method discussed previously and are given in Table VIII-3. The majority of the sample was the expected $\mathrm{L}_{2}$ chromium-modified titanium trialuminide. Unexpected was the presence of the $\mathrm{Ti}_{2} \mathrm{AlN}$ phase, especially in the amount observed. The only possible sources of nitrogen, other than the very small amount observed in the as-cast material by Nic [7, 116] (this material utilized the Master Alloy as a starting powder), are the possibility of nitrogen pickup in the glove box as discussed previously, and incorporation of nitrogen during the CIP process. The TiN and $\mathrm{Al}_{2} \mathrm{O}_{3}$ are near the detection limits and might be considered common for this type of processing. 
Table VIII-3 Volume Fraction Results for Sample MM3h

\begin{tabular}{l|cccc} 
Sample & $\mathrm{L}_{2}$ & $\mathrm{Ti}_{2} \mathrm{AlN}$ & $\mathrm{TiN}$ & $\mathrm{Al}_{2} \mathrm{O}_{3}$ \\
\cline { 2 - 5 } MM3h & $\mathbf{0 . 9 6 5 0}$ & $\mathbf{0 . 0 2 5 9}$ & $\mathbf{0 . 0 0 0 5}$ & $\mathbf{0 . 0 0 8 6}$
\end{tabular}

C. RMMXh9TiAr Annealed Powder XRD Analysis

The volume fractions and lattice parameter of the $\mathrm{L}_{2}$ intermetallic are important to the discussion of the proper chemistry to provide a two-phase structure that is the target of this work consisting of the $\mathrm{L}_{2}$ intermetallic and a complex carbide.

\section{Phase Volume Fractions}

Phase volume fractions were determined for the annealed powders for each RMM time by means of the XRD direct comparison method (as noted, the $15 \mathrm{~h} \mathrm{RMM}$ time annealed powder was not analyzed because of contamination). Intensities (peak areas) were determined for the peaks from all phases by profile fitting the diffraction peaks with a Pearson VII function. The volume fractions were derived from an average of the direct comparison determination for each pair of peaks used in the analysis. Typically up to 5 peaks were used for each phase in the analysis; fewer peaks were used in cases of very low peak intensities and overlaps that could not be deconvoluted satisfactorily. Calculations for each phase in each sample are based on an average using the same $\{\mathrm{hkl}\}$ peaks.

The volume fractions are listed in Table VIII-4 for all identified phases in the annealed powders for each RMM time. There is a steady increase in the amount of carbide, with a structural change from the " 211 " or h-phase to the " 312 " or n-phase carbide at an RMM time of about $10 \mathrm{~h}$. This could reflect demands on compositions requirements of the various phases present given that the carbon content is changing. After $20 \mathrm{~h}$ the carbide content still increases, but at a much lower rate. The annealed $10 \mathrm{~h}$ RMM time has near the desired phases for the composite, namely the chromiummodified cubic trialuminide (designated as $\mathrm{L}_{2}$ in Table VIII-4) with a complex carbide. 


\begin{tabular}{|c|c|c|c|c|c|c|c|c|c|}
\hline $\begin{array}{l}\text { RMM } \\
\text { Time } \\
\text { (h) }\end{array}$ & $\mathbf{L 1}_{2}$ & $\overline{\mathbf{A l}_{3} \mathbf{T i}}$ & 211 & 312 & $\overline{\mathbf{A l}_{2} \mathbf{T i}}$ & $\mathrm{Ti}_{3} \mathrm{Al}$ & $\mathbf{A l}_{2} \mathbf{O}_{3}$ & $\mathrm{AlCr}_{2}$ & $\mathrm{Al}_{8} \mathrm{Cr}_{5}$ \\
\hline $0^{*}$ & 0.830 & 0.114 & & & & 0.003 & 0.005 & & 0.005 \\
\hline 0.5 & 0.594 & & 0.014 & & 0.366 & 0.005 & 0.01 & 0.005 & \\
\hline 1 & 0.677 & & 0.022 & & 0.285 & 0.005 & 0.011 & 0.002 & \\
\hline 5 & 0.745 & & 0.073 & & 0.166 & 0.005 & 0.011 & & \\
\hline 10 & 0.873 & & 0.097 & 0.015 & & & 0.012 & & \\
\hline 20 & 0.693 & 0.113 & & 0.156 & & & 0.013 & & 0.025 \\
\hline 25 & 0.645 & 0.148 & & 0.167 & & & 0.014 & & 0.028 \\
\hline 30 & 0.536 & 0.252 & & 0.165 & & & 0.016 & & 0.032 \\
\hline 40 & 0.412 & 0.363 & & 0.171 & & & 0.012 & & 0.042 \\
\hline
\end{tabular}

* Initial mixture also contains 0.044 elemental titanium

Appearance of a significant amount of $\mathrm{Al}_{3} \mathrm{Ti}$ appears at an RMM time of 20 hours and coincides with changes in the structure (and consequently the stoichiometry) of the carbide phase from a 211 to 312 phase. Additionally, the amount of this phase increases by a factor of 3 with the increased RMM time from 20 - 40 hours, again suggesting increasing demands on the distribution of titanium and aluminum within phases in the material as carbon content increases. Further, the $\mathrm{Al}_{8} \mathrm{Cr}_{5}$ phase is also observed with the appearance of $\mathrm{Al}_{3} \mathrm{Ti}$ phase, with content increasing with amount of $\mathrm{Al}_{3} \mathrm{Ti}$, suggesting a change in the distribution of chromium within the associated phases. All of the samples have a small amount of $\mathrm{Al}_{2} \mathrm{O}_{3}$, which appears to increase slightly with RMM time. This phase is also present in the initial powder, which is not annealed, because of oxygen contamination of the Reading starting powders. Although it is possible that the annealing treatment can also introduce small amounts of oxygen. 


\section{Lattice Parameter}

The lattice parameters of phases in the annealed powders were determined with the Scintag Inc. DMS-NT crystallography software option. Figure VIII-2 shows the variation in the $\mathrm{L}_{2}$ intermetallic lattice parameter for the annealed powders as a function of the RMM time. The error bars represent the estimated standard deviation reported from the software. The lattice parameter, $0.39588 \mathrm{~nm}$, of the initial mixture (or $0 \mathrm{~h} \mathrm{RMM}$ time) is larger than the $0.39565 \mathrm{~nm}$ value found for the $\mathrm{L} 1_{2}$ Standard sample using the same method. This difference could be attributed to the presence of other phases based on aluminum and titanium, which affect the $\mathrm{L}_{2}$ intermetallic composition, as well as interstitial impurity elements, like oxygen and carbon, present in the Reading Alloy Inc. powder. Additionally, the powder is created using a rapid cooling which may influence stoichiometry and homogeneity. The lattice parameter for the $0.5 \mathrm{~h} \mathrm{RMM}$ time is the smallest value among all samples, $0.395804 \mathrm{~nm}$ (but still larger than the standard). This sample had the largest volume fraction of $\mathrm{Al}_{2} \mathrm{Ti}$. The increase in lattice parameter for the 1 and $5 \mathrm{~h} \mathrm{RMM}$ times is in conjunction with a systematic decrease in the volume fraction of the $\mathrm{Al}_{2} \mathrm{Ti}$ phase. The $\mathrm{Al}_{2} \mathrm{Ti}$ phase is no longer present in the $10 \mathrm{~h}$ sample, nor are there any chromium-based phases. In fact, the $10 \mathrm{~h}$ RMM time sample contains the least number of phases, the only other secondary phases are complex carbides and a small amount of $\mathrm{Al}_{2} \mathrm{O}_{3}$. The lattice parameter for the $20 \mathrm{~h} \mathrm{RMM} \mathrm{sample} \mathrm{was} \mathrm{approximately}$ equal to the original mixture and the lattice parameter increases systematically with increasing RMM time for longer times. This coincides with a systematic increase in the $\mathrm{DO}_{22} \mathrm{Al}_{3} \mathrm{Ti}$ phase, coupled with an increase in the $\mathrm{Ti}_{3} \mathrm{AlC}_{2}(312)$, both of which can affect the $\mathrm{L}_{2}$ intermetallic composition. 


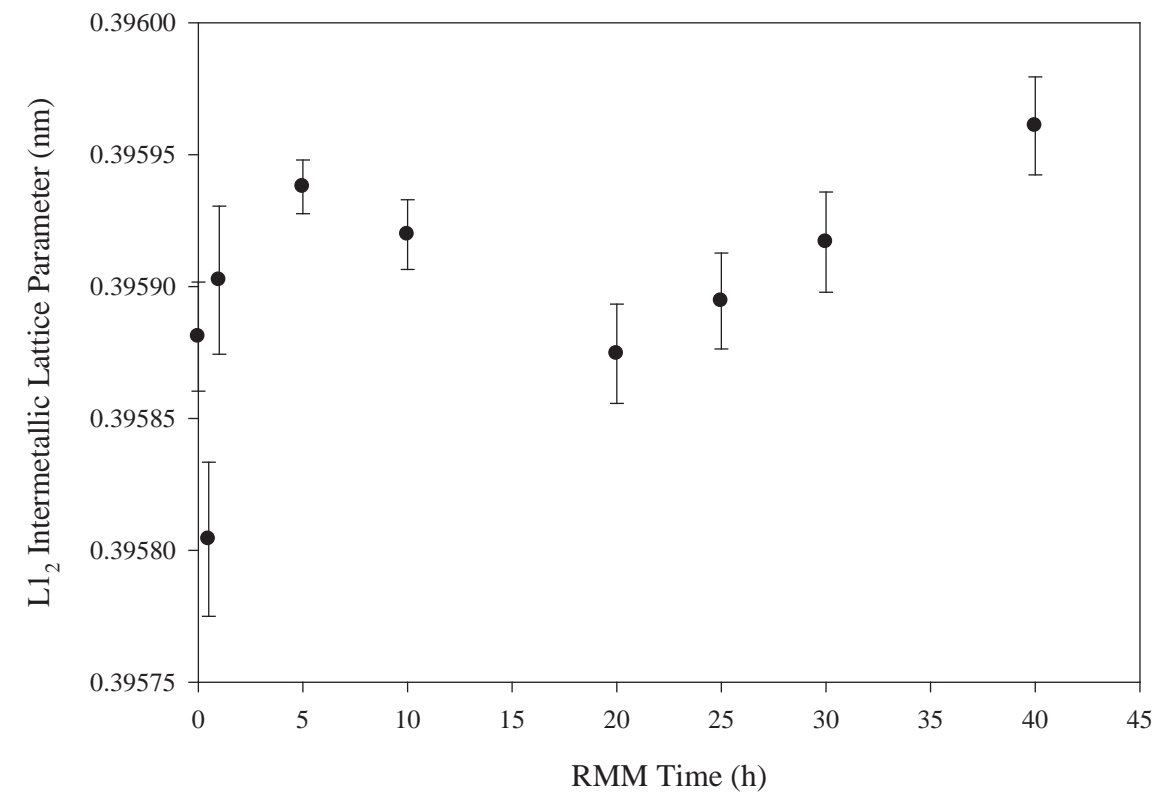

Figure VIII-2 $\mathrm{LI}_{2}$ Intermetallic Lattice Parameter of Annealed Powders for all RMM Times

\section{Phase Analysis of Consolidated Material}

\section{HIP Process RMM20h8.2TiAir800QH15m}

As noted, this sample designated as 20/800/MA-A, the powder for this type of consolidated material was RMM in an air environment and the material was consolidated using the Quick HIP process at a temperature of $800{ }^{\circ} \mathrm{C}$ for $15 \mathrm{~min}$. In this analysis the JCPDS-ICDD or created card numbers are shown in parenthesis. The XRD pattern shown in Figure VIII-3 establishes substantial amounts of the $\mathrm{L}_{2}$ chromium-modified titanium trialuminide (00-0001) and a solid solution of $\mathrm{DO}_{22} \mathrm{Al}_{3} \mathrm{Ti}$ (37-1449) (the solid solution conclusion was based on the systematic diffraction peak position shifts to a lower dspacing). Other phases identified were a $\operatorname{Ti}(\mathrm{C}, \mathrm{N})$ phase with diffraction peaks falling between those for TiC (32-1383) and TiN (38-1420), as well as a small amount of $\mathrm{Ti}_{2} \mathrm{Al}(\mathrm{C}, \mathrm{N})$ with diffraction peaks falling between those for $\mathrm{Ti}_{2} \mathrm{AlC}$ (29-95) and $\mathrm{Ti}_{2} \mathrm{AlN}$ (18-70). Also identified was a significant amount of $\mathrm{Al}_{8} \mathrm{Cr}_{5}$ (29-15) in the material. There 
are no detectable diffraction peaks from any oxide phases, in particular aluminum oxide; this is significant since the material was processed in an air environment.

Three diffraction peaks with measurable intensity could not be identified with the JCPDS-ICDD database. These three unidentified diffraction peaks out of a total of fiftyeight diffraction peaks are located at $\sim 33.8^{\circ}, 38.3^{\circ}$ and $60.4^{\circ} 2 \theta$ with the $\sim 38.3^{\circ}$ diffraction peak being the most intense. The diffraction peak at $\sim 60.4^{\circ} 2 \theta$ is very close, but at a slightly lower angle and with a greater intensity than the $\{220\}$ for $\operatorname{Ti}(\mathrm{C}, \mathrm{N})$ or the (110) for $\mathrm{Ti}_{2} \mathrm{Al}(\mathrm{C}, \mathrm{N})$. In addition, diffraction peaks from $\mathrm{AlN}$ overlap with other diffraction peaks which open the possibility that it could be present in a very small amount.

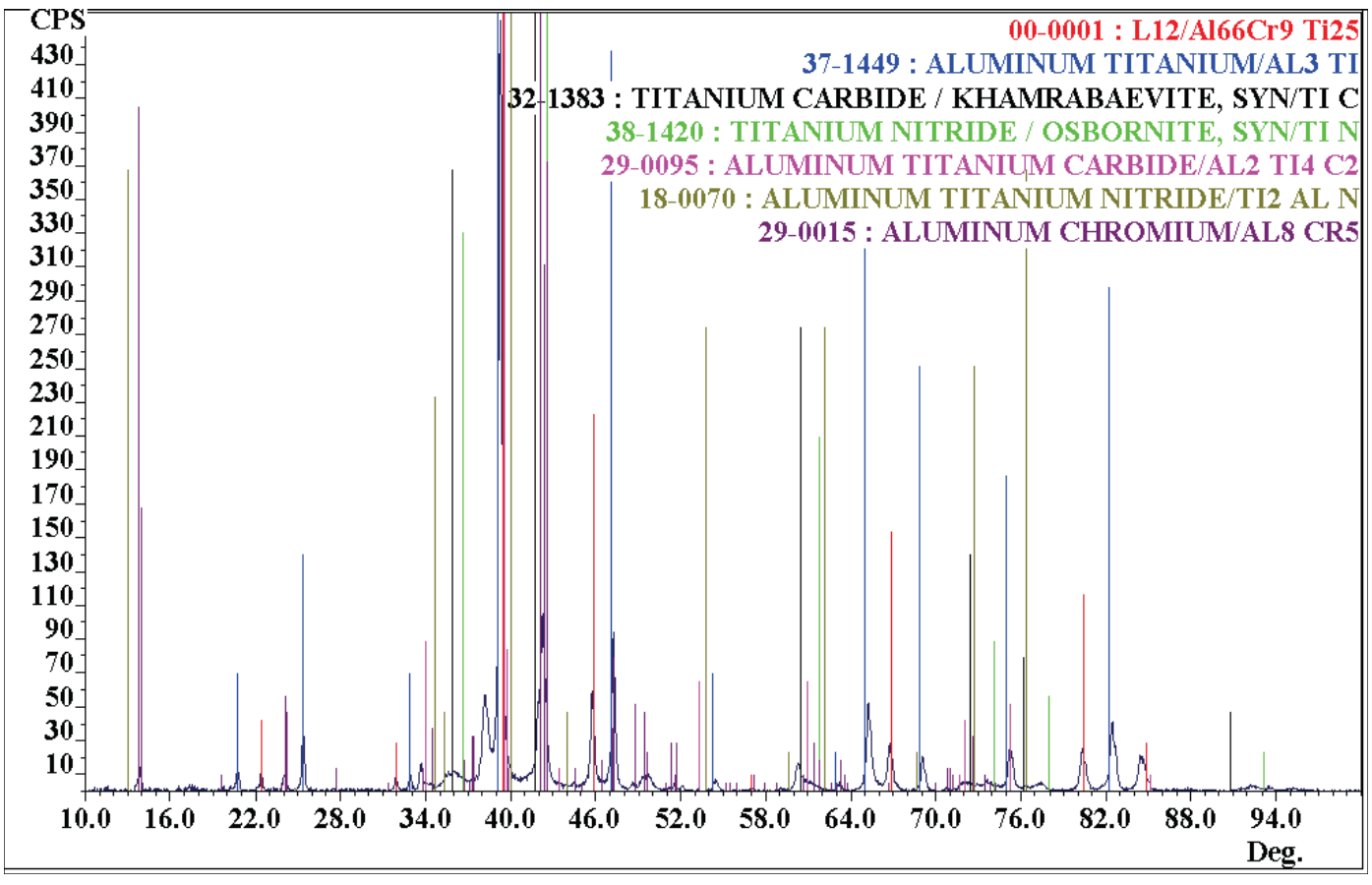

Figure VIII-3 XRD Pattern for HIP Sample 20/800/MA-A with Phase Identification

\section{HIP Process RMM20h8.2TiAr1000QH2h}

In this case, 20/1000/MA, the powder was consolidated using the Quick HIP process at $1000{ }^{\circ} \mathrm{C}$ for $1 \mathrm{~h}$. As expected, the dominant phase is the $\mathrm{L} 1_{2}$ chromiummodified titanium trialuminide (00-0001). Two complex carbide phases were present, $\mathrm{Ti}_{3} \mathrm{AlC}_{2}$ (00-0312) and $\mathrm{Ti}_{2} \mathrm{AlC}(29-95)$. However, it should be noted that several of the 
diffraction peaks for both carbide phases were found to be slightly shifted from data given in [138] and in the JCPDS-ICDD database. These diffraction peak position changes suggest solid solution behavior and/or stoichiometry effects. Based on the known isomorphous structures with these same compounds with nitrogen substituted with carbon and the presence of a small amount of nitrogen, shown in the chemical analysis, a portion of these shifts are most likely due to a solid solution effect with nitrogen. However, based on the low amount of nitrogen and the fact that extra aluminum is not added to the process, and is present in most phases, stoichiometry changes can also influence the lattice parameter. Also found was $\mathrm{AlCr}_{2}$ (29-16) and a small amount of $\mathrm{Al}_{2} \mathrm{O}_{3}$ (10-173). There were four unidentified diffraction peaks located at $\sim 23.4^{\circ}, 25.2^{\circ}, 27.5^{\circ}$, and $35.5^{\circ}$ $2 \theta$, with intensities on the order of $2 \mathrm{cps}$. The diffraction peaks at $\sim 25.2^{\circ}$ and $27.5^{\circ}$ were both very broad (greater than $\left.1^{\circ}\right)$.

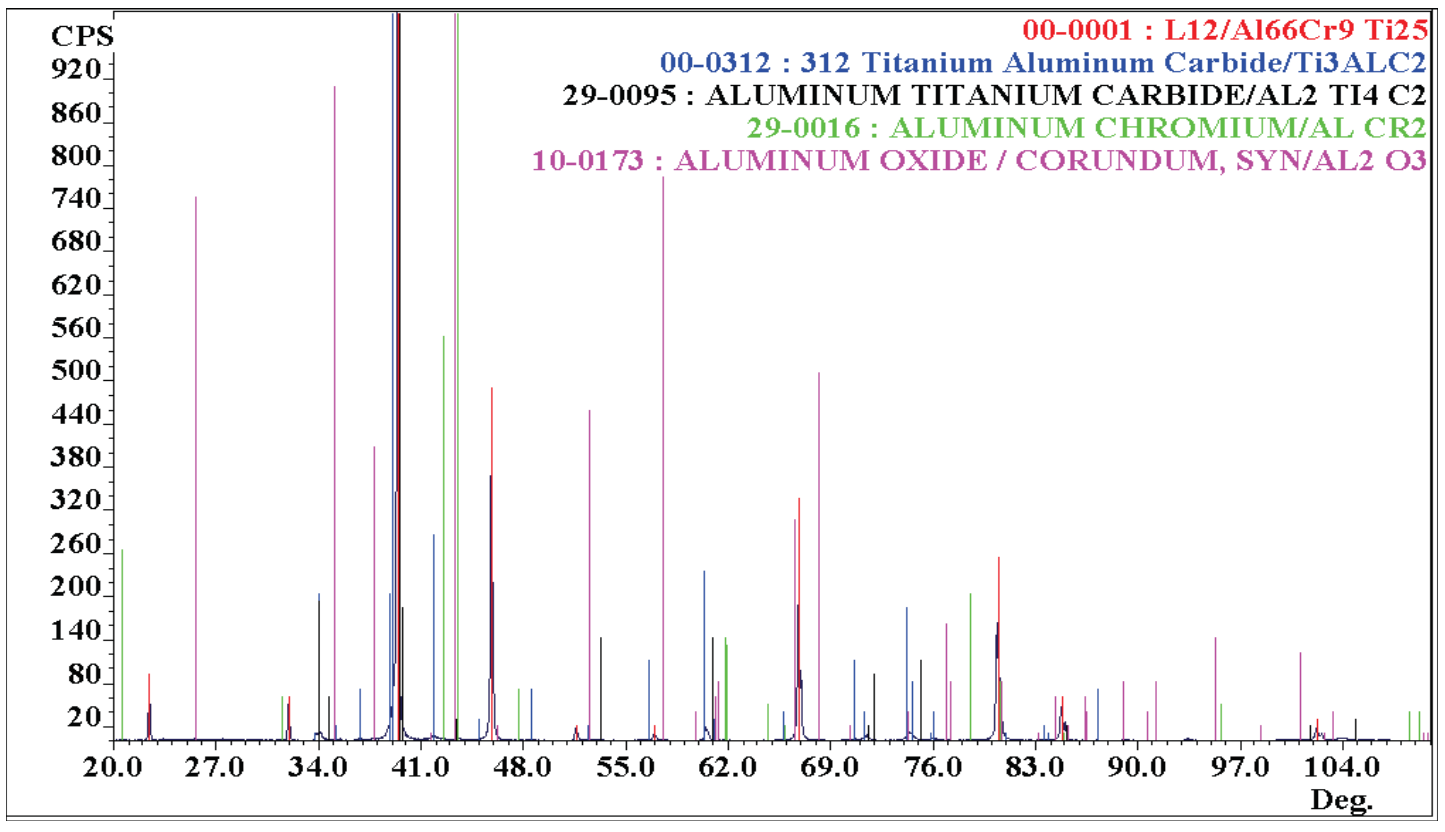

Figure VIII-4 XRD Pattern for HIP Sample 20/1000/M with Phase Identification

\section{HIP Process RMM20h9TiAr1000QH2h}

This powder, 20/1000/RA, was also consolidated using the Quick HIP process at $1000{ }^{\circ} \mathrm{C}$ for $2 \mathrm{~h}$. The dominant phase was again the $\mathrm{L}_{2}$ chromium-modified titanium trialuminide (00-0001). The major carbide phase was the $\mathrm{Ti}_{3} \mathrm{AlC}_{2}(00-0312)$; however, 
several of the diffraction peaks were again shifted from data given in [138], as discussed a portion of the shift is most likely a result of a solid solution with a slight amount of nitrogen substituting with carbon. However, as mentioned this shift is significant and the competition for the aluminum between the $\mathrm{L} 1_{2}$ intermetallic and this phase indicates a majority of this shift maybe caused by stoichiometry effects. Also present was a small amount of $\mathrm{DO}_{22} \mathrm{Al}_{3} \mathrm{Ti}$ (37-1449), again with shifts in the diffraction peaks indicating a lower d-spacing than published in JCPDS-ICDD database. Also found were two very small diffraction peaks (most intense $\sim 6 \mathrm{cps}$ ) with peak positions between those for the two most intense diffraction peaks of the isomorphous phases $\mathrm{Ti}_{3} \mathrm{AlC}(17-438)$ and $\mathrm{Ti}_{3}$ AlN (37-1140). This phase is plausible since the initial starting Reading Alloy powder contained a very small amount of nitrogen in addition to the possible introduction from the glove box operation. The presence of the $\mathrm{DO}_{22} \mathrm{Al}_{3} \mathrm{Ti}$ attributed to the carbon in starting Reading powder, which increased the amount of carbide phase formed and diminished the amount of titanium available for the $\mathrm{L}_{2}$ intermetallic. Typically with $\mathrm{DO}_{22} \mathrm{Al}_{3} \mathrm{Ti}$ present the $\mathrm{Al}_{8} \mathrm{Cr}_{5}$ phase appears. However, there is no strong evidence that this phase is present in any detectable amount. Also present was a very small amount of $\mathrm{Al}_{2} \mathrm{O}_{3}$ (10-173) and a detectable amount of what appeared to be the phase AlFe (33-20) with only two peaks detectable with a low intensity of $1 \mathrm{cps}$ for the most intense diffraction peak. Finally, one unidentified diffraction peak located at $\sim 35.5^{\circ}$ with an intensity of $\sim 1$ cps was observed. 


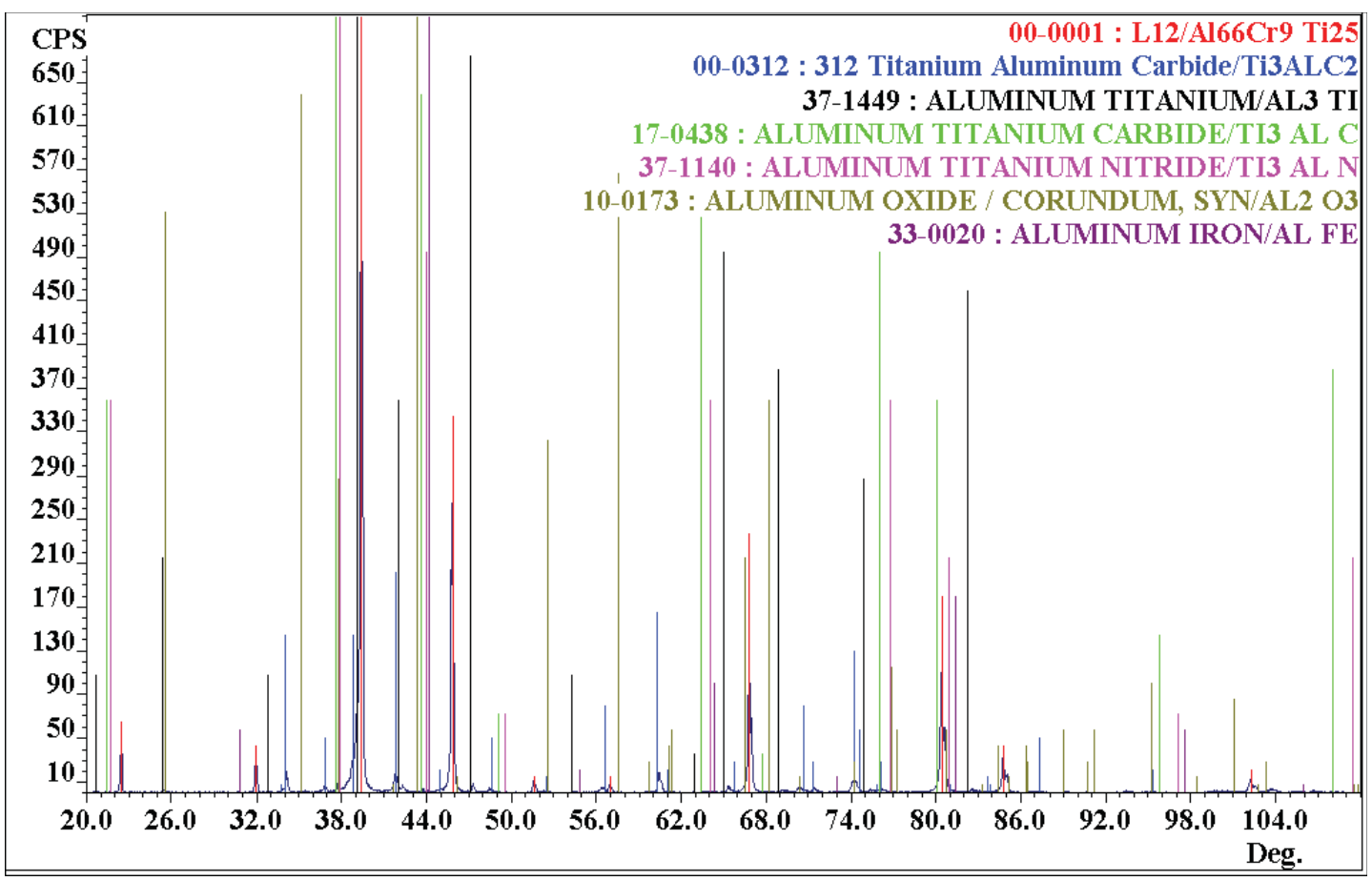

Figure VIII-5 XRD Pattern for HIP Sample 20/1000/RA with Phase Identification

4. Consolidated Materials from Systematic Increases in Excess Titanium and RMM Time in an Argon Atmosphere High Carbide Content (Long RMM Time Processing in Argon)

In order to produce higher carbide contents, the powders were processed for longer RMM times, with more excess titanium. As discussed in Chapter II.A.5.d)II.B.3, the RMM time was increased by integer multiples of $20 \mathrm{~h}$, with the base amount of excess titanium increased by the same integers (recall the Reading starting powders used a base value of 9 wt. pct.). The chosen RMM times for high carbide contents consolidated powders are $40 \mathrm{~h}, 60 \mathrm{~h}, 100 \mathrm{~h}$, and $120 \mathrm{~h}$. XRD phase analysis will be presented for all of the all high carbide consolidated samples.

\section{a) HIP Process RMM40h18TiAr1000QH2h}

After this powder, 40/1000/RA, was consolidated with the Quick HIP process at $1000{ }^{\circ} \mathrm{C}$ for $2 \mathrm{~h}$, the dominant phase was the $\mathrm{L} 1_{2}$ chromium-modified titanium trialuminide (00-0001). The carbide phase was the $\mathrm{Ti}_{3} \mathrm{AlC}_{2}(00-0312)$, with several of the 
diffraction peaks shifted from data given in [138], again as discussed most likely is a solid solution with a slight amount of nitrogen substituting with carbon. However, is should be emphasized that this shift is significant and most likely the majority is a result of stoichiometry effects especially considering the larger amount of this phase would indicate the need for more aluminum and again a competition with the $\mathrm{L} 1_{2}$ intermetallic which contains the source of aluminum. There were two very small diffraction peaks $(\leq \sim 3 \mathrm{cps})$ that can be associated with the first two most intense diffraction peaks of an isomorphous phase mixture consisting of $\mathrm{Ti}_{3} \mathrm{AlC}(17-438)$ and $\mathrm{Ti}_{3} \mathrm{AlN}$ (37-1140). There appeared to be slightly more $\mathrm{Al}_{2} \mathrm{O}_{3}$ (10-173), based on intensity, than observed with the other consolidated materials. A detectable amount of the phase AlFe (33-20) was present, but only two diffraction peaks were detectable with a low intensity of $1 \mathrm{cps}$ for the most intense diffraction peak. There was evidence of $\mathrm{AlCr}_{2}$ (29-15), but only the strongest intensity diffraction peak was visible implying a very small amount. There were three unidentified diffraction peaks located at $\sim 33.5^{\circ}, 35.5^{\circ}$, and $37.4^{\circ} 2 \theta$, all with intensities $\leq 2 \mathrm{cps}$.

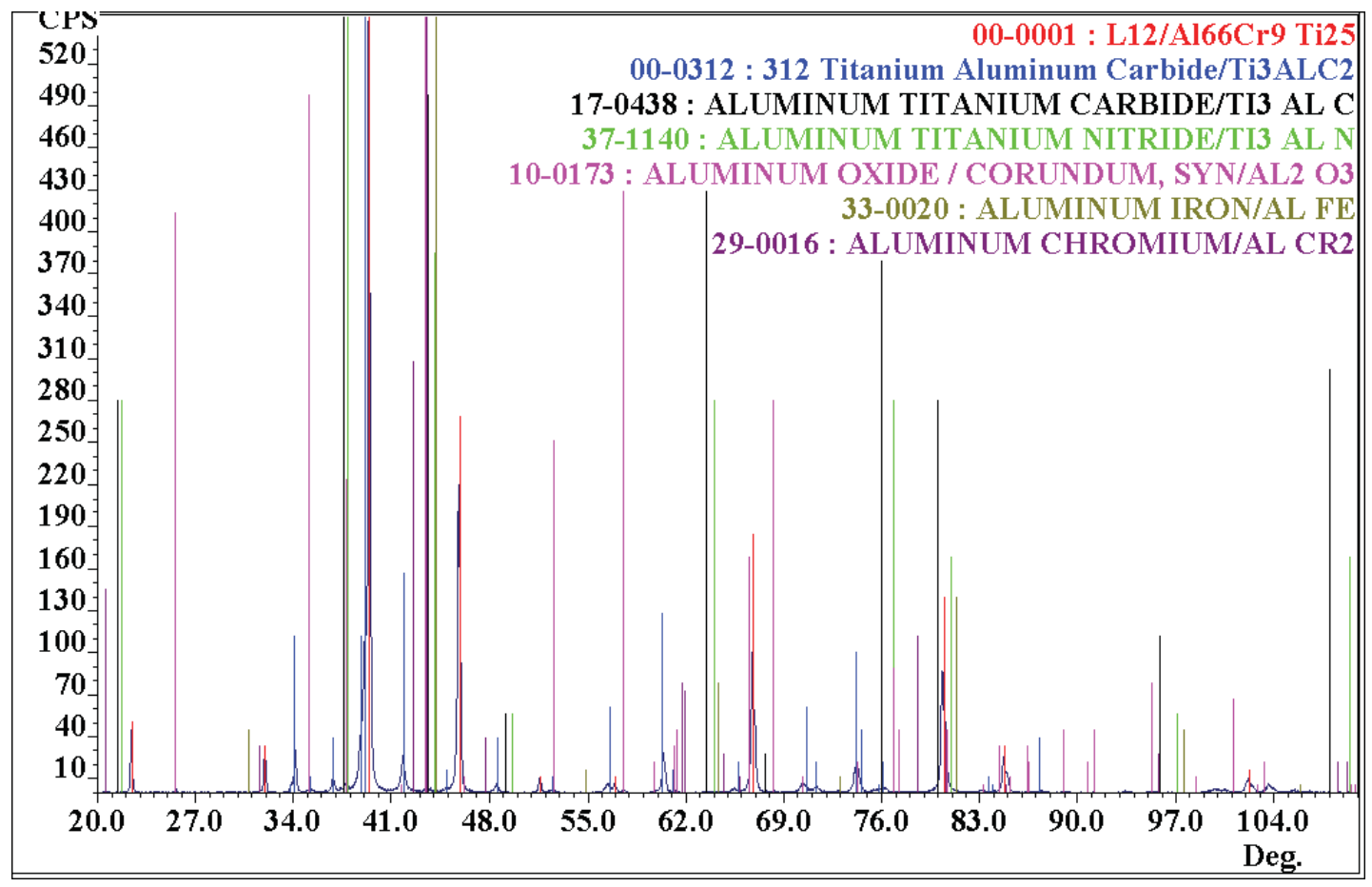

Figure VIII-6 XRD Pattern for HIP Sample 40/1000/RA with Phase Identification 


\section{b) HIP Process RMM60h27TiAr1000QH2h}

Again, this powder, 60/1000/RA, was consolidated using the conventional HIP process at $1000{ }^{\circ} \mathrm{C}$ for $2 \mathrm{~h}$. The dominant phase was the $\mathrm{L}_{2}$ chromium-modified titanium trialuminide (00-0001), with the major carbide phase being $\mathrm{Ti}_{2} \mathrm{AlC}$ (29-95), with indications of a small amount of the $\mathrm{Ti}_{3} \mathrm{AlC}_{2}(00-0312)$ phase. There was a noticeable increase in diffraction peak intensities for the complex carbide phases, indicating an increased amount as expected. Additionally, the diffraction peaks for these phases are significantly shifted compared to known positions in the JCPDS-ICDD and reference [138], given the small amount of nitrogen that was detected in some of these materials a majority of these shifts are due to stoichiometry effects most likely from potential aluminum variations. Also present were small amounts of $\mathrm{AlCr}_{2}$ (29-16), $\mathrm{DO}_{22} \mathrm{Al}_{3} \mathrm{Ti}$ (371449), and $\mathrm{Al}_{2} \mathrm{O}_{3}$ (10-173). As before the $\mathrm{DO}_{22} \mathrm{Al}_{3}$ Ti has diffraction peaks shifted slightly indicating a smaller d-spacing than indicated in the JCPDS-ICDD database. There was an extremely small intensity peak at the position corresponding to the highest intensity peak for AlFe (33-20) phase suggesting the presence of this phase. Finally, there are three unidentified diffraction peaks at $\sim 26.2^{\circ}, 58.8^{\circ}$, and $62.3^{\circ} 2 \theta$, all having intensities of $\leq 1$ cps. 


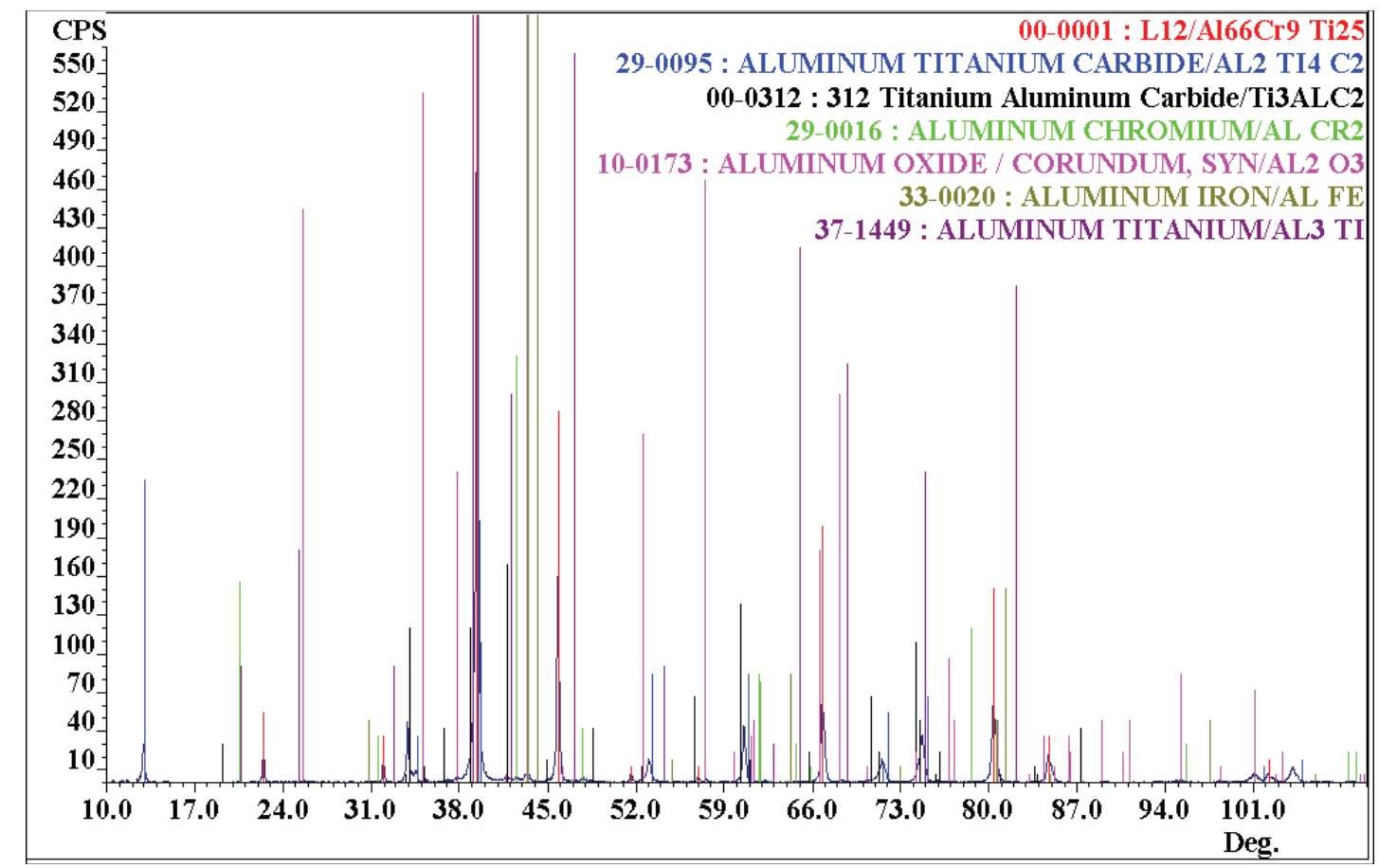

Figure VIII-7 XRD Pattern for HIP Sample 60/1000/RA with Phase Identification

\section{c) HIP Process RMM100h45TiAr1000QH2h}

This powder, 100/1000/RA, was consolidated using the conventional HIP process at $1000{ }^{\circ} \mathrm{C}$ for $2 \mathrm{~h}$, and again the dominant phase was the $\mathrm{L} 1_{2}$ chromium-modified titanium trialuminide (00-0001). The major carbide phase was $\mathrm{Ti}_{2} \mathrm{AlC}$ (29-95) with the presence of a small amount of the $\mathrm{Ti}_{3} \mathrm{AlC}_{2}$ (00-0312) phase. As expected, the intensities of the diffraction peaks for the complex carbide phases increased further. As before there was a significant shift in the diffraction peaks for the complex carbides and given the large amount of these phases the majority of this is attributed to stoichiometry effects (as opposed to the small amount of the solute nitrogen) most likely due to aluminum deficiencies. There was a very small amount of $\mathrm{TiC}(32-1383)$ present in this sample. Of interest is that this sample contains all three of three of the major carbides identified in this study and the $\mathrm{TiC}$ phase had only been observed previously at lower consolidation temperatures. Both the $\mathrm{TiC}$ and $\mathrm{Ti}_{3} \mathrm{AlC}_{2}$ diffraction peaks were very broad indicating a very small crystallite size in comparison to the $\mathrm{Ti}_{2} \mathrm{AlC}$ carbide phase. Also present was a small amount of $\mathrm{AlCr}_{2}$ (29-16) and $\mathrm{Al}_{2} \mathrm{O}_{3}$ (10-173). The amount of $\mathrm{AlCr}_{2}$ phase appeared 
to have increased based on peak intensities compared to all previous diffraction scans. A small diffraction peak at $\sim 44^{\circ} 2 \theta$ was associated with a solid solution AlFe (33-20) phase since this peak appears at a slightly lower angle than shown on the JCPDS-ICDD data card. Other peaks for the phase could not be identified because of overlap with more intense diffraction peaks from other phases and low intensities for those that do not overlap. It was concluded based on structure and lattice parameter that this phase is a $(\mathrm{Al}, \mathrm{Ti}) \mathrm{Fe}$ solid solution with the titanium giving rise to the increased lattice parameter. This conclusion follows from the lattice parameter of $0.2976 \mathrm{~nm}$ for TiFe and 0.28954 for AlFe with the lattice parameter of this solid solution phase falling in between these values. Several very small diffraction peaks could not be satisfactorily resolved and remain unidentified. These occurred at $\sim 26.1^{\circ}(<1 \mathrm{cps}), 30.4^{\circ}, 37.2^{\circ}, 44.0^{\circ}$, and $58.8^{\circ}$ $2 \theta$ all no more than $2 \mathrm{cps}$ of peak intensity. One unusual feature is the diffraction peak at $\sim 30.4^{\circ} 2 \theta$ is very broad with an $\sim 1.2^{\circ}$ FWHM peak width.

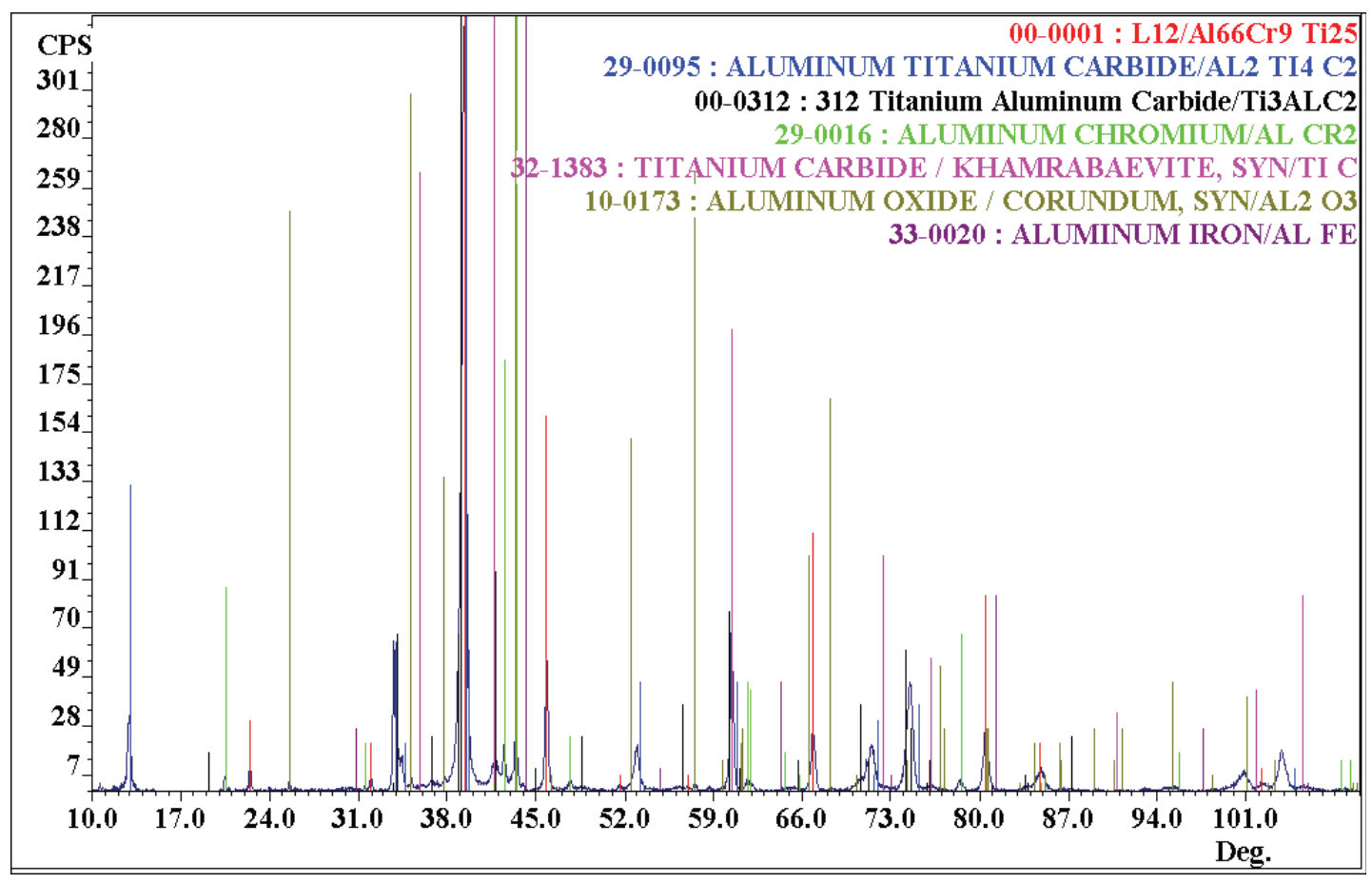

Figure VIII-8 XRD Pattern for HIP Sample 100/1000/RA with Phase Identification 


\section{d) HIP Process RMM120h54TiAr1000QH2h}

This powder, 120/1000/RA, was also consolidated using the conventional HIP process at $1000{ }^{\circ} \mathrm{C}$ for $2 \mathrm{~h}$. In all previously consolidated samples the most intensely diffracting phase has been the $\mathrm{L}_{2}$ intermetallic. However, with this consolidated sample the most intense diffraction occurred from TiC (32-1383). The most intense $\mathrm{L}_{2}$ chromium-modified trialuminide (00-0001) peak was only slightly stronger than the most intense peak for the $\mathrm{Ti}_{3} \mathrm{AlC}_{2}(00-0312)$ phase, and much less than that for the $\mathrm{TiC}$ phase. As before there was a significant shift in the diffraction peaks for the complex carbides and given the large amount of these phases the majority of this is attributed to stoichiometry effects (as opposed to the small amount of the solute nitrogen), again most likely due to aluminum deficiencies. Additionally, there is a significant shift to higher $2 \theta$ (or lower d-spacing) in the $\mathrm{TiC}$ phase. This can be attributed to a carbon deficiency and was be discussed in more detail in Chapter IV.A.1.b)(1)(a). Also present in small amounts were $\mathrm{Al}_{8} \mathrm{Cr}_{5}$ (29-15) and $\mathrm{Al}_{2} \mathrm{O}_{3}$ (10-173). There are several unidentified diffraction peaks at $\sim 21.6^{\circ}$ (6 cps), $25.8^{\circ}$ (6 cps), 32.9 $(1 \mathrm{cps}), 59.4^{\circ}$ (4 cps), $66.1^{\circ}(1$ cps), and $79.8^{\circ}$ ( 2 cps) $2 \theta$, with the intensities indicating very small amount(s) of unidentified phases.

In addition to the above-mentioned diffraction peaks there were several diffraction peaks that were able to be indexed on the basis of an ordered BCC structure, with the most intense diffraction peak almost $50 \%$ as intense as the most intense TiC diffraction peak, indicating a significant amount of the phase. Given the dominance of $\mathrm{TiC}$ in the diffraction pattern and small amount of chromium-bearing phases, it was speculated that this phase would contain significant amounts of titanium and chromium. In addition, XRF analysis has shown a $6.3 \mathrm{wt}$. pct. iron in this material with no other phases identified as iron bearing. Therefore, it was assumed that the iron resides in this phase. The only known chromium and titanium intermetallic phases have a $\mathrm{Cr}_{2} \mathrm{Ti}$ stoichiometry and are FCC or hexagonal. On the other hand, titanium and iron (TiFe) form an ordered BCC structure with a lattice parameter of $0.2975 \mathrm{~nm}$. However, the lattice parameter determined for the observed ordered $\mathrm{BCC}$ phase in this material was 
$0.299 \mathrm{~nm}$. It is speculated based on the known solubility of chromium in iron, and the fact chromium additions to iron typically increase the lattice parameter, that this phase is a $\mathrm{Ti}(\mathrm{Fe}, \mathrm{Cr})$. The possibility also exists that aluminum may be present, which would most likely substitute for titanium since AlFe alloys have the ordered BCC structure, but with a slightly smaller lattice parameter, $\sim 0.290 \mathrm{~nm}$.

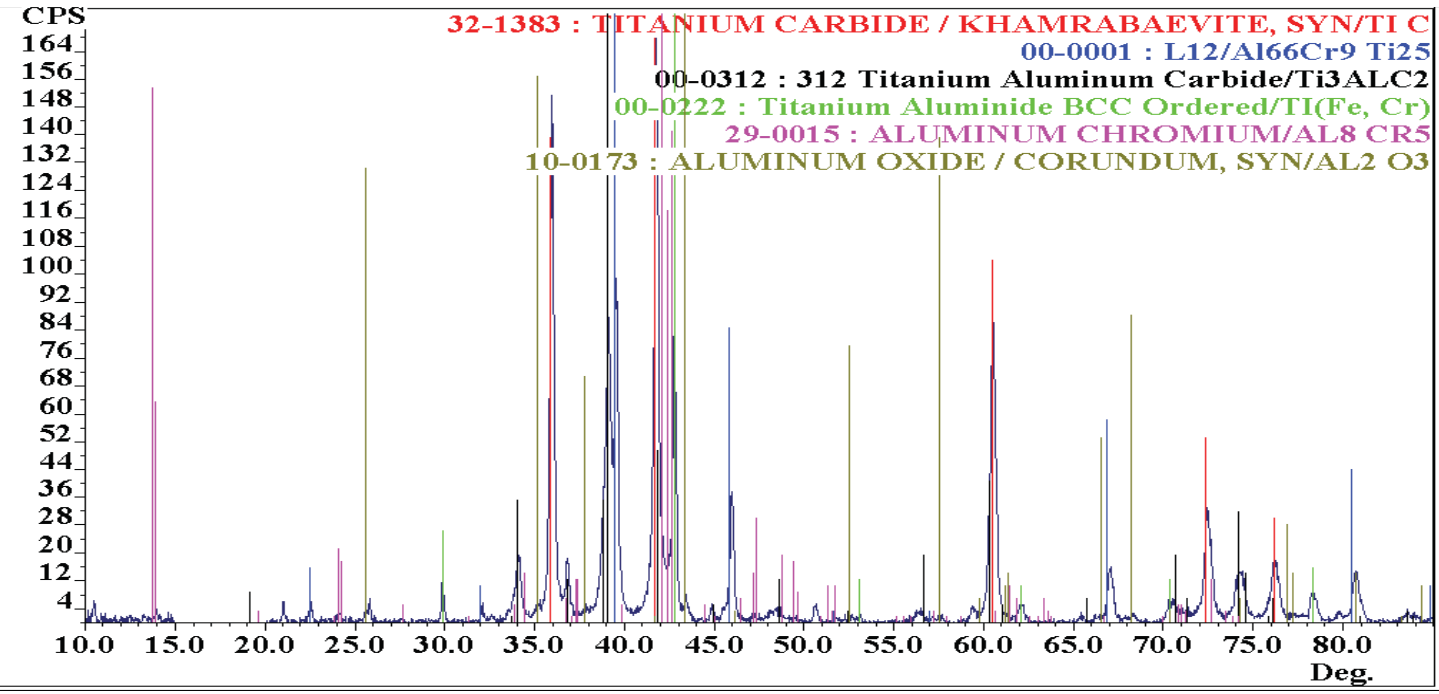

Figure VIII-9 XRD Pattern for HIP Sample 120/1000/RA with Phase Identification

\section{E. NGB Calculation for RMA Processing}

\section{RMA20h8.2TiAr Elemental Starting Powder}

Since it appears certain crystal defects, particularly dislocation movement, contribute to the NGB formation, any ductile phases could participate in the formation of these NGB regions provided large polycrystalline regions of these ductile materials are present. For this sample, these would likely include titanium and chromium, in addition to the $\mathrm{L}_{2}$ intermetallic. Again, this depends on whether the titanium and chromium have a physical particle sizes or regions larger than the crystallite size to permit formation of GBs within the particles. Size estimates, determined with the Scherrer method, gave appropriate crystallite sizes for chromium of $11 \mathrm{~nm}$ and about $6 \mathrm{~nm}$ for titanium. Both of these are larger than that of the $\mathrm{L}_{2}$ intermetallic. For milling times of about $20 \mathrm{~h}$ or longer it is suggested that the chromium and titanium phases are likely mostly isolated 
single crystal particles with the crystallite sizes representing the average size of these particles.

Case 1 is determined in a similar manner to that in the RMMXh9TiAr Series. However, for Case 2 all ductile phases can contain the NGB; i.e. there are no single crystal particles. As before the $\mathrm{TiC}$ and $\mathrm{TiH}_{1.92}$ result from nucleation events being inert in connection with the calculation of the volume of NGB region. In Case 2, one important assumption that must be made concerns the distribution of the NGB region in the various phases in which they are produced. Data for this distribution has not been determined; however, a good assumption is that the NGB region forms in linear relation to the volume fraction of each constituent phase. This assumption appears to pose problems since the chemistry is different between the phases, but these differences would appear in both the numerator and denominator of Equation (IV.4).

The first step is the calculation of the intensity arising from the NGB region, which is simply the difference between the integrated background area of the as-milled powder and the integrated background area of the annealed powder shown in Equation (IV.5), this is the same for both cases and empirically corrects for the $\mathrm{K}$ factor as discussed in terms of Equation (IV.4). The next step for Case 1 is calculation of the volume fraction of the NGB region within the $\mathrm{L}_{2}$ intermetallic (noted as ductile phase in Table VIII-5) phase in the same manner as with the RMMXh9TiAr powders only additionally summing the new phases. In Case 2, the amount of the NGB region within all ductile phases with these crystalline areas added to form the value of $I_{\text {Total }}$ defined in Equation (VIII.1) which becomes the denominator of Equation (IV.4) (this accounts for diffraction peak integrated areas of ductile phases, i.e. the $\mathrm{L}_{2}$ intermetallic, chromium, and titanium) with the numerator defined by Equation (IV.5) yielding the volume of NGB region within the ductile phases in Table VIII-5 for both Case 1 and Case 2 .

$$
\int_{10}^{100} I_{\text {Total }}(2 \theta) d \theta=\int_{10}^{100} f_{N G B}(2 \theta) d \theta+\int_{10}^{100}\left[I_{L 1_{2}}(2 \theta)+I_{T i}(2 \theta)+I_{C r}(2 \theta)\right] d \theta
$$




\begin{tabular}{|c|c|c|c|c|c|}
\hline Case & $\begin{array}{c}\text { fRMM- } \\
\text { Background }(2 \theta)\end{array}$ & $\begin{array}{c}f_{\text {Anneal- }} \\
\text { Background }(2 \theta)\end{array}$ & $f_{\mathrm{NGB}}(2 \theta)$ & $\begin{array}{c}\text { Ductile } \\
\text { Phase Area }\end{array}$ & $\begin{array}{c}\text { Vol. Fraction } \\
\text { NGB in } \\
\text { Ductile Phases } \\
\text { VNGB-Ductile }\end{array}$ \\
\hline 1 & 278.2 & 228.7 & 49.5 & 201.5 & 0.197 \\
\hline 2 & 278.2 & 228.7 & 49.5 & 256.5 & 0.162 \\
\hline
\end{tabular}

A similar procedure will be applied to the RMA20h8.2TiAr sample that was used for the RMMXh9TiAr Series to determine the corrected volume fractions with the addition of the new phases. The calculation for Case 1 requires Equation (VIII.2) which accounts for the all phases present in the material applying the appropriate $\mathrm{X}$ correction factor. $V_{N G B}$ can be found with Equation (IV.8) used earlier for correction of volume fraction data of the RMMXh9TiAr Series assuming the entire NGB region is within the $\mathrm{L} 1_{2}$ intermetallic. Once $V_{N G B}$ is determined and entered into Equation (VIII.2) the correction factor $\mathrm{X}$ can be found to be 0.881 for Case 1 , and can be used to determine the corrected volume fractions. A similar procedure determines the corrected volume fractions for Case 2 which assumes the NGB region is present in all ductile phases using the appropriate $V_{N G B-D u c t i l e}$ variable given in Table VIII-5. The modified expression to determine the corrected volume fractions for the added phases present is shown in Equation (VIII.2). Calculation of $\mathrm{X}$ is accomplished by substituting for $V_{N G B}$ in Equation (VIII.2) with the known parameters from Equation (VIII.3) and algebraically solving for $\mathrm{X}$. The value of correction factor $\mathrm{X}$ for Case 2 is 0.883 that can be applied to correct the original volume fractions.

$$
\begin{gathered}
1=\left(V_{L 1_{2}} \times \mathrm{X}\right)+\left(V_{T i C} \times \mathrm{X}\right)+\left(V_{T i H_{1.92}} \times \mathrm{X}\right)+\left(V_{T i} \times X\right)+\left(V_{C r}+X\right)+V_{N G B} \\
V_{N G B}=\left(V_{L 1_{2}}+V_{T i}+V_{C r}\right) \times \mathrm{X} \times V_{\text {Ductile- } N G B} \quad \text { (VIII.3) }
\end{gathered}
$$

The results of this analysis are given in Table IV-5 along with the original values determined before taking in to account the NGB region. As might have been expected these results show that the final calculated amount of the NGB region is not sensitive to the presence of secondary ductile phases, particularly if present in small amounts as in 
this case. The most notable result is the amount of the NGB region within the sample, which is approximately one half of that determined for the RMM20h9TiAr sample milled for a similar $20 \mathrm{~h}$ milling time.

\section{F. Role of Grain Boundary Structure in Superplastic Deformation}

The GB diffusion in a material with a NEGB structure is higher than in typical HAGBs with those possessing the NGB structure expected to further enhance the diffusion in nanocrystalline materials. However, what is important is both physically and experimentally the diffusivity of the GBs increases as the structure changes from a HAGB to NEGB and finally to a NGB. More importantly, the amount of GB volume increases as the grain size decreases. Both grain rotation and superplastic behavior are dependent on atomic shuffling of atoms on the GBs and it stands to reason that structural changes that produce more disorder to the GBs can assist in the ease of both grain rotation and superplastic behavior. During the plastic deformation of polycrystals to accommodate the non-homogeneous deformation grain rotation can occur allowing conformity of the grains to deformation directions while maintaining the materials integrity, as the grain size is refined down to the lower nanometer range the deformation eventually becomes dominated by GB-mediated plasticity, which has been shown in the presence of high strain rates this analogous to room temperature superplastic behavior.

In atomistic simulations of GB sliding in pure and magnesium doped aluminum bicrystals, it was shown that an increase in GB energy, as determined by the magnesium doped material, provided a clear dependence on the magnitude of sliding on GB energy [272]. No dislocations were observed during the GB sliding and indications are that the natural tendency of a GB to slide depends on the disorder of the GB, as more disorder is related to a higher GB energy. More importantly, this study concluded that a higher energy GB slides at a lower stress with a lower viscosity and has a smaller activation energy. Therefore it would be expected that this GB motion can occur at a lower temperatures. Subsequently, the proposed NGB structure is expected to be highly disordered and hence a large GB energy is expected again promoting GB sliding. Hence 
a combination of the higher GB energy, and large internal strain at the GB interface, and the expected ability for atoms to move on the GB (discussed in Chapter IV.B.1 enhancement of diffusivity at the NGB) contribute to the ability of the material to deform by GB mechanisms.

Superplastic behavior is the result of mass transport along the GB under high stress and temperatures which activate diffusion. Normally superplastic materials are defined as having grain sizes less than $10 \mu \mathrm{m}$ and processed within a strain rate range between $10^{-5}$ to $10^{-1} / \mathrm{s}$ and a temperatures above about $0.5 \mathrm{~T}_{\mathrm{m}}\left({ }^{\circ} \mathrm{K}\right)$ [273]. While the temperatures are often above $0.5 \mathrm{~T}_{\mathrm{m}}\left({ }^{\circ} \mathrm{K}\right)$ the important aspect is the requirement that the grain sizes approach the micrometer level for this property to be observed. These superplastic materials are sensitive to the strain rate with a sigmoidal shape to the logarithmic flow stress with strain rate, and the highest flow stress occurs at the fastest strain rates. The lowest strain rates produces a flow stress behavior, Region I, associated with diffusional creep with the origin of this flow presently unknown. Grain elongation occurs in this region. Region II of the curve, the midsection of the graph, is the superplastic region a linear-like portion of the sigmoidal curve dominated by grain rotation of equiaxed grains with no grain elongation. Here high uniform strains are observed with GB sliding and GB rotation being a substantial amount of the total strain. As the strain rate increases, Region III, terminates at an asymptotic stress value at the largest strain rate. This region is generally associated with conventional recovery controlled dislocation creep (power law creep) with grain elongation and texture occurring during deformation.

Superplastic investigators generally believe grain rotation is a result of unbalanced shear stresses associated with inhomogeneous grain boundary sliding. If grain rotation only occurred in a rigid system cavities would form but cavities are rarely observed in superplastic forming therefore the GB sliding must be accommodated [273]. However, the details of this accommodation mechanism(s) are not well understood with elastic distortions, dislocation activity, and diffusion based mechanisms the prevailing thought. What is important is that the grain size for superplastic deformation must be small or essentially approximately equal to or smaller than the subgrain size that is 
formed during high temperature deformation, this grain size range has been established to be between 0.01 to $10 \mu \mathrm{m}$ [274]. Below the $0.01 \mu \mathrm{m}$ or $10 \mathrm{~nm}$ grain size flow is associated with GB-mediated deformation at room temperature. To grasp the physical meaning of the Raj and Ashby [275] model, describing grain boundary sliding during diffusional creep, between the two smooth but nonplanar mating GB surfaces which mate exactly with each other, a "viscous-oil" model is presented as the intrinsic mechanical properties of the GB. What is interesting is that the NGB could be thought as a mobile fluid of atoms, i.e. changing the intrinsic mechanical properties of the GB. Hayes et al. [276] looked at Coble creep considerations and used values of activation energy based on large-grained materials and hence a false sense of its contribution; however, the change in the GB structure may well alter this activation energy and alter the mechanism for small grain sizes.

Strain rate appears an important aspect of superplastic behavior as described in Equation (VIII.4). Where $D$ is GB diffusivity, $G$ is shear modulus, $b$ is the Burgers vector, $T$ is test temperature, $k$ is Boltzmann constant, $d$ is grain size, $\sigma$ is the flow stress, the exponent $p$ depends on grain size (typically 2), while $n$ is an inverse parameter to the coefficient of strain sensitivity of the flow stress. Important to this equation is the idea that higher strain rates are induced by smaller grain sizes, larger Burgers vector, and increase diffusivity of the GBs allowing manifestation of superplasticity at a relatively low temperature and/or high deformation rates [277]. What is interesting is that materials with NEGBs have been shown to alter properties of materials typically thought of as structural-insensitive such as shear stress, further possibly altering this parameter and subsequently the superplastic temperature. Materials with the NGB structure are expected to further enhance these property changes as well as a progressively higher GB diffusivity. Systematic manipulation of these parameters and grain size should allow observation of superplasticity at progressively lower temperatures typically within strain rate ranges specified for superplastic behavior.

$$
\dot{\varepsilon}=\left(\frac{D G b}{k T}\right)\left(\frac{b}{d}\right)^{p}\left(\frac{\sigma}{G}\right)^{n}
$$


Watanabe [278] discussed the ability of GB engineering for superplasticity concentrating on the near micrometer and larger sizes with the understanding that sliding for individual GBs would be different. Experiments showed that more random GBs induced at the lower grain sizes were both beneficial and detrimental to GB sliding. Despite not providing an engineering model the author speculated that a more robust experimentation would provide an optimal GB structure. The interesting aspect is they demonstrated some ability to vary and increase the fraction of random HAGBs by $40 \%$ to $75 \%$. Todd $[279,280,281]$ has developed theory for fine-grain superplasticity based on GB structure work, Bollmann [44] the displacement shift complete lattice (DSCL derived from CSL), and determined that intrinsic secondary GB dislocations and their reactions are important to superplastic behavior as these typically have Burgers vectors smaller than lattice dislocations. The important aspect is that since this property depends on GB sliding it would be expected that changes to the GB structure can dramatically change this behavior, which as has been shown to occur as the grain size decreases. These GB structural changes are expected to lower the superplastic temperature in a systematic way down to a grain size below $20 \mathrm{~nm}$ where room temperature GB-mediated plasticity is becoming dominant.

Fine-grained Titanium 6A-4V ( $8 \mu \mathrm{m}$ grain size $)$ has a superplastic temperature between 900 and $920^{\circ} \mathrm{C}$ while the same alloy with a $1 \mu \mathrm{m}$ grain size shows a marked reduction in the superplastic temperature to about $775^{\circ} \mathrm{C}$ while the paper describes the benefits of lower temperature forming they do not discuss mechanisms that led to the lower superplastic temperature [282]. Refining the grain size slightly more for this alloy to 0.5 and $0.8 \mu \mathrm{m}$ grain sizes lowered the superplastic temperature to 650 and $750{ }^{\circ} \mathrm{C}$ respectively or a temperature reduction of about $200^{\circ} \mathrm{C}$ by grain refinement [283]. Although this study indicates a trend of a smaller grain size lowering the superplastic activation temperature, a minimum temperature was not determined.

In a study by Valiev et al. [277] on $\mathrm{Ni}_{3} \mathrm{Al}$ processed by equal channel angular pressing (ECAP) to a grain size of about $50 \mathrm{~nm}$, superplasticity manifests itself $400{ }^{\circ} \mathrm{C}$ lower than that in a corresponding microcrystalline specimen. Additionally, a 1420 aluminum alloy created with a 0.3 to $0.4 \mu \mathrm{m}$ grain size showed two to three orders of 
magnitude higher strain rate at $400^{\circ} \mathrm{C}$ than for that from a $5 \mu \mathrm{m}$ grain size alloy. What is of interest is that the $50 \mathrm{~nm}$ grain size material appears to have more of an effect on the temperature of superplasticity and the authors noted all GBs were composed of NEGB structure. Data compiled by Mishra [284] showed a clear linear dependence of grain size reduction and superplastic temperature reduction down to about $100 \mathrm{~nm}[285,286]$. It is suspected that the NEGB structure is beginning to become significant at his grain size but this data was not provided for lower grain sizes.

A 1420 alloy having grain sizes in the sub-micrometer range with values of 0.3 $\mu \mathrm{m}$ shows superplastic behavior at $250{ }^{\circ} \mathrm{C}$ with an $300{ }^{\circ} \mathrm{C}$ temperature required for a 0.9 $\mu \mathrm{m}$ grain size while for the $5 \mu \mathrm{m}$ grain size the superplastic temperature is $400^{\circ} \mathrm{C}$ [287]. More importantly, a decrease in the activation energy of GB diffusion in the $0.3 \mu \mathrm{m}$ grain size over the $0.9 \mu \mathrm{m}$ grain size was observed while the 5 and $20 \mu \mathrm{m}$ grains sizes had similar values. The significance of this activation energy decrease in GB diffusion for the smallest grain size translates into a diffusion coefficient of about 30 times larger than with the larger grained materials.

A similar 1420 alloy with a grain size of $100 \mathrm{~nm}$ was created by SPD having superplastic properties occurring at $250^{\circ} \mathrm{C}$ with this also shown to be the temperature at which grain growth occurs for these fine grains, hypothesizing that the microstructural instability corresponds to the temperature for superplasticity [288]. Of interest in this paper is the fact that at $200^{\circ} \mathrm{C}$ the exponent for the strain rate and flow stress curves determined was 2.5 very close to the ideal of 2 expected for GB sliding and much closer than the 3.6 exponent obtained for the $250^{\circ} \mathrm{C}$ temperature indicating that superplastic properties may well exist at $200{ }^{\circ} \mathrm{C}$ while superplastic behavior at this temperature was not discussed. Valiev et al. [289] determine with a grain size of $50 \mathrm{~nm}$ in $\mathrm{Ni}_{3} \mathrm{Al}$ that GB sliding in superplastic behavior was due to diffusion at lower temperatures, based on HRTEM examination. This study determined the presence of the NEGBs composing nearly $100 \%$ of the GB structure. The lattice dislocation activity in grains was low due to difficulty in generating and moving dislocations inside the lattice of these small grains. Recall lattice dislocation are no longer seen below about $50 \mathrm{~nm}$ or similar to the grain size of the $\mathrm{Ni}_{3} \mathrm{Al}$ alloy in the Valiev et al. study. This study also validated grains are not 
elongated and low defect density suggests behavior around the GBs pointing out that more study on the GB structure and its implications would be fruitful.

While the $100 \mathrm{~nm}$ grain size 1420 alloy and $50 \mathrm{~nm}$ grain size $\mathrm{Ni}_{3} \mathrm{Al}$ both showed much lower superplastic temperatures a nanocrystalline nickel with a $20 \mathrm{~nm}$ grain size had superplastic behavior at $470{ }^{\circ} \mathrm{C}$ below that previously attained [290]. Also of interest was both the nanocrystalline 1420 and $\mathrm{Ni}_{3} \mathrm{Al}$ alloys only doubled in grain size after processing superplastically while the nanocrystalline nickel increase to about a micrometer or nearly 2 orders of magnitude. This corresponds to a $0.36 \mathrm{~T}_{\mathrm{m}}$ the lowest normalized superplastic temperature reported (room temperature for nickel is $0.17 \mathrm{~T}_{\mathrm{m}}$ ). In these studies mentioned, explanations were not provided for the lower superplastic temperatures observed.

The importance of these studies is that as the grain size decreases into the submicrometer range and nanometer range the temperature for superplastic behavior systematically decreases. Supporting this is molecular dynamics studies on material modeled with NEGB structures that determined the activation barriers for plastic deformation are lowered such as; dislocation nucleation, GB sliding via atomic shuffling and migration [189]. While "bulk" lower nanometer materials are difficult to create that are defect free, the data provides a trend of decreasing superplastic temperature with decreasing grain size. While this behavior is strongly dependent on diffusivity of the GB region, as was shown the NGB structure under strain will significantly increase diffusion, thus lowering diffusion temperature adding to the noted effect of lower superplastic temperatures with smaller grain sizes. Additionally, as the grain size decreases the amount of atoms within the GB zone dramatically increases with this effect is relatively unknown. The importance is that a grain size in the true nanometer (below $20 \mathrm{~nm}$ the change begins to be rapid depends on material) superplastic-like properties are observed at room temperature under high strain rates. Implying that the GB structure dramatically effects the deformation mechanisms and hence the mechanical properties (as well as all others) but more importantly may be a key to understanding superplastic behavioral changes with temperature. 


\section{References}

1 A. Raman, and K. Schubert, Z. Metallkde., 56, 991965

2 A. Seibold, Z. Metallkde. 72, 712, 1981

3 K.S. Kumar, and J.R. Pickens, Scripta Met., Vol. 22, 1015, 1988

4 W.O. Powers, and J.A. Wert, Met. Trans. A, Vol. 21A, 1451990

5 U.S. Patent, Serial No. 289, 543 and 331,626, Filed December 1988

6 S. Zhang, J.P. Nic, and D.E. Mikkola, Scripta Met., Vol. 24, 57, 1990

7 J.P. Nic, Structure/Property/Composition Relationships in Cubic Al 3 Ti-Base Intermetallic Alloys, MS Thesis, Michigan Technological University, 1992

8 J.P. Nic, S. Zhang, and D.E. Mikkola, Scripta Met., Vol. 24, 1099, 1990

9 C. T. Sims N.S. Stroloff, and W.C. Hagel, Superalloys II, 3, New York: Wiley, 1986

10 S. Zhang, Ph.D. Dissertation, Michigan Technological University, 1993

11 S.J. Miller, Mechanical Behavior of Chromium-modified $\mathrm{Al}_{3} \mathrm{Ti}$ compared to Ni-Modified $\mathrm{Al}_{3} \mathrm{Ti}_{\text {, and }} \mathrm{Ni}_{3} \mathrm{Al}$, and the Effect Of Quaternary Alloying on the Bend Ductility of $\mathrm{Al}_{65} \mathrm{Cr}_{10} \mathrm{Ti}_{25}$, MS Thesis, Michigan Technological University, 1997

12 K.S. Kumar, $\underline{\text { Structural Intermetallics, }}$ Conference Proceedings, The Minerals Metals \& Materials Society, 87, 1993

13 K.S. Kumar, and S.A. Brown, Scripta. Metall. Mater., Vol. 40, No. 8, 1923, 1992

14 J.H. Schneibel, J.A. Horton, and W.D. Porter, Materials Science and Engineering, A152, 126, 1992

15 Z.L. Wu, and D.P. Pope, Acta Metall. Mater., Vol. 42, No. 2, 519, 1994

16 J.L. Klansky, J.P. Nic, and D.E. Mikkola, Materials Science and Engineering, A152, 132, 1992

17 L.J. Parfitt, J.L. Smialek, J.P. Nic, and D.E. Mikkola, Scripta. Metall., Vol. 25, 727, 1991

18 K. Hirukawa, M. Mabuchi, and Y. Nakayama, Scripta. Metall., Vol. 25, 1211, 1991

19 D.K. Dewald, SBIR Phase I Contract F33615-97-C-5279, Waubik, Inc., 1998

20 D.K. Dewald, and D.E. Mikkola, The Minerals, Metals \& Materials Society, Elevated Temperature Coatings: Science and Technology II, 255, 1996

21 W.E. Frazier, and M.J. Koczak, Dispersion Strengthened Aluminum Alloys, 573, 1988

22 L.S. Darken, Transactions of the ASM, Vol. 54, 600, 1961

23 T. H. Courtney, Mechanical Behavior of Materials, 1990

24 T.N. Baker, Yield, Flow and Fracture of Polycrystals, 1983

25 E.O. Hall, Proced Phys. Soc., B64, 747, 1951

26 A.H. Cottrell, and B.A. Bilby, Proc. Phys. Soc., A, 52, 49, 1949

27 J. D. Eshelby, F.C. Frank, F.R.N. Nabarro, Phil. Mag., 42, 351, 1951

28 N.J. Petch, Journal of the Iron and Steel Institute, 174, 25, 1953

29 C. Suryanarayana, International Materials Reviews, Vol. 40, No. 2, 1995

30 E.M. Schulson, I. Baker, and H.J. Frost, Mat. Res. Soc. Symp. Proc., Vol. 81, 195, 1987 
31 C.S. Pande, R.A. Masumura, and R.W. Armstrong, Nano Structured Materials, Vol.2, Issue 3, 323, 1993

32 A. H. Choksihi, A. Rosen, J. Karch, and H. Gleiter, Scripta Metallurgica, Vol. 23, 1679, 1989

33 A.S. Mohammadabadi, and K. Dehghani, Journal of Materials Engineering and Performance, Vol. 17, Issue 5, 662, October 2008

34 R. O. Scattergood, and C.C. Koch, Scripta Metallurgica, Vol. 27, 1195, 1992

35 H. Chang, C.J. Altstetter, and R.S. Averback, Journal of Materials Research, Vol. 7, No. 11, Nov 1992

36 T.G. Nieh, and J. Wadsworth, Scripta Metallurgica, Vol. 25, 955, 1991

37 A. S. Argon and S. Yip, Philosophical Magazine Letters, Vol. 86, No. 11, 713, November 2006

38 F.R. Morral, Dispersion Strengthening of Metals, Report, April, 1977

39 B.S. Murty, and S. Ranganathan, International Materials Review, Vol. 43, No. 3, 101, 1998

40 E. Orowan, Dislocations in Metals, AIME, 69, 1954

41 G. E. Dieter, Mechanical Metallurgy, McGraw-Hill, 1976

42 H. Gleiter, Progress in Materials Science, 33, No.4, 223, 1989

43 V. Randle, Role of the Coincidence Site Lattice in Grain Boundary Engineering, The Institute of Materials, 1996

44 W. Bollmann, Crystal Defects and Crystalline Interfaces, Springer-Verlag Berlin Heidelber, 1970

45 Metal Interfaces, Seminar on metal interfaces held during the Thirty-Third National Metal Congress and Exposition, October $13^{\text {th }}$ to $19^{\text {th }}$, ASM, Cleveland, 1951

46 K.E. Easterling, D.A. Porter, Phase Transformations in Metals and Alloys, 1981(1984), Van Nostrand Reinhold

47 D.A. Smith, Interface Science, Vol. 4, 11, 1996

48 T. Watanabe, Mechanical Properties and Deformation Behavior of Materials Having Ultra-Fine Microstructures; Porto Novo; Portugal; Pafe, 129, 28 June-10 July 1992

49 R.Z. Valiev, V.Yu. Gertsman, and O.A. Kaibyshev, Physica Status Solidi. A, 97, 11, 1986

50 A.A. Nazarove, A.E. Romanov, and R.Z. Valiev, Acta Metallurgica Materialia, Vol. 41, No. 4, 1033, 1993

51 M. Furukawa, Z. Horita, T.G. Langdon, M. Nemoto, D.J. Smith, and R.Z. Valiev, Journal of Materials Research, Vol. 11, No. 8, Aug 1996

52 R.Z. Valiev, R.K. Islamgaliev, and I.V. Alexandrov, Progress in Materials Science, Vol. 45, 103, 2000

53 R.Z. Valiev, and I.V. Alexandrov, Transactions of the Indian Institute for Metals, 58, 6, 1003, December, 2005

54 H. H. Imai, M.M. Matsumoto, T.T. Miyazaki, K.K. Kato, H.H. Tanida, and T.T Uruga, Chem. Commun. (Camb.), Vol. 47, Issue 12, 3538, March 28, 2011

55 Lucariello, Marialaura; Penazzi, Nerino: Department of Materials Science and Chemical Engineering, Politecnico di Torino; Arca, Elisabetta; Mulas, Gabriele; and Enzo, Stefano. Materials Chemistry and Physics, Vol.•114, Issue 1, 227-234, March 15, 2009

56 H. Gleiter, R. Birringer, H.P. Klein, and P. Marquardt, Physics Letters, Vol. 102A, No. 8, 365, 1984

57 C. Suryanarayana, Journal of Metals, Vol. 54, No. 9, 24, September 2002

58 J. Horvath, R. Birringer, and H. Gleiter, Solid State Communications, Vol. 62, No. 5, 319, 1987

59 S. Schumacher, R. Birringer, R. Straub, and H. Gleiter, Acta Metallurgica, Vol. 37, No. 9, 2485, 1989 
60 E. Hellstern, H.J. Fecht, C. Garland, and W.L. Johnson, Multicomponent Ultrafine Microstructures, Boston Massachusetts, Nov. 30 - Dec. 1, 137, 1988

61 R.H. Wagoner, Metallurgical and Materials Transactions, Vol. 12, No. 12, 2015, 1981

62 N. Hansen, Metallurgical and Materials Transactions, Vol. 32A, 12, 2917, December 2001

63 V.S. Ananthan, T. Leffers, and N. Hansen, Materials Science and Technology, Vol.7, Number 12, 1069, December 1991

64 J.A. Brusso, Substructure Development and Associated Strengthening in Mo-33Re Deformed by Shock Loading and Rolling, MS, Michigan Technological University, 1986

65 J.A. Brusso, R.N. Wright, and D.E. Mikkola, Materials Science and Engineering, Vol. A104, 85, 1988

66 M. Umemoto, Y. Todaka, and K. Tsuchiya, Ultra Fine Grain Materials III Symposium, Warrendale PA, 241, TMS 2004

67 X.Z. Liao, Y.H. Zhao, S.G. Srinivasan, F. Zhou, E.J. Lavernia, M.I. Baskes, H.F. Xu. R.Z. Valiev, and Y.T. Zhu, Ultra Fine Grain Materials III Symposium, Warrendale PA, 3, TMS 2004

68 M.P. Liu, H.J. Roven, T. Ungark, L. Balogh, M. Murashkin, and R.Z. Valiev, Materials Science Forum, Vols. 584$586,528,2008$

69 A.G. Truckner, An X-ray Diffraction Study of the Effects of Annealing on the Substructure of Cold Worked $\alpha$-CuGe, MS Michigan Technological University, 1966

70 D.A. Rigney, Annual Review of Materials Science, Vol. 18, 141, 1988

71 C.J. Youngdahl, J.R. Weertman, R.C. Hugo, and H.H. Kung, Scripta Materialia, Vol. 44, 1475, 2001

72 M. Ke, S.A. Hackney, W.W. Milligan, and E.C. Aifantis, Nanostructured Materials, Vol. 5, No. 6, 689, 1995

73 H. van Swygenhoven, M. Spaczer, and A. Caro, Acta Materialia, Vol. 47, No. 10, 3117, 1999

74 K. Kadau, T. Germann, P.S. Lomdahi, B.L. Holian, et al, Metallurgical and Materials Transactions, Vol. 35A, 9, 2719, Sep. 2004

75 J. Schietz, F.D. DiTolla, and K.W. Jacobson, Nature, Vol. 391, 561, February $5^{\text {th }}, 1998$

76 Z. Shan, E.A. Stach, J.M.K. Wiezorek, J.A. Knapp, D.M. Follstaedt, and S.X. Mao, Science, Vol. 305, No. 5684, 654, 30 July 2004

77 Milligan, W. W. "Mechanical behavior of bulk nanocrystalline and ultrafine-grain metals." Comprehensive Structural Integrity. Elsevier, Amsterdam 529 (2003)

78 M.A. Meyers, A. Mishra, and D.J. Benson, JOM, Vol. 58, No. 4, 41, April 2006

79 J.S. Benjamin, Metallurgical Transactions, Vol. 1, 2943, October, 1970

80 J.S. Benjamin, Materials Science Forum, Vols. 88-90, 1, 1992

81 C. Suryanarayana, Progress in Materials Science, Vol. 46, 1, 2001

82 M. R. Valezi, S. H. Mir Shah Ghassemi, and A. Shokuhfar, Materials Science-Poland, Vol. 26, No. 3, 2008

83 G. Kaupp, CrystEngComm, Vol. 5, 117, 2003

84 T. H. Courtney, and D. Maurice, Metallurgical and Materials Transactions, Vol. 27A, 1999, July 1996

85 M.N. Cisneros, H.F. López, H. Mancha, E. Rincó, D. Vázquez, M.J. Péze, and S.D. De La Torre, Metallurgical and Materials Transactions, Vol. 36A, Issue 5, 1309, May 2005

86 C.C. Koch, O.B. Cavin, C.G. Mckamey, and J.O. Scarbough, Appl. Phys. Lett. 43, 1017, December 1, 1983

87 Alan Arias, Chemical Reactions of Metal Powders with Organic and Inorganic Liquids during Ball Milling, NASA TN D-8015, NASA, Washington D.C., September 1975 
88 C. Suryanarayana, Mechanical Alloying and Milling, 1995

89 G.B. Schaffer, P.G. McCormick, Appl. Phys. Lett., 55, 45, 1989

90 G.B. Schaffer, P.G. McCormick, Metallurgical Transactions A, Vol. 21A, 2789, 1990

91 M.L. Luton, C.S. Jayanth, M.M. Disko, S. Matras, and J. Vallone, Mat. Res. Soc. Symp. Proc., 132, 79, 1989

92 J.D. Whittenberger, C.E. Lowell, A. Garg, and M.G. Hebsur, Mat. Res. Soc. Symp. Proc., Vol. 364, 579, 1995

93 J.D. Whittenberger, E. Arzt, and M.J. Luton, Mat. Soc. Symp. Proc., Vol. 194, 1990

94 J.D. Whittenberger, Structural Intermetallics, Conference Proceedings, The Minerals Metals \& Materials Society, 819, 1993

95 G. Jangg, New Materials by Mechanical Alloying, Ed. By A. Ayzi and L. Schultz, 39, 1988

96 S. Srinivasan, P.B. Desch, and R.B. Schwarz, Scripta Metallurgica, Vol. 25, 2513, 1991

97 K.Y. Lee, and J.H. Ahn, Materials Science and Engineering, A229, 63, 1997

98 M. Heilmaier, H. Saage, and J. Eckert, Materials Science and Engineering, A239-240, 652, 1997

99 P.S. Gilman, and J.S. Benjamin, Ann. Rew. Mater. Sci., 13, 279, 1983

100 T.H. Courtney, Materials Transactions, JIM, Vol. 36, No. 2, 110, 1995

101 T.H. Courtney, Reviews in Particulate Materials, Vol. 2, 63, 1994

102 G. Martin, and E. Gaffet, Journal de Physique, Vol. 51, C4-71, 1990

103 M.A. Shtremel, Metal Science and Heat Treatment, Vol. 44, No. 7-8, 324, 2002

104 L. Lü, M.O. Lai, Mechanical Alloying, Kluwer Academic Publishers, 1998

105 L. Lu, M.O. Lai, and S. Zhang, Journal of Materials Processing Technology, Vol. 67, 100, 1997

106 H. Gleiter, Acta Metallurgica, Vol.16, 455, 1968

107 E.W. Hart, Acta Metallurgica, Vol. 5, 597, 1957

108 A.K. Bhattacharya, and E. Arzt, Scripta Metallurgica, Vol. 28, 395, 1993

109 R.B. Schwarz, Materials Science Forum, Vols. 226-272, 665, 1998

110 Y. Estrin, and E. Rabkin, Scripta Materialia, Vol. 39, No. 12, 1731, 1998

111 A.L. Ruoff, and R.W. Balluffi, Journal of Applied Physics, Vol. 34, No. 7, 1848, July 1963

112 K.S. Kumar, M.S. Dipietro, and J.D. Whittenberger, Acta. Metall. Mater., Vol. 41, No. 5, 1979

113 M. Otsuki, and N.S. Stoloff, Scripta Metallurgica, Vol. 26, 325, 1992

114 D. K. Dewald, Private Communication, Waubik, Inc., Hancock MI

115 JCPDS-ICCD X-ray Powder Diffraction Data File sets 1-47

116 J.P. Nic, Structure/Property/Composition Relationships in Cubic Al 3 Ti-Base Intermetallic Alloys, MS Thesis, Michigan Technological University, 1992

117 B.D. Cullity, Elements of X-ray Diffraction, 1978

118 L. E. Alexander, H. P. Klug, X-ray Diffraction Procedures, 1974

119 J. Payne, VOLFRACT program-MY650 project, Michigan Technological University, 1992

120 D.T. Cromer, and J.B. Mann, Acta Cryst., A24, 321, 1968

121 P. Villars, and L.D. Calvert, Pearson's Handbook of Crystallographic Data for Intermetallic Phases, 1985 
122 N.F.M. Henry, K. Lonsdale, International Tables for X-ray Crystallography, 1952

123 J.P. Nic, Structure and Mechanical Behavior of (Al, Cr)3Ti Alloys, Ph.D. Dissertation, Michigan Technological University, 1994

124 S. B. Robie, Senior Applications Scientist, Scintag Inc., private communication

125 D.E. Mikkola, Private Communication, Professor Emeritus, Michigan Technological University

126 A. Guinier, X-ray Diffraction in Crystals, Imperfect Crystals, and Amorphous Bodies. 1963

127 B.E. Warren, Progress in Metal Physics, 8, 147, 1959

128 B.E. Warren, B.L. Averbach, Journal of Applied Physics, Vol. 23, Number 4, 497, 1952

129 B. E Warren, X-ray Diffraction, 1969

130 C.N.J. Wagner, Technical Report No.15 to Office of Naval Research Contract NONR 609 (43), Department of Engineering and Applied Science, Yale University, 1966

131 S. Enzo, S. Polizzi, and A. Benedetti, Zeitschrift für Kristallographie, R. Oldenbourg Verlag, München, 170, 275 287, 1985

132 S. Zhang, D.E. Mikkola, and W.W. Milligan, Scripta Metallurgica et Materialia, Vol. 27, Issue 8, 1073m October 1992

133 R.B. Schwartz, S. Srinivasan, P.B. Desch, Materials Science Forum, Vols. 88-90, 595, 1992

134 R.A. Varin, D. Wexler, A. Calka, and L. Zbroniec, Intermetallics, Vol. 6, 547, 1998

135 J. A. Brusso, Use of Short Duration Shock Pulses to Examine the Deformation Behavior of 21-6-9 Stainless Steel and the "Boron Effect" in Ni3Al, Ph.D. Dissertation, Michigan Technological University, 1991

136 M. Mhadhbi, M. Khitouni, L. Escoda, J.J. Suñol, and M. Dammak, Journal of Nanomaterials, Article ID. 712407, 1, Vol. 2010

137 E.A. Laitila, M. Woodford, Optimizing Image J Determinations for SEM Particle Size Measurements Research and Engineering Program, Academy of Applied Science, Grant Number 11-39, MTU, 2011

138 N.V. Tzenov, and M.W. Barsoum, Journal of the American Ceramic Society, Vol. 83 No. 4, 825, 2000

139 V. Raghavan, Journal of Phase Equilibria and Diffusion, Vol. 27, No. 2, 148, 2006

140 J. J. Le Claire, Michigan Technological University, Ph.D. Dissertation, 2002

141 D. Hull and D.J.Bacon, Introduction to Dislocations, Pergamon Press, 1984

142 J.J. Le Claire, Michigan Technological University, M.S. Thesis, 1998

143 Y.C. Zhou, and X.H. Wang, Materials Research Innovations, Vol. 5, Number 2, 87, October 2001

144 Y.C. Zhou, and X.H. Wang, Materials Research Innovations, Vol. 5, 87, 2001

145 K.K. Chawla, Composite Materials, Springer-Verlag New York, Inc., 1987

146 W.D. Callister, Jr., and D.G. Rethwisch, Fundamentals of Materials Science and Engineering an Integrated Approach, John Wiley \& Sons, 2008

147 Bingchu Mei, Xuewen Xu, Jiaoqun Liu, and Jun Liu, Xiyou Jinshu Cailiao yu Gongcheng (Rare Metal Materials and Engineering), Vol. 34, Issue 5, 684, May 2005

148 M. Radovic, M.W. Barsoum, A. Ganguly, T. Zhen, P. Finkel, S.R. Kalidindi, and L. Lara-Curzio, Acta Materialia, Vol. 54, 2757, 2006

149 D. Dewald, M. Austin, D.E. Mikkola, In: Dahotre NB, Hampikian JM, editors. Proceedings of the $12^{\text {th }}$ International Conference on Surface Modification Technologies. Materials Park, Oh, ASM International; 7, 1999 
150 D. Dewald, M. Austin, E. Laitila, D. Mikkola, Journal of Thermal Spray Technology, 10, 111, 2001

151 J.W. Byeon, J. Liu, M. Hopkins, W. Fischer, N. Garimella, K.B. Park, M.P. Brady, M. Radovic, T. El-Raghy, and Y. H. Sohn, Oxidation of Metals, Vol. 68, Numbers 1-2, 97, August 2007

152 http://www.its.caltech.edu/ feynman/plenty.html

153 http://www-03.ibm.com/press/us/en/pressrelease/40970.wss\#release, IBM press release May 1, 2013

154 J.A. Eastman, and L.J. Thompson, Mat. Res. Soc. Symp. Proc., Vol. 153, 27, 1989

155 R.W. Siegel, and G.J. Thomas, Ultramicroscopy, Vol. 40, 376, 1992

156 T. Egami, and S.J.L. Billinge, Underneath the Bragg Peaks: Structural Analysis of Complex Materials, Pergamon Press, 2003

157 A.J.C. Wilson, Elements of X-ray Crystallography, Addison Wesley, 1970

158 W.A. Wooster, Diffuse X-ray Reflections from Crystals, Oxford University Press, 1962

159 X. Zhu, R. Birringer, U. Herr, and H. Gleiter, Physical Review B, Vol. 35, No. 17, 9085, 1987

160 T. Haubold, R. Birringer, B. Lengeler, and H. Gleiter, Physics Letters A, Vol. 35, No. 8-9, 461, 1989

161 J.A. Eastman, and L.J. Thompson, Mat. Res. Soc. Symp. Proc., Vol. 153, 27, 1989

162 E.A. Laitila, D.E. Mikkola, Employing X-ray scattering to characterize materials with grain sizes in the nanoregime, Powder Diffraction, Vol. 23, Issue 2, June 2008

163 W. Ruland, Acta Crystallographica, 14, 1180, 1961

164 C.J. Quinn, and D.L. Kohlstedt, Journal of American Ceramic Society, Vol. 67, Issue 5, 305, 1984

165 J. Jung. And S. Kang, Powder Metallurgy, Vol. 47, No. 1, 93, March 2004

166 D. Turnbull, and R.E. Cech, Journal of Applied Physics, Vol. 21, 804, August 1950

167 H.J. Fecht, E. Hellstern, Z. Fu and W.L. Johnson, Metallurgical Transactions A, Vol. 21A, 2333, 1990

168 J. Eckert, J.C. Holzer, C.E. Krill, and W.L. Johnson, Journal of Materials Research, Vol. 7, No. 7, July 1992

169 E. Hellstern, H.J. Fecht, Z. Fu, and W.L. Johnson, Journal of Applied Physics, Vol. 65, No. 1, 305, 1989

170 C.C. Koch, J.S.C. Jang, P.Y. Lee, New Materials by Mechanical Alloying Techniques, E. Arzt and L. Schultz, editors, 101, Oberursel : DGM Informationsgesellschaft, 1989

171 M.A. Bernier, D.C Joy, and M. Schmidt, Mechanical Alloying for Structural Applications, Vancover, British Columbia, Canada, Sep 20, 1993

172 S.S. Nayak, and B.S. Murty, Materials Science and Engineering, Vol. 367 A, 218, 2004

173 W.W. Milligan, S.A. Hackney, M. Ke, and E.C. Aifantis, NanoStructured Materials, Vol. 2, 267, 1993

174 P. Keblinski, S.R. Phillpot, D. Wolf, and H. Gleiter, Acta Mater., Vol. 45, No. 3, 987, 1997

175 B. Fultz, and H.N. Frase, Hypefine Interactions, Vol. 130, 81, 2000

176 R.Z. Valiev, and I.V. Alexandrov, Materials Science Forum, Vols. 321-324, 577, 2000

177 S. Ranganathan, R. Divakar, and V.S. Raghunathan, Scripta Materialia, Vol. 44, 1169, 2001

178 H. Gleiter, Acta Materialia, Vol. 48, 1, 2000

179 V. Yamakov, D. Wolf, S.R. Phillpot, A.K. Mukherjee, and H. Gleiter, Nature Materials, Vol. 3, 43, January 2004

180 D.Wolf, V. Yamakov, S.R. Phillpot, A. Mukherjee, and H. Gleiter, Acta Materialia, Vol. 53, 1, 2005

181 W. Rosenhain, and J.C.W. Humfrey, Journal of the Iron and Steel Institute, Vol. 87, 219, 1913 
182 W. Rosenhain, and D. Ewen, Journal of the Institute of Metals, Vol. 10, 119, 1913

183 J.Y. Huang, A.Q. He, Y.K. Wu, H.Q. Ye, and D.X. Li, Journal of Materials Science, 31, 4165, 1996

184 J.Y. Huang, X.Z. Liao, Y.T. Zhu, F. Zhou, and E.J. Lavernia, Ultra Fine Grain Materials III Symposium, Warrendale PA, 17, TMS 2004

185 X. Savage, G. Wilde, S. Divinsky, Z. Horita, and R.Z. Valiev, Materials Science and Engineering A, Vol. 540, 1, 2012

186 H. Van Swygenhoven, D. Farkas, and A. Caro, Physical Review B, Vol. 62, No.2, 831, July 2000

187 H. Van Swygenhoven, P.M. Derlet, A.G. Frøseth, S. Van Petegem, Z. Budrovic, and A. Hasnaoui, Materials Research Society Symposium Proceeding, Materials Research Society, Vol. 821, 285, 2004

188 A. Hasnaoui, H. Van Swygenhoven, and P.M. Derlet, Acta Materialia, Vol. 50, 3927, 2002

189 R.J. Tucker, and D.L. McDowell, International Journal of Plasticity, Vol. 27, 841, 2011

190 R.Z. Valiev, V.Yu. Gertsman, and O.A. Kaibyshev, Physica Status Solidi. A, 97, 11, 1986

191 I.V. Alexandrov, A.R. Kilmametov, K. Lu, R.Z. Valiev, and K. Zhang, J. Phys.D: Appl. Phys. 30, 3008, 1997

192 I.V. Alexandrov, and R.Z. Valiev, Materials Science Forum, Vols. 321-324, 577, 2000

193 R.Z. Valiev, R.K. Islamgaliev, and I.V. Alexandrov, Progress in Material Science, 45, 103, 2000

194 R.W. Balluffi, G.R. Woolhouse, and Y. Komem, The Nature and Behavior of Grain Boundaries,. Symposium, TME-AIME, 41, October 18-19, 1971

195 J.W. Wyrzykowski, and M.W. Grabski, Philosophical Magazine A, Vol. 53, No. 4, 505, 1986

196 E. A. Laitila, M.S. Keefer, Chemistry Changes to RMM Processing of L12 Intermetallics, Research and Engineering Program, Academy of Applied Science, Grant Number 14-21, MTU, 2014

197 I. Kkaur, and W. Gust, Fundamentals of Grain and Interphase Boundary Diffusion, Ziegler Press, Stuttgart 1 West Germany, 1989

198 H.V. Atkinson, and B.A. Rickinson, Hot Isostatic Pressing, J. Wood (ed.), The Adam Hilger Series on New Manufacturing Processes and Materials, Adam Hilger, Bristol, Philadelphia and New York, 34, 1991

199 L. Lu, M.O. Lai, and S. Zhang, Journal of Materials Processing Technology, Vol. 67, 100, 1997

200 R.Z. Valiev, A.I. Pshenichnyuk, and A.A. Nazarov, Engineering Materials, Vols. 97-98, 59, 1995

201 Yu.R. Kolobov, G.P. Grabovetskaya, I.V. Ratochka, E.V. Kabanova, E.V. Naidenkin, and R.C. Lowe, Annales de Chimie Science des Materiaux, Vol. 21, Nos. 6-7, 483, 1996

202 A.G. Kesarev, V.V. Kondrat'ev, and I.L. Lomaev, The Physics of Metals and Metallography, Vol. 109, No. 4, 329 , 2010

203 C.C. Koch, and K. Yamada, Proceedings of the $2^{\text {nd }}$ International Conference on Structural Applications of Mechanical Alloying, Vancouver, British Columbia, Canada, 20, September 1993

204 Y. Minamino, S. Saji, K. Hirao, K. Ogawa, H. Araki, Y. Miyamoto, and T. Yamane, Materials Transactions, JIM, Vol. 37, No. 2, 130, 1996

205 B. Gilbert, F. Huang, H. Zhang, G. A. Waychunas, and J.F. Banfield, Science, Vol. 305, 5684, 651, 30 July 2004

206 V. Yamakov, D. Wolf, S.R. Phillpot, A.K. Mukherjee, and H. Gleiter, Philosophical Magazine Letters, Vol. 83, No. 6, 385, 2003

207 V. Yamakov, D. Wolf, S.R. Phillpot, A.K. Mukherjee, and H. Gleiter, Philosophical Magazine Letters, Vol. 83, No. 6, 385, 2003

208 I.M. Lifshitz, Soviet Physics, JETP, Vol. 17, 909, 1963 
209 A.A. Nazarov, A.E. Romanov, and R.Z. Valiev, Acta Metall. Mater., Vol. 41, No. 4, 1033, 1993

210 W. Boas, and M.E. Hargreaves, Proceedings of the Royal Society of London, Vol. A193, 89, 1948, V.M. Urie and H.L. Wain, Journal of Institute of Metals, Vol. 81, 153, 1952

211 E.V. Kozlov, N.A. Koneva, N.A. Popova, and A.N. Zhdanov, Russian Metallurgy, Vol. 2010, No. 10, 867, 2010

212 A.S. Mohammadabadi, and K. Dehghani, Journal of Materials Engineering and Performance, Vol. 17, Issue 5, 662, October, 2008

213 R. Monzen, and N. Takada, Materials Science and Engineering, Vol. A234-236, 202, August 301997

214 D.V. Bachurin, A.A. Nazarov, O.A. Shendrova, and D.W. Brenner, Materials Science and Engineering, Vol. A359, 247, 2003

215 R.B. Schwarz, Materials Science Forum, Vols. 226-272, 665, 1998

216 T.H. Courtney, Reviews in Particulate Materials, Vol. 2, 63, 1994

217 D.R. Maurice, Local Modeling of Mechanical Alloying, Ph.D, Michigan Technological University, 1992

218 H. J. Fecht, Nanostructure Materials, Vol. 6, 33, 1995

219 J.S. Benjamin, and T.E. Volin, Metallurgical Transactions, Vol. 5, 1929, August 1974

220 A. Belyakov, T. Sakai, H. Miura, and K. Tsuzaki, Philosophical Magazine A, Vol. 81, No. 11, 2629, 2001

221 E.D. Doyle, and R.L. Aghan, Metallurgical Transactions B, Vol. 6B, 143, March 1975

222 K. Wang, N.R. Tao, G. Liu, J. Lu, and K. Lu, Acta Materialia, Vol. 54, 5281, 2006

223 A. Kelly, Strong Solids, Oxford, Calrendon Press, 1966

224 M. Mhadhbi, M. Khitouni, L. Escoda, J.J. Suñol, and M. Dammak, Journal of Nanomaterials, Vol. 2010, Article ID $712407,1,2010$

225 J. Zhu, and M. Mujahid, Materials Science and Technology, Vol. 21, No. 8, 925, August 2005

226 Y. Lu, J.Y. Huang, C. Wang, S. Sun, and J. Lou, Nature Nanotechnology, Vol. 5, 281, March 2010

227 N. Bay, Metal Construction, Vol. 18, No. 6, 369, 1986

228 D.A. Rigney, L.H. Chen, M.G.S. Naylor, and A.R. Rosenfield, Wear, Vol. 100, 195, 1984

229229 P. Heilmann, J. Don, T.C. Sun, D.A. Rigney, Wear, vol. 91, 171, 1983

230 P. Heilmann, W.A.T. Clark, and D.A. Rigney, Acta Metallurgica, Vol. 31, No. 8, 1293, 1983

231 G.B. Schaffer, and P.G. McCormick, Materials Science Forum, Vols. 88-90, 779, 1992

232 N.J. Calos, J.S. Forrester, and G.B. Schaffer, Journal of Solid State Chemistry, Vol. 122, Article No. 133, 273, 1996

233 A.N. Streletskii, I.V. Kolbanev, D.G. Permenov, I.V. Povstugar, A.B. Borunova, A.Yu. Dolgoborodov, M.N. Makhov, and P.Yu. Butyagin, Reviews on Advanced Materials Science, Vol. 18, 353, 2008

234 D. Osso, O. Tillement, A. Mocelling,G. Le Caer, O. Babushkin, and T. Lindbäck, Journal of the European Ceramic Society, Vol. 15, 1207, 1995

235 Y. Chen, A. Calka, and J.S. Williams, Materials Science and Engineering, Vol. A187, 51, 1994

236 T. Itsukaichi, K. Masuyama, M. Umemoto, I. Okane, and J.G. Cabanas-Moreno, J. Mater. Res., Vol. 8, No. 8, 1817, 1993

237 G. Kaupp, CrystEngComm, Vol. 5, 117, 2003

238 S. J. Gregg, and K.S. Sing, Adsorption, Surface Area and Porosity, Academic Press, 1, 1967 
239 C.R. Copeland, Suppression and Dispersion of Airborne Dust and Nanoparticles, Ph.D, Michigan Technological University, 2007

240 L. Liu, L. Lu, L. Chen, Y. Qin, and L.D. Zhang, Metallurgical and Materials Transactions, Vol. 30A, No. 4, April 1999

241 I.M. Campbell, Catalysis at Surfaces, Chapman and Hall, New York NY, 1988

242 E. A. Laitila, H. Aho, Hydrogen Storage in Nickel Powders by Reactive Mechanical Milling, Research and Engineering Program, Academy of Applied Science, Grant Number 03-115, MTU, 2003

243 I. Chorkdendorff, J.W. Niemantsverdriest, Concepts of Modern Catalysis and Kinetics, Wiley-VCH Verdag GmbH \& Co. KGaA, Weinheim, 2003

244 S. Dahl, A. Logadotii, R. Egberg, J.H. Larsen, I. Chorkendorff, E. Törnquist, and J.K. Nørskov, Physics Review Letters, Vol. 83, 1814, 1999

245 A.V. Ruban, B. Hammer, P. Stoltze. K.W. Jacobsen, H.L. Shriver, and J. K. Nørskov, Journal Of Molecular Catalysis, Vol. A115, 421, 1997

246 E. A. Laitila, D. J. Isaacson, Reactive Mechanical Alloying of Pure Iron Powders, Research and Engineering Program, Academy of Applied Science, Grant Number 05-56, MTU, 2005

247 J.R. Greer, J. D. Jang, and X. W. Gu, JOM, Vol. 64, No. 10, 1241, 2012

248 D. Dewald, M. Austin, E.A. Laitila, and D.E. Mikkola, Journal of Thermal Spray Technology, Vol. 10, No. 1, 111, 2001

249 X.H. Wang, and Y.C. Zhou, Oxidation of Metals, Vol. 59, Nos. 3/4, 303, April 2003

250 X.H. Wang, and Y.C. Zhou, Corrosion Science, Vol. 45, 891, 2003

251 J. Wang, and Y. Zhou, Annual Review of Materials Research, Vol. 39,415, 2009

252 Shibo Guo, Qiping Kang, Jun Liu, and Xuanhui Qu, Rare Metals, Vol. 29, No. 4, 376, August 2010

253 M.A. Pietzka, and J.C. Schuster, Journal of Phase Equilibria, Vol. 15, No. 4, 392, August, 1994

254 H.E. Pettermann, H.J. Bohm, and J. Alcala, Metallurgical and Materials Transactions, Vol. 33A, No. 10, 3187, October 2002

255 G.N. Greaves, A.L. Green, R.S. Lakes, and R. Rouxel, Nature Materials, Review Article, Vol. 10, 823, November 2011

256 A. Yeganeh-Haeri, D.J. Weidner, and J.B. Parise, Science, Vol. 257, 650, 31 July 1992

257 F. Ren, E.D. Case, J.R. Sootsman, M.G. Kanatzidis, H. Kong, C. Uher, E. Lara-Curzio, and R.M. Trejo, Acta Materialia, Vol. 56, 5954, 2008

258 Anonymous, Adjustable CTE Value of Metal Matrix Composite, Composite Manufacturing, Vol. 20, No, 4, 14, Fourth Quarter 2004

259 Li-Hui Tian, Cheng-Xin Li, chang-Jiu Li, and Guan-Jun Yang, Journal of Thermal Spray Technology, Vol. 21, No. 3-4, 689, June 2012

260 M.W. Barsoum, M. Ali, and R. El-Raghy, Metallurgical and Materials Transactions, Vol.31 A, No. 7, 1857, 2000

261 S.S. Brenner, Journal of Applied Physics, Vol. 27, No. 12, 1484, December 1956

262 R.J. Arsenault, Metal Matrix Composites: Mechanisms and Properties, Editors R.K. Everett, R.J. Arsenault, Academic Press Inc., San Diego CA, 79, 1991

263 M.W. Barsoum, Progress in Solid State Chemistry, Vol. 28, 201, 2000

264 X.H. Wang, and Y.C. Zhou, Acta Materialia, Vol. 50, 3141, 2002 
265 K.K. Chawla, Composite Materials Science and Engineering, Springer-Verlag Inc., New York, 1987

266 Y.C. Zhou, and X.H. Wang, Materials Research Innovations, Vol. 5, 87, 2001

267 X.H. Wang, and Y.C. Zhou, Acta Materialia, Vol. 50, 3141, 2002

268 N. Hansen, Acta Metallurgica, Vol. 25, 863, 1977

269 P.D. Funkenbusch, and T.H. Courtney, Acta Metallurgica, Vol. 33, No. 5, 913, 1985

270 E.A. Laitila, David Szyszkoski, Copper TiC Conductors by Reactive Mechanical Milling, Research and Engineering Program, Acadamey of Applied Science, Grant Number 08-28, MTU, 1998

271 B.R. Murphy, and T.H. Courtney, Journal of Materials Research, Vol. 14, No. 11, 4274, November 1999

272 R. Monzen, and N. Takada, Materials Science and Engineering, Vol. A234-236, 202, August 301997

273 J. Pilling, and N. Ridly, Superplasticity in Crystalline Solids, The Institute of Metals, London, England, 1989

274 T.G. Langdon, Materials Science and Engineering, Vol. A166, 67, 1993

275 R. Raj, and M.F. Ashby, Metallurgical Transactions, Vol. 2, 1113, 1971

276 R.W. Hayes, David Witkin, Fei Zhou, and E.J. Lavernia, Acta Materialia, Vol. 52, 4259, 2004

277 R.Z. Valiev, R.K. Islamgaliev, and N.F. Yunusova, Metal Science and Heat Treatment, Vol. 48, Nos. 1-2, 47, 2006

278 T. Watanabe, Materials Science Forum, Vols. 304-306, 421, 1999

279 R.I. Todd, Superplasticity, 60 Years after Pearson, Proceedings of the Conference organized on Behalf of the Superplastic Forming Committee of the Manufacturing Division of the Institute of Materials, Minerals, and Mining, Ed. N. Ridley, 1994

280 R.I. Todd, Superplasticity and Superplastic Forming 1998, San Antonio Texas, The Minerals Metals and Materials Society, 13, February 1998

281 R.I. Todd, Materials Science and Technology, Vol. 16, Nos. 11-12, 1287, November/December 2000

282 L.D. Hefti, JOM, Vol. 62, No. 5, 42, May 2010

283 O.A. Kaibyshev, R.V. Safiullin, R. Ya. Lutfullin, O.R. Raliakhmetov, R.M. Galeyev, A. Dutta, T. Raghu, and G.G. Saha, Materials Science and Technology, Vol. 22, No. 3, 22, March 2006

284 R.S. Mishra, JOM, Vol. 53, No. 3, 23, March 2001

285 R.Z. Valiev, A.V. Korznikov, and R.R. Mulyukov, Material Science and Engineering, Vol. A168, 141, 1993

286 R.S. Mishra et al., International Symposium Gamma Titanium Aluminides, ed. Y.W. Kim, R. Wagnor, and M. Yamuguchi, 57, Warrendale PA: TIMS, 1995

287 M.M. Myshlyaev, M. Yu. Gryaznov, and V.N. Chuvildeev, Russian Metallurgy, Vol. 2011, No. 9, 882, 2011

288 R.S. Mishra, R.Z. Valiev, S.X. McFaddenm, R.K. Islamgaliev, and, A.K. Mukherjee, Philosophical Magazine A, Vol. 81, No. 1, 25, 2001

289 R.Z. Valiev, C. Song, S.X. McFaddenm, A.K. Mukherjee, and R.S. Mishra, Philosophical Magazine A, Vol. 81, No. 1, 25, 2001

290 S.X. McFaddenm, R.S. Mishra, R.Z. Valiev, A.P. Zhilyaev, and A.K. Mukherjee, Letters to Nature, Vol. 398, 684, April 22, 1999 